\title{
WestVirginiaUniversity
}

THE RESEARCH REPOSITORY @ WVU

Graduate Theses, Dissertations, and Problem Reports

2006

\section{Effects of the electric Kelvin force on spray cooling performance}

Shannon Lynnette Glaspell

West Virginia University

Follow this and additional works at: https://researchrepository.wvu.edu/etd

\section{Recommended Citation}

Glaspell, Shannon Lynnette, "Effects of the electric Kelvin force on spray cooling performance" (2006). Graduate Theses, Dissertations, and Problem Reports. 1729.

https://researchrepository.wvu.edu/etd/1729

This Thesis is protected by copyright and/or related rights. It has been brought to you by the The Research Repository @ WVU with permission from the rights-holder(s). You are free to use this Thesis in any way that is permitted by the copyright and related rights legislation that applies to your use. For other uses you must obtain permission from the rights-holder(s) directly, unless additional rights are indicated by a Creative Commons license in the record and/ or on the work itself. This Thesis has been accepted for inclusion in WVU Graduate Theses, Dissertations, and Problem Reports collection by an authorized administrator of The Research Repository @ WVU. For more information, please contact researchrepository@mail.wvu.edu. 


\title{
Effects of the Electric Kelvin Force on Spray Cooling Performance
}

\author{
Shannon Lynnette Glaspell \\ Thesis submitted to the \\ College of Engineering and Mineral Resources \\ at West Virginia University \\ in partial fulfillment of the requirements \\ for the degree of \\ Master of Science \\ in \\ Aerospace Engineering
}

\author{
Committee: \\ John M. Kuhlman, Ph.D., Chair \\ Donald D. Gray, Ph. D. \\ Wade Huebsch, Ph. D. \\ Gary Morris, Ph. D. \\ Kirk Yerkes, Ph. D. \\ Department of Mechanical and Aerospace Engineering \\ Morgantown, West Virginia \\ 2006
}

Keywords: Spray Cooling, Electric Kelvin Force, Electrohydrodynamics, EHD 


\section{Abstract \\ Effects of the Electric Kelvin Force on Spray Cooling Performance \\ Shannon Lynnette Glaspell}

Spray cooling is an efficient thermal management technique that may possibly be improved by employing an electric Kelvin force to control the fluid motion and delay the onset of critical heat flux. In the present study, a spray cooling apparatus consisting of a spray chamber (housing a spray nozzle and electrically heated surface) and a flow generating base package has been built and tested for varying nozzle-to-heater distances and also varying electrode geometries for a range of spray conditions and electrode voltages. The two part goal was to first determine the qualitative sensitivity of the distance between the heated surface and nozzle exit, and second, to determine the magnitude of the effects of the electric Kelvin force on the performance of the spray cooling apparatus for the various electrode geometries.

An experimental nozzle-to-heater spacing comparison performance test indicated that confined flows provide less efficient cooling than unconfined flows but are less dependent on the nozzle-to-heater distance. An experimental and numerical study of four cap electrode designs showed no significant effect of the electric Kelvin force on spray cooling performance. This may have been due to the non-optimal electrode geometries and also possibly due to the properties of the FC-72 fluid used. A redesigned electrode configuration that has been designed using the electric field module of the multiphysics code CFD ACE+ and the use of a different fluid, HFE-7000, showed a cooling effect of the heated surface as well as an increase of approximately $5 \%$ in the convection heat transfer coefficient. 


\section{Table of Contents:}

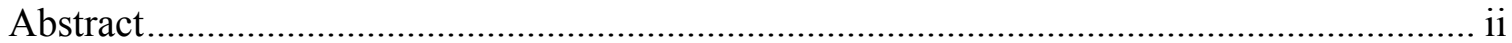

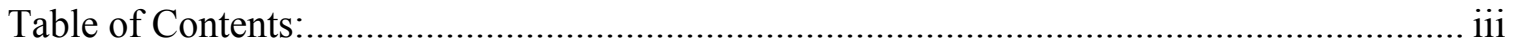

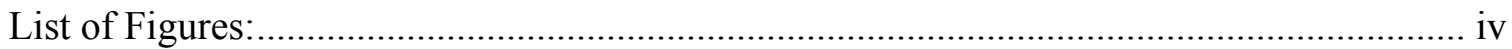

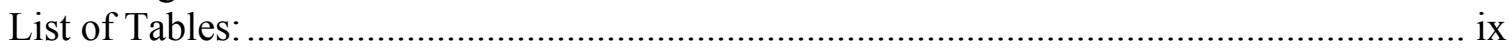

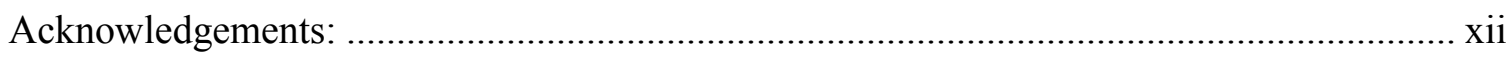

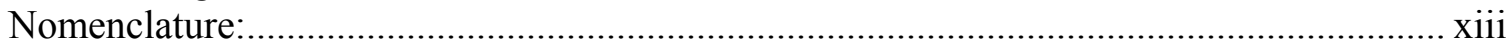

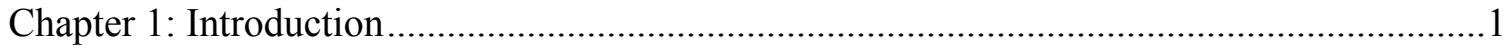

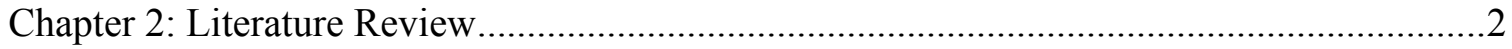

2.1 Importance of Advancing Thermal Management Techniques...............................

2.2 Comparison of Thermal Management Techniques ................................................

2.3 The Physics of Spray Cooling............................................................................ 7

2.4 The Parameters that Affect Spray Cooling ........................................................... 15

2.5 Numerical Simulation Studies of Spray Cooling .................................................22

2.6 The Physics and Parameters that Affect Electrohydrodynamics ...........................24

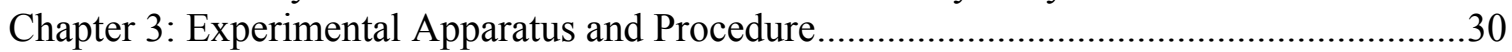

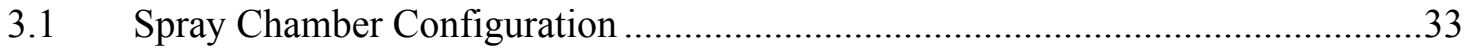

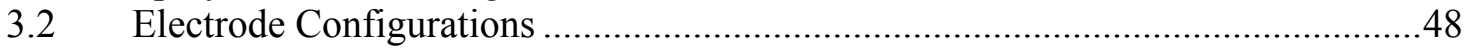

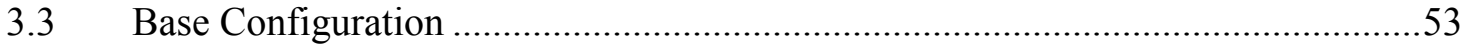

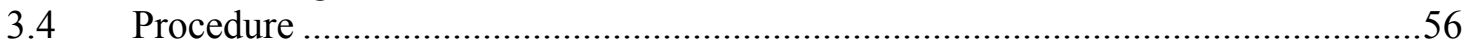

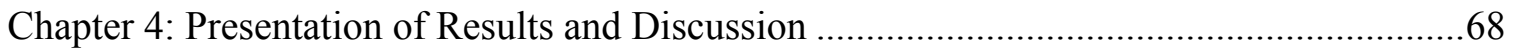

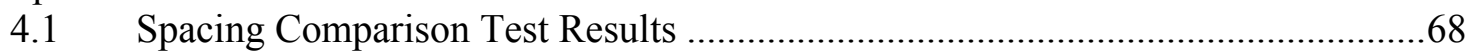

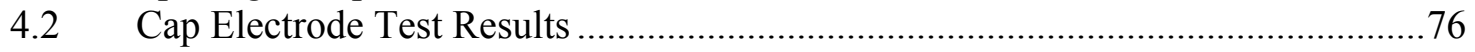

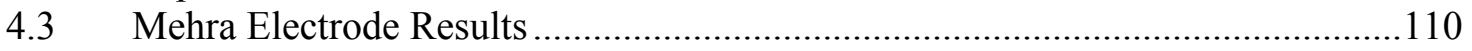

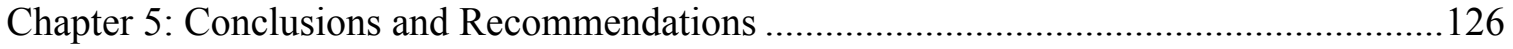

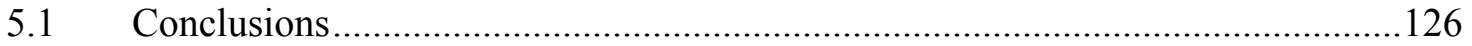

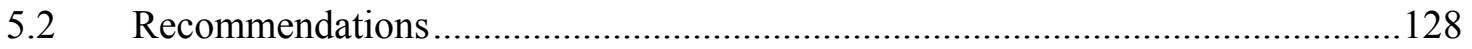

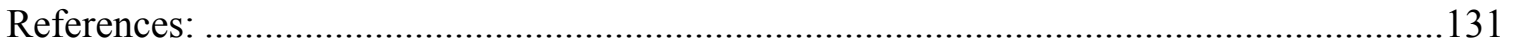

Appendix A: Thick Film Resistor (TFR) Composition and Layer Thickness Study using

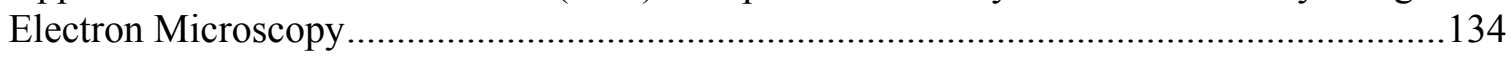

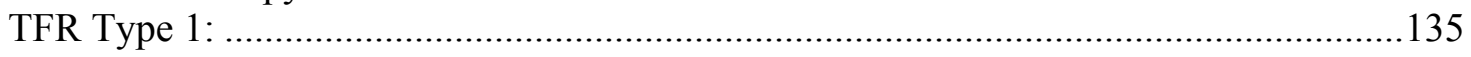

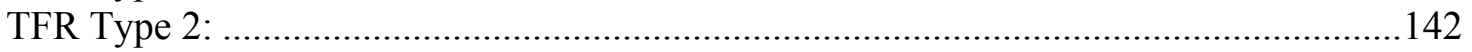

Appendix B: Test Plans for Spacing Comparison and Electrode Performance Studies ........148

Appendix C: Derivation and Solutions of Energy Equations for the TFR Pedestals ............151

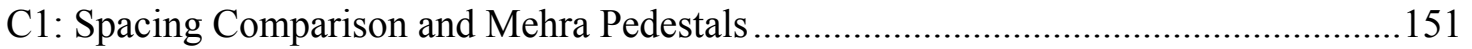

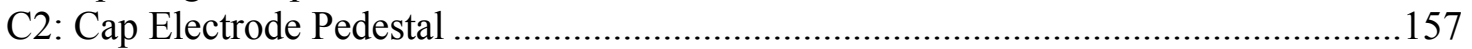

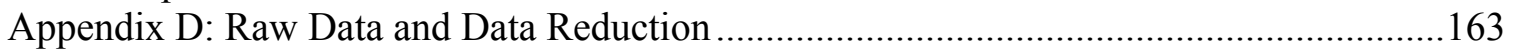

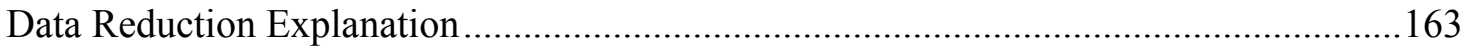

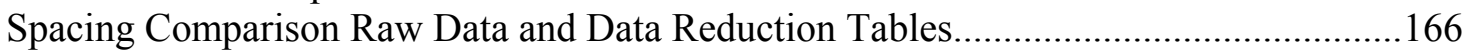

Cap Electrode Averaged Raw Data and Data Reduction Tables....................................184

Mehra Electrode Averaged Raw Data and Data Reduction Tables..................................208

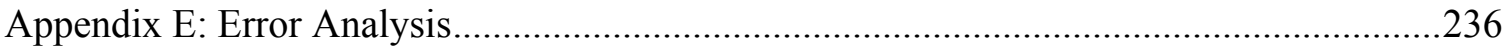

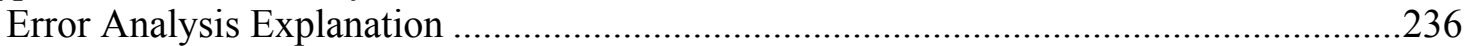

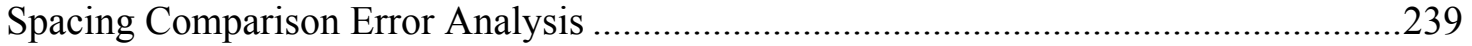

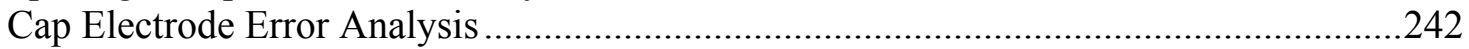

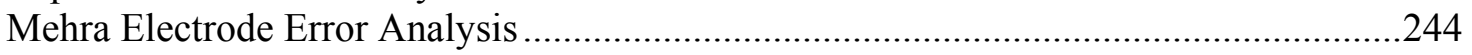




\section{List of Figures:}

Figure 2.1: Comparison of Thermal Management Techniques Using Various Fluids (Mudawar, 2000)6 Figure 2.2:Typical Spray Cooling Heat Transfer Curve Showing 3 Regions (redrawn from Yang, $1993)$

Figure 2.3: Photograph of Ridges Present on the Film Surface (Yang, 1993) ...................................... 9

Figure 2.4: Photograph of Vapor Bubbles in the Film Surface (Yang, 1993)...................................... 9

Figure 2.5: Heat Transfer Mechanisms in Spray Cooling (Chow et al., 1997).................................. 10

Figure 2.6: High Speed Video Images and Schematic of the Evolution of New Bubbles from the

Rupture of a Previous Bubble (Mesler and Mailen, 1977)............................................................... 11

Figure 2.7: Photograph of CHF on the Spray Cooling Surface (Yang, 1993) .................................... 12

Figure 2.8: Forced Convection Boiling at High Heat Fluxes with Entrainment and Deposition of

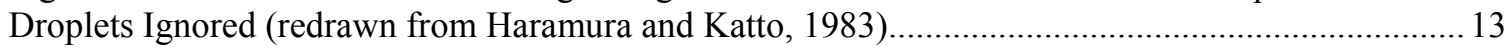

Figure 2.9: Macrolayer Dry-out Model for Spray Cooling CHF (Chow et al., 1997) .......................... 14

Figure 2.10: Dryout Beginning at the Edges and Receding Inward (Yang, 1993).............................. 14

Figure 2.11: Comparison of Air, Fluorochemical Liquids, and Water for Natural Convection, Forced

Convection, and Boiling (redrawn from Mudawar, 2000)........................................................... 16

Figure 2.12: Comparison of a) Low Density, Low Weber Number and b) High Density, High Weber

Number Sprays (Estes and Mudawar, 1995).................................................................................. 18

Figure 2.13: Effects of Surface Roughness at the Same Flow Conditions (redrawn from Tilton, 1989)

214: Effects of Surface Roughnes (Sehmbey et al, 195)

Figure 2.14: Effects of Surface Roughness (Sehmbey et al., 1995) ............................................... 20

Figure 2.15: Heat Flux as a Function of Surface Temperature for (A) Embedded Structures and (B)

Extended Surfaces in Degassed Conditions (Silk et al., 2006) ......................................................... 21

Figure 2.16: Spray Profiles at a) 0.66, b) 0.99, and c) $1.32 \mathrm{~ms}$ After Start of Injection with the Liquid

Phase (Dark Areas) Superimposed onto the Vapor Phase (Grover and Dennis, 2001)....................... 23

Figure 2.17: Vapor Patterns under the Influence of Different Gravitational and Electric Fields (Marco

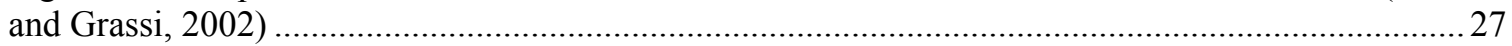

Figure 2.18: Vapor Bubble Behavior under the Influence of Different Gravitational and Electric Fields

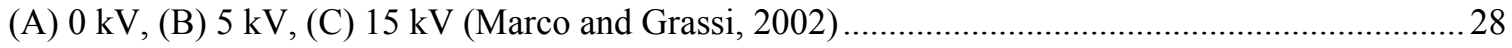

Figure 2.19: Vapor Bubble Behavior in Microgravity under the Influence of (A) No Magnetic Kelvin

Force and (B) the Magnetic Kelvin Force (approximately $20 \mu \mathrm{N}$ ) (Kuhlman et al., 2005)................. 28

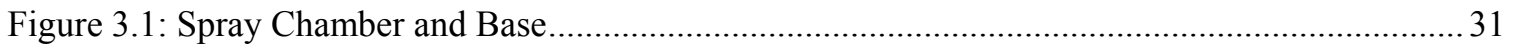

Figure 3.2: Schematic of Apparatus Flow Loop (modified from Kreitzer, 2006) ................................ 32

Figure 3.3: Main Spray Chamber Configuration (Dimensions in Inches) .......................................... 34

Figure 3.4 Chamber Side Views Showing A) Nozzle Assembly and Plug, B) Pressure Transducer and

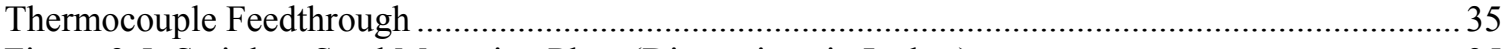

Figure 3.5: Stainless Steel Mounting Plate (Dimensions in Inches) ................................................... 35

Figure 3.6: Spray Chamber with Mounting Plates and Aluminum Frame............................................. 36

Figure 3.7: Spray Chamber with Copper Water Cooling Loop Attached .............................................. 37

Figure 3.8: Insulator Seal High Voltage Feedthrough with Pin Connector ........................................... 37

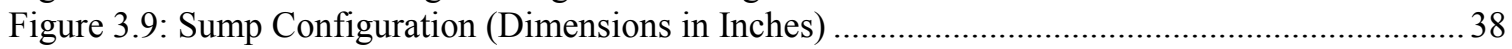

Figure 3.10: Polycarbonate and Aluminum Sump with Pipe Fittings Attached ................................... 39

Figure 3.11: Spacing Comparison Pedestal Assembly with Unattached TFR ...................................... 39

Figure 3.12: Cap Electrode Pedestal (Dimensions in Inches) .......................................................... 40

Figure 3.13: Cap Electrode Pedestal Thermocouple Locations (Hunnell, 2005 and Courtesy of Mr.

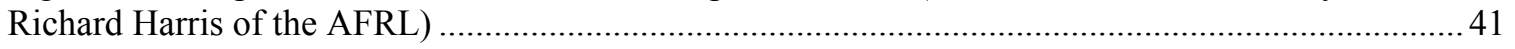

Figure 3.14: Cap Electrode Pedestal Top Showing Attached Wafers and Thermocouple Labels ........ 42

Figure 3.15: Cap Electrode Pedestal Assembly ................................................................................. 42

Figure 3.16: Backup Cap Electrode Pedestal Thermocouple Locations .............................................. 43

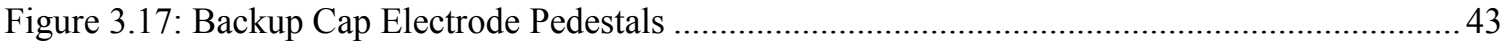

Figure 3.18: Top of Mehra Electrode Pedestal without $2 \mathrm{~mm}$ Alumina Layer ................................... 45 


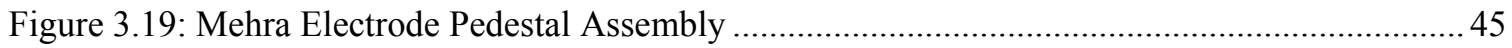

Figure 3.20: Approximate Thermocouple Locations within the Chamber...........................................46

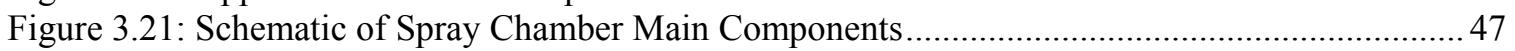

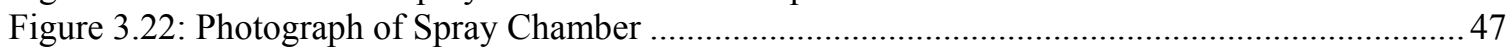

Figure 3.23: ProE Renderings of Four Cap Electrode Designs.......................................................... 48

Figure 3.24: Photographs of Sides and Bottoms of Four Cap Electrode Designs with Pin Connectors 48

Figure 3.25: Cap Design from Baysinger, 2004 (Dimensions in Inches) ............................................ 49

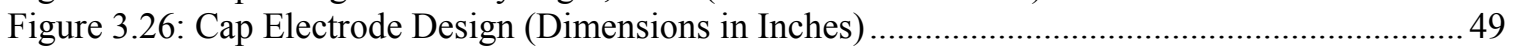

Figure 3.27: Thick Top Half Cap Electrode Attached to Sump ............................................................ 50

Figure 3.28: Nozzle Electrode Rendering and Schematic (Dimensions in Inches)...............................51

Figure 3.29: Cap Electrode Rendering and Schematic (Dimensions in Inches) ................................... 51

Figure 3.30: Pedestal Electrode Rendering and Schematic (Dimensions in Inches).............................51

Figure 3.31: Modified Cap Rendering and Schematic (Dimensions in Inches) ....................................52

Figure 3.32: Cover Rendering and Schematic (Dimensions in Inches) .............................................52

Figure 3.33: Electrode Arrangement around Sump, Pedestal, and Nozzle Showing a Solid Rendering

(left) and Transparent View (right) of Design by Mehra (2006) .....................................................52

Figure 3.34: Photograph of Electrode Arrangement in the Spray Chamber ........................................5 53

Figure 3.35: Original Base and Spray Chamber Configuration by Hunnell (2005).............................. 54

Figure 3.36: Agilent Data Acquisition System Addition and Protek DC Power Supply ......................55

Figure 3.37: Glassman High Voltage Power Supply Addition Mounted in Cabinet with Astron Power

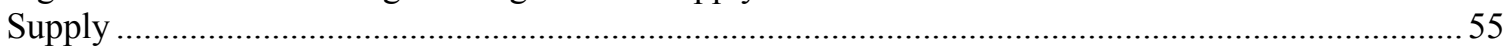

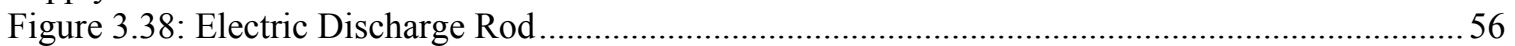

Figure 3.39: Experimental Configuration of a) Confined Case and b) Unconfined Case for Spacing

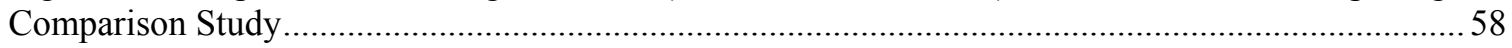

Figure 3.40: Full Length Cap Electrode inside Chamber with High Voltage Feedthrough Attached to

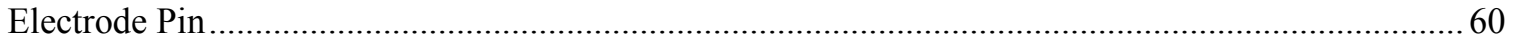

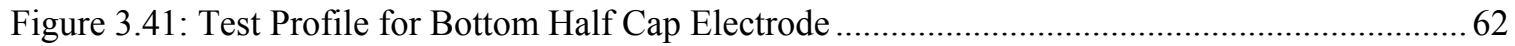

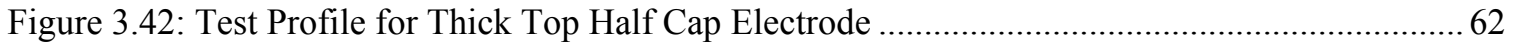

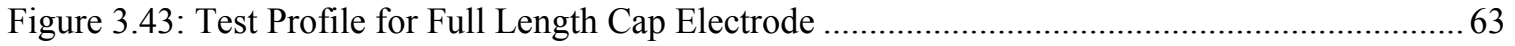

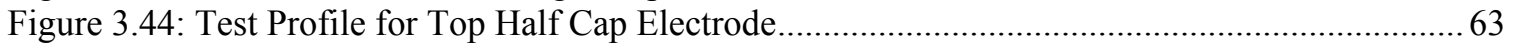

Figure 3.45: Test Profile for Full Length Electrode with HFE-7000 as the Working Fluid .................6 64

Figure 3.46: Mehra Electrodes inside Chamber with High Voltage Feedthrough Attached to Electrode

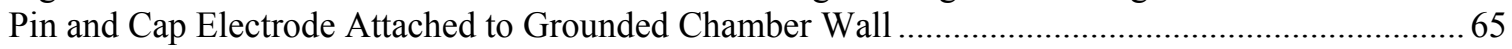

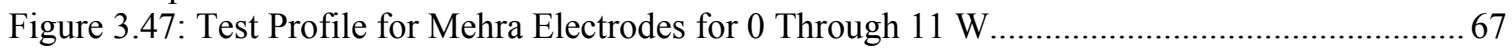

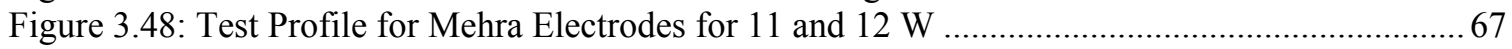

Figure 4.1: Heat Flux in $\mathrm{W} / \mathrm{m}^{2}$ as a Function of the Temperature Difference between the Surface and the Top of the Pedestal in Degrees Celsius for the Confined Case ....................................................... 69 Figure 4.2: Non-dimensional Heat Flux as a Function of the Non-dimensional Temperature Difference between the Surface and the Pedestal Top for the Confined Case ..................................................... 70 Figure 4.3: Convection Heat Transfer Coefficient in W/m $\mathrm{m}^{2} \mathrm{~K}$ as a Function of Heat Flux in W/m $\mathrm{m}^{2}$ for

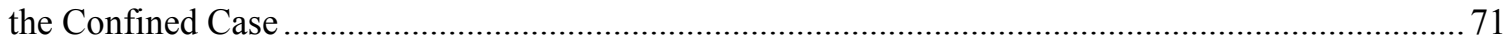
Figure 4.4: Nusselt Number as a Function of Non-dimensional Heat Flux for the Confined Case ...... 71 Figure 4.5: Heat Flux in $\mathrm{W} / \mathrm{m}^{2}$ as a Function of the Temperature Difference between the Surface and the Top of the Pedestal in Degrees Celsius for the Unconfined Case ................................................. 72 Figure 4.6: Non-dimensional Heat Flux as a Function of the Non-dimensional Temperature Difference between the Surface and the Pedestal Top for the Unconfined Case ................................................. 73 Figure 4.7: Convection Heat Transfer Coefficient in $\mathrm{W} / \mathrm{m}^{2} \mathrm{~K}$ as a Function of Heat Flux in $\mathrm{W} / \mathrm{m}^{2}$ for

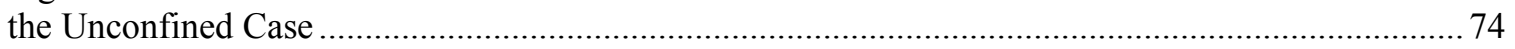
Figure 4.8: Nusselt Number as a Function of Non-dimensional Heat Flux for the Unconfined Case .. 74 Figure 4.9: Heat Flux in $\mathrm{W} / \mathrm{m}^{2}$ as a Function of the Temperature Difference between the Surface and the Top of the Pedestal in Degrees Celsius for the Bottom Half Cap Electrode. 
Figure 4.10: Non-dimensional Heat Flux as a Function of the Non-dimensional Temperature Difference between the Surface and the Pedestal Top for the Bottom Half Cap Electrode Figure 4.11: Heater Resistance in Ohms as a Function of Surface Temperature in Degrees Celsius for the Bottom Half Cap Electrode Before and After the Formation of a Crack ...................................... 78 Figure 4.12: Convection Heat Transfer Coefficient in W/m $\mathrm{m}^{2} \mathrm{~K}$ as a Function of Heat Flux in W/ $/ \mathrm{m}^{2}$ for

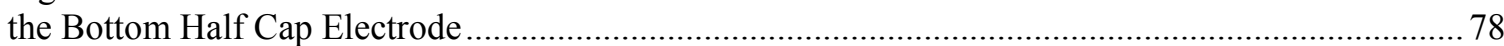
Figure 4.13: Nusselt Number as a Function of Non-dimensional Heat Flux for the Bottom Half Cap Electrode

Figure 4.14: Heat Flux in $\mathrm{W} / \mathrm{m}^{2}$ as a Function of the Temperature Difference between the Surface and the Top of the Pedestal in Degrees Celsius for the Thick Top Half Cap Electrode .............................. 80 Figure 4.15: Non-dimensional Heat Flux as a Function of the Non-dimensional Temperature Difference between the Surface and the Pedestal Top for the Thick Top Half Cap Electrode ............. 80 Figure 4.16: Convection Heat Transfer Coefficient in W/m $\mathrm{m}^{2} \mathrm{~K}$ as a Function of Heat Flux in W/.... for

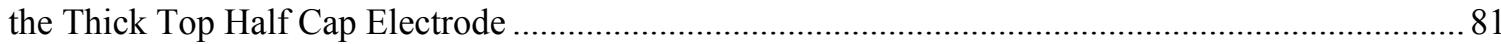
Figure 4.17: Nusselt Number as a Function of Non-dimensional Heat Flux for the Thick Top Half Cap

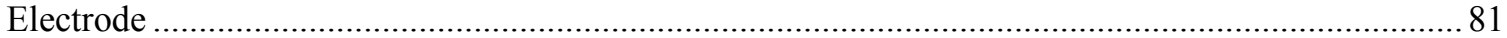
Figure 4.18: Heat Flux in $\mathrm{W} / \mathrm{m}^{2}$ as a Function of the Temperature Difference between the Surface and the Top of the Pedestal in Degrees Celsius for the Full Length Cap Electrode .................................... 82 Figure 4.19: Non-dimensional Heat Flux as a Function of the Non-dimensional Temperature Difference between the Surface and the Pedestal Top for the Full Length Cap Electrode..... Figure 4.20: Convection Heat Transfer Coefficient in W/m $\mathrm{m}$ as a Function of Heat Flux in $\mathrm{W} / \mathrm{m}^{2}$ for the Full Length Cap Electrode

Figure 4.21: Nusselt Number as a Function of Non-dimensional Heat Flux for the Full Length Cap Electrode

Figure 4.22: Heat Flux in $\mathrm{W} / \mathrm{m}^{2}$ as a Function of the Temperature Difference between the Surface and the Top of the Pedestal in Degrees Celsius for the Top Half Cap Electrode ........................................ 85 Figure 4.23: Non-dimensional Heat Flux as a Function of the Non-dimensional Temperature Difference between the Surface and the Pedestal Top for the Top Half Cap Electrode ...................... 85 Figure 4.24: Convection Heat Transfer Coefficient in W/m $\mathrm{m}^{2} \mathrm{~K}$ as a Function of Heat Flux in W/m $/ \mathrm{m}^{2}$ for

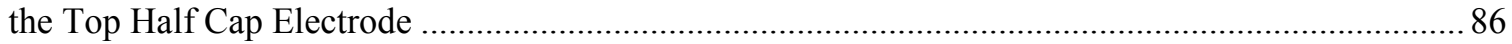
Figure 4.25: Nusselt Number as a Function of Non-dimensional Heat Flux for the Top Half Cap

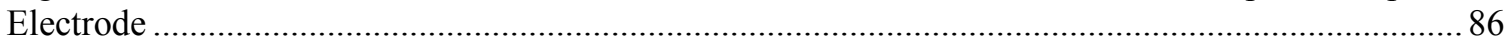
Figure 4.26: Electric Field Lines for Bottom Half Cap Electrode ......................................................... 89 Figure 4.27: Electric Potential for the Bottom Half Cap Electrode ..................................................... 89 Figure 4.28: Magnitude of the Electric Kelvin Force in the x-direction for the Bottom Half Cap Electrode

Figure 4.29: Magnitude of the Electric Kelvin Force in the y-direction for the Bottom Half Cap Electrode

Figure 4.30: Electric Kelvin Force per Mass with all FC-72 inside the cap (top) and FC-72 only in the Spray Cone and Above the Pedestal (bottom) as a Function of the Pedestal Radius for Five Distances from the Heater Surface for the Bottom Half Cap Electrode

Figure 4.31: Electric Field Lines for Thick Top Half Cap Electrode.................................................. 93

Figure 4.32: Electric Potential for the Thick Top Half Cap Electrode................................................. 93

Figure 4.33: Magnitude of the Electric Kelvin Force in the x-direction for the Thick Top Half Cap

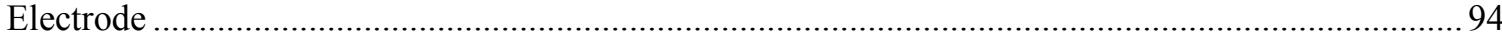

Figure 4.34: Magnitude of the Electric Kelvin Force in the y-direction for the Thick Top Half Cap

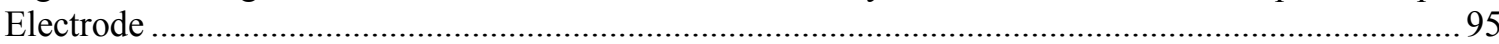
Figure 4.35: Electric Kelvin Force per Mass with all FC-72 inside the cap (top) and FC-72 only in the Spray Cone and Above the Pedestal (bottom) as a Function of the Pedestal Radius for Five Distances

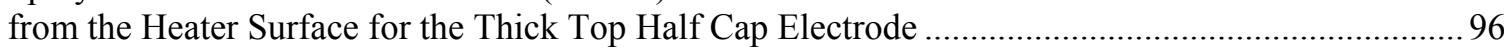
Figure 4.36: Electric Field Lines for Full Length Cap Electrode....................................................... 97 Figure 4.37: Electric Potential for the Full Length Cap Electrode......................................................... 97 
Figure 4.38: Magnitude of the Electric Kelvin Force in the x-direction for the Full Length Cap Electrode

Figure 4.39: Magnitude of the Electric Kelvin Force in the y-direction for the Full Length Cap Electrode

Figure 4.40: Electric Kelvin Force per Mass with all FC-72 inside the cap (top) and FC-72 only in the Spray Cone and Above the Pedestal (bottom) as a Function of the Pedestal Radius for Five Distances from the Heater Surface for the Full Length Cap Electrode

Figure 4.41: Electric Field Lines for Top Half Cap Electrode.

Figure 4.43: Magnitude of the Electric Kelvin Force in the x-direction for the Top Half Cap Electrode

Figure 4.44: Magnitude of the Electric Kelvin Force in the y-direction for the Top Half Cap Electrode

103

Figure 4.45: Electric Kelvin Force per Mass with all FC-72 inside the cap (top) and FC-72 only in the Spray Cone and Above the Pedestal (bottom) as a Function of the Pedestal Radius for Five Distances from the Heater Surface for the Top Half Cap Electrode.

Figure 4.46: Gradient of the Electric Field Squared Field Lines for the Full Length Cap Electrode.. 106 Figure 4.47: Heat Flux in $\mathrm{W} / \mathrm{m}^{2}$ as a Function of the Temperature Difference between the Surface and the Top of the Pedestal in Degrees Celsius for the Full Length Cap Electrode with HFE-7000 as the Working Fluid.

Figure 4.48: Non-dimensional Heat Flux as a Function of the Non-dimensional Temperature

Difference between the Surface and the Pedestal Top for the Full Length Cap Electrode with HFE-

7000 as the Working Fluid.

Figure 4.49: Convection Heat Transfer Coefficient in W/m $\mathrm{m}^{2} \mathrm{~K}$ as a Function of Heat Flux in $\mathrm{W} / \mathrm{m}^{2}$ for

Full Length Cap Electrode with HFE-7000 as the Working Fluid. ... 108

Figure 4.50: Nusselt Number as a Function of Non-dimensional Heat Flux for the Full Length Cap

Electrode with HFE-7000 as the Working Fluid.

Figure 4.51: Electric Field Lines for Mehra Electrode Configuration...

Figure 4.52: Electric Potential for the Mehra Electrode Configuration

Figure 4.53: Magnitude of the Electric Kelvin Force per unit Mass in the x-direction for the Mehra

Electrodes.

Figure 4.54: Magnitude of the Electric Kelvin Force per unit Mass in the y-direction for the Mehra

Electrodes.

Figure 4.55: Electric Kelvin Force per Mass in the x-direction as a Function of the Pedestal Radius for Three Distances from the Pedestal Surface for the Mehra Electrodes ............................................ 113 Figure 4.56: Electric Kelvin Force per Mass in the y-direction as a Function of the Pedestal Radius for Four Distances from the Pedestal Surface for the Mehra Electrodes. 114 Figure 4.57: Gradient of the Electric Field Squared Lines for the Mehra Electrodes ......................... 114 Figure 4.58: Heat Flux in $\mathrm{W} / \mathrm{m}^{2}$ as a Function of the Temperature Difference between the Surface and the Top of the Pedestal in Degrees Celsius for the Mehra Electrodes at Voltages up to $23 \mathrm{kV}$......... 116

Figure 4.59: Enlarged View of the Highest Heat Flux in Figure 4.58 ............................................ 116

Figure 4.60: Non-dimensional Heat Flux as a Function of the Non-dimensional Temperature Difference between the Surface and the Pedestal Top for the Mehra Electrodes at Voltages up to 23 $\mathrm{kV}$.......

Figure 4.61: Enlarged View of the Highest $\mathrm{G} \Delta$ in Figure 4.60.....

Figure 4.62: Temperature Differences between 0 and $23 \mathrm{kV}$ as a Function of Increasing Electrode Voltages for Varying Heater Powers ............................................................................................. 118 Figure 4.63: Temperature Differences between 0 and $23 \mathrm{kV}$ as a Function of Decreasing Electrode Voltages for Varying Heater Powers

Figure 4.64: Non-dimensional Temperature Differences between 0 and $23 \mathrm{kV}$ as a Function of Increasing Electrode Voltages for Varying Heater Powers...

Figure 4.65: Temperature Differences between 0 and $23 \mathrm{kV}$ as a Function of Decreasing Electrode Voltages for Varying Heater Powers 
Figure 4.66: Convection Heat Transfer Coefficient in $\mathrm{W} / \mathrm{m}^{2} \mathrm{~K}$ as a Function of Heat Flux in $\mathrm{W} / \mathrm{m}^{2}$ for

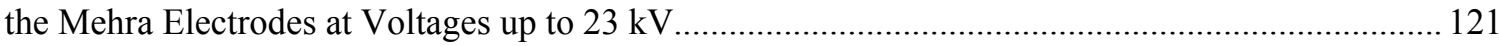

Figure 4.67: Enlarged View of the Highest Heat Flux in Figure 4.66 ............................................. 121 Figure 4.68: Nusselt Number as a Function of Non-dimensional Heat Flux for the Mehra Electrodes at

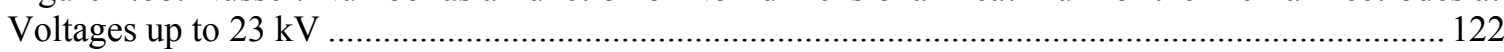

Figure 4.69: Enlarged View of the Highest G $\Delta$ in Figure 4.68 ....................................................... 122 Figure 4.70: Heat Transfer Coefficient Differences between 0 and $23 \mathrm{kV}$ as a Function of Increasing Electrode Voltages for Varying Heater Powers ......................................................................... 123 Figure 4.71: Heat Transfer Coefficient Differences between 0 and $23 \mathrm{kV}$ as a Function of Decreasing

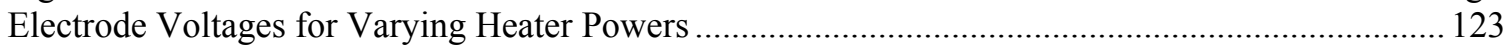
Figure 4.72: Nusselt Number Differences between 0 and $23 \mathrm{kV}$ as a Function of Increasing Electrode Voltages for Varying Heater Powers 124 Figure 4.73: Nusselt Number Differences between 0 and $23 \mathrm{kV}$ as a Function of Decreasing Electrode Voltages for Varying Heater Powers ............................................................................................ 124

Figure A1: Polished Samples of TFR Type 1 (left) and TFR Type 2 (right) Heaters......................... 134 Figure A2: TFR Type 1 at 140x Magnification Showing the Alumina and Glass Layers with

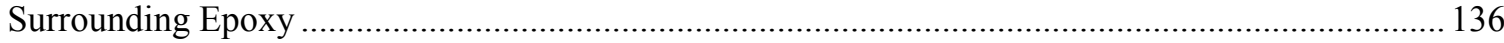
Figure A3: TFR Type 1 X-Ray Analysis at 140x Magnification...................................................... 136 Figure A4: TFR Type 1 Chemical Analysis at 140x Magnification Showing a) Thin layer of Silver b) Sulfur Layer c) Silicon Layer d) Large Layer of Aluminum e) Composite Image .............................. 137 Figure A5: TFR Type 1 at 1500x Magnification Showing the Conductive Heater Layer ................... 138 Figure A6: X-Ray Analysis at 1500x Magnification ......................................................................... 138 Figure A7: Chemical Analysis at 1500x Magnification Showing a) Layer of Silver b) Layer of Sulfur c) Layer of Silicon d) Layer of Aluminum e) Composite Image .................................................... 139

Figure A8: TFR Type 1 at 3000x Magnification Showing the Conductive Heater Layer .................. 139

Figure A9: X-Ray Analysis at 3000x Magnification Showing Point mostly Silver ........................... 140 Figure A10: Chemical Analysis at 3000x Magnification Showing a) Layer of Silver b) Layer of Sulfur c) Layer of Silicon d) Layer of Aluminum e) Composite Image ...................................................... 140

Figure A12: TFR Type 2 at 150x Magnification Showing the Alumina Layer ................................. 143

Figure A13: X-Ray Analysis at 150x Magnification ...................................................................... 143

Figure A14: Chemical Analysis at 150x Magnification Showing a) Silver Throughout b) Layer of

Sulfur c) Layer of Silicon d) Layer of Aluminum e) Nickel f) Composite Image .............................. 144

Figure A15: TFR Type 2 at 1000x Magnification Showing the Conductive Layer............................ 145

Figure A16: X-Ray Analysis at 1000x Magnification .................................................................. 145

Figure A17: Chemical Analysis at 150 Magnification Showing a) Silver Throughout b) Layer of

Sulfur c) Layer of Silicon d) Layer of Aluminum e) Composite Image .............................................. 146

Figure B1: Spacing Comparison Test Plan at $0.029 \mathrm{~m}^{3} / \mathrm{hr}(7.72 \mathrm{GPH}) \mathrm{FC}-72 \ldots \ldots \ldots \ldots \ldots \ldots \ldots \ldots \ldots \ldots . . . . . . . . . . . . . . .148$ Figure B2: Cap Electrode Test Plan at $0.029 \mathrm{~m}^{3} / \mathrm{hr}(7.72 \mathrm{GPH})$ of FC-72 and $0.016 \mathrm{~m}^{3} / \mathrm{hr}(4.23 \mathrm{GPH})$ of HFE-7000

Figure B3: Mehra Electrode Test Plan at $0.014 \mathrm{~m}^{3} / \mathrm{hr}(3.8 \mathrm{GPH})$ of HFE-7000 150

Figure C1: Energy Equation Derivation Schematic for the Spacing Comparison and Mehra Pedestals

Figure C2: Respective Geometry, Equations, and Boundary Conditions for Each Layer of the Spacing

Comparison and Mehra Pedestals ................................................................................................ 154

Figure C3: Energy Equation Derivation Schematic for the Cap Electrode Pedestal......................... 157

Figure C4: Respective Geometry, Equations, and Boundary Conditions for Each Layer of the Cap

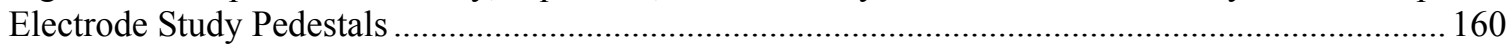

Figure D1: Plot of Thermal Conductivity vs. Temperature from the F-chart Program Data .............. 165 


\section{List of Tables:}

Table 2.1: Comparison of Thermal Management Techniques ............................................................ 4

Table 2.2: Parameters Influencing EHD-Enhanced Nucleate Boiling Heat Transfer (redrawn from

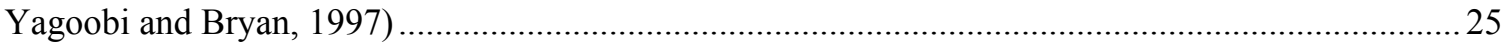

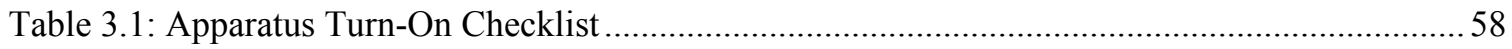

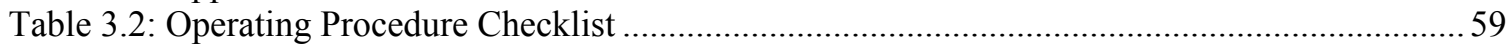

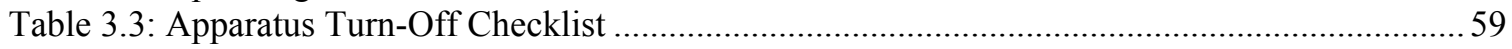

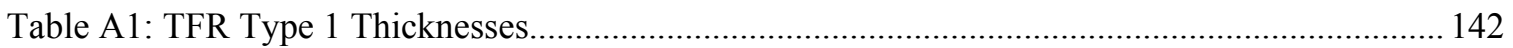

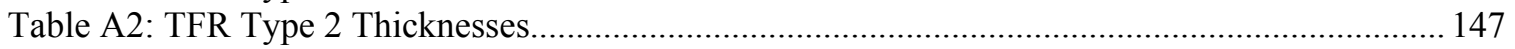

Table D1: Thicknesses and Thermal Conductivity of TFR Layers..................................................... 164

Table D2: Power, Flow Rates, and Pressure Data for Confined 9 mm Spacing ................................. 167

Table D3: Room and Pedestal Temperatures for Confined $9 \mathrm{~mm}$ Spacing ......................................... 167

Table D4: Chamber Temperatures for Confined 9 mm Spacing....................................................... 167

Table D5: Power, Flow Rates, and Pressure Data for Confined $11 \mathrm{~mm}$ Spacing ............................... 168

Table D6: Room and Pedestal Temperatures for Confined $11 \mathrm{~mm}$ Spacing ..................................... 168

Table D7: Chamber Temperatures for Confined $11 \mathrm{~mm}$ Spacing...................................................... 168

Table D8: Power, Flow Rates, and Pressure Data for Confined 13 mm Spacing ............................... 169

Table D9: Room and Pedestal Temperatures for Confined $13 \mathrm{~mm}$ Spacing .................................... 169

Table D10: Chamber Temperatures for Confined 13 mm Spacing................................................. 169

Table D11: Power, Flow Rates, and Pressure Data for Confined $15 \mathrm{~mm}$ Spacing ............................. 170

Table D12: Room and Pedestal Temperatures for Confined $15 \mathrm{~mm}$ Spacing ..................................... 170

Table D13: Chamber Temperatures for Confined $15 \mathrm{~mm}$ Spacing .................................................... 170

Table D14: Power, Flow Rates, and Pressure Data for Confined $17 \mathrm{~mm}$ Spacing ............................. 171

Table D15: Room and Pedestal Temperatures for Confined $17 \mathrm{~mm}$ Spacing .................................. 171

Table D16: Chamber Temperatures for Confined $17 \mathrm{~mm}$ Spacing................................................... 171

Table D17: Power, Flow Rates, and Pressure Data for Unconfined $9 \mathrm{~mm}$ Spacing .......................... 172

Table D18: Room and Pedestal Temperatures for Unconfined $9 \mathrm{~mm}$ Spacing .................................. 172

Table D19: Chamber Temperatures for Unconfined $9 \mathrm{~mm}$ Spacing ................................................. 172

Table D20: Power, Flow Rates, and Pressure Data for Unconfined $11 \mathrm{~mm}$ Spacing ......................... 173

Table D21: Room and Pedestal Temperatures for Unconfined $11 \mathrm{~mm}$ Spacing ................................. 173

Table D22: Chamber Temperatures for Unconfined $11 \mathrm{~mm}$ Spacing................................................. 173

Table D23: Power, Flow Rates, and Pressure Data for Unconfined $13 \mathrm{~mm}$ Spacing ......................... 174

Table D24: Room and Pedestal Temperatures for Unconfined $13 \mathrm{~mm}$ Spacing ............................... 174

Table D25: Chamber Temperatures for Unconfined $13 \mathrm{~mm}$ Spacing................................................. 174

Table D26: Power, Flow Rates, and Pressure Data for Unconfined $15 \mathrm{~mm}$ Spacing ......................... 175

Table D27: Room and Pedestal Temperatures for Unconfined $15 \mathrm{~mm}$ Spacing ................................. 175

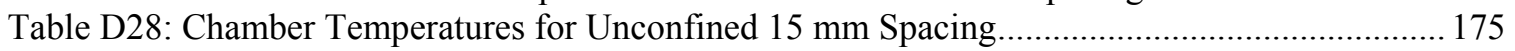

Table D29: Power, Flow Rates, and Pressure Data for Unconfined $17 \mathrm{~mm}$ Spacing ......................... 176

Table D30: Room and Pedestal Temperatures for Unconfined $17 \mathrm{~mm}$ Spacing ................................. 176

Table D31: Chamber Temperatures for Unconfined $17 \mathrm{~mm}$ Spacing................................................. 176

Table D32: Power, Flow Rates, and Pressure Data for Unconfined 13 mm \#2 Spacing ................... 177

Table D33: Room and Pedestal Temperatures for Unconfined $13 \mathrm{~mm}$ \#2 Spacing............................. 177

Table D34: Chamber Temperatures for Unconfined 13 mm \#2 Spacing ............................................ 177

Table D35: Reduced Data for Confined $9 \mathrm{~mm}$ Spacing................................................................. 178

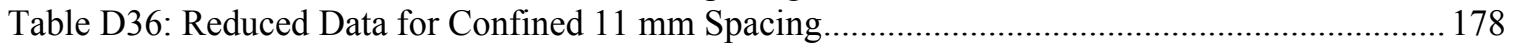

Table D37: Reduced Data for Confined 13 mm Spacing................................................................ 179

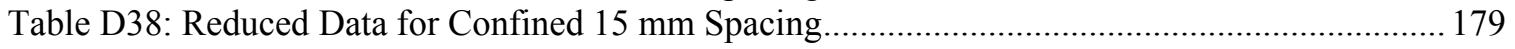

Table D39: Reduced Data for Confined 17 mm Spacing................................................................. 180 
Table D40: Reduced Data for Unconfined 9 mm Spacing ............................................................. 180

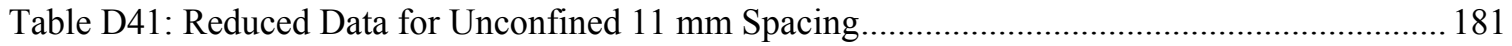

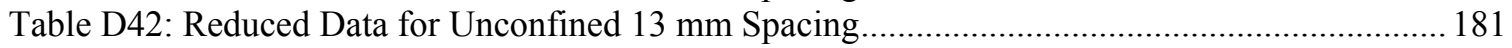

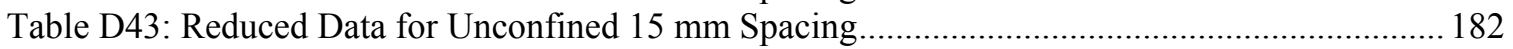

Table D44: Reduced Data for Unconfined 17 mm Spacing........................................................... 182

Table D45: Reduced Data for Unconfined 13 mm \#2 Spacing ..................................................... 183

Table D46: Averaged Heater and Pressure Raw Data for Bottom Half Electrode.............................. 184

Table D47: Averaged Pedestal Temperature Raw Data for Bottom Half Electrode............................ 185

Table D48: Averaged Chamber Temperature Raw Data for Bottom Half Electrode .......................... 186

Table D49: Averaged Heater and Pressure Raw Data for Thick Top Half Electrode.......................... 187

Table D50: Averaged Pedestal Temperature Raw Data for Thick Top Half Electrode ....................... 188

Table D51: Averaged Chamber Temperature Raw Data for Thick Top Half Electrode...................... 189

Table D52: Averaged Heater and Pressure Raw Data for Full Length Electrode................................ 190

Table D53: Averaged Pedestal Temperature Raw Data for Full Length Electrode ............................. 191

Table D54: Averaged Chamber Temperature Raw Data for Full Length Electrode............................ 192

Table D55: Averaged Heater and Pressure Raw Data for Top Half Electrode ................................... 193

Table D56: Averaged Pedestal Temperature Raw Data for Top Half Electrode ................................. 194

Table D57: Averaged Chamber Temperature Raw Data for Top Half Electrode ................................ 195

Table D58: Averaged Heater and Pressure Raw Data for Full Length Electrode with HFE-7000 ..... 196

Table D59: Averaged Pedestal Temperature Raw Data for Full Length Electrode with HFE-7000 .. 197

Table D60: Averaged Chamber Temperature Raw Data for Full Length Electrode with HFE-7000 198

Table D61: Reduced Data for Bottom Half Electrode 0 kV Runs ................................................... 199

Table D62: Reduced Data for Bottom Half Electrode $3 \mathrm{kV}$ Run.................................................... 199

Table D63: Reduced Data for Bottom Half Electrode $6 \mathrm{kV}$ Run.......................................................200

Table D64: Reduced Data for Thick Top Half Electrode 0 kV Runs ................................................ 200

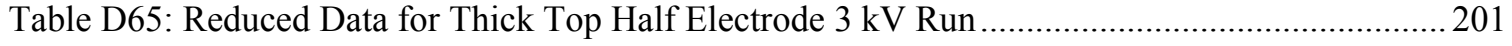

Table D66: Reduced Data for Thick Top Half Electrode 6 kV Run ................................................... 201

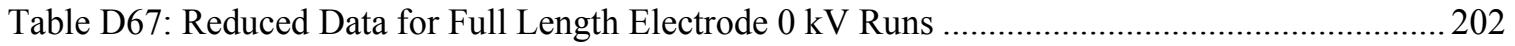

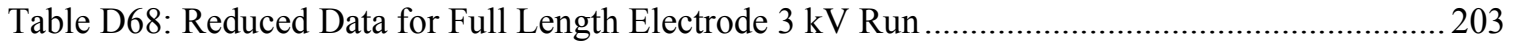

Table D69: Reduced Data for Full Length Electrode 6 kV Run ........................................................ 203

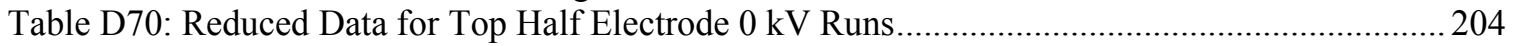

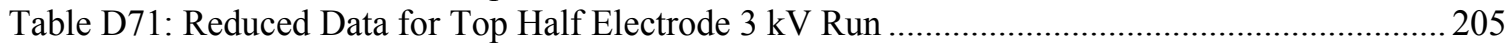

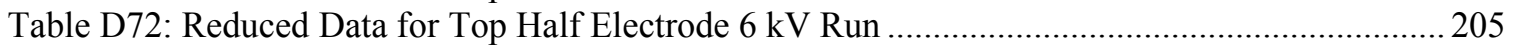

Table D73: Reduced Data for Full Length Electrode 0 kV Runs with HFE-7000 ..............................206

Table D74: Reduced Data for Full Length Electrode 3 kV Run with HFE-7000 ...............................206

Table D75: Reduced Data for Full Length Electrode 6 kV Run with HFE-7000 ............................... 207

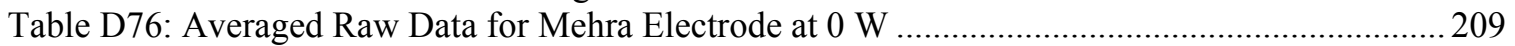

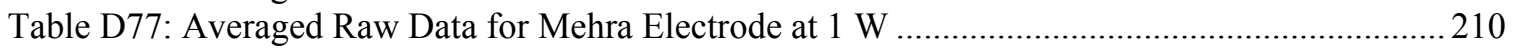

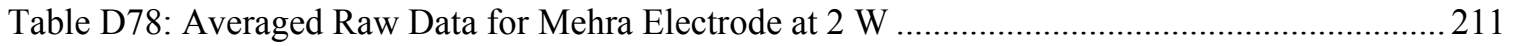

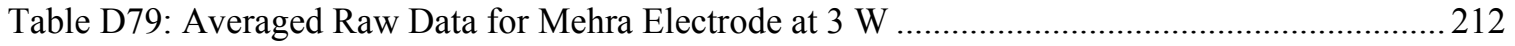

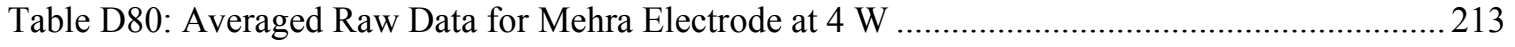

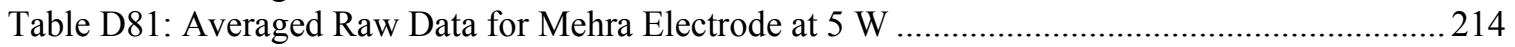

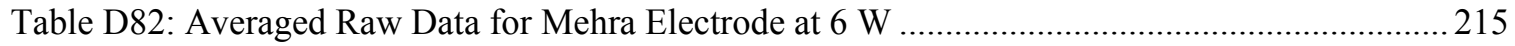

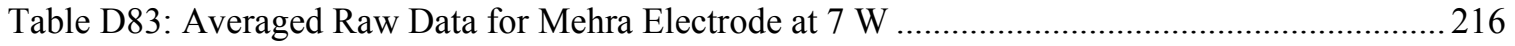

Table D84: Averaged Raw Data for Mehra Electrode at $8 \mathrm{~W}$........................................................... 217

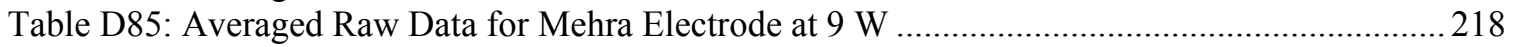

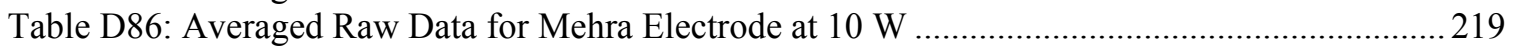

Table D87: Averaged Raw Data for Mehra Electrode at $11 \mathrm{~W}$ (first) ...............................................220

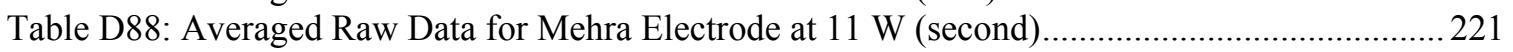

Table D89: Averaged Raw Data for Mehra Electrode at $12 \mathrm{~W}$.........................................................222

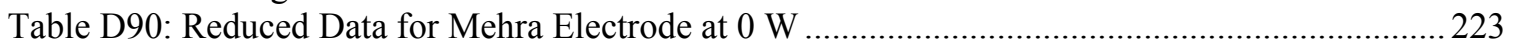

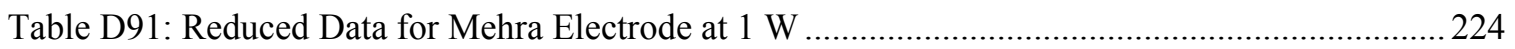

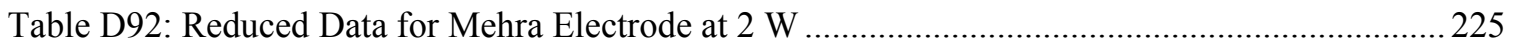




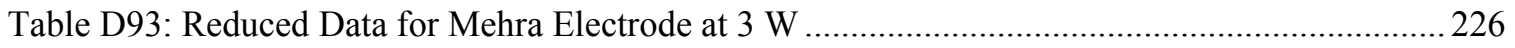

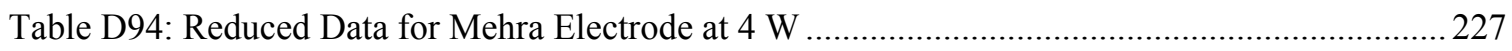

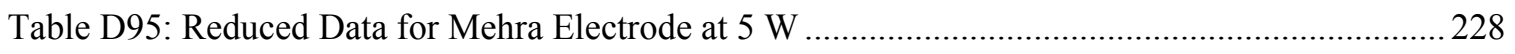

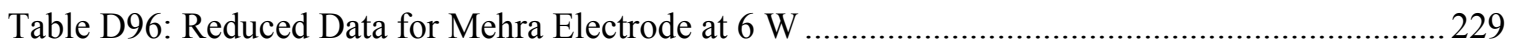

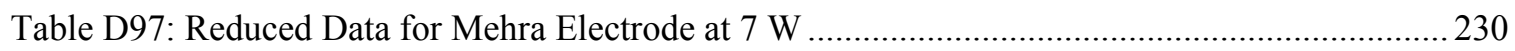

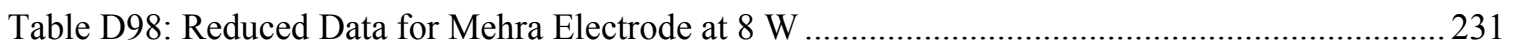

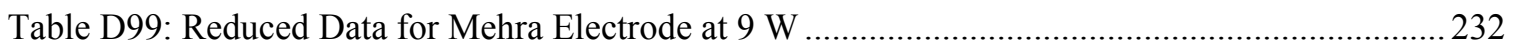

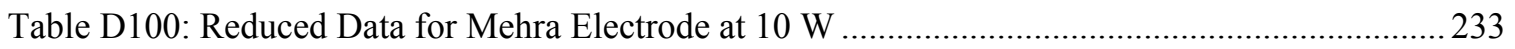

Table D101: Reduced Data for Mehra Electrode at $11 \mathrm{~W}$ (averaged) ................................................234

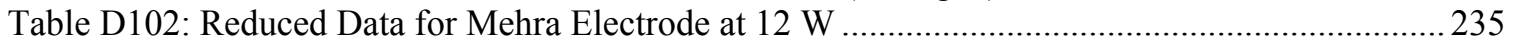

Table E1: Equipment and Errors Used in Spacing Comparison and Electrode Studies......................236

Table E2: Error Values for Confined $9 \mathrm{~mm}$ Spacing with Respective Units ......................................240

Table E3: Error Values for Confined $9 \mathrm{~mm}$ Spacing in Percentages .................................................240

Table E4: Error Values for Unconfined $9 \mathrm{~mm}$ Spacing with Respective Units ..................................241

Table E5: Error Values for Unconfined $9 \mathrm{~mm}$ Spacing in Percentages ............................................241

Table E6: Error Values for Top Half Cap Electrode with Respective Units at $0 \mathrm{kV}$..........................243

Table E7: Error Values for Top Half Cap Electrode in Percentages at $0 \mathrm{kV}$.....................................243

Table E8: Error Values for Mehra Electrode with Respective Units at $23 \mathrm{kV}$.................................245

Table E9: Error Values for Mehra Electrode in Percentages at $23 \mathrm{kV}$............................................246 


\section{Acknowledgements:}

I would like to thank the Air Force Office of Scientific Research for providing funding for this project and also to Dr. Kirk Yerkes of the Air Force Research Laboratory in Dayton, $\mathrm{OH}$ for his mentoring throughout this project and in my time spent during my internship. Thanks also to the team at AFRL: Mr. Richard Harris, Mr. John Tennett, Mr. Travis Michalak, Mrs. Kerri Baysinger, Mr. Levi Elston, Ms. Bekah Puterbaugh, and Ms. Sarah Trent for all their help, support, and laughs.

I would like to thank Mr. Cliff Judy and Mr. Chuck Coleman for their machining and electrical expertise. I would like to also thank Mr. Paul Kreitzer and Mr. Andy Hunnell for their work with the experimental apparatus and Mr. Deepak Mehra for all of his hard work in providing the numerical simulations for this report. Thank you also to Mr. John Sakacsi for helping me with the SEM study.

I would especially like to thank my other committee members and professors Dr. Donald D. Gray, Dr. Wade Huebsch, and Dr. Gary Morris for agreeing to guide me through my Masters degree and other college endeavors. Thank you also to Dr. John Kuhlman, my advisor, mentor, and friend for directing me in both my academic and professional career decisions and for the numerous hours he has spent to make this project a success.

Finally I would like to thank my family and Derek for their love, support, and comfort in every aspect of my life. I couldn't have done it without you all and I love you very much. 


\section{Nomenclature:}

\begin{tabular}{|c|c|c|}
\hline A & Surface Area & Subscripts \\
\hline Amb & Ambient & fluid FC-72 or HFE-7000 \\
\hline $\mathrm{b}$ & Pedestal Radius & glass TFR glass layer \\
\hline B & Magnetic Induction & TFR heater layer \\
\hline $\mathrm{CHF}$ & Critical Heat Flux & interface \\
\hline $\mathrm{D}$ & Diameter & Toward Bottom of Pedestal \\
\hline$E$ & Electric Field & surface \\
\hline $\mathrm{f}$ & Fractional Loss Down Pedestal & Saturation \\
\hline$f$ & Electro-magnetic Body Force per Volume & sub TFR substrate layer \\
\hline FS & Full Scale & Toward Top of Pedestal \\
\hline $\mathrm{g}$ & Gravity, Volumetric Heat Generation & $\infty$ wall side of the pedestal \\
\hline $\mathrm{G} \Delta$ & $\begin{array}{l}\text { Non-dimensional Heat Flux Parameter: } \\
(1-f) Q / \pi b\left(T_{\text {sat }}-T_{\text {swall }}\right) k_{h t r}\end{array}$ & $\infty$ top top of the pedestal \\
\hline $\mathrm{h}$ & Convection Heat Transfer Coefficient & \\
\hline $\mathrm{H}$ & Layer Thickness, Magnetic Field & \\
\hline $\mathrm{H}_{\mathrm{fg}}$ & Latent Heat of Vaporization & \\
\hline HV & High Voltage & \\
\hline I & Heater Current & \\
\hline $\mathrm{J}$ & Current Density & \\
\hline $\mathrm{k}$ & Thermal Conductivity & \\
\hline 1 & Length of Plate & \\
\hline $\mathrm{Nu}$ & Nusselt Number: $h b / k_{\text {fluid }}$ & \\
\hline q & Heat Flux & \\
\hline $\mathrm{Q}$ & Heater Power & \\
\hline SEM & Scanning Electron Microscope & \\
\hline $\mathrm{T}$ & Temperature & \\
\hline TFR & Thick Film Resistor & \\
\hline $\mathrm{u}$ & Velocity of the Saturated Liquid & \\
\hline $\mathrm{V}$ & Velocity, Voltage & \\
\hline We & Weber Number: $\rho V^{2} D / \sigma$ & \\
\hline $\mathrm{z}$ & Distance & \\
\hline$\delta_{\mathrm{c}}$ & Critical Thickness of the Liquid Film & \\
\hline$\Delta$ & Difference & \\
\hline$\varepsilon_{0}$ & Permittivity of Free Space & \\
\hline$\kappa$ & Relative Permittivity & \\
\hline$\kappa_{\mathrm{m}}$ & Relative Permeability & \\
\hline$\mu_{0}$ & Permeability of Free Space & \\
\hline$\rho$ & Density & \\
\hline$\rho_{e}$ & Free Electric Charge per Volume & \\
\hline$\sigma$ & Surface Tension & \\
\hline$\theta$ & $\begin{array}{l}\text { Non-dimensional Temperature Parameter: } \\
\left(T_{s}-T_{\text {otop }}\right) /\left(T_{\text {sat }}-T_{\text {owall }}\right)\end{array}$ & \\
\hline
\end{tabular}




\section{Chapter 1: Introduction}

Requirements of advancing technology are pushing the envelope of currently available methods of removing vast amounts of heat from small areas. Spray cooling is an efficient thermal management technique that may be improved by employing an electric Kelvin force to control the fluid motion and delay the onset of critical heat flux. However, spray cooling, as well as electrohydrodynamics, rely on many varying parameters that are currently being studied (both experimentally and numerically) to identify the key mechanisms that could advance the technology.

In the present study, a spray cooling apparatus consisting of a spray chamber housing a spray nozzle and electrically heated surface, and a flow generating base package has been built. It has been tested for varying electrode geometries for a range of spray conditions. The goals of these tests were to detect a definite effect from the electric Kelvin force on spray cooling and to define the parameters that impact performance the most. These tests, performed at West Virginia University, were sponsored by the Air Force Office of Scientific Research (AFOSR) in collaboration with the Air Force Research Laboratory (AFRL) and are a continuation of a previous study by Hunnell (2005).

The objectives of this thesis are to: review the current state of thermal management technologies (specifically spray cooling), outline the design and construction of the apparatus and electrodes, present data and results from the three sets of tests performed (nozzle-to-heater spacing comparison, cap electrode performance comparison, and Mehra electrode performance results), and discuss conclusions that can be made from the study. 


\section{Chapter 2: Literature Review}

Chapter 2 consists of a review of previous work that relates to the current study. Topics investigated include a demonstration of the importance of advancing the area of thermal management techniques, a comparison of methods (pool boiling, heat pipes, jet impingement, channel flow boiling, and spray cooling), the physics and parameters that affect spray cooling (type of fluid, nozzle parameters, surface shape, and amount of splashing), numerical simulation studies, and the effects of electrohydrodynamics (EHD) on heat transfer.

\subsection{Importance of Advancing Thermal Management Techniques}

Thermal management of emerging technologies has become a key issue in advancing state-of-the-art designs from consumer electronics to future aircraft and space power systems or weapon platforms, and the techniques used to cool these high power systems must be addressed. The heat flux of a typical desktop computer processor is almost $50 \mathrm{~W} / \mathrm{cm}^{2}$, a super computer over $75 \mathrm{~W} / \mathrm{cm}^{2}$, and proposed laser weapon platforms are on the order of 200 to $1000 \mathrm{~W} / \mathrm{cm}^{2}$. These compare to the heat flux of an ordinary electric kitchen stove of only about $6 \mathrm{~W} / \mathrm{cm}^{2}$ (Shedd and Pautsch, 2005 and Mahefkey et al., 2004).

Of course there have been many proposed methods of dealing with high heat flux cooling requirements including pool boiling, heat pipes, jet impingement, channel flow boiling, and spray cooling. All of these rely on the physics of fluid behavior as well as the understanding of principles related to heat transfer. While single phase techniques have been studied, it has been shown that for high heat flux applications, methods involving phase change are superior due to the relatively small increase in device 
temperature (Mudawar, 2000). However, there is still much to be learned about the varying parameters governing each technique. NASA, for one, has organized workshops focusing on identifying essential research issues that have determined that "...multiphase flow and phase change play a crucial role in many of these advanced technology concepts" (Singh, 2003). These forums even went as far as to describe the understanding of phase change components as "critical" (or must be resolved in order to achieve missions) and also described the technology to enhance critical heat flux as "severely limiting” (Motil and Singh, 2004). When discussing military aircraft power systems, Mahefkey et al. (2004) describes thermal management and limited payload capacity as one of the primary design challenges facing engineers.

\subsection{Comparison of Thermal Management Techniques}

The debate over which method of thermal management can produce the best results is ongoing and shifts significantly based on the specific application because “...system considerations always play a paramount role in determining the most suitable cooling scheme" (Mudawar, 2000). Table 2.1 shows a list of some thermal management techniques and are discussed below. 
Table 2.1: Comparison of Thermal Management Techniques

\begin{tabular}{|c|c|c|c|l|l|}
\hline Technique & Mechanism & $\begin{array}{c}\text { Heat Flux } \\
\left(\mathbf{W} / \mathbf{c m}^{2}\right)\end{array}$ & $\begin{array}{c}\text { Temp. } \\
\left({ }^{\circ} \mathbf{C}\right)\end{array}$ & Disadvantages & $\begin{array}{c}\text { Typical } \\
\text { Fluids }\end{array}$ \\
\hline Pool Boiling & Evaporation & & & $\begin{array}{l}\text { Orientation } \\
\text { Variable Gravity }\end{array}$ & \\
\hline Heat Pipes & Evaporation & $<10-20$ & & Variable Gravity & $\begin{array}{l}\text { R134a } \\
\text { Ammonia }\end{array}$ \\
\hline $\begin{array}{c}\text { Loop Heat } \\
\text { Pipes }\end{array}$ & Evaporation & $10-20$ & & Variable Gravity & $\begin{array}{l}\text { R134a } \\
\text { Ammonia }\end{array}$ \\
\hline $\begin{array}{c}\text { Jet } \\
\text { Impingement }\end{array}$ & $\begin{array}{c}\text { Convection } \\
(\& \text { Evaporation })\end{array}$ & $100-1000$ & 20 & $\begin{array}{l}\text { Delicate Surfaces } \\
\text { Uneven Heating } \\
\text { Flow Management }\end{array}$ & $\begin{array}{l}\text { FC-72 } \\
\text { R134a } \\
\text { Ammonia } \\
\text { Water }\end{array}$ \\
\hline Flow Boiling & $\begin{array}{c}\text { Convection \& } \\
\text { Evaporation }\end{array}$ & $100-1000$ & 20 & $\begin{array}{l}\text { Flow Physics } \\
\text { Geometries }\end{array}$ & $\begin{array}{l}\text { FC-72 } \\
\text { R134a } \\
\text { Ammonia } \\
\text { Water }\end{array}$ \\
\hline $\begin{array}{c}\text { Spray } \\
\text { Cooling }\end{array}$ & $\begin{array}{c}\text { Convection \& } \\
\text { Evaporation }\end{array}$ & $100-1000$ & 100 & $\begin{array}{l}\text { Flow Management } \\
\text { Nozzle Pressures }\end{array}$ & $\begin{array}{l}\text { FC-72 } \\
\text { R134a } \\
\text { Ammonia } \\
\text { Water }\end{array}$ \\
\hline
\end{tabular}

Pool Boiling: Pool boiling is a passive evaporative cooling technique that relies on buoyancy forces to carry vapor bubbles away from a heated surface. Thermosyphons, for instance, cool components with the use of pool boiling. The heated surface is submerged in a coolant and as heat is transferred to the coolant, it boils and rises. The vapor condenses and falls back into the coolant pool. Orientation of the heated surface and variable gravity conditions can affect the performance of pool boiling systems (Baysinger, 2004).

Heat Pipes: An evaporator and condenser are used to passively transport heat in a heat pipe. These sealed vessels are partially filled with a liquid and the internal walls of the pipe are lined with a wick (a porous medium). Heat is applied to one end of the pipe and the liquid begins to evaporate and vapor moves to the cooler end. The vapor 
condenses and is transported back to the heated side by the wick through capillary action. Loop heat pipes are similar, but separate the vapor and liquid paths. In general, the effectiveness of heat pipes decrease with decreasing lengths (Lasance and Simons, 2005). Both types of heat pipes have a disadvantage in a variable gravity (hypergravity) condition which can cancel out the capillary pressures on the wick and reduce the heat transport performance (Gu et al., 2004).

Jet Impingement: Jet impingement uses convective cooling (and often evaporative cooling as well), making it more effective than pool boiling alone, but does not work well for components that are delicate due to the high velocity of the liquid impacting the heated surface. Also, it has been shown to cool the surface unevenly (Hunnell, 2005 and Bernardin et al., 1997).

Flow Boiling: Channel flow boiling presents problems with critical heat flux decrease due to large vapor void ratios or dryout and decreased subcooling in the downstream regions of the flow (Mudawar, 2000). Heat transfer augmentation devices (HTAD) use a method of mixing the gas and liquid in a channel, such as a helical ribbon or variable diameter section of pipe, to increase the liquid contact with the heated surface (Parang et al., 2003) and have been proposed as a method of increasing the critical heat flux. However, due to liquid-vapor stratification, vapor stagnation or counterflow causing liquid blockage, and the specific geometries required, channel flow applications are limited (Mudawar, 2000).

Spray Cooling: Spray cooling has advantages over these other methods of cooling because it uses both evaporative cooling and forced convection as its primary mechanisms. However, spray cooling requires flow management unlike the passive 
methods discussed (pool boiling and heat pipes). Also for spray cooling, a higher nozzle pressure drop is needed compared to jet impingement (Lasance and Simons, 2005). When compared to channel flow, the apparatus used for spray cooling can become more intricate and relies on more variables. Figure 2.1 is a chart comparing various thermal management techniques and some common liquids used. Note that free convection only techniques give the lowest heat transfer coefficients, followed by forced convection and boiling. Spray cooling provides the largest heat transfer coefficient of between approximately 0.75 and $8 \mathrm{~W} / \mathrm{cm}^{2}{ }^{\circ} \mathrm{C}$ (depending on the type of fluid). The physics and the parameters that affect spray cooling are explained in depth within the next two sections.

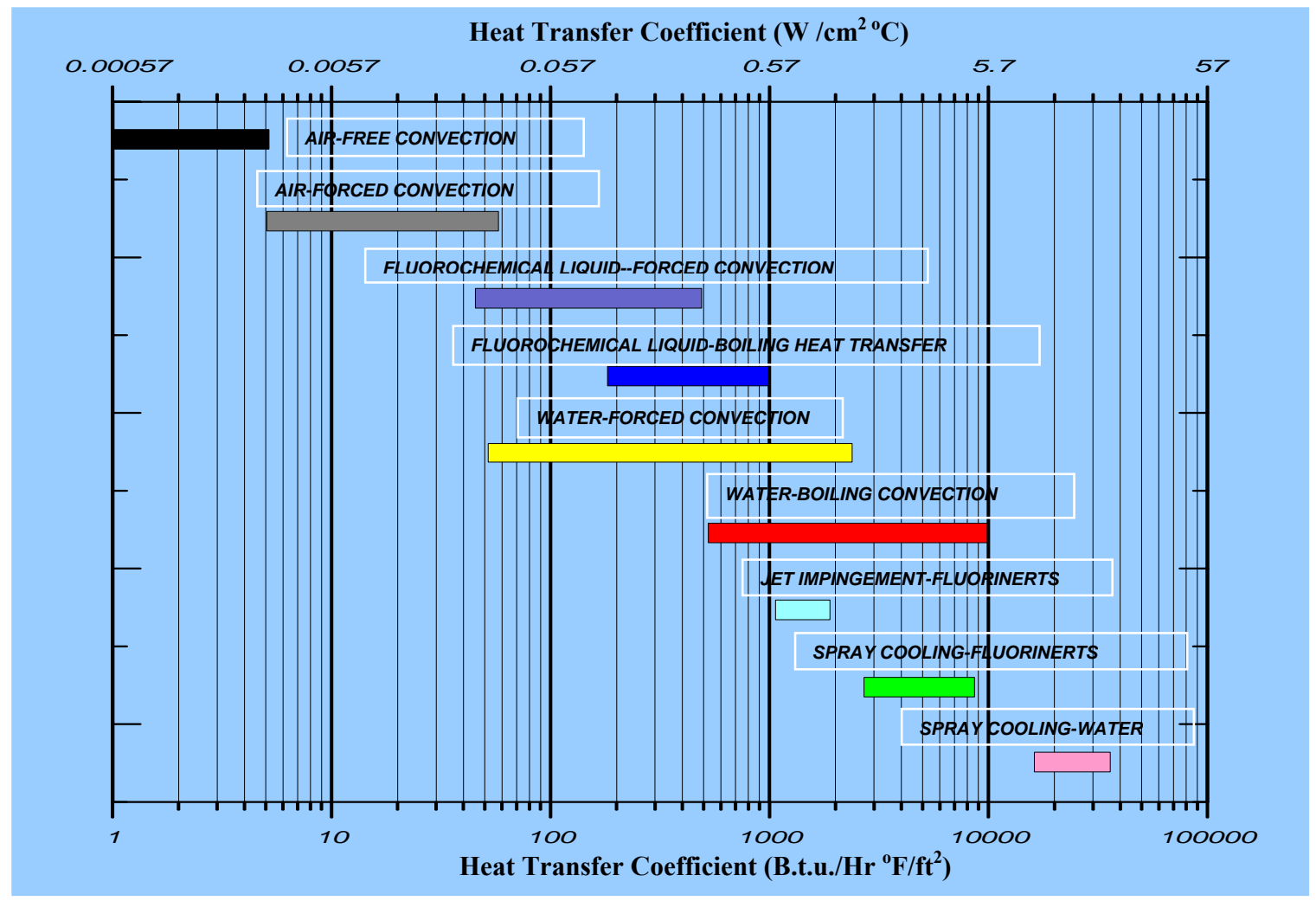

Figure 2.1: Comparison of Thermal Management Techniques Using Various Fluids (Mudawar, 2000) 


\subsection{The Physics of Spray Cooling}

Spray cooling has been used for many applications including the quenching of metals and the cooling of electrical components. It has great promise in the fields of aircraft and spacecraft thermal management due to the lack of dependence on buoyancy forces according to Tilton (1989), and has been studied in microgravity conditions. However, later studies have revealed that some types of spray cooling (i.e. mist cooling) can depend on buoyancy due to the low velocities of the liquid or other parameters of an individual study. Spray cooling uses a nozzle (or array of nozzles) directed at a heated surface. The liquid emanating from the nozzle is broken up into droplets and deposited onto the hot surface where the droplets interact with one another and form a thin liquid film. The liquid film has a large tangential momentum at high spray volume fluxes and the bulk motion of the liquid film is thought to efficiently re-wet regions of local dryout. Once this cooling process begins, three stages can be identified and are illustrated in Figure 2.2 on a typical heat flux versus surface temperature curve for spray cooling. 


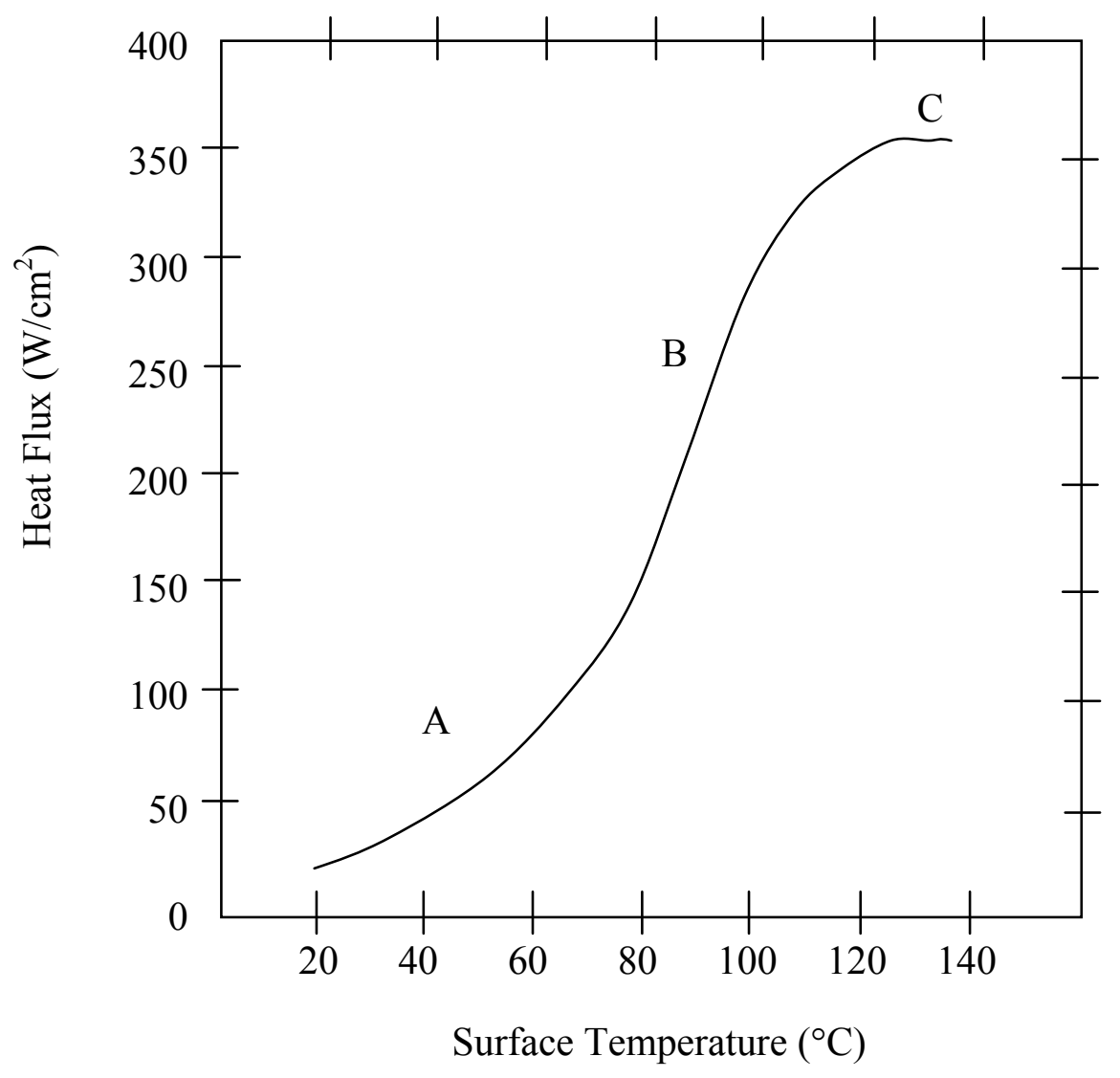

Figure 2.2:Typical Spray Cooling Heat Transfer Curve Showing 3 Regions (redrawn from Yang, 1993)

Region A: This region from Figure 2.2, corresponds to forced convection and evaporation due to the impinging droplets removing heat at the liquid/vapor interface.

Region B: In this region, the two-phase phenomenon is initiated as more and more heat is transferred to the liquid film from the heated surface. The thermophysics in this region is not clearly understood. Yang (1993) observed boiling with the subsequent formation of ridges and then vapor bubbles on the liquid-vapor interface as shown in Figure 2.3 and Figure 2.4. These photographs were taken from beneath the spray impingement area through a clear heater. 


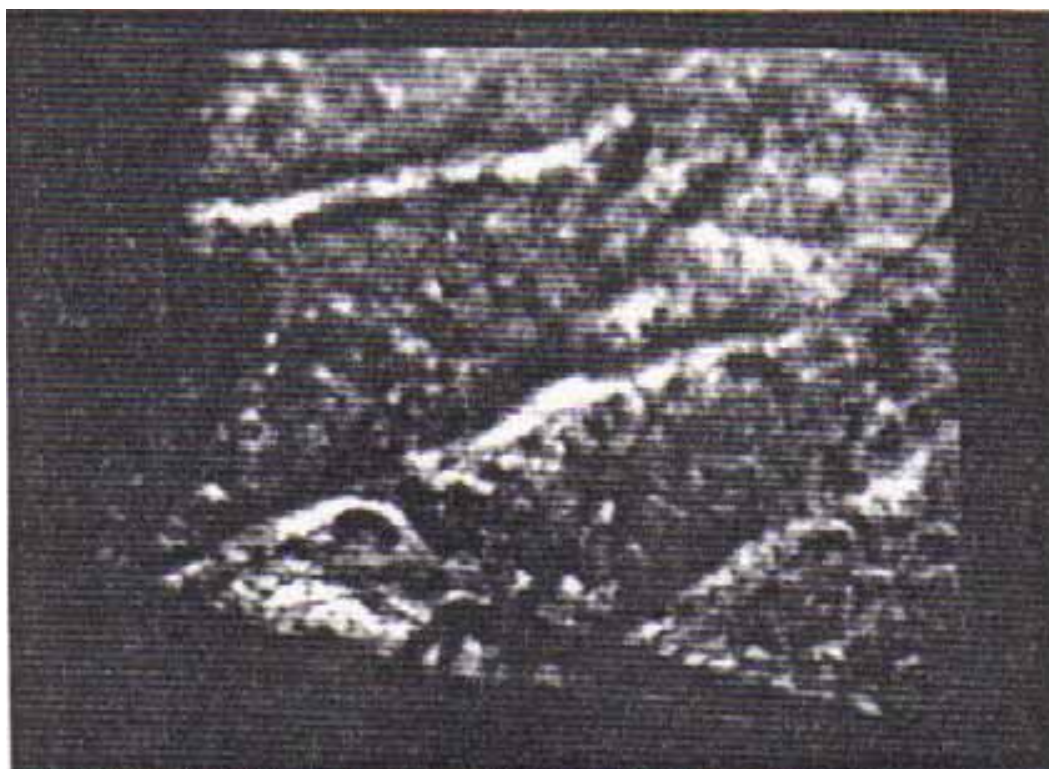

Figure 2.3: Photograph of Ridges Present on the Film Surface (Yang, 1993)

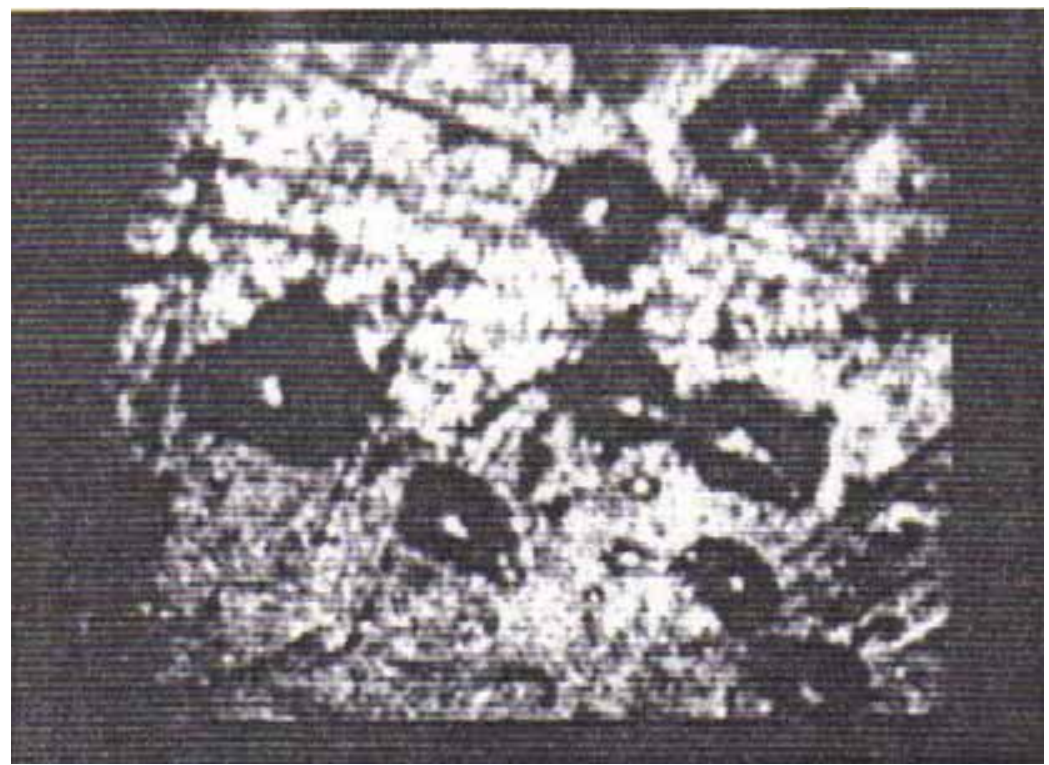

Figure 2.4: Photograph of Vapor Bubbles in the Film Surface (Yang, 1993)

A schematic taken from Chow et al. (1997) illustrates the nucleation and convection in Figure 2.5. This also shows an example of secondary nuclei studied by Mesler and Mailen (1977) that are believed to arise from entrained vapor created by bursting (venting) bubbles. Figure 2.6 shows some high speed video images and a rendering that support this theory that new bubbles form from the rupturing of previous 
bubbles. For spray cooling, the premature bursting of bubbles near the free surface due to impinging drops also may cause secondary nucleation and account for the significantly higher number of nucleation sites and higher heat transfer coefficients for thin film boiling over pool boiling (Chow et al., 1997).

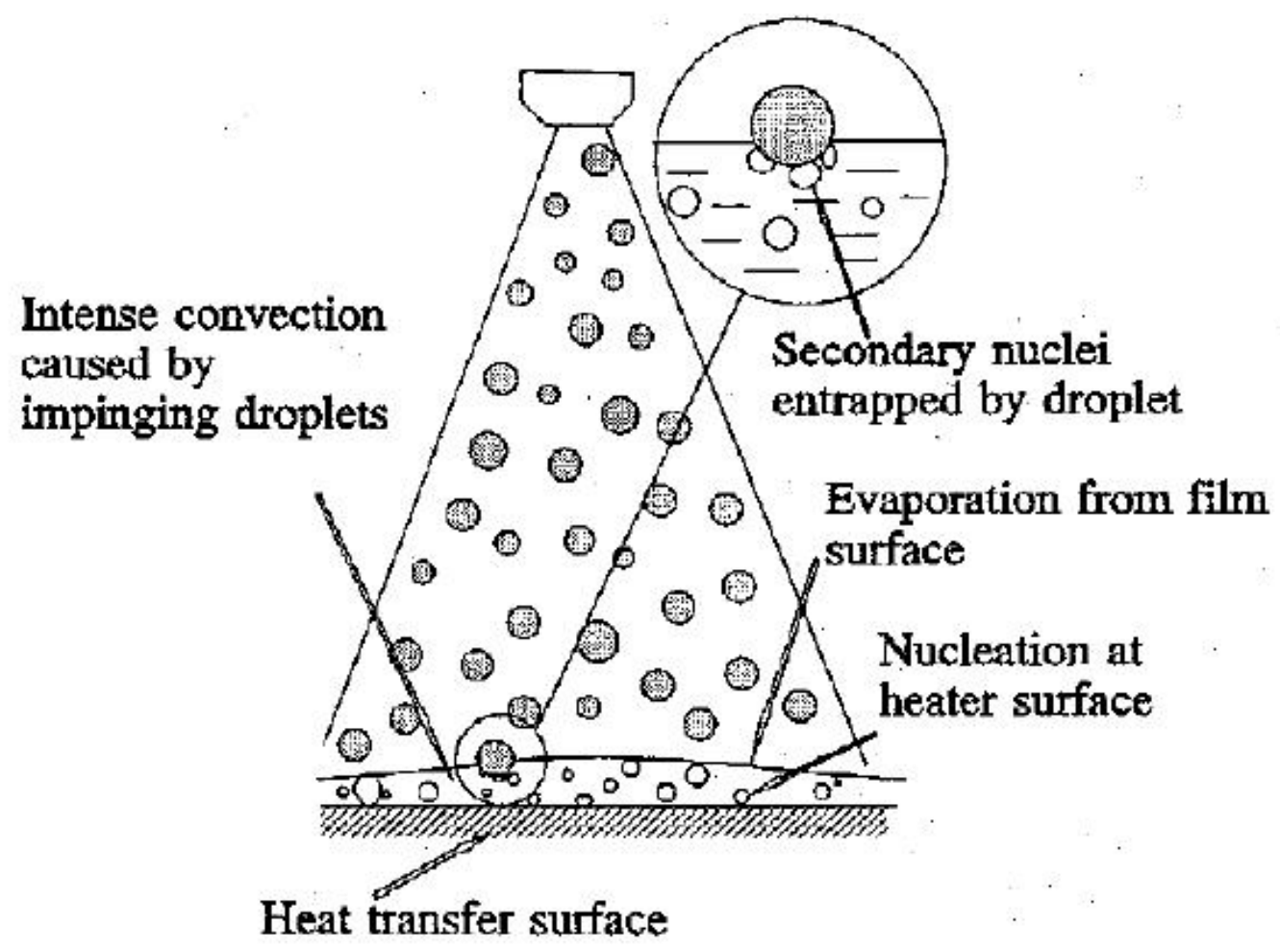

Figure 2.5: Heat Transfer Mechanisms in Spray Cooling (Chow et al., 1997) 

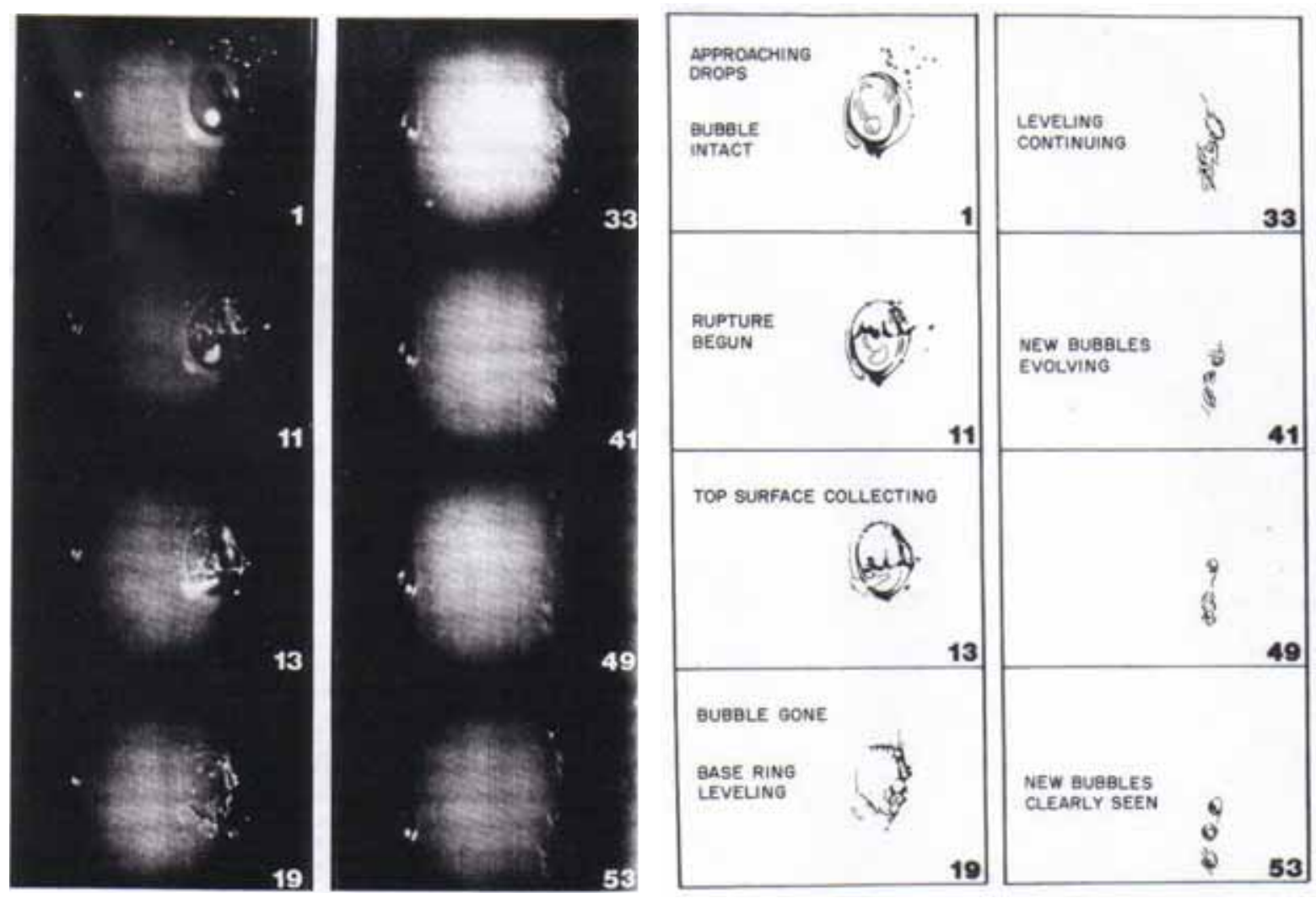

Figure 2.6: High Speed Video Images and Schematic of the Evolution of New Bubbles from the Rupture of a Previous Bubble (Mesler and Mailen, 1977)

Region $\mathrm{C}$ : In Figure 2.2, region $\mathrm{C}$ represents the critical heat flux (CHF). This point is reached in spray cooling when the surface temperature continues to rise without an increase in the heat flux. Hunnell (2005) states that critical heat flux can occur when the vapor mass flux production is greater than the rate of droplets impinging on the surface. However, other parameters can affect the on-set of critical heat flux such as the wetting of the surface and the surface temperature. A picture of a spray cooling experiment reaching critical heat flux is shown in Figure 2.7 (taken from Yang, 1993). 


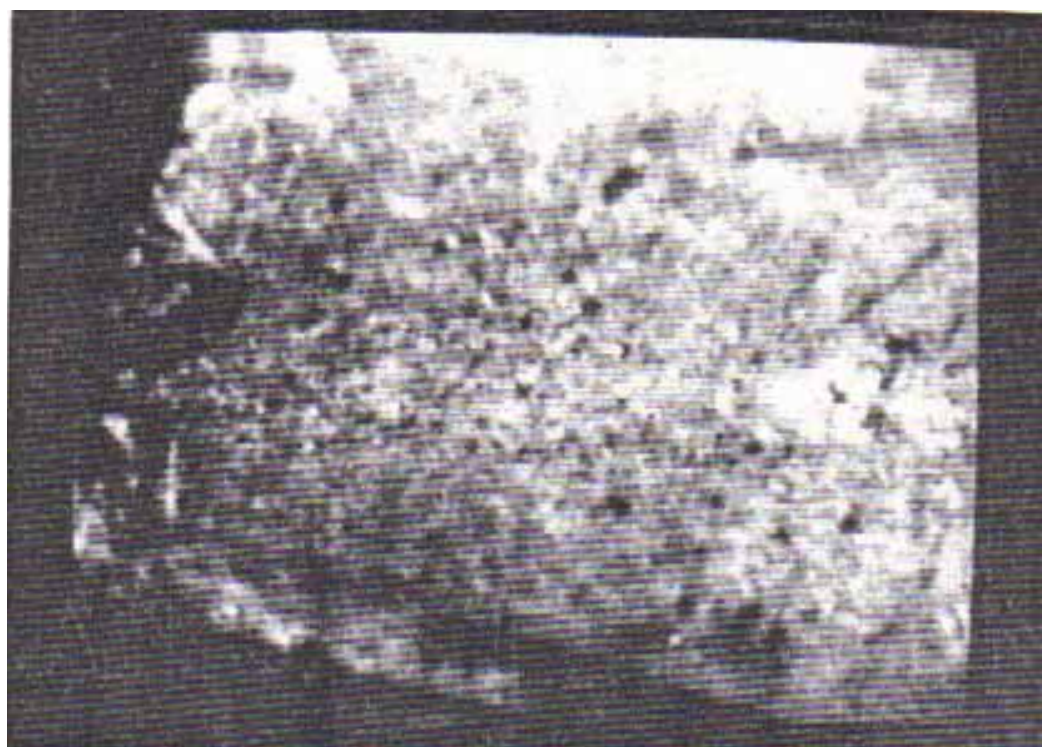

Figure 2.7: Photograph of CHF on the Spray Cooling Surface (Yang, 1993)

A macrolayer dry-out model was originally developed by Haramura and Katto (1983). The effect of forced convection on critical heat flux was studied for a vertical, flat plate in an upward parallel flow as shown in Figure 2.8. In this figure, the liquid film decreases in thickness in the direction of the flow due to evaporation. A large vapor bubble blankets the liquid film and is fed by vapor flowing from numerous vapor stems distributed throughout the liquid film. An analytical equation for the heat balance between the heat flux (q) and length of the plate (l) to the density of the liquid $\left(\rho_{1}\right)$, critical thickness of the liquid film $\left(\delta_{\mathrm{c}}\right)$, velocity of the saturated liquid $(\mathrm{u})$, and latent heat of vaporization $\left(\mathrm{H}_{\mathrm{fg}}\right)$ was developed by Haramura and Katto (1983) and is shown in Equation 2.1. 


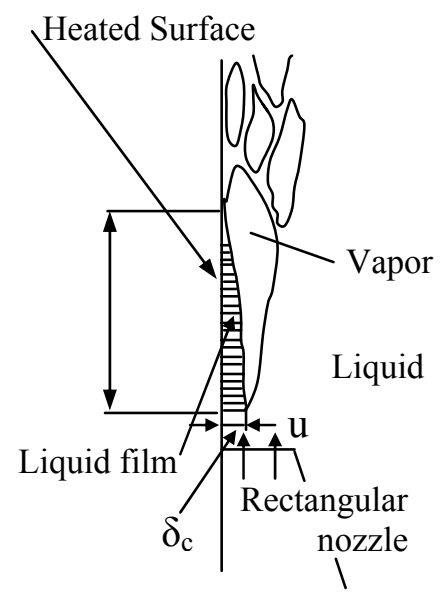

Figure 2.8: Forced Convection Boiling at High Heat Fluxes with Entrainment and Deposition of Droplets Ignored (redrawn from Haramura and Katto, 1983)

$$
q l=\rho_{1} \delta_{c} u H_{f g}
$$

The schematic shown in Figure 2.9, taken from Chow et al. (1997), shows an illustration of macrolayer dry-out for spray cooling. As vapor bubbles form and combine at the heater surface, incoming droplets or internal pressures break the bubbles and blow away the liquid above the vapor. A macrolayer forms on the heater surface and dryout occurs if the rate of incoming droplets is insufficient to wet the whole surface before boiling and convection dry out the surface. Yang (1993) observed that the dryout process occurs first at the edges of the heated surface and migrates inward as the heat flux is increased as shown in Figure 2.10. 

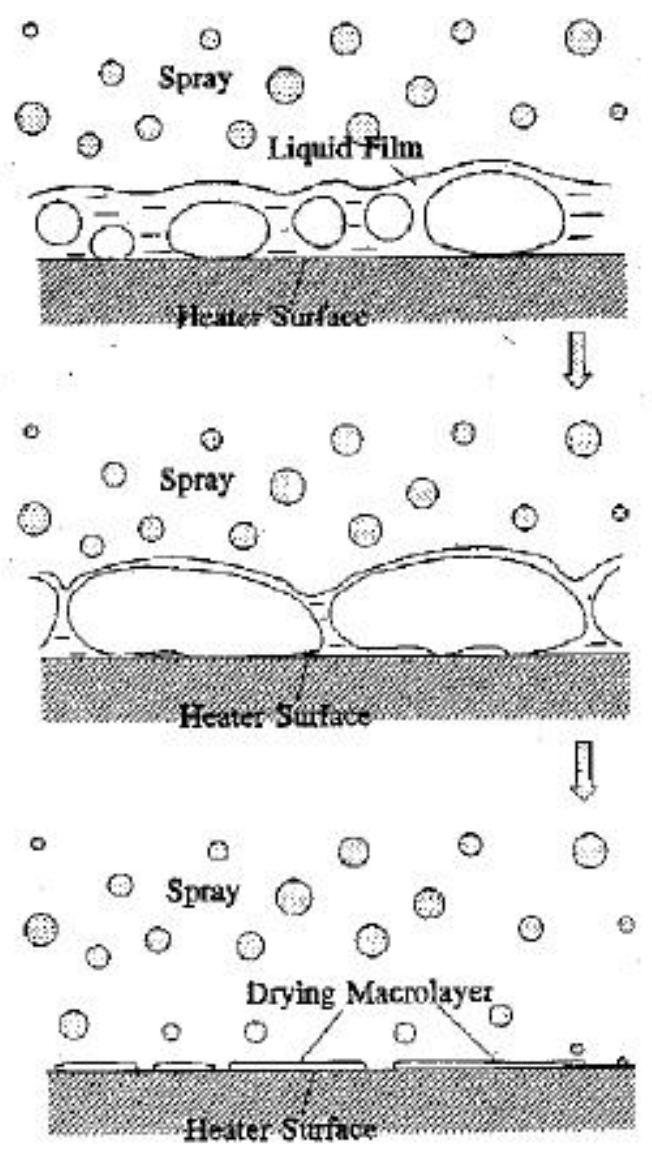

Figure 2.9: Macrolayer Dry-out Model for Spray Cooling CHF (Chow et al., 1997)
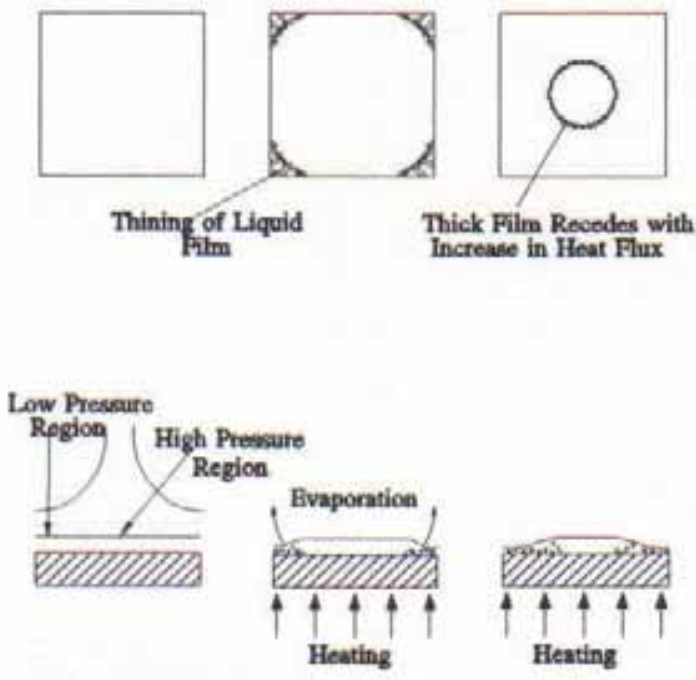

Figure 2.10: Dryout Beginning at the Edges and Receding Inward (Yang, 1993) 


\subsection{The Parameters that Affect Spray Cooling}

If the mechanisms causing critical heat flux become better understood, their negative effects can be minimized and more efficient thermal management can be achieved. Several parameters are already known to affect spray cooling, such as the type of fluid, the nozzle parameters, the heater surface, and splashing. Research to determine the specific effects of these and other mechanisms is ongoing.

Fluid: Air is by far the most economical and readily available cooling fluid, but has poor thermal transport properties. Water continues to be the most effective cooling fluid, but its high electrical conductivity excludes it from applications requiring direct contact with electrical systems unless they are given special insulating coatings. Dielectric fluorochemical liquids such as FC-72, HFE-7000, FC-87, etc., have inferior thermal transport properties as compared to water, but can be substituted in these instances (Mudawar, 2000). Figure 2.11 (taken from Mudawar, 2000) shows a comparison of heat transfer coefficients of these three fluids for natural convection, forced convection, and boiling. Note that the two-phase methods produce higher heat transfer coefficients than the single-phase methods as stated above and also shown in Figure 2.1. 

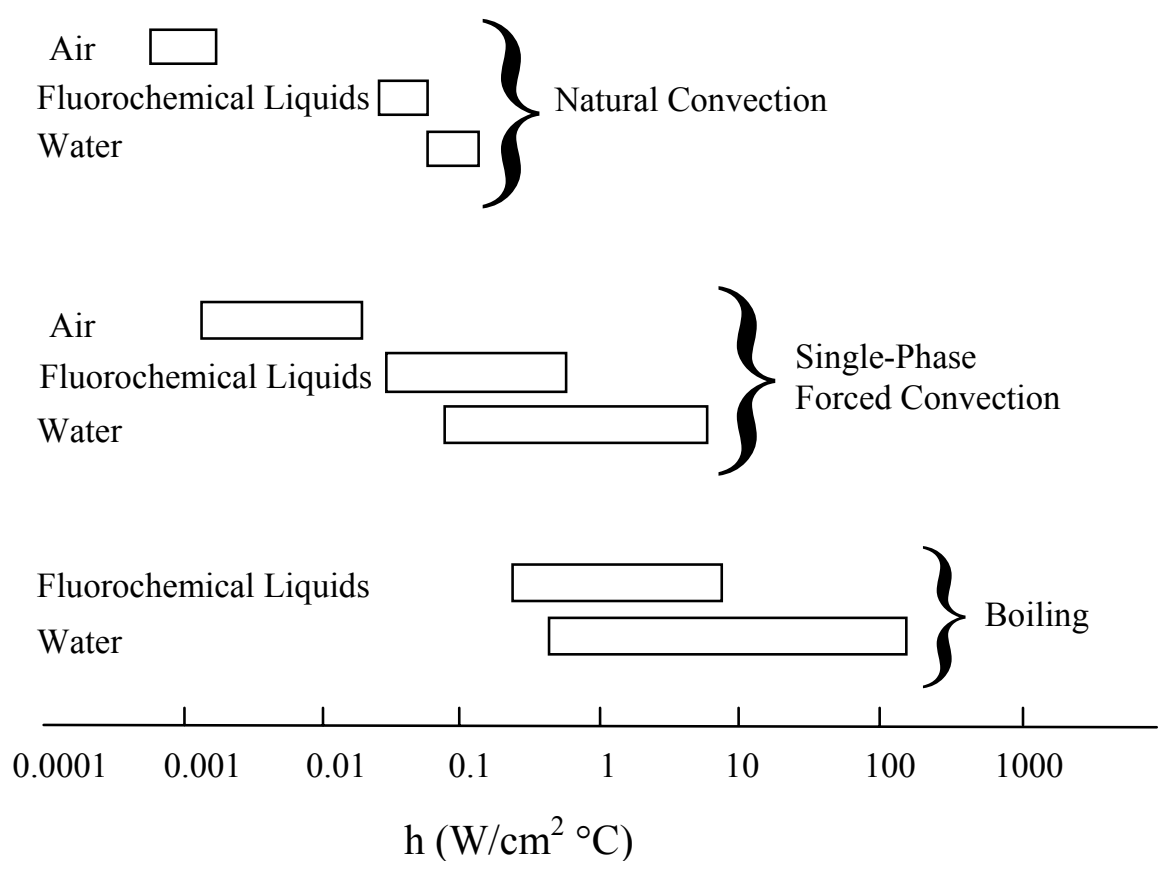

Figure 2.11: Comparison of Air, Fluorochemical Liquids, and Water for Natural Convection, Forced Convection, and Boiling (redrawn from Mudawar, 2000)

Nozzle Parameters: The nozzles for use in spray cooling can vary greatly. Common spray nozzles are classified as pressure atomized or gas-assisted atomized sprays. (Other types exist such as sonic, ultrasonic, electrostatic, etc, but are not discussed here.) Pressure sprays supply high pressure liquid through an orifice while gasassisted sprays introduce a high-pressure stream of air to aid in liquid breakup. Mudawar (2000) and Chow et al. (1997) agree that gas-assisted atomized sprays provide superior cooling performance compared to pressure sprays, but both note that the gas becomes difficult to separate from a dielectric liquid in a closed loop system. Chow et al. (1997) also states that pressure sprays are the most widely used method of spray generation, but require a minimum pressure difference to atomize the liquid. This can pose a problem if 
low flow rates are desired for a specific application because a very small orifice diameter would be required, thus increasing the likelihood of blockage.

Various nozzles also produce different spray patterns, droplet sizes, and spray densities. Spray shapes come in full cone, hollow cone, flat, square, and many other forms and can be chosen to meet specific heater surface geometries. The Sauter mean diameter (or volume-surface mean diameter) is used as a measure of individual droplet diameter within a spray. It is defined in Equation 2.2 as the ratio of the cube of the volume mean diameter to the square of the surface mean diameter, and its value is equivalent to the diameter of a drop having the same volume to surface ratio as the entire spray. In a study conducted using a Phase Doppler Particle Analyzer (PDPA), the critical heat flux was found to be greater for nozzles that produce smaller drops (Estes and Mudawar, 1995). High density sprays, corresponding to a high volumetric flux and therefore a high Weber number (defined in Equation 2.3 as proportional to the ratio of the inertial forces to the surface tension forces), are defined as sprays that provide enough impinging liquid to sustain a liquid film such that the surface remains wetted. According to Chow et al. (1997), high density water sprays achieve a higher heat flux than low density or dilute sprays (corresponding to a low volumetric flux and therefore a low Weber number) with water.

$$
\begin{aligned}
& \text { Sauter Mean Diameter }=\frac{(\text { Volume Mean Diameter })^{3}}{(\text { Surface Mean Diameter })^{2}} \\
& W e=\frac{\rho V^{2} D}{\sigma} \approx \frac{\text { Inertial Forces }}{\text { Surface Tension Forces }}
\end{aligned}
$$

Figure 2.12 (taken from Estes and Mudawar, 1995) illustrates a dilute spray and a dense spray and it can be seen that the evaporation efficiency is much higher for the 
dilute spray because the relatively low frequency of impinging droplets does not suppress the nucleation. This explains why the volumetric flux and/or Weber number has a greater significance over drop velocity due to the cumulative effect of multiple drop impingements rather than the momentary, local interaction of an individual drop (Estes and Mudawar, 1995) as demonstrated in studies conducted by Deb and Yao (1989).
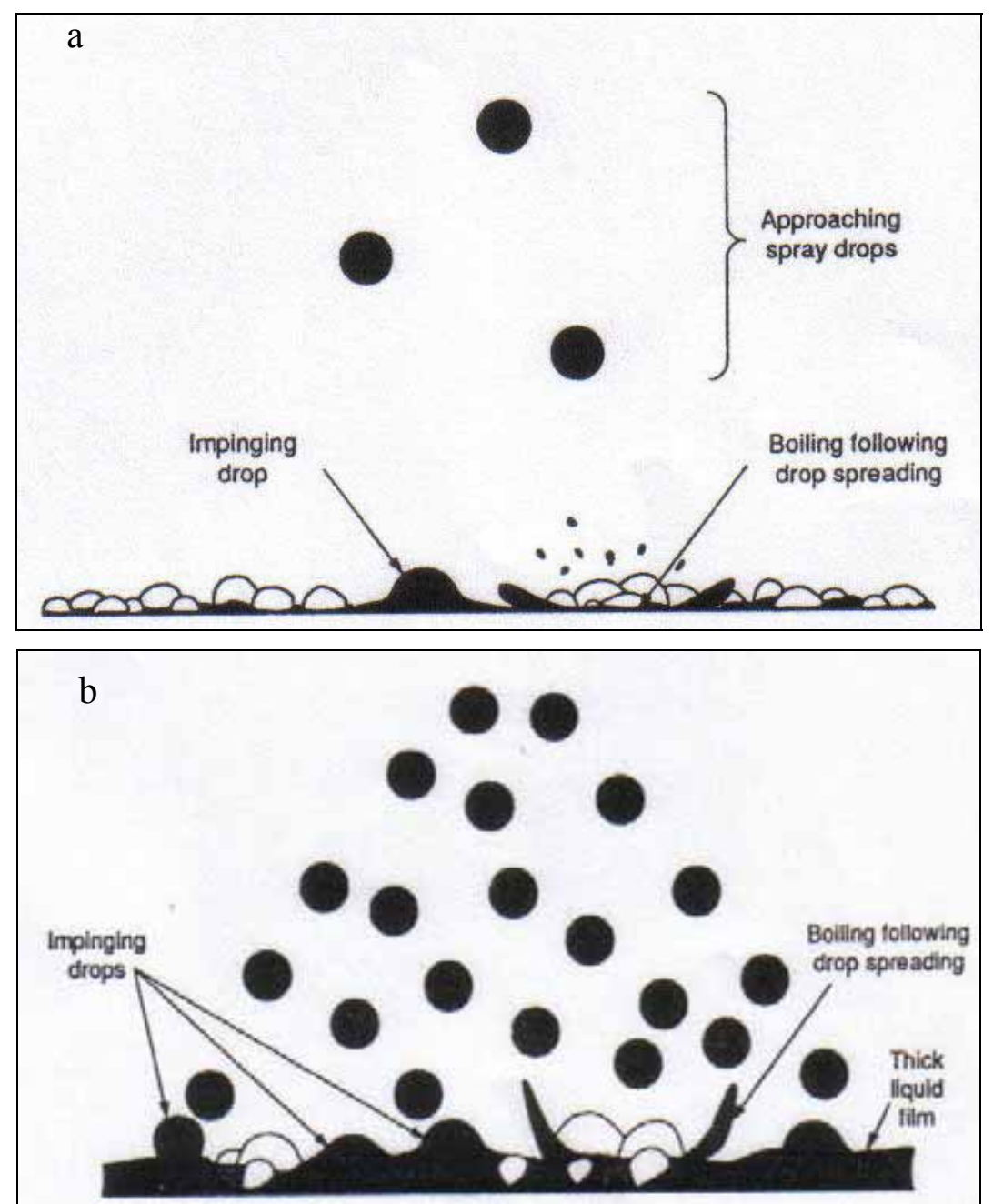

Figure 2.12: Comparison of a) Low Density, Low Weber Number and b) High Density, High Weber Number Sprays (Estes and Mudawar, 1995)

The distance between the nozzle exit and the heated surface also has an effect on spray cooling and critical heat flux. Critical heat flux is a maximum that occurs when the 
spray covers the full area of the heater surface (Mudawar, 2000). This condition can be found using simple geometry and knowing the spray angle.

Another consideration is that nominally identical nozzles with the same model numbers, from the same manufacturer often produce very different spray parameters (Chow, 1997) and each nozzle should therefore be tested to determine the most accurate spray data.

Heater Surface: Since nucleate boiling is a primary mechanism of spray cooling, it is only natural that the shape of the heater surface plays a role in heat flux. Increased surface roughness provides more potential nucleation sites and boiling at lower superheats (Chow et al., 1997). Tilton (1989) and Sehmbey et al. (1995) both observed increasing heat flux with increasing surface roughness. The results from Tilton (1989) are shown in Figure 2.13, and the results from Sehmbey et al. (1995) are shown in Figure 2.14 .

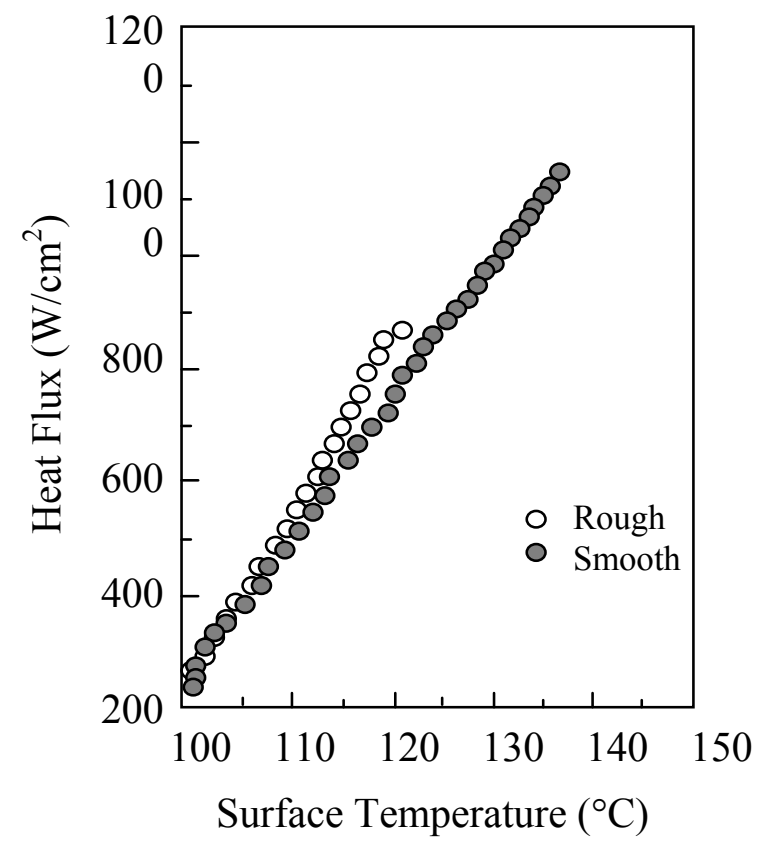

Figure 2.13: Effects of Surface Roughness at the Same Flow Conditions (redrawn from Tilton, 1989) 


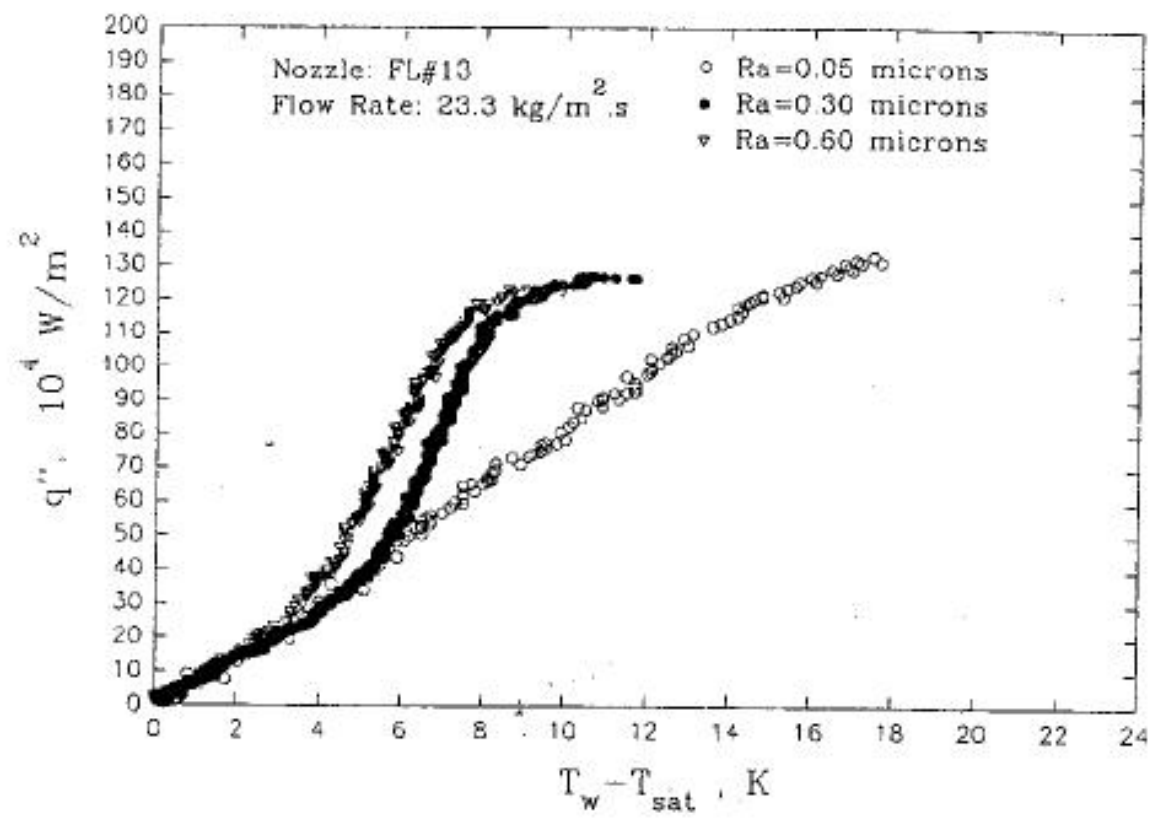

Figure 2.14: Effects of Surface Roughness (Sehmbey et al., 1995)

Horacek et al. (2005) found that the wall heat flux correlated with the contact line length and suggested that geometric structuring of the heater surface could improve the control and magnitude of the heat flux. Silk et al. (2006) studied the effects of surface enhancements such as embedded structures and compound extended surfaces (with the highest CHF obtained using straight fins and porous tunnels). This study found that CHF could be increased (with respect to a flat surface) in degassed and gassy conditions by up to $77 \%$ and $62 \%$, respectively. Figure 2.15 shows an example of the results from Silk et al. (2006) for embedded and extended surfaces in degassed conditions. 

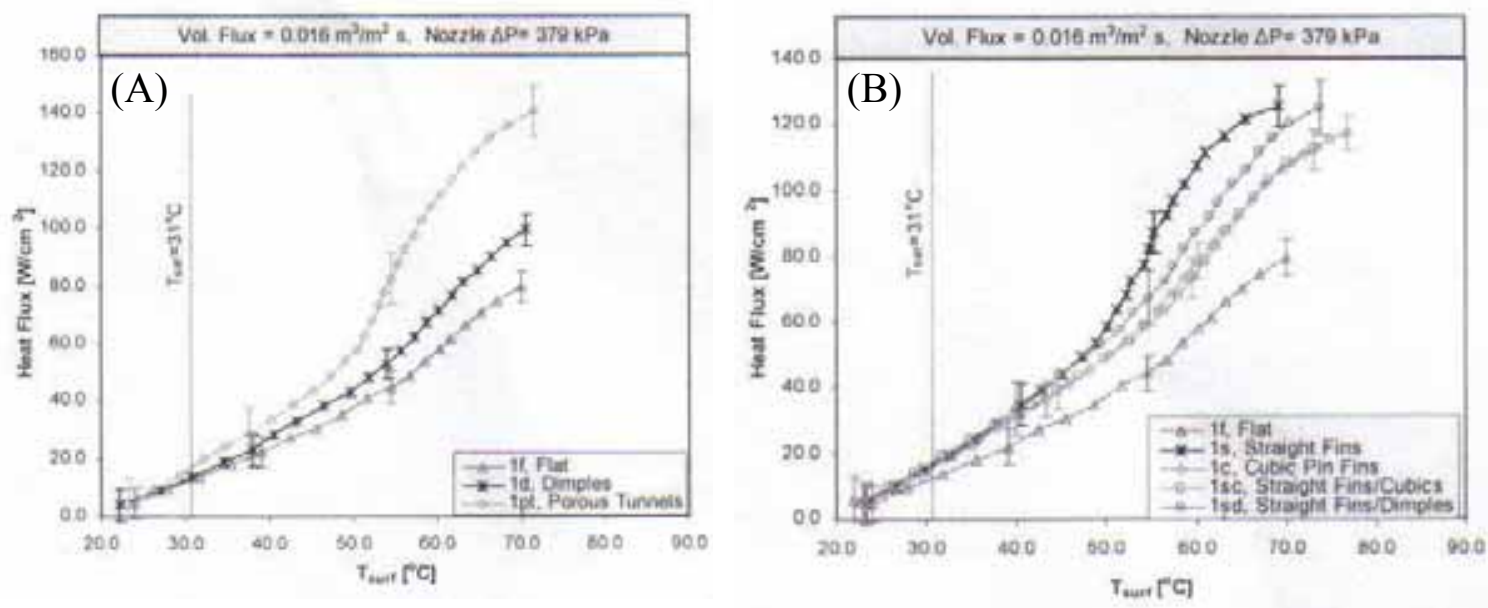

Figure 2.15: Heat Flux as a Function of Surface Temperature for (A) Embedded Structures and (B) Extended Surfaces in Degassed Conditions (Silk et al., 2006)

Splashing: The splashing of droplets also plays a role in the heat transfer characteristics of spray cooling. The liquid loss due to splashing is usually relatively low, but Tilton (1989) shows that as the heat flux was increased, the amount of liquid splashing also increased. Tilton attributed the splashing to an increase of bursting bubbles in the film. Chow et al. (1997) argue that this could also be due to droplet entrainment in the escaping vapor. This increase in splashing with an increase in heat flux contradicted a later study by Yang (1993). Yang used an air-atomizing nozzle which could account for the differences since the added air flow entrains droplets and can inhibit splashing (Chow et al., 1997).

In summary, spray cooling is considered an optimal method of high-heat flux thermal management due to its combined primary mechanisms of forced convection (due to spray drop impingement) and nucleate boiling. Many parameters complicate the behavior of spray cooling including the type of liquid used, the nozzle geometry and position, surface roughness, and splashing. Better understanding of the management of these parameters can give insight into the enhancement of the critical heat flux point. 


\subsection{Numerical Simulation Studies of Spray Cooling}

There have been several numerical simulation studies aimed at investigating spray cooling characteristics. Many of these have focused on individual mechanisms present within the complex physics of spray cooling and are therefore difficult to combine. Selvam et al. (2005) state that a "...focused effort to develop a comprehensive numerical model is a prime importance to this field," and have begun the development of models to simulate spray cooling from the fluid discharge at the nozzle exit through the convection of the fluid at the hot surface. Baysinger (2004) conducted a study using finite element software to model the conduction in an experimental spray cooling apparatus to examine the effects of volumetric flow rate, heat transfer rate, and orientation of heated surfaces on the heat transfer coefficient. Rahman et al. (1990) developed a numerical solution procedure for the computation of free surface, thin film flows in terrestrial and zerogravity environments. These types of flows are difficult to simulate using a finitedifference method because the geometry of the free surface is not known and the profile does not fit into Cartesian or cylindrical coordinates. Grover and Dennis (2001) investigated the impingement of spray droplets onto a surface to specifically aid in the advancement of automotive technologies, but the computer codes generated can be useful in many areas involving spray cooling. An illustration of an impinging spray for a fuel injector simulation as it contacts a surface (taken from Grover and Dennis, 2001) is shown in Figure 2.16. 


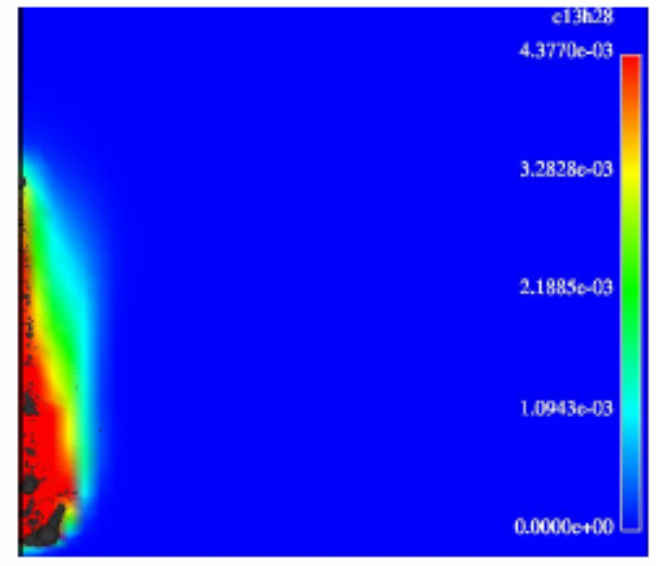

(a)

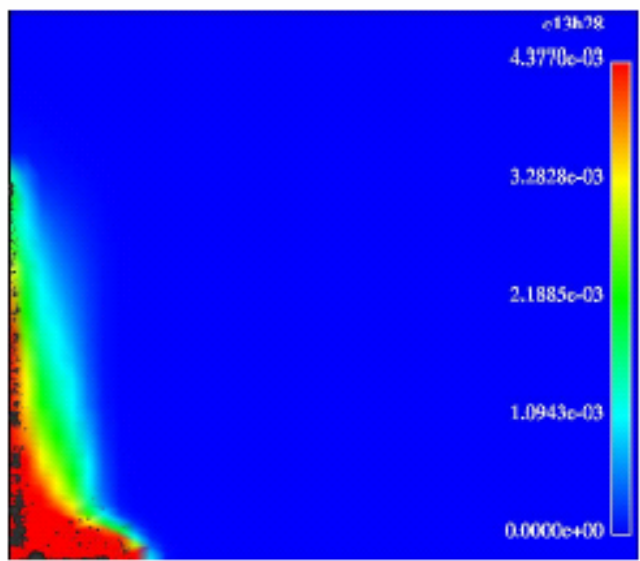

(b)

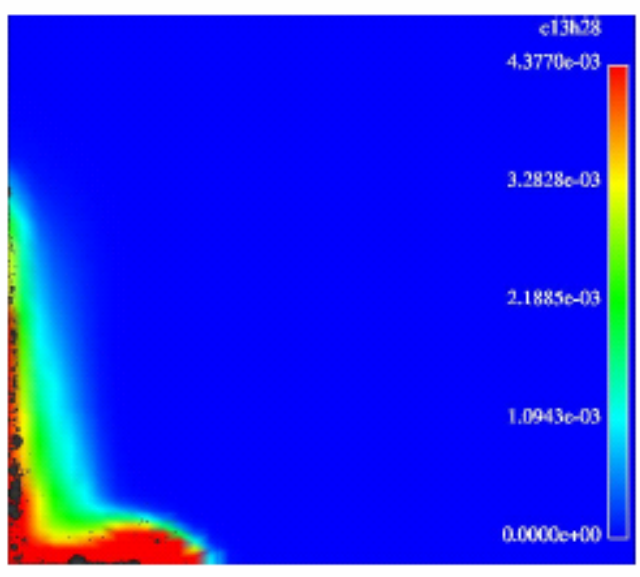

(c)

Figure 2.16: Spray Profiles at a) 0.66, b) 0.99, and c) $1.32 \mathrm{~ms}$ After Start of Injection with the Liquid Phase (Dark Areas) Superimposed onto the Vapor Phase (Grover and Dennis, 2001) 


\subsection{The Physics and Parameters that Affect Electrohydrodynamics}

Electrohydrodynamics (EHD) is the study of the dynamics of electrical forces on fluids. In the presence of an electrostatic field, dielectric fluids can be induced to flow due to forces exerted by the field on the susceptible fluid. In short, the electrical energy can be converted to kinetic energy. This phenomenon can be exploited in the case of spray cooling to aid in removal of vapor bubbles from the heated surface and thus delay dryout conditions and increase the critical heat flux. It is noted in Marco and Grassi (2002) that on Earth, gravity is likely to mask the effects of other force fields (electric, magnetic, or acoustic) and in the absence of gravity, these other force fields can be used to create a simulated gravity to increase heat transfer.

Yagoobi and Bryan (1999) give examples where EHD would be an effective method of increasing heat and mass transfer including the control of two-phase heat transfer in refrigeration and process industries as well as in microgravity conditions with few moving parts and low vibration. In these situations, EHD techniques have advantages such as rapid control of enhancement by varying the electric field, being simple and nonmechanical, being suitable for special environments (space), being applicable to single and multi-phase flows, and having minimal power consumption (Yagoobi and Bryan, 1999). Comprehensive reviews of EHD heat transfer enhancement in liquids are given by Jones (1978) and Allen and Karayiannis (1995).

When cooling a heated surface as in spray cooling or pool boiling, EHD may increase the liquid film motion which resupplies the coolant to the hot surface, and the EHD forces can also break up the vapor film patches that start to form (Yagoobi and 
Bryan, 1997). Many parameters influence the heat transfer in EHD-enhanced nucleate boiling as shown in Table 2.2.

Table 2.2: Parameters Influencing EHD-Enhanced Nucleate Boiling Heat Transfer (redrawn from Yagoobi and Bryan, 1997)

\begin{tabular}{|l|l|l|l|l|}
\hline \multicolumn{1}{|c|}{ Fluid Properties } & \multicolumn{1}{|c|}{ Electrical } & \multicolumn{1}{c|}{ Geometrial } & \multicolumn{1}{c|}{ Operating } & \multicolumn{1}{c|}{ Misc. } \\
\hline Electrical conductivity & DC applied voltage & Electrode design & Heat flux & Impurities \\
\hline Electrical permittivity & AC applied voltage & Electrode spacing & Wall superheat & Oils \\
\hline Surface tension & Uniform field & Smooth surface & Saturation temp. & Additives \\
\hline Thermal conductivity & Non-uniform field & Enhance surface & & \\
\hline Latent heat & & Well configuration & & \\
\hline Specific heat & & Tube bundle type & & \\
\hline Viscosity & & & & \\
\hline Density & & & & \\
\hline
\end{tabular}

The general electromagnetic body force per volume can be written as shown in Equation 2.4 (Hughes and Young, 1966) and explained as in Gray and Kuhlman, 2006.

$$
f=\rho_{e} E-\left(\frac{\varepsilon_{0}}{2}\right) E^{2} \nabla \kappa+\left(\frac{\varepsilon_{0}}{2}\right) \nabla\left[E^{2} \frac{\partial \kappa}{\partial \rho} \rho\right]+J \times B-\left(\frac{\mu_{0}}{2}\right) H^{2} \nabla \kappa_{m}+\left(\frac{\mu_{0}}{2}\right) \nabla\left[H^{2} \frac{\partial \kappa_{m}}{\partial \rho} \rho\right]
$$

$$
\begin{aligned}
& \text { Electro- }=\text { Coulomb Permittivity Electro- Pondero- Permeability Magneto- } \\
& \text { magnetic }=\text { Coulomb }- \text { Gradient }+ \text { stictive }+ \text { motive }- \text { Gradient }+ \text { strictive } \\
& \text { Body Force Force Force Force Force Force Force }
\end{aligned}
$$

The first term on the right represents the Coulomb force on free charges and can be used to control charged spray droplets. The second is the permittivity gradient force and the third is the electrostrictive force. These two can be rewritten as shown in Equation 2.5.

$-\left(\frac{\varepsilon_{0}}{2}\right) E^{2} \nabla \kappa+\left(\frac{\varepsilon_{0}}{2}\right) \nabla\left[E^{2} \frac{\partial \kappa}{\partial \rho} \rho\right]=\left(\frac{\varepsilon_{0}}{2}\right)(\kappa-1) \nabla E^{2}-\nabla\left(\left(\frac{\varepsilon_{0}}{2}\right) E^{2} \frac{\partial[(\kappa-1) / \rho]}{\partial(1 / \rho)}\right)$

In Equation 2.5, the first term on the right side is the electric Kelvin force. Its coefficient is always positive which means it is always directed toward higher electric field regions. The second term on the right side of Equation 2.5 is the gradient of an electric pressure and when permittivity is proportional to density (in most cases) is zero. 
The fourth term in Equation 2.4 is the ponderomotive force which is negligible for electrically insulating coolants such as FC-72 and HFE-7000. The fifth and sixth terms in Equation 2.4 are known as the permeability gradient force and the magnetostrictive force and are analogous to the second and third terms but for magnetic forces rather than electrical. For this study, there are no magnetic fields and the spray droplets are not being charged, so the dominating term is the electric Kelvin force.

A team of researchers (Marco and Grassi, 2002) has done extensive work on film boiling in the presence of an externally imposed electric field. This team has performed experiments involving a heated wire or capillary tube with orifice submerged in a liquid in laboratory, parabolic flight, sounding rocket, and drop shaft settings. Electric fields of up to $30 \mathrm{kV}$ were applied around the heater or tube. It was concluded that the fields (g or E) did not have a major effect on the nucleate boiling heat transfer coefficient but did significantly change the vapor pattern by reducing bubble size and increasing detachment frequency as can be seen in Figure 2.17. Also, CHF was increased significantly. 


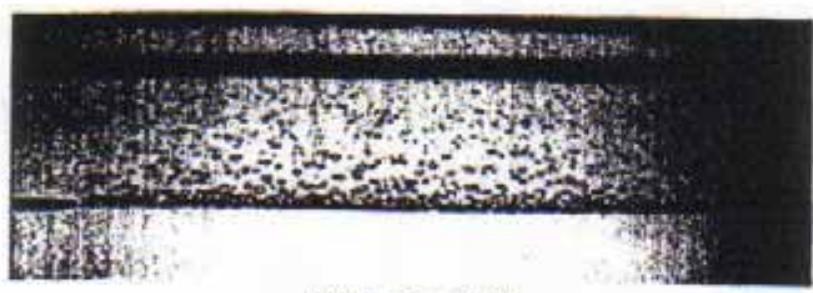

(A) $g=280.0 \mathrm{kV}$

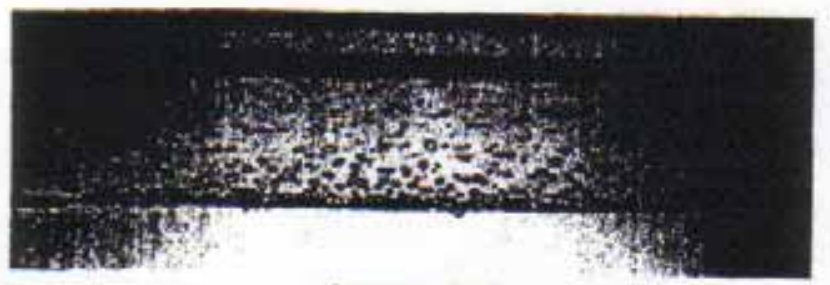

(A) $g=80.0 \mathrm{kV}$

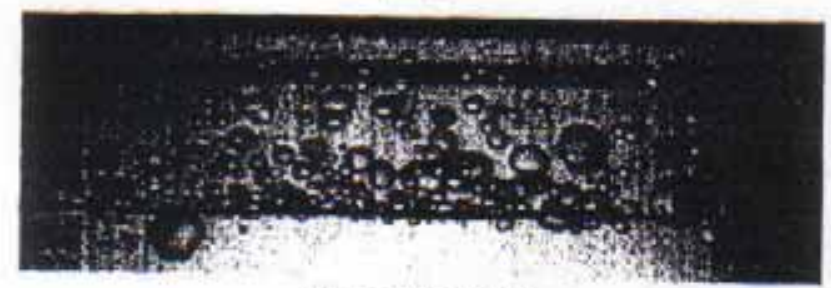

(C) $g=0.0280 .0 \mathrm{kV}$

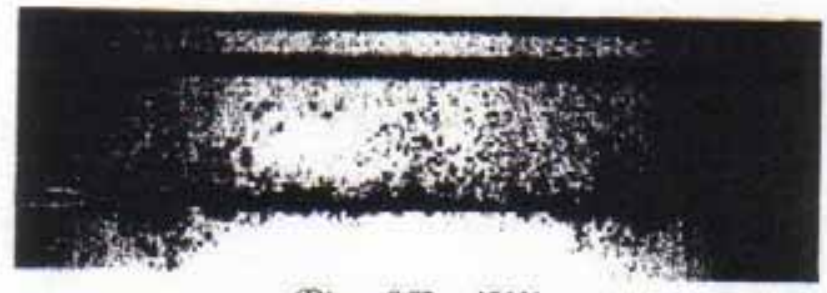

(D) $g=0.0280 .10 \mathrm{kV}$

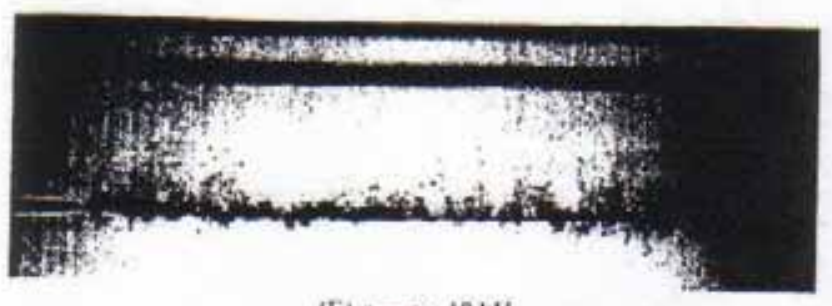

(E) $g=80.10 \mathrm{kV}$

Figure 2.17: Vapor Patterns under the Influence of Different Gravitational and Electric Fields (Marco and Grassi, 2002)

The behavior of vapor bubbles in a liquid was also studied in the presence of an electric field and Figure 2.18 shows three electric field levels $(0 \mathrm{kV}, 5 \mathrm{kV}$, and $15 \mathrm{kV})$ in microgravity and Earth gravity. Similar trends were also seen by Kuhlman et al. (2005) using the analogous magnetic field as shown in Figure 2.19. 


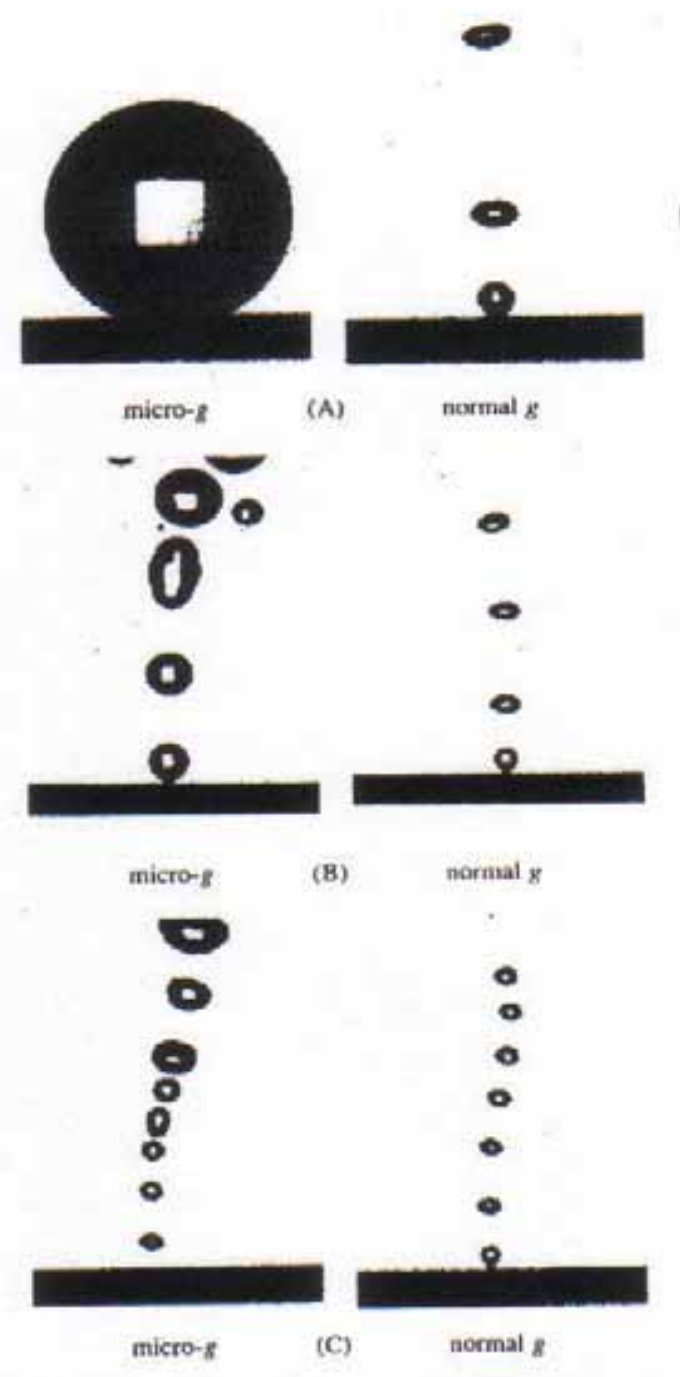

Figure 2.18: Vapor Bubble Behavior under the Influence of Different Gravitational and Electric Fields (A) $0 \mathrm{kV}$, (B) $5 \mathrm{kV}$, (C) $15 \mathrm{kV}$ (Marco and Grassi, 2002)

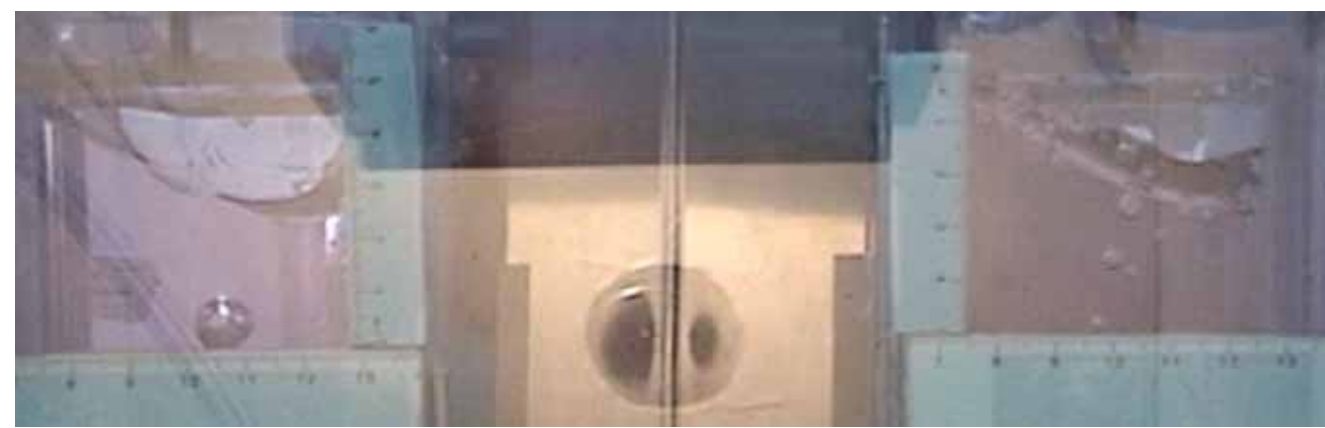
(A) No Magnet
(B) Magnet

Figure 2.19: Vapor Bubble Behavior in Microgravity under the Influence of (A) No Magnetic Kelvin Force and (B) the Magnetic Kelvin Force (approximately $20 \mu \mathrm{N}$ ) (Kuhlman et al., 2005) 
Marco and Grassi concluded that critical heat flux can be very sensitive to both gravity and electric fields. Therefore, the study of spray cooling using electrohydrodynamics to alter the flow pattern may prove useful in increasing the thermal management regime. 


\section{Chapter 3: Experimental Apparatus and Procedure}

The experimental apparatus used for this study consists of a base, a spray chamber, and a variety of electrodes as seen in Figure 3.1. The spray cooling fluids used for the tests were FC-72 and HFE-7000. These are both fluorinert electronic liquids from 3M. FC-72 has a boiling point of $56^{\circ} \mathrm{C}$ at $1 \mathrm{~atm}$, a vapor pressure of $30.9 \mathrm{kPa}$, and a thermal conductivity of $0.057 \mathrm{~W} / \mathrm{m}^{\circ} \mathrm{C}$ at $25^{\circ} \mathrm{C}$. HFE-7000 has a boiling point of $34{ }^{\circ} \mathrm{C}$ at $1 \mathrm{~atm}$, a vapor pressure of $64.4 \mathrm{kPa}$, and a thermal conductivity of $0.075 \mathrm{~W} / \mathrm{m}^{\circ} \mathrm{C}$ at 25 ${ }^{\circ} \mathrm{C}$. Water is also used in a separate flow loop to cool the spray chamber. The entire flow schematic can be seen in Figure 3.2. The FC-72 or HFE-7000 is pumped from a reservoir and into the spray chamber where it impinges on a heated surface. An electrode (or group of electrodes) is placed near the heated surface to attempt to control the motion of the impinging fluid. Another pump removes the liquid from the chamber and passes it through a bypass loop or heat exchanger back to the reservoir. Flowrates and pressures are measured at points indicated in Figure 3.2. The water in the cooling loop is pumped from a reservoir through coils that wrap around the outside of the chamber. It then passes through a heat exchanger and back to the reservoir. Flowrates and pressures of the water are also measured at points indicated.

This chapter details the design and construction of the experimental spray chamber apparatus in Section 3.1 and the electrode configurations in Section 3.2. The experimental base used for this study was designed and constructed as outlined by Hunnell (2005), but several modifications were made to this base and are also summarized here in Section 3.3. The procedure used to collect each set of data is also discussed in Section 3.4. 


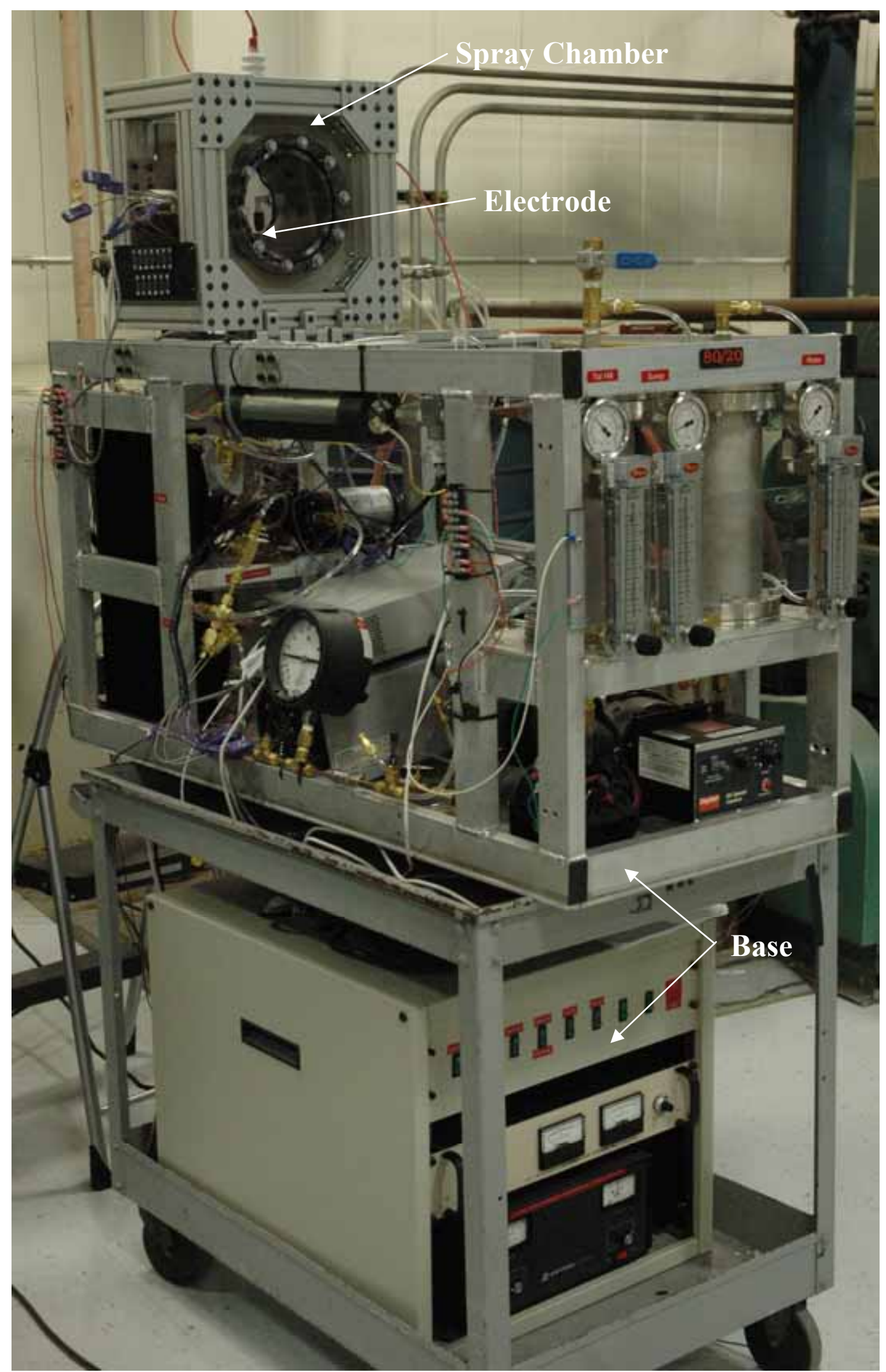

Figure 3.1: Spray Chamber and Base 


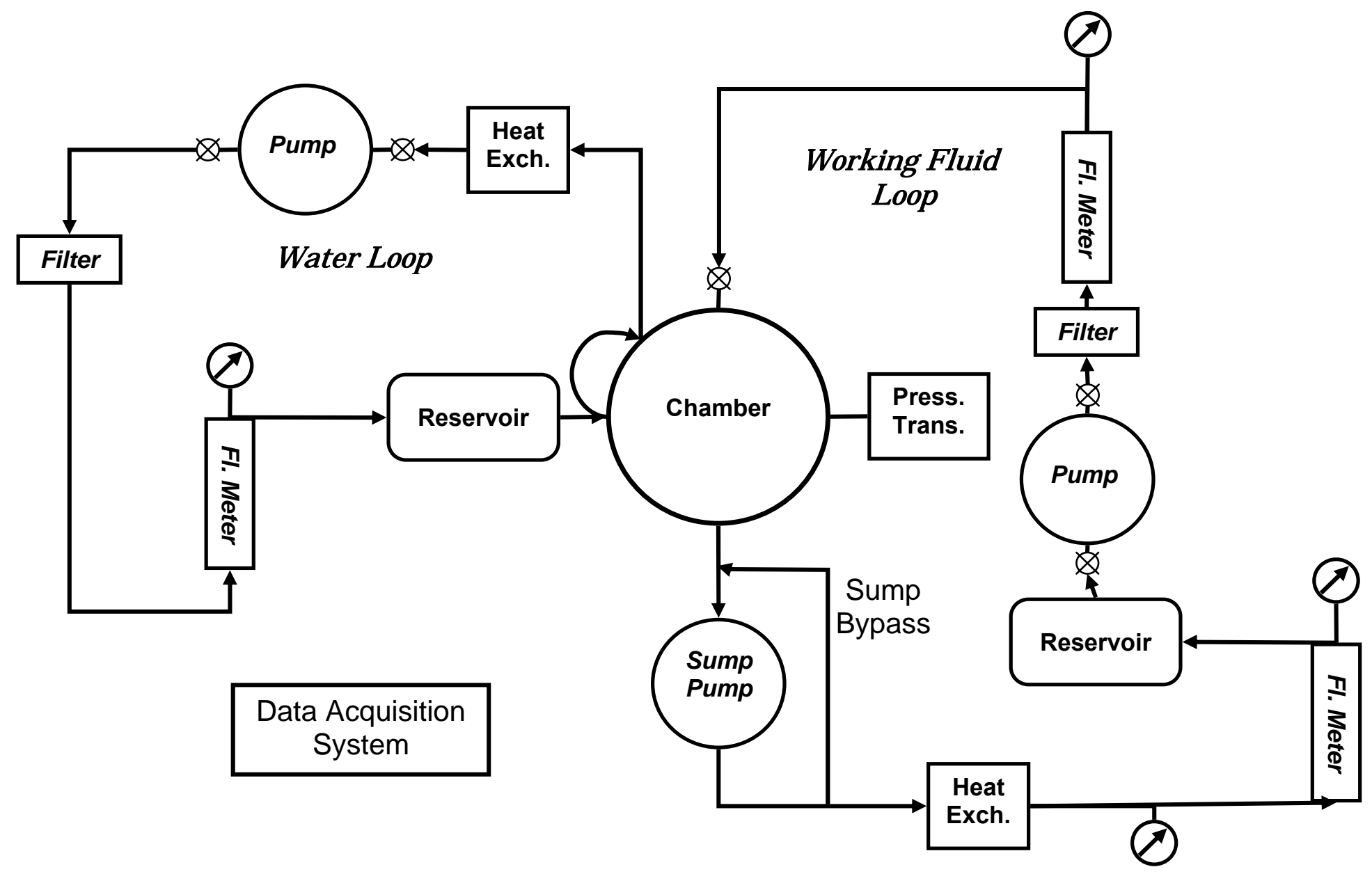

Figure 3.2: Schematic of Apparatus Flow Loop (modified from Kreitzer, 2006) 


\subsection{Spray Chamber Configuration}

The spray chamber configuration shown in Figure 3.3 was constructed from stainless steel. This chamber houses the spray nozzle, heated surface, and electrodes and is mounted on top of the experimental base. The main chamber area has an inner diameter of six inches, is six inches deep, and has a wall thickness of 0.109 inches. The open ends have fixed flanges that are eight inches in diameter with a 20-bolt-hole pattern (tapped 5/16-24) in each for attaching 1.3-inch thick, clear, polycarbonate viewports. Two additional ports, with rotatable flanges, are connected to the main chamber. The top port is 2.5 inches in diameter with a 4.5 -inch flange (8-bolt-hole pattern, tapped 5/16-24) and the bottom is 1.5 inches in diameter with a 2.75 -inch flange (6-bolt-hole pattern, tapped 1/4-28). Four NPT ports are included on the main chamber to accommodate a pressure transducer, thermocouple feedthrough, and flow nozzle feedthrough. The other NPT port is extra.

The pressure transducer is an Omega PX303-200A5V (with a range of 0 to 200 psia) and fits into a 1/4" NPT port (NPT \#2 in Figure 3.3); the thermocouple feedthrough is a Conax MHC5-062-A6-V and fits into a 1/2" NPT port (NPT \#3 in Figure 3.3). Both of these ports are located on the same side of the chamber on the horizontal centerline. The nozzle feedthrough fits into a $1 / 2$ " NPT port located opposite the pressure transducer and thermocouple feedthrough (NPT \#1 in Figure 3.3). The copper tubing leading to the nozzle is 0.25 inch OD and the nozzle is a Spraying Systems Co. Full Jet 1/8G-PVC. This nozzle produces a full cone spray with droplets that have the average diameter of 48 microns at 9.0 GPH of FC-72 (Yerkes, 2006). A PTFE, 90 degree pipe fitting attaches the nozzle to the copper tubing. The additional 1/2" NPT port (NPT \#4 in Figure 3.3) was 
sealed with a stainless steel hex head plug. Photographs of the pressure transducer, thermocouple feedthrough, nozzle assembly, and plug attached to the chamber are shown in Figure 3.4

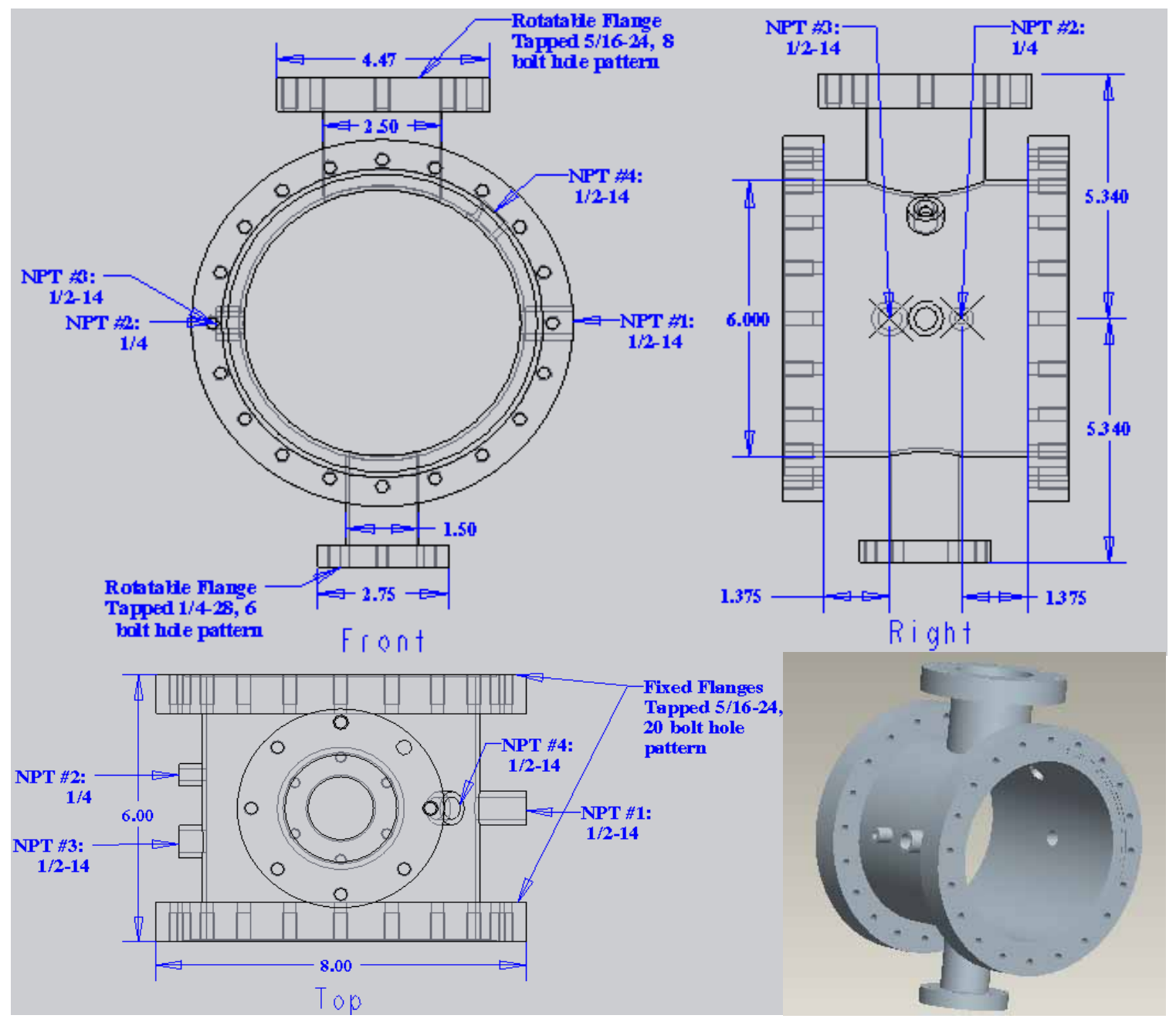

Figure 3.3: Main Spray Chamber Configuration (Dimensions in Inches) 

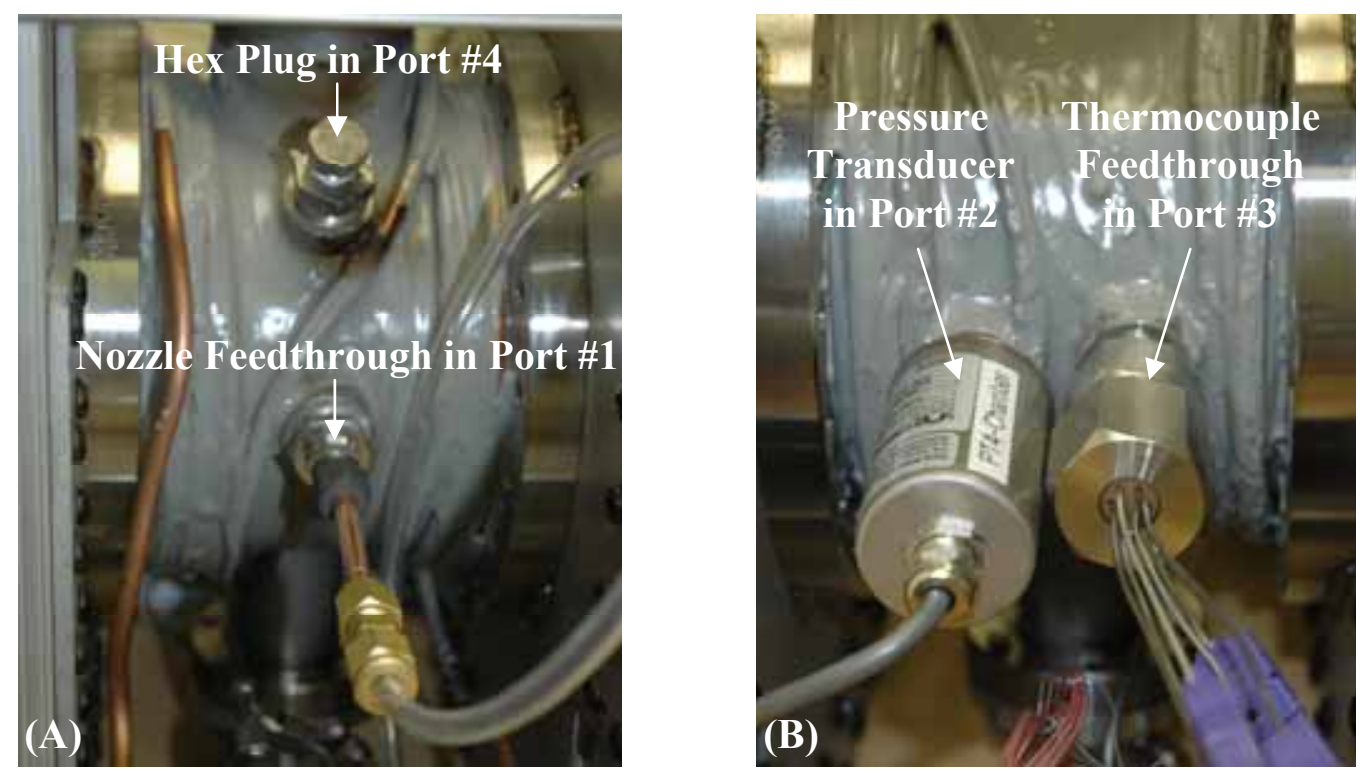

Figure 3.4 Chamber Side Views Showing A) Nozzle Assembly and Plug, B) Pressure Transducer and Thermocouple Feedthrough

The 8-inch flanges on the main chamber were welded to two stainless steel mounting plates cut with a water jet to the pattern shown in Figure 3.5. The frame of the chamber, made from 10-inch long, 2-inch square 80/20 aluminum extrusions and brackets, was connected to these plates with $1 / 4-20$ bolts and nuts as shown in Figure 3.6.

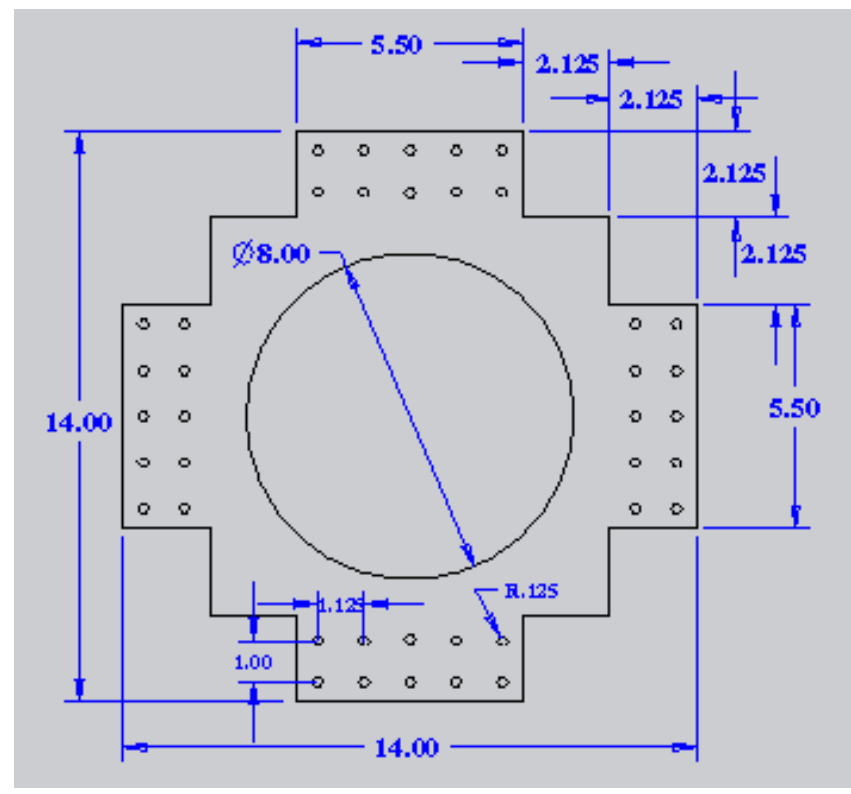

Figure 3.5: Stainless Steel Mounting Plate (Dimensions in Inches) 


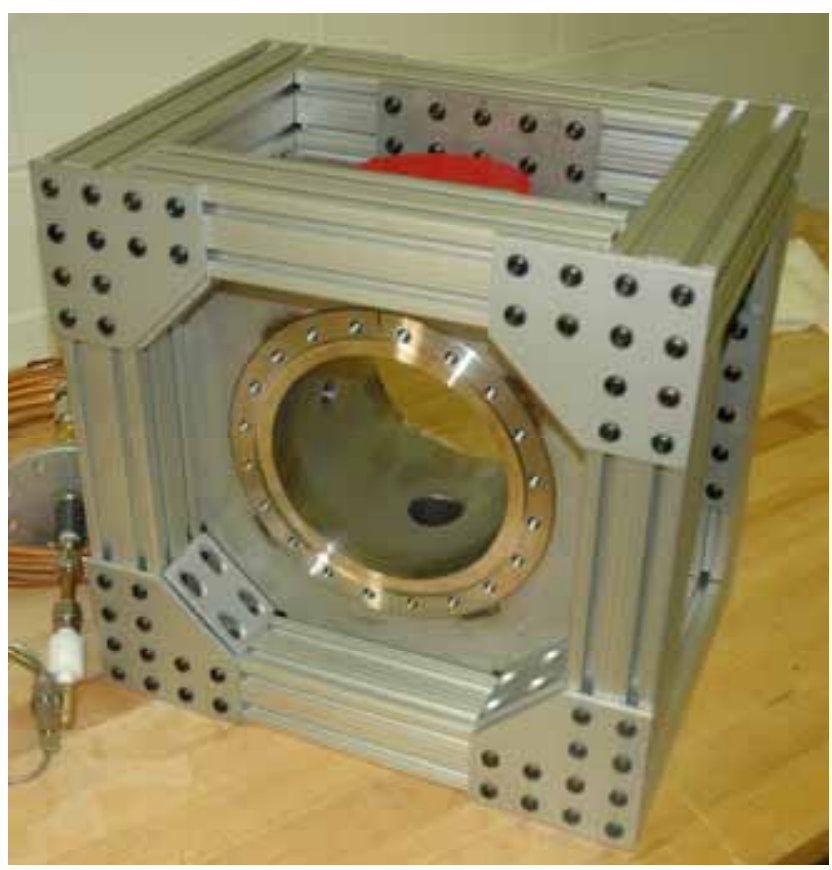

Figure 3.6: Spray Chamber with Mounting Plates and Aluminum Frame

The copper tubing used for the water cooling loop shown in Figure 3.2 was wrapped around the main spray chamber and held into place using Masterbond EP21ANHT epoxy baked for 40 minutes at 300 degrees F. This epoxy has an exceptionally high thermal conductivity (22 BTU in $/ \mathrm{ft}^{2} \mathrm{hr}$ F) to aid in heat transfer from the chamber to the water coolant and also has excellent electrical insulation properties to aid in isolating the high voltage in the chamber. Figure 3.7 shows the spray chamber with the water cooling loop attached. 


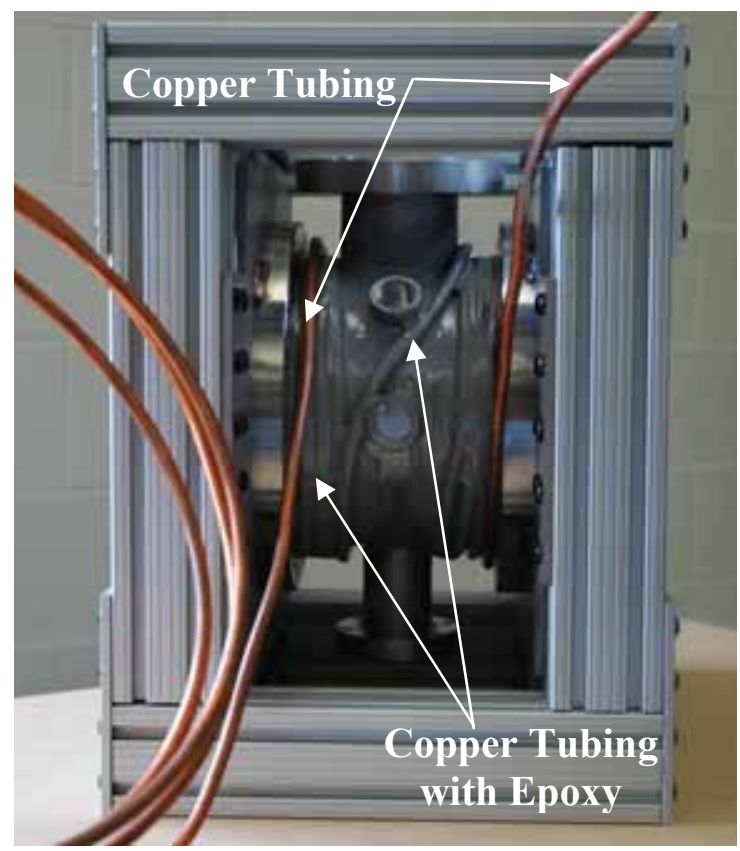

Figure 3.7: Spray Chamber with Copper Water Cooling Loop Attached

A high voltage feedthrough was chosen to fit into the 2.5 -inch port on the top of the main chamber. It is an Insulator Seal 9442012 and is shown in Figure 3.8. It is rated to $30 \mathrm{kV}$. A pin connector, also shown in Figure 3.8, is attached to the end of feedthrough with a crimp ring. This connector then attaches to a test electrode with a wire. The conductor was bent and shortened slightly to fit correctly into the test chamber, and it was also covered in heat shrink tubing. These modifications are not shown in Figure 3.8. The other end of the high voltage feed through attaches to a high voltage power supply with an Alden high voltage plug and receptacle that is also rated to $30 \mathrm{kV}$.

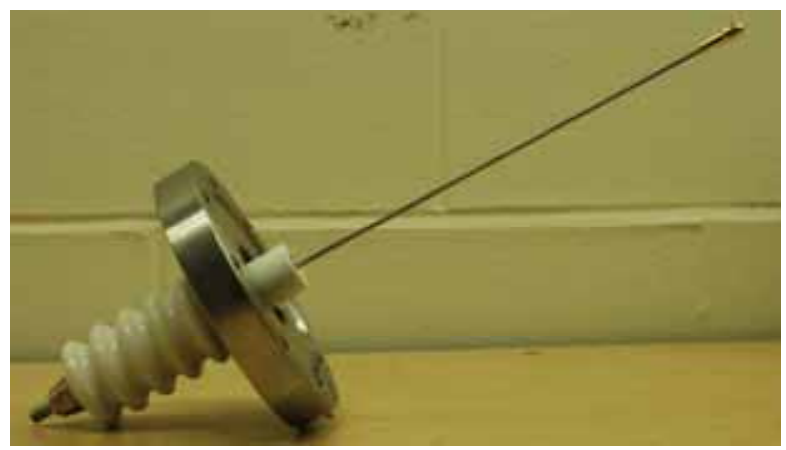

Figure 3.8: Insulator Seal High Voltage Feedthrough with Pin Connector 
A sump was designed to fit into the 1.5 -inch port on the bottom of the main chamber to drain excess fluid and is modeled after the sump used in Baysinger et al. (2004). This sump was originally constructed entirely of polycarbonate, but due to a crack that developed in the plastic base, the bottom portion was reconstructed from aluminum and the two were pinned together. A schematic of the sump dimensions is show in Figure 3.9 and a photograph is shown in Figure 3.10.

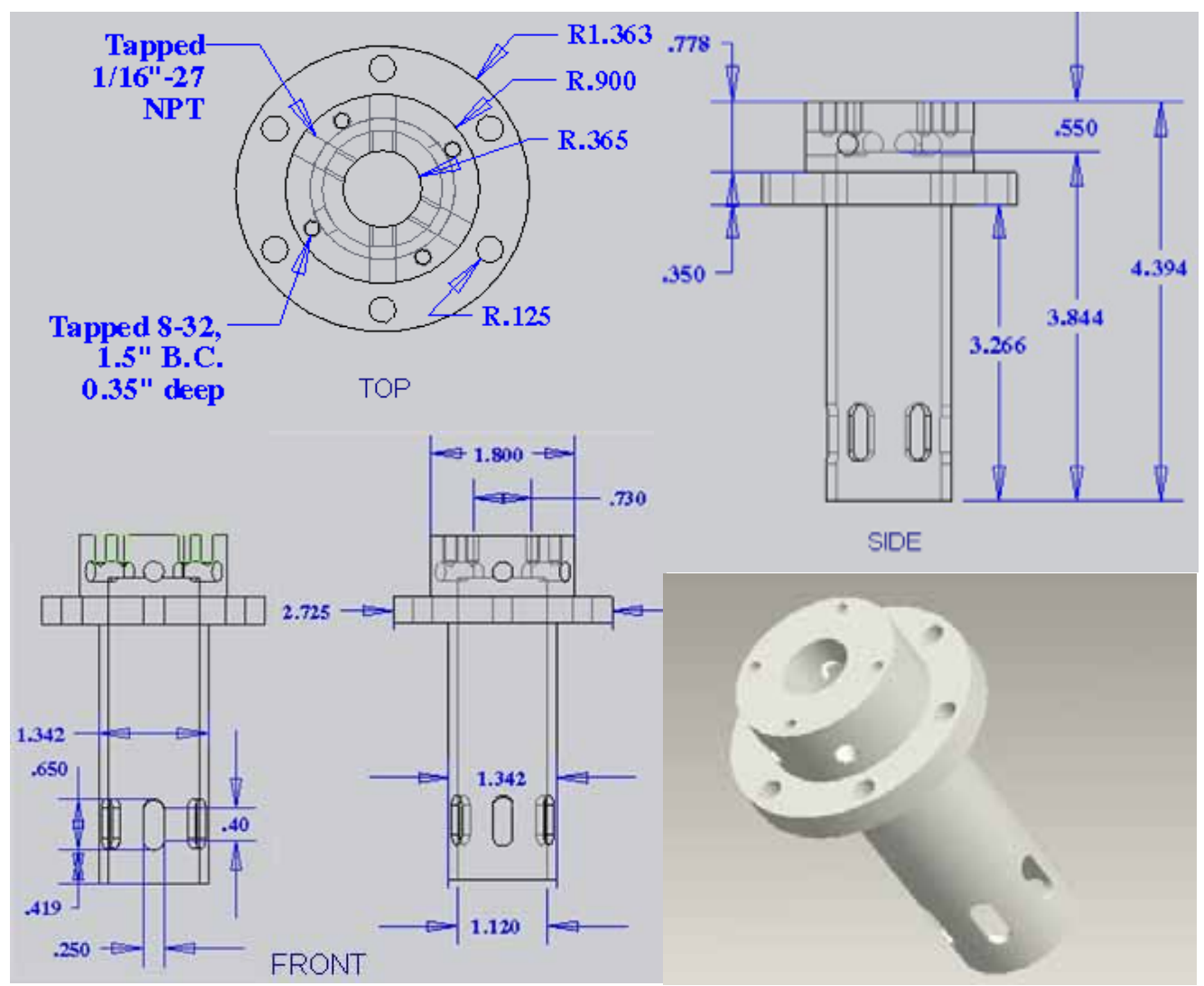

Figure 3.9: Sump Configuration (Dimensions in Inches) 


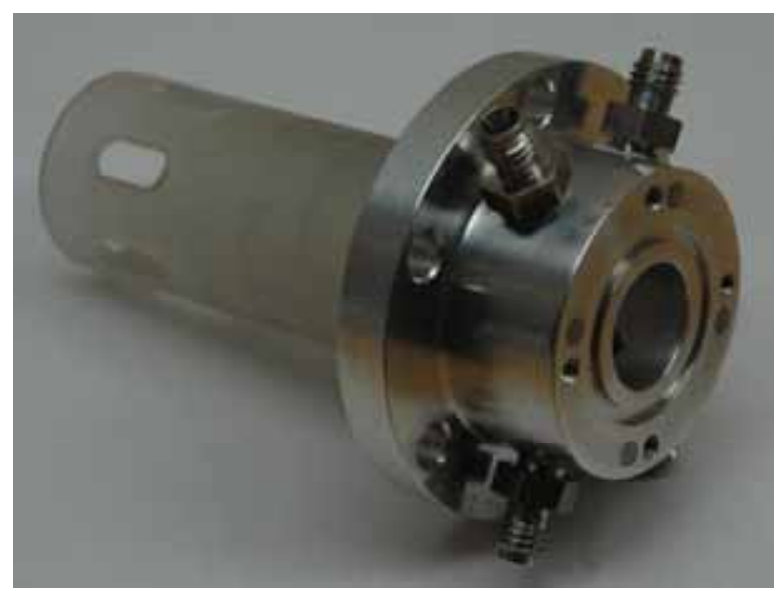

Figure 3.10: Polycarbonate and Aluminum Sump with Pipe Fittings Attached

This sump was designed to house one of three types of pedestal heater assemblies. The heated surface is attached to the top of the pedestal and thermocouples are embedded within them. The first type of pedestal built by Hunnell, 2005, was used in the spacing comparison test, and was made of glass and is shown in Figure 3.11 (before the attachment of the TFR heater).

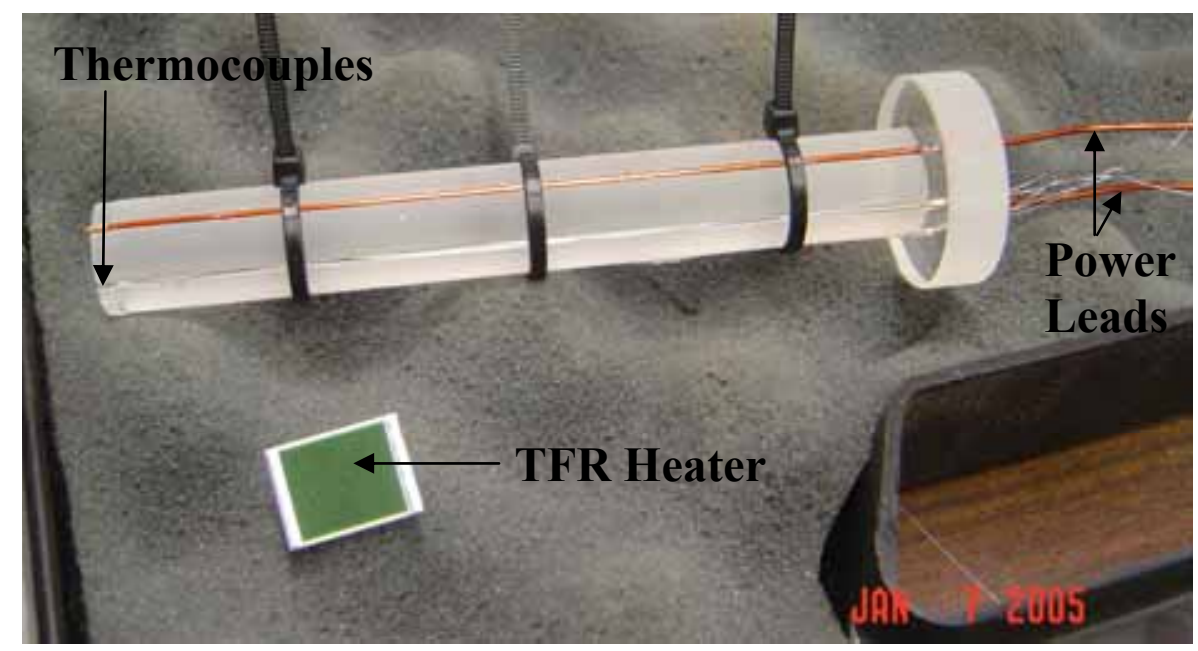

Figure 3.11: Spacing Comparison Pedestal Assembly with Unattached TFR

The second type was built from the same design for the cap electrode comparison study but was made from PTFE. It has seven thermocouples embedded within it. The dimensions for both of these pedestals (the spacing comparison glass pedestal and the cap 
electrode PTFE pedestal) and the positions of the thermocouples are shown in Figure 3.12 and Figure 3.13, respectively. Thin (approximately $1 \mathrm{~mm}$ ) wafers were cut and scored as shown in Figure 3.13 and recombined as indicated in Figure 3.14. These wafers were cut in order to correctly position the thermocouples in between each of them. The rod, wafers, and base were attached together on the PTFE pedestal by first treating the plastic with an etching agent and then using an epoxy adhesive. A thick film resistor (TFR) heater from Mini-Systems, Inc. was attached to the top of the pedestals with epoxy and connected to a power supply with copper wire and EPO-TEK H20E electrically conductive epoxy as shown in Figure 3.15. A study of the TFR heater composition is given in Appendix A. (A Type 1 TFR with a top glass layer was used for the spacing comparison pedestal and a Type 2 TFR without the top glass layer was used for the cap electrode pedestal.)

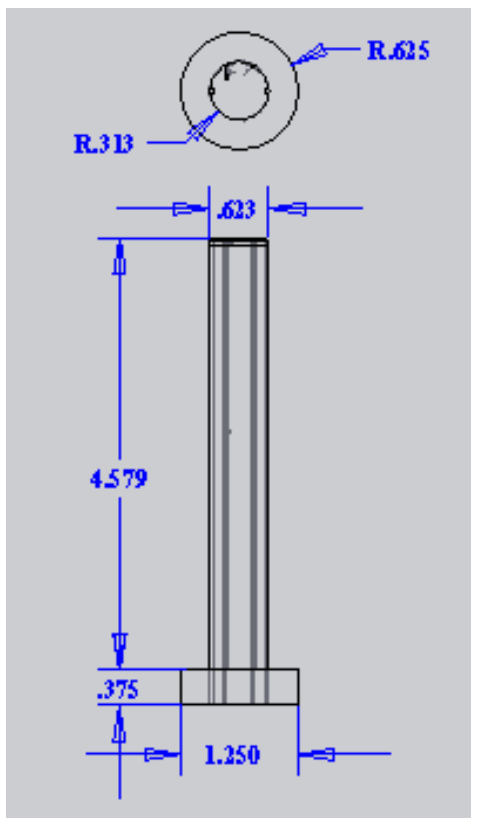

Figure 3.12: Cap Electrode Pedestal (Dimensions in Inches) 
Section at top surface

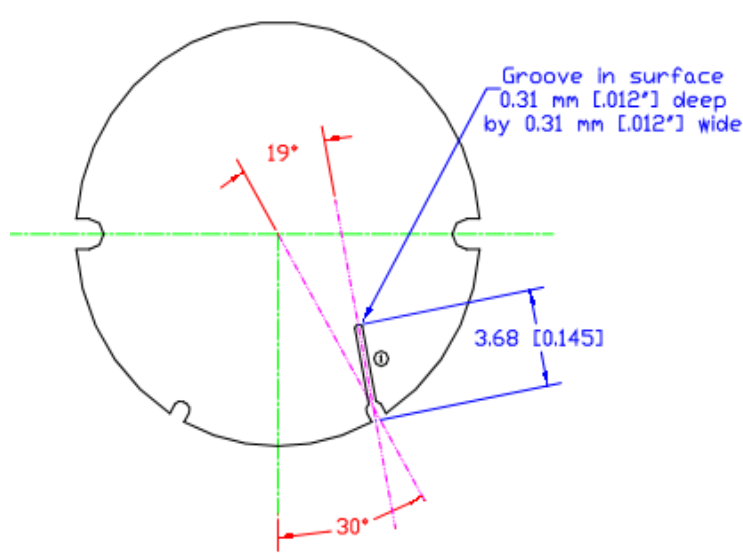

Section at $1 \mathrm{~mm}$ depth

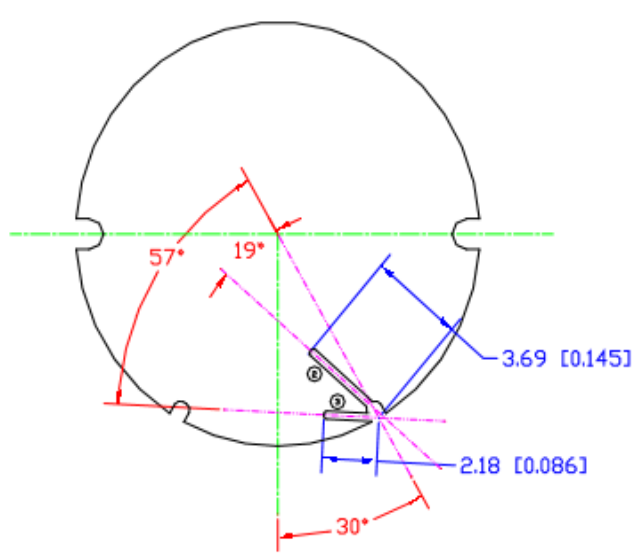

Section at $50 \mathrm{~mm}$ depth
Section at 2 mm depth

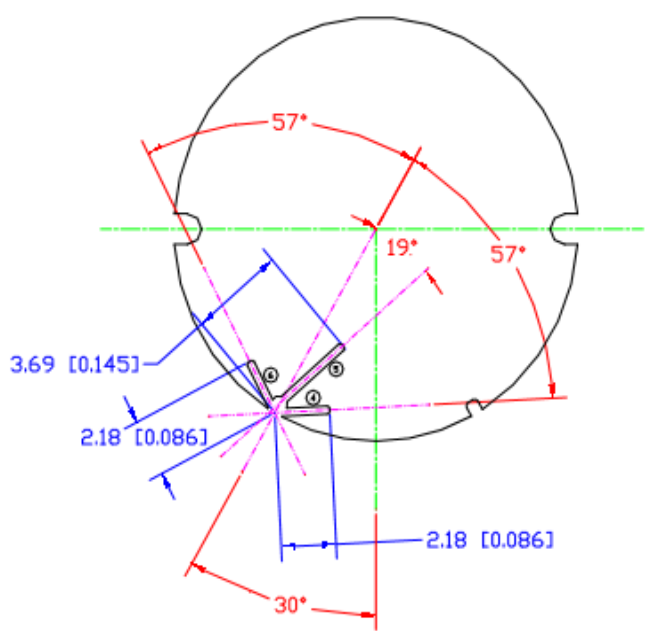

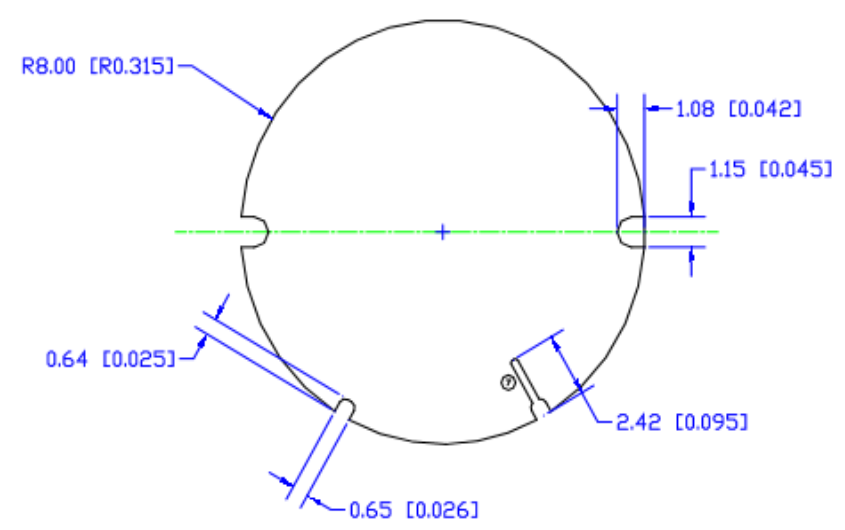

Figure 3.13: Cap Electrode Pedestal Thermocouple Locations (Hunnell, 2005 and Courtesy of Mr. Richard Harris of the AFRL) 


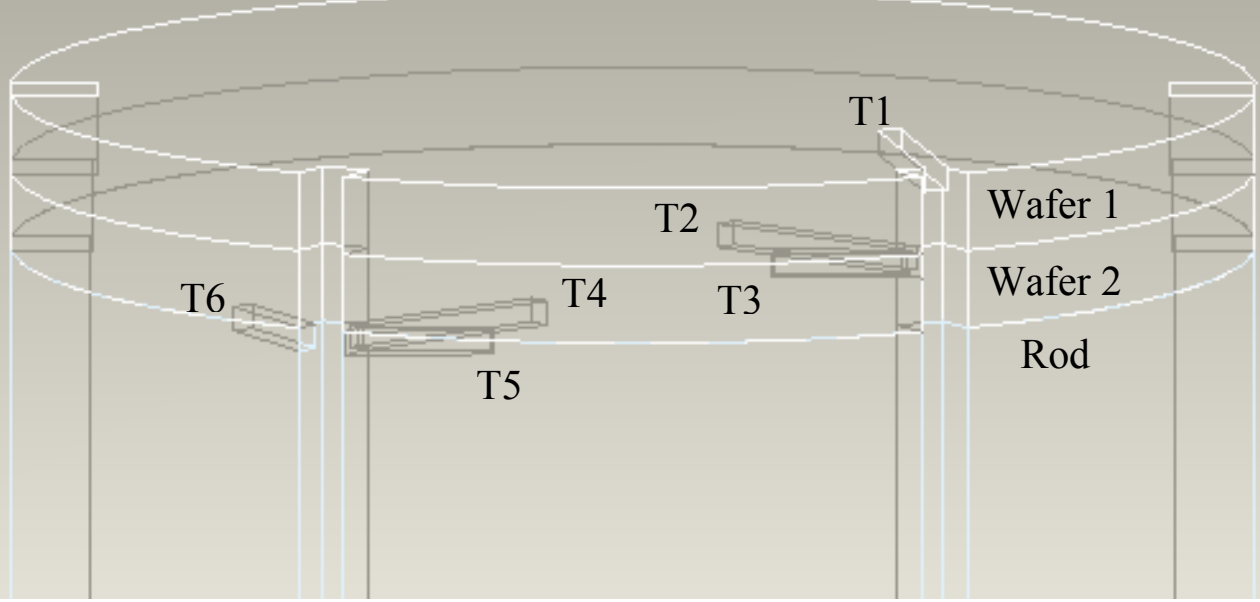

Figure 3.14: Cap Electrode Pedestal Top Showing Attached Wafers and Thermocouple Labels

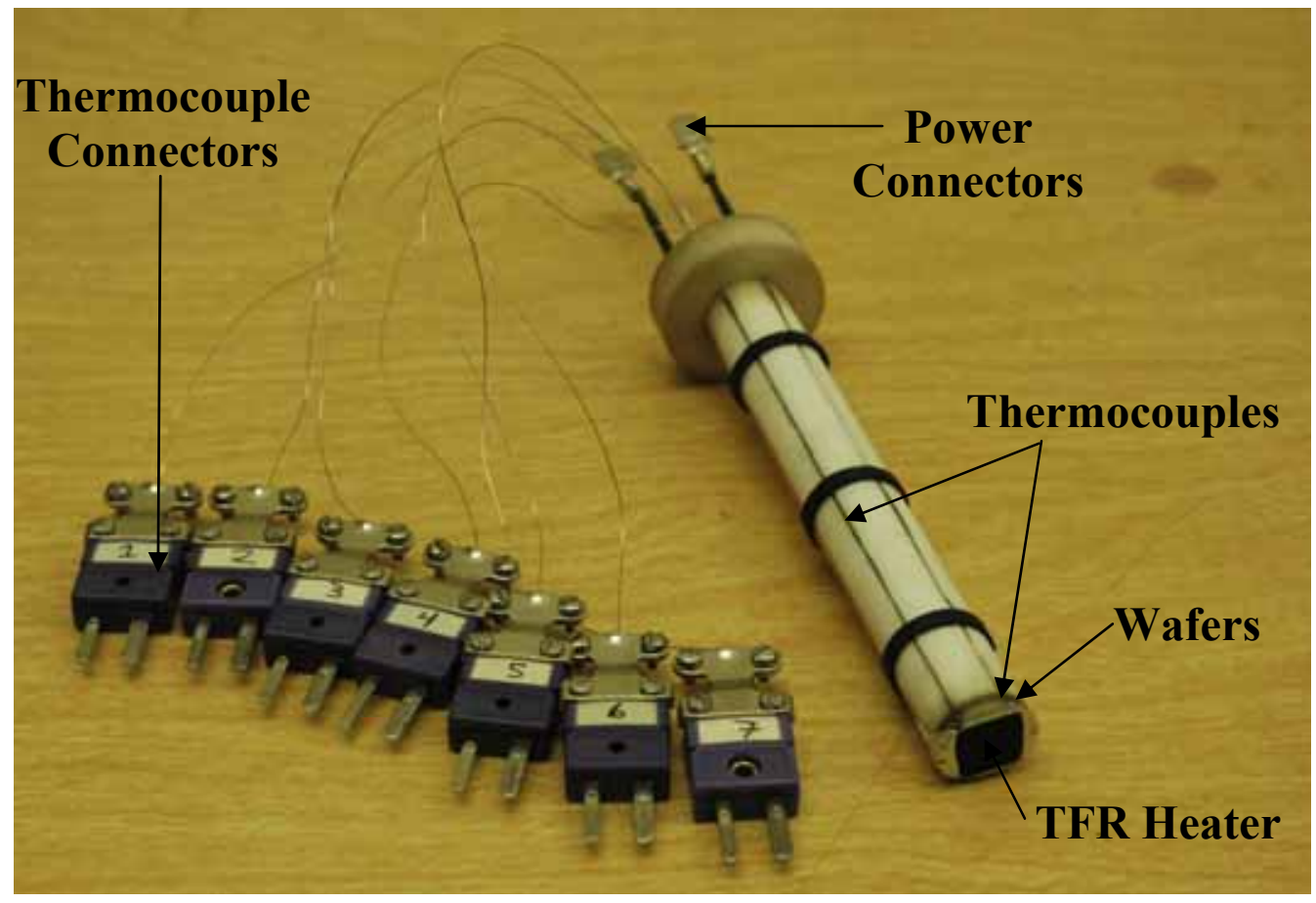

Figure 3.15: Cap Electrode Pedestal Assembly 
Two other pedestals very similar to this cap electrode pedestal were also built as backups. One had a Type 2 TFR (no glass layer) attached while the other had a Type 1 (glass layer). For these pedestals, no wafers were used and the rods were lengthened by 2 $\mathrm{mm}$ so that the total length remained the same. Five thermocouples were placed directly under the TFR heaters in the pattern shown in Figure 3.16. Note that the location of T1 is the same as the main cap electrode pedestal shown in Figure 3.14. The pedestals are shown in Figure 3.17.

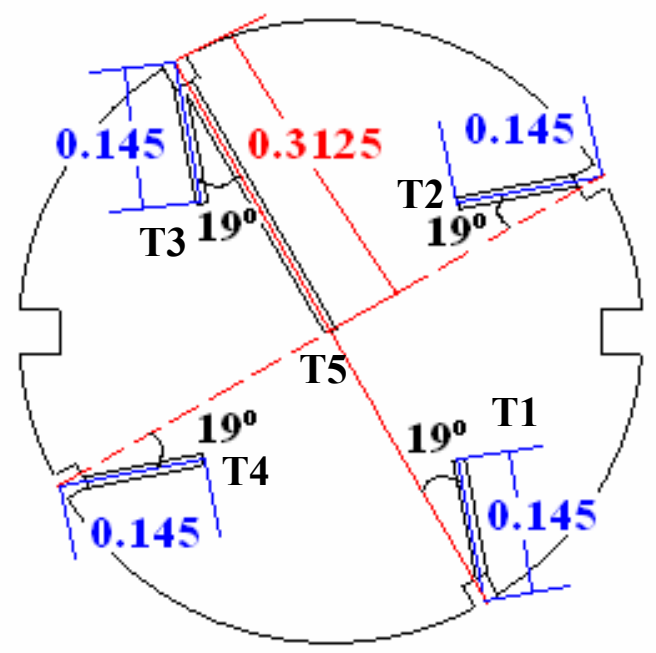

Figure 3.16: Backup Cap Electrode Pedestal Thermocouple Locations

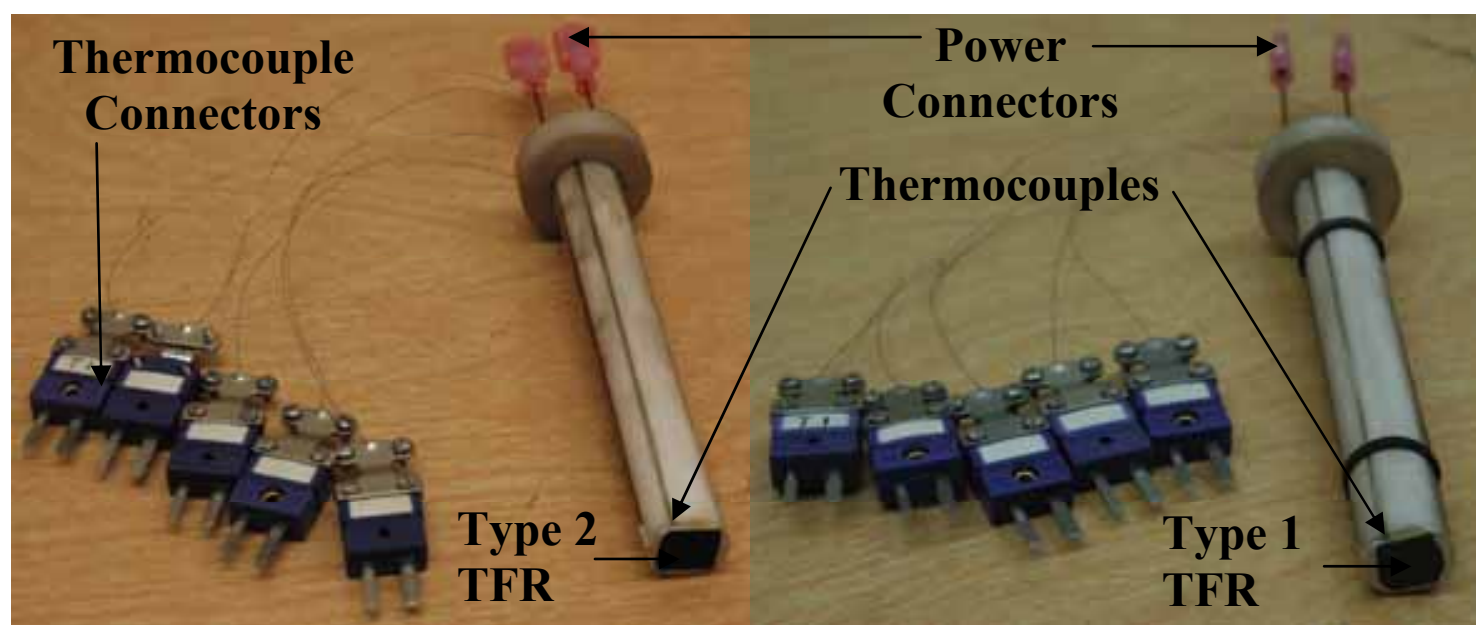

Figure 3.17: Backup Cap Electrode Pedestals 
The third type of pedestal is also made of PTFE, but was modified to accommodate the Mehra electrode design geometry described in Section 3.2. The TFR heater is surrounded at its outer radius by $1 \mathrm{~mm}$ of PTFE by machining an indentation into the top of the rod. The PTFE rod is also $1 \mathrm{~mm}$ shorter than the spacing comparison or cap electrode pedestal rod. However, a piece of alumina, $2 \mathrm{~mm}$ thick, was glued above the heater. This material is thermally conductive (thermal conductivity of approximately $33 \mathrm{~W} / \mathrm{m} \mathrm{K}$ ), yet electrically insulating (volume resistivity greater than $10^{14} \Omega \mathrm{cm}$ ) allowing the TFR to heat the top surface of the pedestal while preventing electrostatic breakdown due to the geometry of the Mehra electrodes. This pedestal has five thermocouples embedded within it in the same arrangement as shown in Figure 3.16. The location of $\mathrm{T} 1$ is below the TFR heater and is the same as the other pedestals. However, the other thermocouples (T2 through T5) are positioned above the heater with a thin layer of epoxy insulating them from the heater surface. Figure 3.18 shows the Mehra electrode assembly without the alumina top and Figure 3.19 shows the full Mehra electrode assembly. 


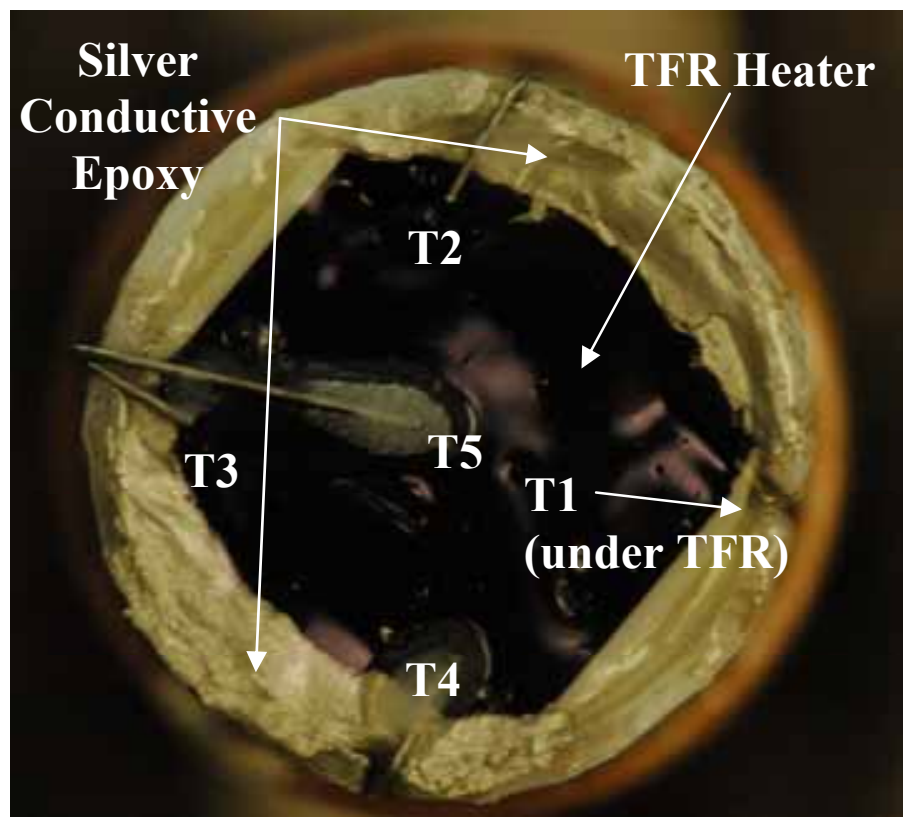

Figure 3.18: Top of Mehra Electrode Pedestal without $2 \mathrm{~mm}$ Alumina Layer

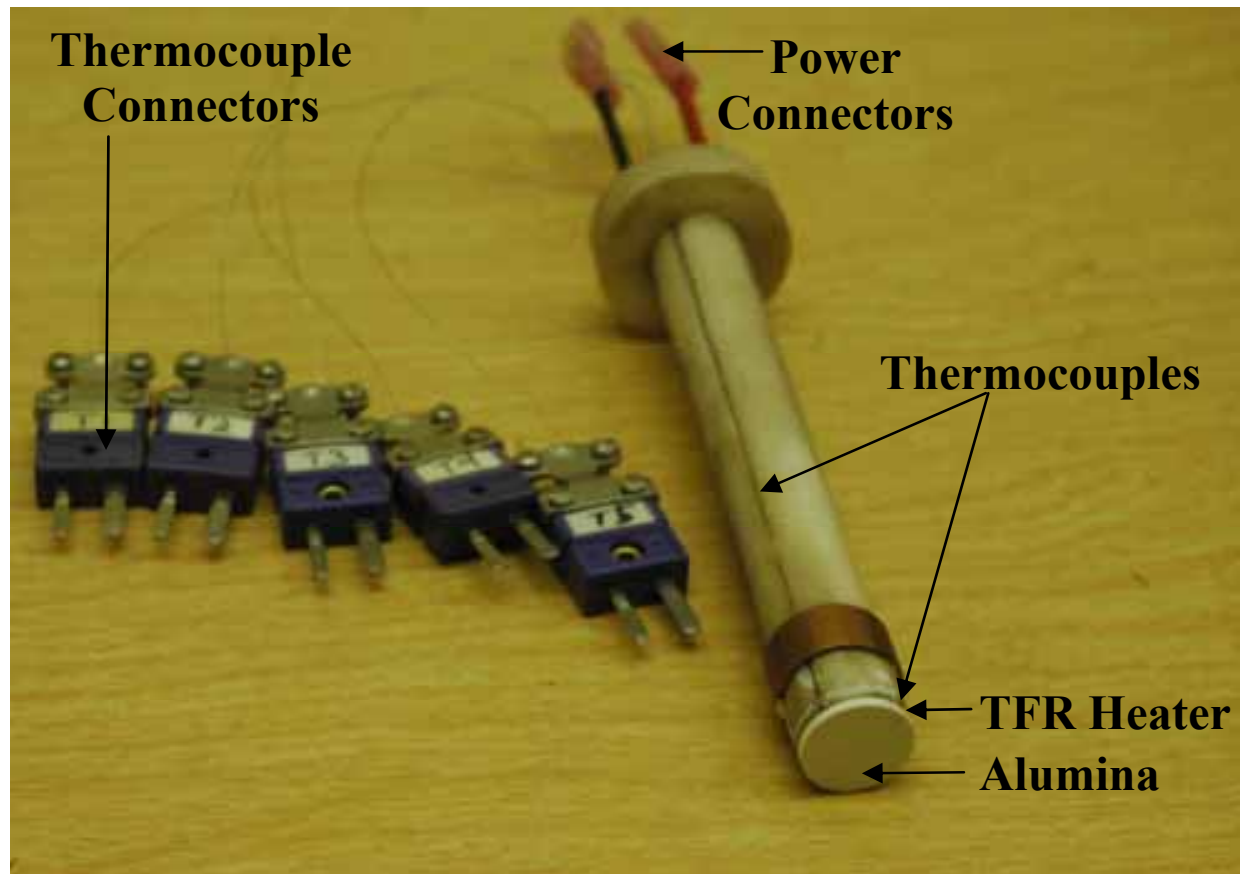

Figure 3.19: Mehra Electrode Pedestal Assembly 
Six additional thermocouples are mounted in the chamber area and their locations are shown in Figure 3.20 and are labeled T8 through T13. An additional thermocouple, T14, is positioned near the nozzle inlet to measure the inlet spray temperature. Note that T11 is below the plane of the heater surface in an effort to position it further from the electrodes to avoid electrical breakdown.

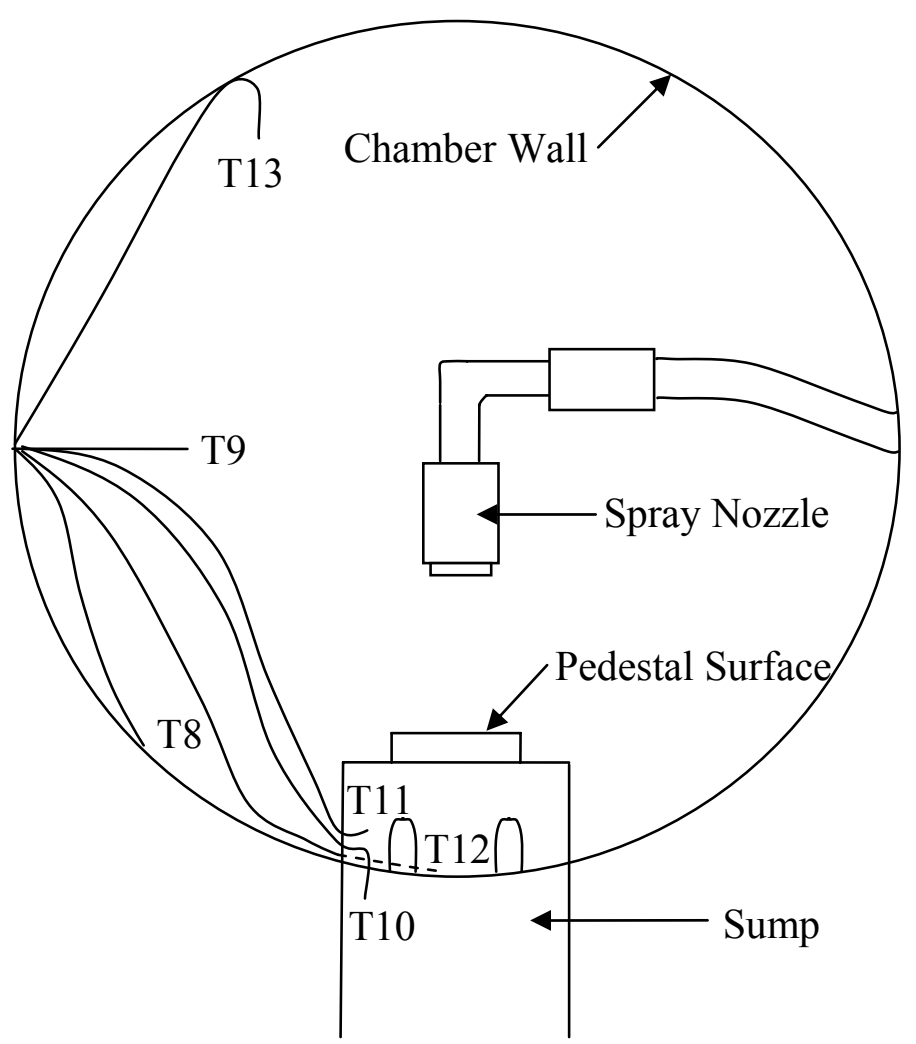

Figure 3.20: Approximate Thermocouple Locations within the Chamber

Three bulkheads for the thermocouple, high voltage, FC-72 (or HFE-7000) and water flow loop connections were attached to the frame of the chamber. These can be seen in the schematic and photograph of the entire spray chamber apparatus shown in Figure 3.21 and Figure 3.22, respectively. 


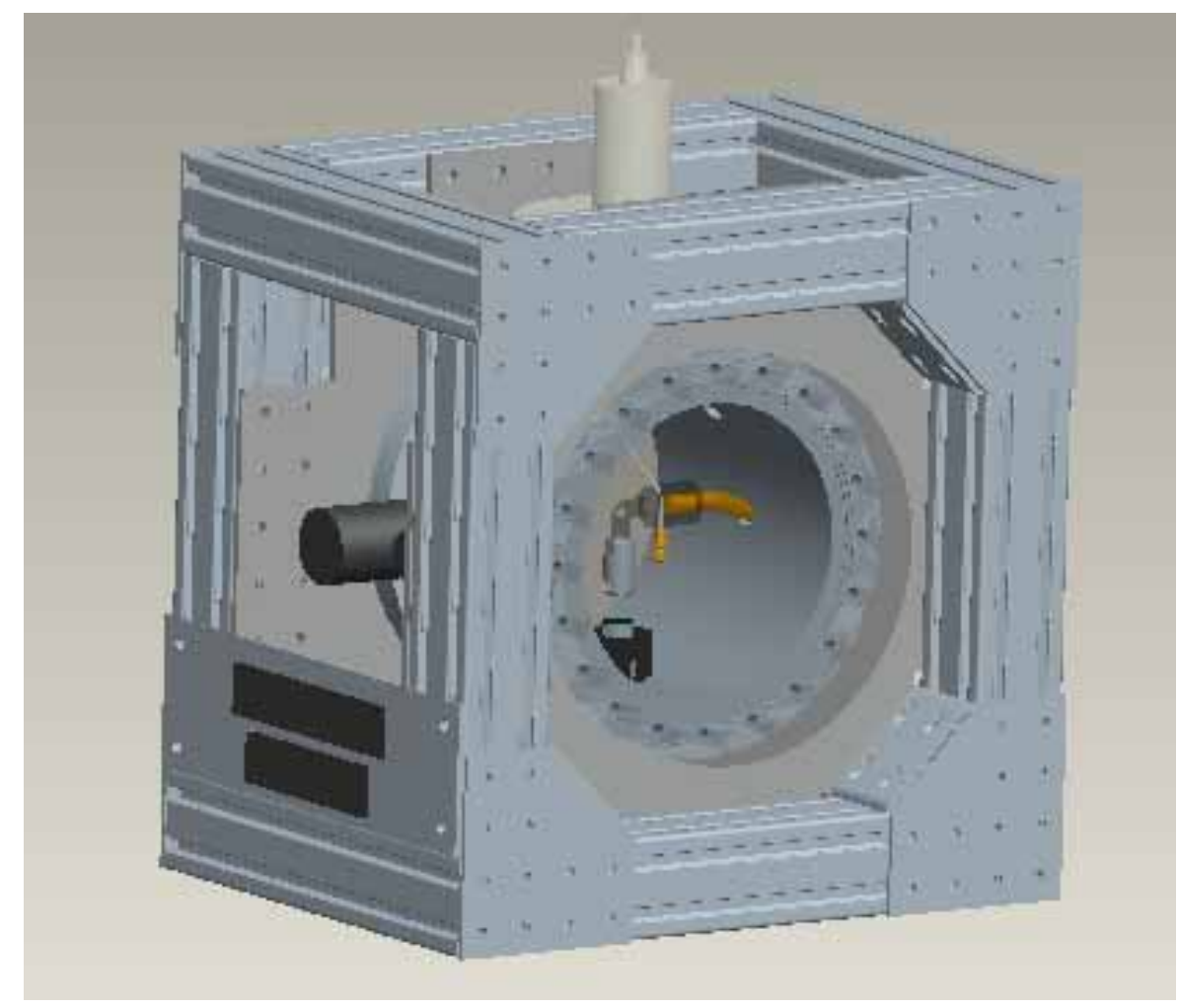

Figure 3.21: Schematic of Spray Chamber Main Components

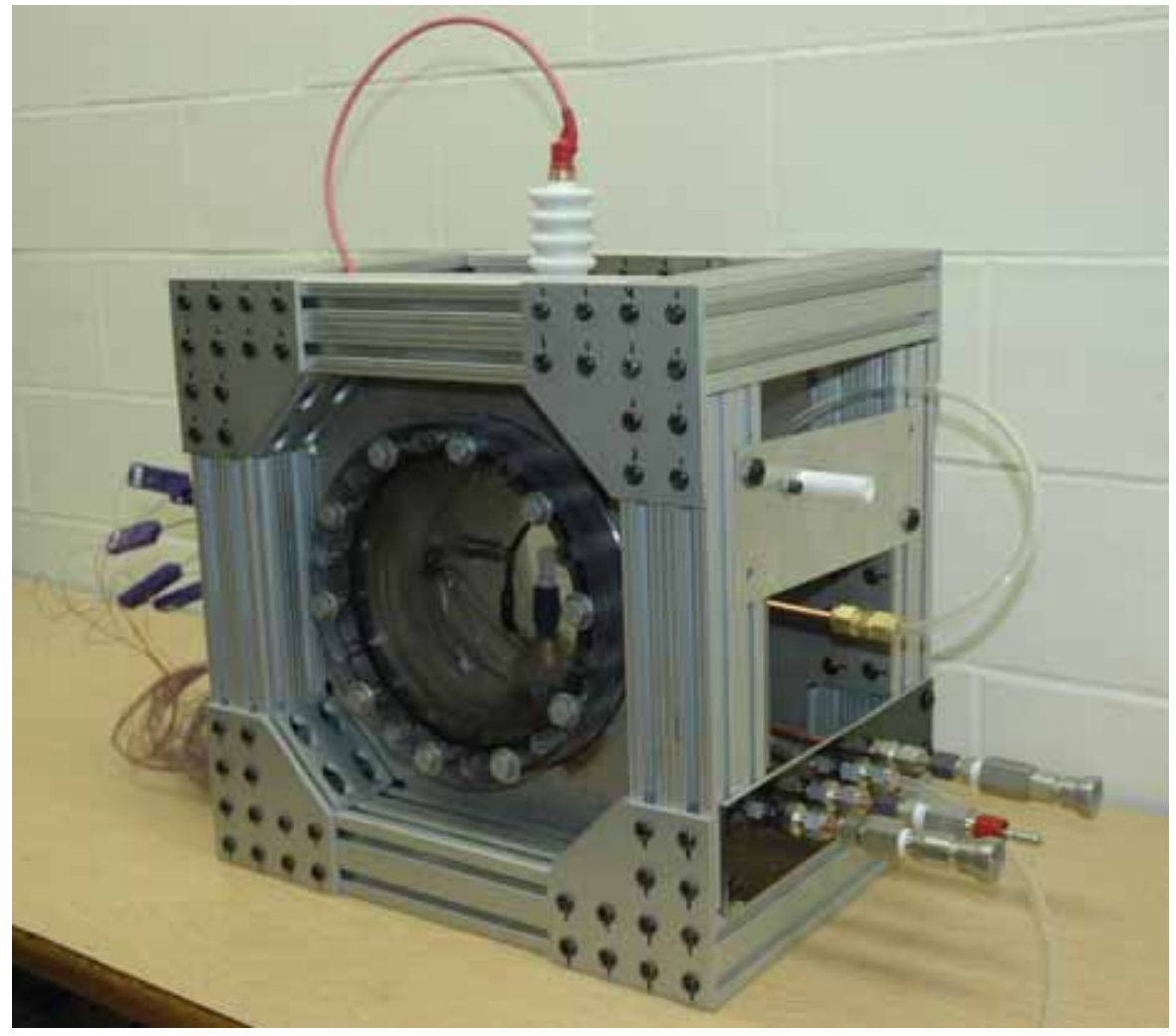

Figure 3.22: Photograph of Spray Chamber 


\subsection{Electrode Configurations}

Several different electrode designs were developed. The first set of designs are shown in Figure 3.23 and Figure 3.24, are made of polycarbonate and brass, and are modeled from the cap design from Baysinger, 2004 (shown here in Figure 3.25). The dimensions for the designs are shown in Figure 3.26. The cap electrodes were given brief names as shown below according to their geometries to help in quickly identifying them and will be referred to as such.

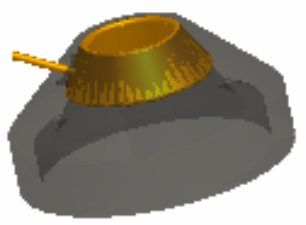

Top Half

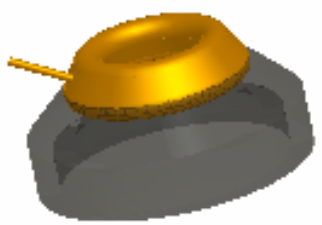

Thick Top Half

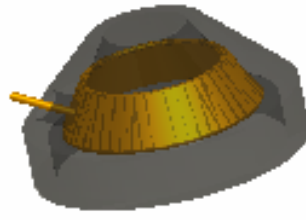

Bottom Half

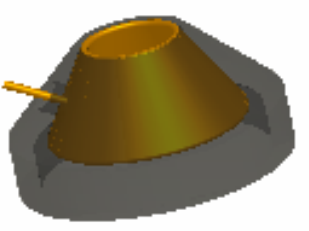

Full Length

Figure 3.23: ProE Renderings of Four Cap Electrode Designs

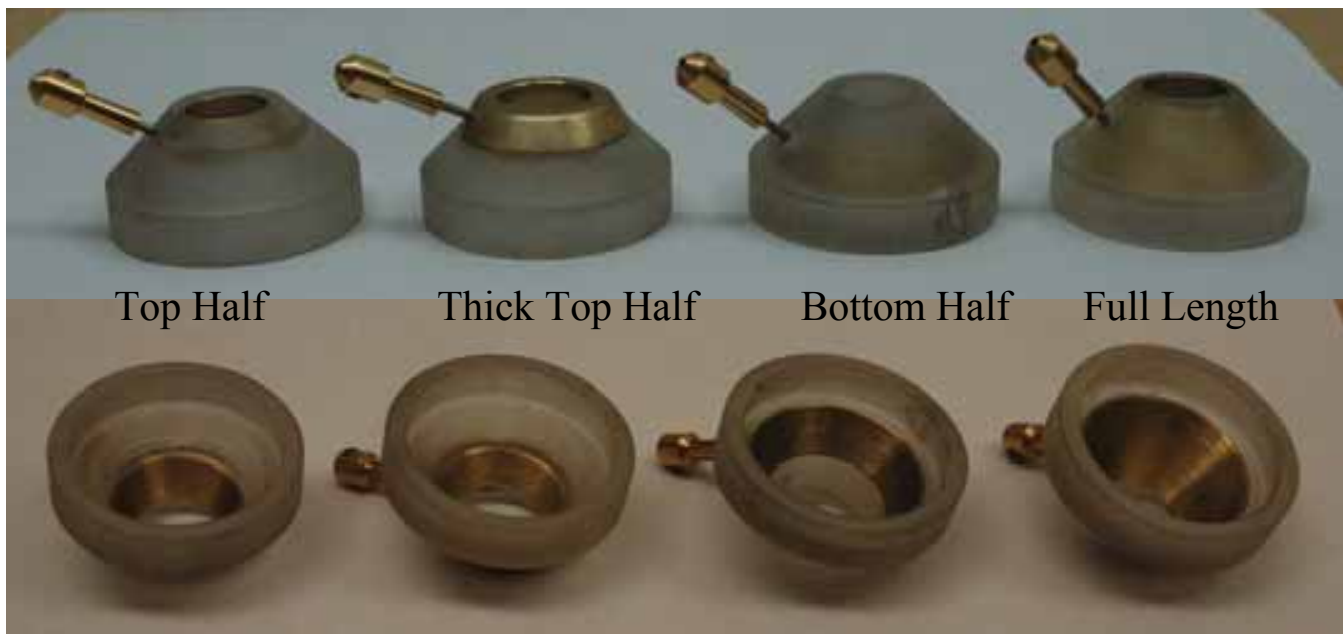

Figure 3.24: Photographs of Sides and Bottoms of Four Cap Electrode Designs with Pin Connectors 

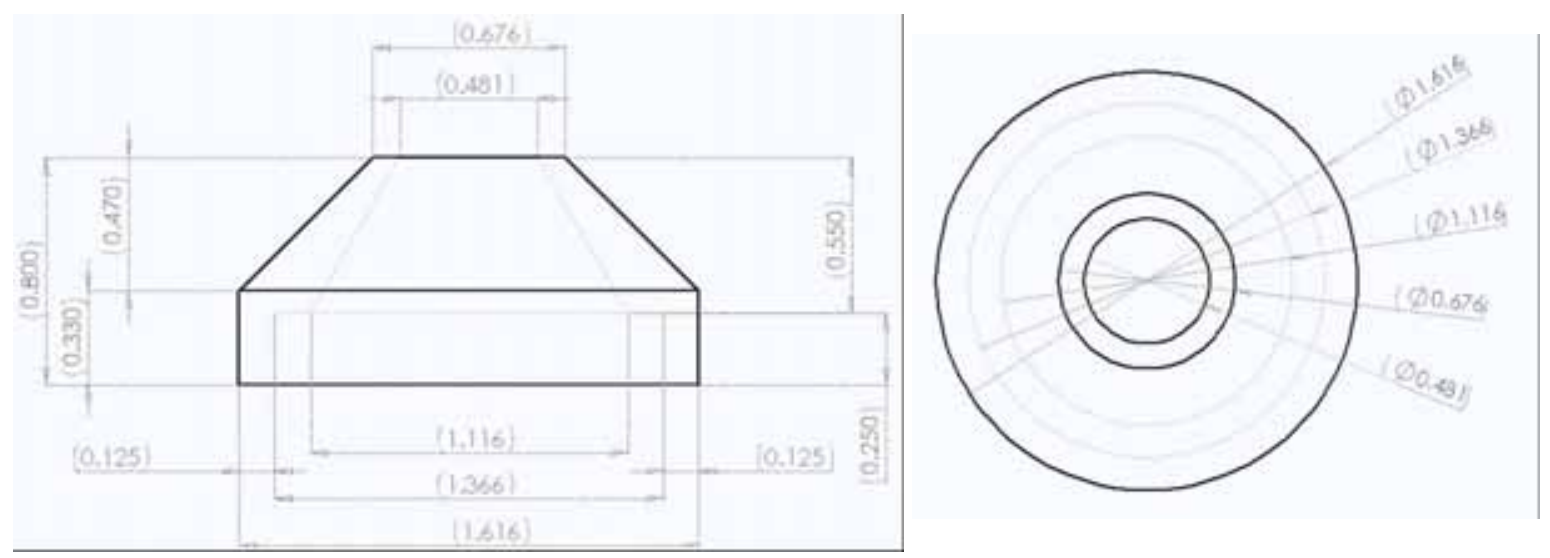

Figure 3.25: Cap Design from Baysinger, 2004 (Dimensions in Inches)

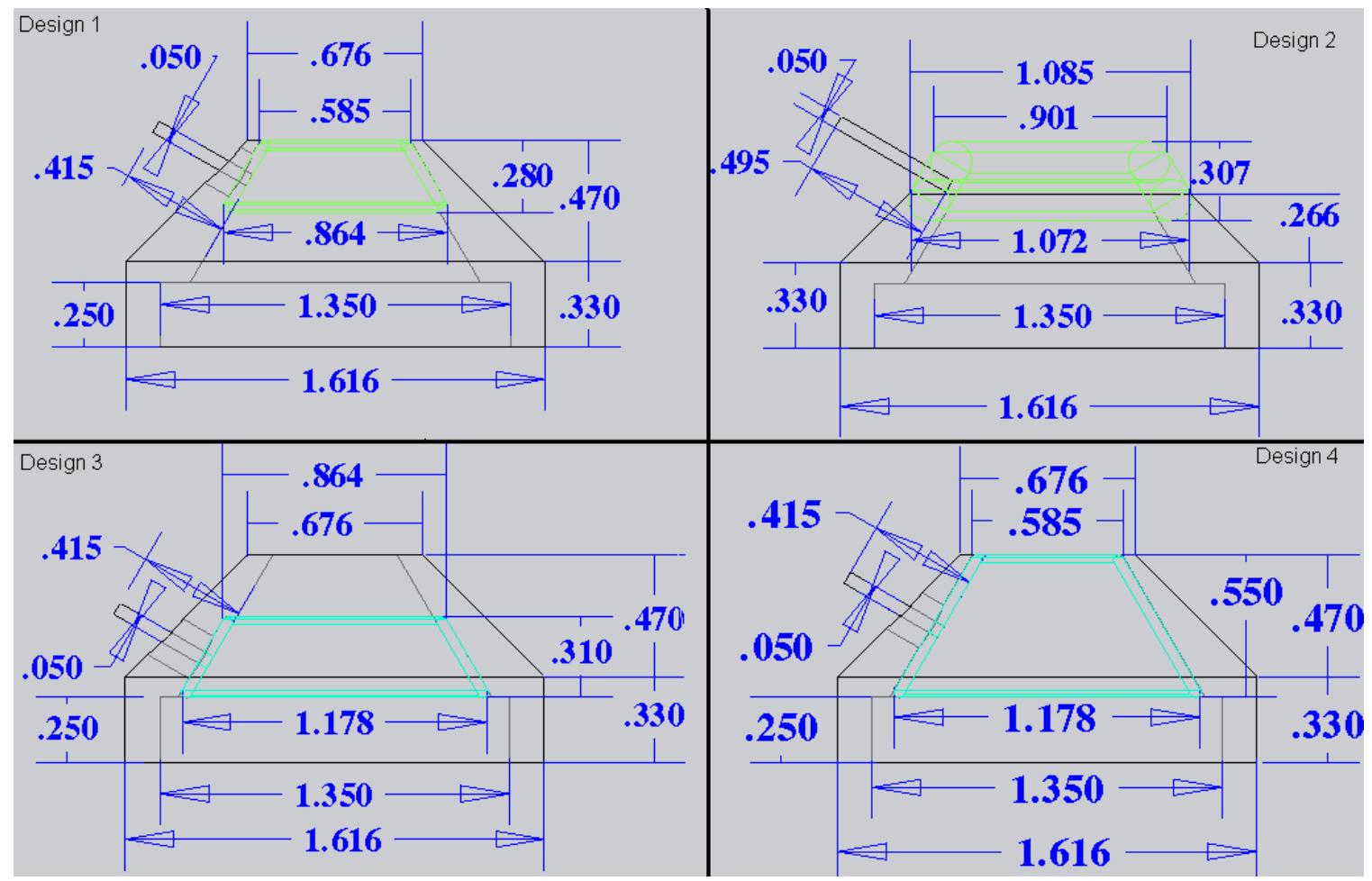

Figure 3.26: Cap Electrode Design (Dimensions in Inches)

Each of the four designs has similar internal dimensions with the difference being the size and location of the brass electrode. Design 1 (top half) has an electrode with a thickness of 0.04 inches placed at the top half of the internal cap slope. Design 2 (thick top half) has the electrode in the same position but with a thickness of 0.16 inches. Design 3's (bottom half) electrode is at the bottom half of the internal cap slope and has a 
thickness of 0.04 inches. Design 4's (full length) electrode covers the entire internal cap slope and has a thickness of 0.04 inches. The polycarbonate portion attaches to the sump (described above) with a set screw in the position shown in Figure 3.27. The brass electrode portion attaches to the high voltage feedthrough using a 0.05 inch pin and connector (from Insulator Seal) also shown in Figure 3.27. A wire links this connector to a similar connector on the high voltage feedthrough in the spray chamber.

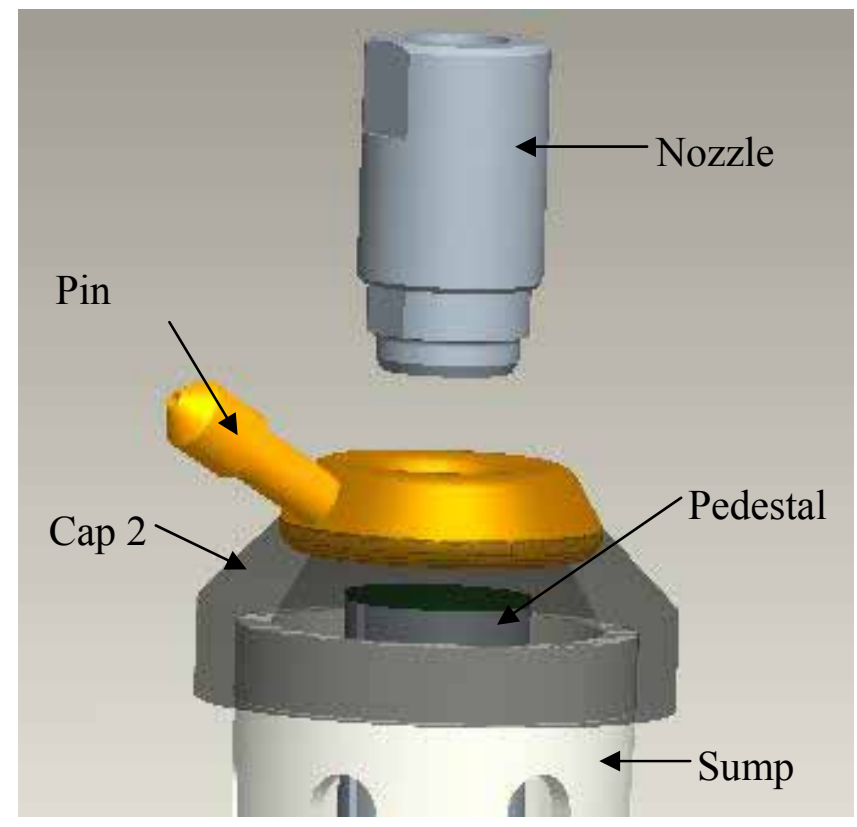

Figure 3.27: Thick Top Half Cap Electrode Attached to Sump

The second electrode configuration consists of three copper electrodes arranged around the surface of the pedestal. This design was developed conceptually by Mehra (2006) using CFD-Ace+ with the design goal of achieving as large of an upwards electric Kelvin force normal to the top pedestal surface as possible. Schematics of the nozzle electrode, cap electrode, and pedestal electrode for this design are shown in Figure 3.28, Figure 3.29, and Figure 3.30, respectively. A modified cap was designed and built to accommodate the shape of the cap electrode as shown in Figure 3.31 and a plastic cover 
was also built to help prevent electrostatic breakdown between the nozzle and cap electrodes and is shown in Figure 3.32. The arrangement of these components is shown in Figure 3.33 and a photograph of the assembled configuration is shown in Figure 3.34. The nozzle electrode is attached to the high voltage power supply while the cap electrode is grounded to the chamber wall and the pedestal electrode is grounded to the TFR heater.

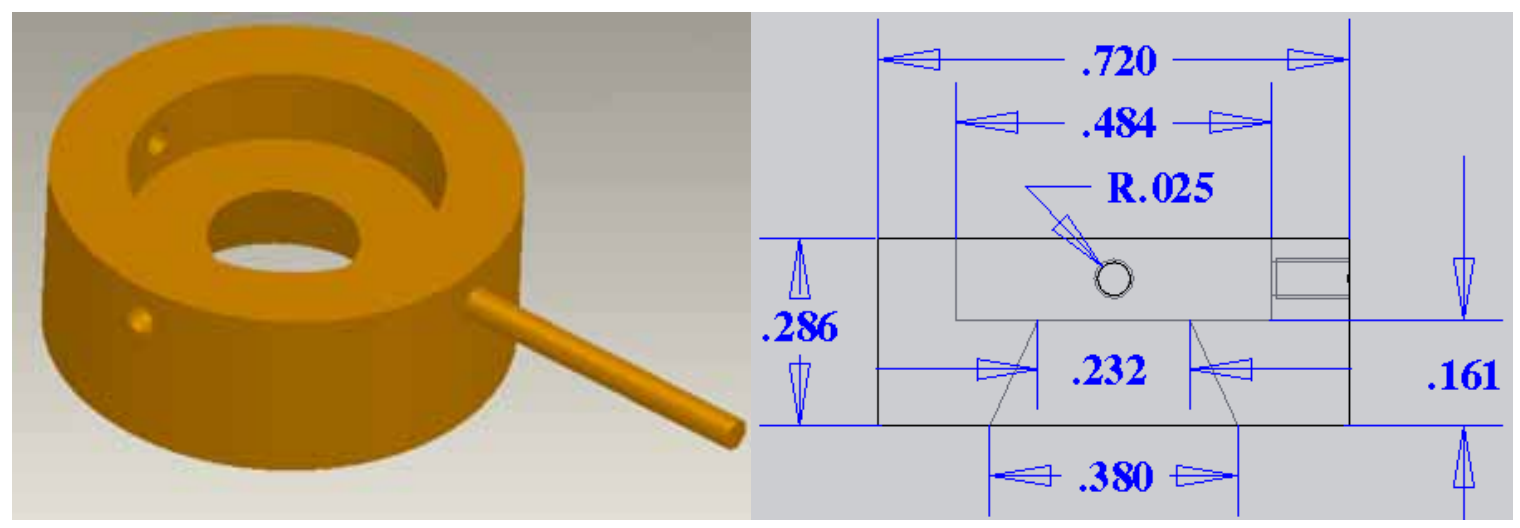

Figure 3.28: Nozzle Electrode Rendering and Schematic (Dimensions in Inches)

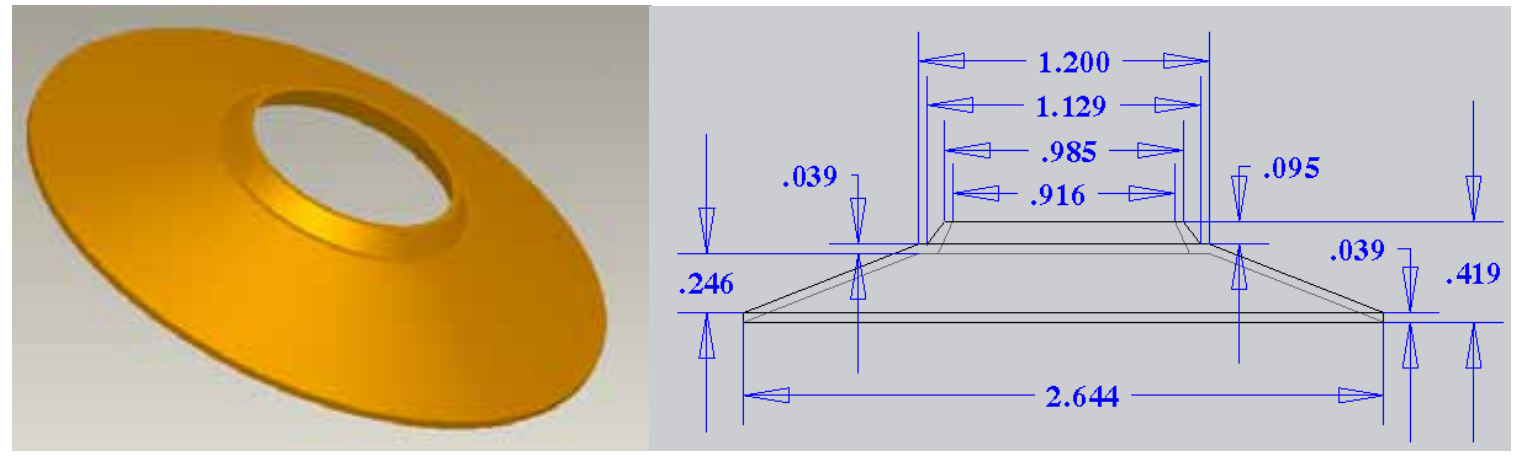

Figure 3.29: Cap Electrode Rendering and Schematic (Dimensions in Inches)

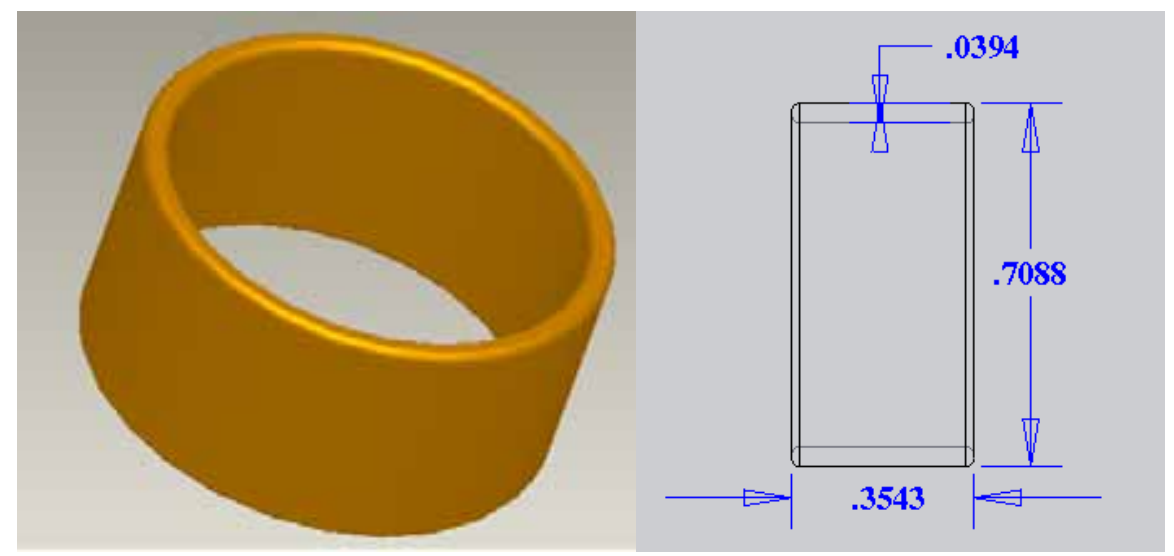

Figure 3.30: Pedestal Electrode Rendering and Schematic (Dimensions in Inches) 

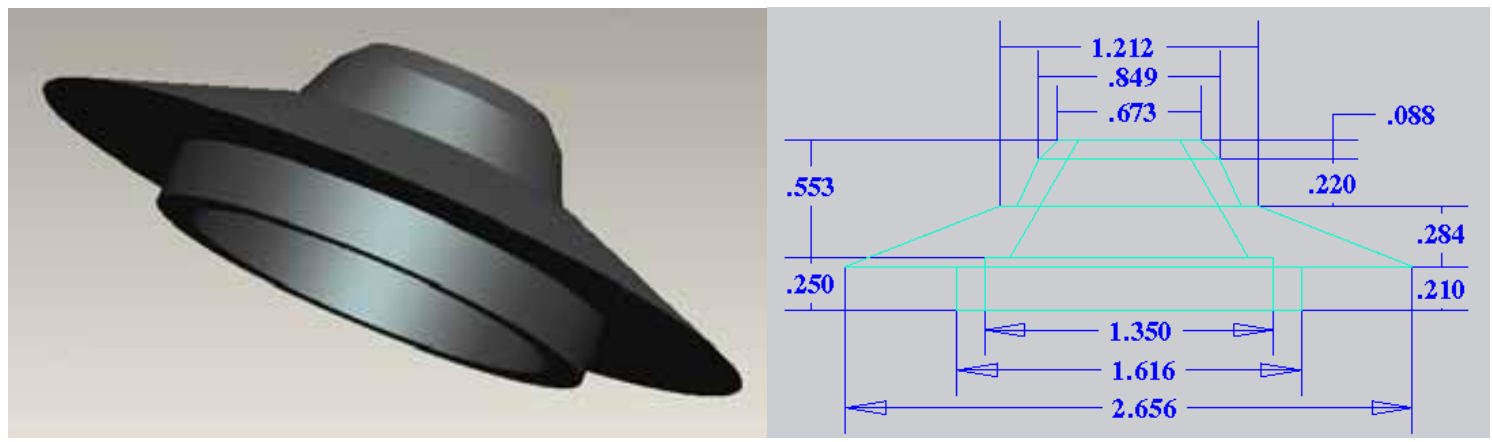

Figure 3.31: Modified Cap Rendering and Schematic (Dimensions in Inches)

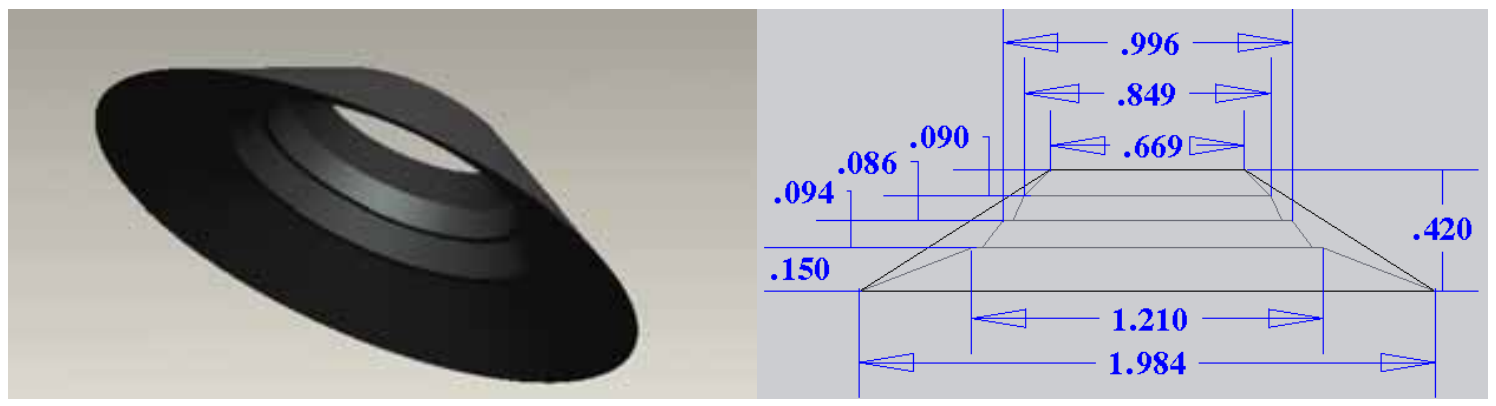

Figure 3.32: Cover Rendering and Schematic (Dimensions in Inches)

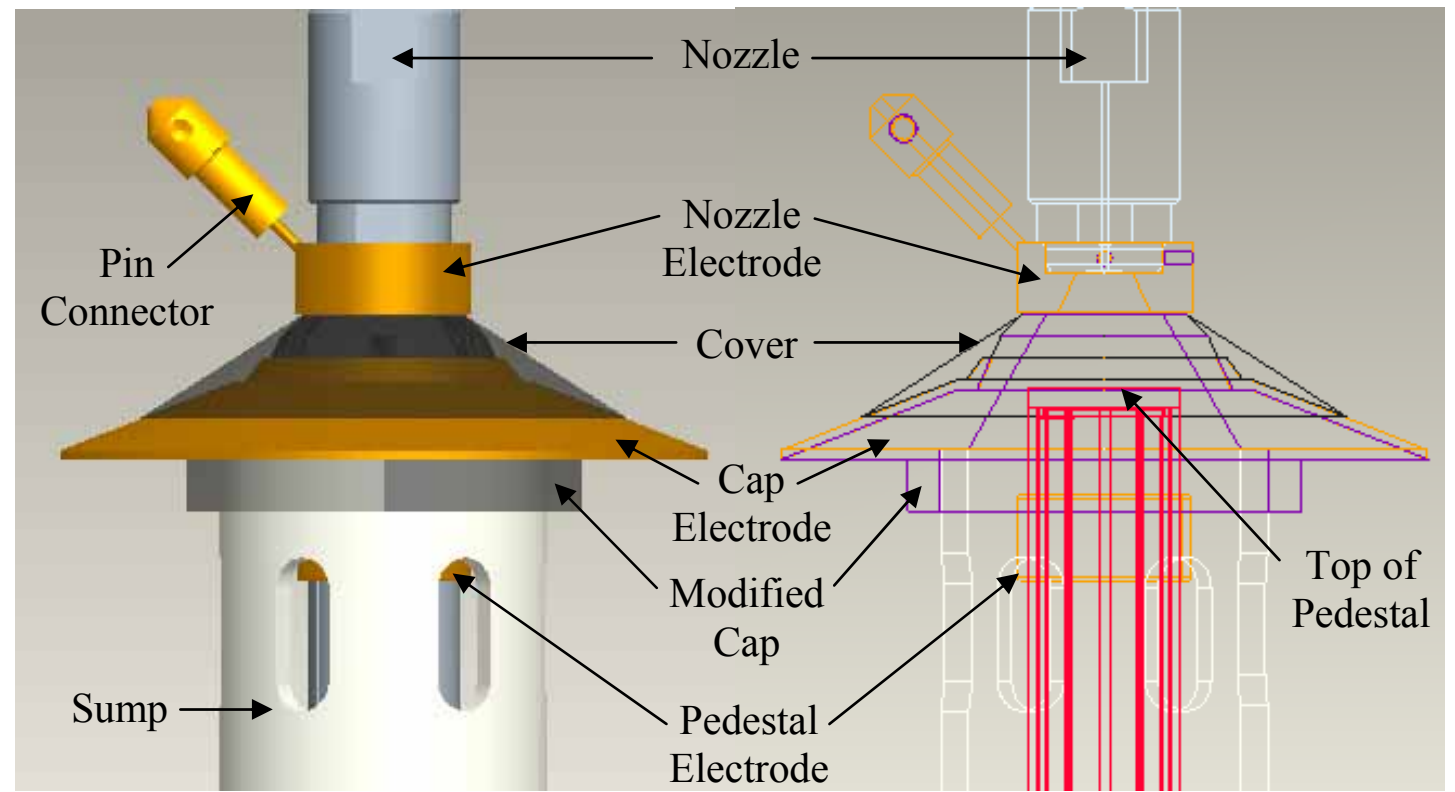

Figure 3.33: Electrode Arrangement around Sump, Pedestal, and Nozzle Showing a Solid Rendering (left) and Transparent View (right) of Design by Mehra (2006) 


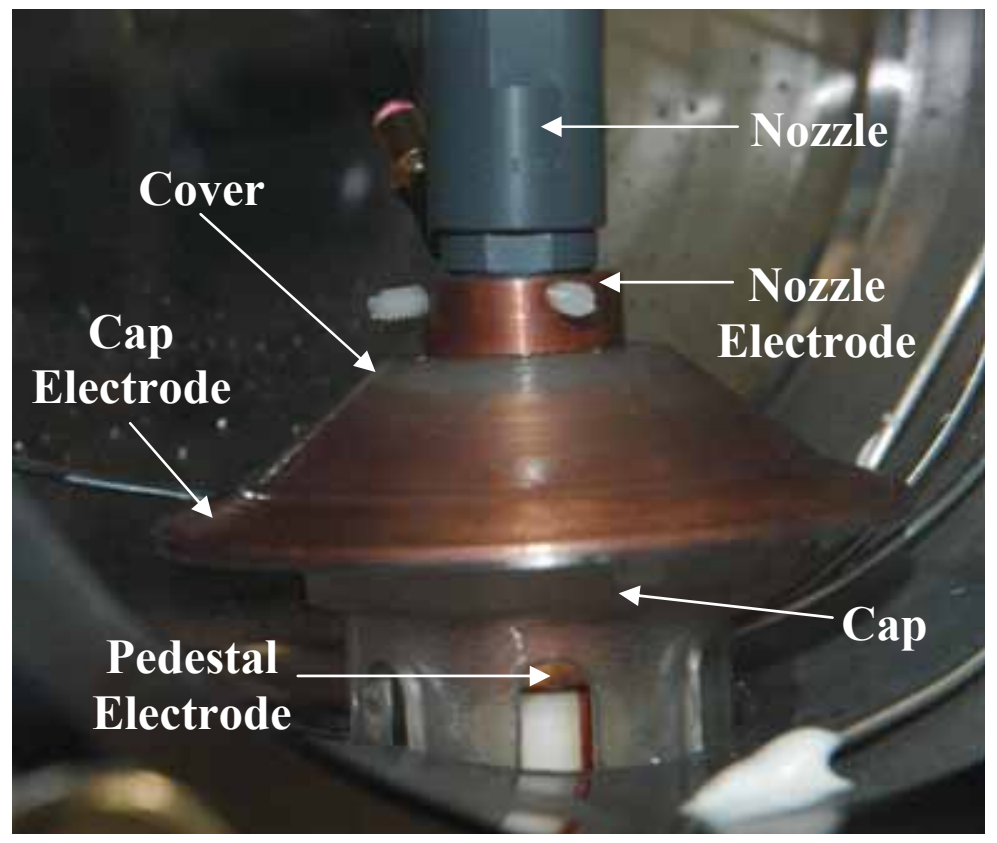

Figure 3.34: Photograph of Electrode Arrangement in the Spray Chamber

\subsection{Base Configuration}

The purpose of the base for this apparatus is to provide the spray chamber with the necessary flow of the working fluid, e.g. FC-72 or HFE-7000, and the chamber coolant fluid, water. It also houses the power supplies and data acquisition system. Inside this frame there are series of pumps, pressure gages and transducers, heat exchangers, filters, flow meters, and reservoirs as shown in the flow schematic in Figure 3.2 that are used to manage and measure the fluid flows. The basic setup of this base is the same as given in Hunnell (2005) with some key modifications that have primarily been made by Kreitzer (2006). A photograph of the current configuration is shown in Figure 3.1 and a photograph of the original setup is shown in Figure 3.35. 


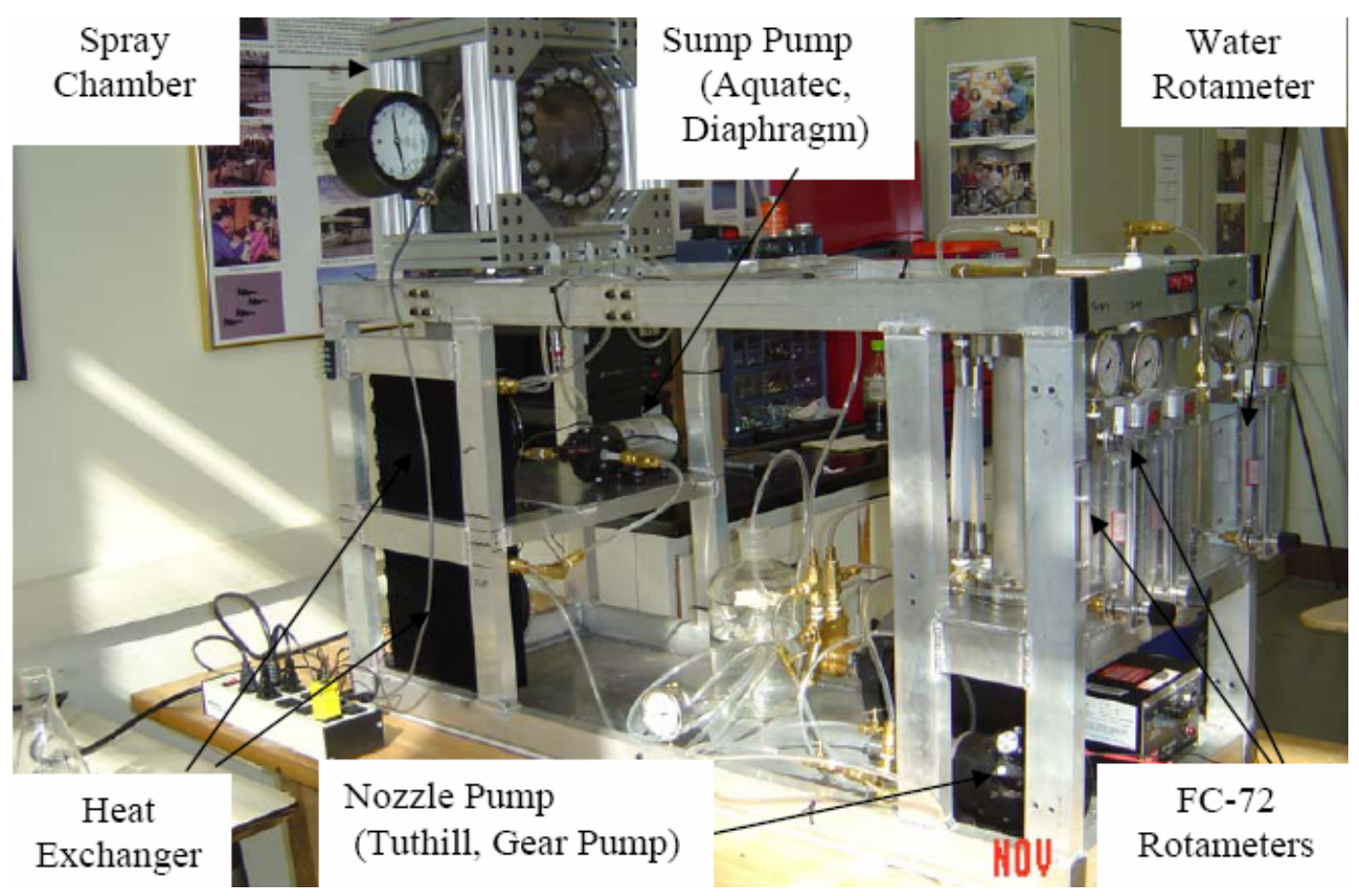

Figure 3.35: Original Base and Spray Chamber Configuration by Hunnell (2005)

The first modification made was to remove the air accumulator from the FC-72 flow loop to prevent the large loss of fluid associated with allowing the system to vent to the atmosphere. Closing the system also helps maintain a more constant air saturation level within the FC-72 (or HFE-7000) fluid and removes an additional variable from the study. A bypass was added to the flow loop in order to allow proper balancing of the nozzle and sump flow rates.

An Agilent 34970A data acquisition system was installed as explained by Kreitzer (2006). This item was placed with the Protek 5050S DC Power Supply (which provides adjustable power to the thick film resistor heater on the top of the pedestal) as shown in Figure 3.36. 


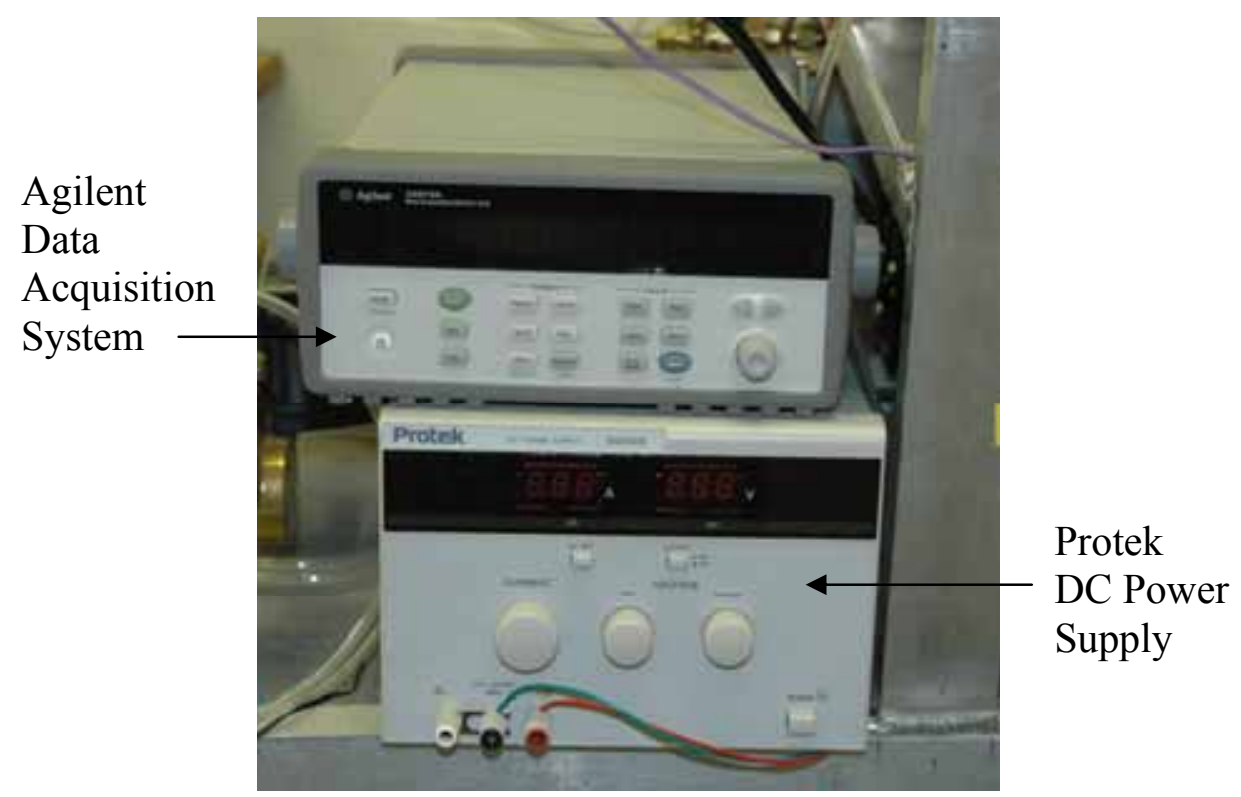

Figure 3.36: Agilent Data Acquisition System Addition and Protek DC Power Supply

One final modification to the base is the addition of a high voltage power supply from Glassman High Voltage Inc. (model number PS/EL30R01.5). This power supply was mounted in a table top rack cabinet as shown in Figure 3.37 along with the Astron VLS-25M DC power supply (which provides power to the pumps and pressure transducers). It supplies up to $\pm 30 \mathrm{kV}$ to the electrodes mounted in the spray chamber.

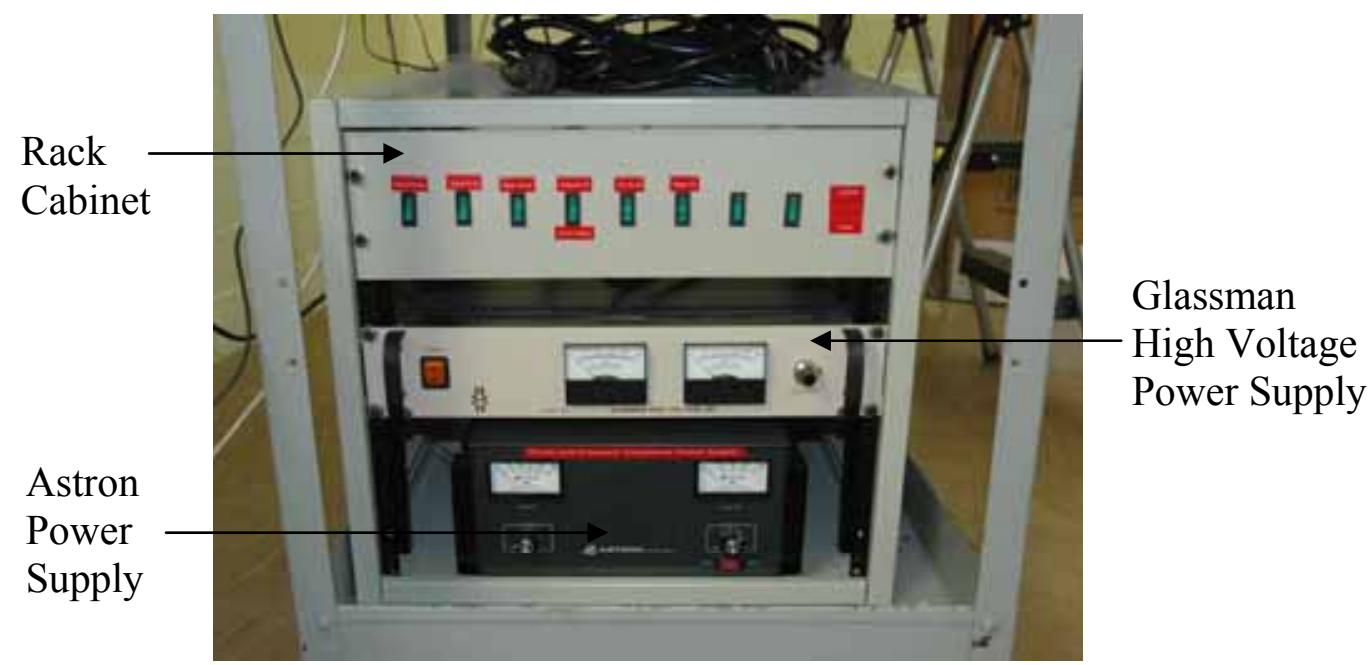

Figure 3.37: Glassman High Voltage Power Supply Addition Mounted in Cabinet with Astron Power Supply 
The rotameters used to measure the flow rates of the FC-72 (or HFE-7000) and the water that were on the original base could measure up to $12 \mathrm{GPH}$ of water. These were replaced with rotameters having a range of 0 to $20 \mathrm{GPH}$ of water. Corrections were made in calculations for the corresponding fluid in each rotameter using the density ratios.

For safety, a discharge rod, shown in Figure 3.38, was made to attach to ground and protect from the possibility of electric shock from the high voltages. In the case of a portion of the apparatus becoming charged, the rod could be held at a safe distance and used to attach the apparatus to a ground.

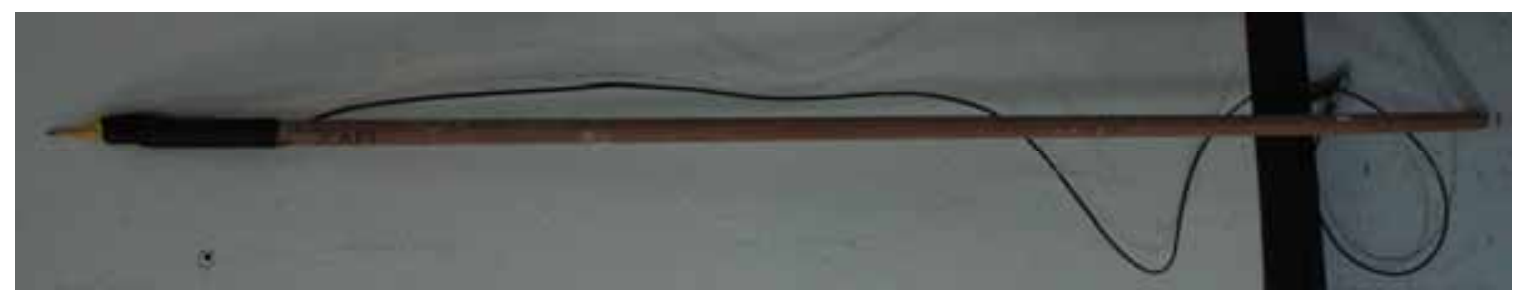

Figure 3.38: Electric Discharge Rod

\subsection{Procedure}

Two different procedures were used to take the data. The first set of data that compares the effects of the spacing of the nozzle exit to the heater surface was obtained before the addition of the data acquisition system as described in the previous section. Therefore, the turn-on and operating procedure was very similar to that outlined in Hunnell (2005). One exception to those procedures was that since the air accumulator component was replaced with a bypass loop, the corresponding procedural steps were altered to include adjusting the bypass valve. Also, for the spacing tests, only one flow rate was studied $\left(0.029 \mathrm{~m}^{3} / \mathrm{hr}\right.$ or $7.72 \mathrm{GPH}$ of $\left.\mathrm{FC}-72\right)$ so the corresponding procedural steps were modified to include the adjustment of the spacing between the nozzle and the 
heater. Test plans for the spacing comparison and electrode studies can be seen in Appendix B.

\section{Spacing Comparison Procedure}

A comparison of the effects of spacing between the PVC spray nozzle exit and the TFR heater was performed for both confined and unconfined cases. The geometry of both cases can be seen in Figure 3.39. The first set of data recorded was for the confined case using a straight cap from Hunnell's (2005) study. Tests were conducted at spacings ranging from $9 \mathrm{~mm}$ to $17 \mathrm{~mm}$ (in $2 \mathrm{~mm}$ increments). This range was chosen based on the $13 \mathrm{~mm}$ nozzle spacing configuration used previously by both the team at AFRL, Baysinger (2004), and by Hunnell (2005). The apparatus was turned on, set to a spray flow rate of $0.029 \mathrm{~m}^{3} / \mathrm{hr}(7.72 \mathrm{GPH})$ of FC-72 with no power applied to the heater $(\mathrm{Q}=0)$, and allowed to run for approximately 45 minutes in order to reach steady state. The atmospheric pressure was recorded using a mercury barometer. The pedestal, chamber, and room temperatures, pressures, and flow rates were recorded for each spacing at power levels of $0 \mathrm{~W}$ to $70 \mathrm{~W}$ (in $10 \mathrm{~W}$ increments). Data for the unconfined case was acquired the same way, but an additional run at the $13 \mathrm{~mm}$ spacing was done at the end of the tests to check repeatability for power levels from $0 \mathrm{~W}$ to $70 \mathrm{~W}$ as well as at $86.5 \mathrm{~W}$. This highest power level corresponded to a temperature of $99.3{ }^{\circ} \mathrm{C}$ at the substrate and pedestal interface $\left(T_{\text {int }}\right)$ and was the highest temperature attainable before the temperature cut-off activated at $100{ }^{\circ} \mathrm{C}$. The energy equation and appropriate boundary conditions for the pedestal used in the spacing comparison are shown in Appendix C. Data from these tests were reduced as shown in Appendix D, along with derivations of the data reduction equations. 


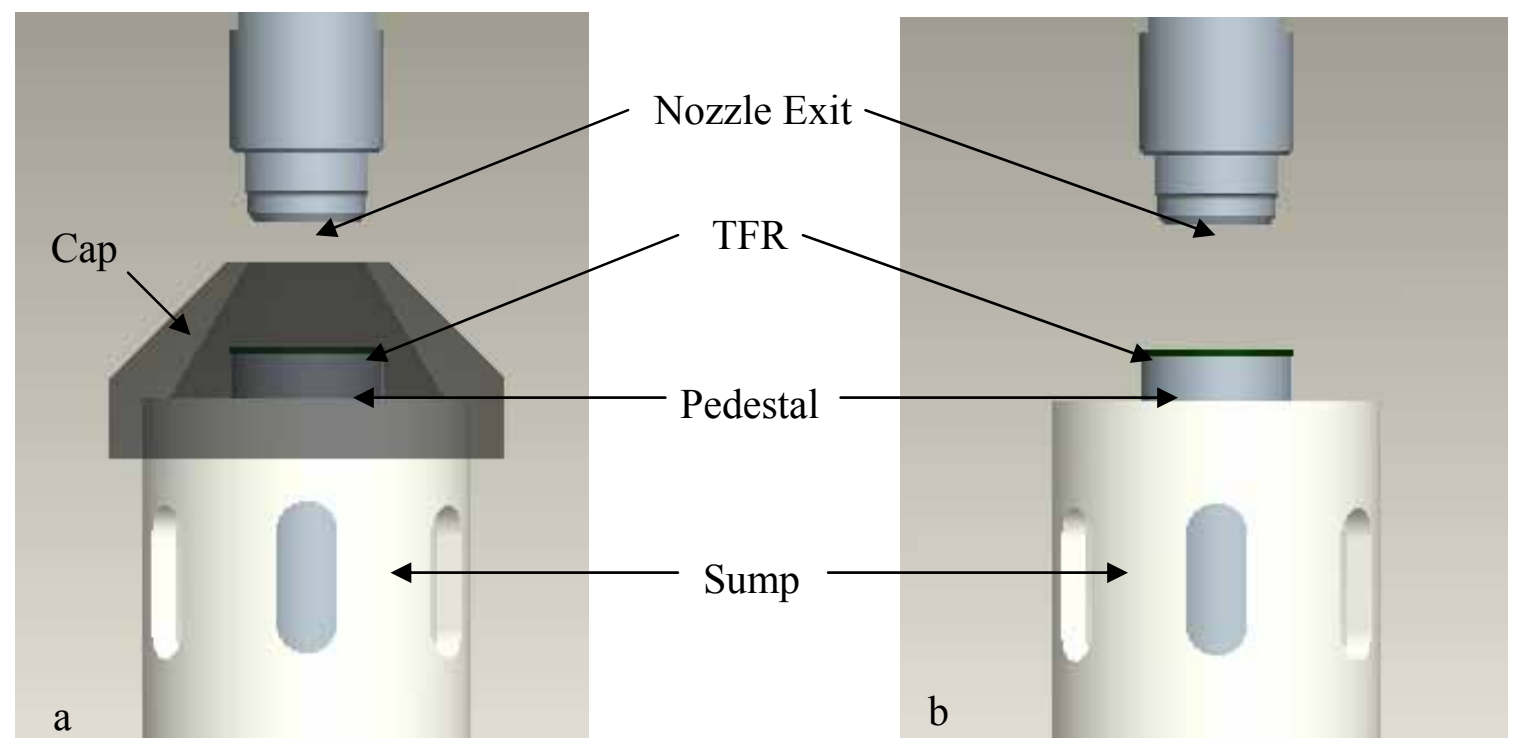

Figure 3.39: Experimental Configuration of a) Confined Case and b) Unconfined Case for Spacing Comparison Study

\section{Cap Electrode Performance Procedure}

The electrode cap tests were performed after the modifications to the base (removal of the air accumulator and addition of a data acquisition system and high voltage power supply) were made. Therefore, the Turn-On Checklist for the apparatus was modified, and is shown in Table 3.1. The Operating Procedure also changed and is shown in Table 3.2. A Turn-Off Checklist was also developed for safety reasons when dealing with the high voltage power supply and is shown in Table 3.3.

\section{Table 3.1: Apparatus Turn-On Checklist}

1. Arrange electrode configuration and attach to high voltage feedthrough in chamber

2. Align nozzle and secure viewports

3. Attach ground wire from high voltage power supply and discharge rod to ground

4. Plug apparatus into electrical outlet and turn on power strip

5. Connect high voltage plug to chamber

6. Connect quick disconnect tubes for FC-72 (or HFE-7000) to chamber

7. Assure rotameters are fully open

8. Check all other switches and valves for proper positioning

9. Turn on spray nozzle pump, sump pump, water pump, and heat exchanger fans

10. Turn on heater and high voltage power supplies

11. Turn on laptop and data acquisition 
12. Attach data acquisition to laptop and start software

Table 3.2: Operating Procedure Checklist

1. Start data acquisition and observe temperature and pressure values for steadiness

2. Turn knob on spray nozzle pump to set desired flowrate and observe rotameter values for steadiness

3. Turn knob on heater power supply to set desired heater power

4. Turn knob on high voltage power supply to set desired electrode voltage

5. Repeat steps 2 through 4 as needed for each test condition

6. Stop data acquisition and save information

\section{Table 3.3: Apparatus Turn-Off Checklist}

1. Turn off data acquisition and detach from laptop

2. Turn off high voltage and heater power supplies

3. Turn off heat exchanger fans, water pump, sump pump, and spray nozzle pump

4. Disconnect quick disconnect tubes for FC-72 (or HFE-7000) from chamber

5. Disconnect high voltage plug from chamber and touch it to the tip of the discharge rod

6. Touch the discharge rod to the metal in the high voltage receptacle on chamber

7. Turn off power strip and unplug apparatus from electrical outlet

The maximum high voltage input limit was chosen as $6 \mathrm{kV}$ for these cap electrodes based on an electrostatic breakdown estimation for air at 1 atm pressure. In general, the upper limit before breakdown occurs is $30 \mathrm{kV}$ when there is a $1 \mathrm{~cm}$ distance between the charged electrode and ground with air as the medium. There is a gap of 0.11 in for these configurations which corresponds to an expected breakdown at approximately $8.4 \mathrm{kV}$. Choosing $6 \mathrm{kV}$ as a maximum high voltage input gives a safety factor of approximately 1.4. Also, the actual breakdown voltage is estimated to be slightly higher than $8.4 \mathrm{kV}$ since the FC-72 or HFE-7000 (which are dielectric fluids) flow between the charged electrode and ground and insulates better than air alone.

Before the data acquisition system was connected and the electrode caps tested, a non-operational pedestal was installed in the chamber. Each electrode was placed in the chamber and attached to the sump with the set screw. The high voltage feedthrough was connected to the electrode pin as shown in Figure 3.40. 


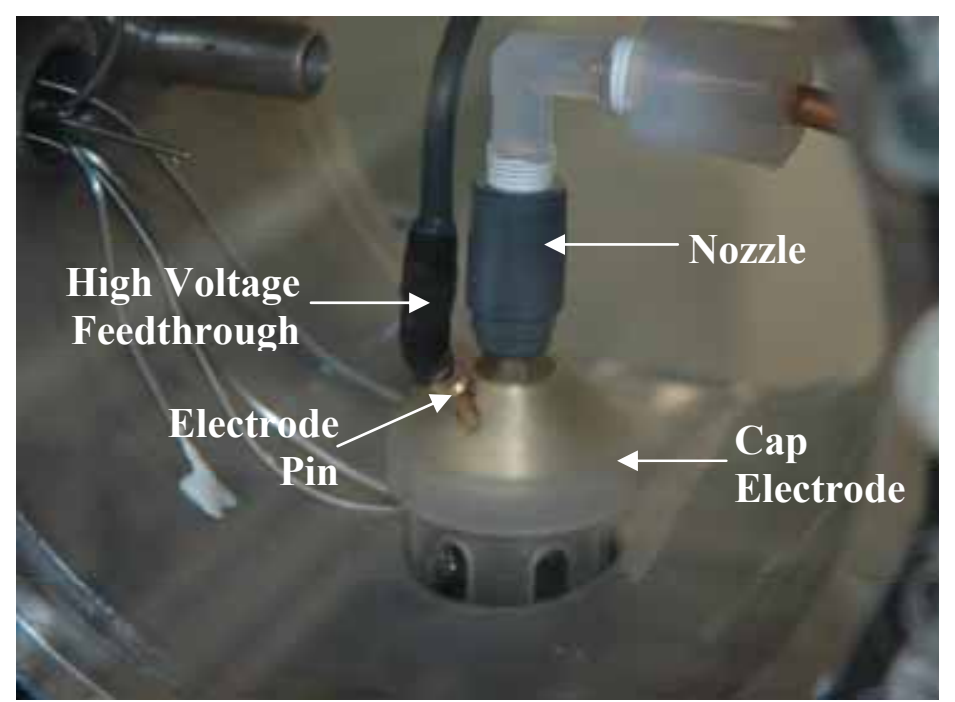

Figure 3.40: Full Length Cap Electrode inside Chamber with High Voltage Feedthrough Attached to Electrode Pin

The spray apparatus was turned on and the high voltage power supply was incrementally adjusted in a range from $0 \mathrm{kV}$ to $6 \mathrm{kV}$. (The polarity of the high voltage power supply was set to a negative value, but it is assumed that the sign of the polarity would not affect the overall outcome of the tests.) The current output on the high voltage power supply and the chamber interior were observed for signs of electrostatic breakdown. None were observed. The PTFE pedestal was then installed and each cap electrode was tested. Appendix B gives the full test plan for electrodes. Only one flowrate (the highest of $0.029 \mathrm{~m}^{3} / \mathrm{hr}$ or $7.72 \mathrm{GPH}$ of FC-72) was tested with each of the cap electrodes.

The test profiles from the data acquisition system for the bottom half, thick top half, full length, and top half electrodes with FC-72 as the working fluid are shown in Figure 3.41 through Figure 3.44, respectively. The heater power, Q, and the thermocouple one temperature, $\mathrm{T} 1$, can be read on the left primary axis and the high voltage, $\mathrm{HV}$, can be read on the right secondary axis. For each test, the TFR heater was 
tested up to near critical heat flux in $10 \mathrm{~W}$ increments first with no high voltage input. The TFR heater was then brought back to $0 \mathrm{~W}$ and tested again in $10 \mathrm{~W}$ increments with the high voltage being adjusted in $3 \mathrm{kV}$ increments at each heater power setting. Note that for the bottom half electrode (Figure 3.41), the heater power was increased to nearly $100 \mathrm{~W}$ while the other three tests reach only $70-75 \mathrm{~W}$. This test was the first performed and was used to help determine at what approximate heater power the onset of critical heat flux occurred. The TFR on the PTFE pedestal unknowingly cracked at this high power and the high voltage test occurring directly afterward produced a temperature profile with much lower values. This test was still considered valid due to an analysis of the heater resistance that showed the resistance remained relatively constant during the high voltage test (although higher than before the crack developed). Also note that for the bottom half electrode that the high voltage tests were begun at $50 \mathrm{~W}$ rather than at 0 $\mathrm{W}$ as in the other three tests. This was done because it was first assumed that any effects would not be seen at the lower heater powers, but was then changed to include the full range. Data from these tests were reduced as shown in Appendix D, with an error analysis shown in Appendix E. 


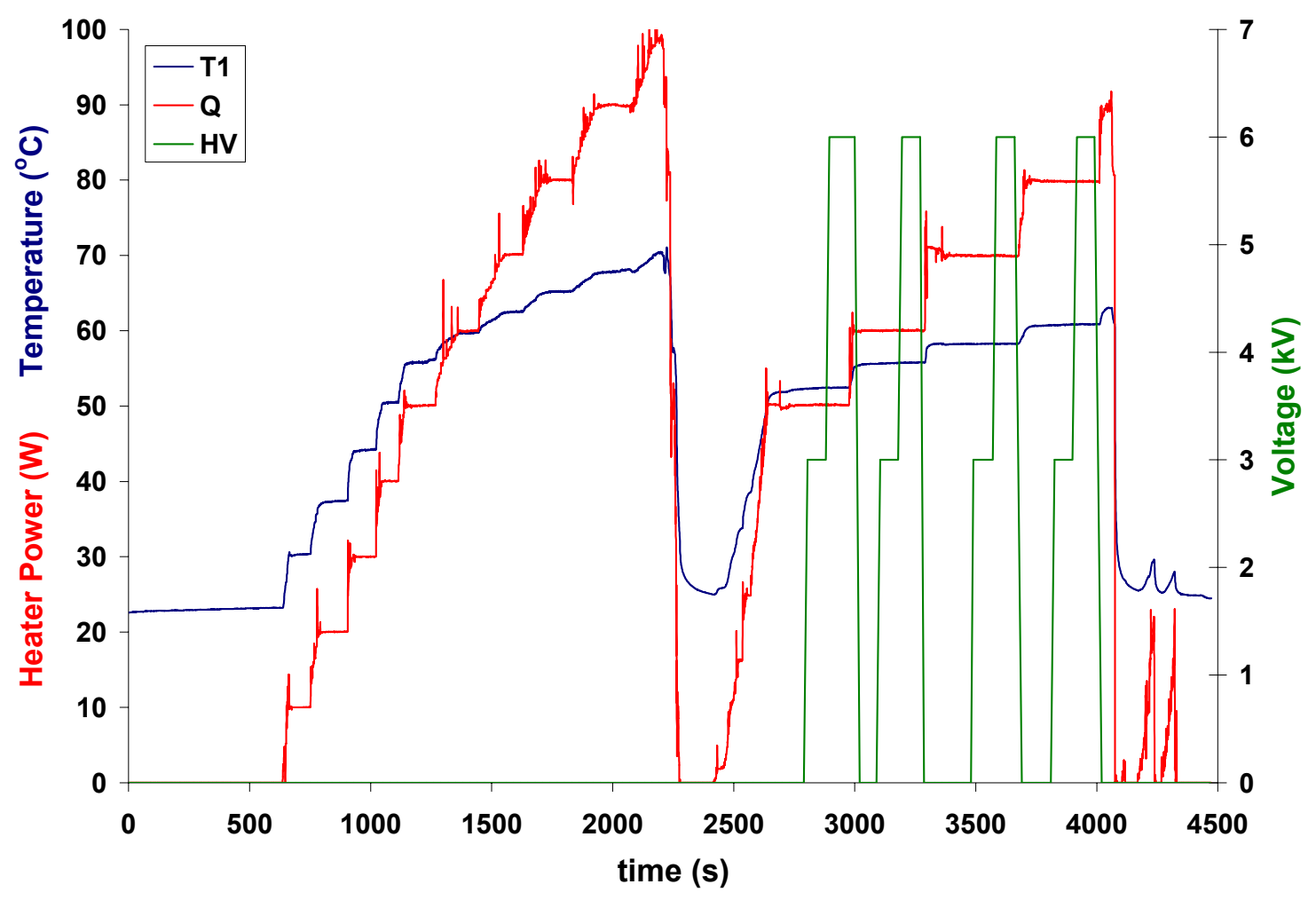

Figure 3.41: Test Profile for Bottom Half Cap Electrode

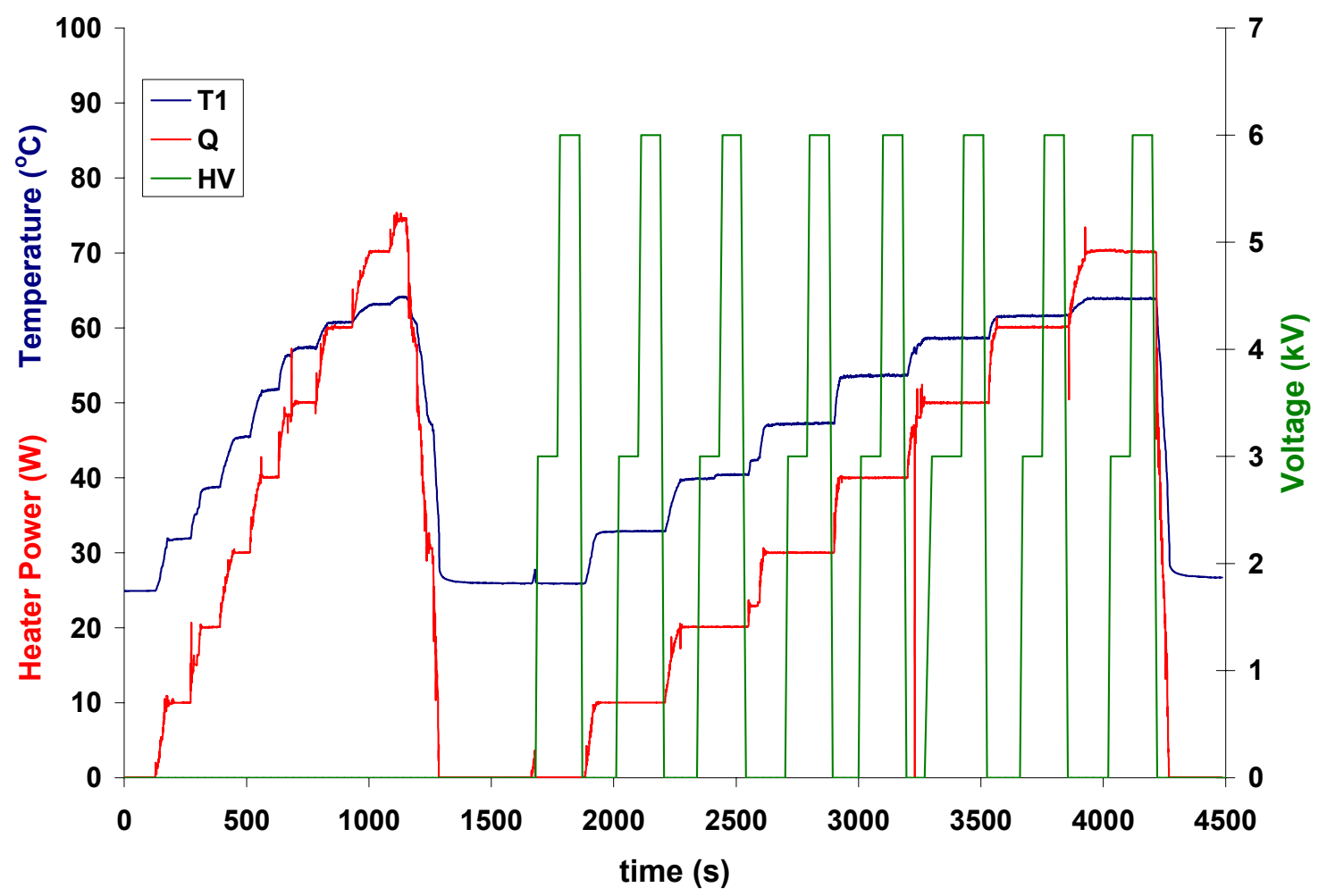

Figure 3.42: Test Profile for Thick Top Half Cap Electrode 


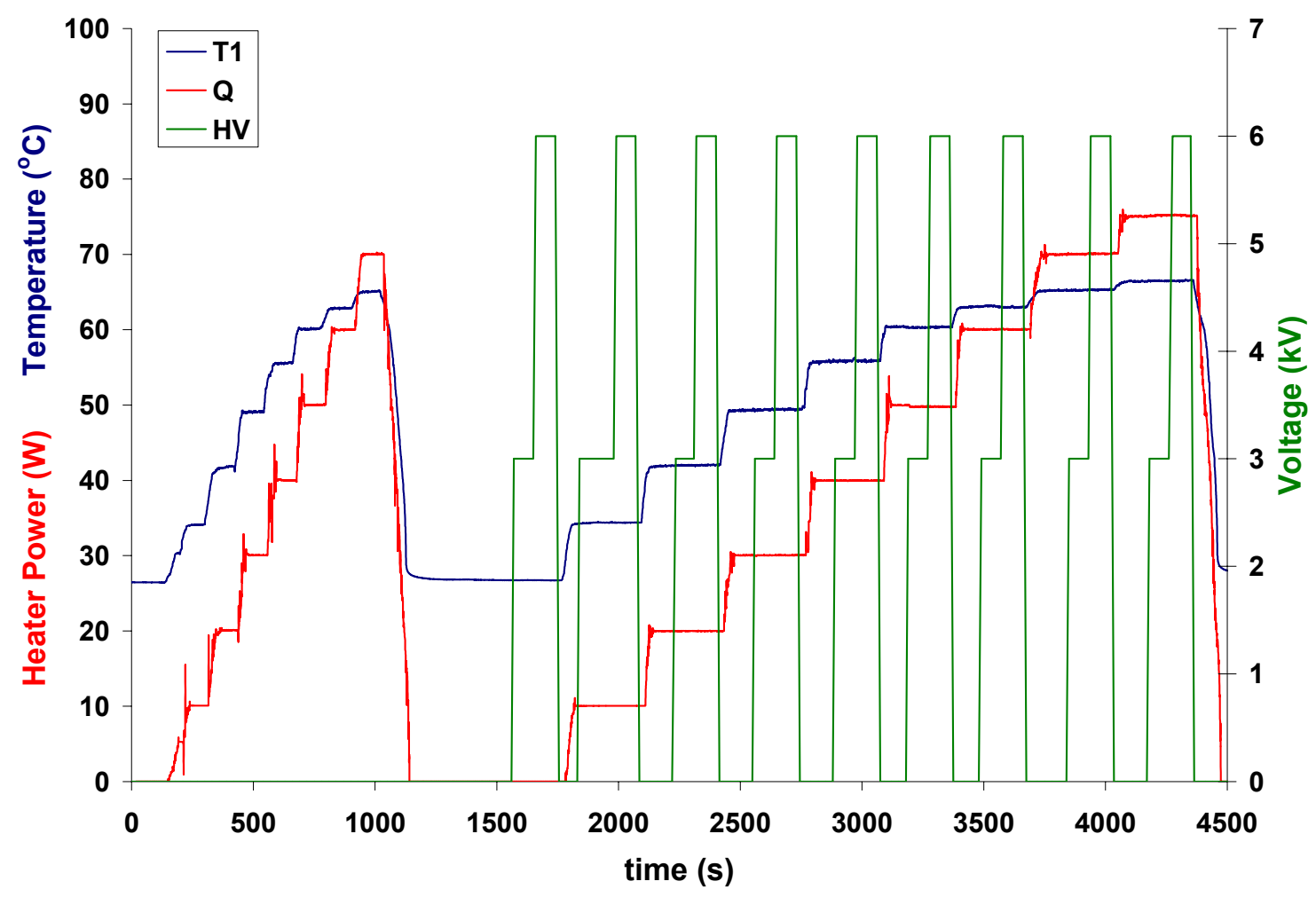

Figure 3.43: Test Profile for Full Length Cap Electrode

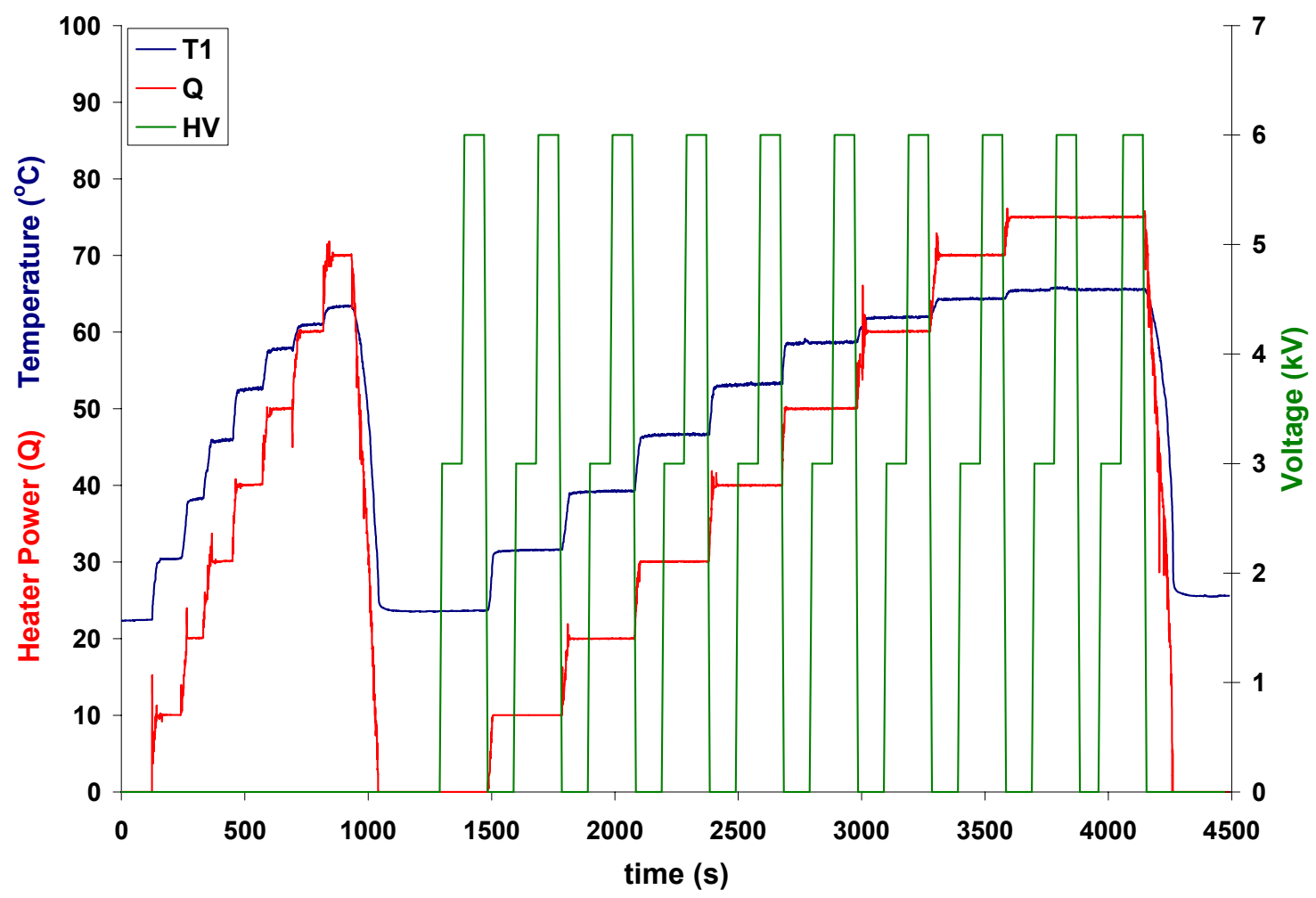

Figure 3.44: Test Profile for Top Half Cap Electrode 
In addition to the four cap electrodes being studied with FC-72 as the working fluid, the FC-72 flow loop was drained and replaced with HFE-7000. However, only the full length cap electrode was tested with this fluid and the test profile is shown below in Figure 3.45 .

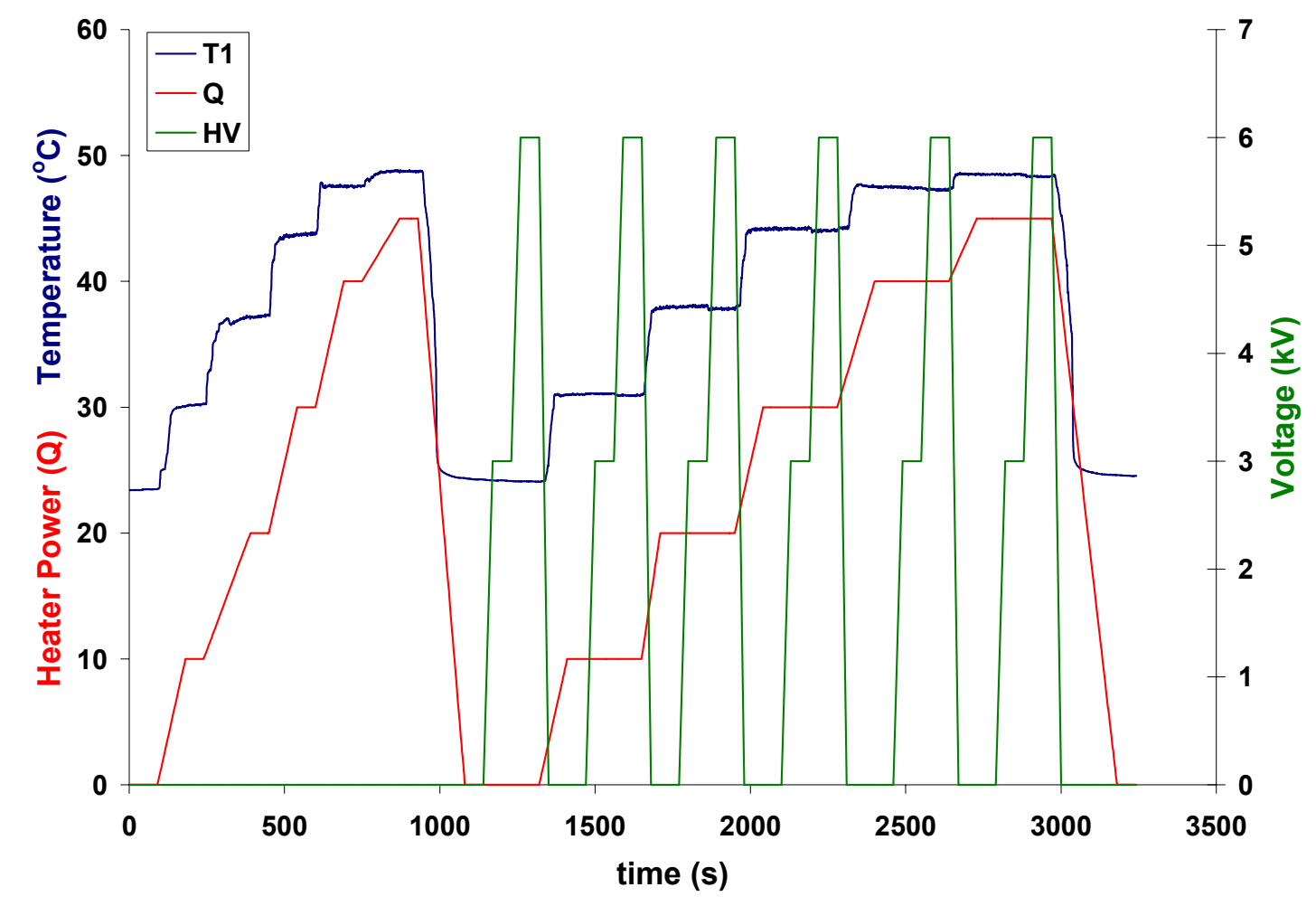

Figure 3.45: Test Profile for Full Length Electrode with HFE-7000 as the Working Fluid

\section{Mehra Electrode Performance Procedure}

The Mehra electrode tests were also performed after the modifications to the base (removal of the air accumulator and addition of a data acquisition system and high voltage power supply) were made. The Turn-On Checklist, Operating Procedure, and Turn-Off Checklist remained the same.

The maximum high voltage input limit was chosen as $23 \mathrm{kV}$ for this electrode configuration based on the electrostatic breakdown estimation for air at 1 atm pressure 
that for a distance of $1 \mathrm{~cm}$ between the charged electrode and ground, the allowable voltage is $30 \mathrm{kV}$. For the Mehra electrode, there was an estimated a gap of 0.32 in which corresponds to an expected breakdown at approximately $24 \mathrm{kV}$. Choosing $23 \mathrm{kV}$ as a maximum high voltage input gives a safety factor of approximately 1.0. Also, the actual breakdown voltage is estimated to be slightly higher than $24 \mathrm{kV}$ since the HFE-7000 (which is dielectric fluid) flows between the charged electrode and ground and insulates better than air alone.

Before the data acquisition system was connected and the Mehra electrode configuration tested, the non-operational pedestal was installed in the chamber. The nozzle and cap electrodes were placed in the chamber and attached to the nozzle and sump (respectively) with the set screws. The high voltage feedthrough was connected to the electrode pin on the nozzle electrode. The ground wire from the cap electrode was connected to the inner chamber wall using a piece of spring steel to form a press fit. These connections are shown in Figure 3.46.

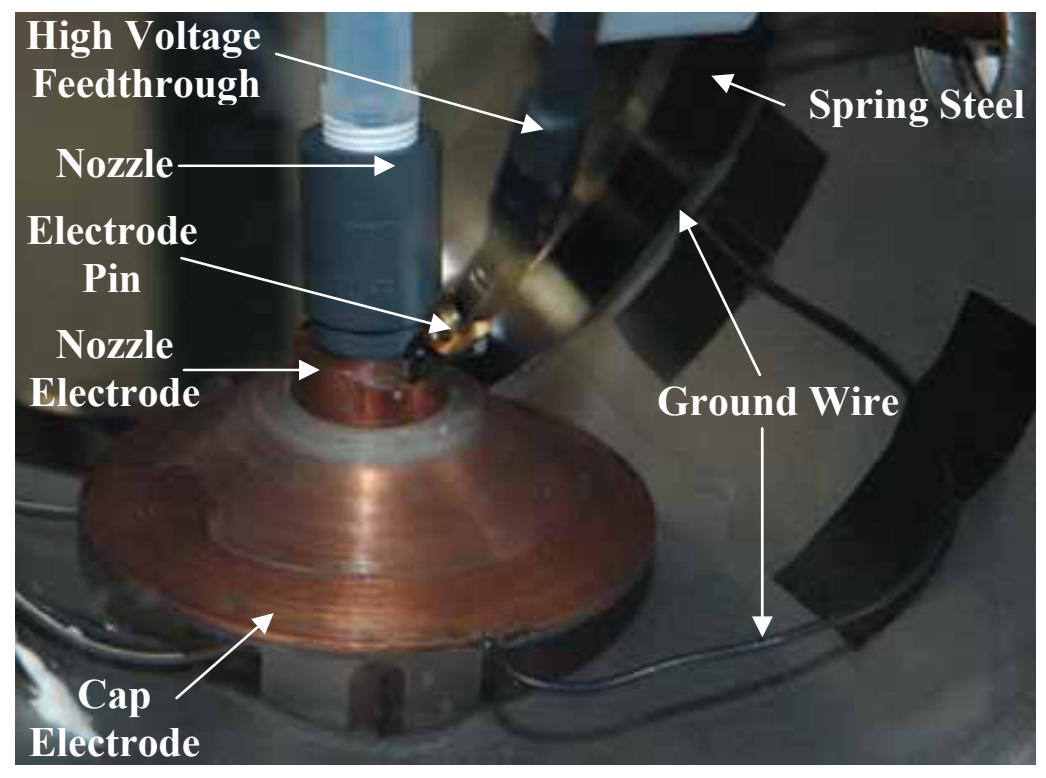

Figure 3.46: Mehra Electrodes inside Chamber with High Voltage Feedthrough Attached to Electrode Pin and Cap Electrode Attached to Grounded Chamber Wall 
The spray apparatus was turned on and the high voltage power supply was incrementally adjusted in a range from $0 \mathrm{kV}$ to $23 \mathrm{kV}$. (The polarity of the high voltage power supply was again set to a negative value, but it is assumed that the sign of the polarity would not affect the overall outcome of the tests.) The current output on the high voltage power supply and the chamber interior were observed for signs of electrostatic breakdown. None were observed, so the PTFE pedestal was installed and each cap electrode was then tested up to electrode voltage inputs of $23 \mathrm{kV}$. Appendix B gives the full test plan for the Mehra electrodes. Only one flowrate $\left(0.014 \mathrm{~m}^{3} / \mathrm{hr}\right.$ or $3.8 \mathrm{GPH}$ of HFE-7000) was tested.

The test profile from the data acquisition system is shown in two parts. Heater powers of 0 to $11 \mathrm{~W}$ were tested first and are shown in Figure 3.47, while the test profile for a repeat run at $11 \mathrm{~W}$ and also $12 \mathrm{~W}$ were performed on a different day and are shown in Figure 3.48. The thermocouple one temperature, T1, can be read on the left primary axis and the high voltage, $\mathrm{HV}$, and the heater power, $\mathrm{Q}$, can be read on the right secondary axis. The TFR heater was tested up to a heater power of $12 \mathrm{~W}$ in $1 \mathrm{~W}$ increments. The maximum of $12 \mathrm{~W}$ was chosen because a power level of $13 \mathrm{~W}$ corresponded to a thermocouple one temperature reading of above $150{ }^{\circ} \mathrm{C}$ which activated the temperature cutoff switch. Data from these tests were reduced as shown in Appendix D, with an error analysis shown in Appendix E. 


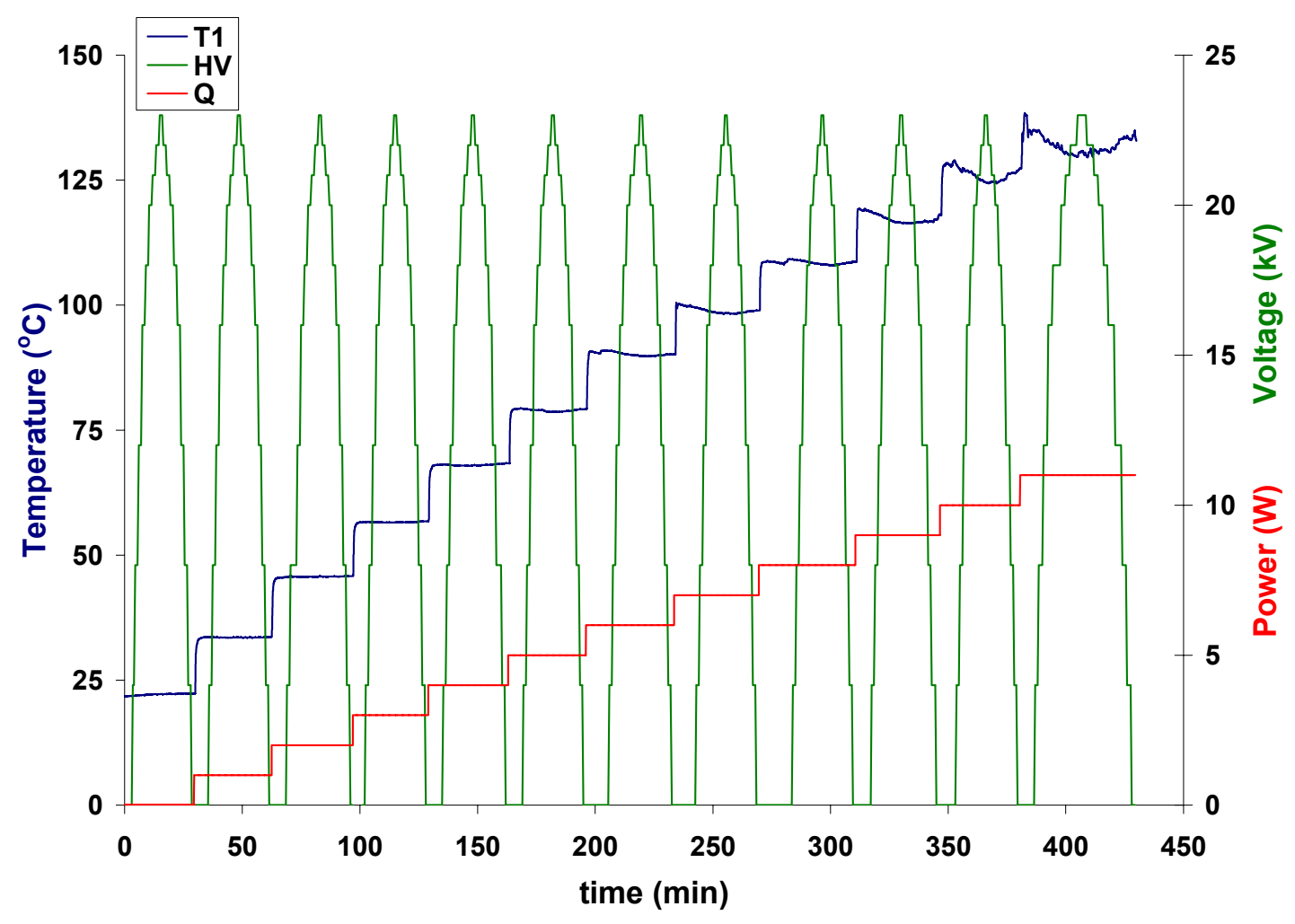

Figure 3.47: Test Profile for Mehra Electrodes for 0 Through $11 \mathrm{~W}$

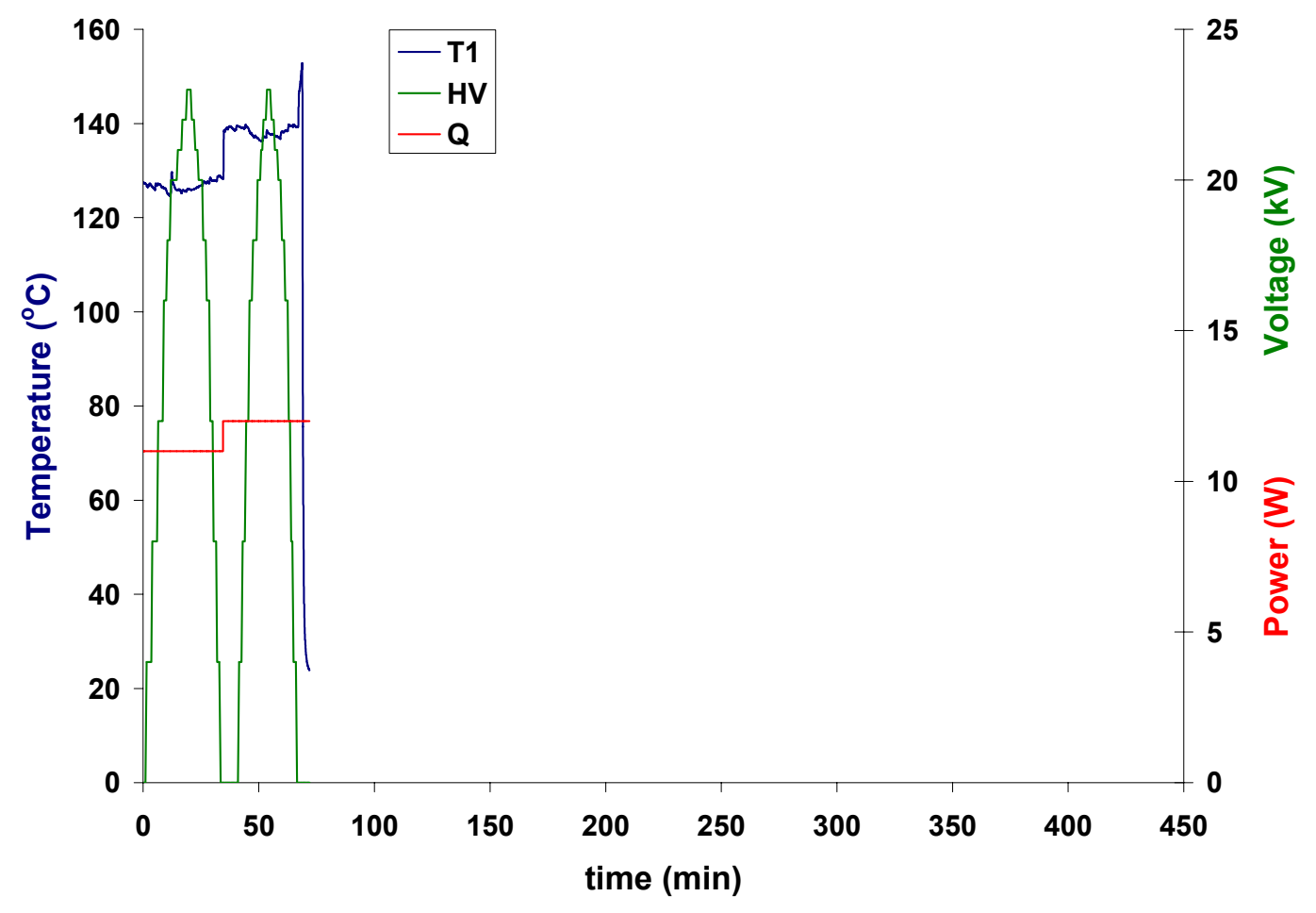

Figure 3.48: Test Profile for Mehra Electrodes for 11 and $12 \mathrm{~W}$ 


\section{Chapter 4: Presentation of Results and Discussion}

This chapter presents the results obtained from the nozzle-to-heater spacing performance comparison, cap electrode performance tests, and Mehra electrode performance tests. Section 4.1 gives the results obtained for the confined and unconfined cases for five different nozzle-exit-to-heater-surface spacings and heater powers ranging from $0 \mathrm{~W}$ to $70 \mathrm{~W}$. Section 4.2 presents the experimental results obtained for the four initial cap electrode geometries for a range of a high voltage input of 0 to $6 \mathrm{kV}$ and heater powers reaching near critical heat flux. Also presented in this section are a summary of results of a numerical simulation study (Mehra, 2006) of the predicted electric fields for the four cap electrodes and a discussion of possible factors contributing to the experimental and numerical results. Section 4.3 presents the experimental performance results and a summary of the predicted electric field results for the Mehra electrode configuration.

\subsection{Spacing Comparison Test Results}

For the two flow conditions (confined and unconfined flow), five different nozzleto-heater spacings $(9 \mathrm{~mm}, 11 \mathrm{~mm}, 13 \mathrm{~mm}, 15 \mathrm{~mm}$, and $17 \mathrm{~mm})$ were tested at heater powers ranging from $0 \mathrm{~W}$ to $70 \mathrm{~W}$ (and in one case $86.5 \mathrm{~W}$ ) using a PVC nozzle at a flow rate of $0.029 \mathrm{~m}^{3} / \mathrm{hr}(7.72 \mathrm{GPH})$ of FC-72, and data were recorded as described Chapter 3 . The raw data was reduced as shown in Appendix D, and the error analysis shown in Appendix E has been used to estimate the experimental uncertainty in these results. For the confined study, a plot of the heat flux in $\mathrm{W} / \mathrm{m}^{2}$ as a function of the difference between the heater surface temperature, $\mathrm{T}_{\mathrm{s}}$, and the temperature at the top of the pedestal, $\mathrm{T}_{\infty, \text { top }}$, in 
degrees Celsius was created and is shown in Figure 4.1. A non-dimensional plot showing the non-dimensional heat flux, G $\Delta$ (defined in Appendix D), versus the non-dimensional temperature difference between the heater surface and the liquid film on top of the pedestal is shown in Figure 4.2. As can be seen for each spacing, the temperature difference increases non-linearly with heat flux. The heater surface is hottest at the $9 \mathrm{~mm}$ spacing while it is coolest at the $17 \mathrm{~mm}$ spacing. The other spacings fall very closely together between these, but not in ascending order as would be assumed. The discrepancy is within experimental uncertainty in the temperature and heat flux measurements and may be due to errors in realigning the nozzle to be concentric with the heater for each different nozzle-to-heater spacing. (The error bars are shown on the 9 mm spacing curves below.) Also, the nozzle distance was realigned with an approximate error of $0.5 \mathrm{~mm}$.

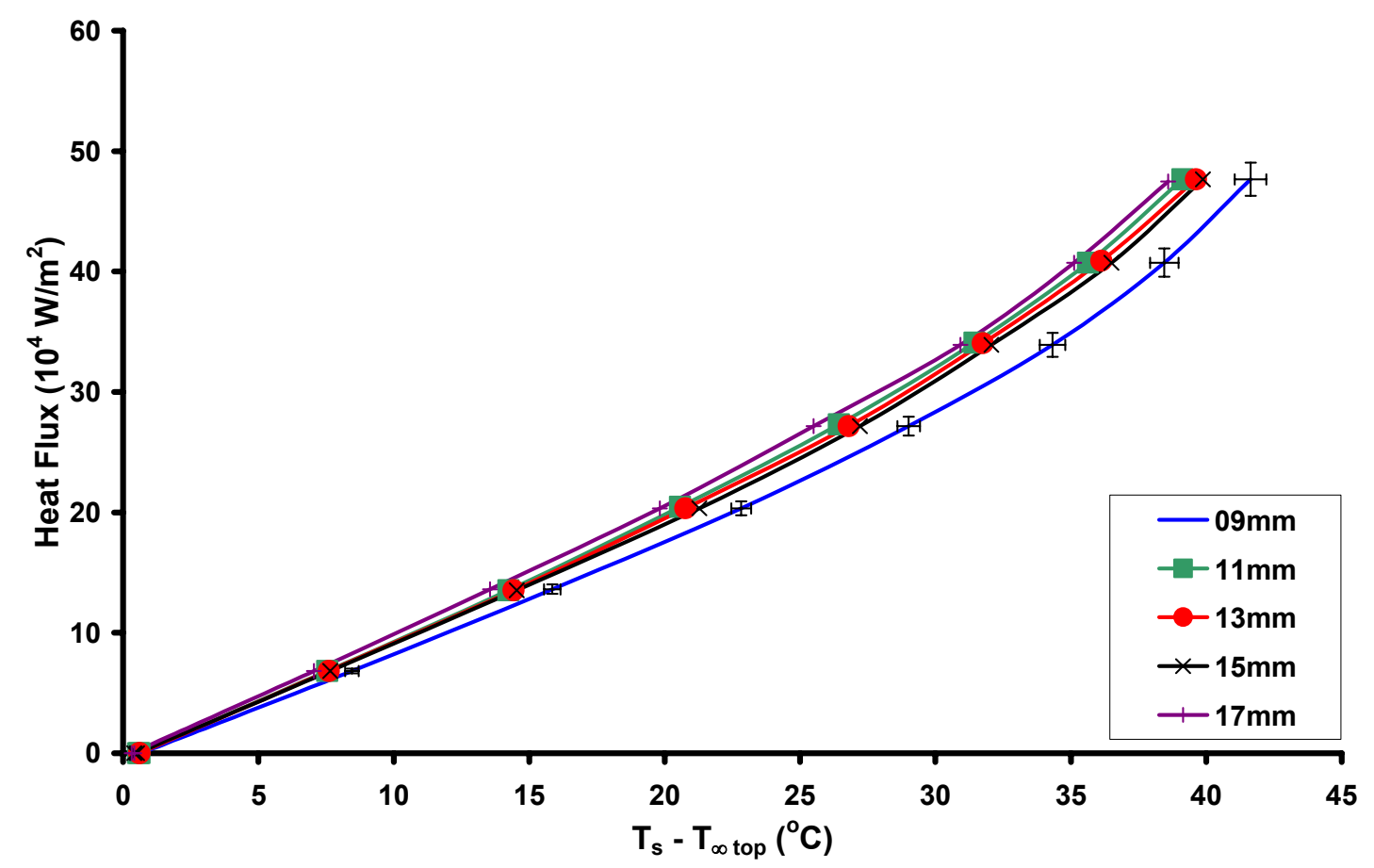

Figure 4.1: Heat Flux in $\mathrm{W} / \mathrm{m}^{2}$ as a Function of the Temperature Difference between the Surface and the Top of the Pedestal in Degrees Celsius for the Confined Case 


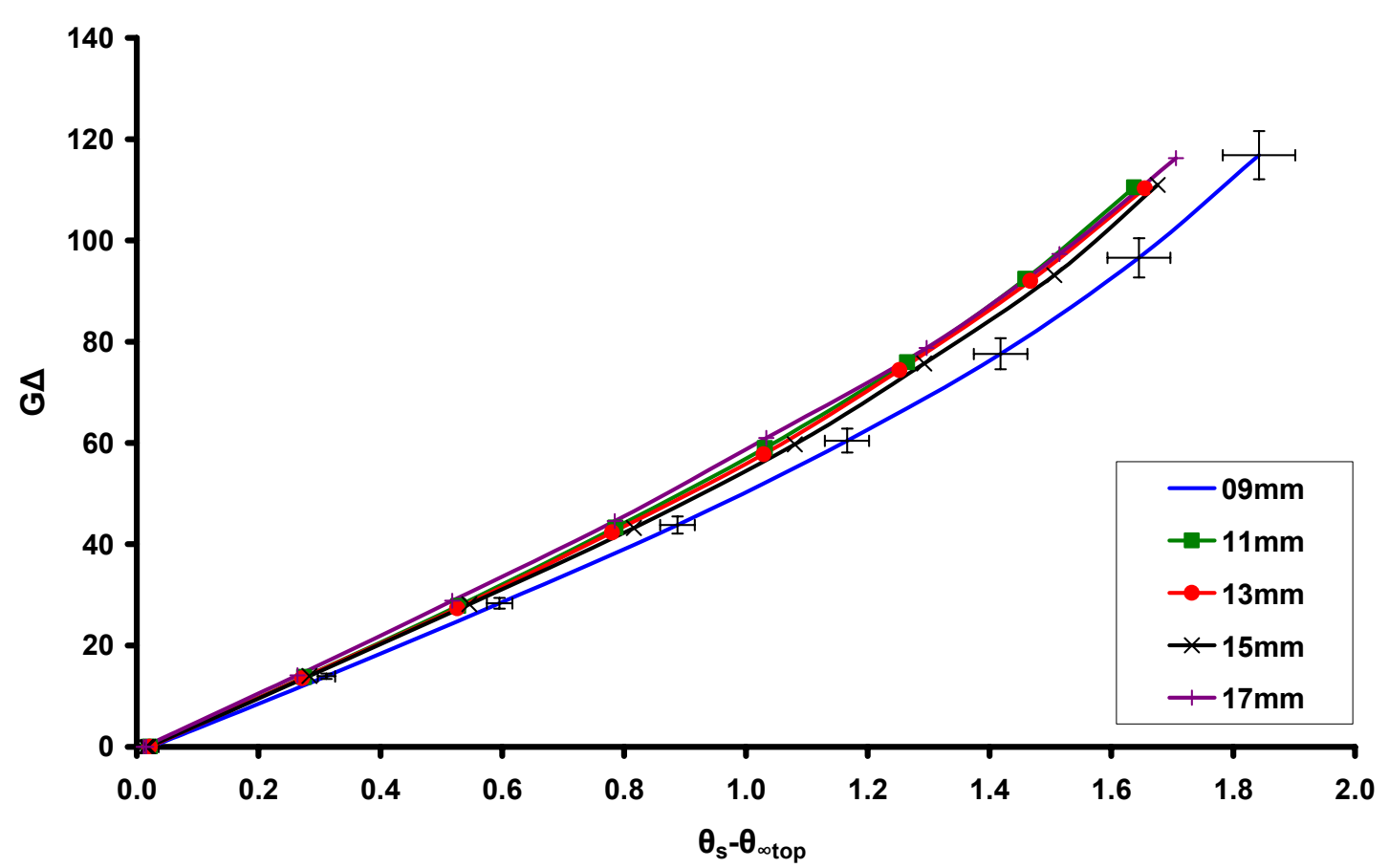

Figure 4.2: Non-dimensional Heat Flux as a Function of the Non-dimensional Temperature Difference between the Surface and the Pedestal Top for the Confined Case

Plots showing the heat transfer coefficient, $\mathrm{h}$, in $\mathrm{W} / \mathrm{m}^{2} \mathrm{~K}$ as a function of the heat flux in $\mathrm{W} / \mathrm{m}^{2}$ and the non-dimensional version (Nusselt number as a function of $\mathrm{G} \Delta$ ) for the confined case are shown in Figure 4.3 and Figure 4.4, respectively. These plots show that the heat transfer coefficient increases with heat flux. The heat transfer coefficient is lowest for the $9 \mathrm{~mm}$ spacing and highest for the $17 \mathrm{~mm}$ spacing. The other values fall between these two. 


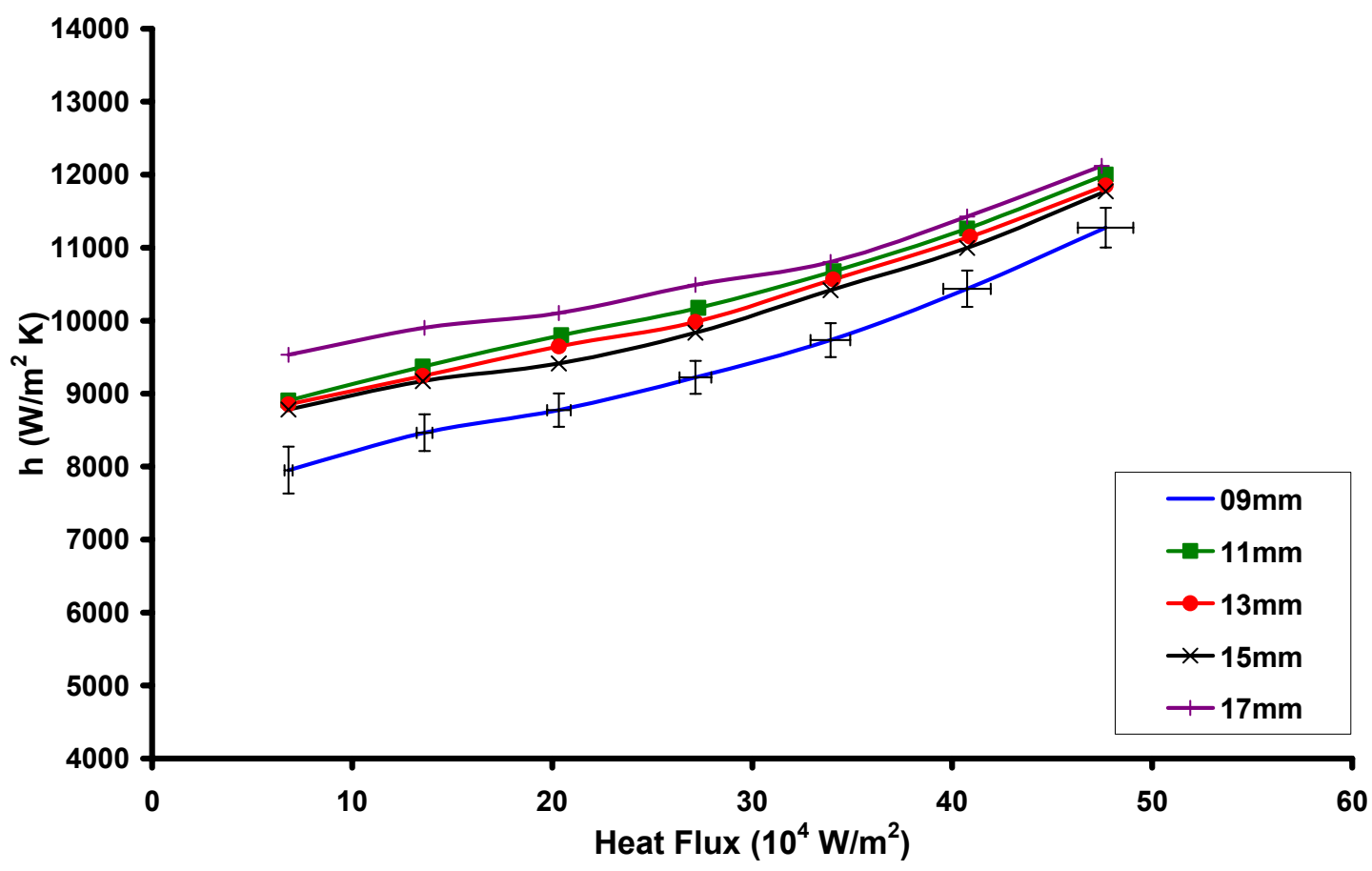

Figure 4.3: Convection Heat Transfer Coefficient in $\mathrm{W} / \mathrm{m}^{2} \mathrm{~K}$ as a Function of Heat Flux in $\mathrm{W} / \mathrm{m}^{2}$ for the Confined Case

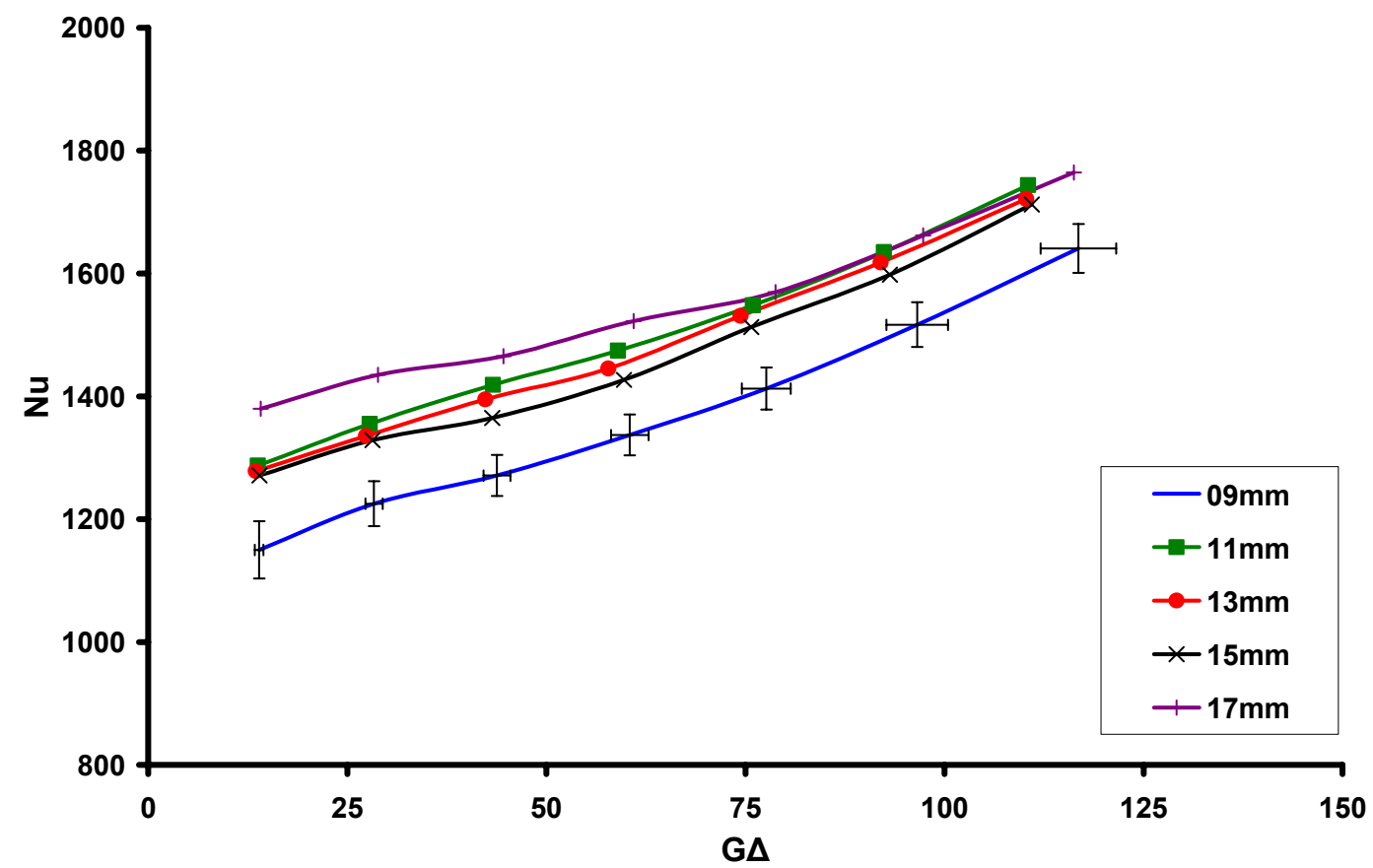

Figure 4.4: Nusselt Number as a Function of Non-dimensional Heat Flux for the Confined Case 
For the unconfined case, the plot of the heat flux in $\mathrm{W} / \mathrm{m}^{2}$ as a function of the difference between the heater surface temperature, $T_{\mathrm{s}}$, and the temperature at the top of the pedestal, $\mathrm{T}_{\infty, \text { top }}$, in degrees Celsius is shown in Figure 4.5. The corresponding nondimensional plot showing the non-dimensional heat flux, G $\Delta$, versus the non-dimensional temperature difference between the heater surface and the liquid film on top of the pedestal is shown in Figure 4.6. The heater surface is hottest at $9 \mathrm{~mm}$ followed by the second $13 \mathrm{~mm}$ spacing test values and the $11 \mathrm{~mm}$ spacing. The coolest heater surface occurred at the first $13 \mathrm{~mm}$ spacing with the 15 and $17 \mathrm{~mm}$ spacing falling very closely below. Note that for the second $13 \mathrm{~mm}$ spacing run, the heat flux was set to nearly $600,000 \mathrm{~W} / \mathrm{m}^{2}$ in an attempt to get closer to critical heat flux. Also note that one of the data points for the first $13 \mathrm{~mm}$ spacing (corresponding to a heater power of approximately $40 \mathrm{~W}$ ) was removed due to a reading error in manually recording the data.

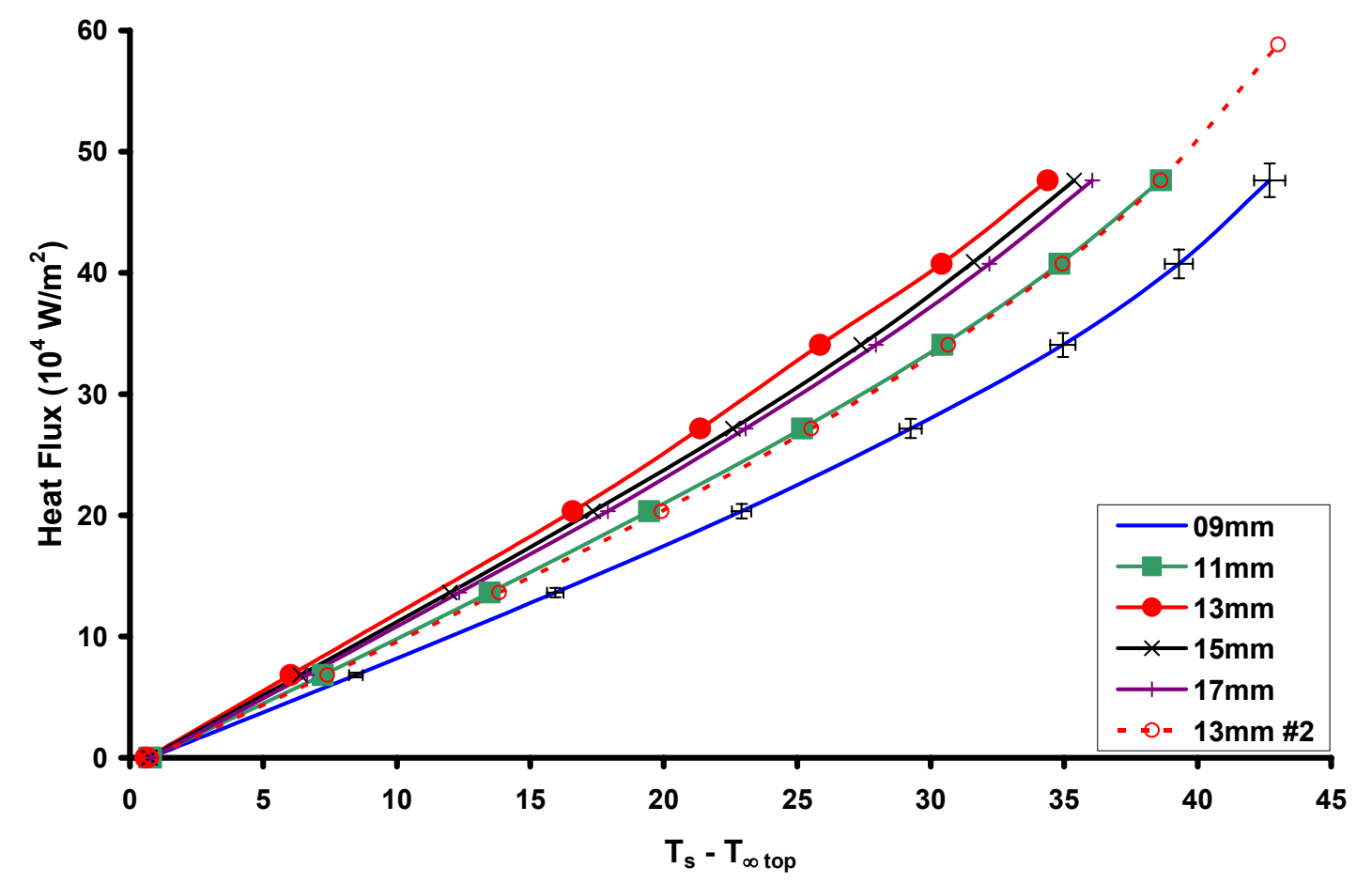

Figure 4.5: Heat Flux in $\mathrm{W} / \mathrm{m}^{2}$ as a Function of the Temperature Difference between the Surface and the Top of the Pedestal in Degrees Celsius for the Unconfined Case 


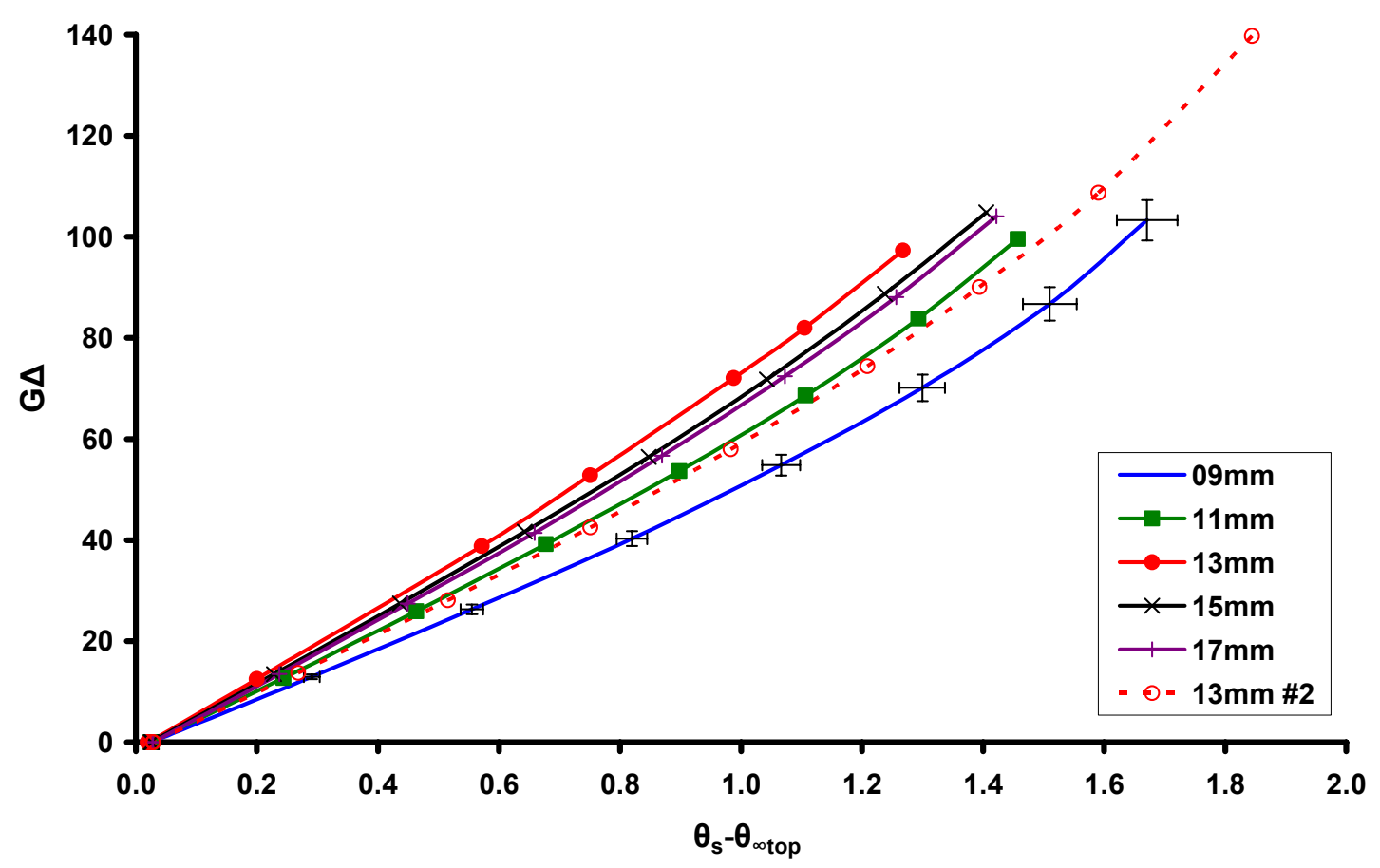

Figure 4.6: Non-dimensional Heat Flux as a Function of the Non-dimensional Temperature Difference between the Surface and the Pedestal Top for the Unconfined Case

Plots showing the heat transfer coefficient, $\mathrm{h}$, in $\mathrm{W} / \mathrm{m}^{2} \mathrm{~K}$ as a function of the heat flux in $\mathrm{W} / \mathrm{m}^{2}$ and the non-dimensional version (Nusselt number as a function of $\mathrm{G} \Delta$ ) for the unconfined case are shown in Figure 4.7 and Figure 4.8, respectively. These also show that the heat transfer coefficient increases with heat flux. The lowest heat transfer coefficient occurred for the $9 \mathrm{~mm}$ spacing and the highest occurred for the first $13 \mathrm{~mm}$ spacing. 


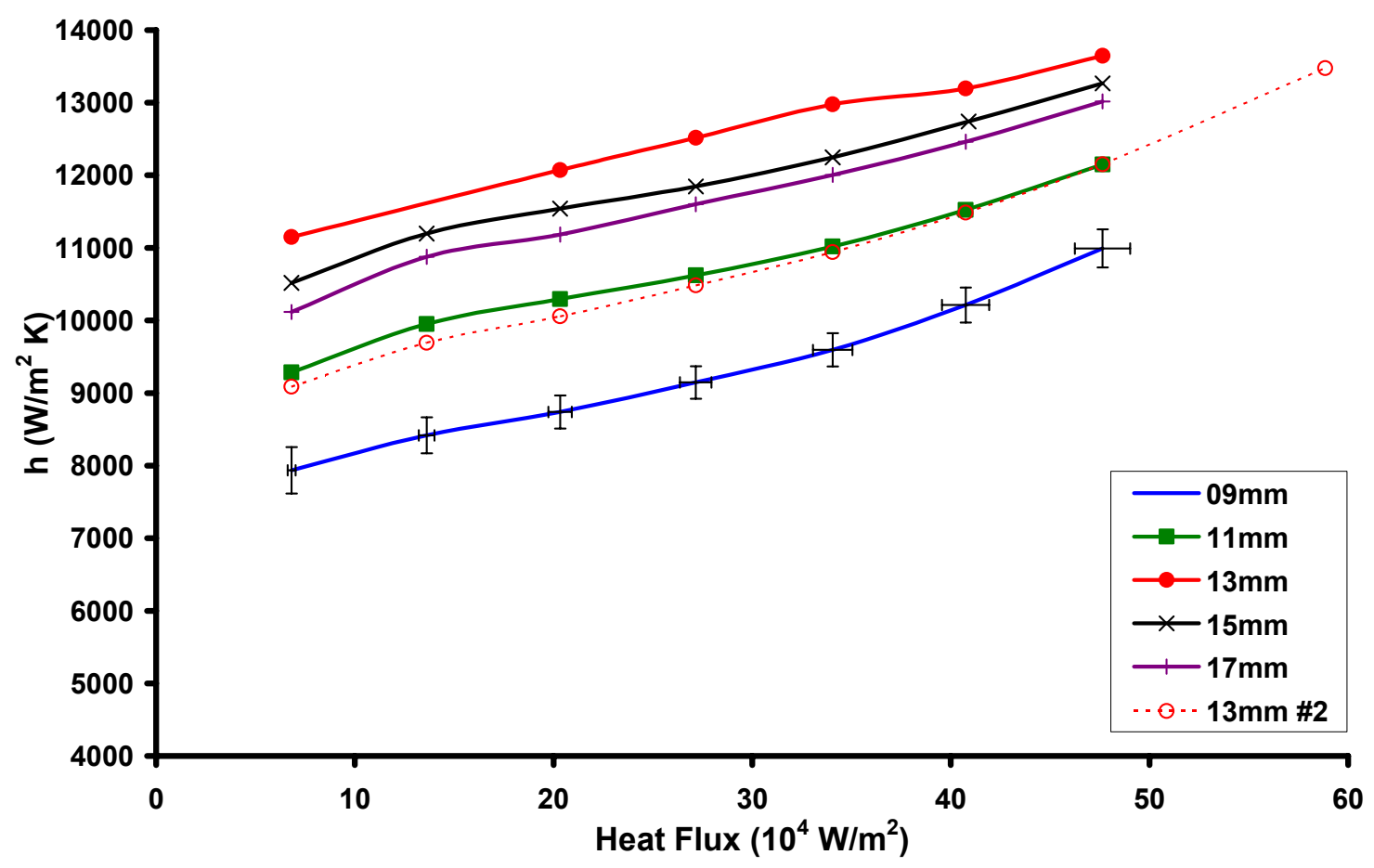

Figure 4.7: Convection Heat Transfer Coefficient in $\mathrm{W} / \mathrm{m}^{2} \mathrm{~K}$ as a Function of Heat Flux in $\mathrm{W} / \mathrm{m}^{2}$ for the Unconfined Case

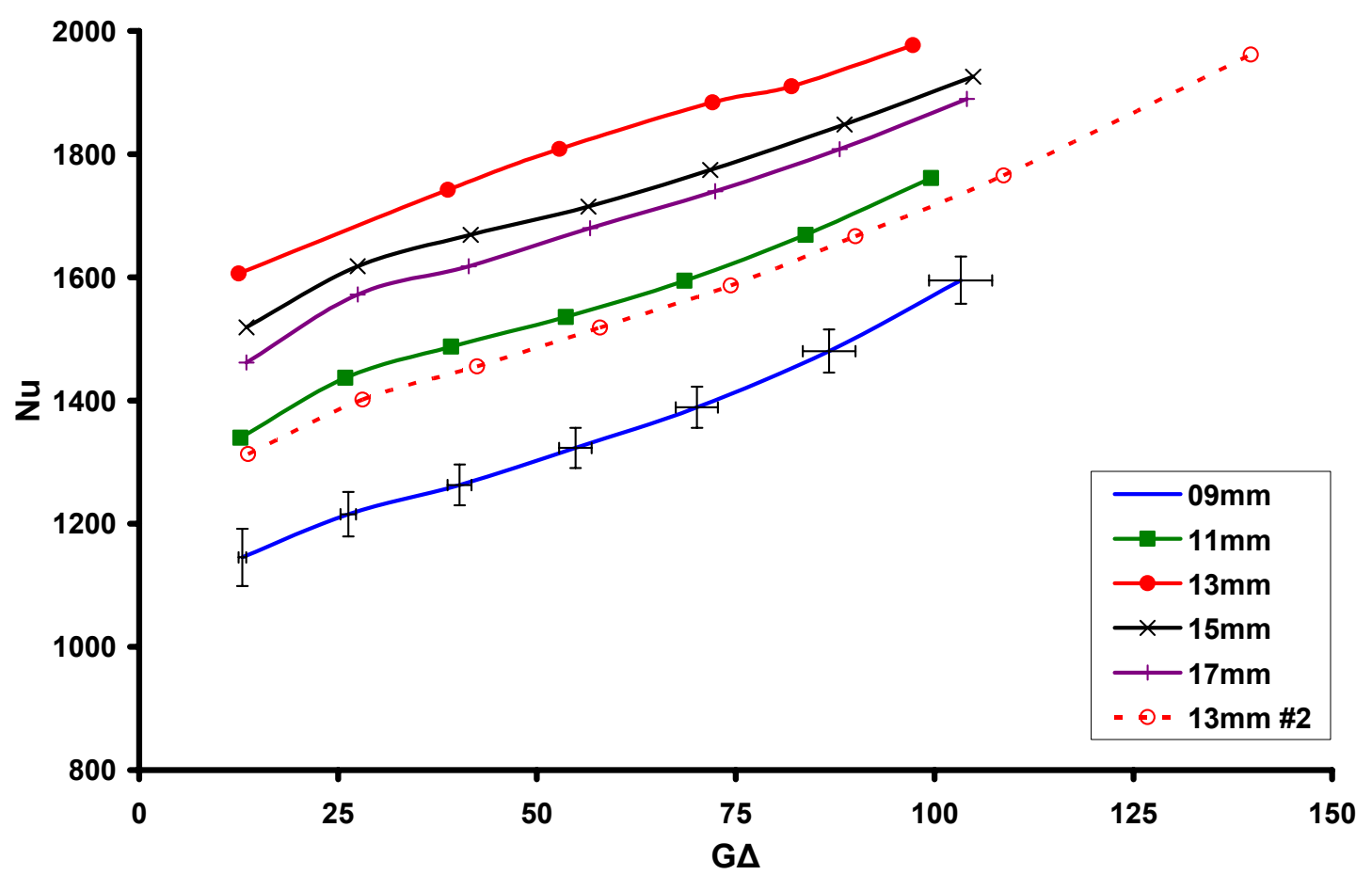

Figure 4.8: Nusselt Number as a Function of Non-dimensional Heat Flux for the Unconfined Case 
When comparing the corresponding plots for the confined and unconfined cases, it can be seen that the curves for each spacing are much more spread out for the unconfined case. This demonstrates that confined geometries are less dependent on the nozzle-to-heater distance. Also, the temperature difference increases more rapidly as heat flux is increased for the confined case. This disagrees with Hunnell (2005) who found that there is more efficient cooling for confined geometries and attributed this to recirculation or rebound of the spray droplets on the internal surface of the cap. The theory that the optimal nozzle-to-heater distance can be found by calculating the spray impact diameter from the nozzle-to-heater distance and spray cone angle is also supported by the data from this study. It may be noted however that for the unconfined study at a heater power of $70 \mathrm{~W}$, the change between the pedestal temperature differences from $9 \mathrm{~mm}$ to $13 \mathrm{~mm}$ (decreasing the distance from calculated optimal) was nearly five times higher than the pedestal temperature differences from $13 \mathrm{~mm}$ to $17 \mathrm{~mm}$ (increasing the distance from calculated optimal). The heat transfer coefficient differences at these same distances were increased by over four times.

Also, note that for the unconfined test, a run at a spacing of $13 \mathrm{~mm}$ was performed at the beginning and end of the set of data. The variability of approximately $20 \%$ between the two may be due to the error in realigning the spray nozzle over the heater surface. 


\subsection{Cap Electrode Test Results}

The four cap electrodes described in Chapter 3 were tested at electrode voltages of $0 \mathrm{kV}, 3 \mathrm{kV}$ and $6 \mathrm{kV}$ at various heater powers. Test parameters were changed as shown in the test profiles presented in Chapter 3. The full run from no power to near critical heat flux at the beginning of each profile is indicated in the plots below with an open diamond symbol. The closed symbols represent data collected at the high voltage setting at each power level.

The first geometry tested was the bottom half electrode; this electrode design has been presented Figure 3.23 and Figure 3.24. A full run at $0 \mathrm{kV}$ with heater powers ranging from 0 to $90 \mathrm{~W}$ was performed as indicated by the open diamond symbols in Figure 4.9 and Figure 4.10. However, during this test, the TFR heater on the PTFE pedestal unknowingly cracked, possibly due to surpassing critical heat flux. The incremental high voltage tests (closed symbols) were performed at heater powers ranging from 50 to $80 \mathrm{~W}$ after the crack developed. This data was not discarded after confirming that the resistance of the heater was nearly constant, although larger, after the crack formed. Figure 4.11 shows a graph of the calculated heater resistances before and after the crack formed. This jump in resistance explains why the temperature values for the full run at $0 \mathrm{kV}$ are much higher at the same heat flux level than for the incremental high voltage runs.

Figure 4.9 and Figure 4.10 show that there is no noticeable effect on pedestal temperatures for the bottom half cap electrode geometry. Also, there is no effect on the heat transfer coefficient (and therefore also Nusselt number) as shown in Figure 4.12 and Figure 4.13. Possible reasons that no effect was observed are discussed below. 


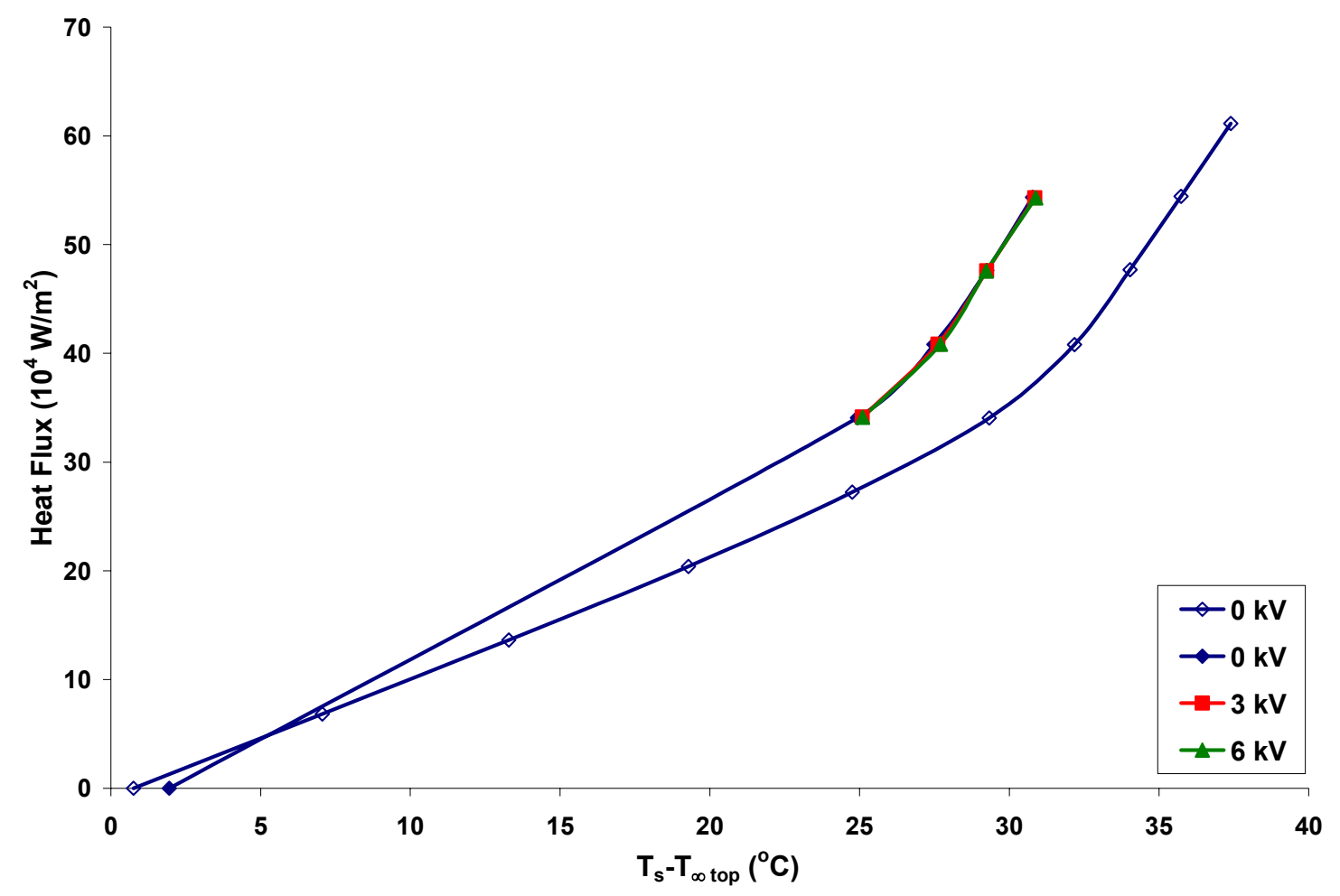

Figure 4.9: Heat Flux in $\mathrm{W} / \mathrm{m}^{2}$ as a Function of the Temperature Difference between the Surface and the Top of the Pedestal in Degrees Celsius for the Bottom Half Cap Electrode

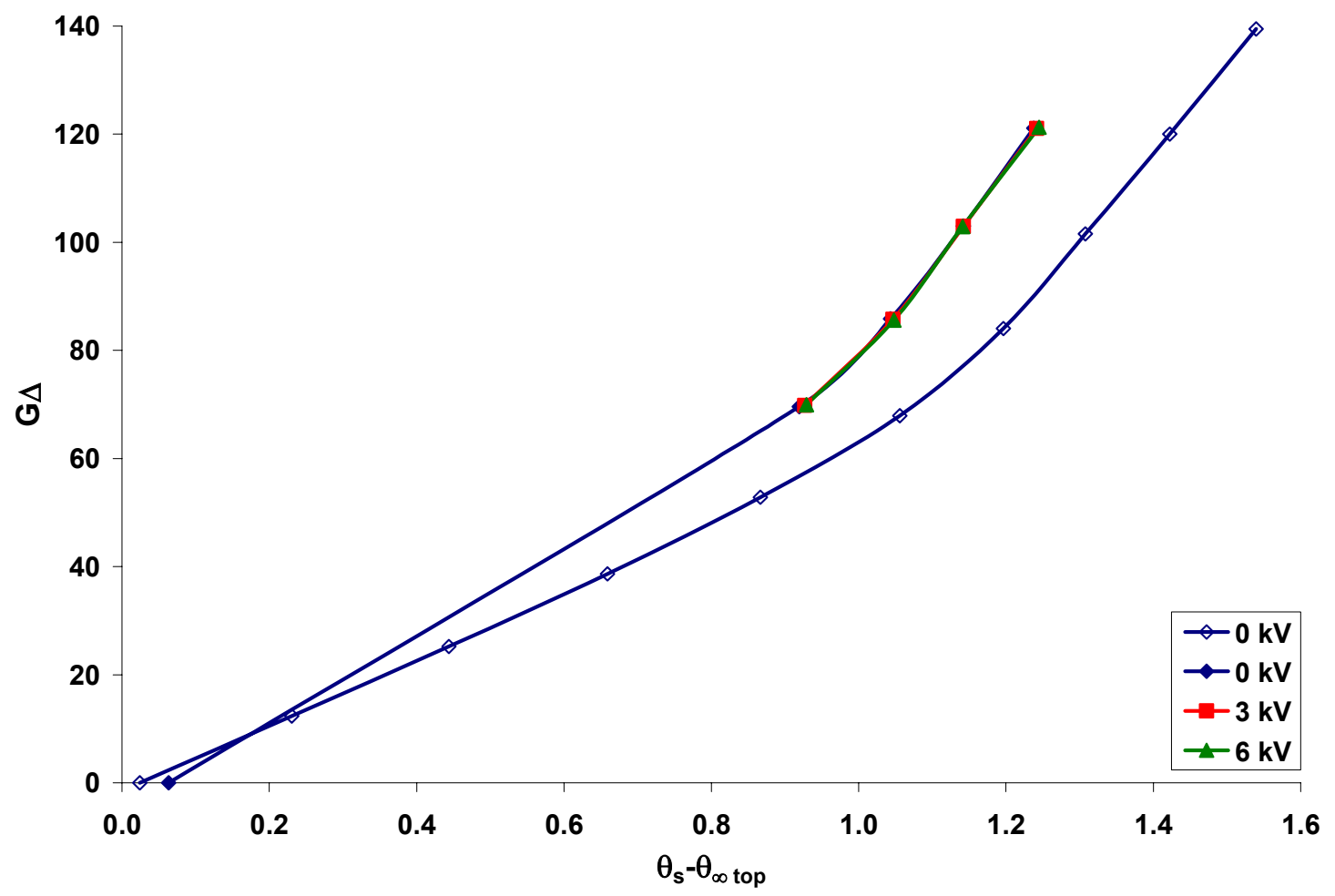

Figure 4.10: Non-dimensional Heat Flux as a Function of the Non-dimensional Temperature Difference between the Surface and the Pedestal Top for the Bottom Half Cap Electrode 


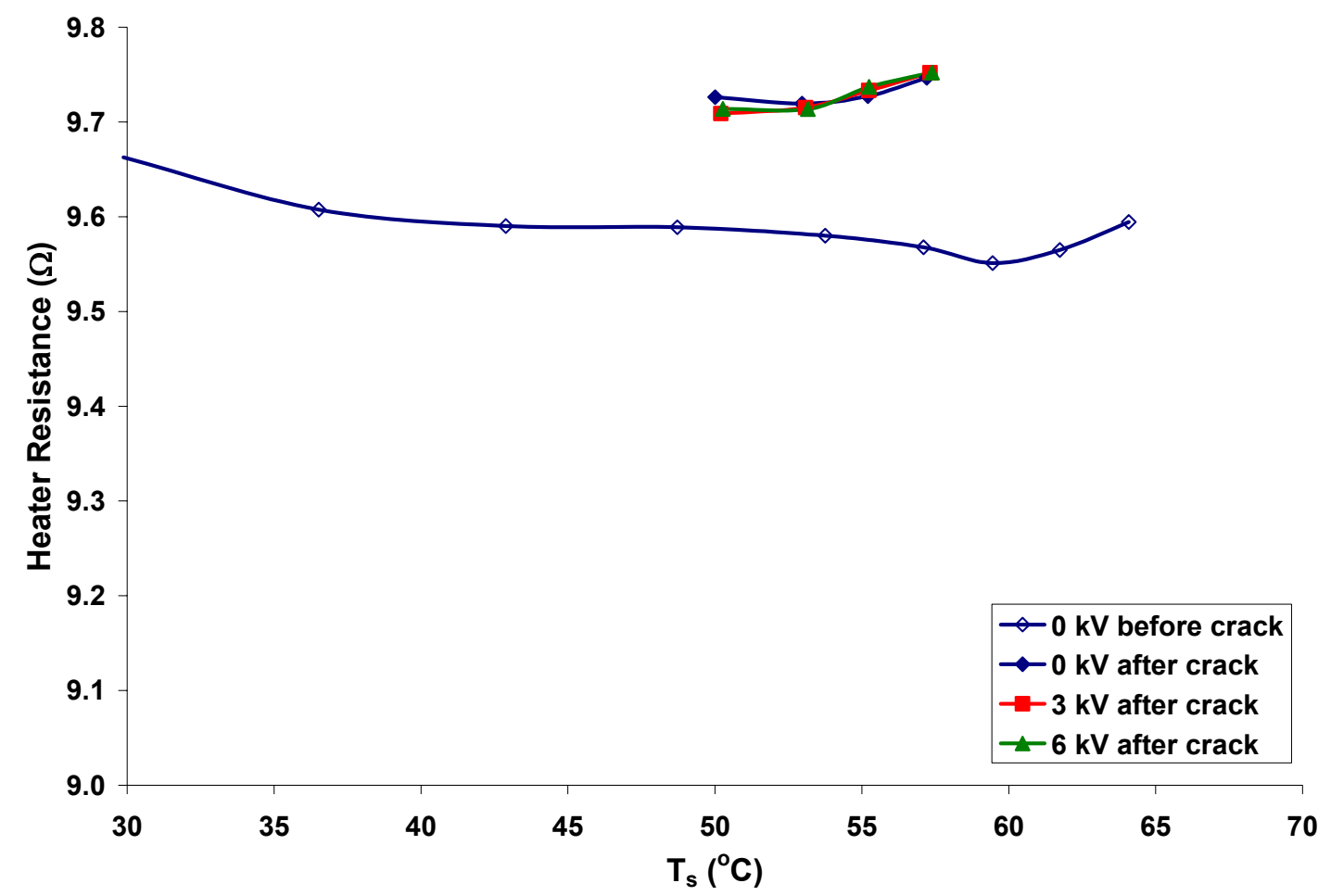

Figure 4.11: Heater Resistance in Ohms as a Function of Surface Temperature in Degrees Celsius for the Bottom Half Cap Electrode Before and After the Formation of a Crack

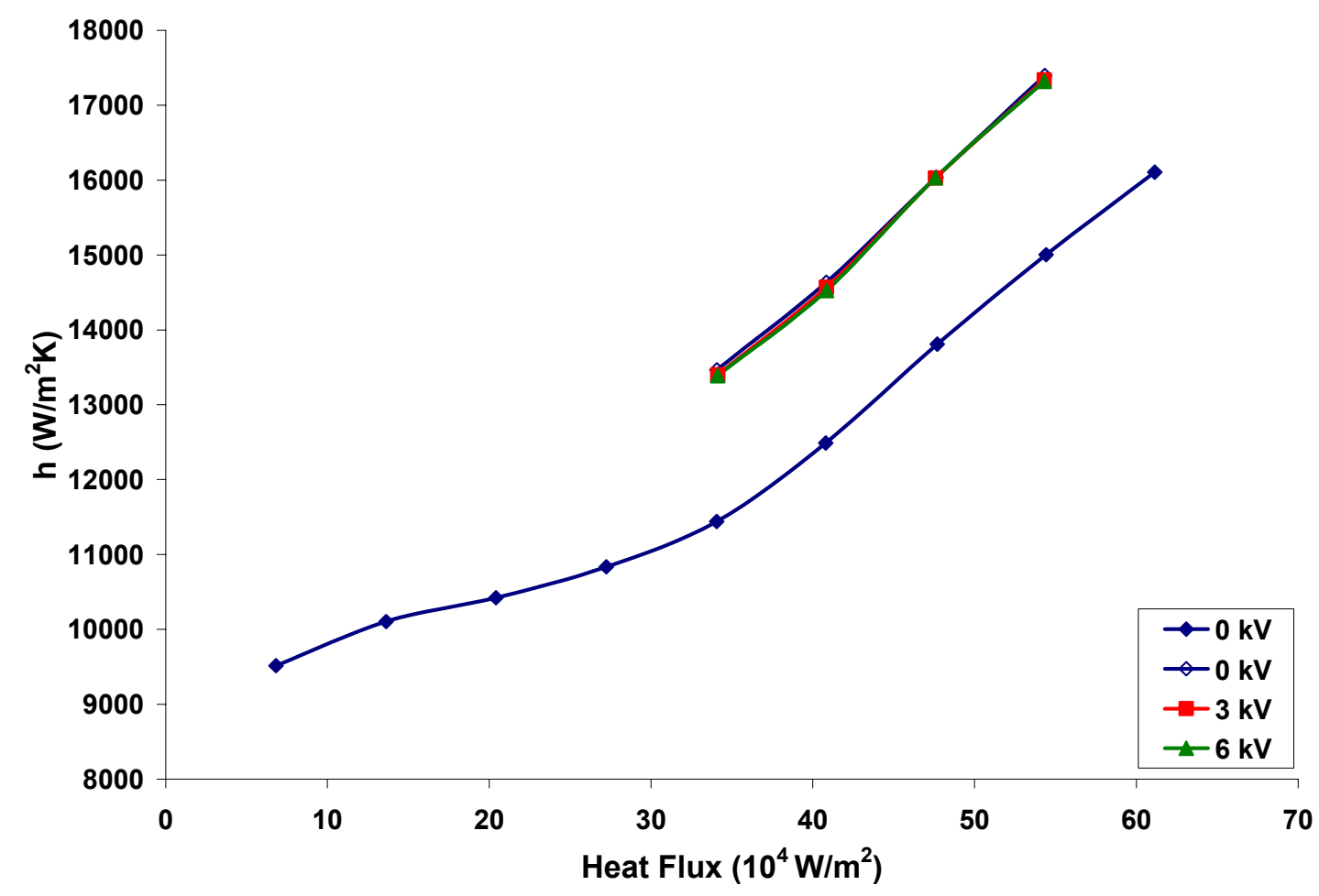

Figure 4.12: Convection Heat Transfer Coefficient in $\mathrm{W} / \mathrm{m}^{2} \mathrm{~K}$ as a Function of Heat Flux in $\mathrm{W} / \mathrm{m}^{2}$ for the Bottom Half Cap Electrode 


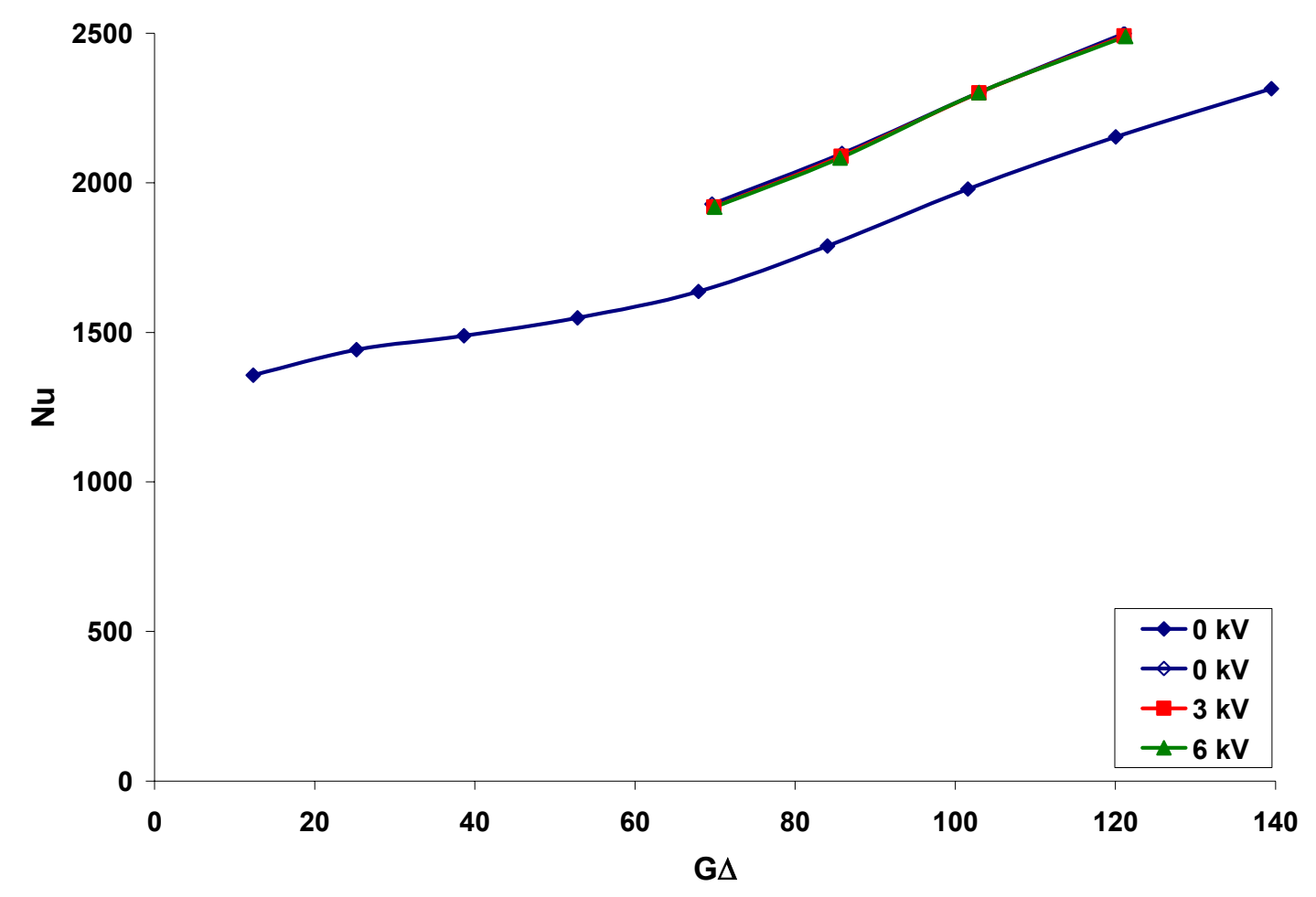

Figure 4.13: Nusselt Number as a Function of Non-dimensional Heat Flux for the Bottom Half Cap Electrode

The second test was performed on the thick top half electrode after replacing the cracked TFR heater. Heater powers ranging from 0 to $70 \mathrm{~W}$ were tested at 0,3 , and 6 $\mathrm{kV}$. Figure 4.14 and Figure 4.15 show that no significant effect was seen on the pedestal temperatures at these conditions. Figure 4.16 and Figure 4.17 also show no effects on the heat transfer coefficient and Nusselt number, respectively. Again, possible causes for the results are discussed below. A plot of the heater resistance versus surface temperature was also created for this electrode and the resistance values were relatively constant for each test ranging from 9.1 to $9.25 \Omega$. Note that the slope of the heat flux versus temperature difference in Figure 4.14 is nearly linear as the heat flux increases, but steepens at approximately $350,000 \mathrm{~W} / \mathrm{m}^{2}$ as critical heat flux (CHF) is approached. It appears that CHF occurs at a heat flux of approximately $500,000 \mathrm{~W} / \mathrm{m}^{2}$. 


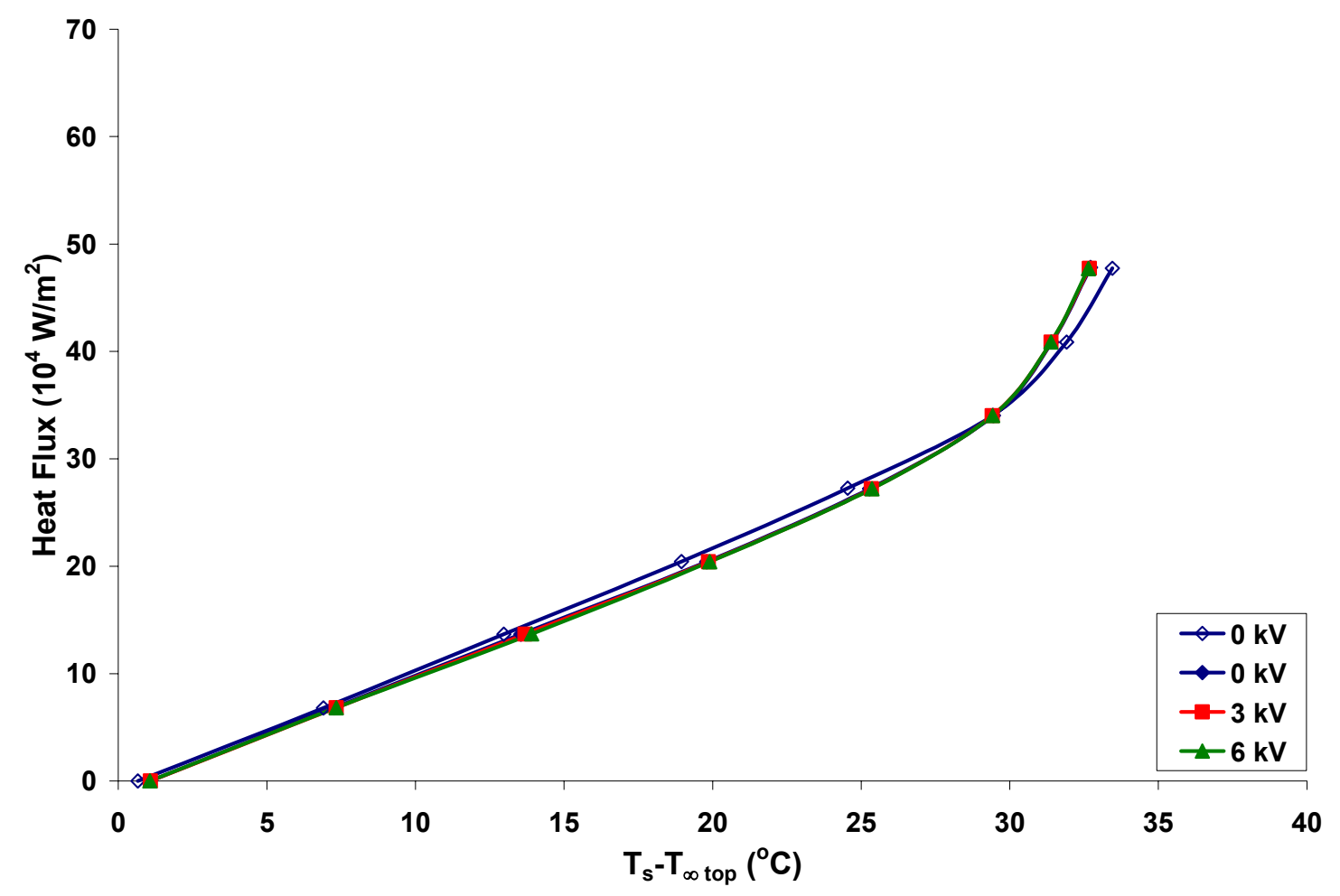

Figure 4.14: Heat Flux in $\mathrm{W} / \mathrm{m}^{2}$ as a Function of the Temperature Difference between the Surface and the Top of the Pedestal in Degrees Celsius for the Thick Top Half Cap Electrode

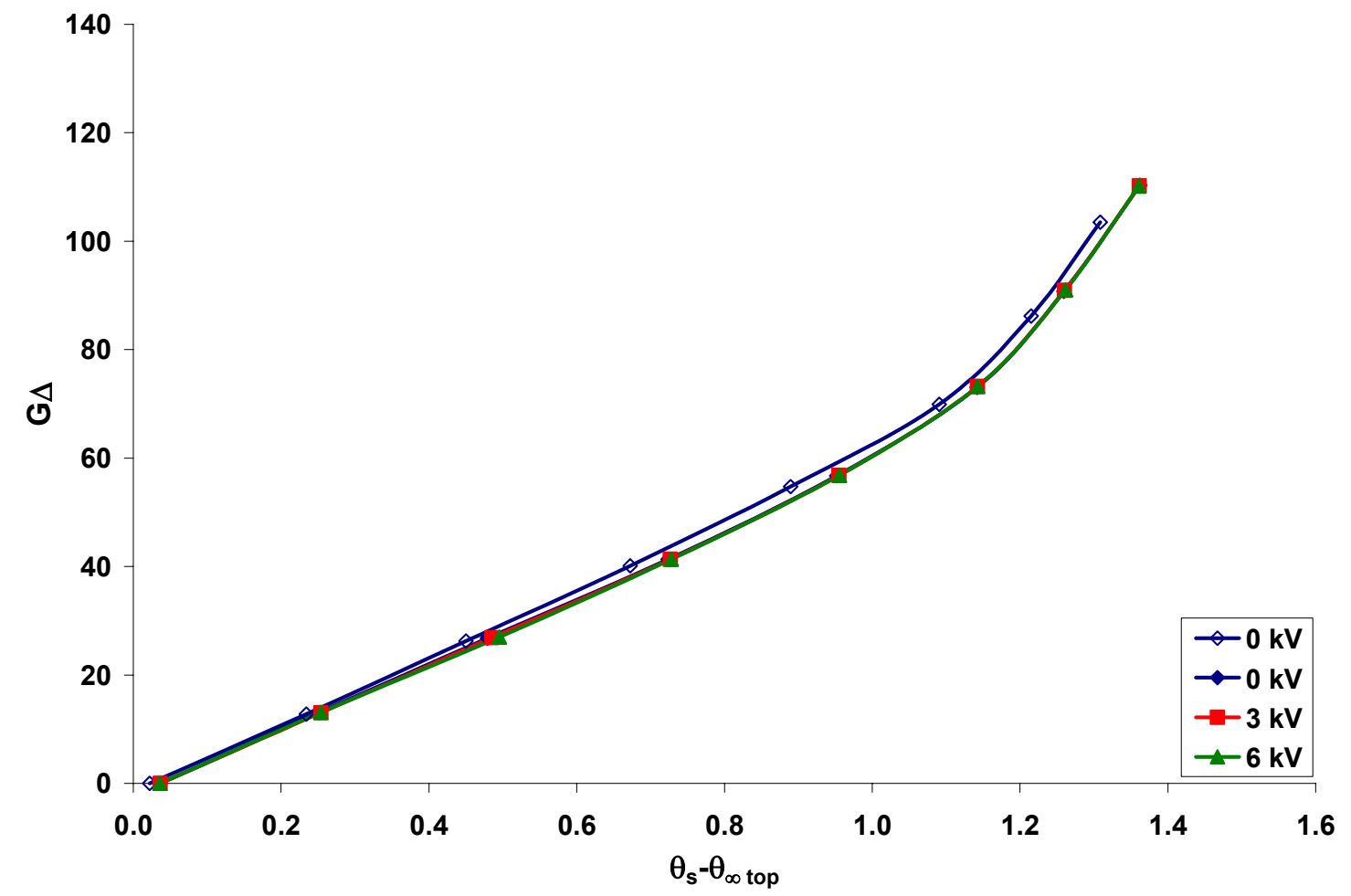

Figure 4.15: Non-dimensional Heat Flux as a Function of the Non-dimensional Temperature Difference between the Surface and the Pedestal Top for the Thick Top Half Cap Electrode 


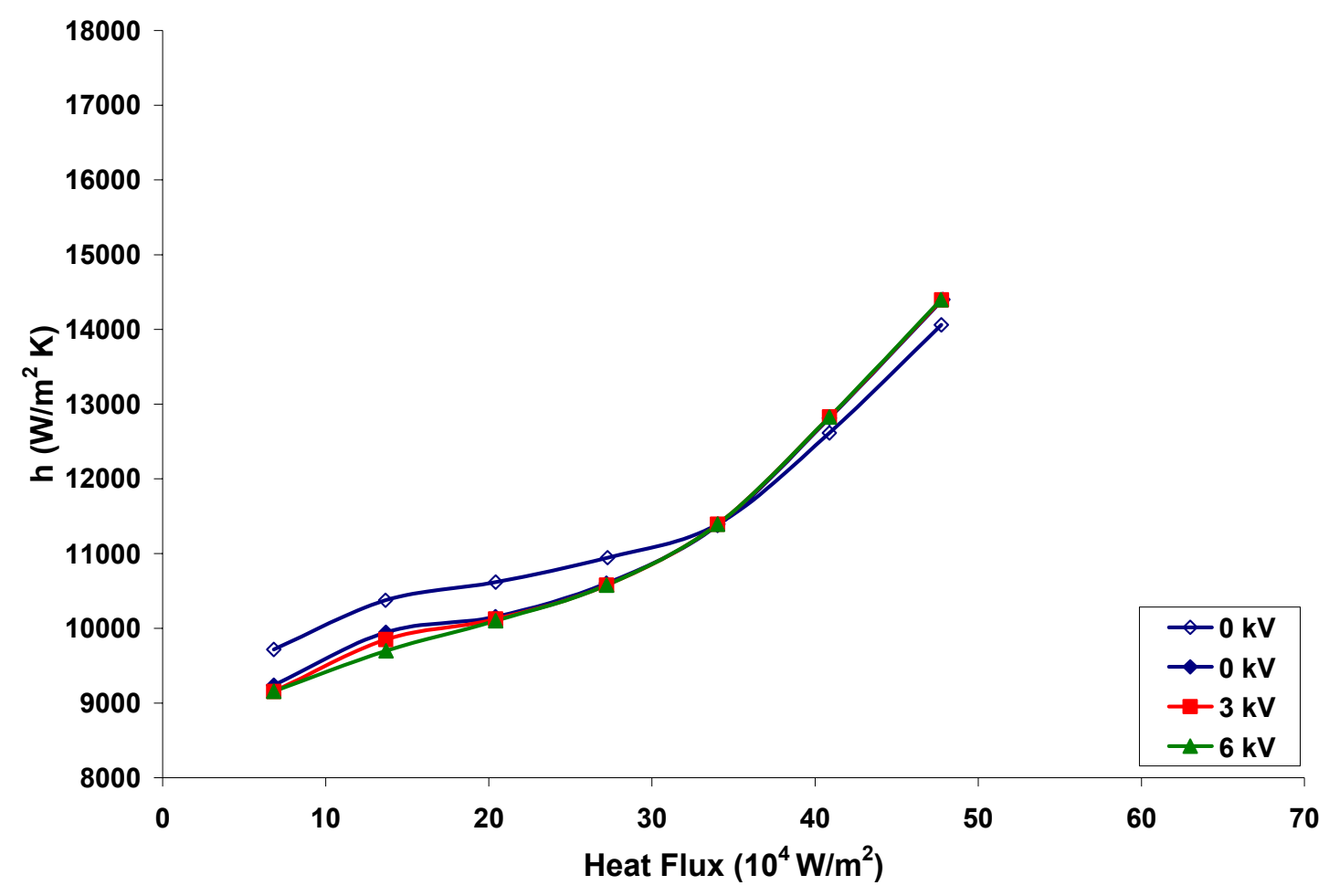

Figure 4.16: Convection Heat Transfer Coefficient in $\mathrm{W} / \mathrm{m}^{2} \mathrm{~K}$ as a Function of Heat Flux in $\mathrm{W} / \mathrm{m}^{2}$ for the Thick Top Half Cap Electrode

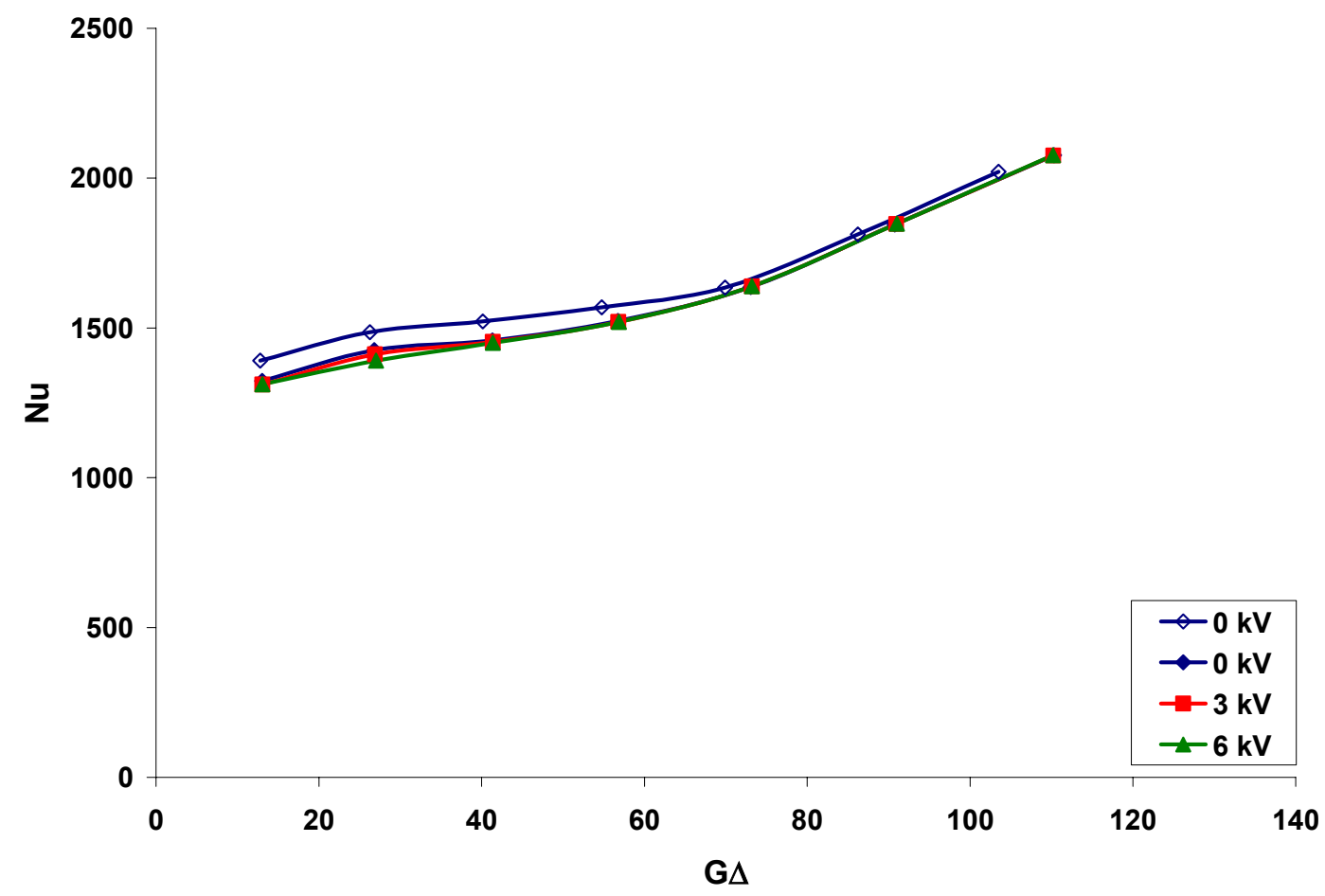

Figure 4.17: Nusselt Number as a Function of Non-dimensional Heat Flux for the Thick Top Half Cap Electrode 
The third cap electrode tested was the full length design as shown in Figure 3.23 and Figure 3.24. The full $0 \mathrm{kV}$ run was performed with a heater power from 0 to $70 \mathrm{~W}$, while the incremental high voltage run was performed from 0 to $75 \mathrm{~W}$ in an attempt to get closer to critical heat flux. This electrode also showed no significant effect for the geometry and high voltage conditions, but was the most repeatable. Each series shown in Figure 4.18 and Figure 4.19 lie almost exactly on top of one another. There is some small variation in the heat transfer coefficient and Nusselt number as seen in Figure 4.20 and Figure 4.21 at the lower heat fluxes, but the plots still show no definite substantial effect of the electrohydrodynamic electric Kelvin force. The heater resistances for this electrode were very consistent for each test and ranged from 9.15 to $9.25 \Omega$.

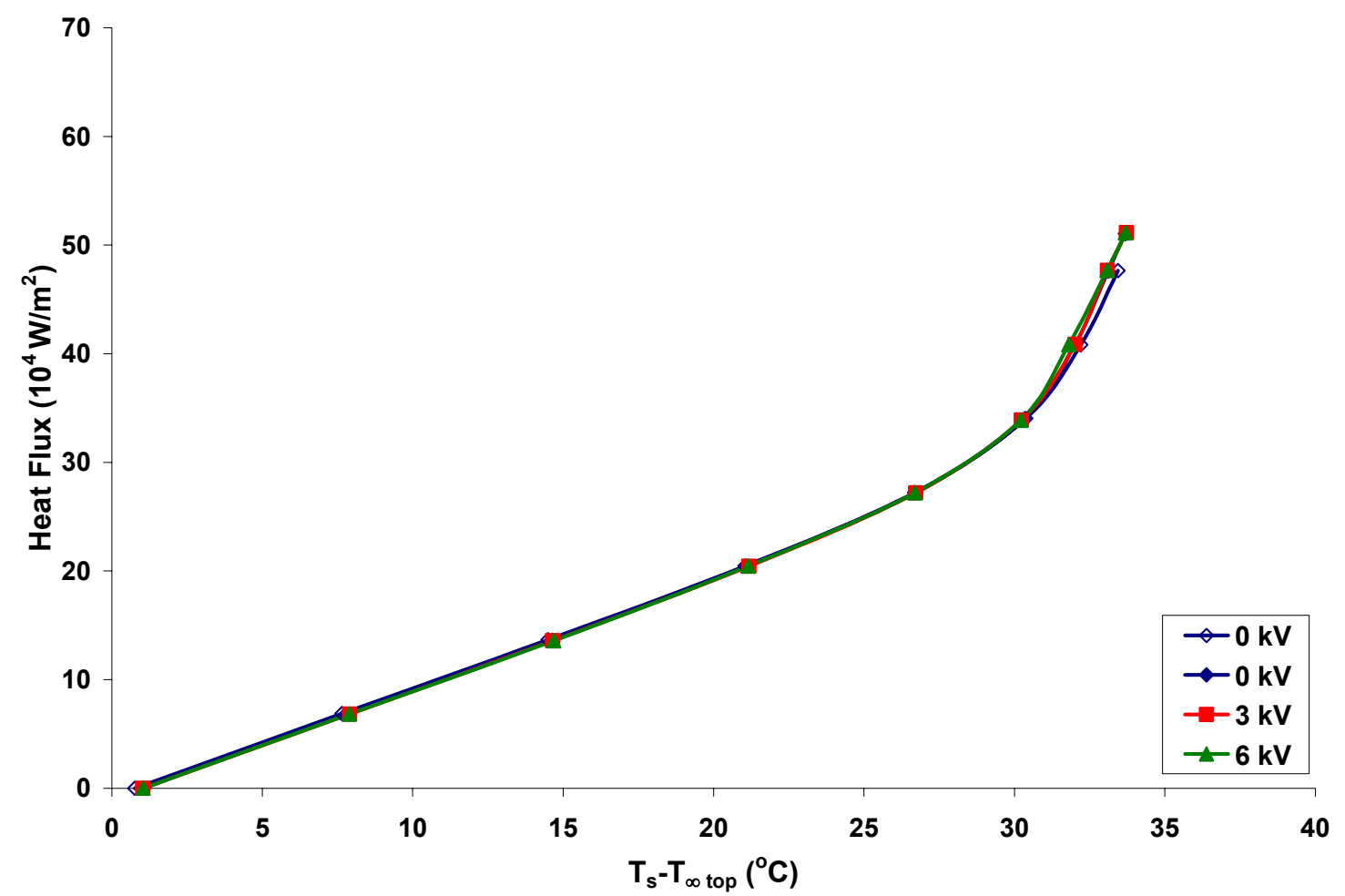

Figure 4.18: Heat Flux in $\mathrm{W} / \mathrm{m}^{2}$ as a Function of the Temperature Difference between the Surface and the Top of the Pedestal in Degrees Celsius for the Full Length Cap Electrode 


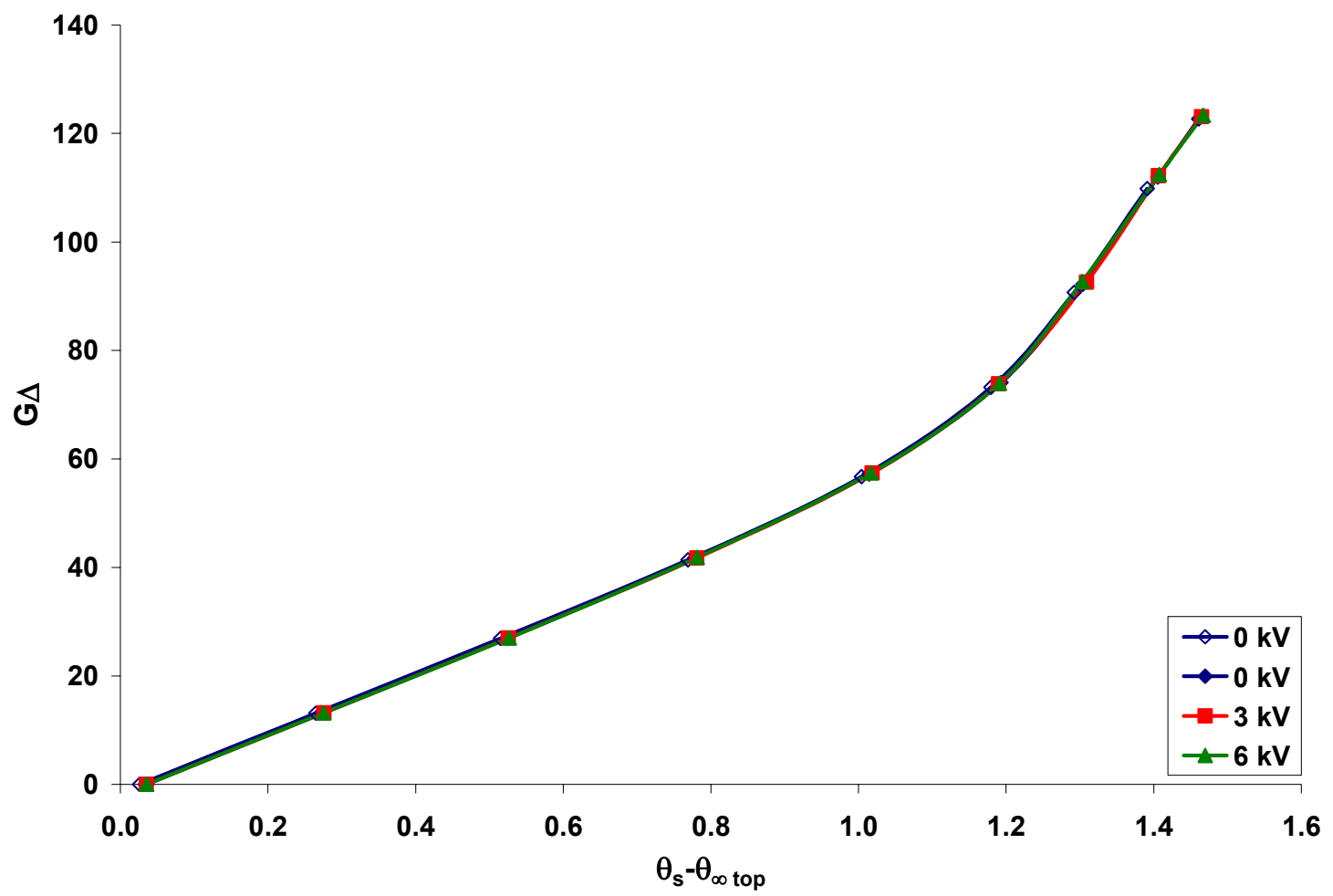

Figure 4.19: Non-dimensional Heat Flux as a Function of the Non-dimensional Temperature Difference between the Surface and the Pedestal Top for the Full Length Cap Electrode

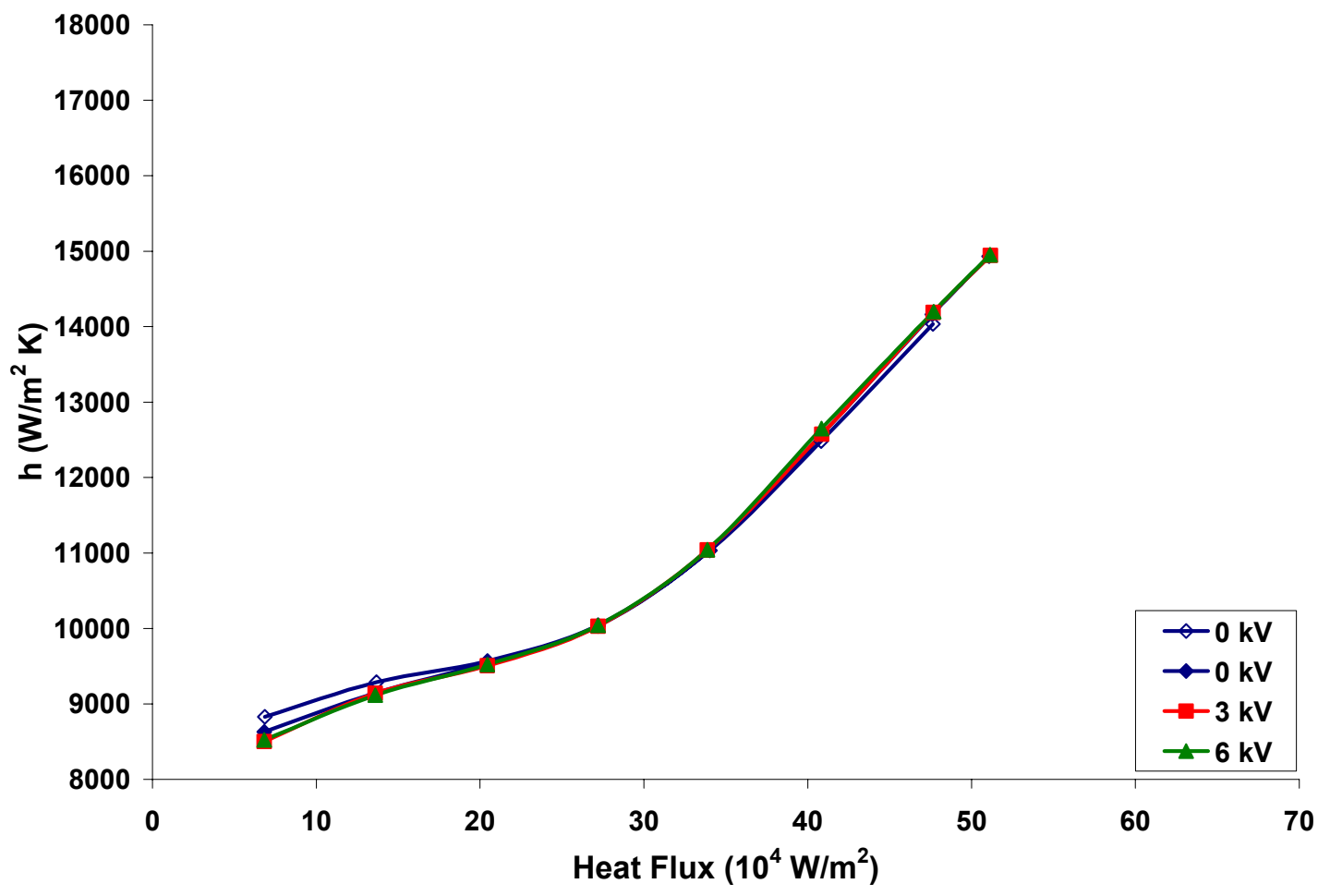

Figure 4.20: Convection Heat Transfer Coefficient in $\mathrm{W} / \mathrm{m}^{2} \mathrm{~K}$ as a Function of Heat Flux in $\mathrm{W} / \mathrm{m}^{2}$ for the Full Length Cap Electrode 


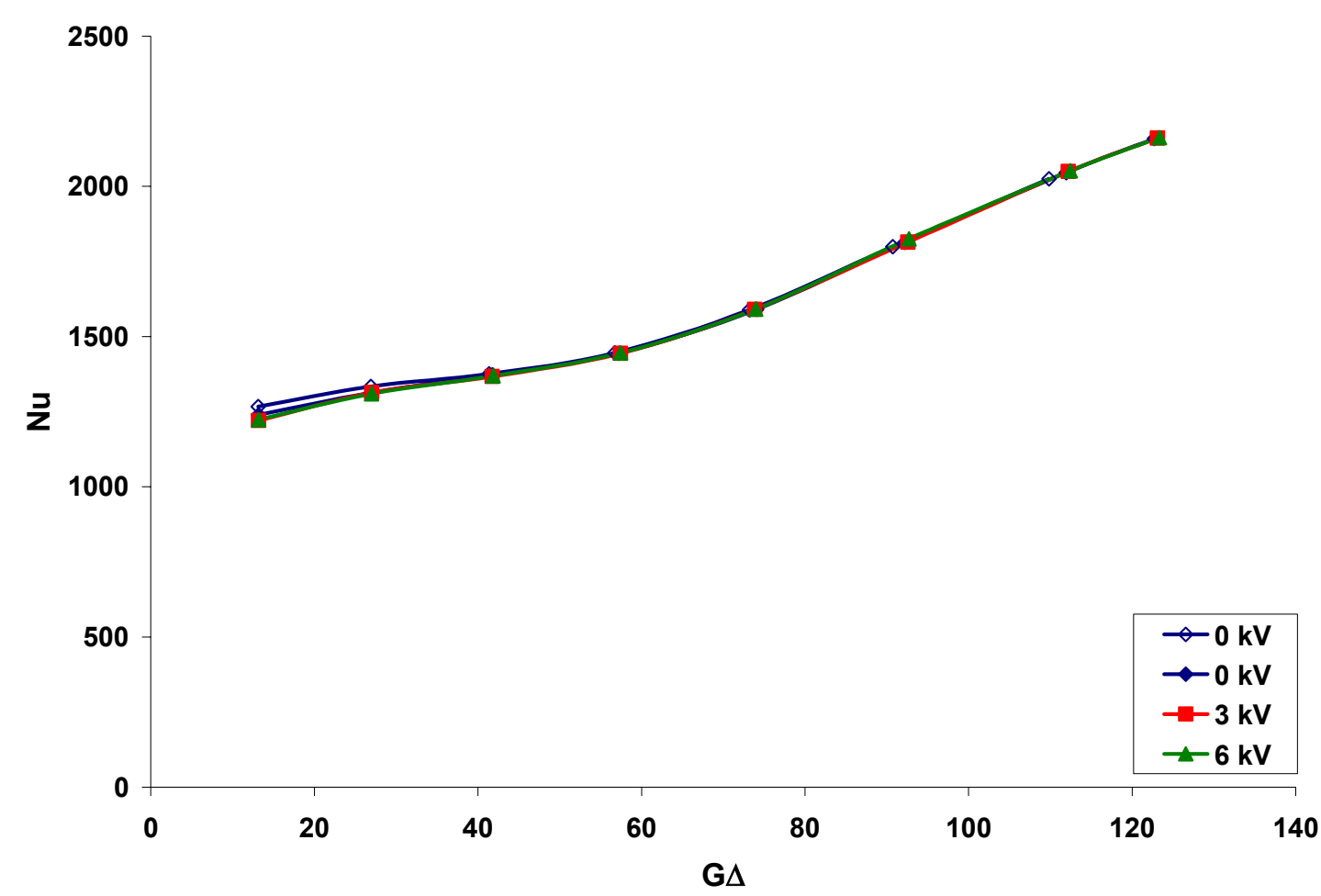

Figure 4.21: Nusselt Number as a Function of Non-dimensional Heat Flux for the Full Length Cap Electrode

The final cap electrode tested was the top half electrode. The heater powers tested were similar to the test profile for the full length electrode. The full $0 \mathrm{kV}$ run had heater powers ranging from 0 to $70 \mathrm{~W}$, while the incremental high voltage runs had heater powers from 0 to $75 \mathrm{~W}$. Figure 4.22 and Figure 4.23 show no effect on the pedestal temperatures. Figure 4.24 and Figure 4.25 show no effect on the heat transfer coefficient and Nusselt number, respectively. An error analysis was performed for the full run at $0 \mathrm{kV}$ and the error bars are shown on the plots. An explanation of the error analysis and the actual error values can be found in Appendix E. Heater resistances for this electrode ranged from 9.05 to $9.3 \Omega$. 


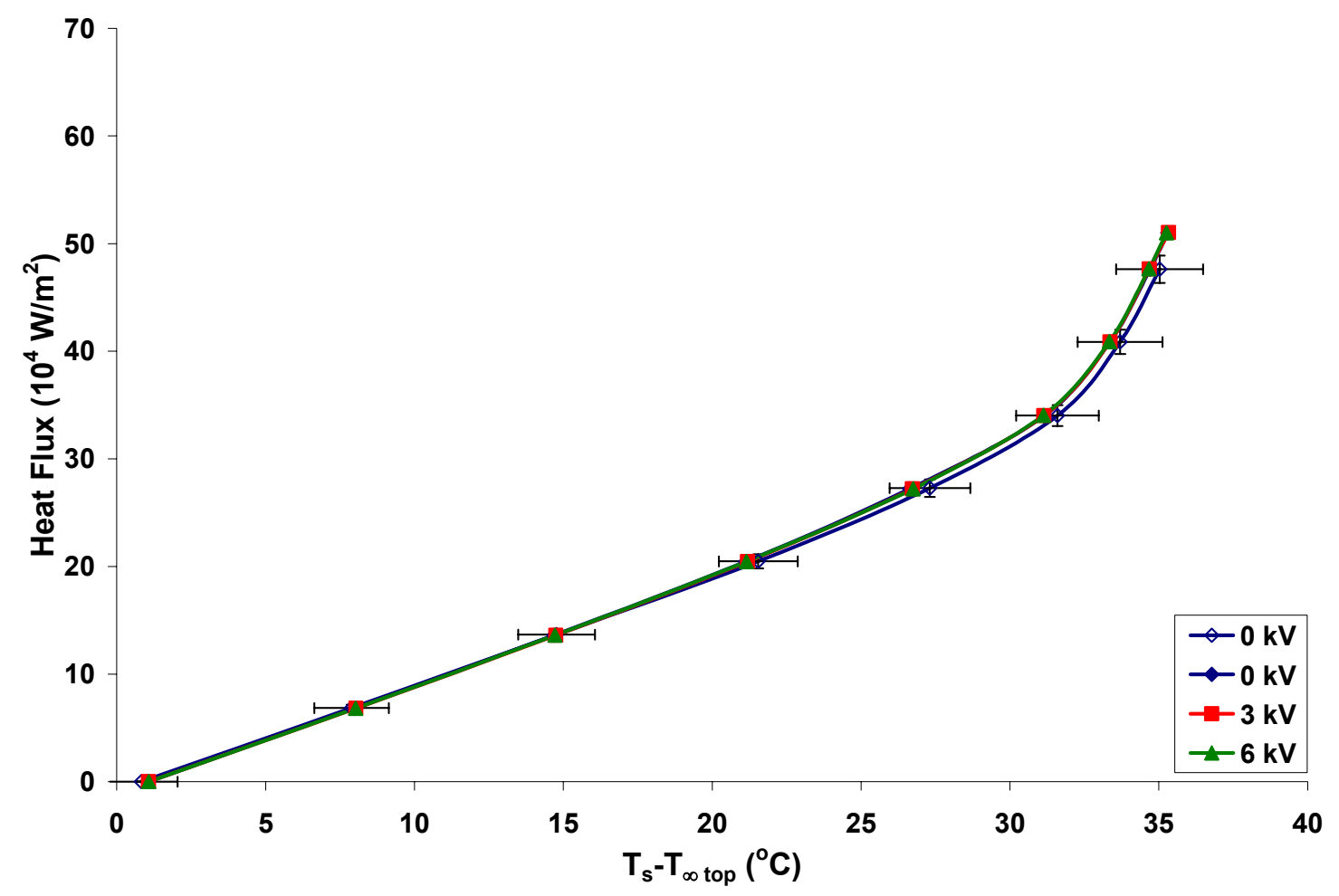

Figure 4.22: Heat Flux in $\mathrm{W} / \mathrm{m}^{2}$ as a Function of the Temperature Difference between the Surface and the Top of the Pedestal in Degrees Celsius for the Top Half Cap Electrode

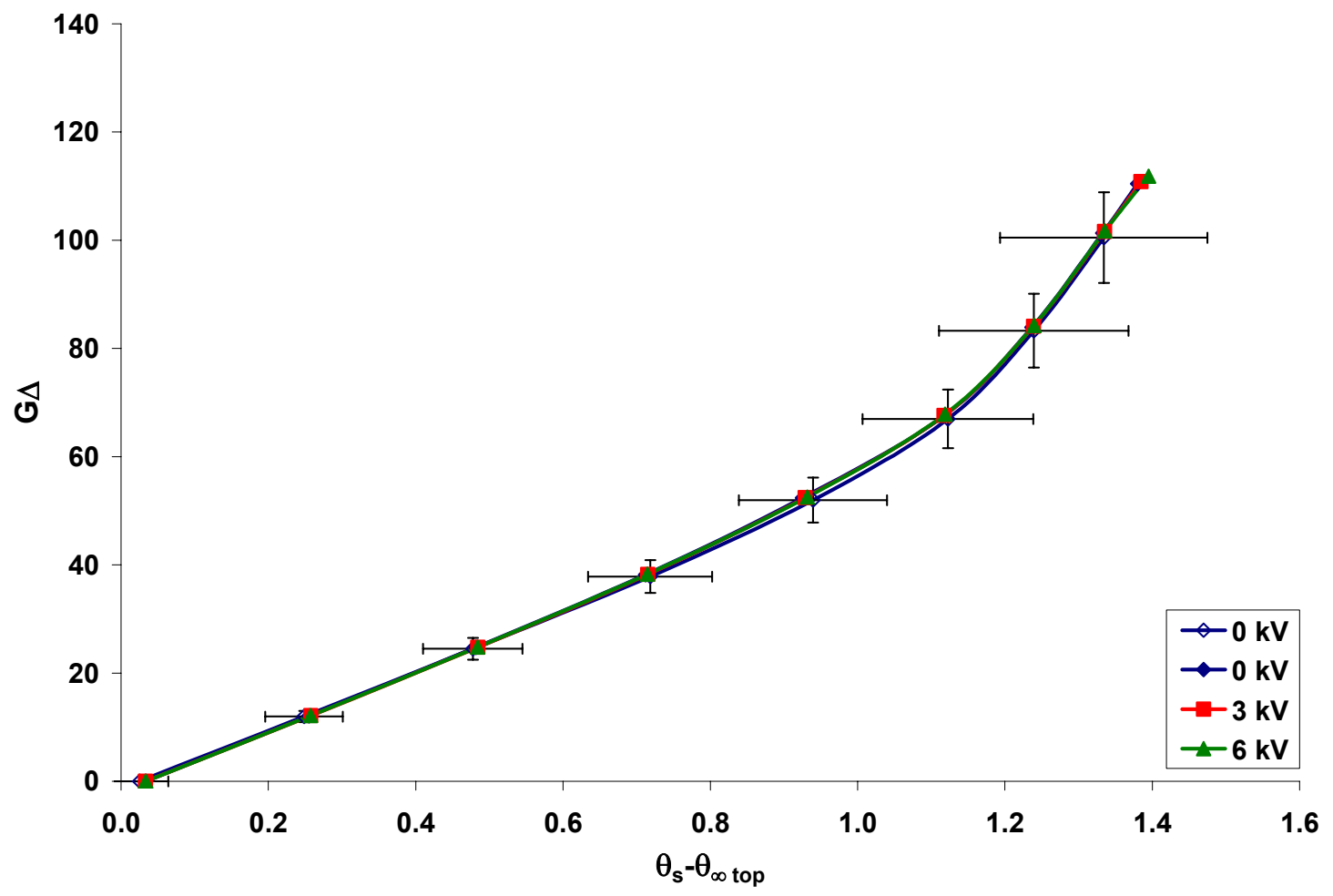

Figure 4.23: Non-dimensional Heat Flux as a Function of the Non-dimensional Temperature Difference between the Surface and the Pedestal Top for the Top Half Cap Electrode 


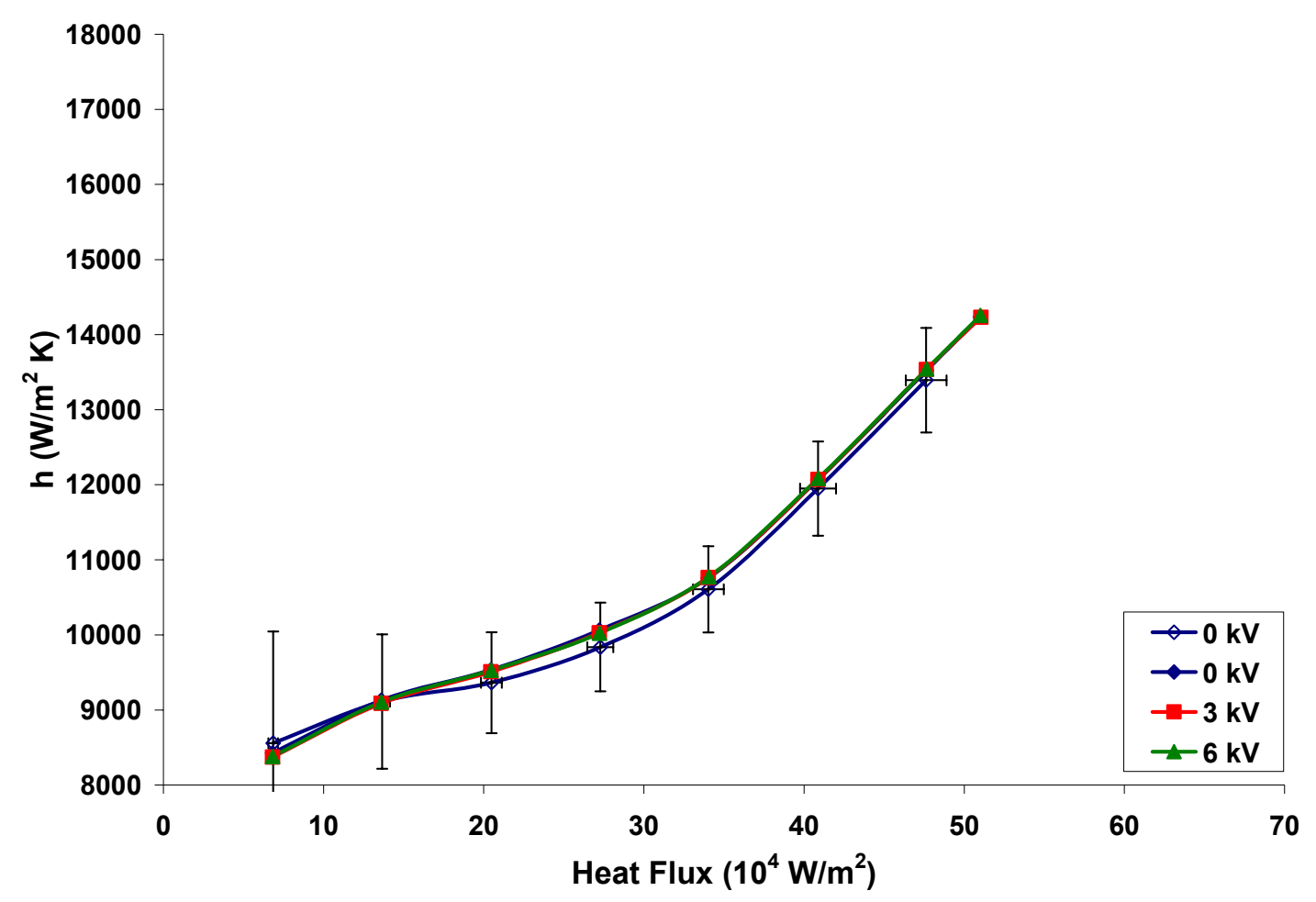

Figure 4.24: Convection Heat Transfer Coefficient in $\mathrm{W} / \mathrm{m}^{2} \mathrm{~K}$ as a Function of Heat Flux in $\mathrm{W} / \mathrm{m}^{2}$ for the Top Half Cap Electrode

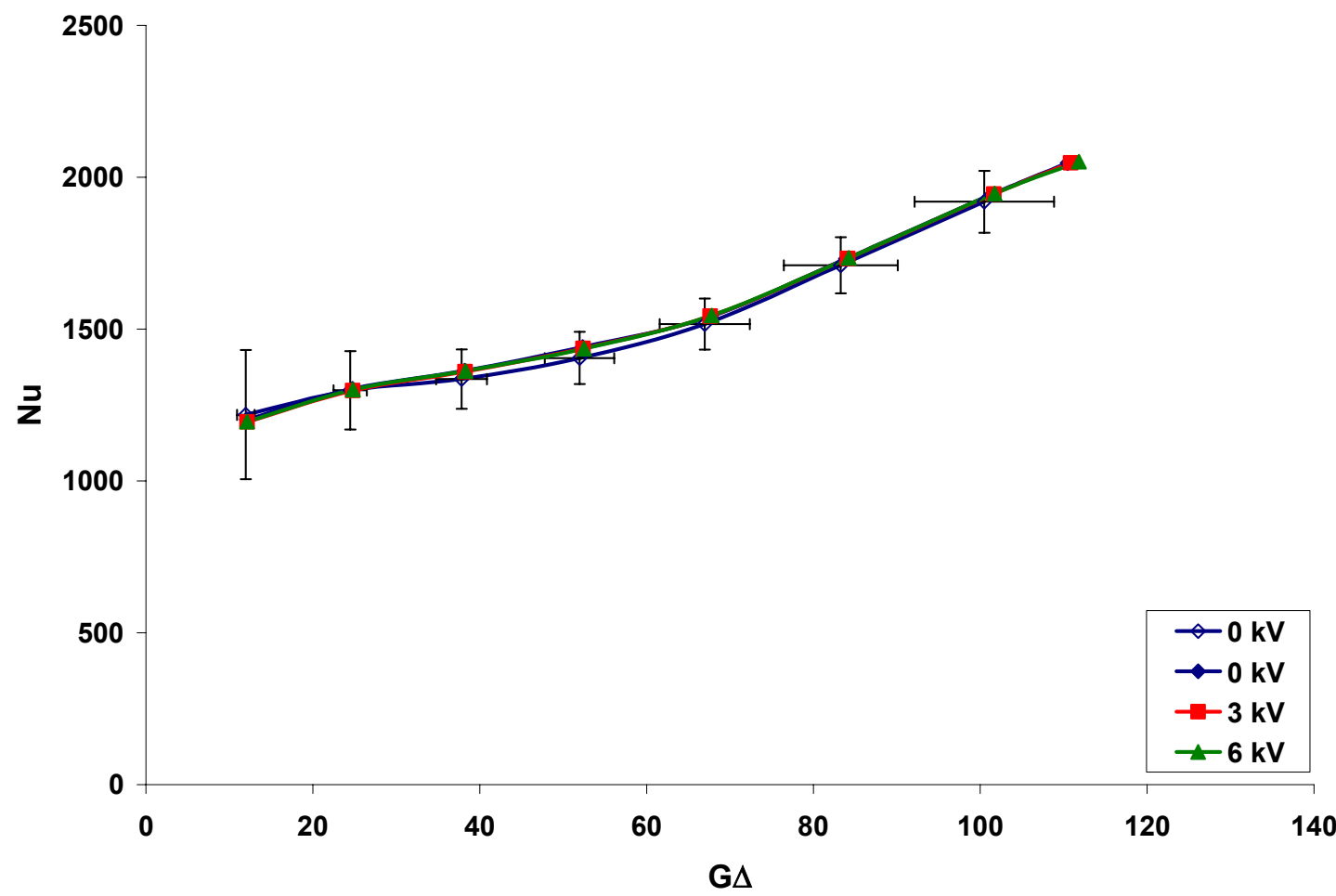

Figure 4.25: Nusselt Number as a Function of Non-dimensional Heat Flux for the Top Half Cap Electrode 
The experimental results obtained for the four cap electrodes with heat fluxes approaching critical heat flux and voltages of up to $6 \mathrm{kV}$ applied to each electrode, showed no noticeable effect from the electric Kelvin force for any of the tests. This result may be due to the non-optimal geometry of the electrode configurations or the relatively low maximum allowable limit of the high voltage applied. The small distance between the charged electrode and heater surface (ground) limited the high voltage input and therefore the strength of the electric field. The limit of $6 \mathrm{kV}$ to prevent electrostatic breakdown may not create an electric field strong enough to produce a measurable change in pedestal temperatures and related values due to the electric Kelvin force.

In order to investigate the cause of the negative experimental results, the cap electrodes were simulated axisymmetrically by Mr. Deepak Mehra (Mehra, 2006) using the CFD Ace+ multiphysics code (ESI CFD Inc., 2005) to solve the electrostatic equations and CFD-View and Tecplot in post processing. An electrode voltage of $6 \mathrm{kV}$ was used for all of these simulations.

Figure 4.26 shows the computed electric field lines for the bottom half cap electrode. Note that the electric field lines are perpendicular to the electrode and ground (pedestal surface). The gap between the sump and the plastic cap in these two figures represents the oval slots cut into the sump. Figure 4.27 shows the computed electric potential from 0 to $6 \mathrm{kV}$ for the bottom half cap electrode. For this simulation, the outer boundary conditions were assumed to be grounded. The chamber wall is in reality slightly further away, but the difference does not change the results inside the cap significantly. Also, the thermocouples, the pressure transducer, the high voltage 
feedthrough, and other movable metal pieces which could affect the electric potential are all located beyond the outer boundary of the simulation.

Figure 4.28 and Figure 4.29 show the magnitude in Newton per kilogram of the accelerations due to the computed electric Kelvin force in the $\mathrm{x}$ and $\mathrm{y}$ directions, respectively (where the positive $\mathrm{x}$ direction is pointing down toward the heater and the positive y direction is the radial direction from the centerline of the pedestal) for the bottom half cap electrode. Each figure is broken into two pieces. The top represents the computed electric Kelvin force when the entire area between the cap electrode and pedestal is filled with FC-72 (and therefore computed assuming those cells possess the properties of FC-72), and the bottom represents the force when only the spray cone and area directly above the pedestal are filled with FC-72 with the other areas assumed to be air. Since, in actuality, the amount of fluid in each area at any given time is unknown, the electric Kelvin forces would lie somewhere between these two extremes. These figures show that the magnitudes of the forces are small and have maximums near the pedestal edges. Figure 4.30 shows plots of the electric Kelvin Force per unit mass in the $\mathrm{x}$ direction as a function of the pedestal radius for the entire area filled with FC-72 (on top) and only the spray cone and area above the heater surface filled with FC-72 (on bottom) for five distances from the top of the heater surface. The thick top half cap electrode simulations are shown in Figure 4.31 through Figure 4.35, the full length cap electrode simulations are shown in Figure 4.36 through Figure 4.40, and the top half cap electrode simulations are shown in Figure 4.41 through Figure 4.45. These all show similar trends. 


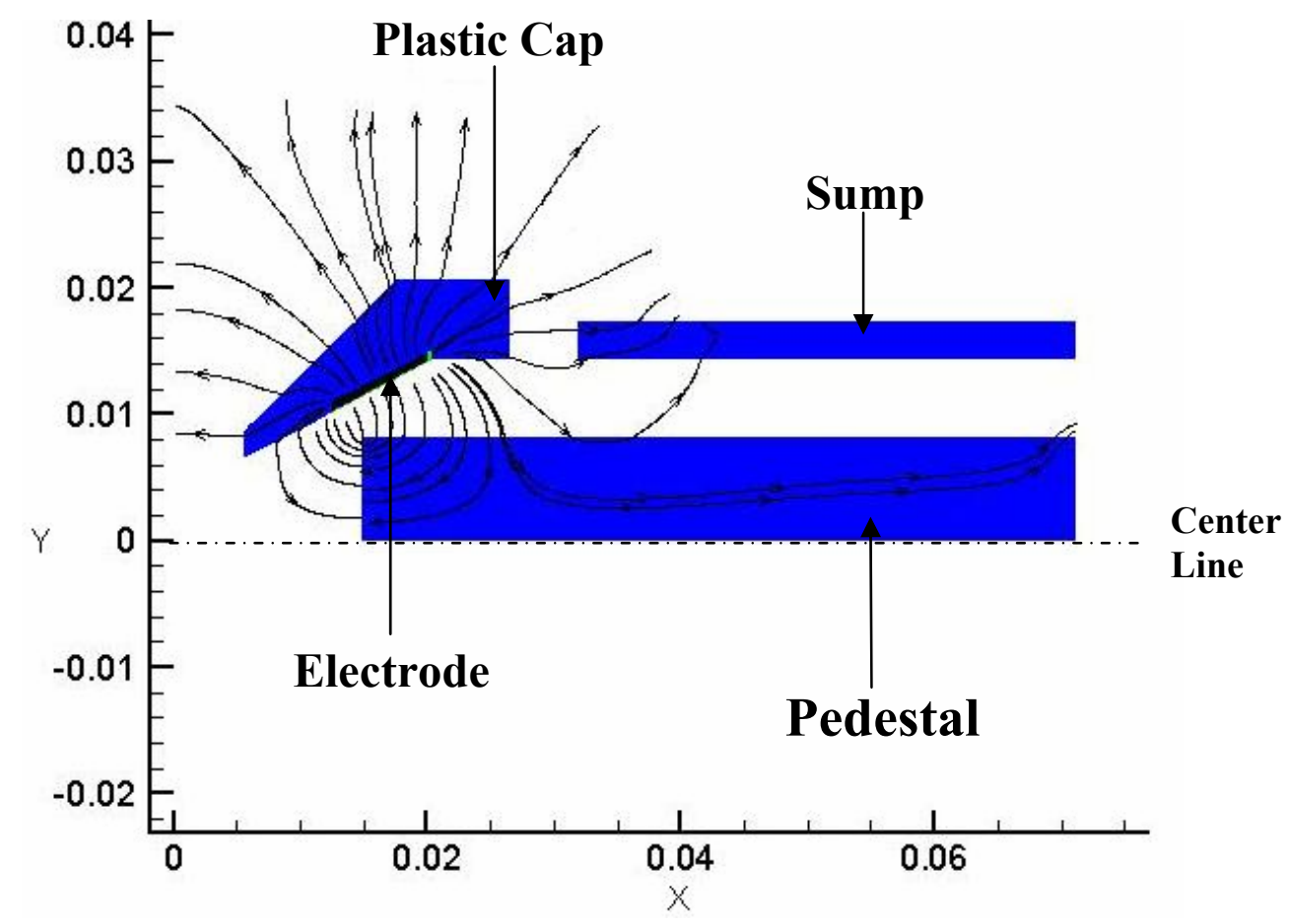

Figure 4.26: Electric Field Lines for Bottom Half Cap Electrode
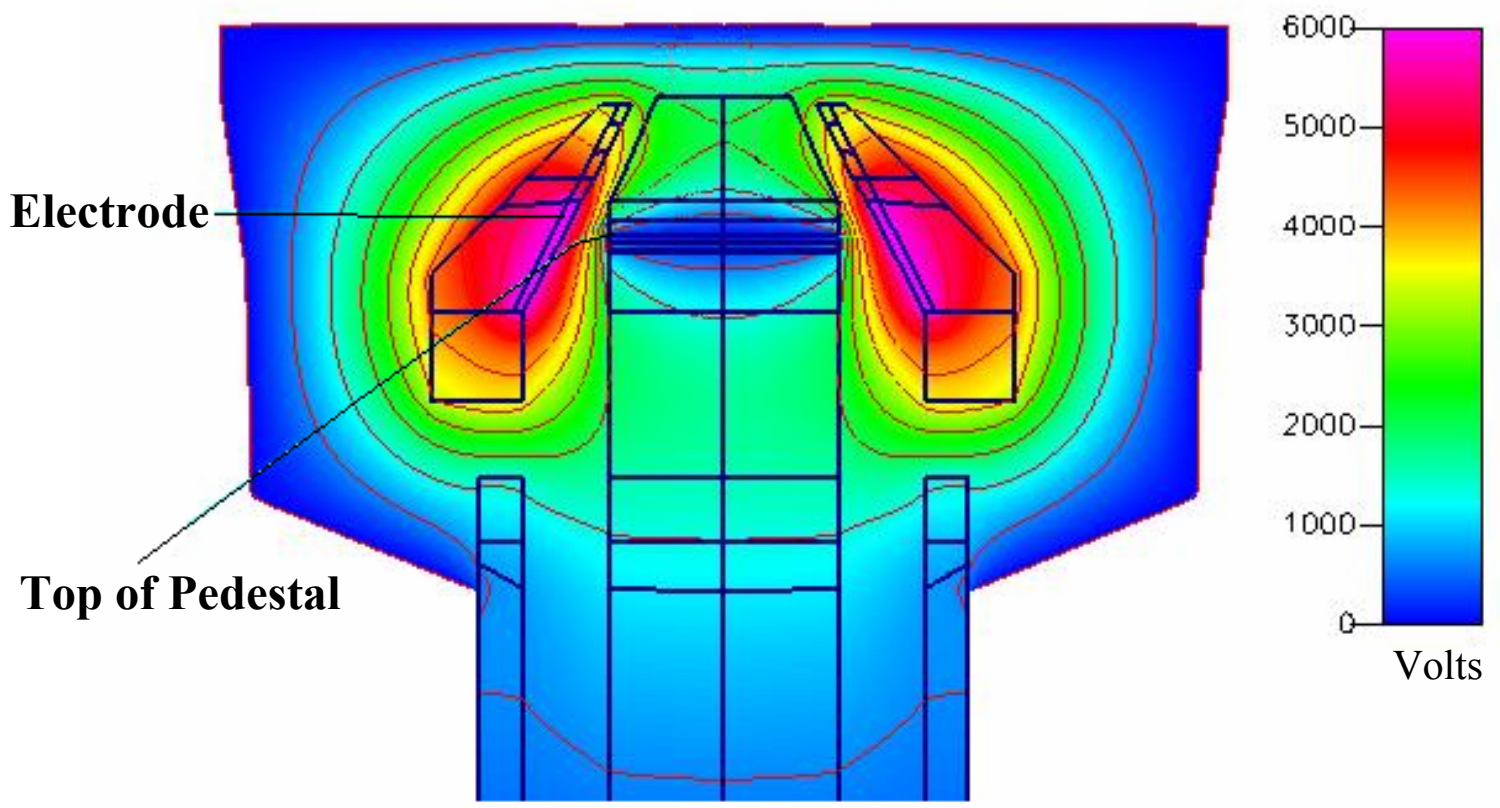

Figure 4.27: Electric Potential for the Bottom Half Cap Electrode 

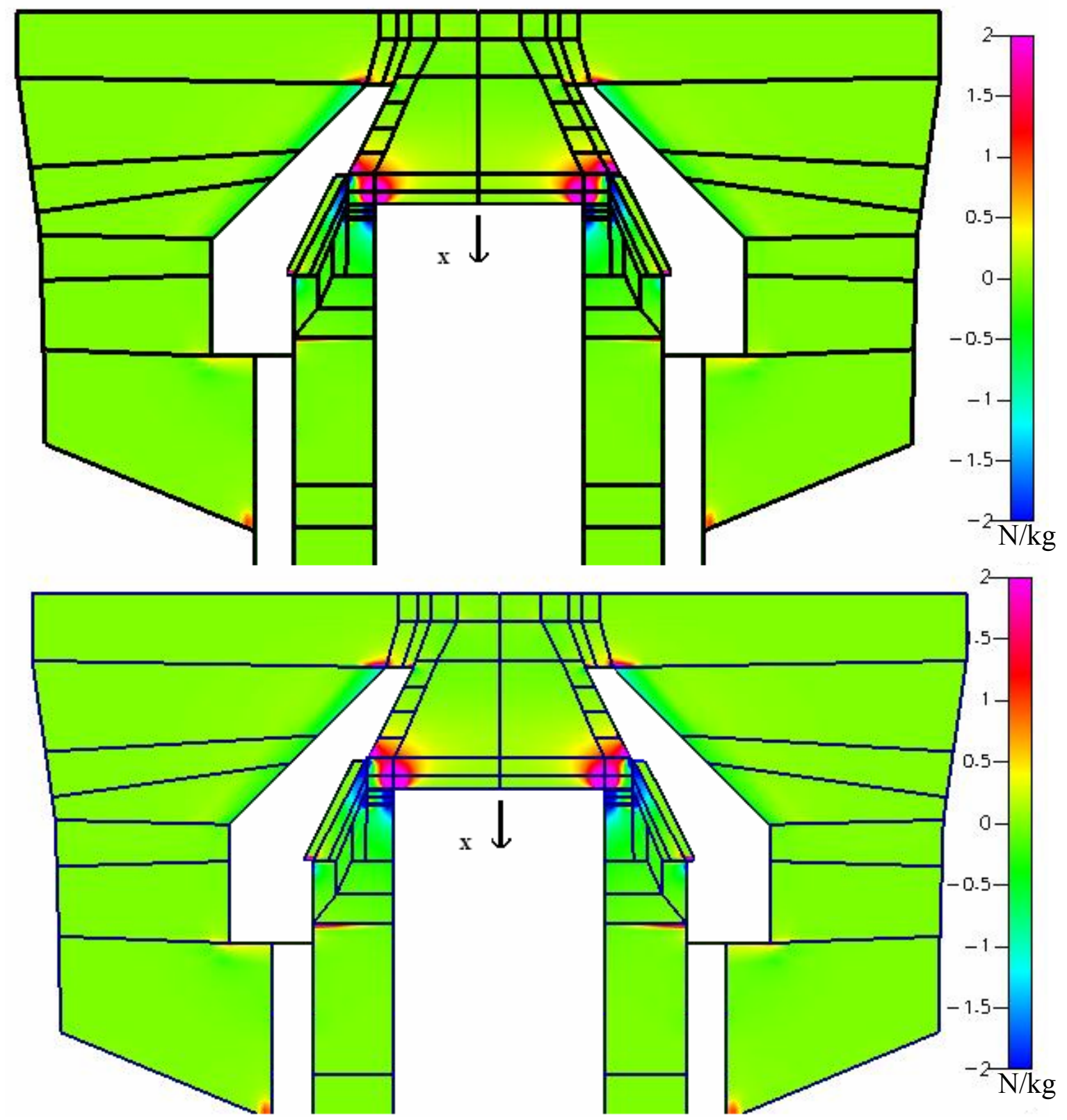

Figure 4.28: Magnitude of the Electric Kelvin Force in the x-direction for the Bottom Half Cap Electrode 

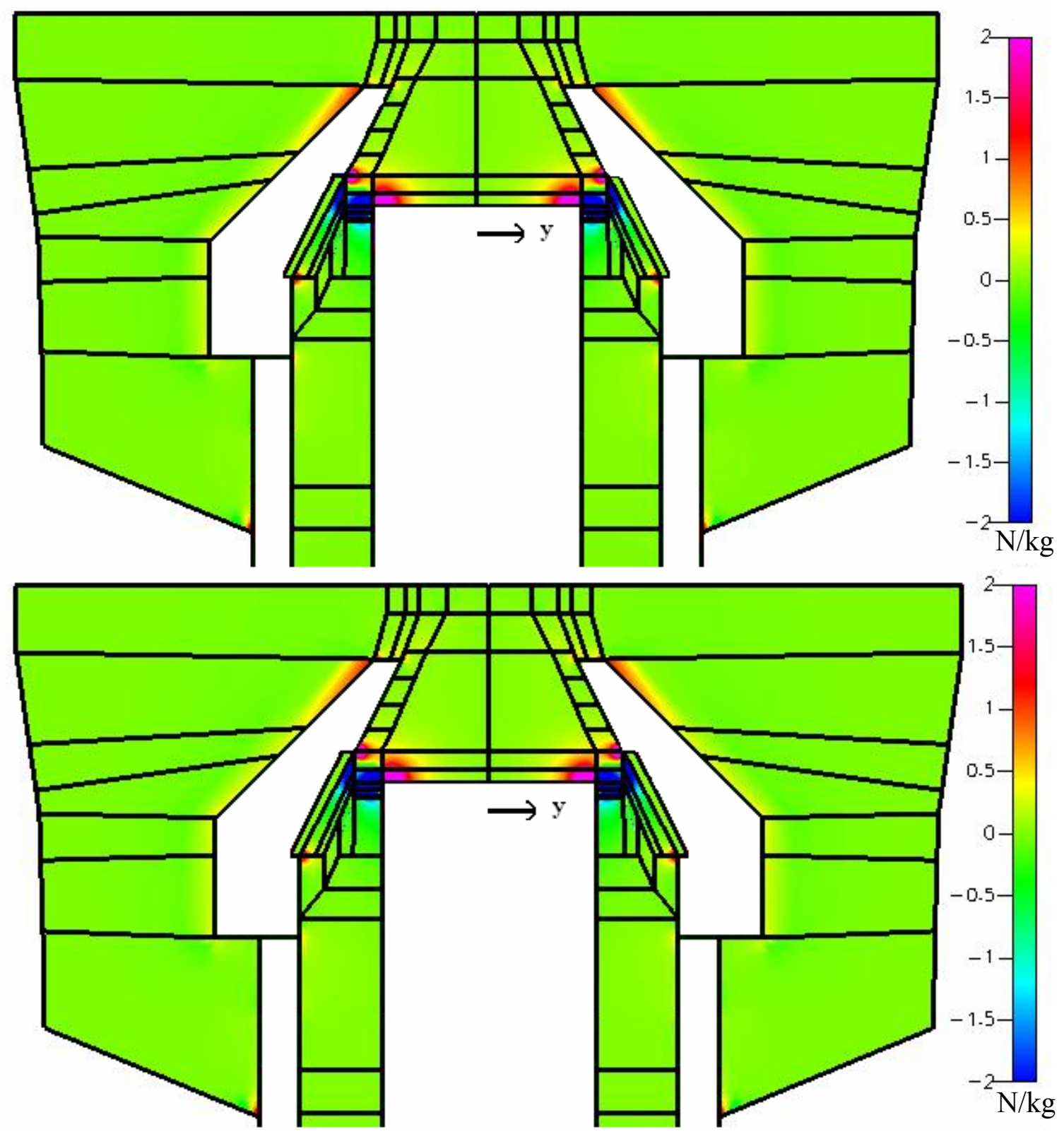

Figure 4.29: Magnitude of the Electric Kelvin Force in the y-direction for the Bottom Half Cap Electrode 

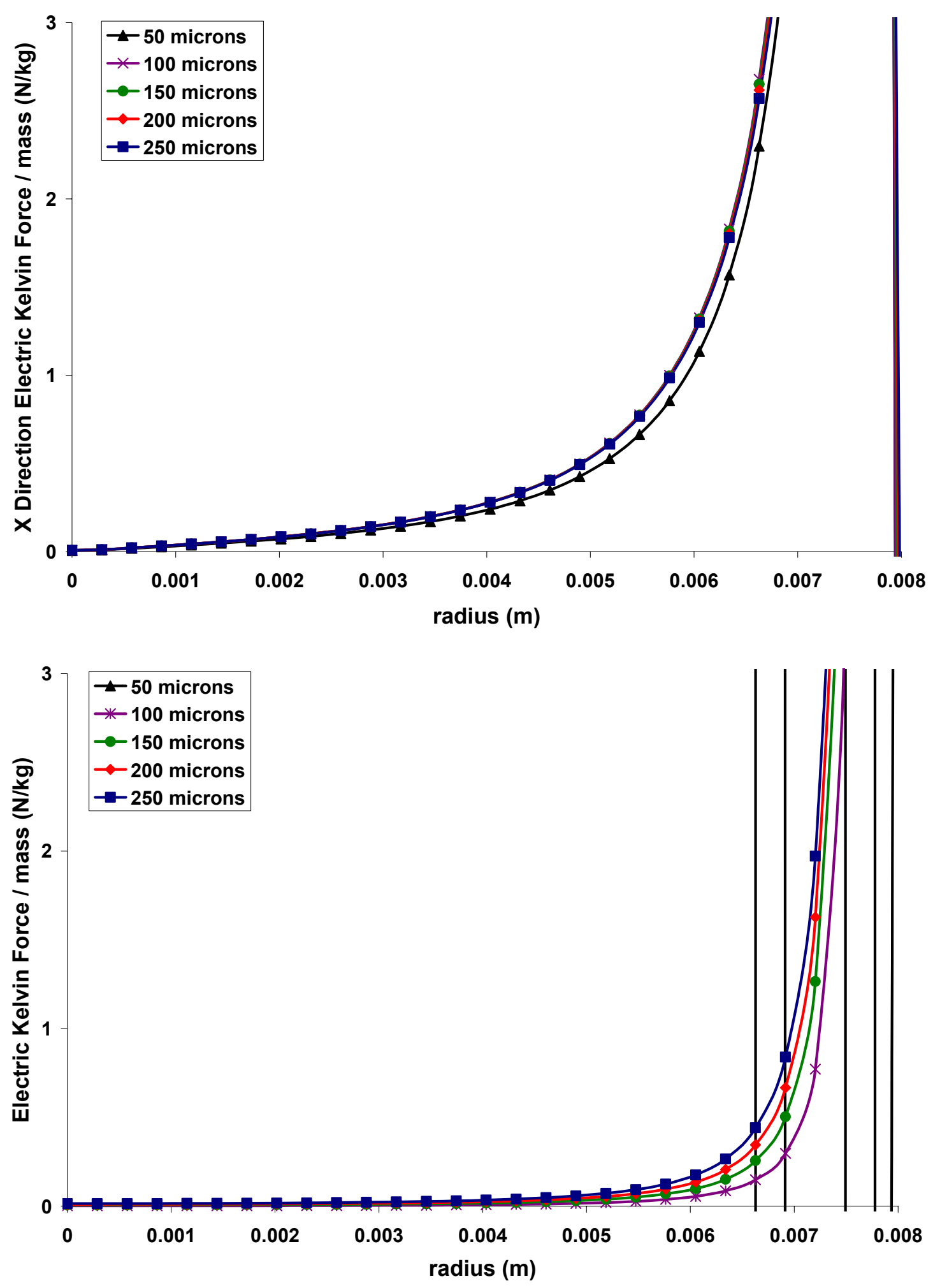

Figure 4.30: Electric Kelvin Force per Mass with all FC-72 inside the cap (top) and FC72 only in the Spray Cone and Above the Pedestal (bottom) as a Function of the Pedestal Radius for Five Distances from the Heater Surface for the Bottom Half Cap Electrode 


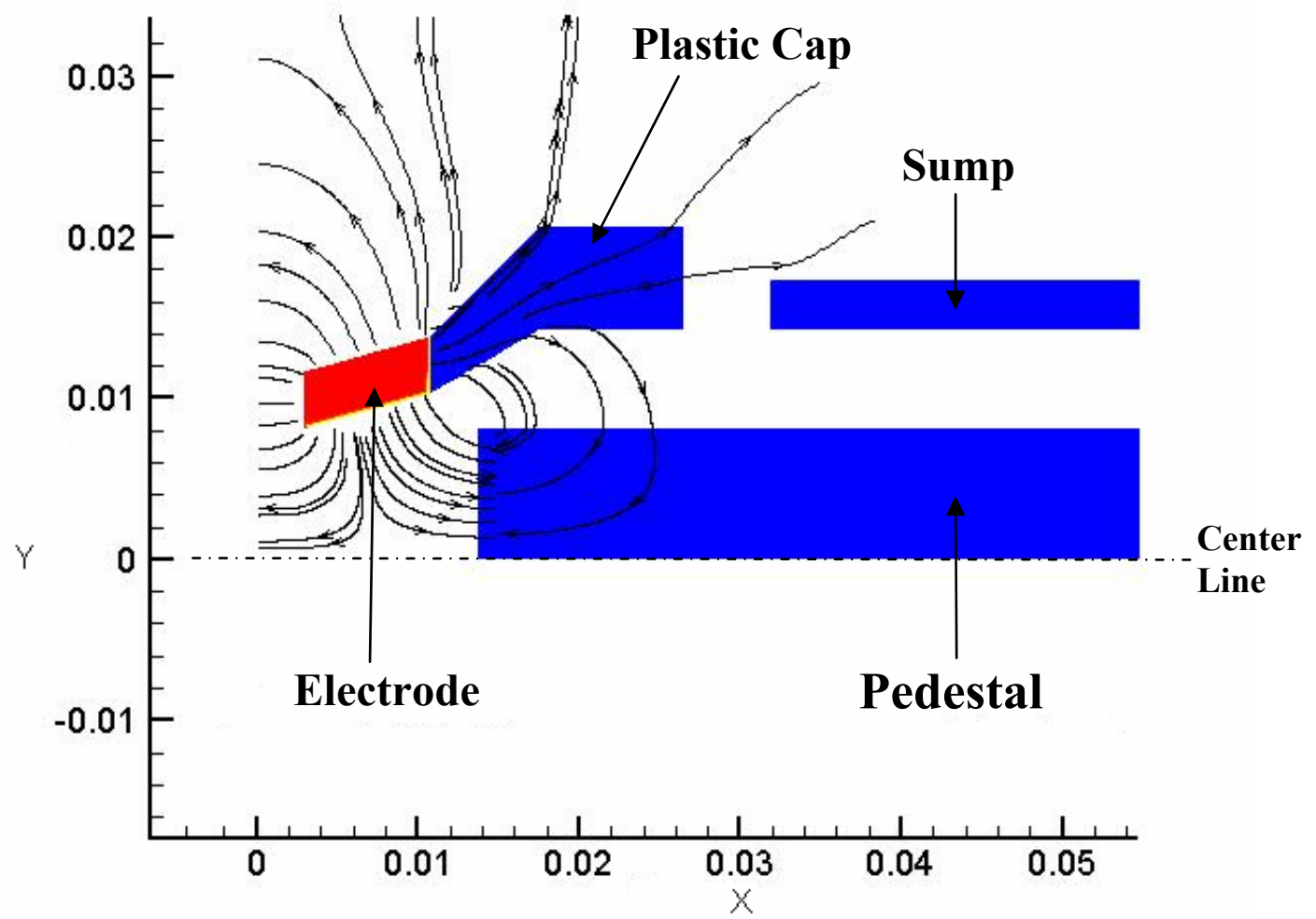

Figure 4.31: Electric Field Lines for Thick Top Half Cap Electrode
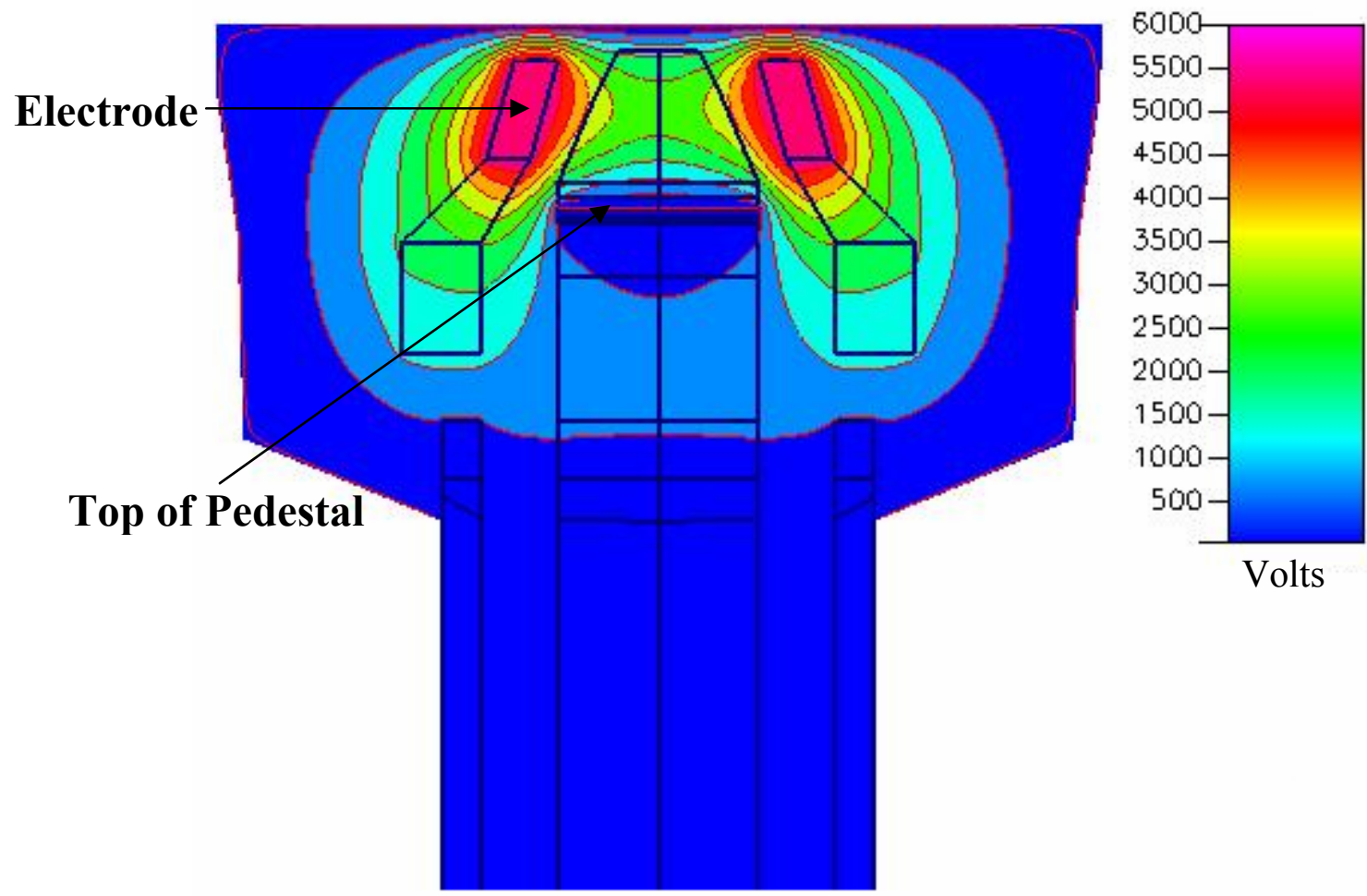

Figure 4.32: Electric Potential for the Thick Top Half Cap Electrode 


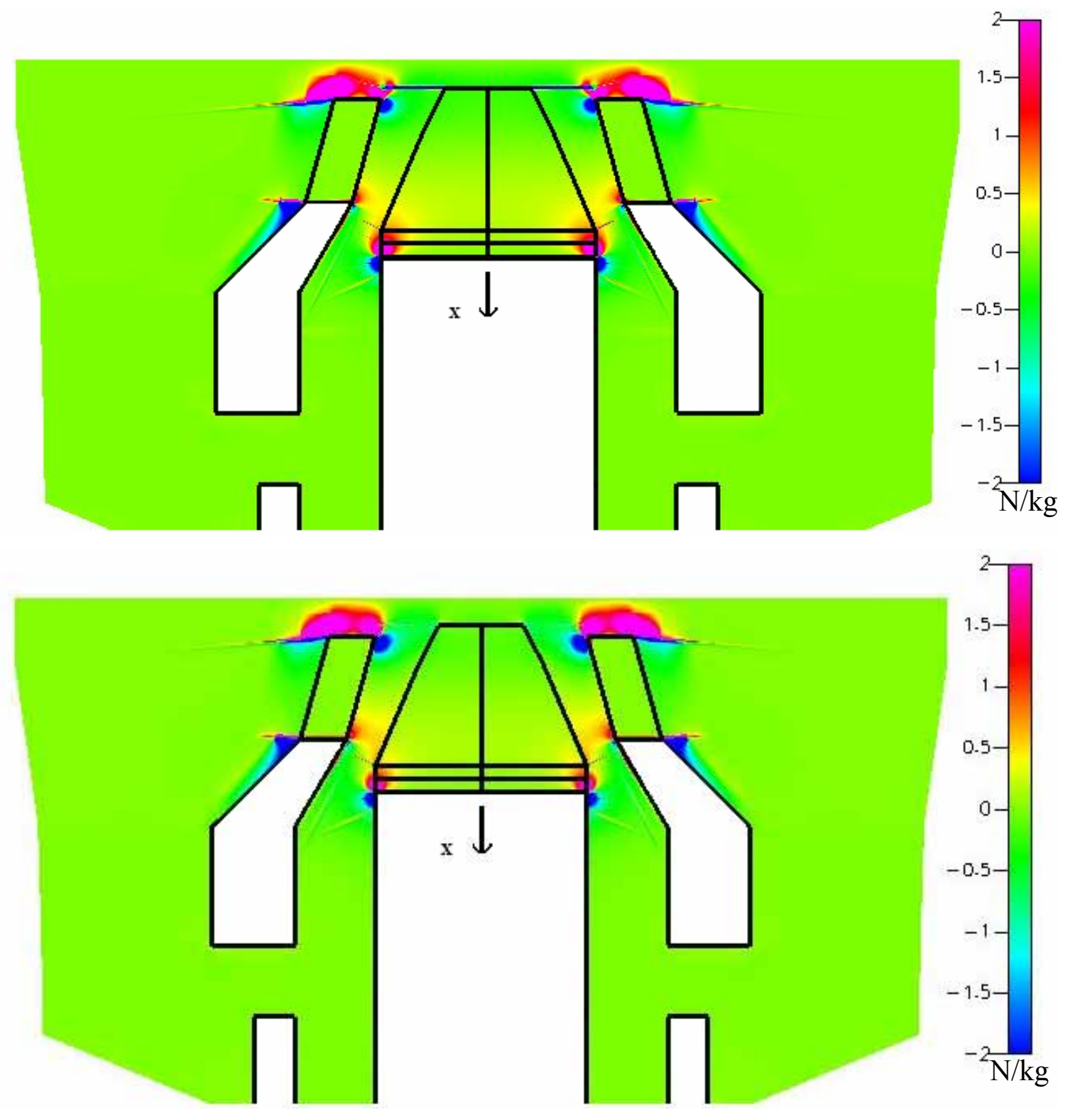

Figure 4.33: Magnitude of the Electric Kelvin Force in the x-direction for the Thick Top Half Cap Electrode 


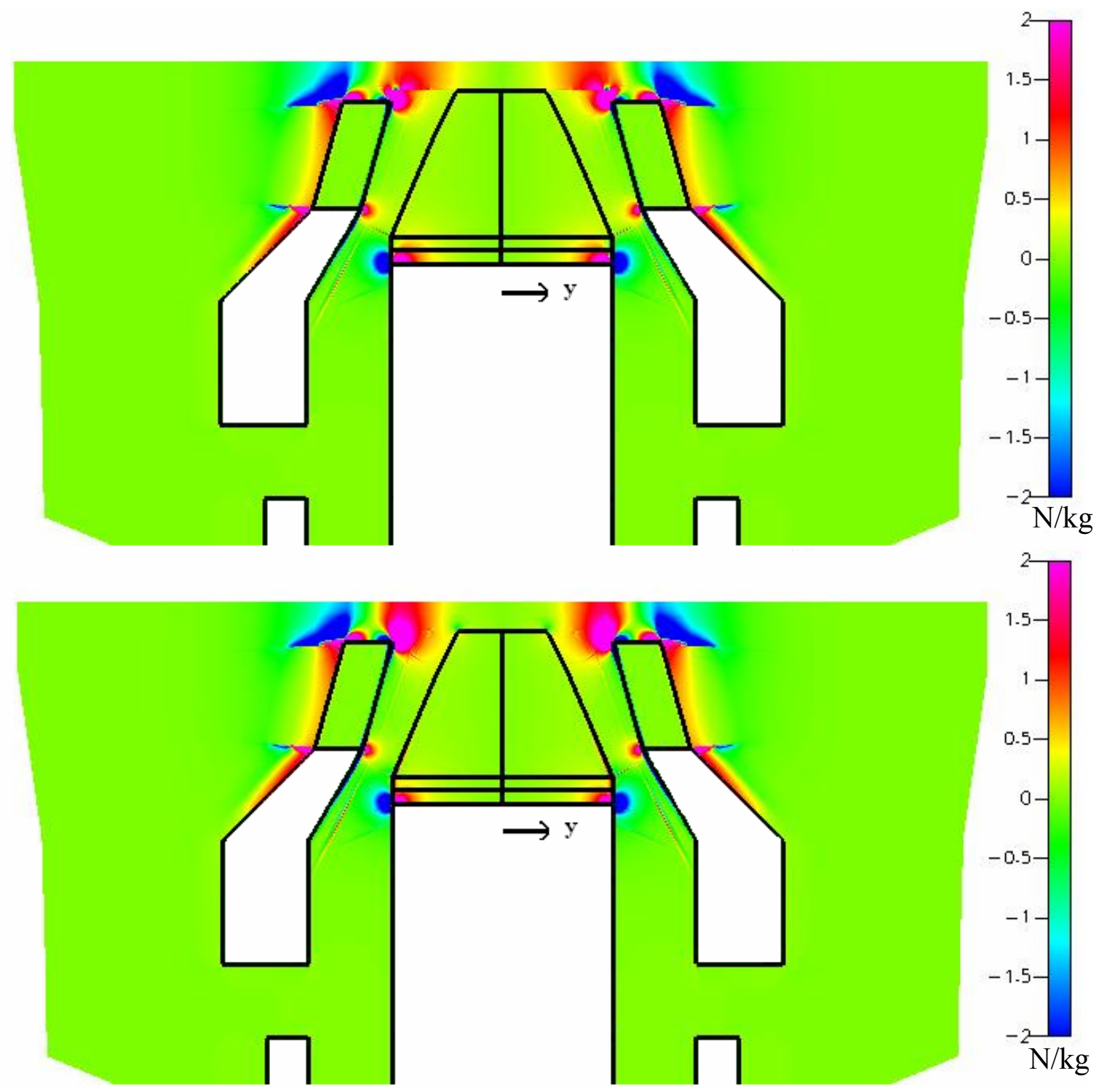

Figure 4.34: Magnitude of the Electric Kelvin Force in the y-direction for the Thick Top Half Cap Electrode 

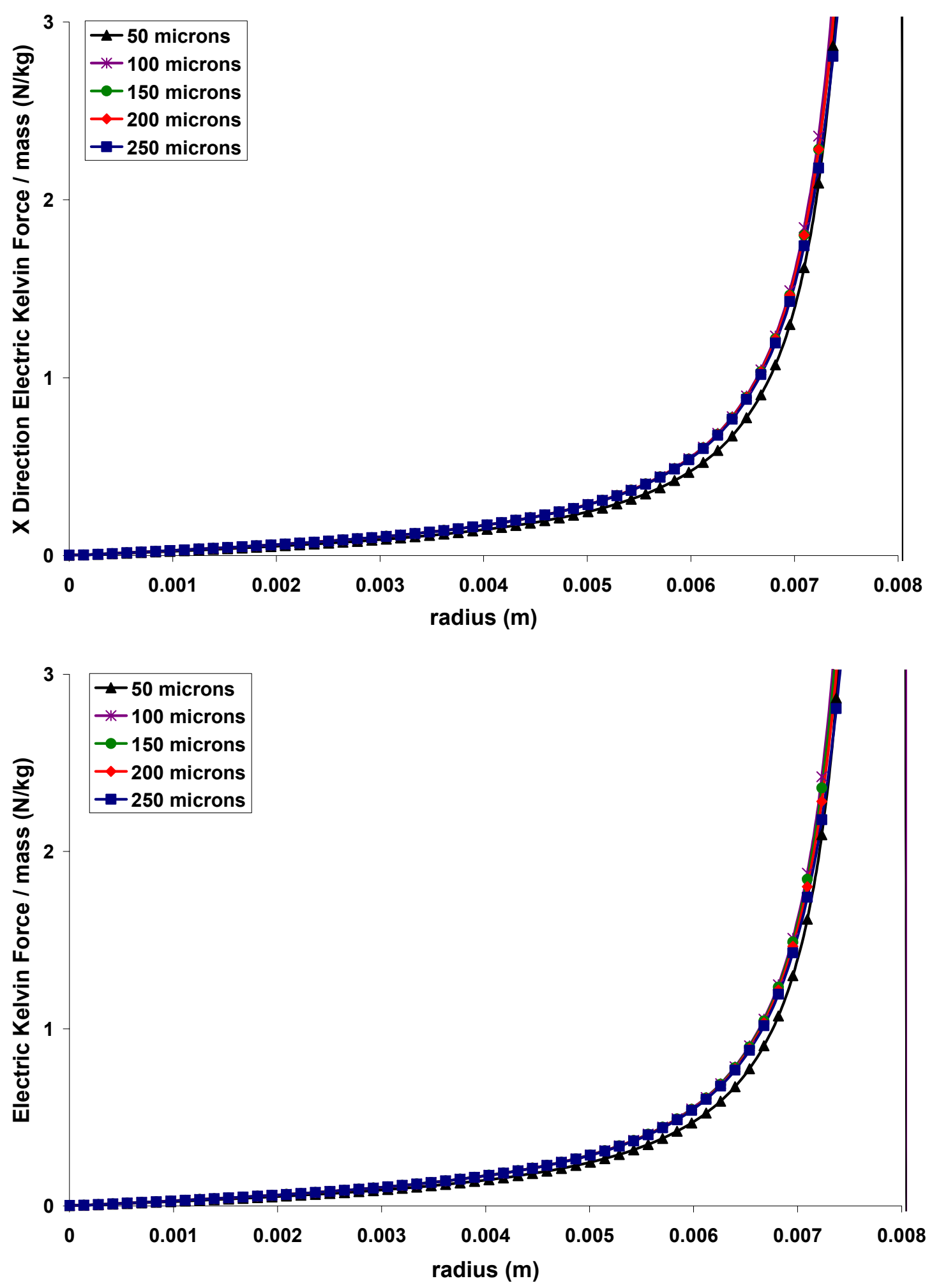

Figure 4.35: Electric Kelvin Force per Mass with all FC-72 inside the cap (top) and FC72 only in the Spray Cone and Above the Pedestal (bottom) as a Function of the Pedestal Radius for Five Distances from the Heater Surface for the Thick Top Half Cap Electrode 


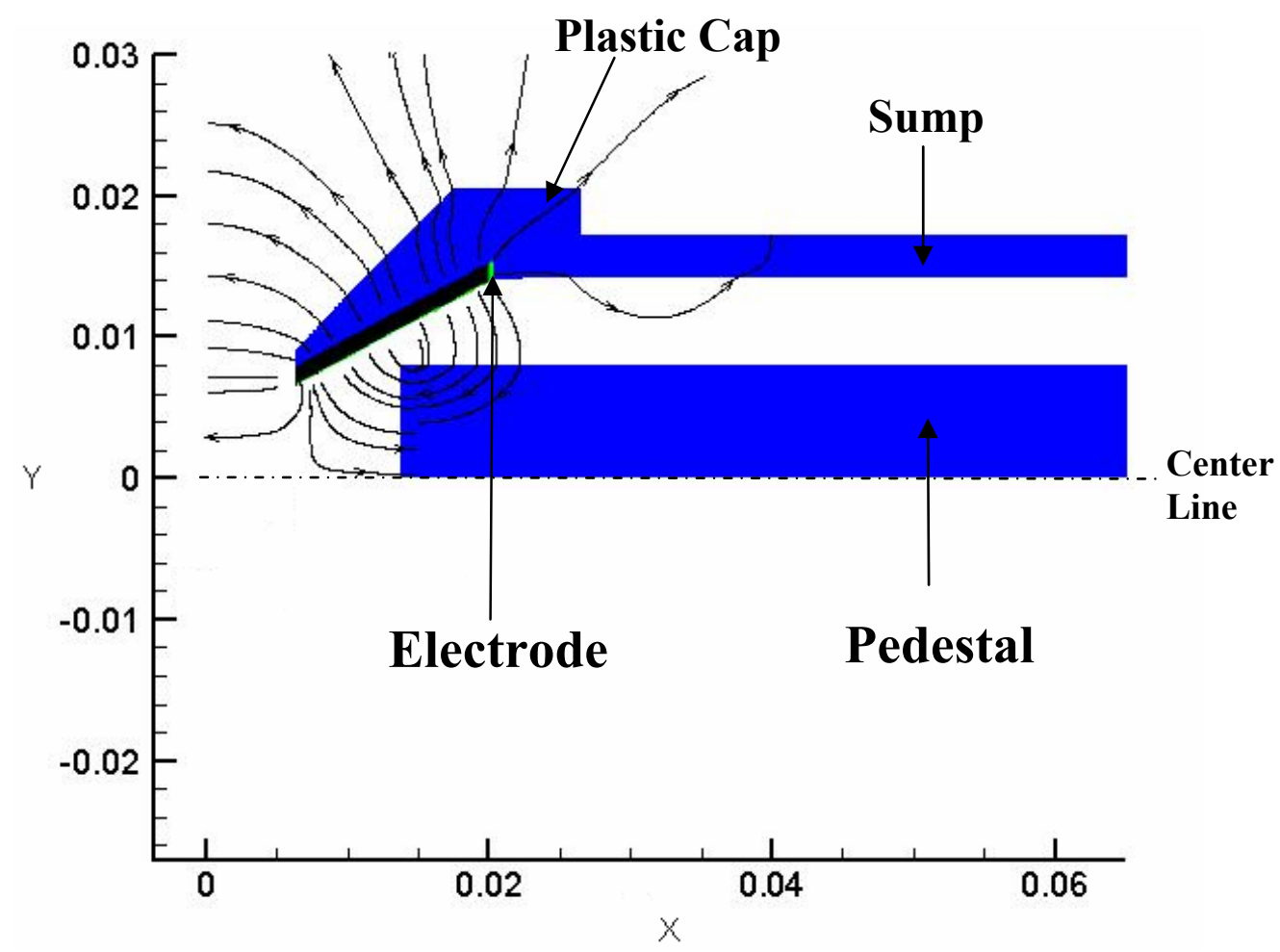

Figure 4.36: Electric Field Lines for Full Length Cap Electrode
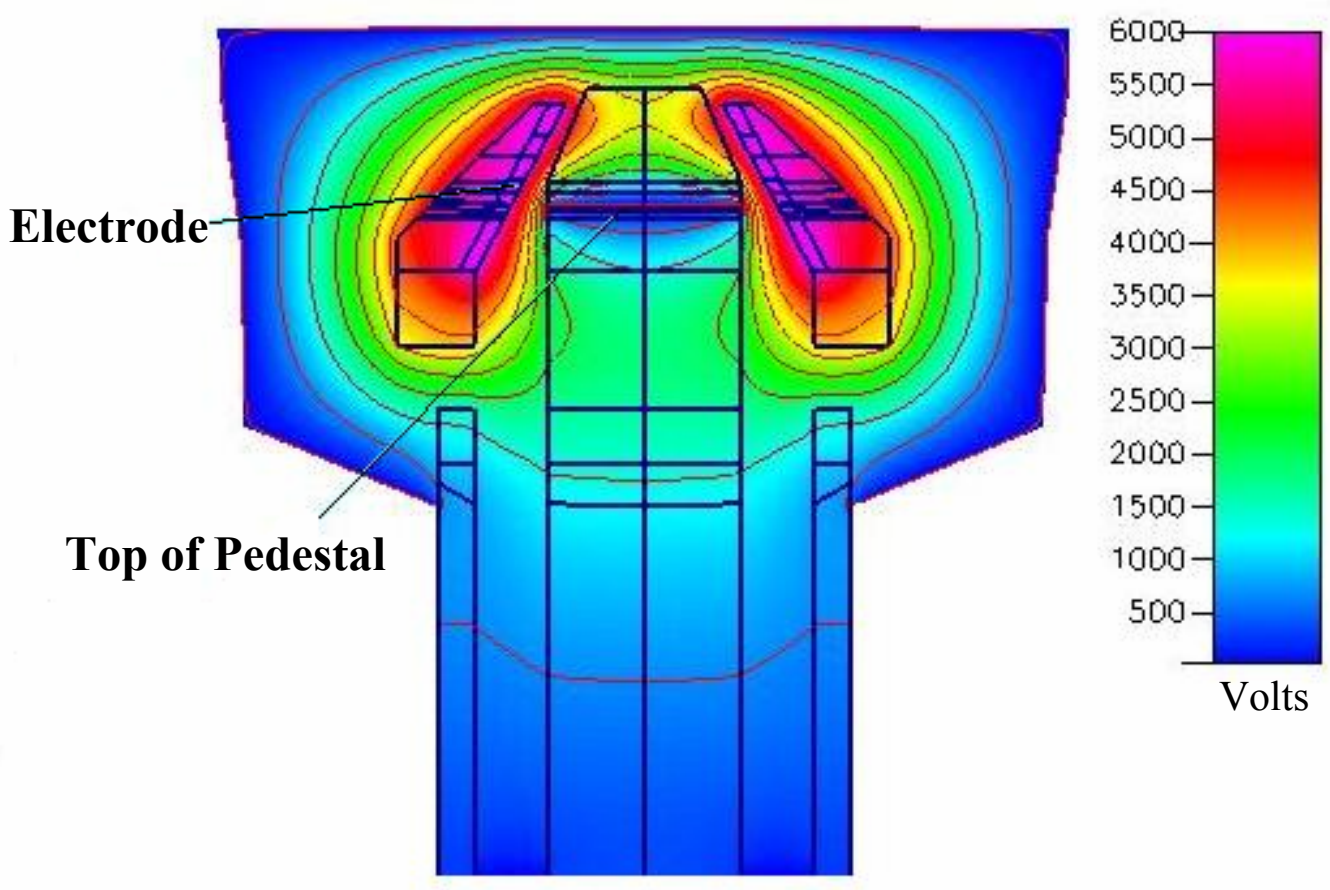

Figure 4.37: Electric Potential for the Full Length Cap Electrode 

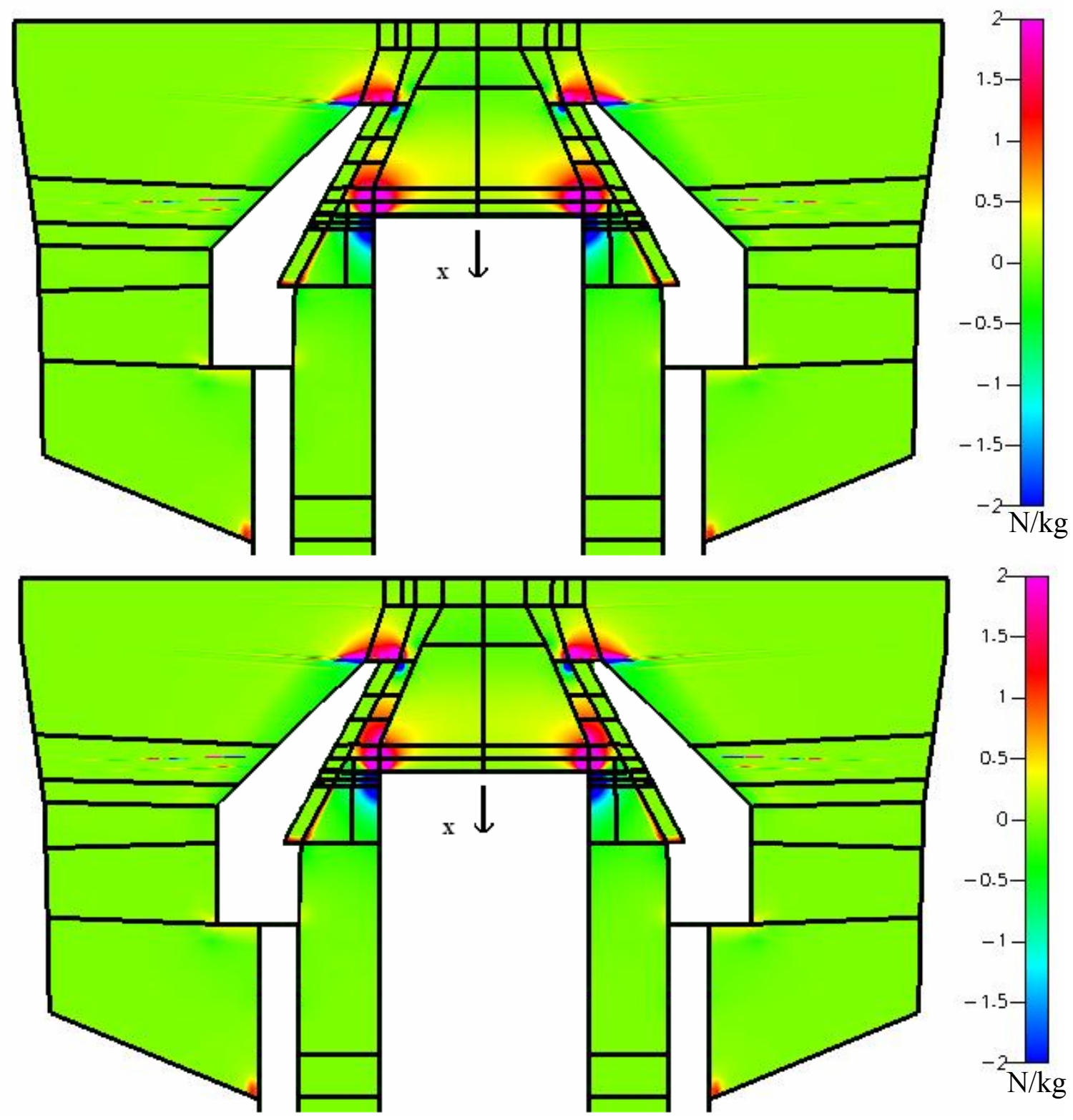

Figure 4.38: Magnitude of the Electric Kelvin Force in the x-direction for the Full Length Cap Electrode 

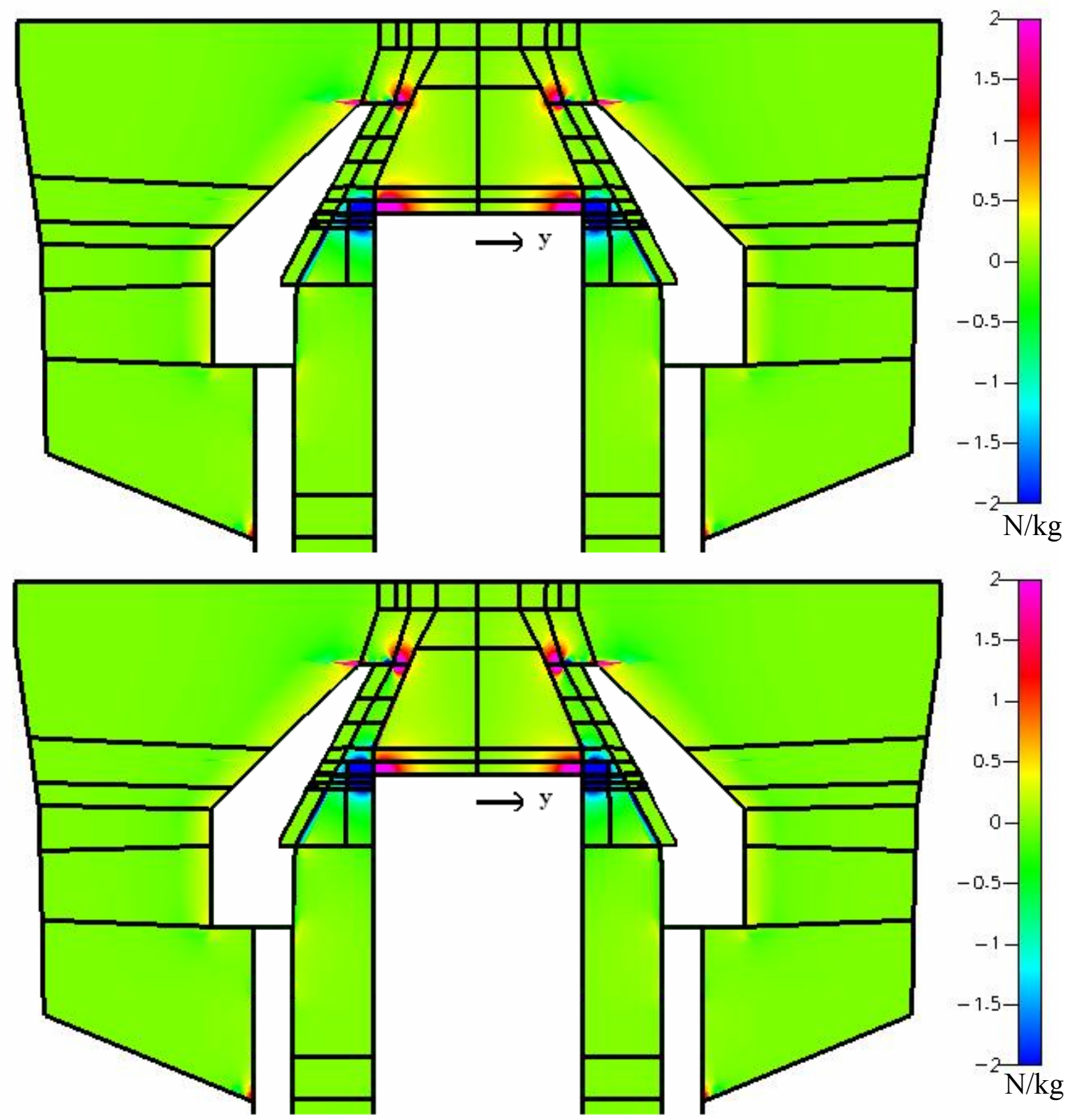

Figure 4.39: Magnitude of the Electric Kelvin Force in the y-direction for the Full Length Cap Electrode 

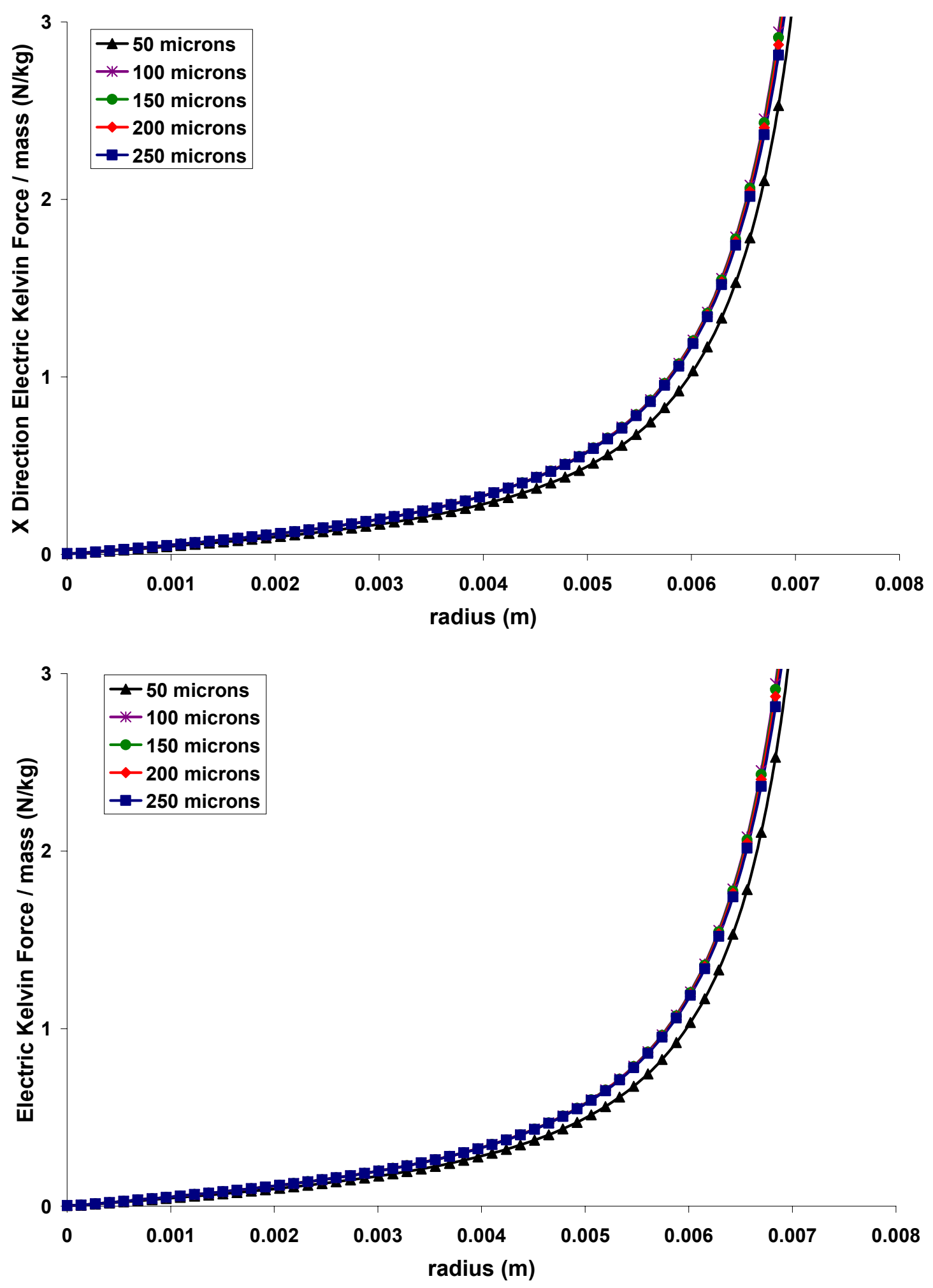

Figure 4.40: Electric Kelvin Force per Mass with all FC-72 inside the cap (top) and FC72 only in the Spray Cone and Above the Pedestal (bottom) as a Function of the Pedestal Radius for Five Distances from the Heater Surface for the Full Length Cap Electrode 


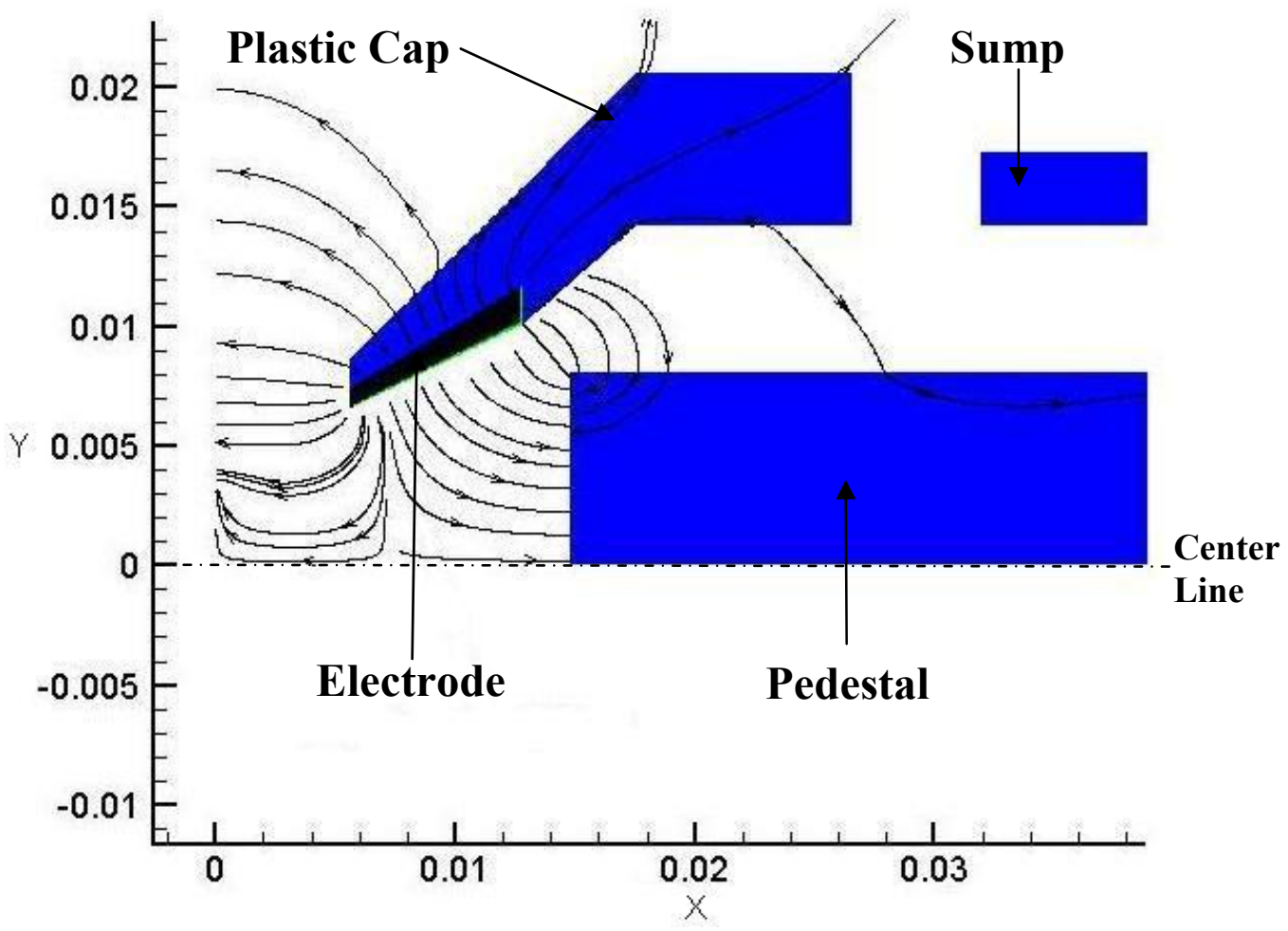

Figure 4.41: Electric Field Lines for Top Half Cap Electrode
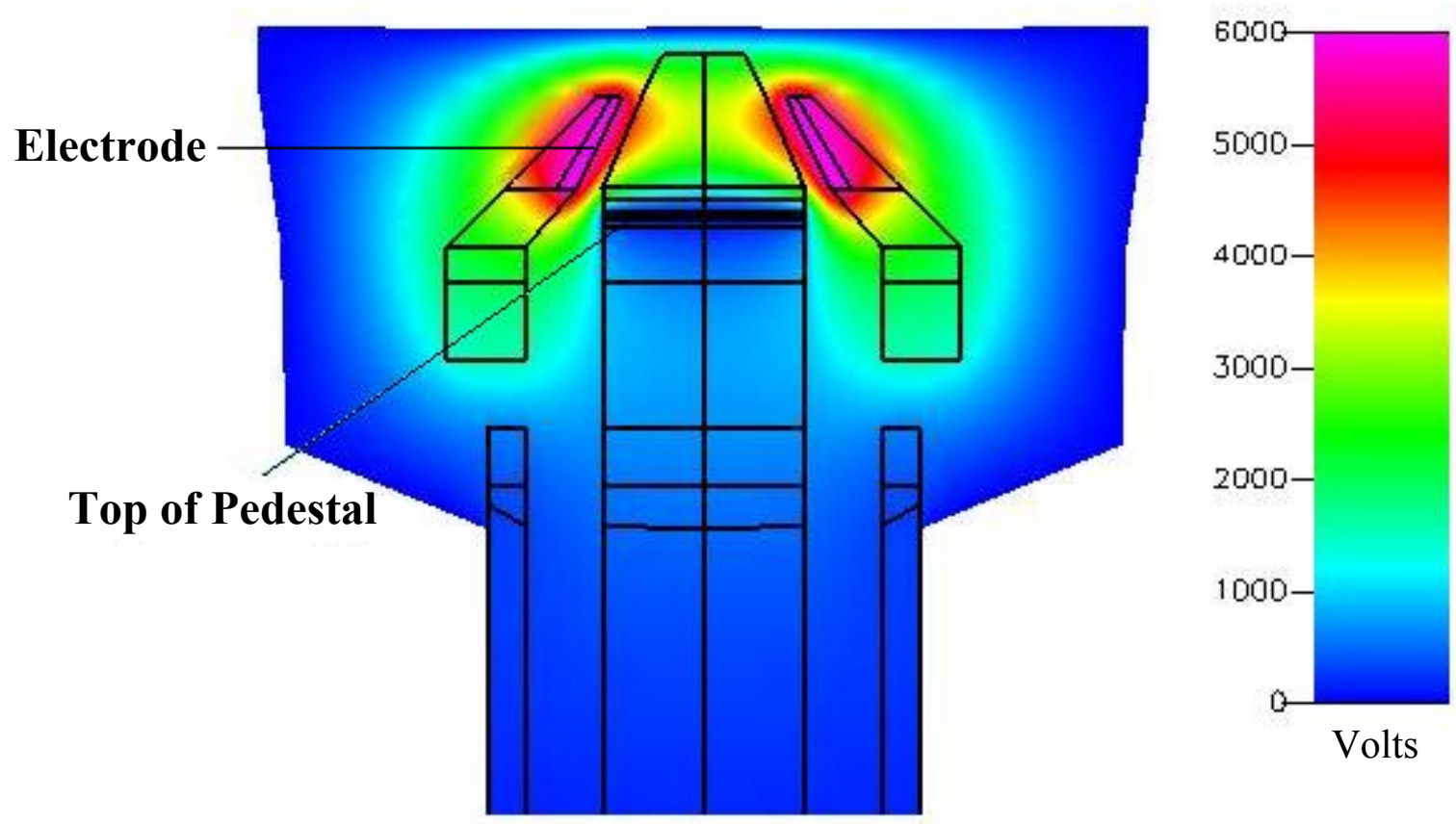

Figure 4.42: Electric Potential for the Top Half Cap Electrode 

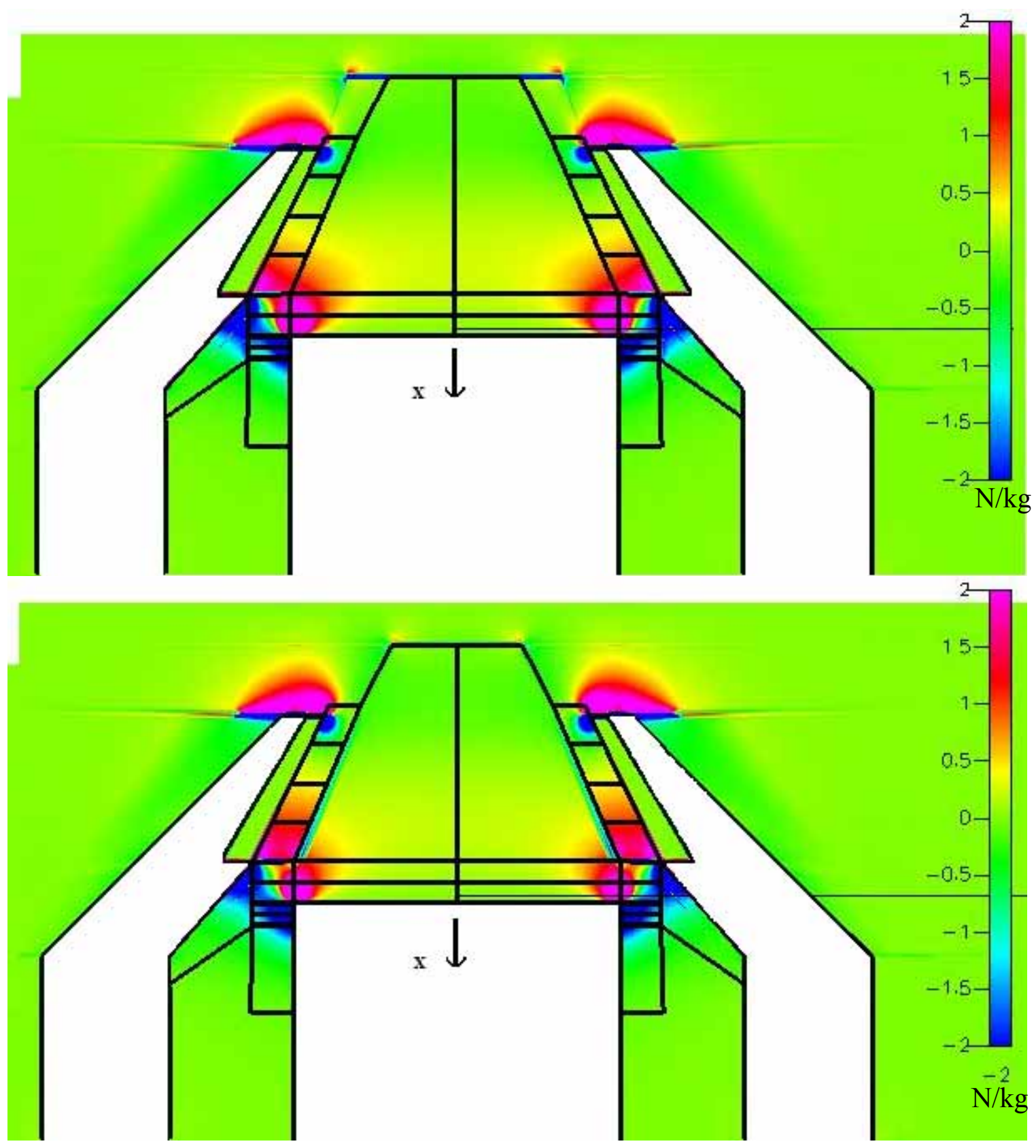

Figure 4.43: Magnitude of the Electric Kelvin Force in the x-direction for the Top Half Cap Electrode 

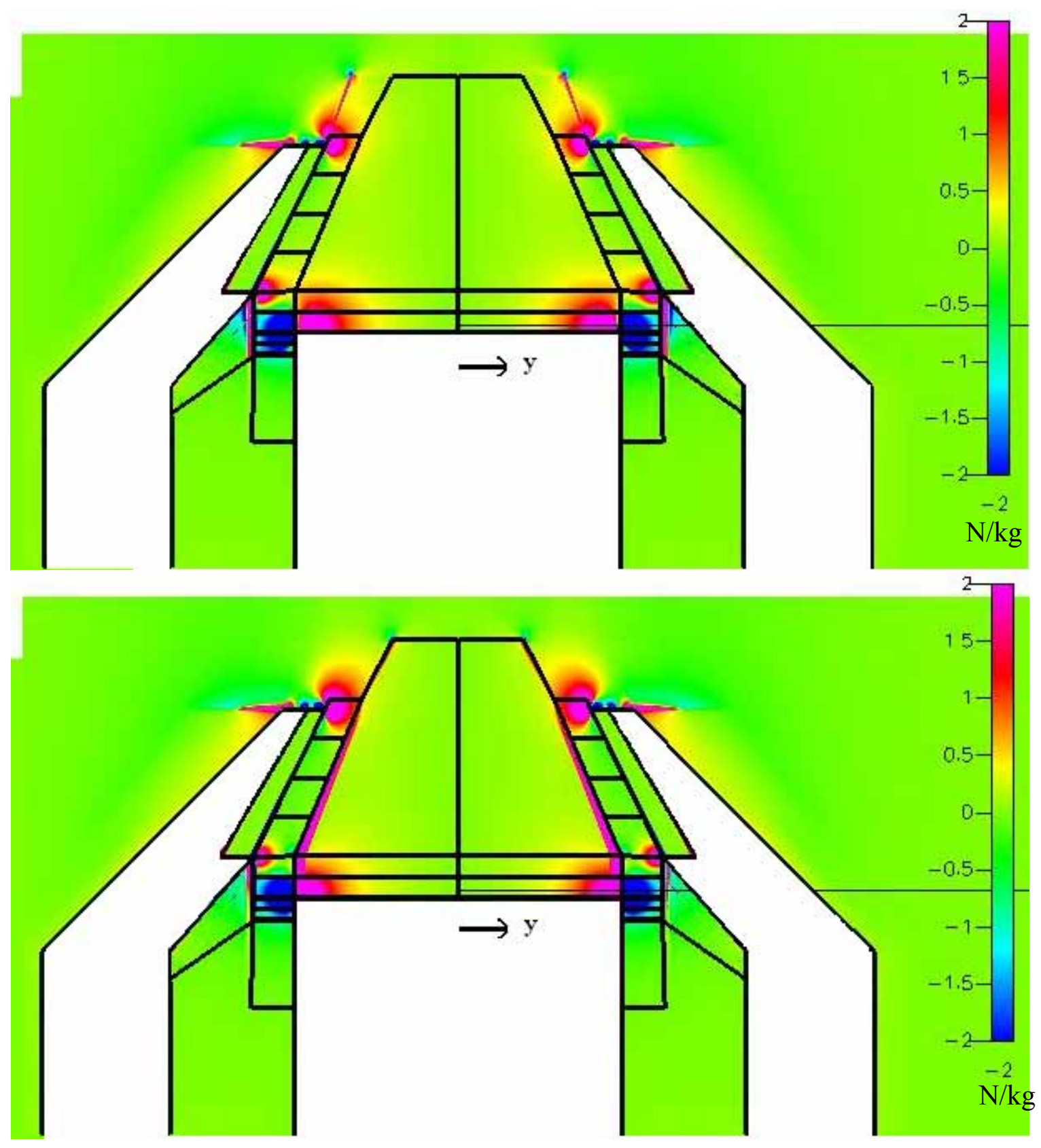

Figure 4.44: Magnitude of the Electric Kelvin Force in the y-direction for the Top Half Cap Electrode 

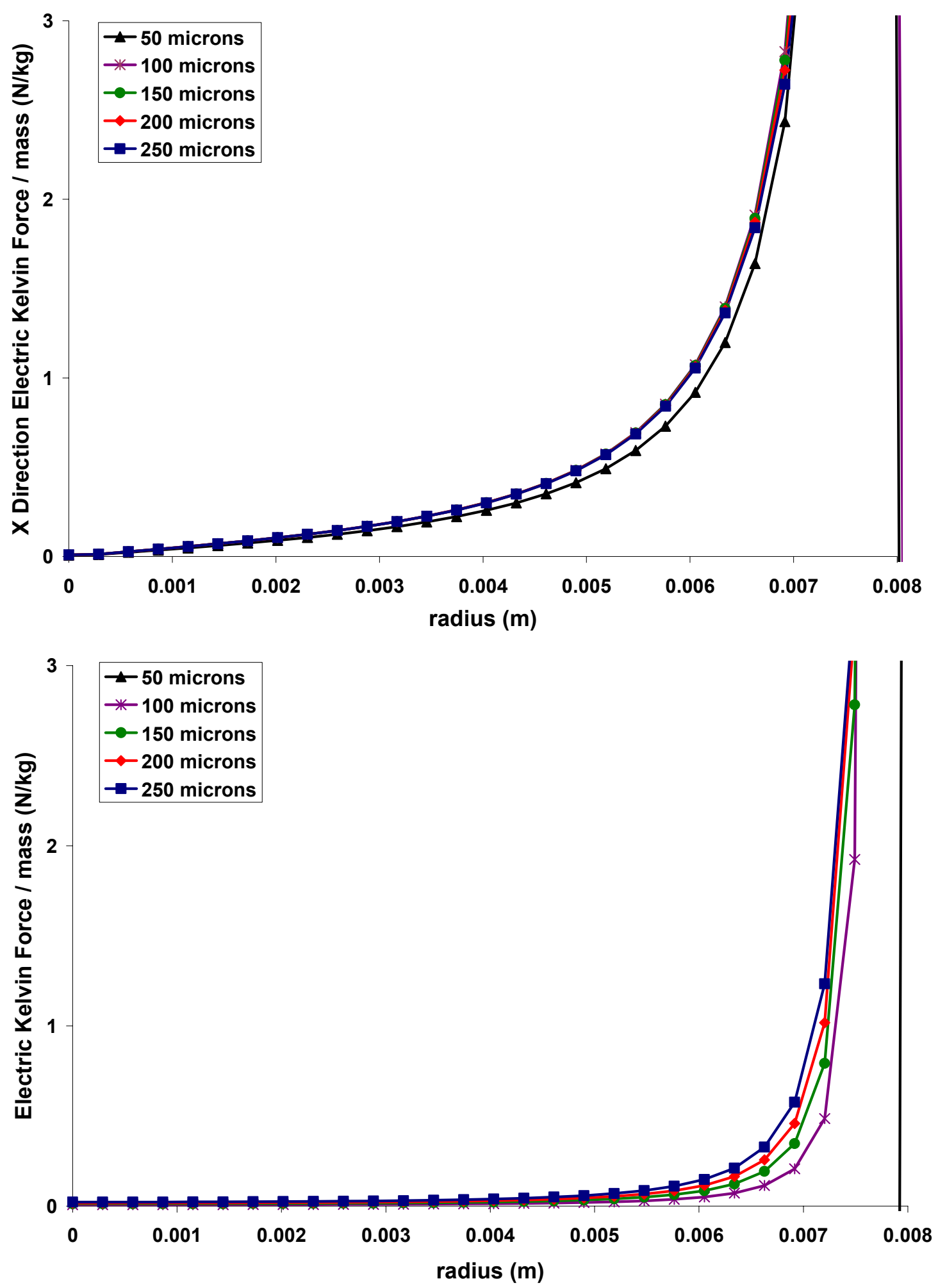

Figure 4.45: Electric Kelvin Force per Mass with all FC-72 inside the cap (top) and FC72 only in the Spray Cone and Above the Pedestal (bottom) as a Function of the Pedestal Radius for Five Distances from the Heater Surface for the Top Half Cap Electrode 
From these simulations based on the relatively small forces that were predicted, no significant effect from the electric Kelvin force was predicted and are all consistent with the negative experimental results. However, these results do indicate that the full length cap electrode produced the largest force across the greatest area of the pedestal.

Several factors could contribute to the negative results obtained from both the experimental tests and numerical simulations. The electric Kelvin force was defined in Chapter 2 as a force proportional to the gradient of the electric field squared (as stated below in Equation 4.1). From this equation, it can be seen that the electric Kelvin force can be increased by increasing the electric field (increasing the applied voltage), increasing the gradient of the electric field (configuring the electrode geometry) or by increasing the electric susceptibility (choosing a fluid with a higher electric susceptibility value).

$$
F_{\text {Kelvin }}=\left(\frac{\varepsilon_{0}}{2}\right)(\kappa-1) \nabla E^{2}
$$

The greatest effect on the surface temperature can be achieved by aligning the gradient of the electric field squared field lines normal to the top pedestal surface. Figure 4.46 shows the gradient of the electric field squared lines for the full length cap electrode. These are not perpendicular to the pedestal surface, but tend to turn out radially close to the surface. 


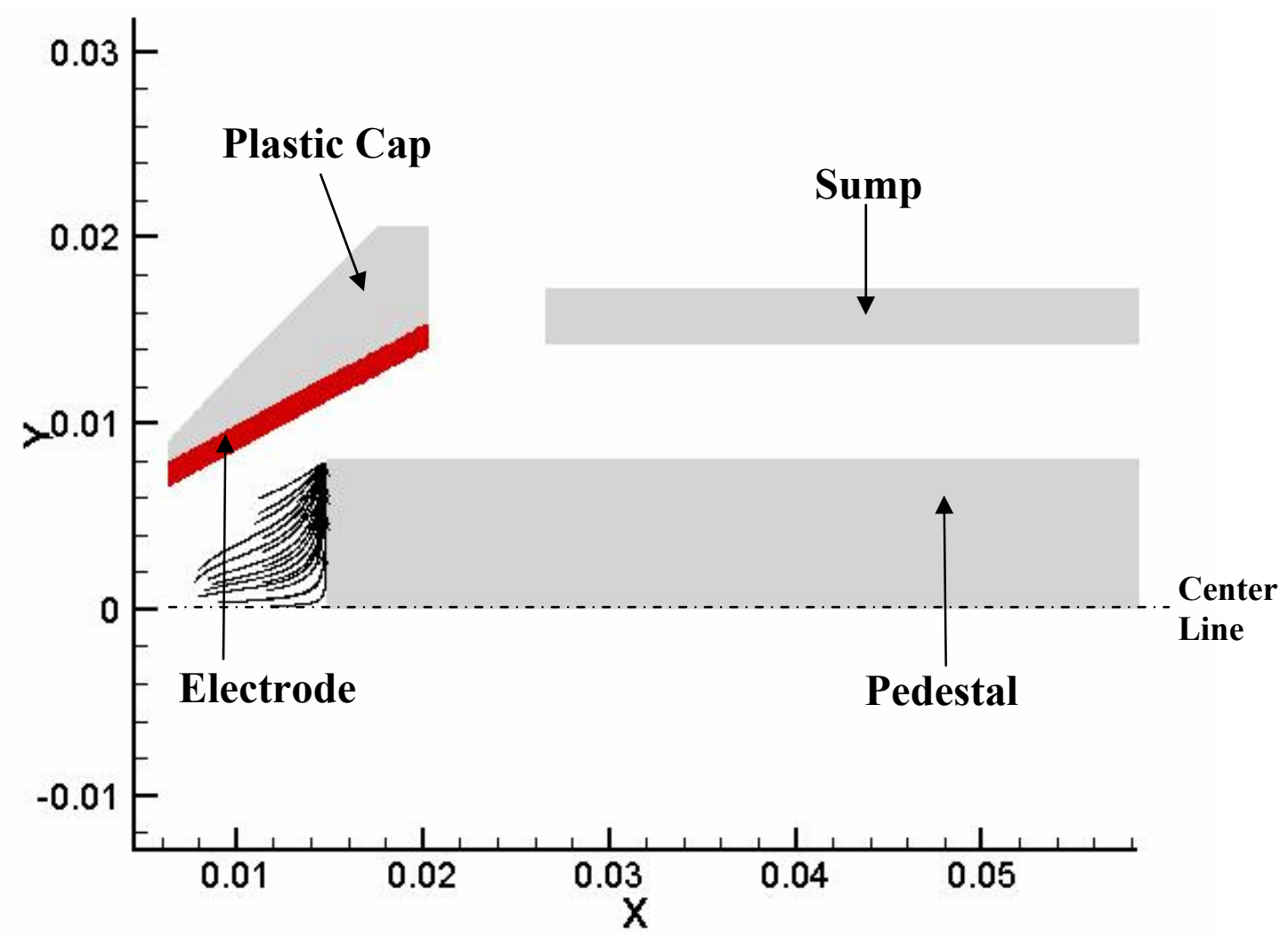

Figure 4.46: Gradient of the Electric Field Squared Field Lines for the Full Length Cap Electrode

For the other three cap electrode simulations, none had gradient of the electric field squared lines that were perpendicular to the pedestal surface. Changing the geometry of the electrodes changes the gradient of the electric field squared lines. Also in the simulations, the magnitudes of the electric Kelvin force in the $\mathrm{x}$ and $\mathrm{y}$ directions were both relatively small. This could be increased by increasing the voltage applied to the electrode. This also would require moving the electrode further from the ground to prevent electrostatic breakdown. From the equation, choosing a fluid with a higher electric susceptibility value would tend to increase the electric Kelvin force. FC-72 has an electric susceptibility of 0.75 , while HFE-7000 has an electric susceptibility of 6.4 . 
To test the theory that HFE-7000 may increase the electric Kelvin force, the FC72 was drained from the apparatus and replaced with HFE-7000. The full length electrode was then retested at a flow rate of $0.016 \mathrm{~m}^{3} / \mathrm{hr}(4.23 \mathrm{GPH})$ of HFE-7000 and electrode voltages of 0,3 , and $6 \mathrm{kV}$. Figure 4.47 shows the measured heat flux as a function of the temperature difference at the top of the pedestal and Figure 4.48 shows the nondimensional heat flux as a function of the non-dimensional temperature difference at the top of the pedestal. The open symbol represents a full run from 0 to $45 \mathrm{~W}$ without changing the voltages, while the closed symbols represent tests performed by increasing the electrode voltage input at each heater power. A plot of the convection heat transfer coefficient as a function of heat flux and a plot of the Nusselt Number as a function the non-dimensional heat flux are shown in Figure 4.49 and Figure 4.50, respectively.

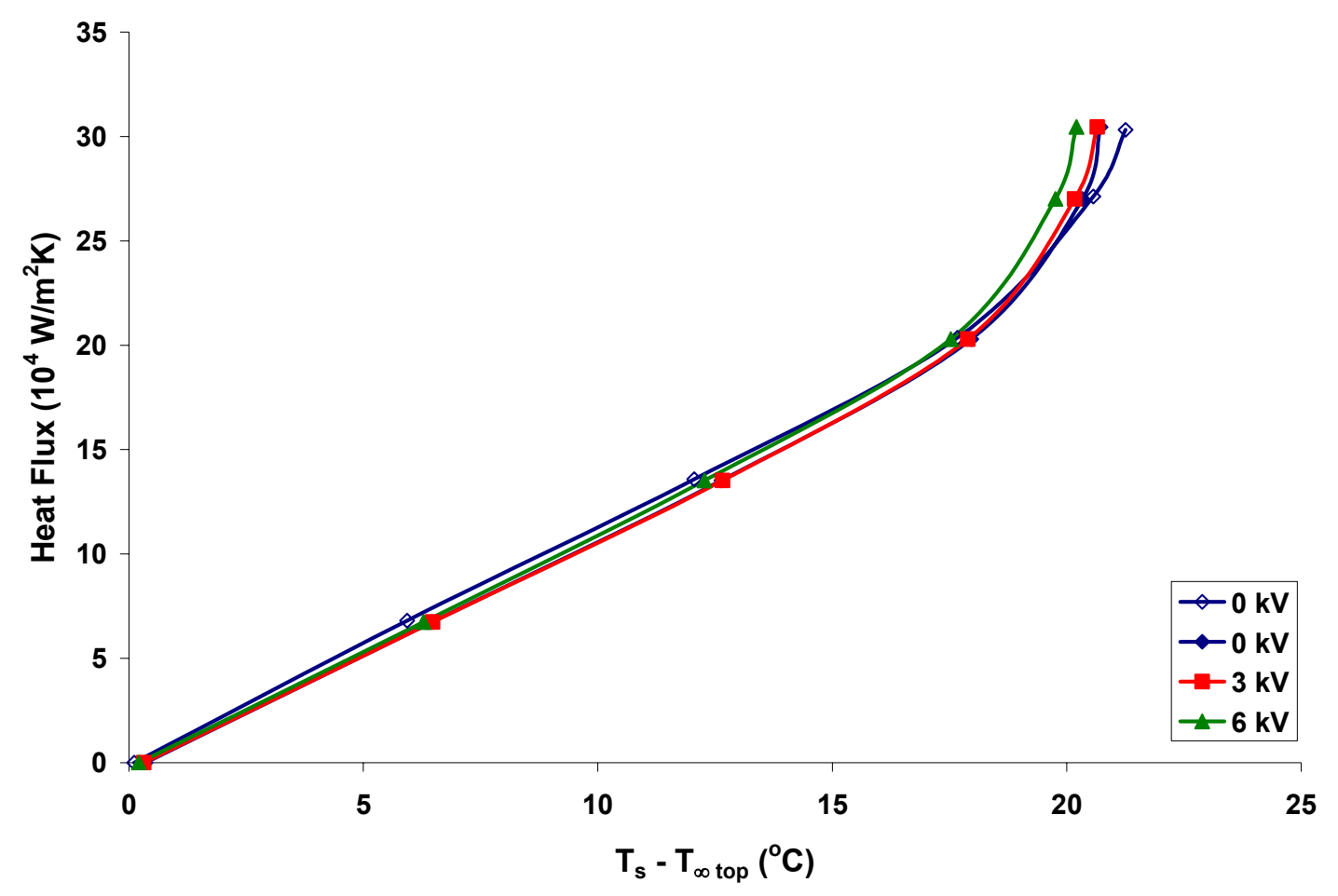

Figure 4.47: Heat Flux in $\mathrm{W} / \mathrm{m}^{2}$ as a Function of the Temperature Difference between the Surface and the Top of the Pedestal in Degrees Celsius for the Full Length Cap Electrode with HFE-7000 as the Working Fluid 


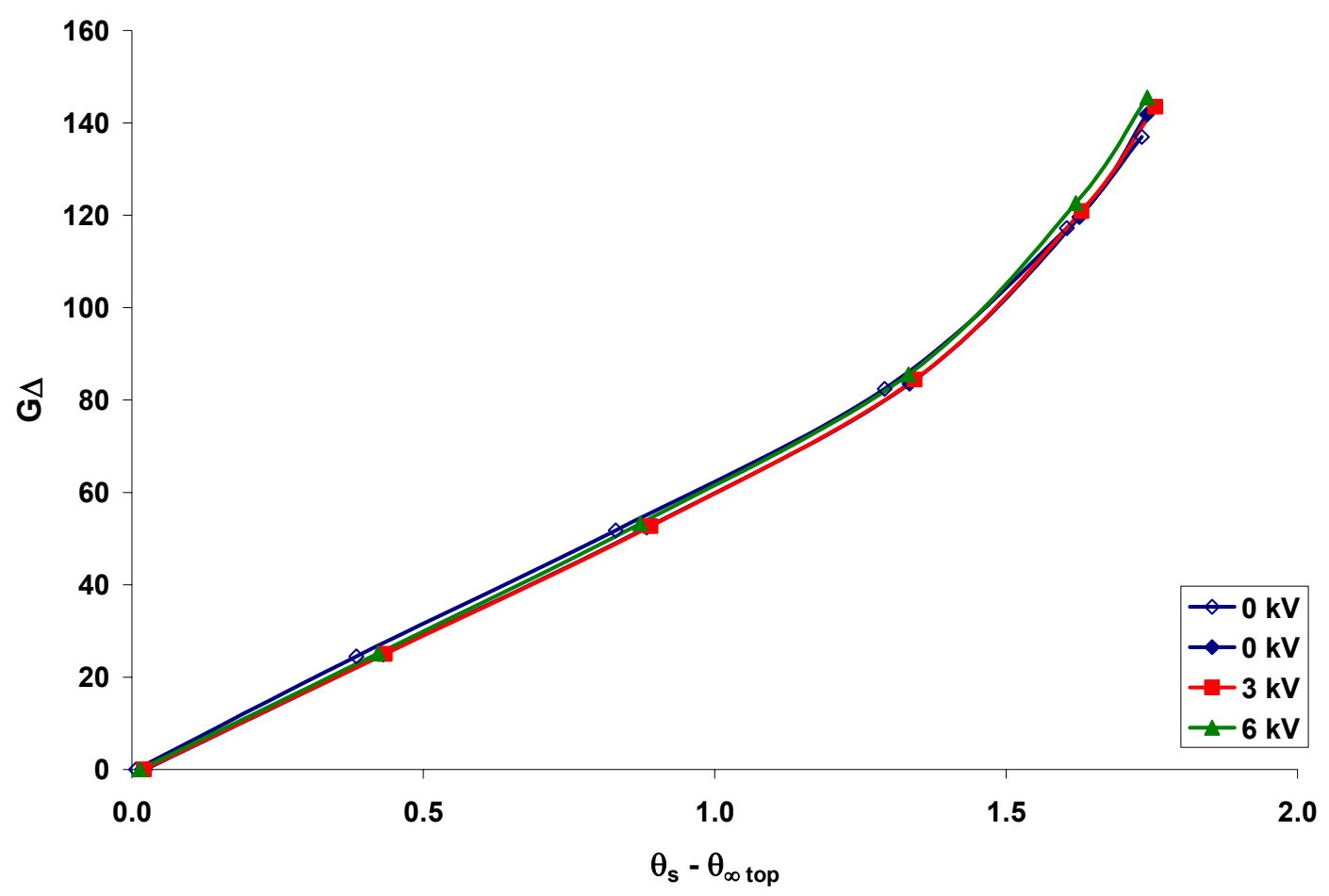

Figure 4.48: Non-dimensional Heat Flux as a Function of the Non-dimensional Temperature Difference between the Surface and the Pedestal Top for the Full Length Cap Electrode with HFE-7000 as the Working Fluid

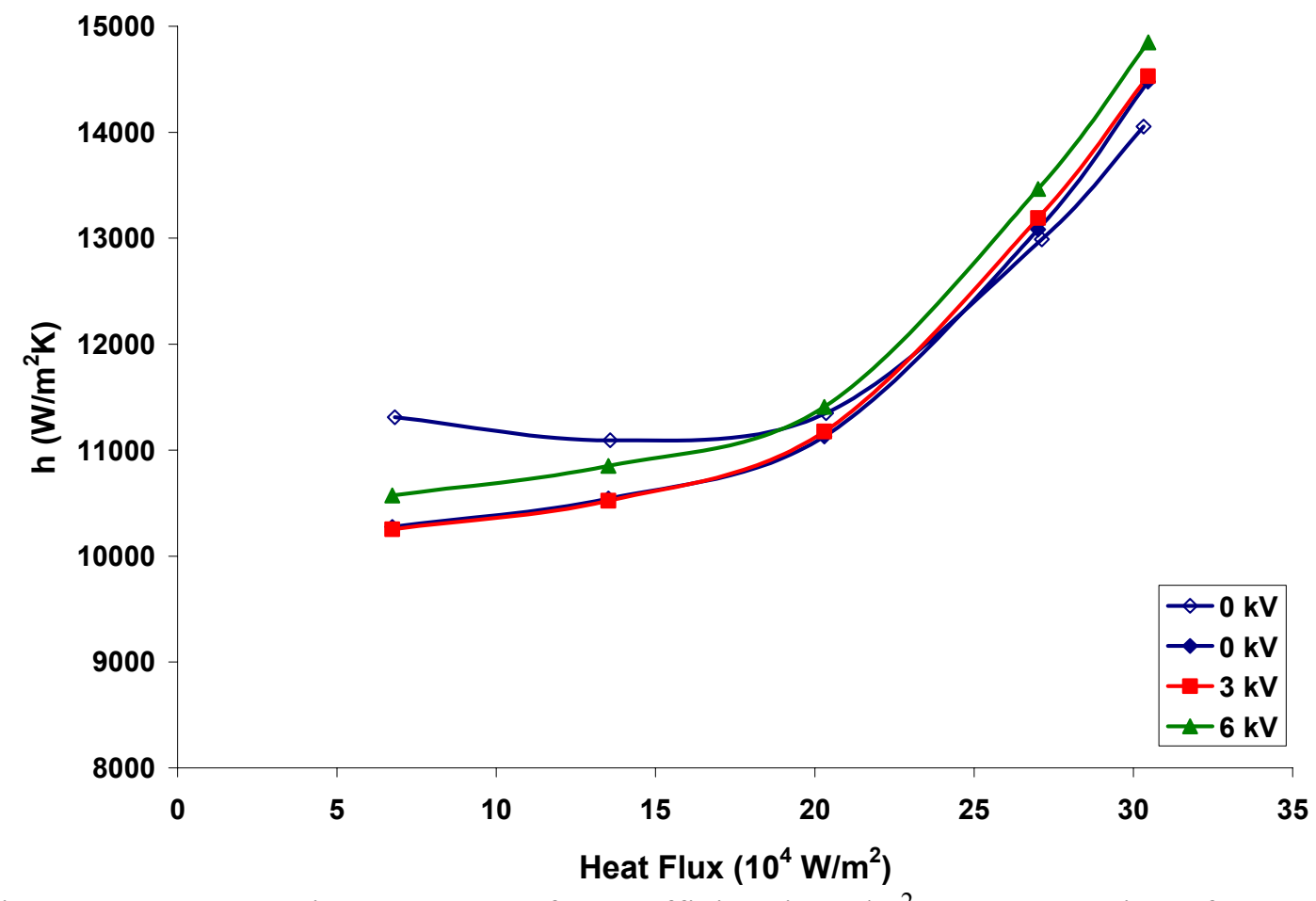

Figure 4.49: Convection Heat Transfer Coefficient in $\mathrm{W} / \mathrm{m}^{2} \mathrm{~K}$ as a Function of Heat Flux in $\mathrm{W} / \mathrm{m}^{2}$ for Full Length Cap Electrode with HFE-7000 as the Working Fluid 


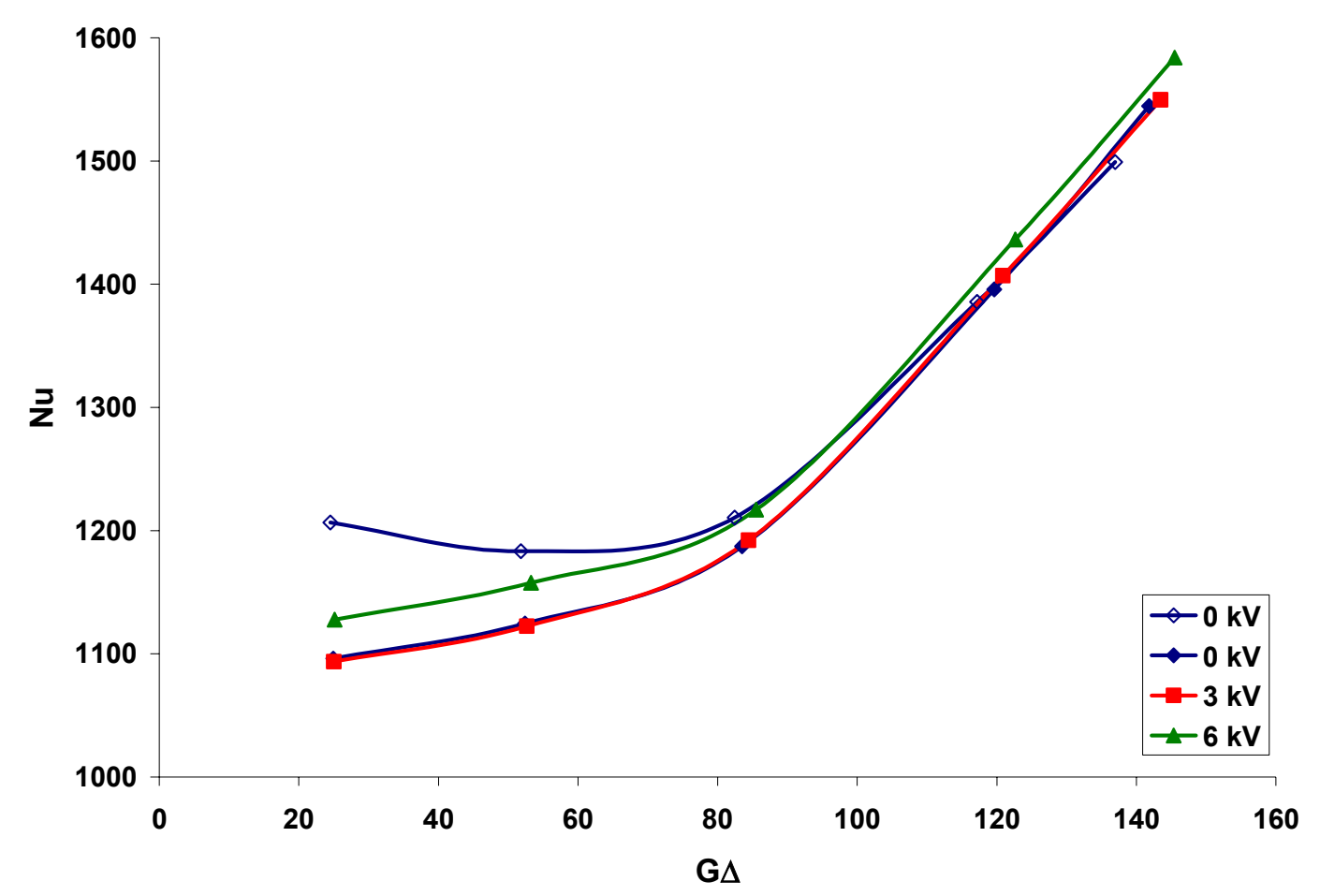

Figure 4.50: Nusselt Number as a Function of Non-dimensional Heat Flux for the Full Length Cap Electrode with HFE-7000 as the Working Fluid

These plots show a slight trend of cooling the pedestal surface with a magnitude of the temperature difference of approximately $0.5{ }^{\circ} \mathrm{C}$ and a magnitude of the heat transfer coefficient of approximately $330 \mathrm{~W} / \mathrm{m}^{2} \mathrm{~K}$. While this full length cap electrode with HFE-7000 as the working fluid shows a slight trend experimentally, note that these magnitudes are relatively small when compared to the magnitudes of the experimental error values. 


\subsection{Mehra Electrode Results}

The Mehra electrode that was described in Chapter 3 (shown in Figure 3.34) was tested both numerically (by Mr. Deepak Mehra) and experimentally in this study. The numerical study is described in detail in Mehra's dissertation along with a short presentation of the results below. The electrode configuration was tested experimentally at increasing electrode voltages up to $23 \mathrm{kV}$ and also at decreasing voltages for various heater powers up to $12 \mathrm{~W}$. Data was recorded as shown in the test profiles presented in Chapter 3.

Figure 4.51 shows the numerically calculated plot of the electric field lines with the electrode configuration. Note that the electric field lines are all normal to the electrode surfaces. The nozzle electrode is at $23 \mathrm{kV}$ and the cap electrode, pedestal electrode, and heater are at ground as shown in the contour plot of the electric potential in Figure 4.52.

Figure 4.53 and Figure 4.54 show the magnitude in Newtons per kilogram of the force per unit mass due to the computed electric Kelvin force in the $\mathrm{x}$ and $\mathrm{y}$ directions, respectively (where just as for the cap electrodes, the positive $\mathrm{x}$ direction is pointing down toward the heater and the positive y direction is the radial direction from the centerline of the pedestal) for the Mehra electrode configuration. The plot represents the computed electric Kelvin force when the entire area between the cap electrode and pedestal is filled with HFE-7000 (and therefore computed assuming those cells possess the properties of HFE-7000) and the other areas are filled with air. These figures show that a significant upward (negative $\mathrm{x}$-direction) force exists across the entire top surface of the pedestal as well as a significant inward (negative y-direction) force at the outer 
radius of the pedestal. To quantify these magnitudes, Figure 4.55 and Figure 4.56 show plots of the computed electric Kelvin Force per unit mass in the x-direction and ydirection (respectively) as a function of the pedestal radius for various distances from the top of the pedestal surface. For both the $\mathrm{x}$ and $\mathrm{y}$ directions, the magnitudes of the electric Kelvin force per unit mass get smaller further from the pedestal surface.

As discussed in the previous section, it is optimal for the gradient of the electric field squared lines to be normal to the pedestal surface. These calculated field lines are shown in Figure 4.57.

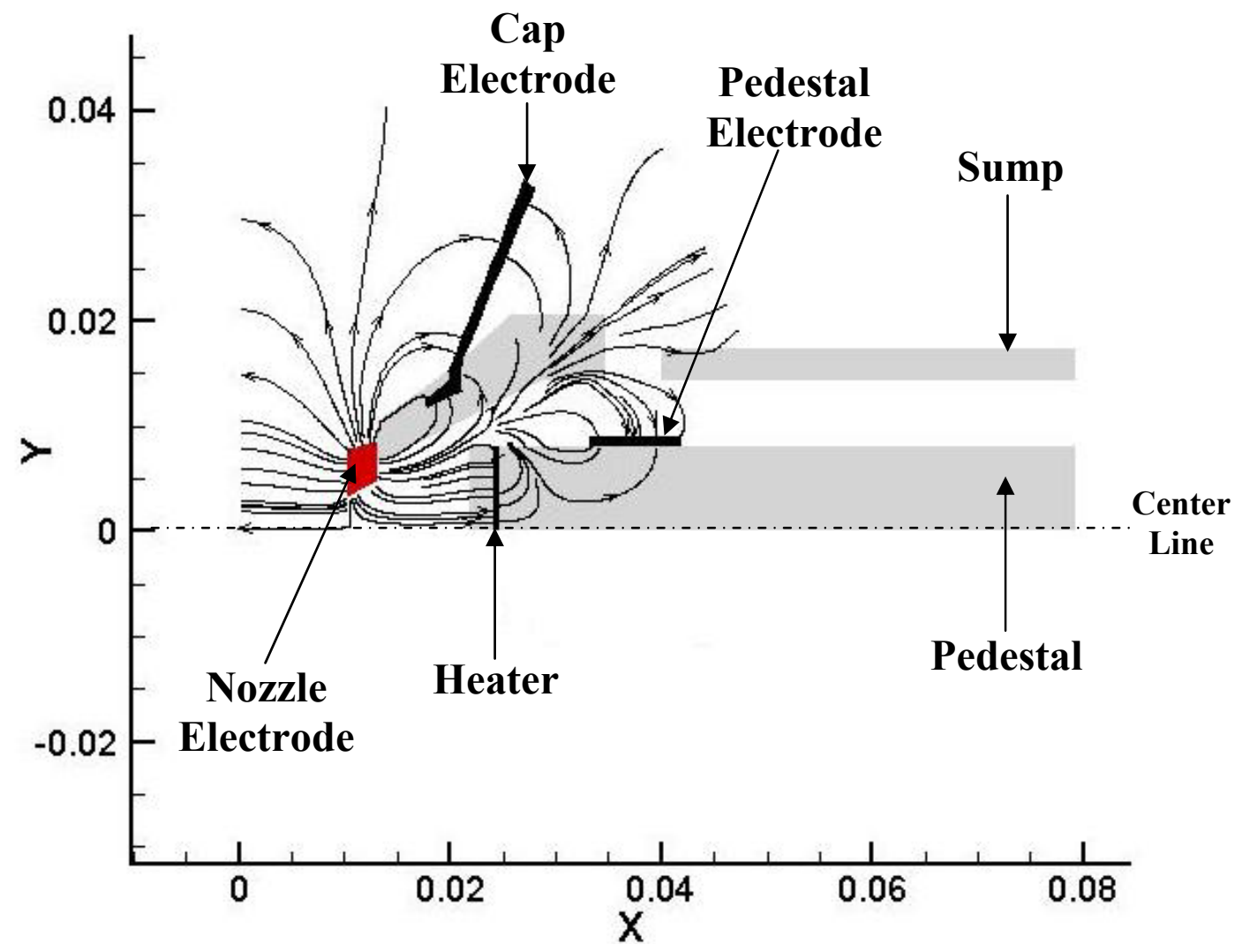

Figure 4.51: Electric Field Lines for Mehra Electrode Configuration 

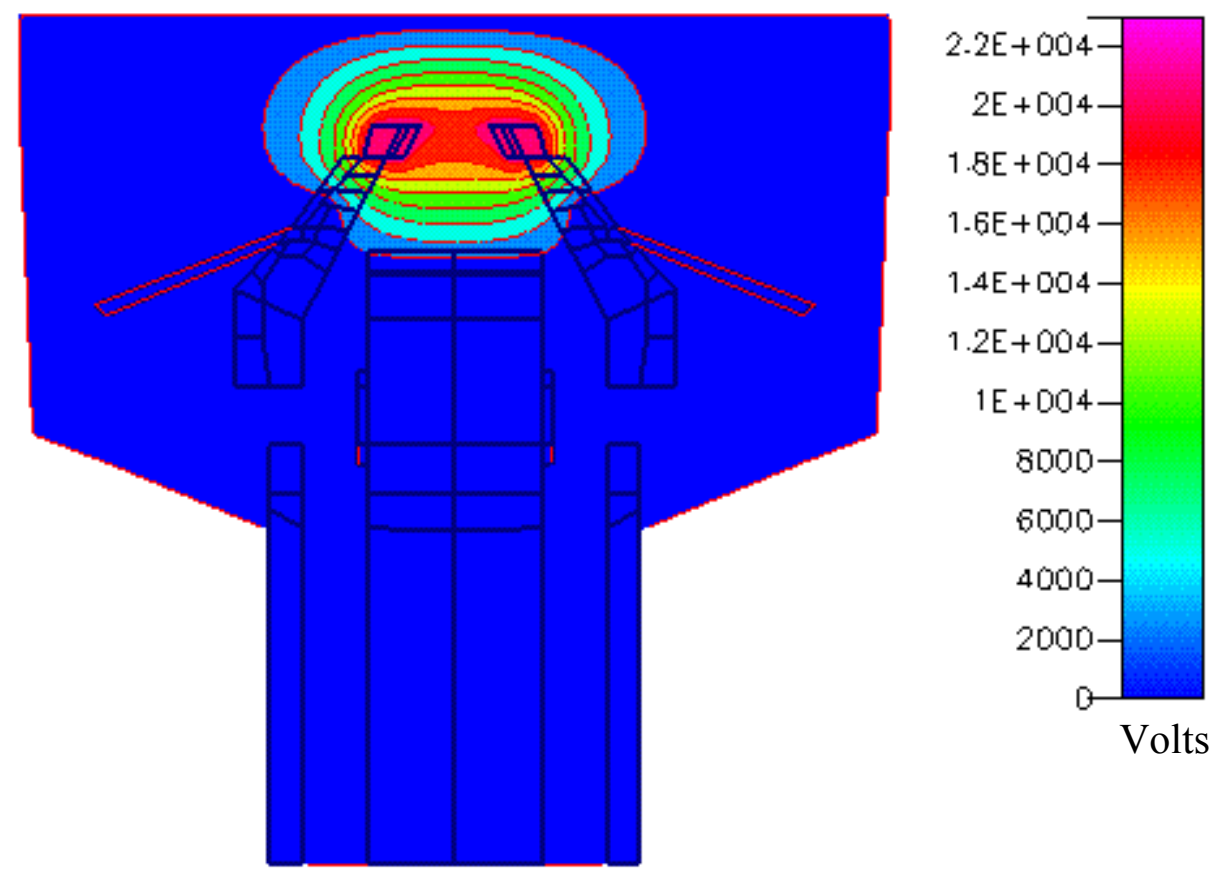

Figure 4.52: Electric Potential for the Mehra Electrode Configuration
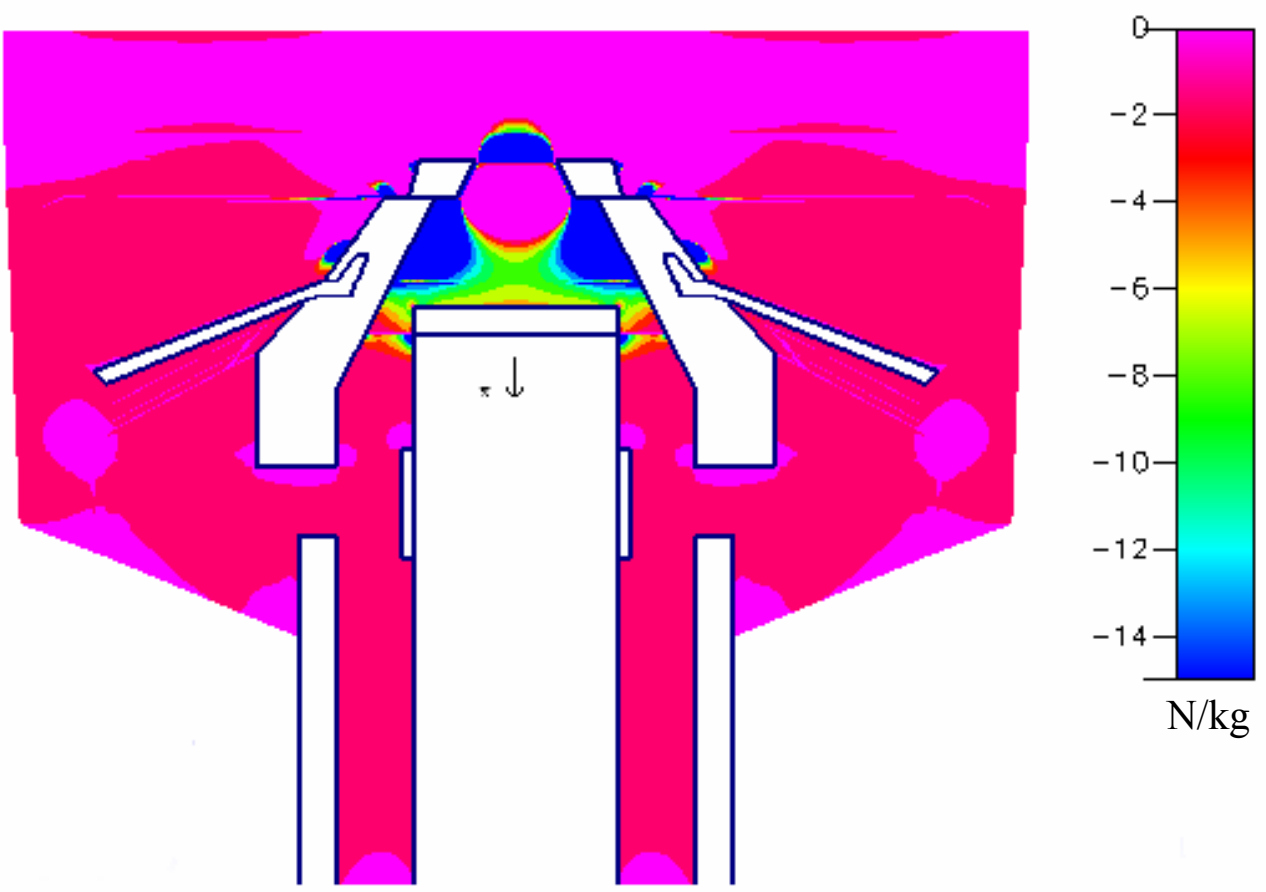

Figure 4.53: Magnitude of the Electric Kelvin Force per unit Mass in the X-direction for the Mehra Electrodes 


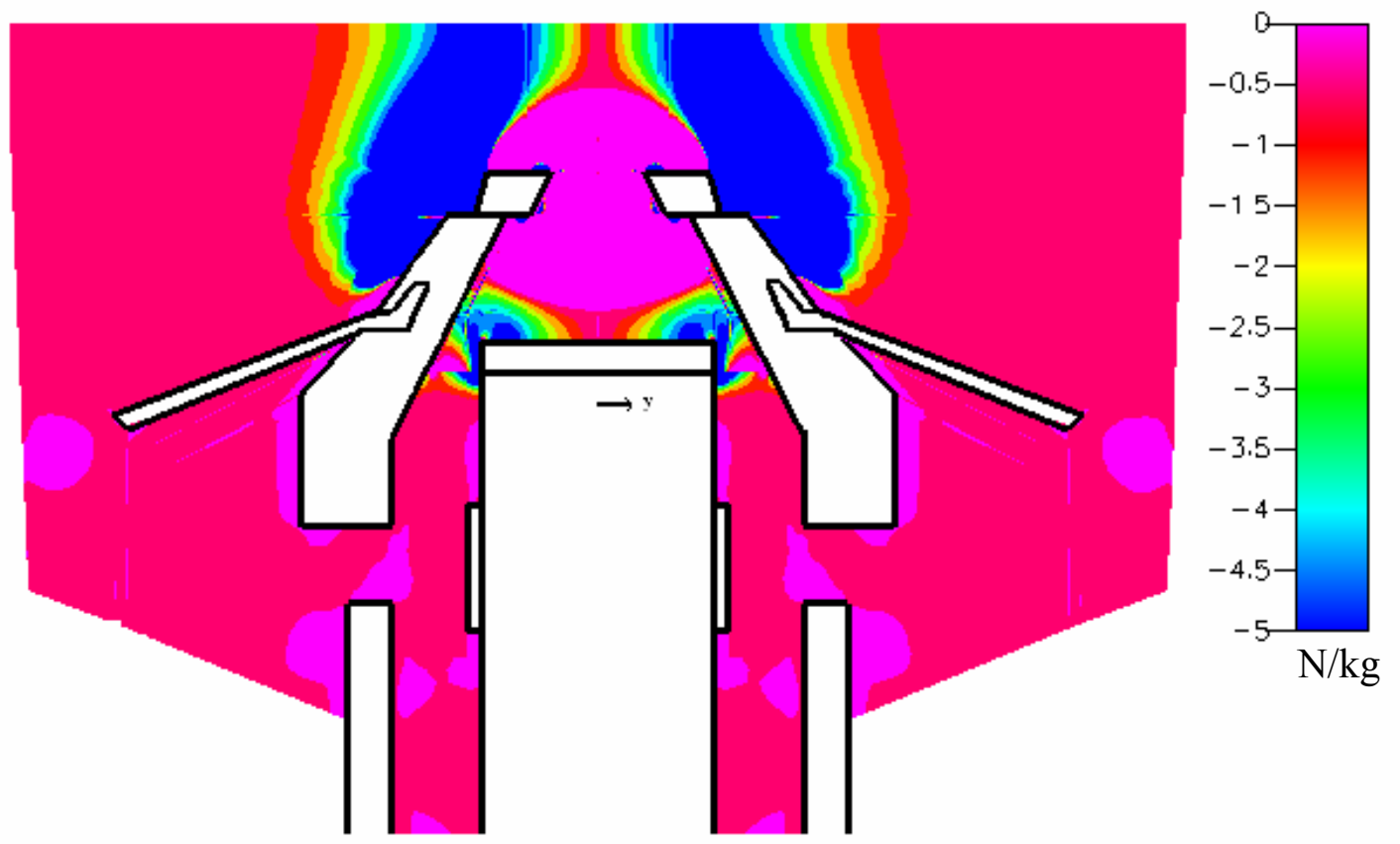

Figure 4.54: Magnitude of the Electric Kelvin Force per unit Mass in the y-direction for the Mehra Electrodes

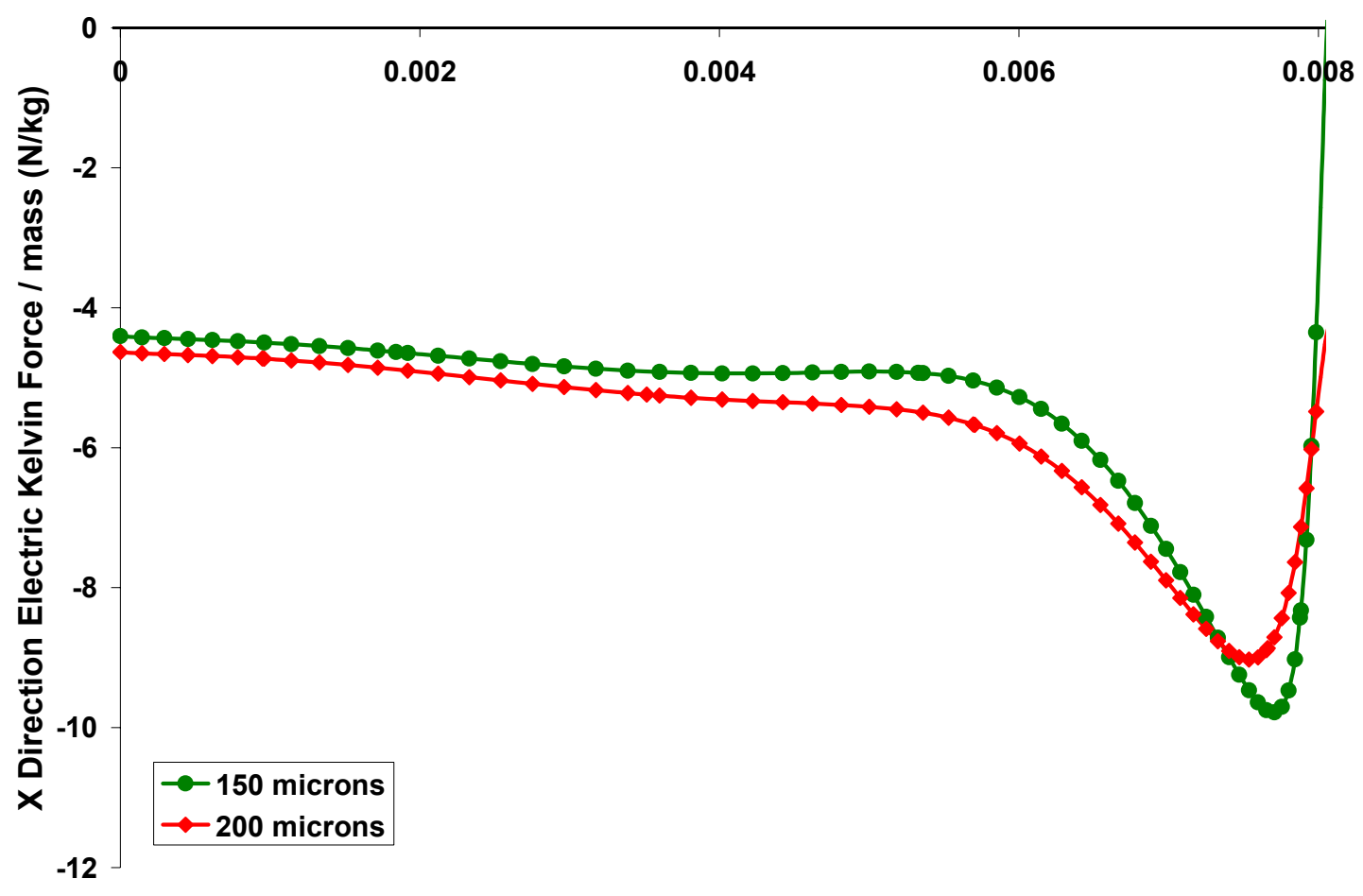

\section{Radius (m)}

Figure 4.55: Electric Kelvin Force per Mass in the x-direction as a Function of the Pedestal Radius for Three Distances from the Pedestal Surface for the Mehra Electrodes 


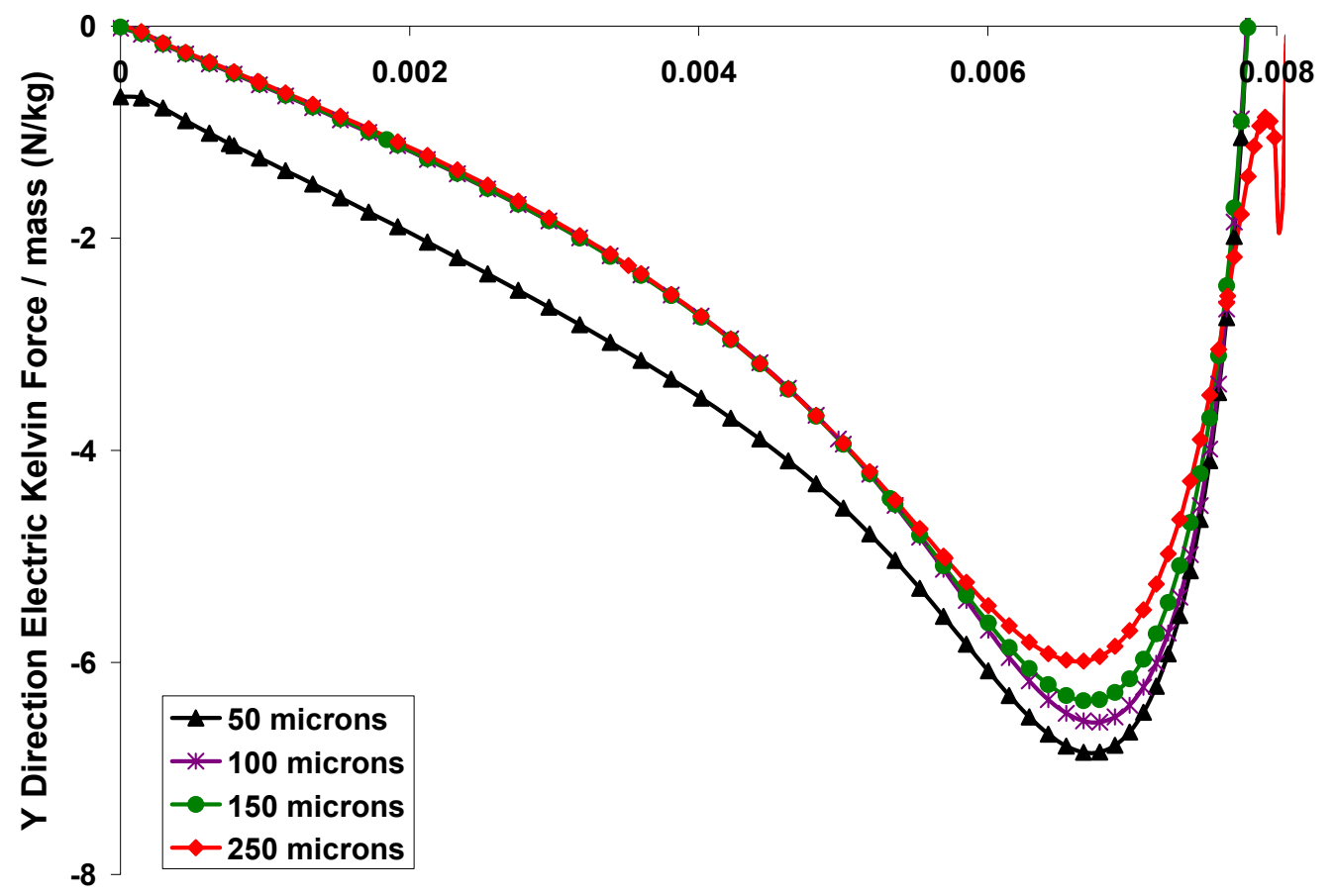

Radius (m)

Figure 4.56: Electric Kelvin Force per Mass in the y-direction as a Function of the Pedestal Radius for Four Distances from the Pedestal Surface for the Mehra Electrodes

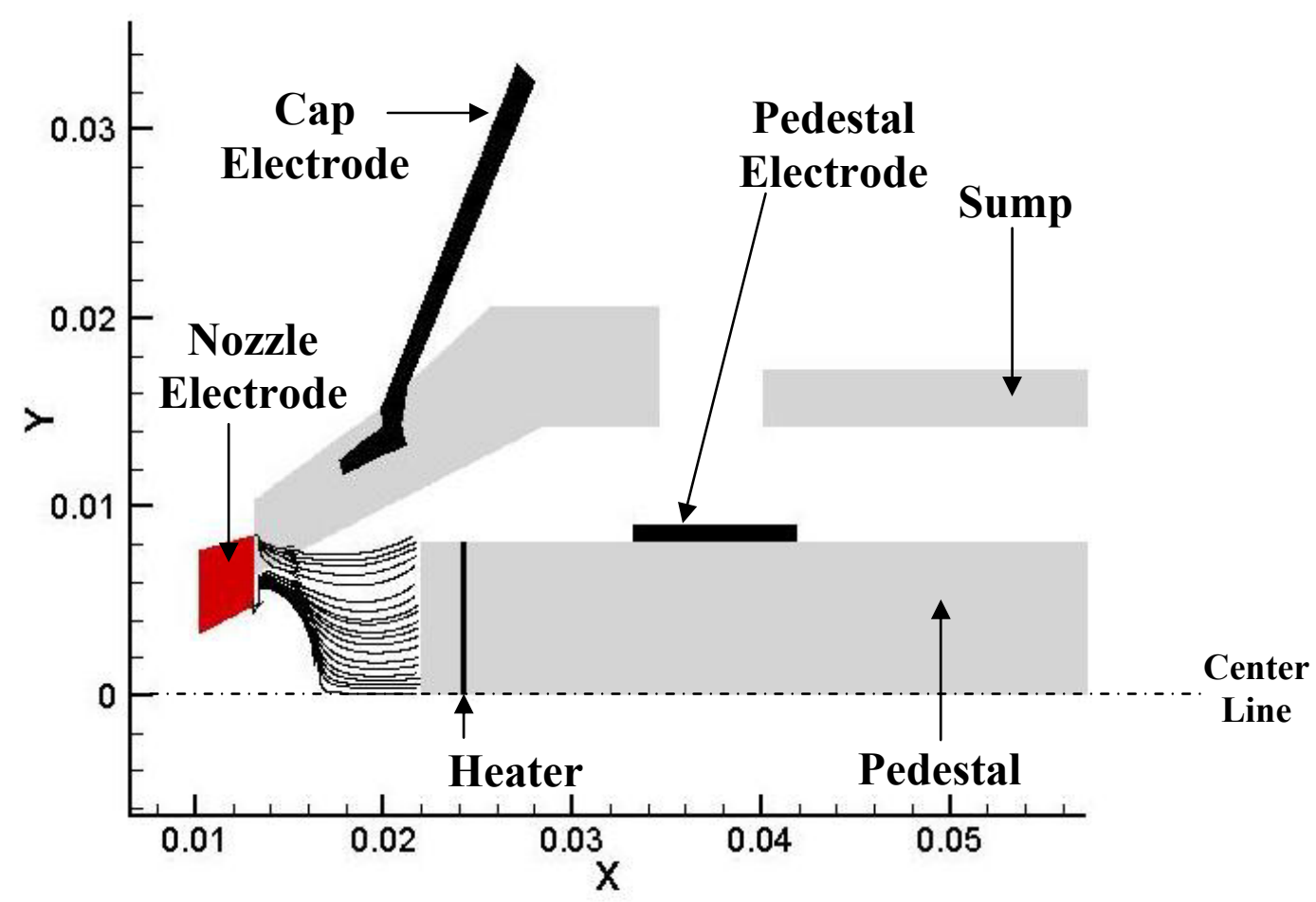

Figure 4.57: Gradient of the Electric Field Squared Lines for the Mehra Electrodes 
From these numerical simulations by Mr. Deepak Mehra, it was predicted that the electric Kelvin force would produce a positive effect on the cooling efficiency of the experimental spray cooling apparatus. The experimental tests on the Mehra electrode as described in Chapter 3, were performed for electrode voltages of up to $23 \mathrm{kV}$ and heater powers of up to $12 \mathrm{~W}$. The closed symbols in the plots below represent the portion of the test profile where the voltages were being increased at each heater power, while the open symbols represent the portion where the voltages were decreased. Figure 4.58 is a plot of the heat flux as a function of the temperature difference at the top of the pedestal for electrode voltages ranging from 0 to $23 \mathrm{kV}$. The curves begin to diverge near the higher heat fluxes showing a cooling effect as the voltage is increased and a subsequent heating effect as the voltage is then decreased. The area indicated in Figure 4.58 corresponds to the enlarged view shown Figure 4.59. (The corresponding non-dimensional versions of these plots are shown in Figure 4.60 and Figure 4.61.) It can be seen that at a heat flux of $120,700 \mathrm{~W} / \mathrm{m}^{2}$, as the electrode voltage is increased from 0 to $23 \mathrm{kV}$, the temperature difference at the top of the pedestal decreases by $1.91{ }^{\circ} \mathrm{C}$. As the electrode voltage is then decreased back to $0 \mathrm{kV}$, the temperature difference increases by $1.46{ }^{\circ} \mathrm{C}$. Note that the temperature difference did not increase back to the same value at the first and last 0 $\mathrm{kV}$ input. This could be due to a general increase in the ambient temperature during the approximately 45 minutes it took to perform the test. The other voltages increase and decrease almost in sequential order between these two maximums. The lower voltages of 0 and $4 \mathrm{kV}$ became out of order as the voltage was being decreased (open symbols), but this discrepancy is within experimental errors and may be due to an inadequate electrode voltage input to produce the effect seen at higher voltages. 


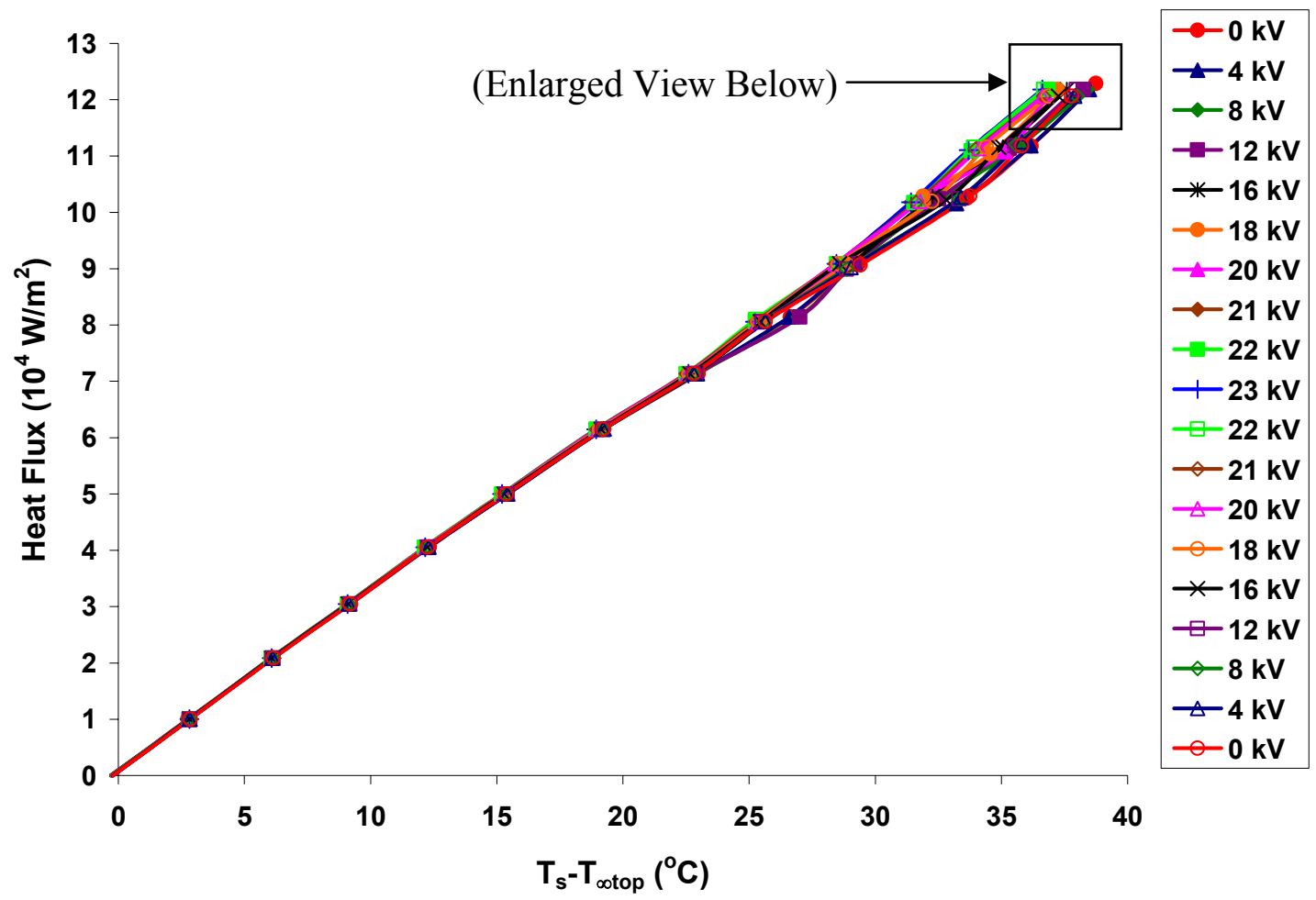

Figure 4.58: Heat Flux in W/m $\mathrm{m}^{2}$ as a Function of the Temperature Difference between the Surface and the Top of the Pedestal in Degrees Celsius for the Mehra Electrodes at Voltages up to $23 \mathrm{kV}$

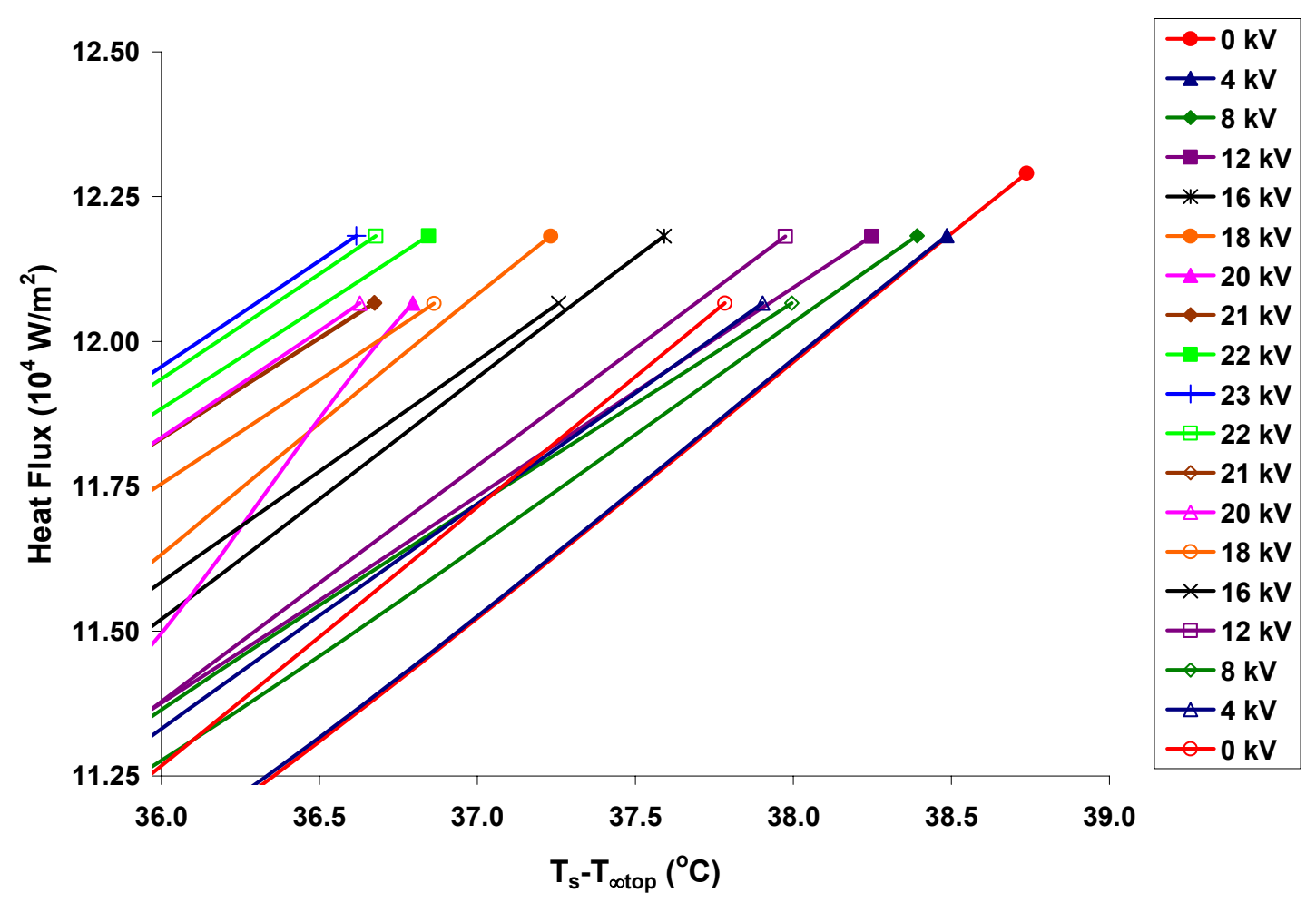

Figure 4.59: Enlarged View of the Highest Heat Flux in Figure 4.58 


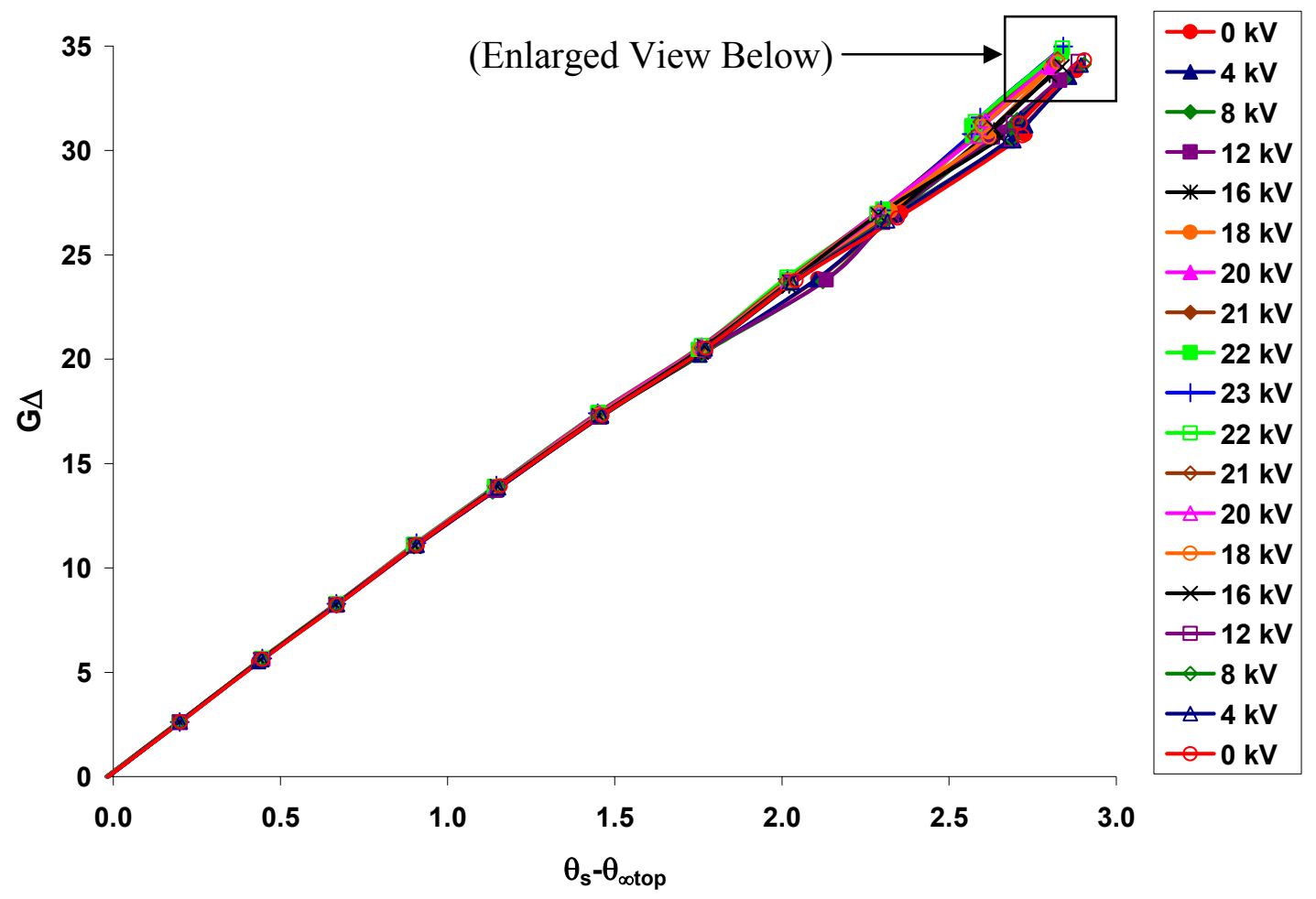

Figure 4.60: Non-dimensional Heat Flux as a Function of the Non-dimensional Temperature Difference between the Surface and the Pedestal Top for the Mehra Electrodes at Voltages up to $23 \mathrm{kV}$

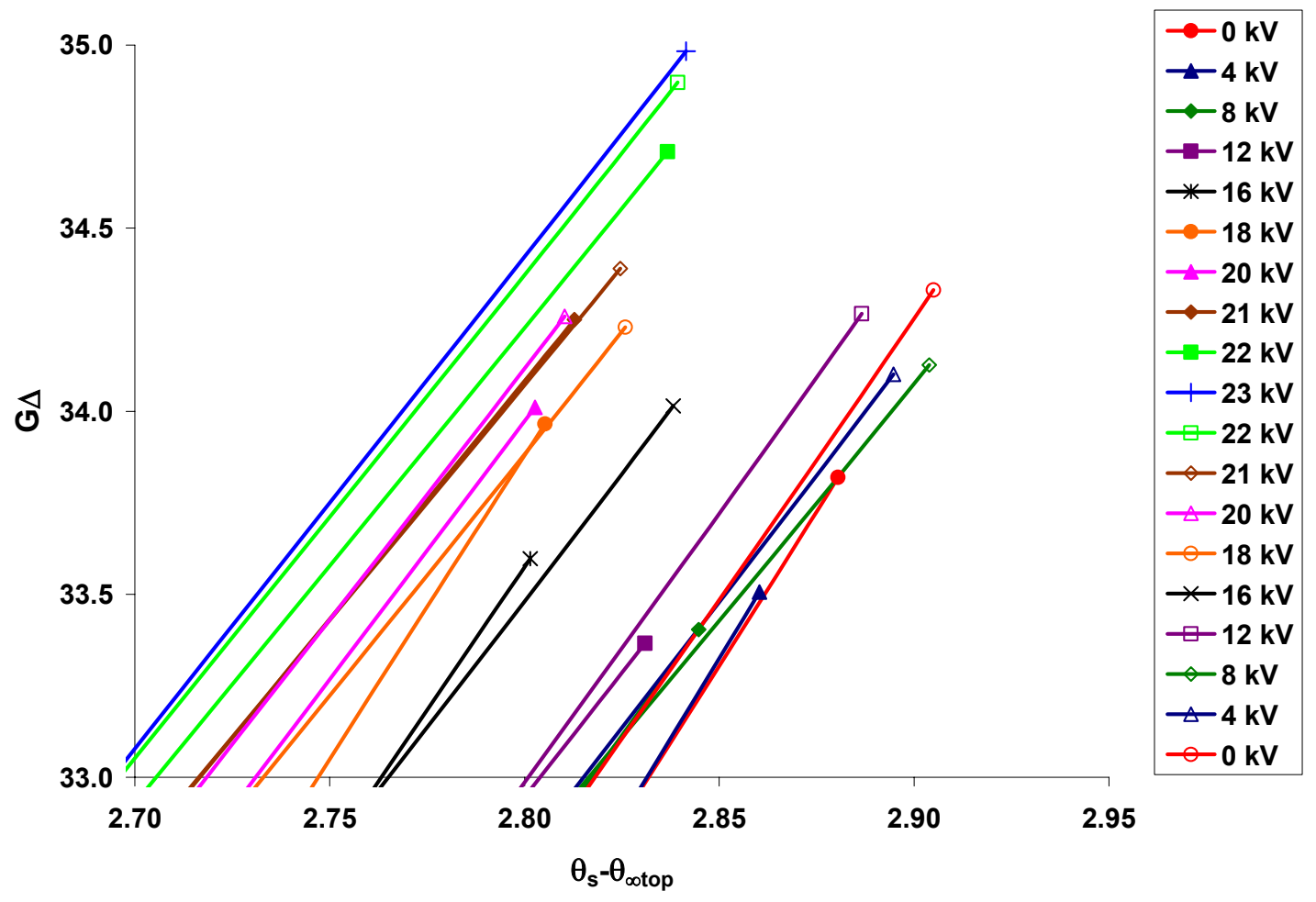

Figure 4.61: Enlarged View of the Highest G $\Delta$ in Figure 4.60 
To better view the effect of the electrode voltage input, the temperature difference at the top of the pedestal at $0 \mathrm{kV},\left(\mathrm{T}_{\mathrm{s}}-\mathrm{T}_{\text {otop }}\right)_{0 \mathrm{kV}}$, was subtracted from the temperature difference at the top of the pedestal at each electrode voltage, $\left(\mathrm{T}_{\mathrm{s}}-\mathrm{T}_{\text {otop }}\right)$, and plotted as a function of the electrode voltage for the various different heater powers. This plot is shown in Figure 4.62 for the increasing electrode voltages and in Figure 4.63 for decreasing voltages. The corresponding non-dimensional plots are shown in Figure 4.64 and Figure 4.65.

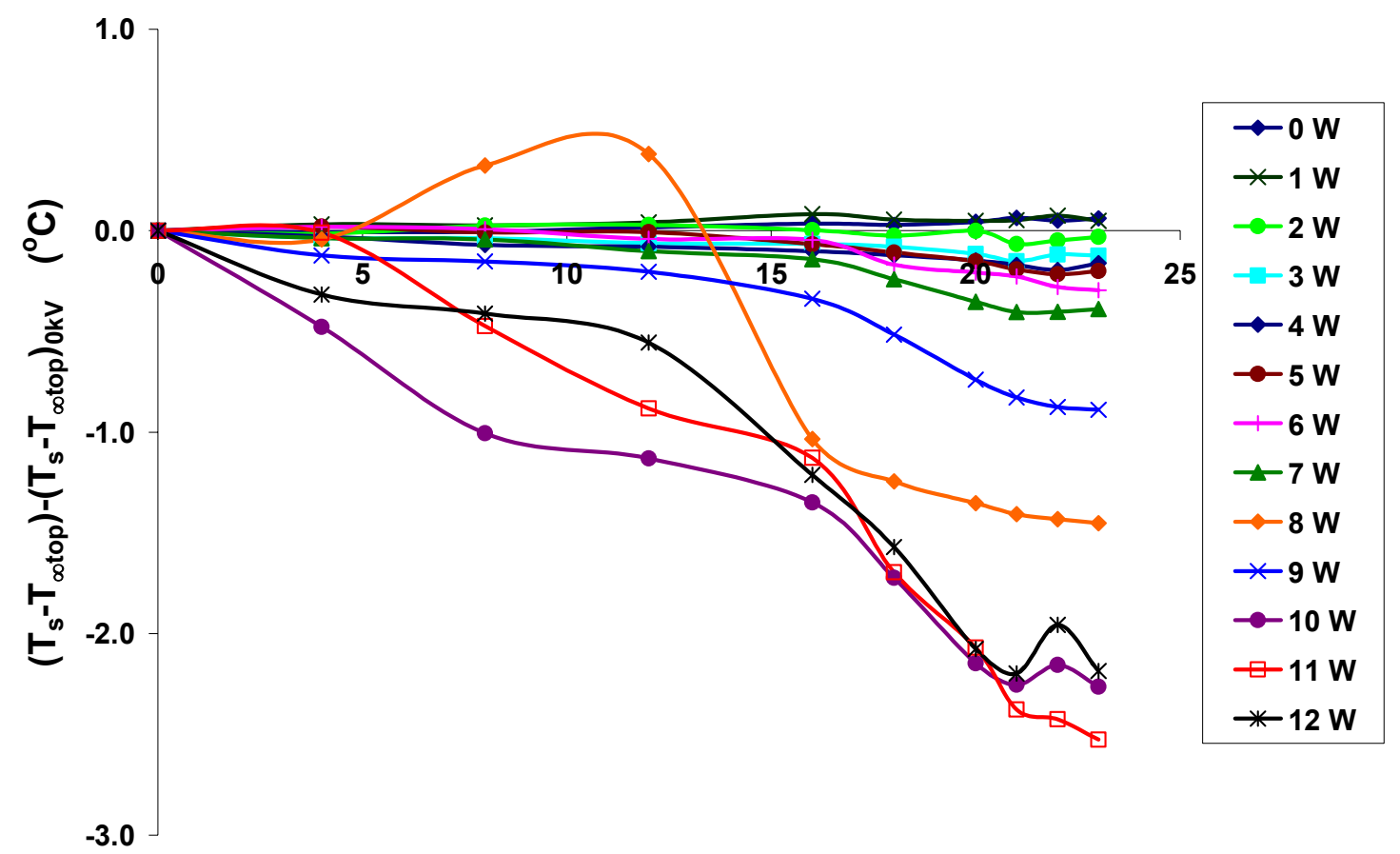

High Voltage Input (kV)

Figure 4.62: Temperature Differences between 0 and $23 \mathrm{kV}$ as a Function of Increasing Electrode Voltages for Varying Heater Powers 


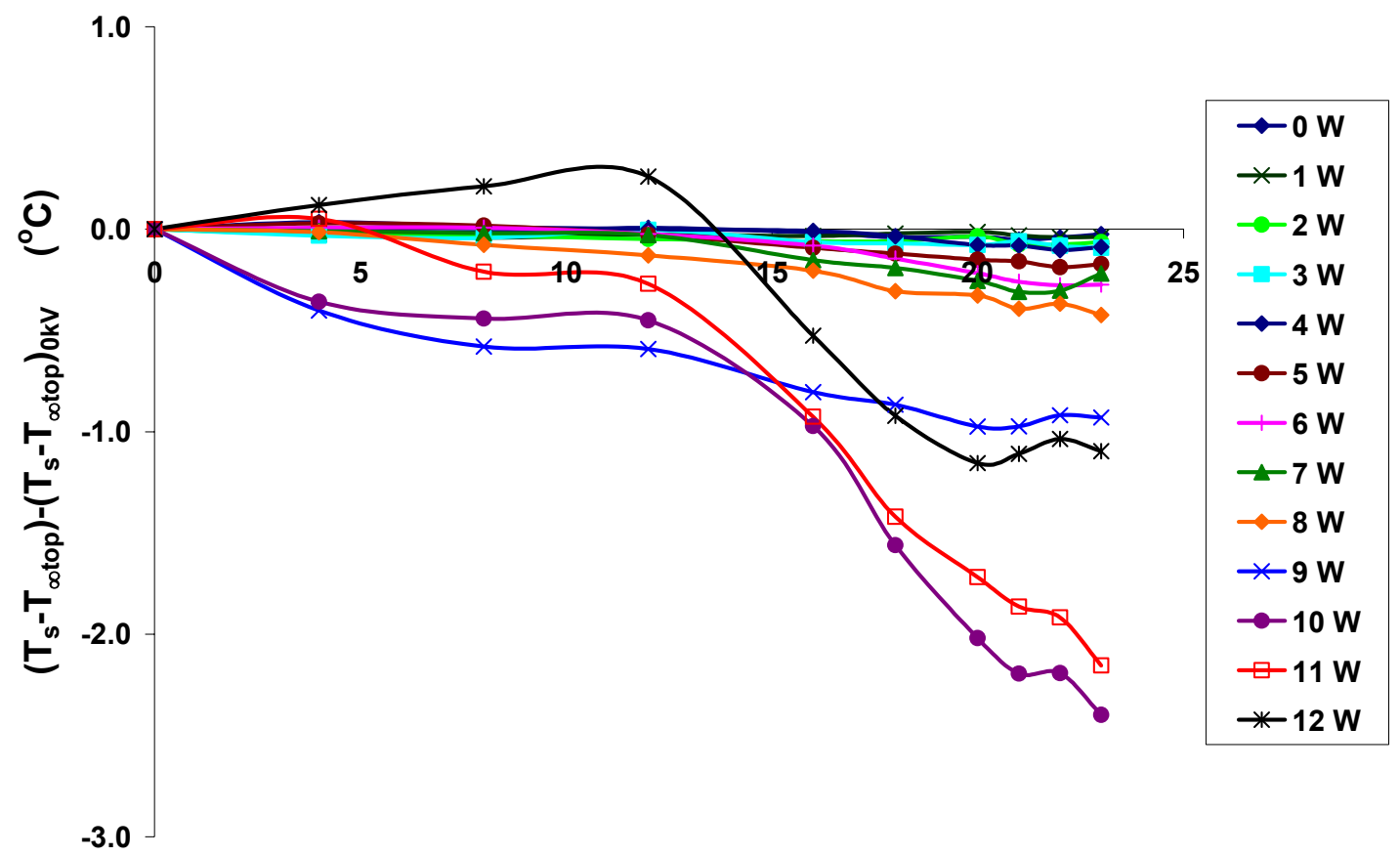

High Voltage Input (kV)

Figure 4.63: Temperature Differences between 0 and $23 \mathrm{kV}$ as a Function of Decreasing Electrode Voltages for Varying Heater Powers

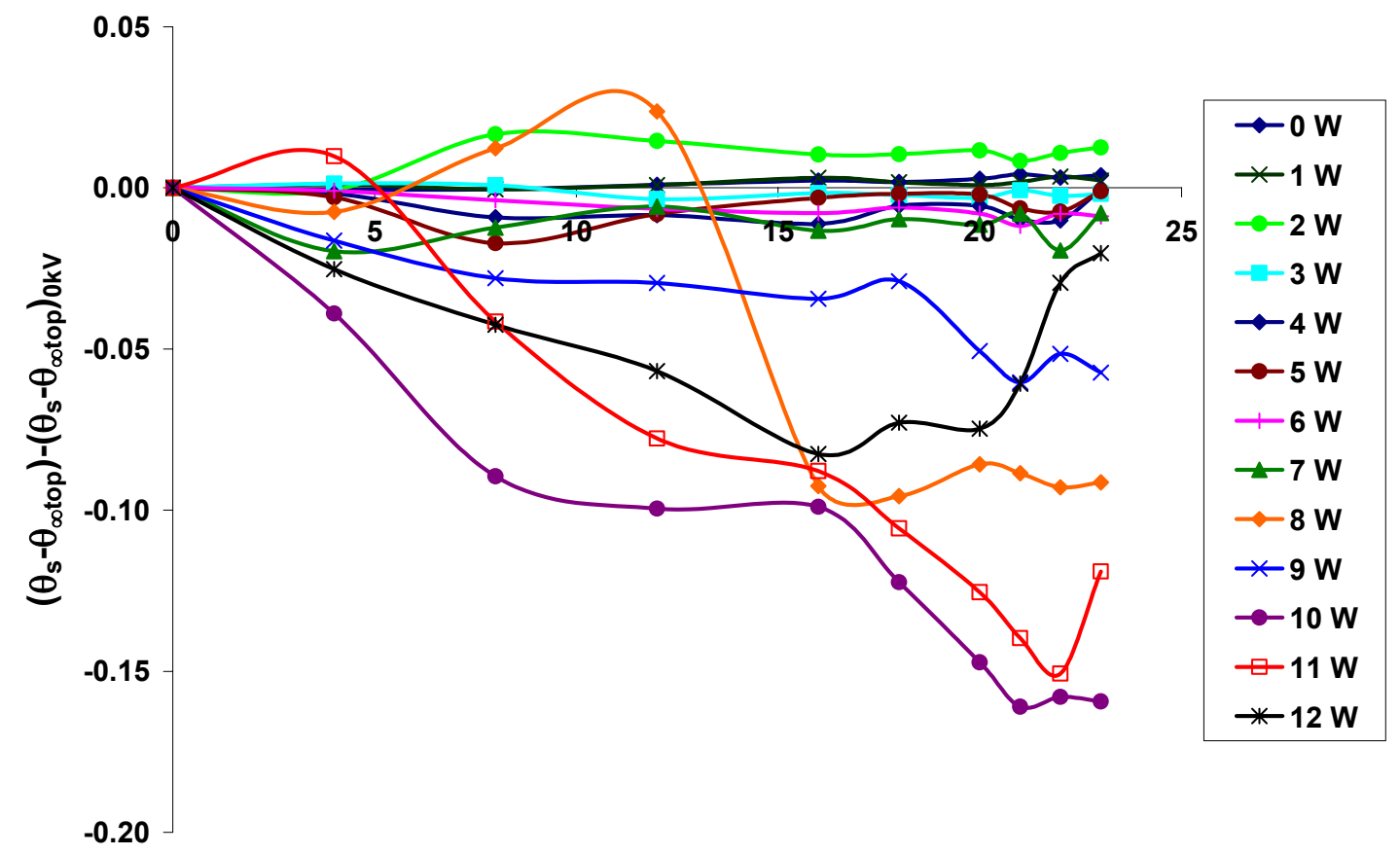

\section{High Voltage Input (kV)}

Figure 4.64: Non-dimensional Temperature Differences between 0 and $23 \mathrm{kV}$ as a Function of Increasing Electrode Voltages for Varying Heater Powers 


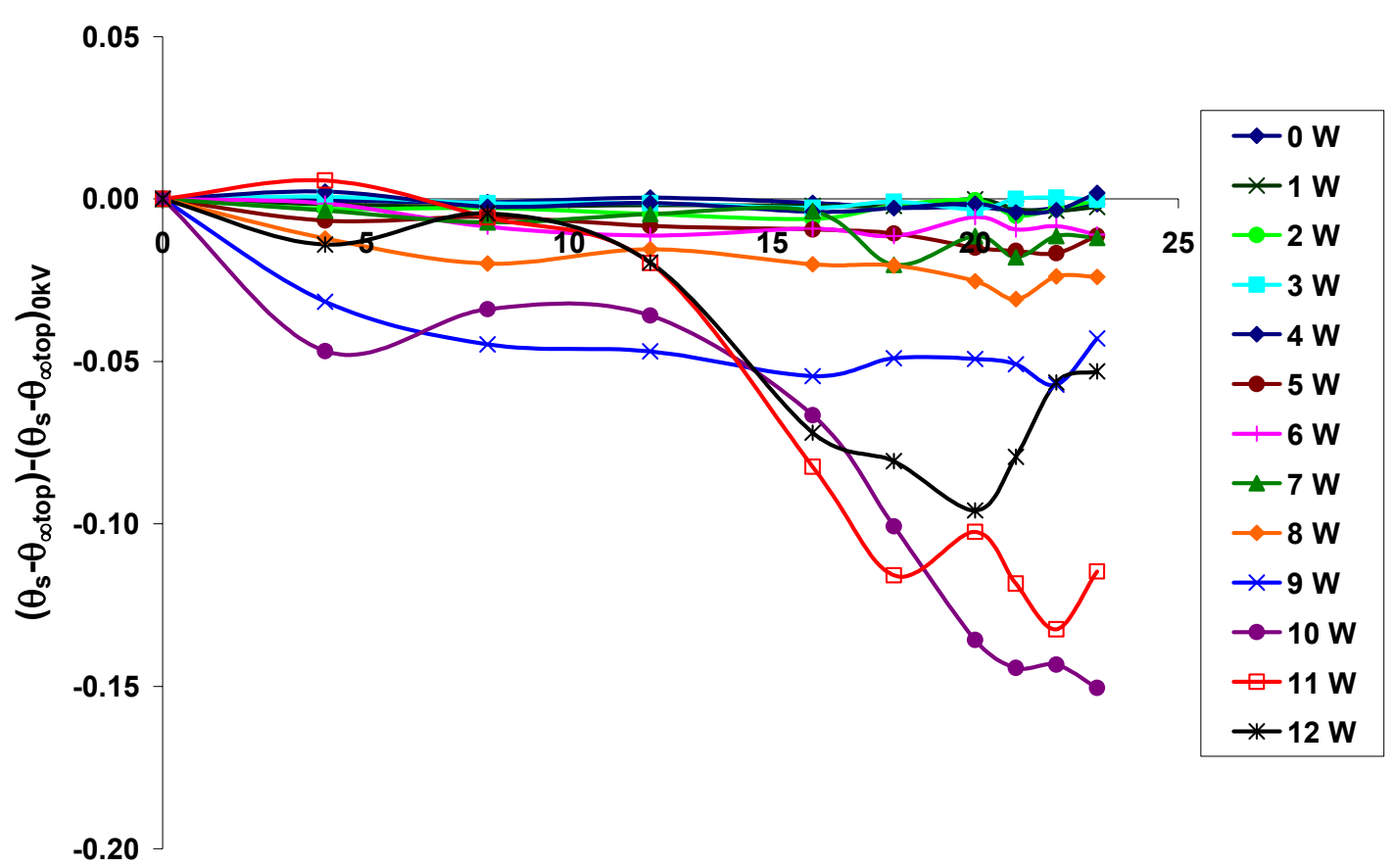

High Voltage Input (kV)

Figure 4.65: Temperature Differences between 0 and $23 \mathrm{kV}$ as a Function of Decreasing Electrode Voltages for Varying Heater Powers

The convection heat transfer coefficients increased as the electrode voltage increased. Figure 4.66 (and the enlarged view in Figure 4.67) indicate that at a heat flux of $120,700 \mathrm{~W} / \mathrm{m}^{2}$, as the electrode voltage was increased from 0 to $23 \mathrm{kV}$, the heat transfer coefficient increased by $165 \mathrm{~W} / \mathrm{m}^{2} \mathrm{~K}$. As the electrode voltage was then decreased back to $0 \mathrm{kV}$, the heat transfer coefficient decreased by $128 \mathrm{~W} / \mathrm{m}^{2} \mathrm{~K}$. The corresponding non-dimensional plots are shown in Figure 4.68 and Figure 4.69. The heat transfer coefficient at $0 \mathrm{kV},\left(\mathrm{h}_{0 \mathrm{kV}}\right)$, was subtracted from the heat transfer coefficient at each electrode voltage, (h), and plotted as a function of the electrode voltage for the varying heater powers. This plot is shown in Figure 4.70 for the increasing electrode voltages and in Figure 4.71 for decreasing voltages. The corresponding non-dimensional plots are shown in Figure 4.72 and Figure 4.73. 


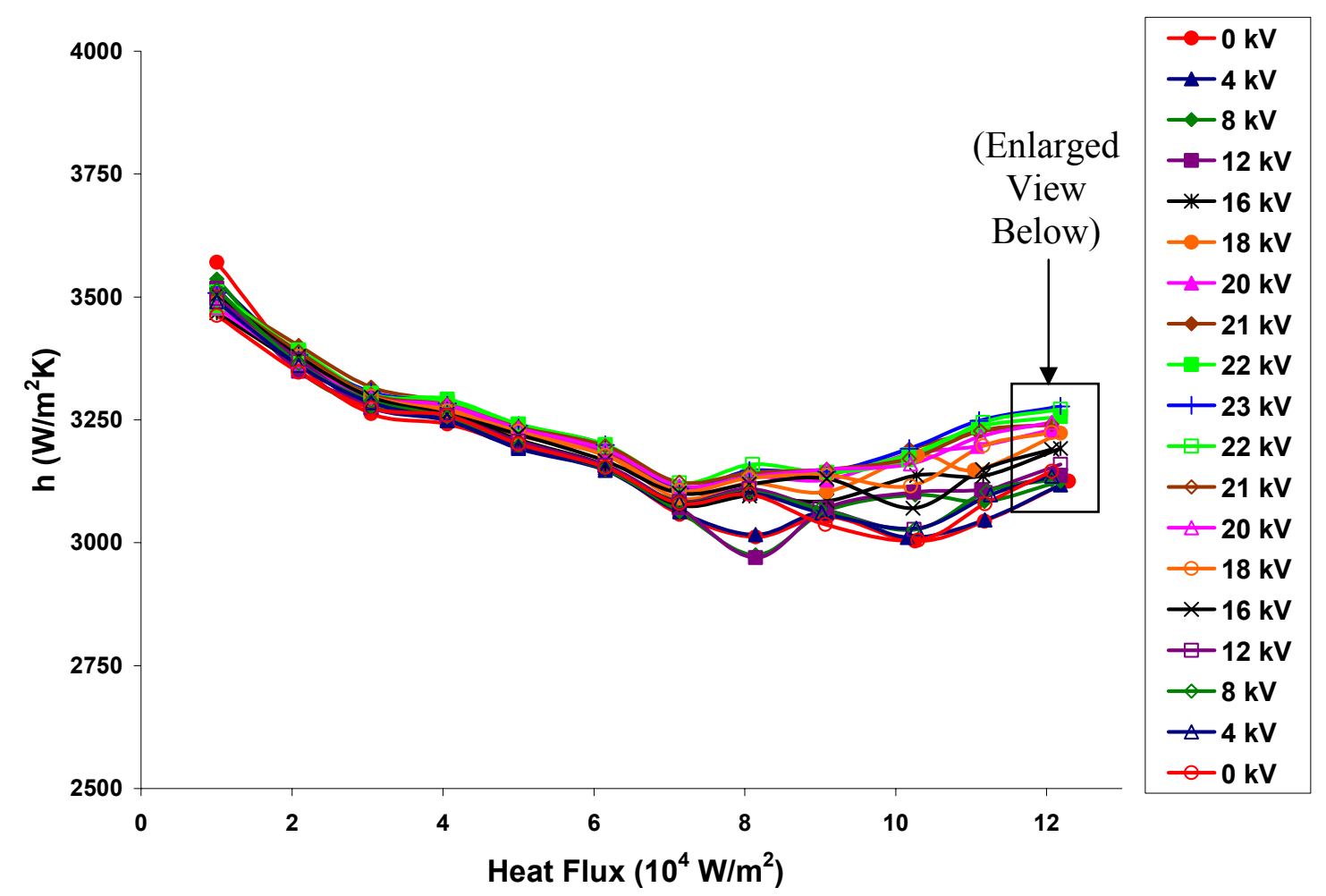

Figure 4.66: Convection Heat Transfer Coefficient in $\mathrm{W} / \mathrm{m}^{2} \mathrm{~K}$ as a Function of Heat Flux in $\mathrm{W} / \mathrm{m}^{2}$ for the Mehra Electrodes at Voltages up to $23 \mathrm{kV}$

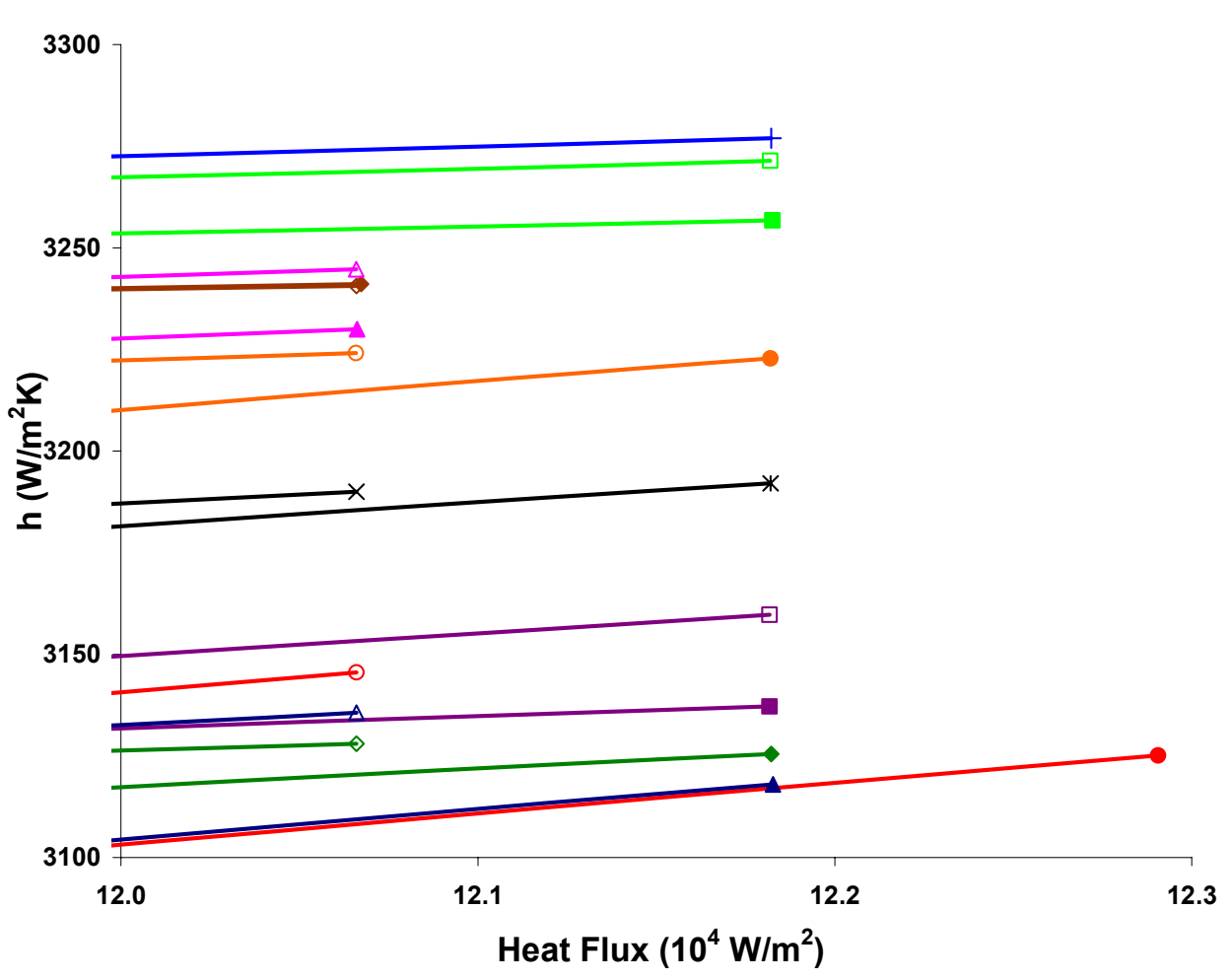

$$
\begin{aligned}
& \rightarrow 0 \mathrm{kV} \\
& \rightarrow-4 \mathrm{kV} \\
& \rightarrow-8 \mathrm{kV} \\
& \rightarrow-12 \mathrm{kV} \\
& \text { * }-16 \mathrm{kV} \\
& -18 \mathrm{kV} \\
& -20 \mathrm{kV} \\
& \rightarrow-21 \mathrm{kV} \\
& -22 \mathrm{kV} \\
& +23 \mathrm{kV} \\
& \square-22 \mathrm{kV} \\
& \neg 21 \mathrm{kV} \\
& \triangle 20 \mathrm{kV} \\
& -18 \text { kV } \\
& * 16 \mathrm{kV} \\
& \square-12 \mathrm{kV} \\
& \neg 8 \mathrm{kV} \\
& \triangle 4 \mathrm{kV} \\
& -0 \mathrm{kV}
\end{aligned}
$$

Figure 4.67: Enlarged View of the Highest Heat Flux in Figure 4.66 


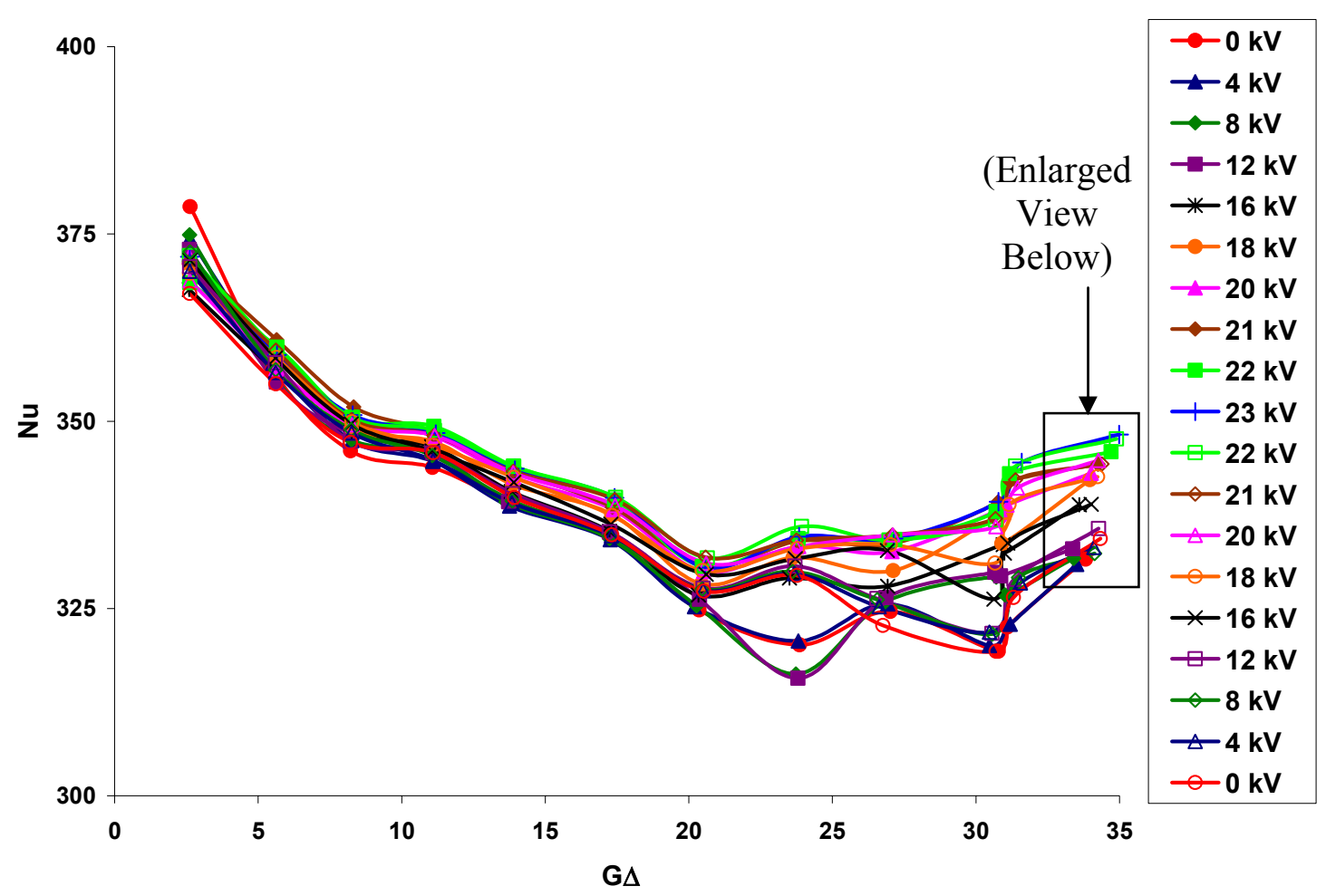

Figure 4.68: Nusselt Number as a Function of Non-dimensional Heat Flux for the Mehra Electrodes at Voltages up to $23 \mathrm{kV}$

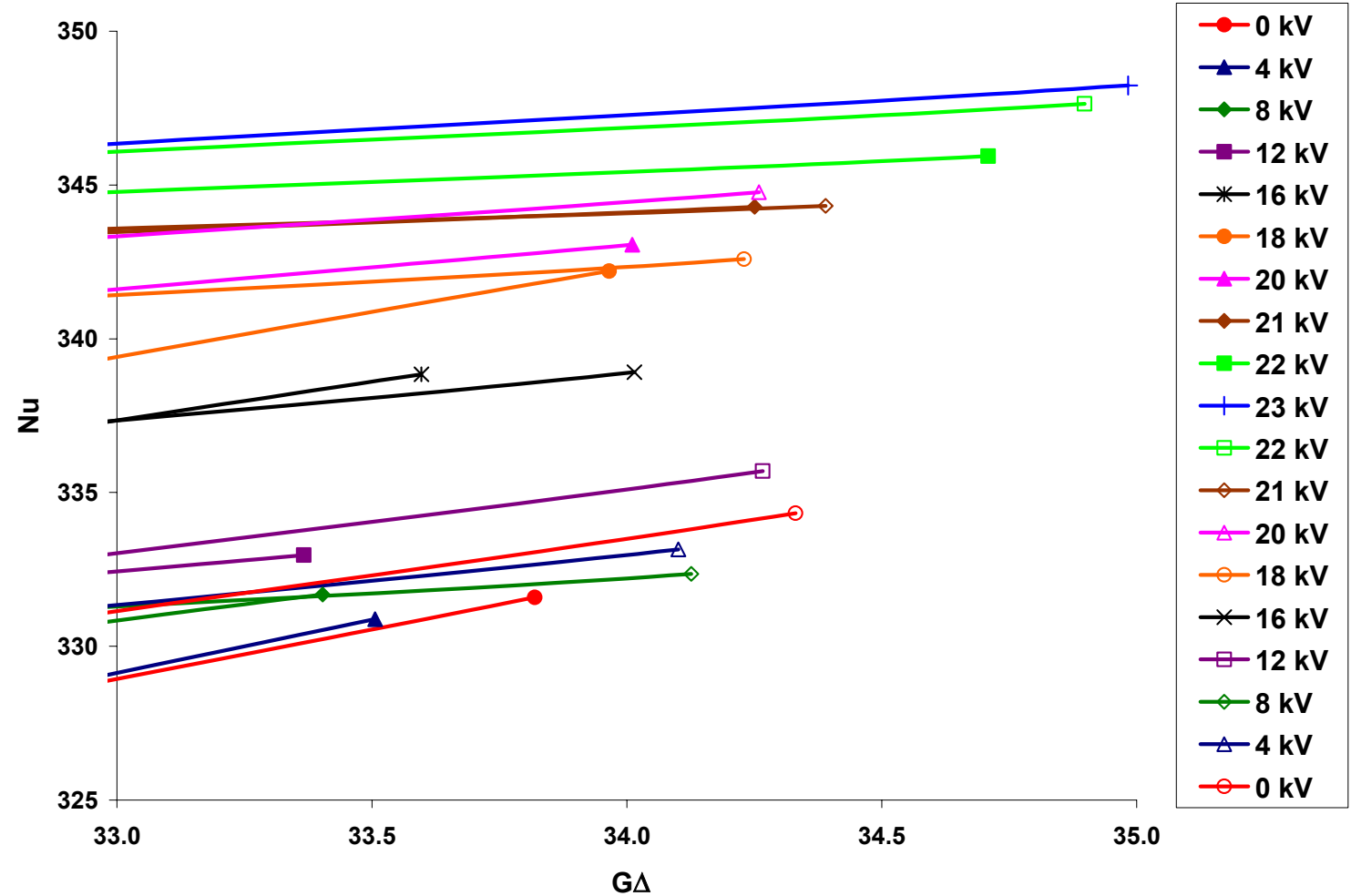

Figure 4.69: Enlarged View of the Highest G $\Delta$ in Figure 4.68 


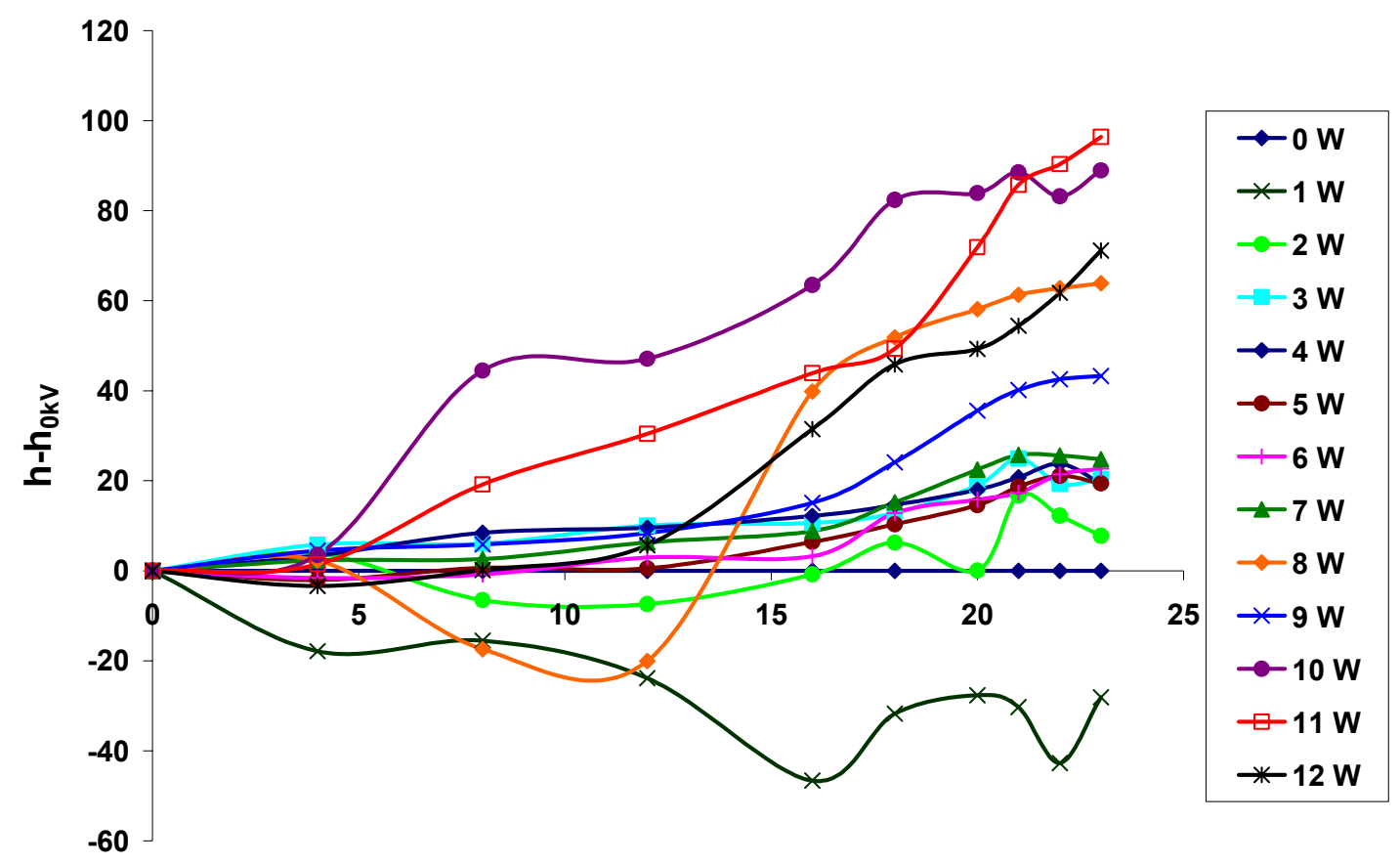

High Voltage Input (kV)

Figure 4.70: Heat Transfer Coefficient Differences between 0 and $23 \mathrm{kV}$ as a Function of Increasing Electrode Voltages for Varying Heater Powers

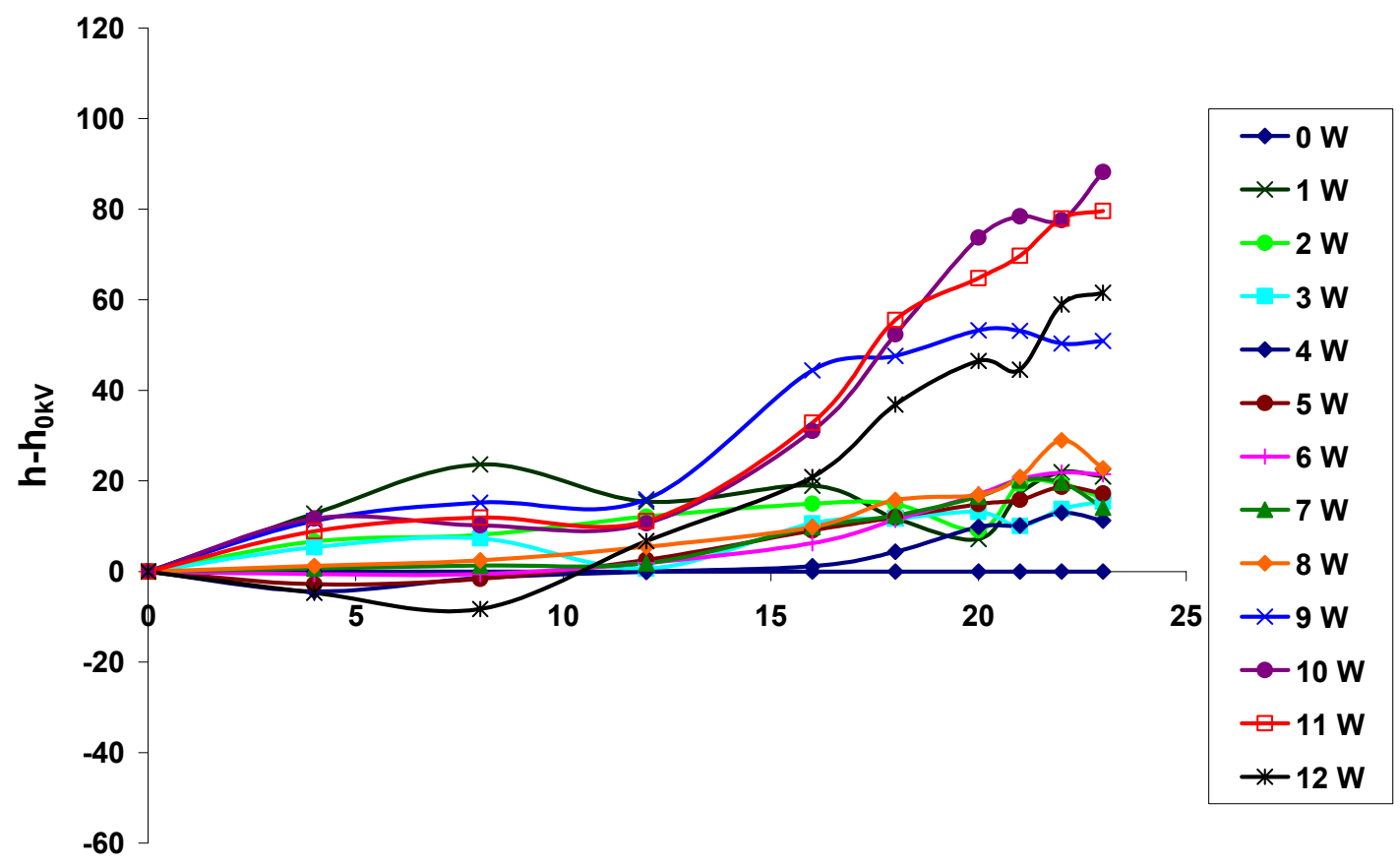

High Voltage Input (kV)

Figure 4.71: Heat Transfer Coefficient Differences between 0 and $23 \mathrm{kV}$ as a Function of Decreasing Electrode Voltages for Varying Heater Powers 


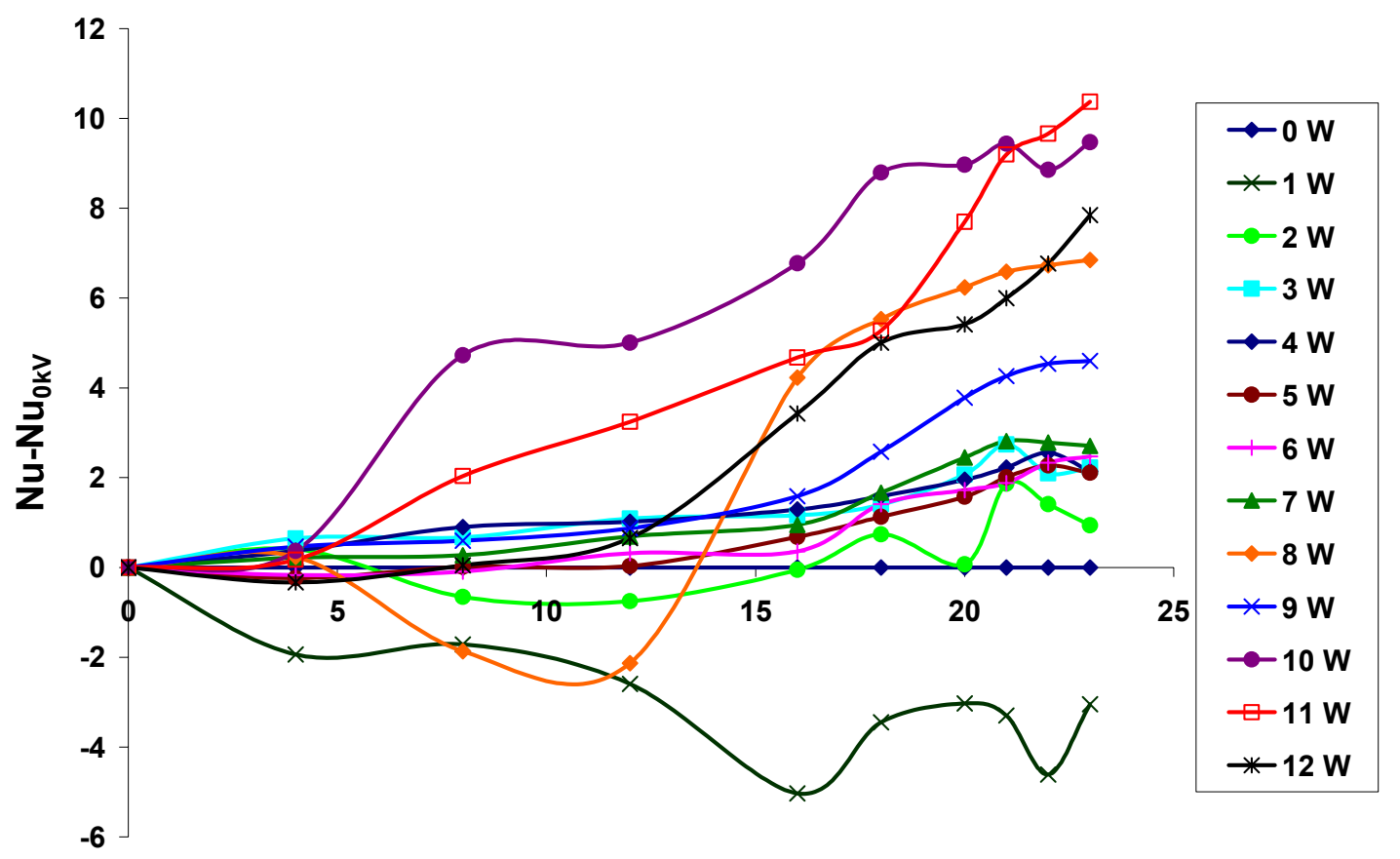

High Voltage Input (kV)

Figure 4.72: Nusselt Number Differences between 0 and $23 \mathrm{kV}$ as a Function of Increasing Electrode Voltages for Varying Heater Powers

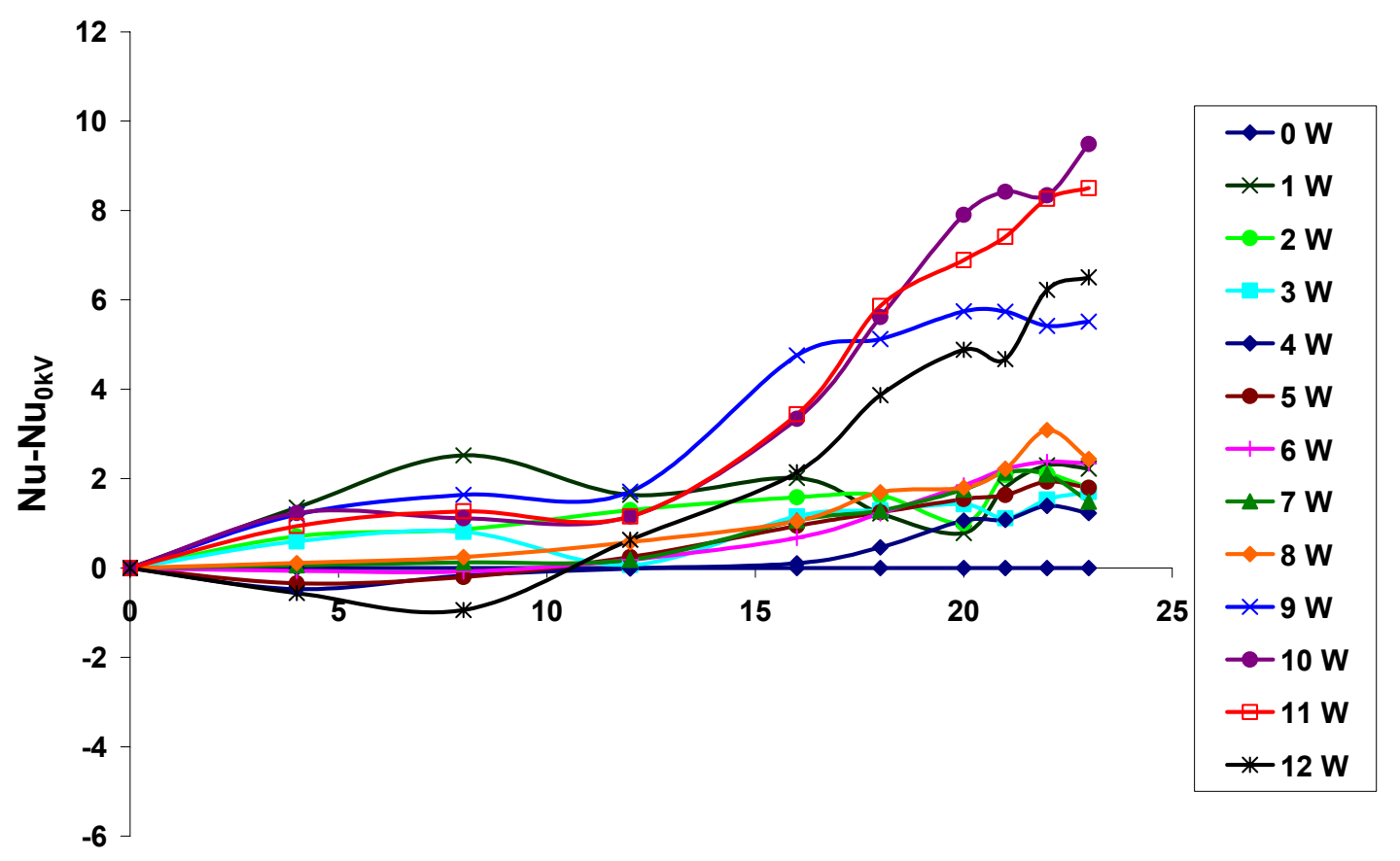

High Voltage Input (kV)

Figure 4.73: Nusselt Number Differences between 0 and $23 \mathrm{kV}$ as a Function of Decreasing Electrode Voltages for Varying Heater Powers 
These results indicate a significant effect on the pedestal temperature (of up to $1.91{ }^{\circ} \mathrm{C}$ ) and heat transfer coefficient (of up to $165 \mathrm{~W} / \mathrm{m}^{2} \mathrm{~K}$ or $5.2 \%$ ) when at higher heat flux and electrode voltage values with the use of the electric Kelvin force produced by the Mehra electrode. While this effect is present at a heat flux of $12 \mathrm{~W}$ where the pedestal surface temperatures did reach above the $34{ }^{\circ} \mathrm{C}$ boiling point of HFE-7000, it can be seen from the plots and values that the spray cooling apparatus was not yet near critical heat flux for the Mehra electrode tests. The maximum heat flux for these experiments was limited due to the temperature cutoff that activated at $13 \mathrm{~W}$. Altering the pedestal to allow cooler operating temperatures may enable higher heat fluxes to be reached in order to study the effects of the Mehra electrode configuration nearer to critical heat flux. It is believed that these effects would become larger at higher heat fluxes. 


\section{Chapter 5: Conclusions and Recommendations}

This chapter presents the conclusions drawn from the results from the current study. Recommendations for future work are also discussed.

\subsection{Conclusions}

A spray cooling apparatus consisting of a spray chamber built for the present study and a flow generating base package modified from Hunnell (2005) has been constructed. This apparatus was employed to study the effects of the spacing between the nozzle exit and heater surface and the effects of the electric Kelvin force for five different electrode configurations using two different working fluids.

The first objective of this study was to experimentally determine the sensitivity of the nozzle-to-heater distance in both confined and unconfined flows and was achieved. The results indicated that the confined flow is less dependent on the nozzle-to-heater spacing but is slightly less efficient at cooling the heated surface when compared to an unconfined flow with similar flow parameters. This disagrees with Hunnell (2005), who proposed that confined flows are more efficient due to the rebounding of spray droplets back onto the heated surface within the confined geometry, thus providing a larger volume of liquid to aid in heat transfer. However, relatively large variations in performance for repeated runs indicated the significant effect on the results due to alignment of the nozzle and heater.

The second objective was to experimentally and/or numerically show a measurable effect of the electric Kelvin force on spray cooling performance. The first series of experimental tests on the four cap electrodes showed no significant effect of the 
electric Kelvin force on spray cooling performance. This is believed to be due to the non-optimal geometries and also possibly due to the properties of the FC-72 fluid used. Numerical simulations of these electrode designs indicated that the geometries were insufficient to produce an electric Kelvin force with a large enough magnitude and proper direction to significantly effect the spray cooling parameters such as pedestal surface temperature and convection heat transfer coefficient. The electrode in each of these designs was so close to the heater surface (ground) that the maximum allowable electrode voltage input was severely limited (to only $6 \mathrm{kV}$ ) in order to prevent electrostatic breakdown. Also, the shape and placement of the electrodes produced gradient of the electric field squared lines that were not normal to the heater surface as would be optimal. For the full length electrode, FC-72 was first used which has a relatively small electric susceptibility of approximately 0.75 . The electric susceptibility is also proportional to the electric Kelvin force. HFE-7000 (which has an electric susceptibility of approximately 6.4) was also used to experimentally test the full length cap electrode. These tests showed a slight improvement in cooling efficiency, but the magnitude was below the estimated level of the experimental errors.

The Mehra electrode test used the conclusions drawn from the cap electrode tests to improve the results. The geometry of the electrode configuration was first optimized using numerical simulations by Mr. Deepak Mehra and then experimentally tested for this study using HFE-7000 at a flow rate of $0.014 \mathrm{~m}^{3} / \mathrm{hr}$ of HFE-7000. The numerical tests indicated that the positioning and magnitudes of the electric Kelvin force per unit mass may produce a significant effect on the spray cooling parameters of the experimental apparatus. The calculated gradient of the electric field squared lines were 
normal to the pedestal surface. The calculated magnitudes of the accelerations everywhere adjacent to the pedestal surface were greater than $4 \mathrm{~N} / \mathrm{kg}$ in an upward direction. The Mehra electrode experimental results showed that at a heat flux of $120,700 \mathrm{~W} / \mathrm{m}^{2}$ and electrode voltage input of $23 \mathrm{kV}$, the temperature difference at the top of the pedestal decreased by a maximum of $1.91{ }^{\circ} \mathrm{C}$ and the heat transfer coefficient increased by a maximum of $165 \mathrm{~W} / \mathrm{m}^{2} \mathrm{~K}$ (or $5.2 \%$ ).

\subsection{Recommendations}

Recommendations from Hunnell (2005) were addressed in this project including the addition of a data acquisition system and new rotameters to the experimental apparatus. Several other recommendations were also attempted. First, a reheater was built and installed on the experimental base just before the spray chamber in order to have the capability of varying the subcooling. However, problems were encountered with the installation of the PID controller for the system and time constraints prevented its use. It is therefore recommended that the reheater be reinstalled with a new PID controller. Decreasing the subcooling of the working fluid allows boiling to occur at a lower flow rate and/or heat flux. Second, in the data reduction calculations, the fractional heat loss down the pedestal was assumed to be 0.015. It was found in Baysinger (2004) that the heat loss varied by up to $1 \%$ from this value and was therefore included in the error analysis for the current study. However, the value found in Baysinger (2004) was specifically for a glass pedestal, while a PTFE pedestal was used in the electrode tests in this study. The thermal conductivity is approximately $1.04 \mathrm{~W} / \mathrm{mK}$ for glass and 0.25 $\mathrm{W} / \mathrm{mK}$ for PTFE. While the difference may not be significant, it is recommended that a 
more accurate value (of approximately 4 times less) of the heat loss down a PTFE pedestal be used in calculations.

Another recommendation is to add a more accurate flow meter to the experimental base. This was also attempted by purchasing and installing a Sponsler turbine meter to be read by the data acquisition system, but problems encountered with a large amount of noise and a lack of time to correct the problem prevented its use in the current work.

The properties of the working fluid can be an important parameter in spray cooling. The amount of dissolved air in the fluid may greatly affect the performance. The dissolved air content of the FC-72 in the WVU experimental apparatus was tested by the AFRL team and found to be approximately $22 \%$. The system is not pressurized and it is assumed the dissolved air content remains relatively constant. However, it is recommended that the dissolved air content be monitored more closely. This can be done in several ways. First, samples could be periodically taken as was done by the AFRL team and analyzed for changes. A second method would be to install a dissolved oxygen meter probe. Initial tests were performed using an air calibrated YSI Inc. Model 50B meter and 5750 BOD non-stirring probe. The instrument works by allowing oxygen (and certain other gases) to flow across a membrane to reach a cathode sensor. This sensor outputs current in proportion to the amount of oxygen present. Initial tests indicated that the probe could provide a dissolved air reading in FC-72 measured in percent of saturation or $\mathrm{mg} / \mathrm{L}$ if a proper amount of movement (flow or stirring) is maintained in the fluid. It is recommended that a newer model field meter be purchased (the 50B meter has been discontinued), tested, and installed on the experimental base in order to monitor the 
dissolved air in the working fluid. Consideration should also be made to close the system and create the means to vary the amount of dissolved air in the fluid. Tests should also be performed with the dissolved oxygen meter using the HFE-7000 working fluid.

One final recommendation is that the Mehra electrode configuration be tested at higher heat fluxes (up to critical heat flux if possible). The pedestal used in the Mehra electrode tests could only withstand a heater power input of $12 \mathrm{~W}$ before the temperature cutoff switch triggered at $150^{\circ} \mathrm{C}$. This temperature cutoff was chosen because the epoxy used to bond the heater and alumina to the PTFE rod had a maximum operating temperature of $177{ }^{\circ} \mathrm{C}$. Modifying the pedestal by choosing an epoxy that is less thermally insulating and/or has a higher operating temperature should allow higher heat fluxes to be reached. Also reducing the thickness of the alumina layer above the heater would allow the heat to more easily conduct to the pedestal surface to be removed by the impinging spray. It is predicted that the cooling effect that occurred at $12 \mathrm{~W}$ would be enhanced at higher heat fluxes. 


\section{References:}

Allen, P. H. G. and Karayiannis, T. G., "Electrohydrodynamic enhancement of heat transfer and fluid flow," Recovery Systems \& CHP, Vol. 15, No. 5, pp. 389-423, 1995.

Baysinger, K. M., Yerkes, K. L., and Michalak, T. E., "Design of a microgravity spray cooling experiment," $42^{\text {nd }}$ AIAA Aerospace Sciences Conference and Exhibit, Reno, NV, January, 2004.

Baysinger, K., "Experimental testing and numerical modeling of spray cooling under terrestrial gravity conditions," M. S. Thesis, Wright State University, Dayton, OH, 2004.

Bernardin, J. D., Stebbins, C. J., and Mudawar, I., "Mapping of impact and heat transfer regimes of water drops impinging on a polished surface," Int. J. Heat Mass Transfer, Vol. 40, pp.247-267, 1997.

Chow, L. C. and Sehmbey, M. S., Pais, M. R., "High heat flux spray cooling," Annual Review of Heat Transfer, Vol. 8, pp. 291-318, Hemisphere Pub. Corp., New York, 1997.

Deb, S. and Yao, S. C., "Analysis on film boiling heat transfer of impacting sprays," Int. J. Heat Mass Transfer, Vol. 32, No. 11, pp. 2099-2112, 1989.

ESI CFD Inc., “CFE-ACE+ 2005 User Manual,” 2005.

Estes, K.A. and Mudawar, I., "Correlation of sauter mean diameter and critical heat flux for spray cooling of small surfaces," Int. J. Heat Mass Transfer, Vol. 38, No. 6, pp. 29852996, 1995.

Gray, D. D. and Kuhlman, J. M., "Electromagnetic control of high-heat flux spray impingement boiling under microgravity conditions," Unpublished Manuscript/Personal Communication, 2006.

Grover, R. O. and Dennis, N. A., "A spray wall impingement model based upon conservation principles," The Fifth International Symposium on Diagnostics and Modeling of Combustion in Internal Combustion Engines, Nagoya, Japan, 2001.

Gu, J.J., Kawaji, M., and Futamata, R., "Effects of gravity on the performance of pulsating heat pipes," AIAA Journal of Thermophysics and Heat Transfer, Vol. 18, No. 3, pp. 370-378, July-September, 2004.

Haramura, Y. and Katto, Y., "A new hydrodynamic model of critical heat flux applicable widely to both pool and forced convection boiling on submerged bodies in saturated liquids," Int. J. Heat Mass Transfer, Vol. 26, No. 2, pp. 389-399, 1983. 
Horacek, B., Kiger, K., Kim, J., "Single nozzle spray cooling heat transfer mechanisms," International Journal of Heat and Mass Transfer, Vol. 48, No. 8, pp. 1425-1438, 2005.

Hughes, W. F. and Young, F. J., "The electromagnetodynamics of fluids," New York, John Wiley \& Sons Inc. 1966.

Hunnell, C. A., "Design, construction, and initial testing of experimental test package for convective spray cooling in terrestrial gravity conditions," M. S. Thesis, West Virginia University, Morgantown, WV, 2005.

Jones, T. B., "Electrohydrodynamically enhanced heat transfer in liquids-a review," Advances in Heat Transfer, Vol. 14, pp. 107-148, 1978.

Kreitzer, P., Personal Communication, 2006.

Kuhlman, J. M., Gray, D. D., Glaspell, S. L., and Kreitzer, P. J., "Positioning of simulated vapor bubbles in microgravity by the Kelvin Force," $38^{\text {th }}$ AIAA Thermophysics Conference, Toronto, Canada, June, 2005.

Lasance, C. and Simons, R. E., "Advances in high-performance cooling for electronics," Electronics Cooling, Vol. 11, No. 4, November, 2005.

Mahefkey, T., Yerkes, K., Donovan, B., and Ramalingam, M. L., "Thermal management challenges for future military aircraft power systems," SAE Technical Paper 2004-013204, Reno, Nevada, November 2004.

Marco, D. P. and Grassi, W., "Motivation and results of a long-term research on pool boiling heat transfer in low gravity," International Journal of Thermal Sciences, Vol. 41, pp. 567-585, 2002.

Mehra, D., Personal Communication, April, 2006.

Mesler, R. and Mailen, G., "Nucleate boiling in thin film liquid films," AIChE Journal, Vol. 23, No. 6, pp. 954-957, 1977.

Motil, B. J. and Singh, B. S., "NASA's microgravity fluid physics strategic research roadmap," 42 $2^{\text {nd }}$ AIAA Aerospace Sciences Meeting and Exhibit, Reno, Nevada, 2004.

Mudawar, I., "Assessment of high-heat flux thermal management schemes," 2000 Intersociety Conference on Thermal Phenomena, Las Vegas, Nevada, 2000.

Parang, M., Tipton, J. B., and Garth, J. D., "Two-phase flow heat transfer under microgravity condition," AIAA $41^{\text {st }}$ Aerospace Sciences Meeting and Exhibit, Reno, Nevada, January, 2003. 
Rahman, M. M., Faghri, A., Hankey, W. L., "Computation of the free surface flow of a thin liquid film at zero and normal gravity," Numerical Heat Transfer, Part A, Vol. 17, No. 1, pp. 53-71, 1990.

Sehmbey, M. S., Chow, L. C., Hahn, O. J., and Pais, M. R., "Effect of spray characteristics on spray cooling with liquid nitrogen," Journal of Thermophysics and Heat Transfer, Vol. 9, No. 4, October-December, 1995.

Selvam, R. P., Bhaskara, S., Balda, J. C., Barlow, F., and Elshabini, A., "Computer modeling of liquid droplet impact on heat transfer during spray cooling," 2005 ASME Summer Heat Transfer Conference, San Francisco, CA, July 2005.

Shedd, T. A. and Pautsch, A. G., "Spray impingement cooling with single- and multiplenozzle arrays part II: visualization and empirical models," International Journal of Heat and Mass Transfer, Vol. 48, No. 15, pp. 3176-3184, July 2005.

Silk, E. A., Kim, J., Kiger, K., "Enhanced surface spray cooling with embedded and compound extended surface structures," ITherm Tenth Intersociety Conference on Thermal and Thermomechanical Phenomena in Electronic Systems, San Diego, 2006.

Singh, B. S., "Multiphase flow and phase change in microgravity: fundamental research and strategic research for exploration of space," Proceedings of ASME FEDSM'03 $4^{\text {th }}$ ASME_JSME Joint Fluids Engineering Conference, Hawaii, 2003.

Tilton, D. E., "Spray cooling," Ph. D. Dissertation, University of Kentucky, Lexington, KY, 1989.

Yagoobi, J. and Bryan, J., "Enhancement of heat transfer and mass transport in singlephase flows with electrohydrodynamics," Advances In Heat Transfer, Vol. 33, pp. 95$186,1999$.

Yang, J., "Spray Cooling with an air atomizing nozzle," Ph. D. Dissertation, University of Kentucky, Lexington, KY, 1993.

Yerkes, K., Personal Communication, March, 2006. 


\section{Appendix A: Thick Film Resistor (TFR) Composition and Layer Thickness Study using Electron Microscopy}

Manufacturer information regarding the layer thicknesses of the thick film resistors (TFR) varied. These thicknesses need to be quantified in order to determine the surface temperature using a heat conduction analysis (shown in Appendix C). In order to determine the composition and layer thickness for the two types of thick film resistors (TFR Type 1 and TFR Type 2), a scanning electron microscope (SEM) was employed, and the information gathered was then compared to the manufacturer information that was available. TFR Type 1 consists of three layers: an aluminum substrate on the bottom, a palladium silver conductive heater layer in the middle, and a glass layer on top. TFR Type 2 has only two layers: an aluminum substrate on the bottom and a palladium silver conductive heater layer on top. Initial photographs at 500 and 1,000 times magnification and initial chemical analysis of specimens that were simply broken and then viewed with the SEM did not yield conclusive information as to the chemical composition or thickness of each layer on either type of TFR heater. Therefore, each piece of TFR was mounted in Buehler Sampl-Kwick fast cure acrylic epoxy and polished in alternating directions using 120, 240, 320, 400, and 600 grit sandpaper. These samples (shown Figure A1) were then re-examined under the SEM microscope.

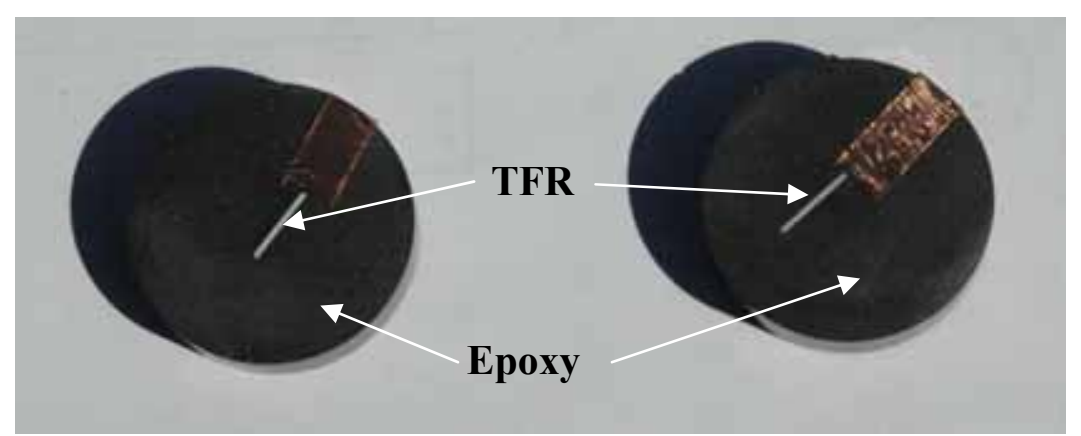

Figure A1: Polished Samples of TFR Type 1 (left) and TFR Type 2 (right) Heaters 


\section{TFR Type 1:}

Based on manufacturer information, the first type of TFR consists of three layers. The bottom substrate layer is made of alumina and is specified as 25 mils (634 microns) thick. The middle layer is a conductive resistor containing palladium silver specified as 14-16 microns thick. The top layer is glass specified as 8-18 microns thick.

The results from the scanning electron microscope at a magnification of 140 (shown in Figure A2) show two distinct layers of glass and alumina along with the surrounding epoxy. The thin conductive layer, i.e. the actual resistive material, is not visible at this magnification. The bright white areas in the alumina in Figure A2 are from the non-conductive alumina becoming charged by the SEM microscope while the black area in the alumina is not charging because it is next to the conductive layer. X-ray spectra were taken in the spots indicated in Figure A2 to confirm the composition of the layers, and these images are shown in Figure A3. The spikes on these figures correspond to a specific element energy level and are labeled on each figure. The small spikes at energy levels 11 and 13 match that of nickel which is present in the epoxy. The chemical analysis results are shown in Figure A4. From these tests at 140 times magnification, the glass layer measures approximately $40 \pm 5$ microns thick and the alumina layer measures approximately $650 \pm 10$ microns thick. 


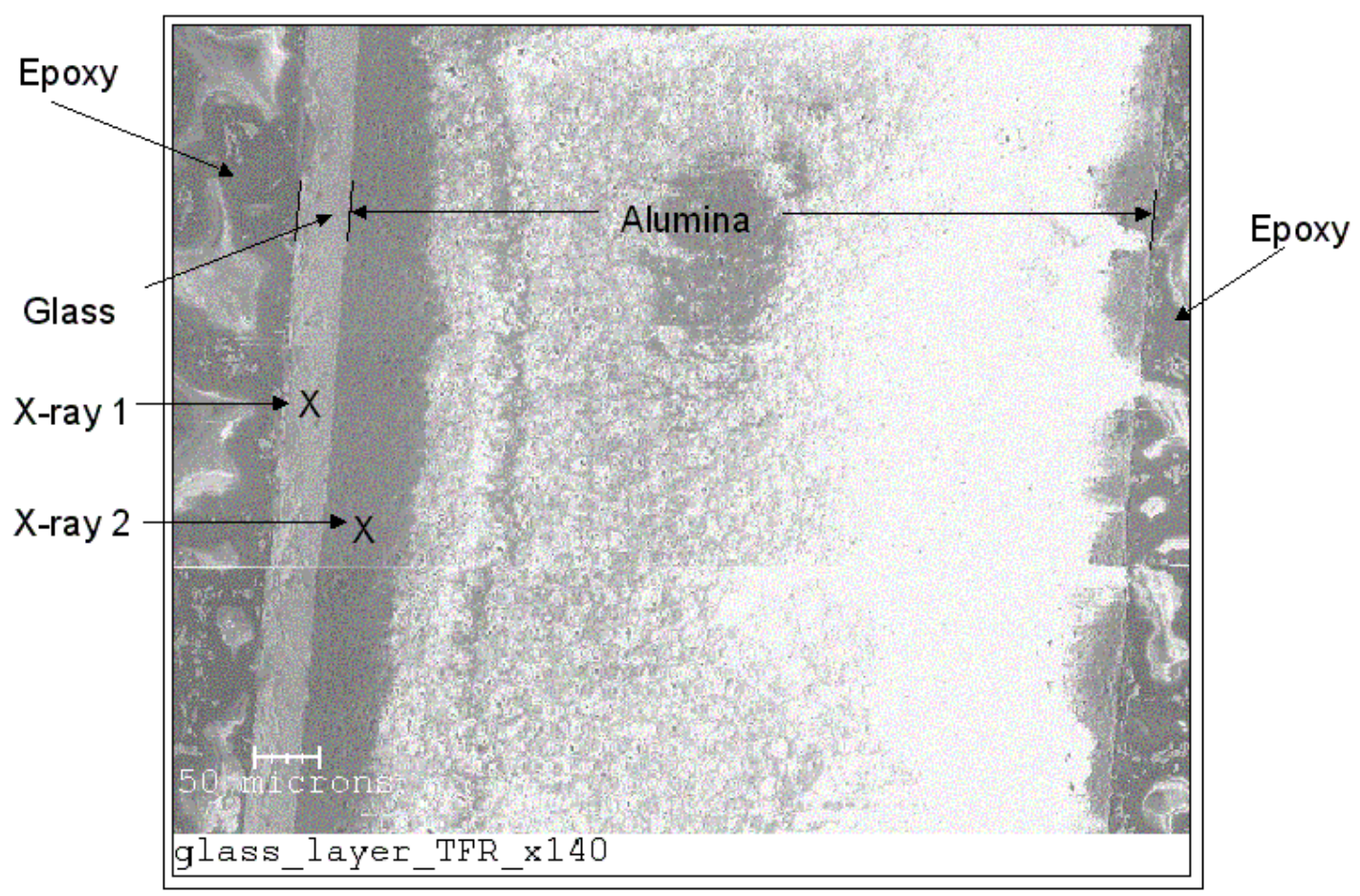

Figure A2: TFR Type 1 at 140x Magnification Showing the Alumina and Glass Layers with Surrounding Epoxy

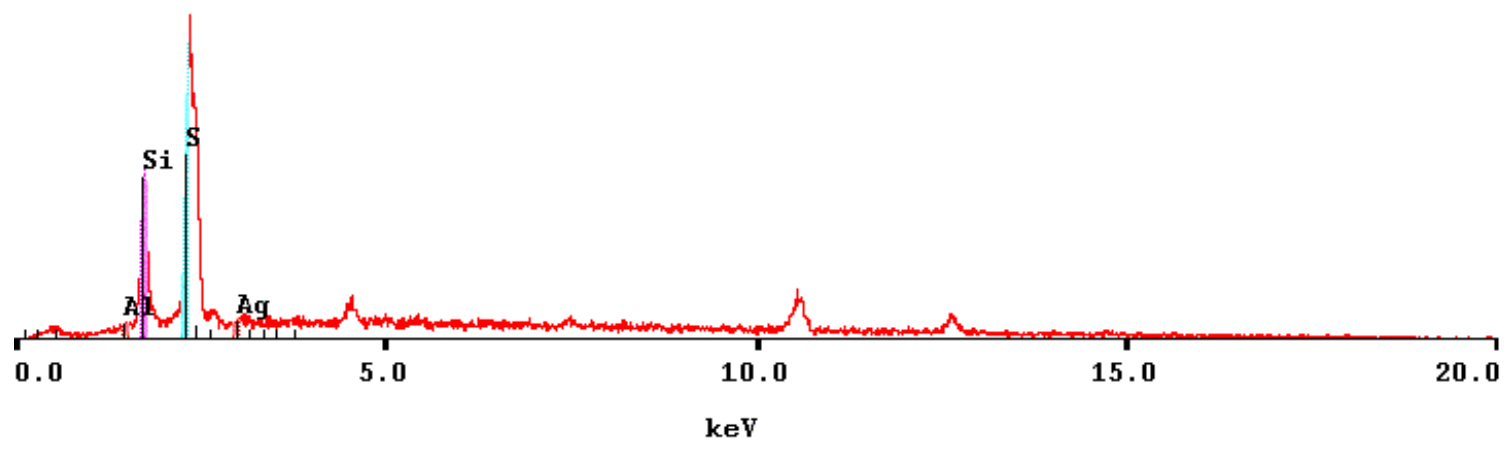

a) X-Ray 1 Showing Point Contains Mostly Silicon and Sulfur (with some nickel)

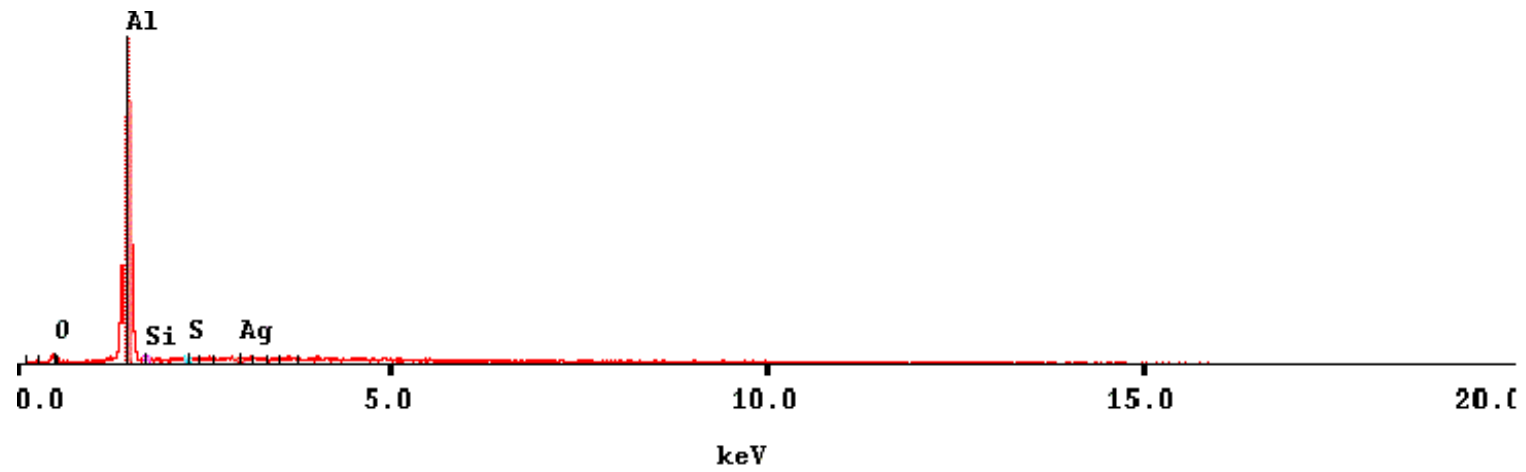

b) X-Ray 2 Showing Point Contains Mostly Aluminum

Figure A3: TFR Type 1 X-Ray Analysis at 140x Magnification 


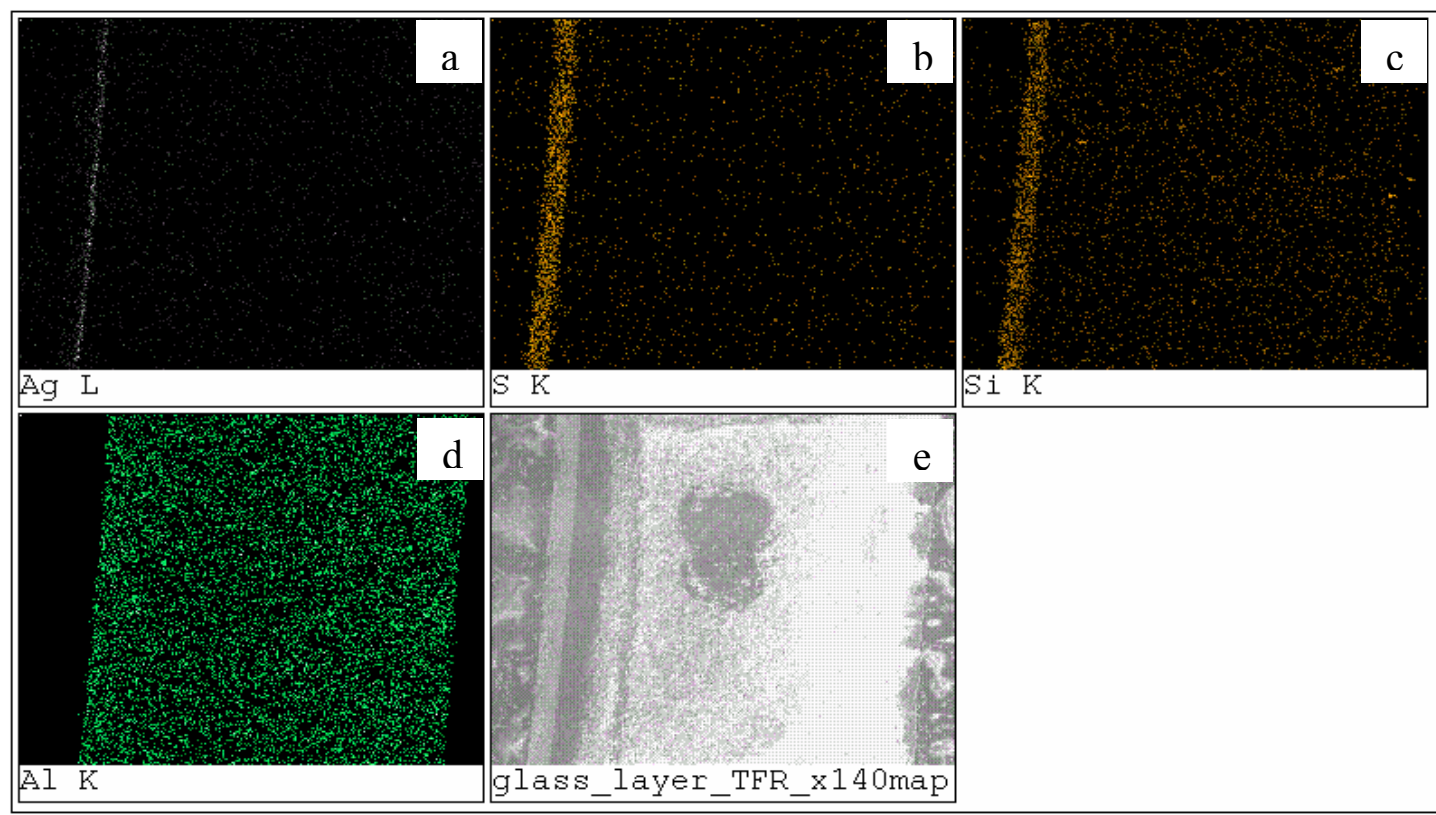

Figure A4: TFR Type 1 Chemical Analysis at 140x Magnification Showing a) Thin layer of Silver b) Sulfur Layer c) Silicon Layer d) Large Layer of Aluminum e) Composite Image

Magnifying the area between the glass layer and aluminum layer further to 1500 times magnification shows the third conductive heater layer as is shown in Figure A5. Xray results of the points shown in this figure are shown in Figure A6 and the chemical analysis is shown in Figure A7. At this high magnification, the edges of the layers begin to become rough and uneven. Also, during polishing, the materials become blended together creating less clearly defined edges and multiple elements appear at points close to the interface area. However, it is clear from Figure A7 that the heater layer is primarily silver with a layer of glass to the left and a layer of alumina to the right. At this magnification of 1500 times, the conductive layer measures approximately only 7 to $8 \pm 3$ microns thick. 


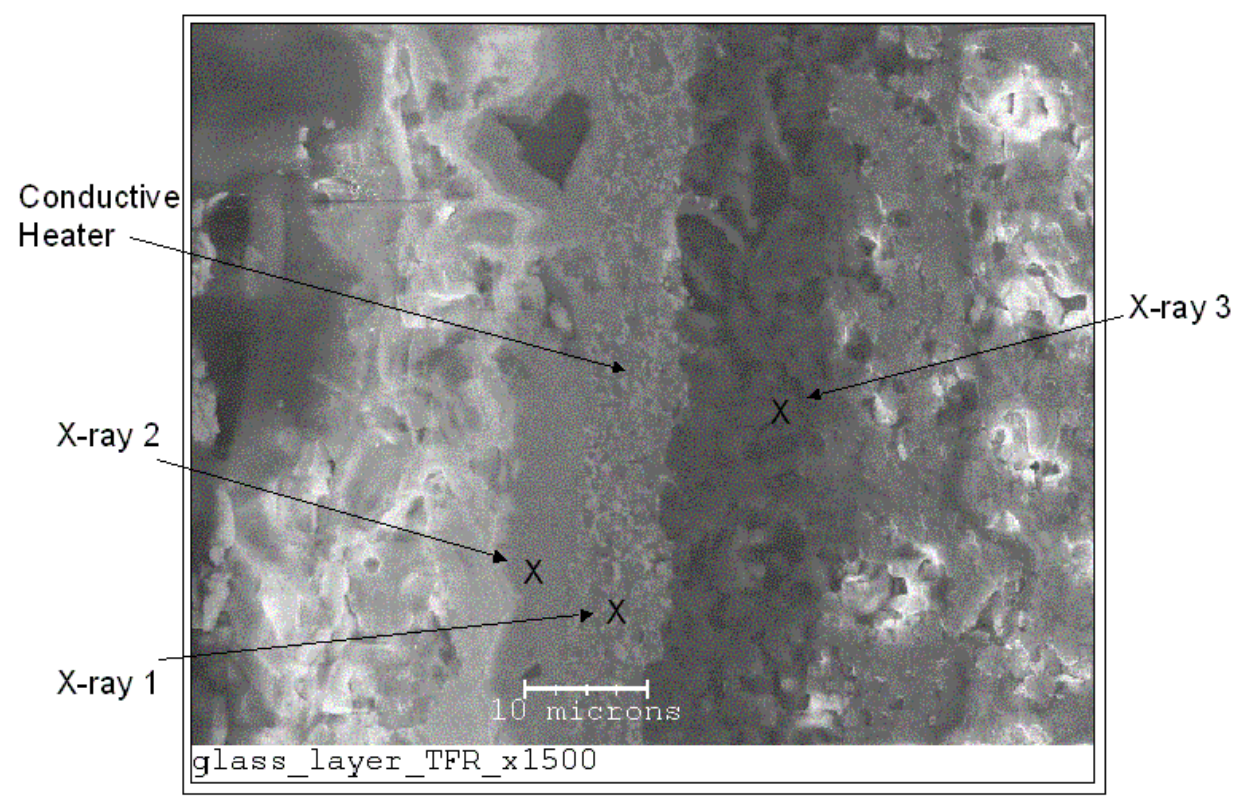

Figure A5: TFR Type 1 at 1500x Magnification Showing the Conductive Heater Layer

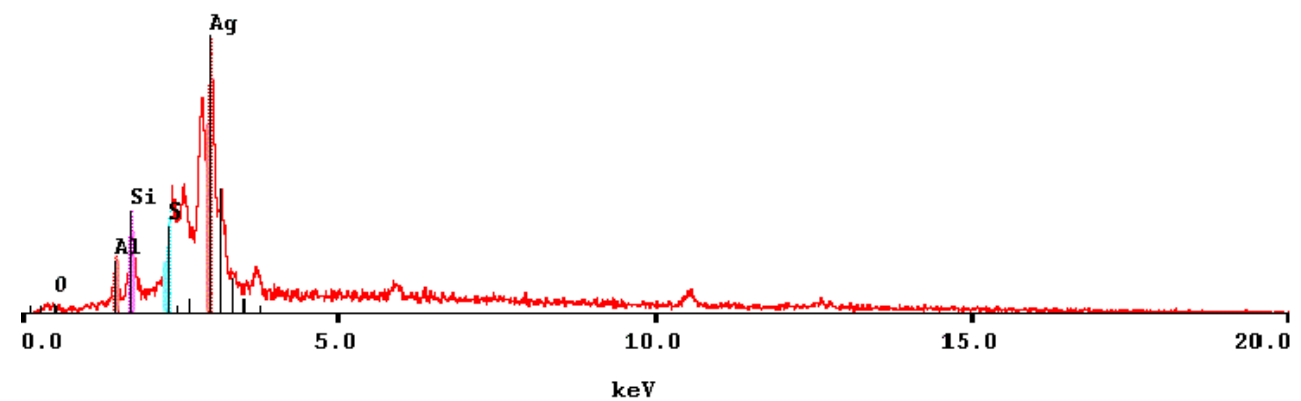

a) X-Ray 1 Showing Point Contains Mostly Silver with Some Aluminum, Silicon, and Sulfur

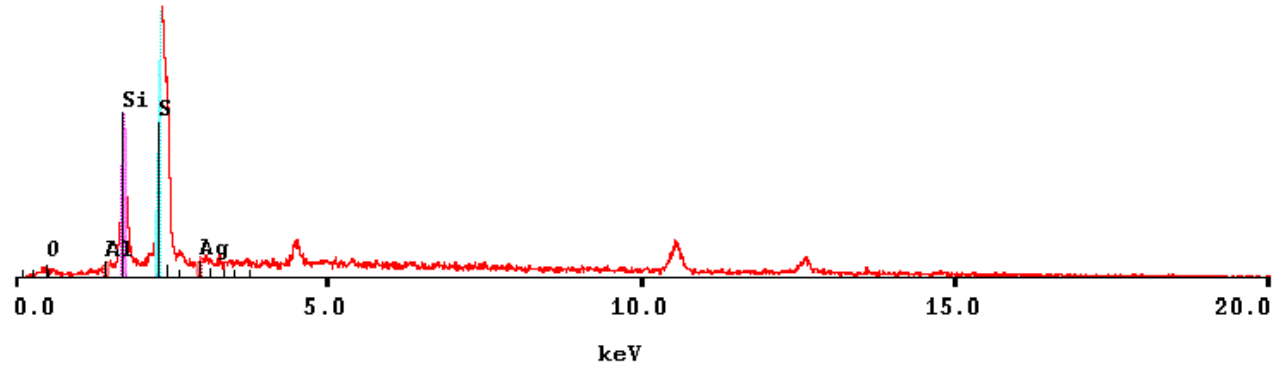

b) X-Ray 2 Showing Point Contains Mostly Silicon and Sulfur

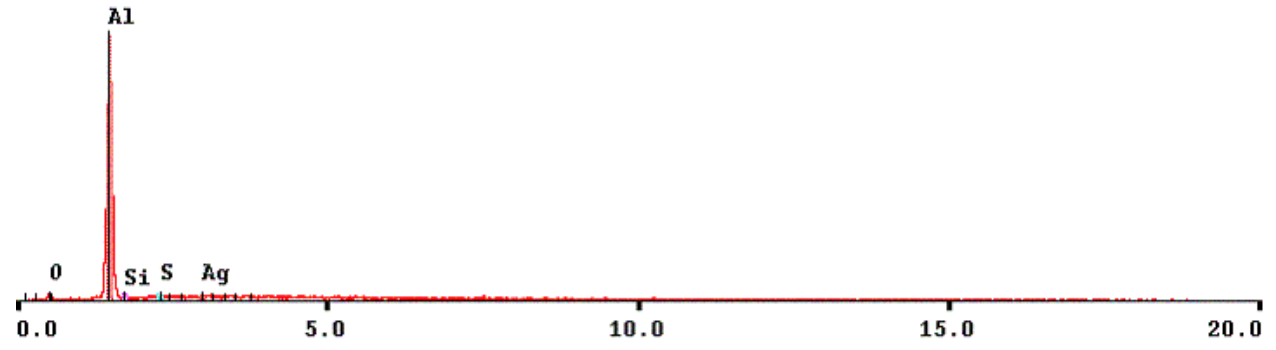

c) X-Ray 3 Showing Point Contains Mostly Aluminum

Figure A6: X-Ray Analysis at 1500x Magnification 


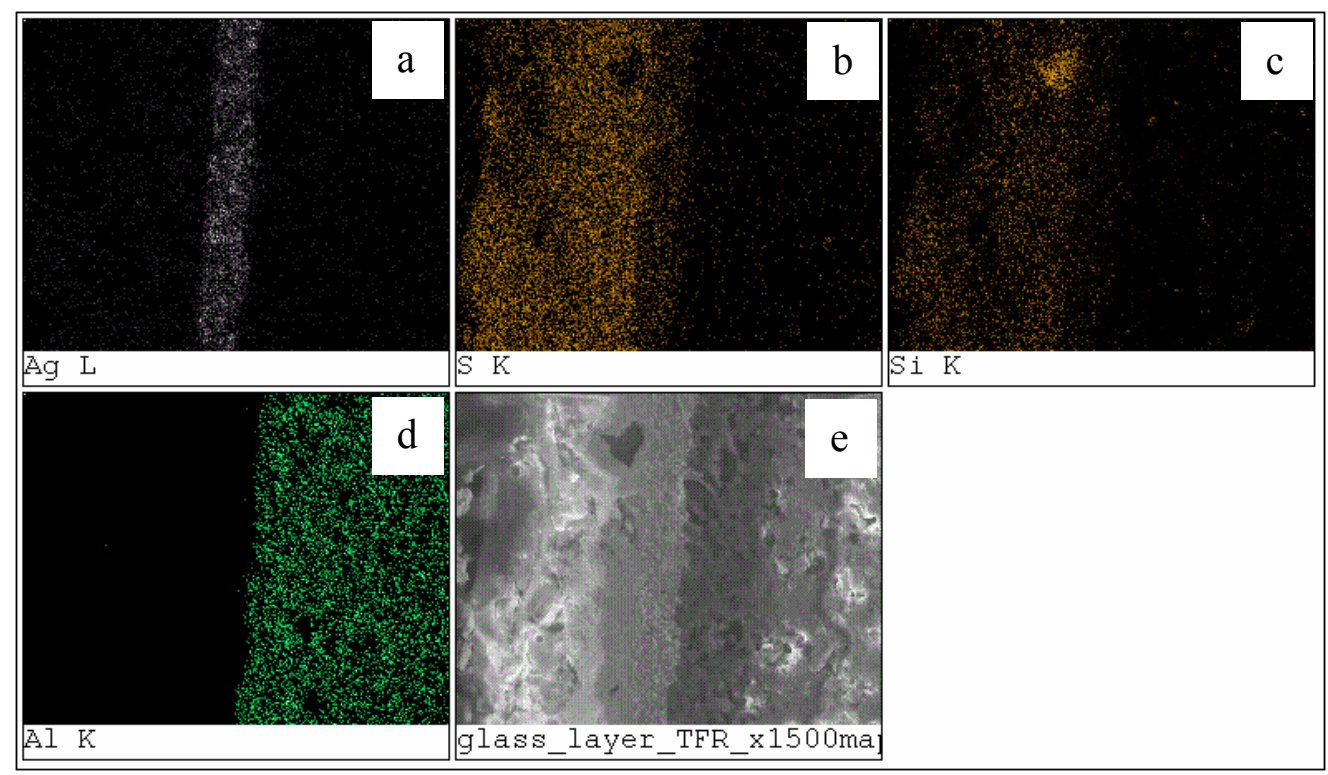

Figure A7: Chemical Analysis at 1500x Magnification Showing a) Layer of Silver b) Layer of Sulfur c) Layer of Silicon d) Layer of Aluminum e) Composite Image

The conductive layer was magnified once more to 3000 times as shown in Figure A8. X-ray results of the point indicated are shown in Figure A9 and the chemical analysis is shown in Figure A10. This magnification confirms that the conductive layer is approximately 7 to 8 microns thick, but decreases the error to \pm 2 microns.

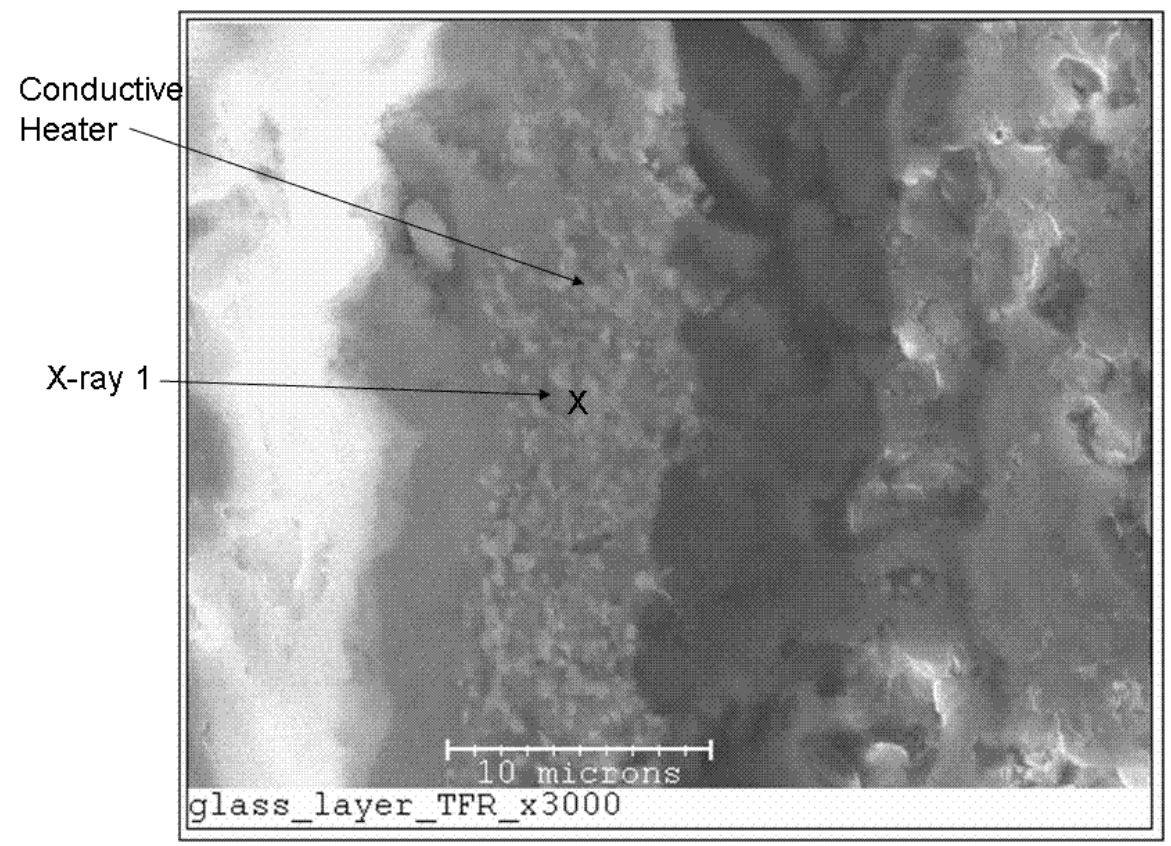

Figure A8: TFR Type 1 at 3000x Magnification Showing the Conductive Heater Layer 


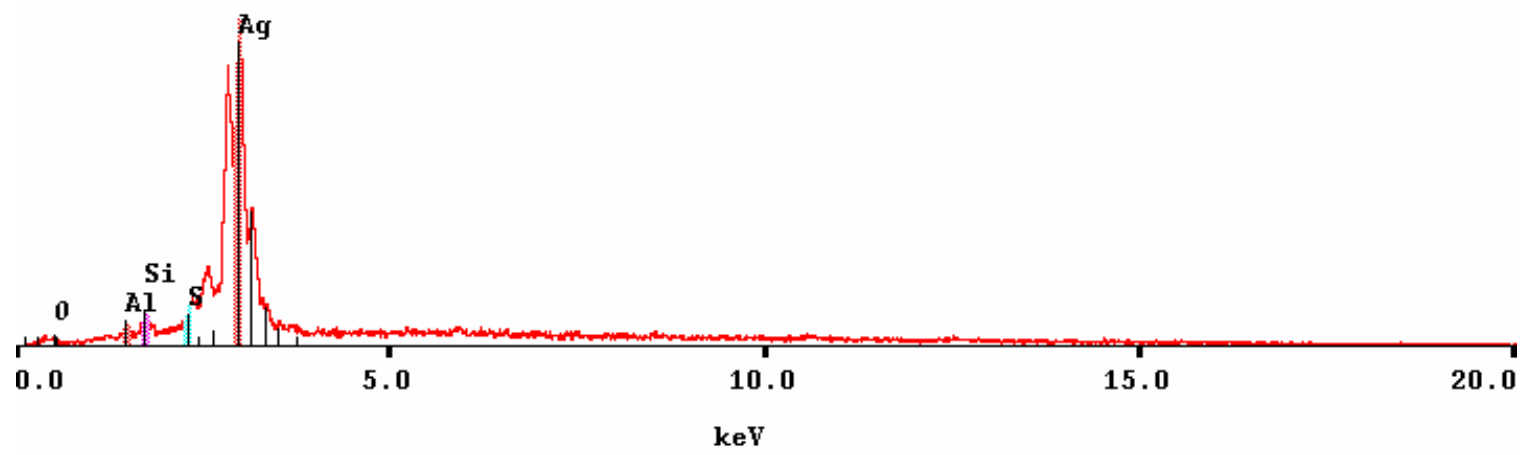

Figure A9: X-Ray Analysis at 3000x Magnification Showing Point mostly Silver

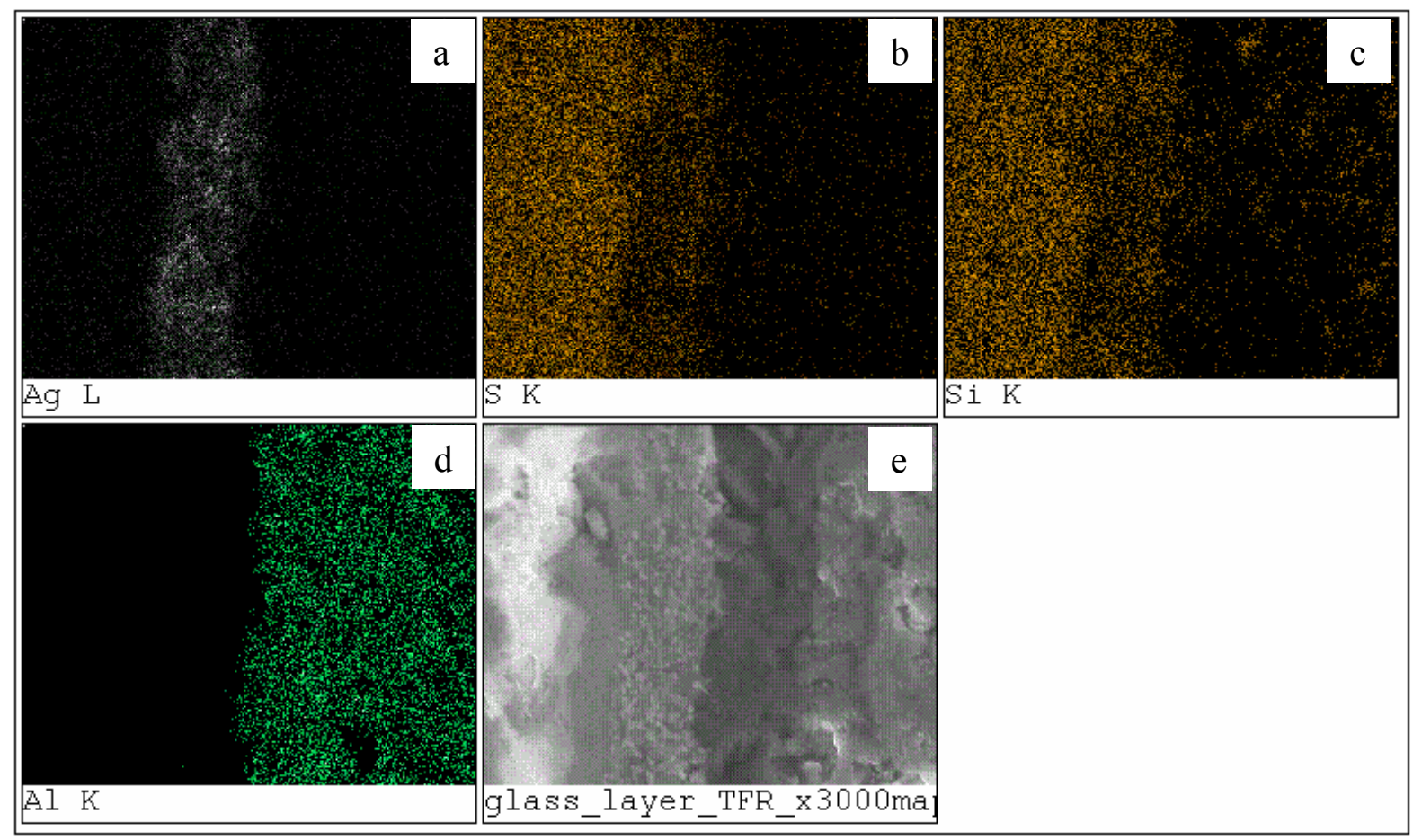

Figure A10: Chemical Analysis at 3000x Magnification Showing a) Layer of Silver b) Layer of Sulfur c) Layer of Silicon d) Layer of Aluminum e) Composite Image

From the tests performed on TFR Type 1, the composition and layer thicknesses were determined. The bottom layer was found to contain aluminum and was $650 \pm 10$ microns thick. The middle layer was found to contain silver (which is highly conductive) and was between 7 and $8 \pm 2$ microns thick. The top layer was found to contain sulfur and silicon (two main components of glass) and was $40 \pm 5$ microns thick. A schematic of these results are shown in Figure A11. 


\begin{tabular}{|c|}
\hline Glass: $40 \pm 5$ microns \\
\hline Heater: $7-8 \pm 2$ microns \\
\hline Alumina: $650 \pm 10$ microns \\
\end{tabular}

Figure A11: TFR Type 1 Schematic of Experimental Results (not to scale)

When compared to the manufacturer's information, the agreement of results varies for each layer. The bottom alumina substrate layer (found to be $650 \pm 10$ microns thick) compared very well to the manufacturer's given thickness of 634 microns. Because the values compared well and because the magnification to view the alumina layer was relatively low, the thickness to be used in data reduction calculations is 634 \pm 10 microns. The middle conductive heater layer was found to be 7 to $8 \pm 2$ microns which is only half of the given thickness of 14-16 microns. However, because the thickness was found at a very high magnification, the conductive heater layer thickness is assumed to be $8 \pm 2$ microns for calculations. The top glass layer differed the most as it was found to be $40 \pm 5$ microns and the manufacturer gave a thickness of 8-18 microns. Because of the high magnification and because the manufacturer stated that sometimes two layers of glass are attached, a glass layer thickness of $40 \pm 5$ microns will be used. To help confirm the final results, a dial caliper was also used to measure the entire thickness of the piece and was found to be approximately 686 microns ( $0.0270 \mathrm{in})$. This compares well to the total calculated thickness (634 microns, plus 8 microns, plus 40 microns, equals 682 microns) of 682 microns $(0.0269$ in). A summary of the TFR Type 1 thicknesses is shown in Table A1. 
Table A1: TFR Type 1 Thicknesses

\begin{tabular}{|c|c|c|c|}
\hline Layer & $\begin{array}{c}\text { Manufacturer } \\
\text { Values }\end{array}$ & $\begin{array}{c}\text { Experimental } \\
\text { Values }\end{array}$ & $\begin{array}{c}\text { Values to be used in } \\
\text { Analysis }\end{array}$ \\
\hline Glass & $8-18$ microns & $40 \pm 5$ microns & $40 \pm 5$ microns \\
\hline Conductive Heater & $14-16$ microns & $7-8 \pm 2$ microns & $8 \pm 2$ microns \\
\hline Alumina Substrate & 634 microns & $650 \pm 10$ microns & $634 \pm 10$ microns \\
\hline
\end{tabular}

\section{TFR Type 2:}

Based on manufacturer information, the second type of TFR consists of two layers. The bottom substrate layer is made of alumina and the second layer is a conductive resistor containing palladium silver. The layer thicknesses were not given. The layer of glass that was on the Type 1 TFR is not present. The results from the scanning electron microscope at a magnification of 150 times (shown in Figure A12) show the alumina layer along with the surrounding epoxy. The thin conductive layer is not visible at this magnification. As with the TFR Type 1 heater, the bright white areas in the alumina are from the non-conductive alumina becoming charged by the SEM microscope while the black area in the alumina is not charging because it is next to the conductive layer. X-rays were taken in the spots indicated to confirm the composition of the layers and are shown in Figure A13. The chemical analysis results are shown in Figure A14. Nickel was included in this chemical analysis but only appeared in the epoxy. At this 150 times magnification, the alumina layer measures approximately 630 \pm 10 microns in thickness. 


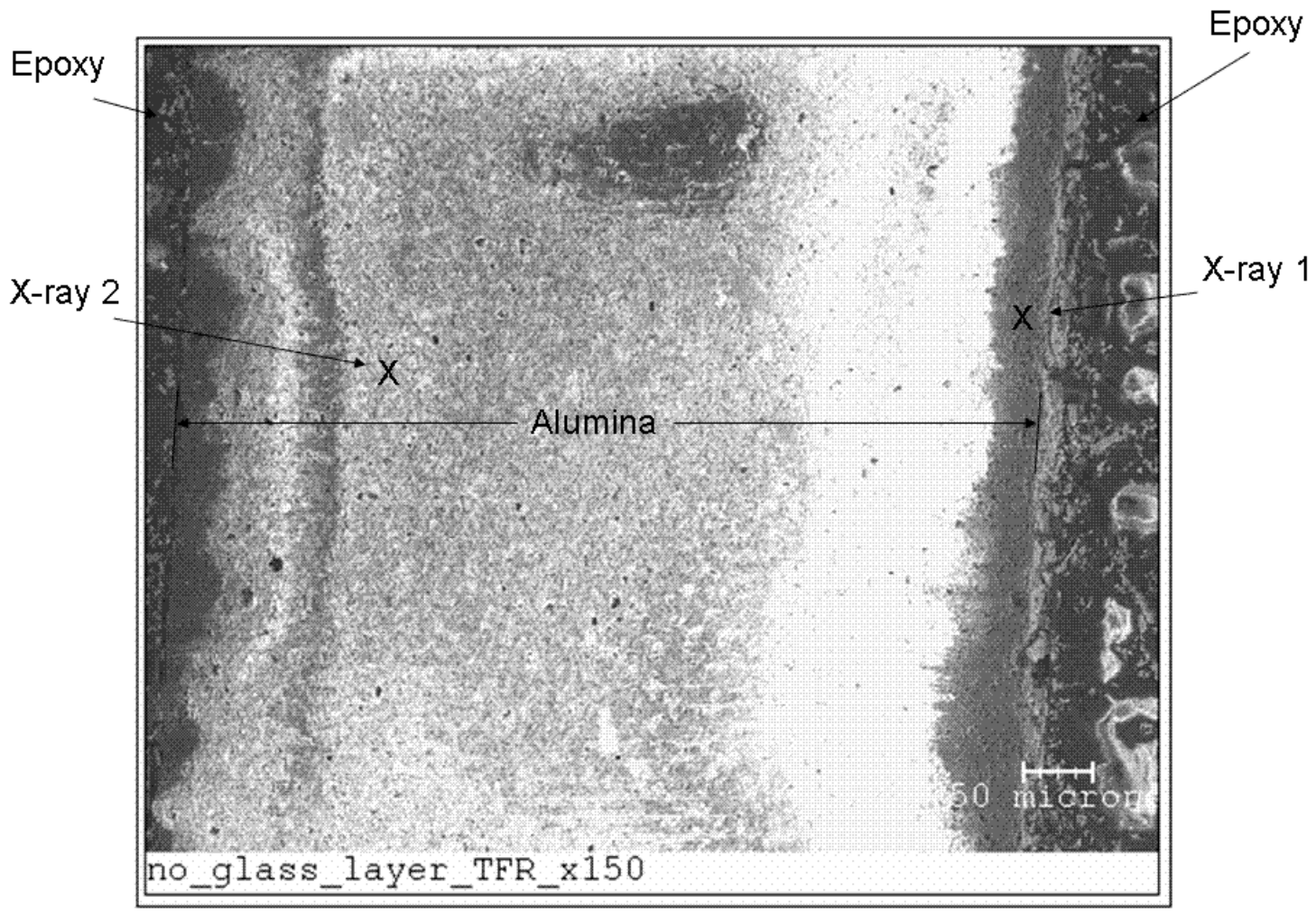

Figure A12: TFR Type 2 at 150x Magnification Showing the Alumina Layer

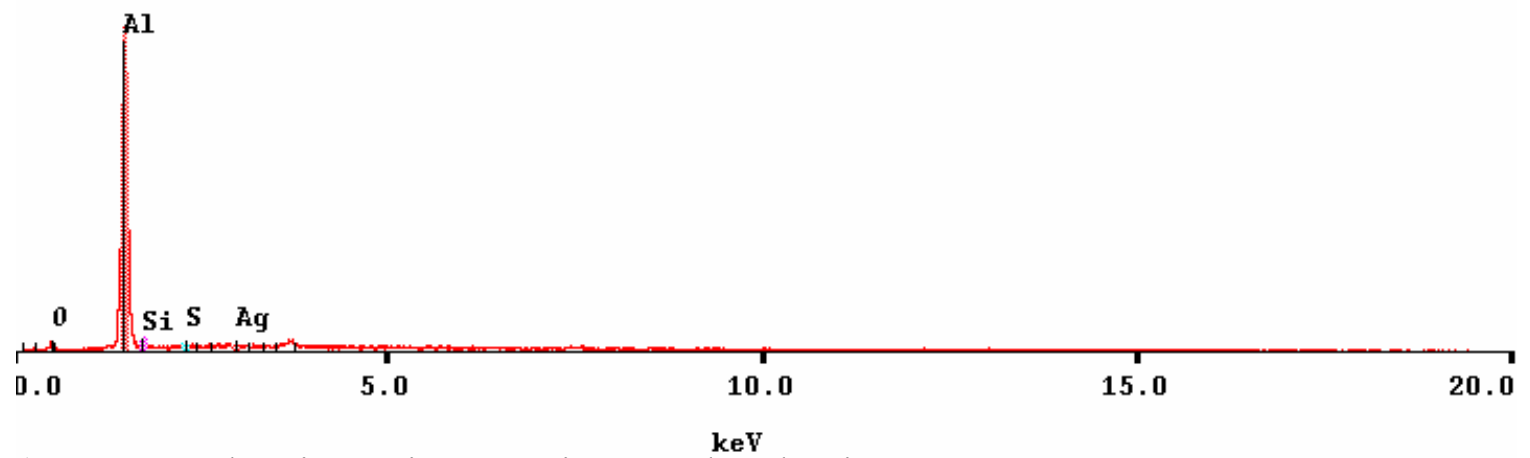

a) X-Ray 1 Showing Point Contains Mostly Aluminum

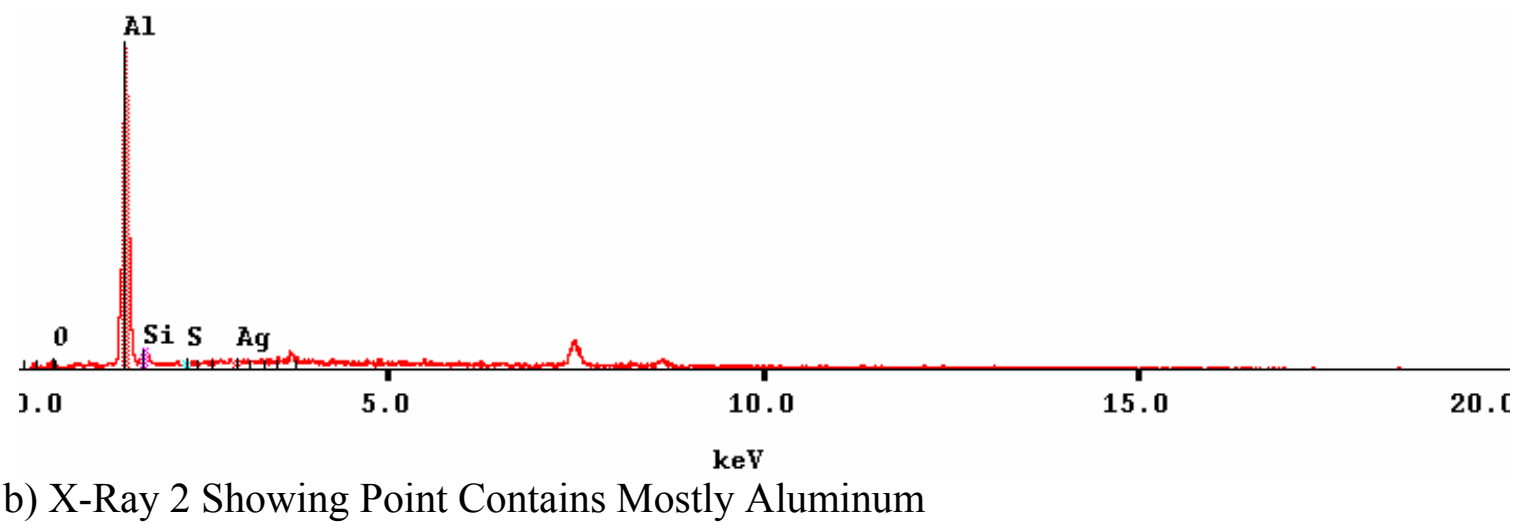

Figure A13: X-Ray Analysis at 150x Magnification 


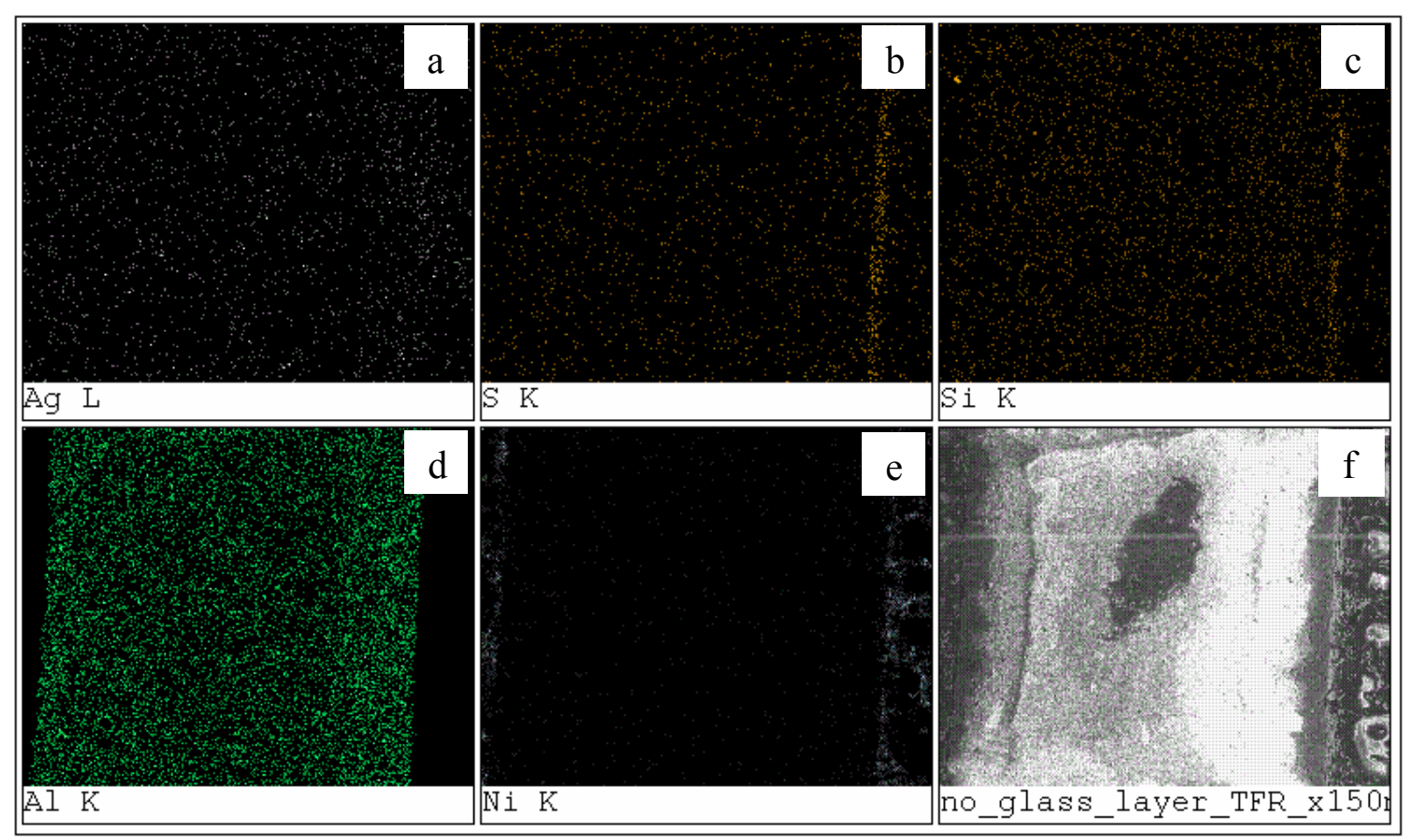

Figure A14: Chemical Analysis at 150x Magnification Showing a) Silver Throughout b) Layer of Sulfur c) Layer of Silicon d) Layer of Aluminum e) Nickel f) Composite Image

Magnifying the edge with the conductive layer to 1000 times magnification shows the very ragged edges between layers and also between the epoxy and the sample as seen in Figure A15. This could be due to polishing. X-rays were taken at the points indicated to help in determining where each layer lies and are shown in Figure A16. X-ray 1 and 3 show several different elements indicating that those points are near the interface. X-ray 2 is clearly in the alumina layer. The chemical analysis is shown in Figure A17. At this magnification the conductive layer is measured to be approximately $15-20 \pm 5$ microns. 


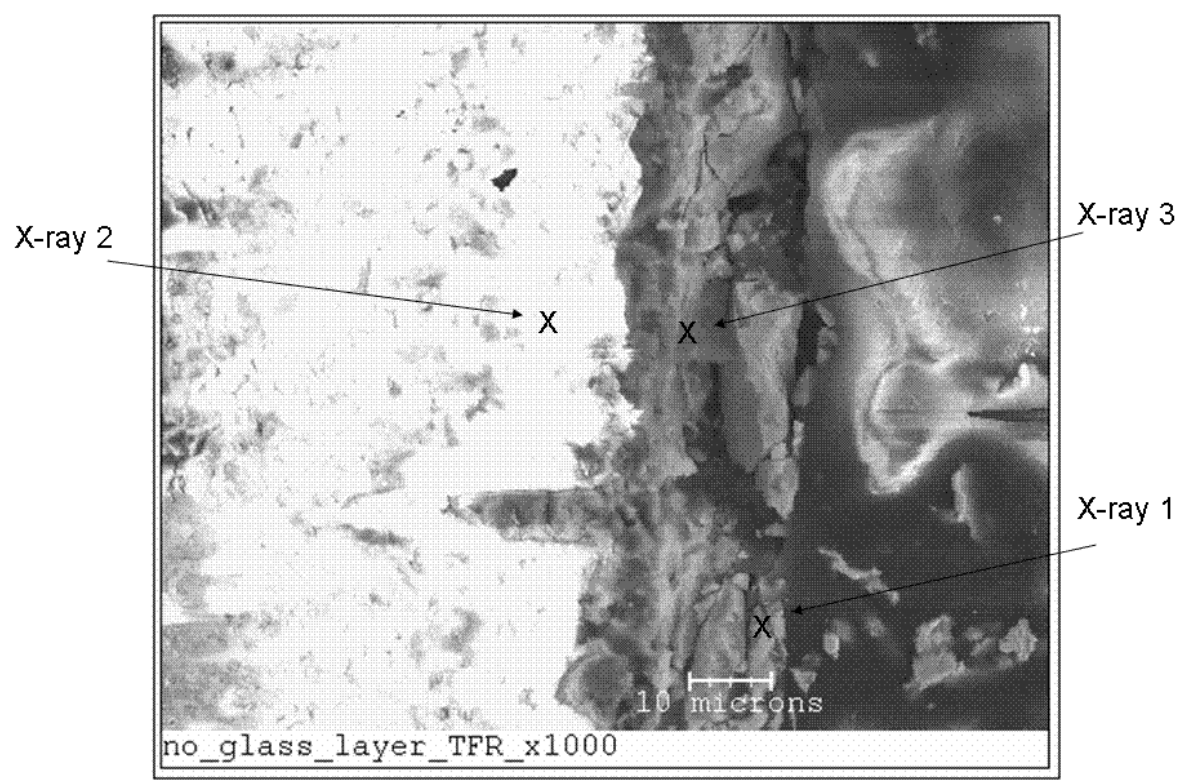

Figure A15: TFR Type 2 at 1000x Magnification Showing the Conductive Layer

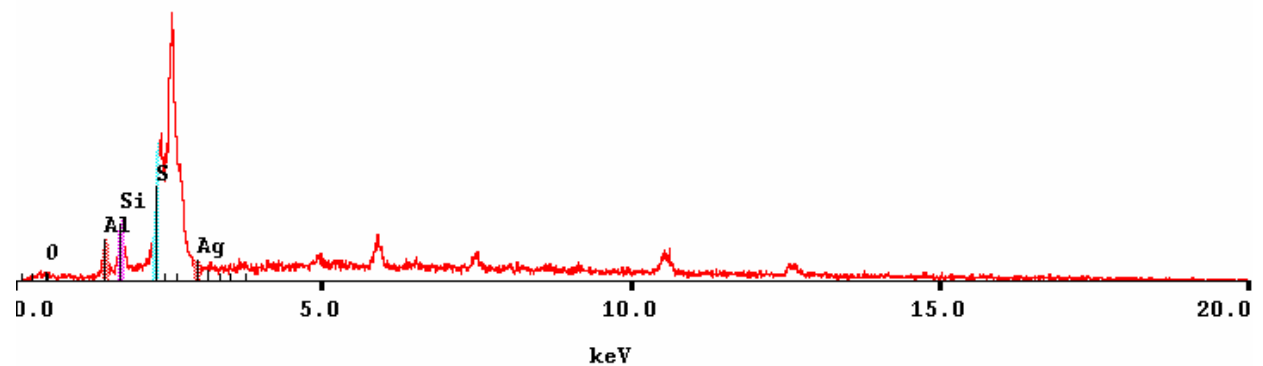

a) X-Ray 1 Showing Point Contains Mixture of Sulfur, Silicon, and Aluminum

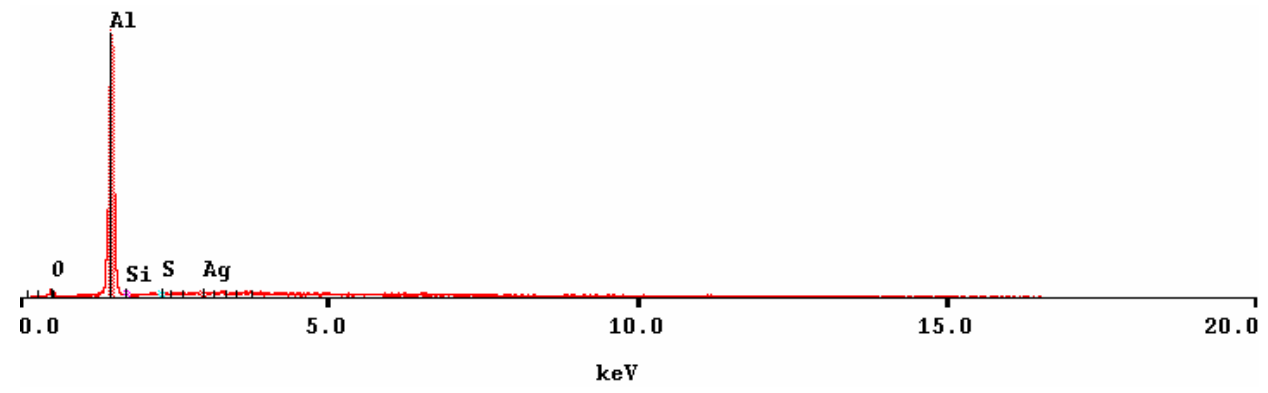

b) X-Ray 2 Showing Point Contains Mostly Aluminum

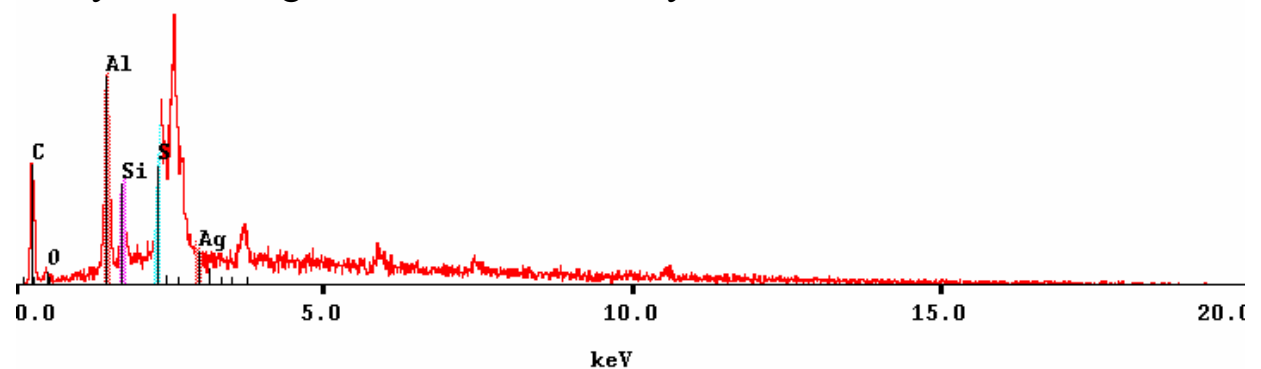

c) X-Ray 3 Showing Point Contains Mixture of Sulfur, Aluminum, Silicon, Carbon, and Silver Figure A16: X-Ray Analysis at 1000x Magnification 


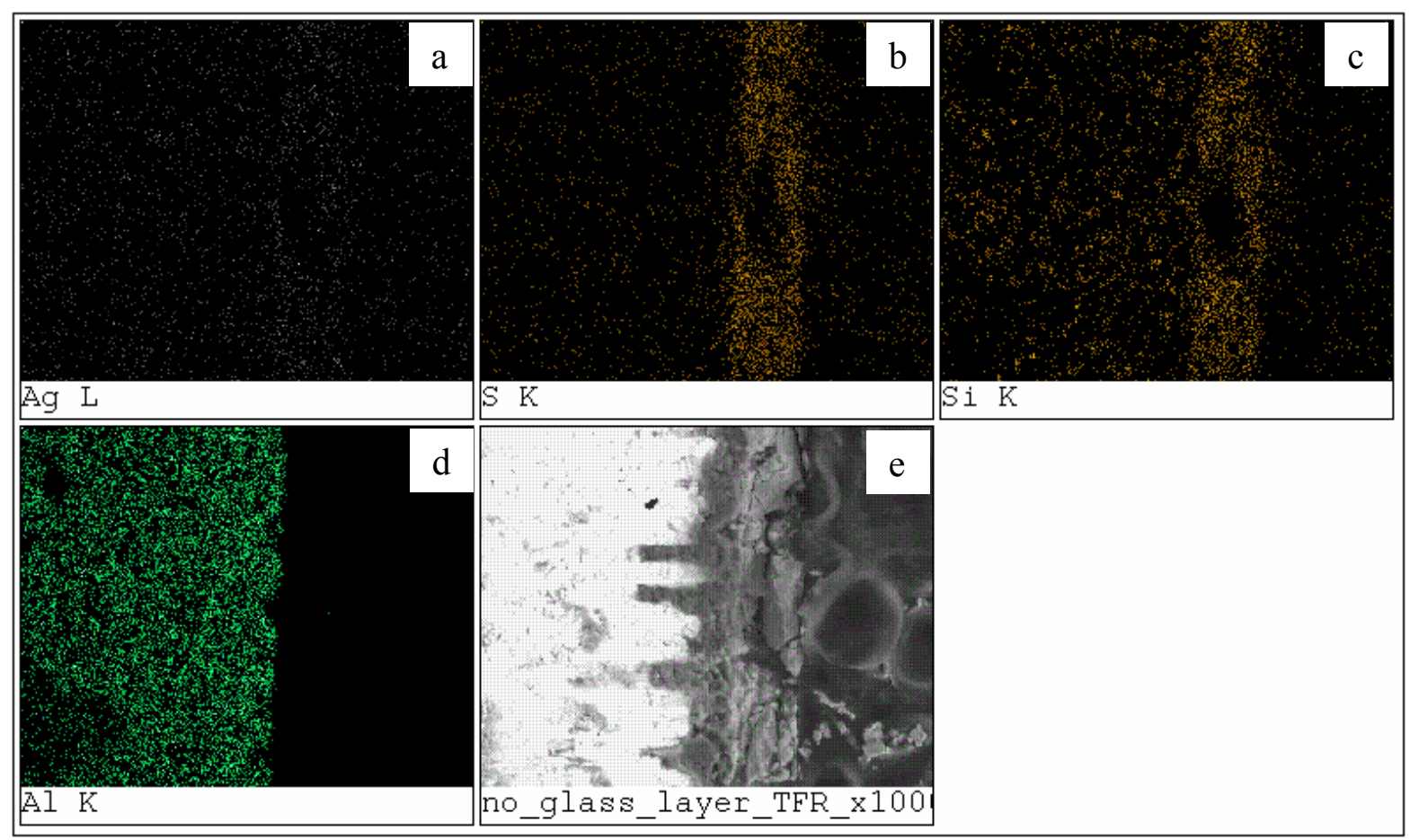

Figure A17: Chemical Analysis at 150 Magnification Showing a) Silver Throughout b) Layer of Sulfur c) Layer of Silicon d) Layer of Aluminum e) Composite Image From the tests performed on the TFR Type 2, the composition and layer thicknesses were determined. The bottom layer was found to contain aluminum and was $630 \pm 10$ microns thick. The top layer was found to contain silver, sulfur, and silicon (and is assumed to be the conductive heater) and was 15-20 \pm 5 microns thick. A schematic of these results are shown in Figure A18. No information was given by the manufacturer for the thickness of each layer and therefore cannot be compared.

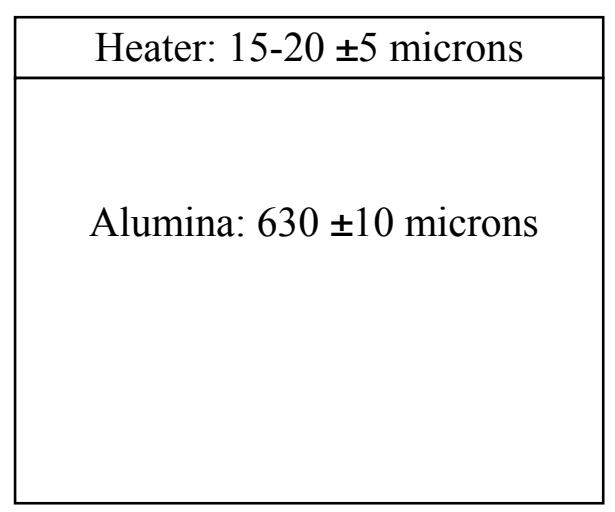

Figure A18: TFR Type 2 Schematic of Experimental Results (not to scale) 
For data reduction calculation purposes, the alumina layer is assumed to have the same given thickness as the Type 1 TFR of $634 \pm 10$ microns. The conductive heater is assumed to have a thickness of $20 \pm 5$ microns. A dial caliper was used to measure the entire thickness of the piece, and it was found to be approximately 660 microns $(0.0260$ in). This agrees very well with the total calculated thickness (634 microns, plus 20 microns, equals 654 microns) of 654 microns (0.0257 in). A summary of the TFR Type 2 thicknesses is shown in Table A2.

Table A2: TFR Type 2 Thicknesses

\begin{tabular}{|c|c|c|c|}
\hline Layer & $\begin{array}{c}\text { Manufacturer } \\
\text { Values }\end{array}$ & $\begin{array}{c}\text { Experimental } \\
\text { Values }\end{array}$ & $\begin{array}{c}\text { Values to be used in } \\
\text { Analysis }\end{array}$ \\
\hline Conductive Heater & None given & $15-20 \pm 5$ microns & $20 \pm 5$ microns \\
\hline Alumina Substrate & None given & $630 \pm 10$ microns & $634 \pm 10$ microns \\
\hline
\end{tabular}




\section{Appendix B: Test Plans for Spacing Comparison and Electrode Performance Studies}

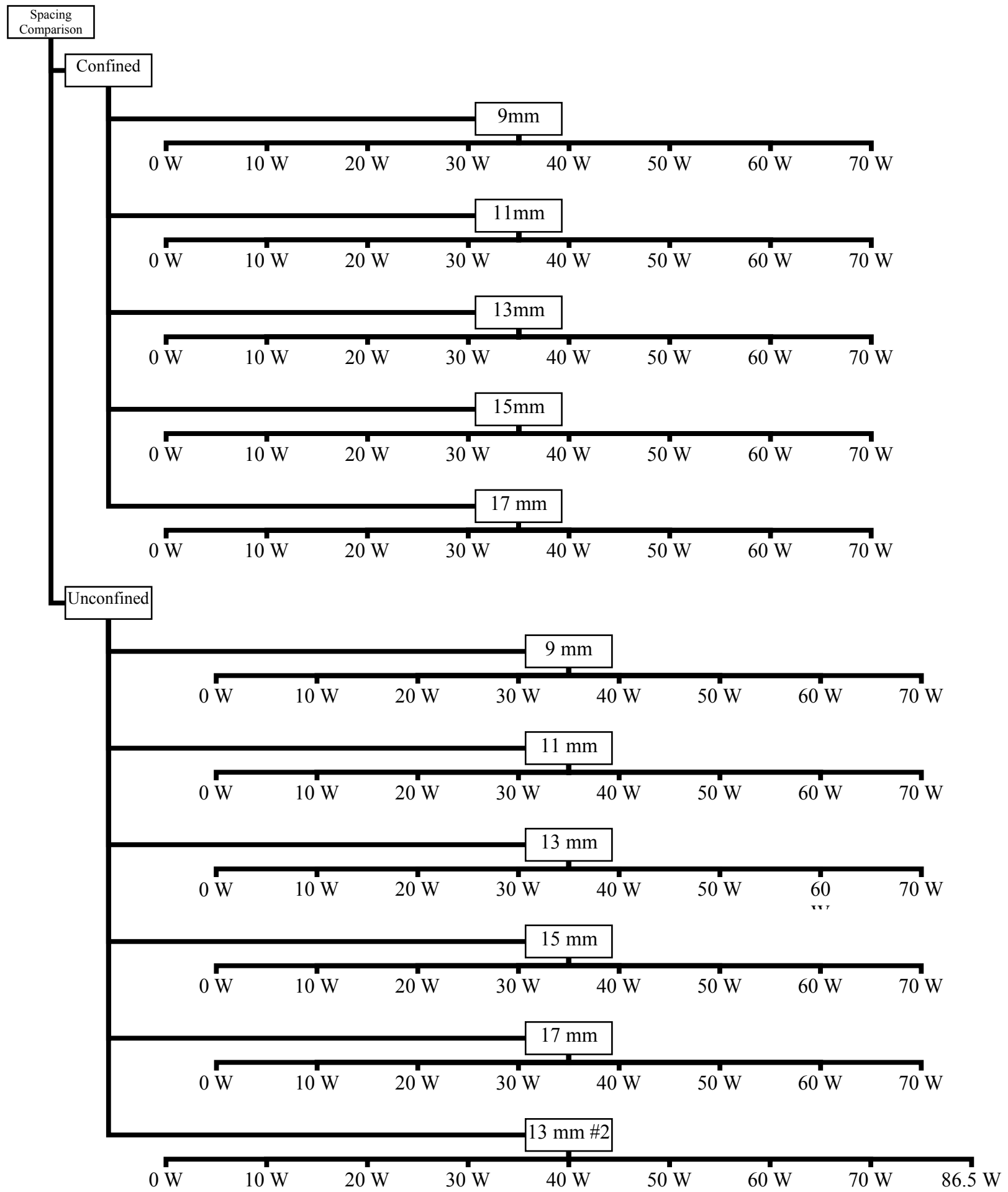

Figure B1: Spacing Comparison Test Plan at $0.029 \mathrm{~m}^{3} / \mathrm{hr}$ (7.72 GPH) FC-72 


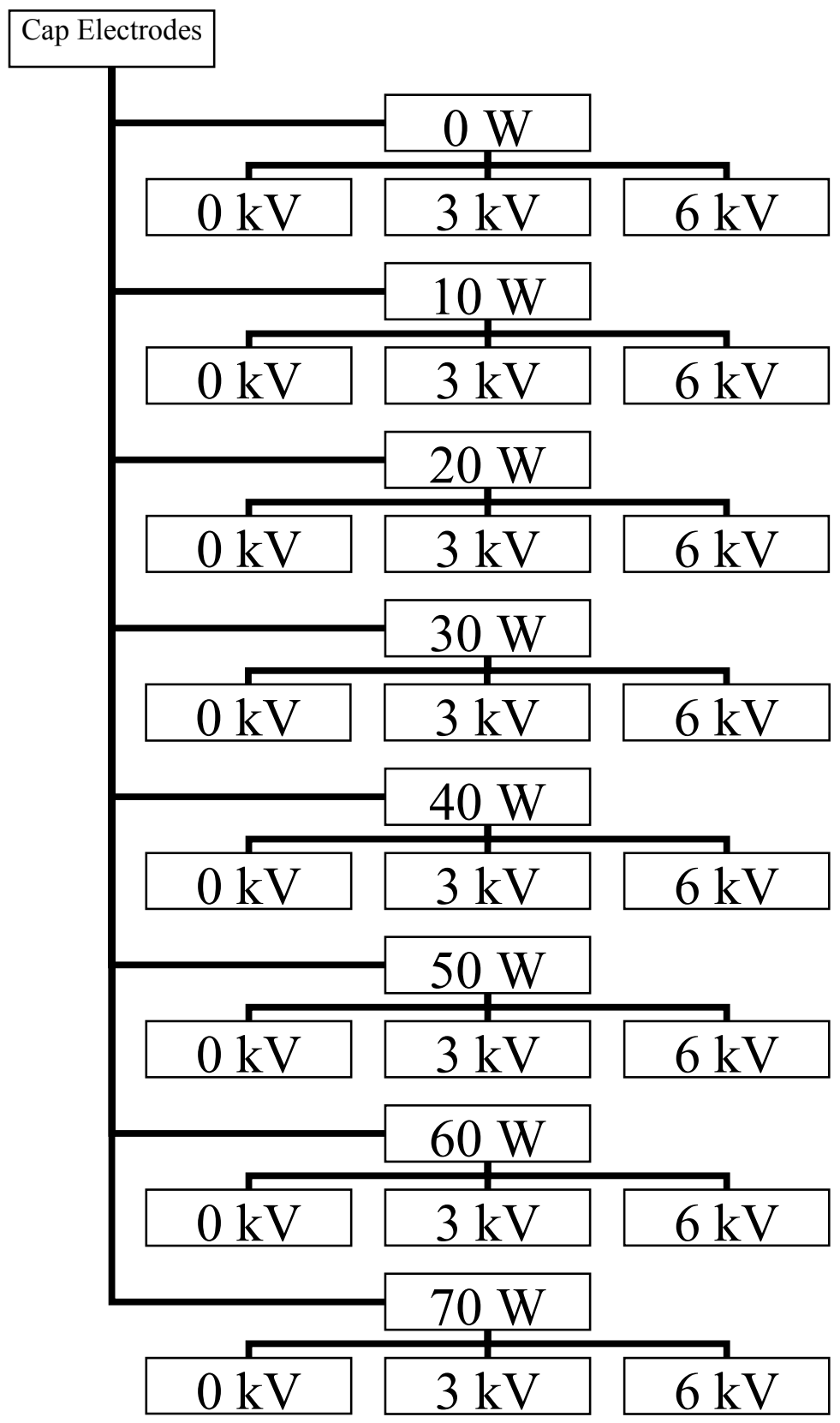

Figure B2: Cap Electrode Test Plan at $0.029 \mathrm{~m}^{3} / \mathrm{hr}(7.72 \mathrm{GPH})$ of FC-72 and $0.016 \mathrm{~m} / \mathrm{hr}$ (4.23 GPH) of HFE-7000 


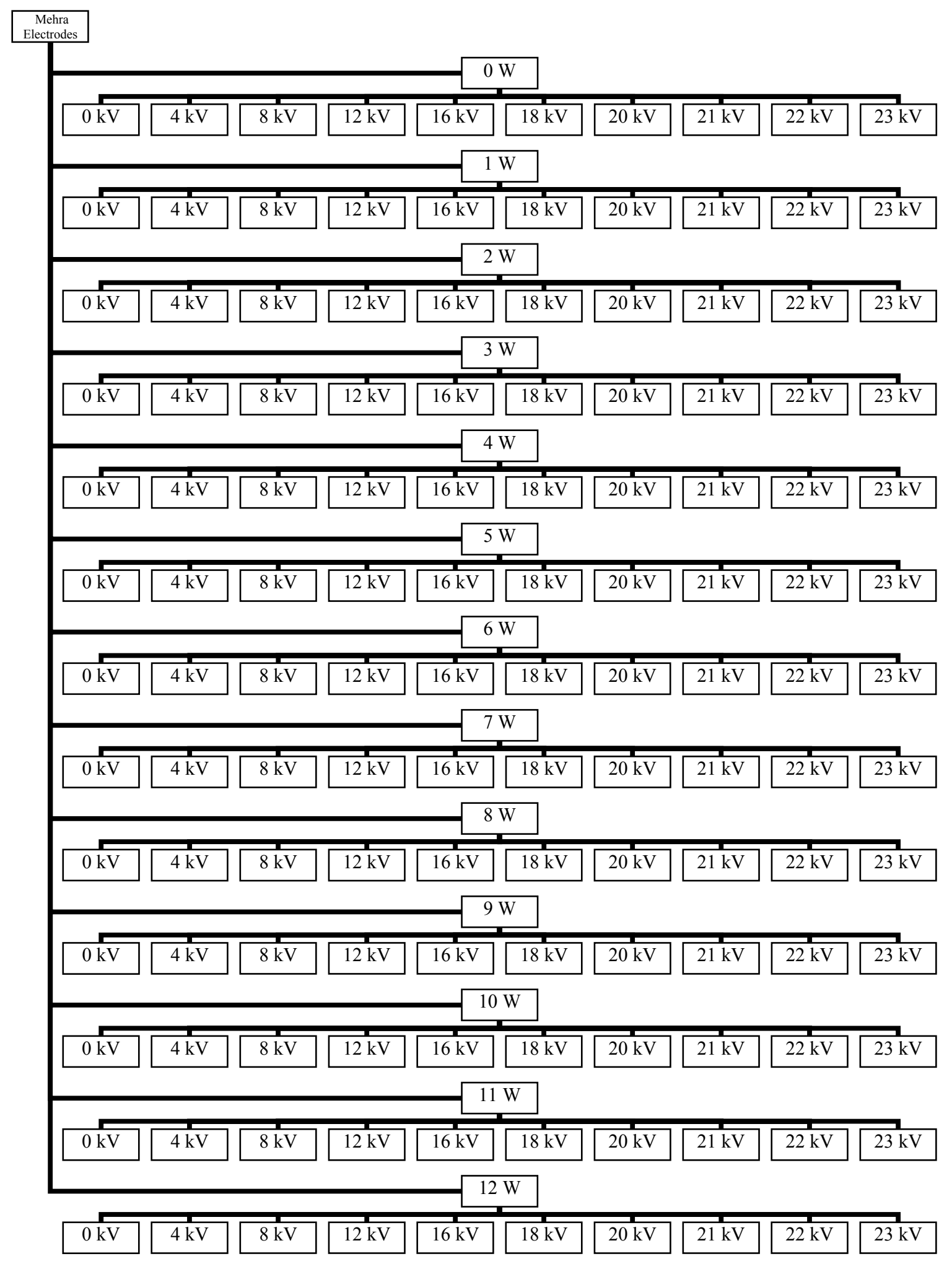

Figure B3: Mehra Electrode Test Plan at $0.014 \mathrm{~m}^{3} / \mathrm{hr}$ (3.8 GPH) of HFE-7000 


\section{Appendix C: Derivation and Solutions of Energy Equations for the TFR Pedestals}

This appendix shows the derivation of the energy equations needed to solve the heat conduction and convection problem associated with the layers of the different TFR pedestals. The pedestal used for the spacing comparison consisted of a glass rod with a Type 1 TFR (with a glass layer). The pedestal used for the Mehra electrode tests consisted of a PTFE rod with a Type 2 TFR (no glass layer) and $2 \mathrm{~mm}$ of alumina above the heater. The conduction and convection problem for these two pedestals were modeled similarly and are shown in the first section below. The pedestal used for the cap electrode tests consisted of a PTFE rod with a Type 2 TFR (no glass layer) and is shown in the second section below.

\section{C1: Spacing Comparison and Mehra Pedestals}

\section{Energy Equation Derivation for Each Layer}

The energy equation for a one-dimensional, steady state, circular layer with insulated sides as shown below in Figure $\mathrm{C} 1$ can be found as follows.

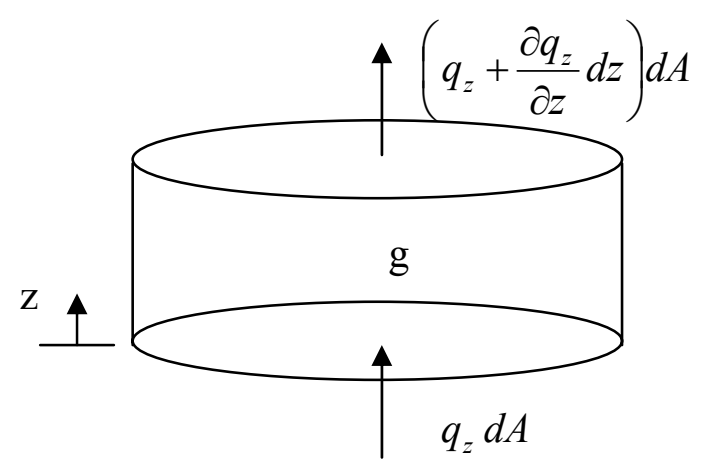

Figure C1: Energy Equation Derivation Schematic for the Spacing Comparison and Mehra Pedestals 
The net heat flow into the system is found by subtracting the heat flow out of the system from the heat flow into the system and including the internal heat generation rate per unit volume, g, as shown in Equation C1. Simplifying yields Equation C2.

$$
\begin{aligned}
& \frac{d q}{d t}=q_{z} d A-\left(q_{z}+\frac{\partial q_{z}}{\partial z} d z\right) d A+g d A d z \\
& \frac{d Q}{d t}=-\frac{\partial q_{z}}{\partial z}+g
\end{aligned}
$$

Using Fourier's Law in the z-direction as shown in Equation C3, assuming steady state as shown in Equation C4, and using a constant thermal conductivity reduces the equation to the energy equation needed to solve for the temperature distribution in the heater layer, Equation C5.

$$
\begin{aligned}
& q_{z}=-k \frac{\partial T}{\partial z} \\
& \frac{d Q}{d t}=0 \\
& 0=\frac{\partial^{2} T}{\partial z^{2}}+\frac{g}{k}
\end{aligned}
$$

For the glass and substrate layers on the spacing comparison pedestal and for the substrate and alumina layer on the Mehra pedestal, there is no internal heat generation and the equation reduces further as shown in Equation C6.

$$
0=\frac{\partial^{2} T}{\partial z^{2}}
$$

\section{Boundary Conditions Defined for Each Layer}

The boundary conditions between each layer come from Fourier's Law which is given in the z-direction as shown in Equation $\mathrm{C} 7$. 


$$
q_{z}=-k \frac{d T}{d z}
$$

Solving for the heat flux and taking into account how the z-direction is defined for each of the three layers (shown in Figure C2) produces five of the six boundary conditions. The notation of $q_{T}$ and $q_{L}$ are also introduced as shown in Equations C8 and C9 to describe the amount of heat moving up and down the pedestal, respectively, from the bottom of the heater, where $\mathrm{f}$ is the heater conduction loss fraction.

$$
\begin{aligned}
& q_{T}=(1-f) q \\
& q_{L}=f q
\end{aligned}
$$

The sixth boundary condition is at the top surface of the glass (or alumina) layer and includes convection. The heat flux at this point can be written as shown in Equation C10.

$$
q=h\left(T_{s}-T_{\infty}\right)
$$

This equation and Fourier's Law can be related to produce the final boundary condition at the top surface of the glass (or alumina) and is shown in Equation C11.

$$
\frac{d T}{d z}+\frac{h}{k} T_{s}=\frac{h}{k} T_{\infty}
$$

The geometry definitions, governing equations and, boundary conditions for the spacing comparison and Mehra pedestal geometries are shown below in Figure C2. 


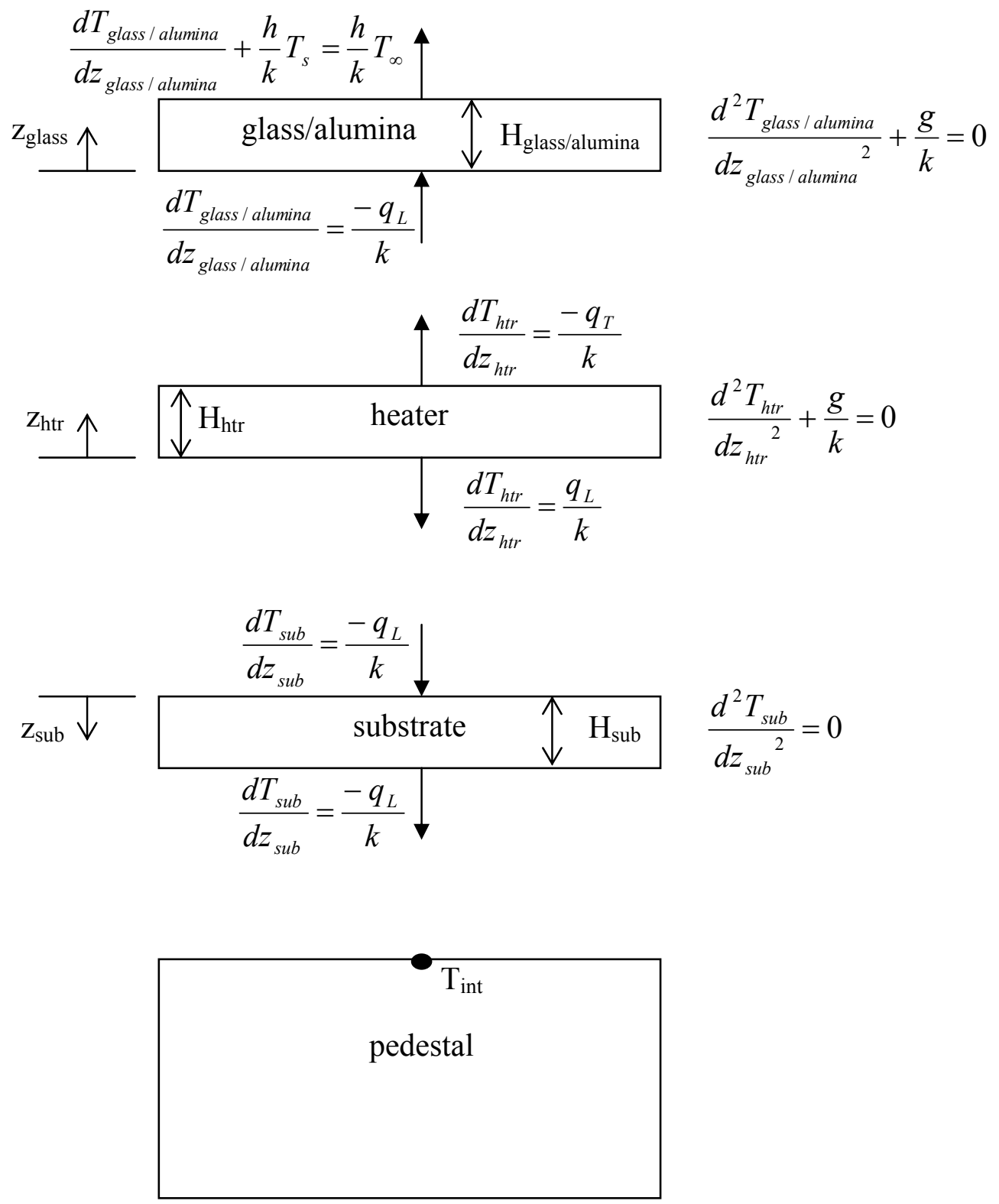

Figure C2: Respective Geometry, Equations, and Boundary Conditions for Each Layer of the Spacing Comparison and Mehra Pedestals

\section{Solutions for Each Layer}

Glass/Alumina Layer: The governing equation for the glass/alumina layer was found from Equation C6 above and includes no internal heat generation. The first boundary condition was found from Equation $\mathrm{C} 11$ above and includes a constant temperature 
condition and convection at the top of the pedestal going in the positive $\mathrm{z}$ direction. The second boundary condition was found from Equation $\mathrm{C} 7$ above and involves onedimensional heat conduction at the glass/alumina and heater interface in the positive $\mathrm{z}$ direction. Contact resistances were neglected. These equations can be used to produce the temperature distribution solution in the glass/alumina layer, Equation C12.

\section{Governing Equation}

$$
\frac{d^{2} T_{\text {glass/alumina }}}{d z_{\text {glass/alumina }}^{2}}+\frac{g}{k}=0
$$

$\underline{\text { Boundary Conditions }}$

$$
\begin{aligned}
& \frac{d T_{\text {glass / alumina }}}{d z_{\text {glass / alumina }}}+\frac{h}{k_{\text {glass / alumina }}} T_{s}=\frac{h}{k_{\text {glass / alumina }}} T_{\infty}, \mathrm{z}=\mathrm{H}_{\text {glass/alumina }} \\
& \frac{d T_{\text {glass / alumina }}}{d z_{\text {glass / alumina }}}=\frac{-q_{L}}{k_{\text {glass / alumina }}} \quad, \mathrm{Z}_{\text {glass/alumina }}=0
\end{aligned}
$$

$\underline{\text { Solution }}$

$$
T_{\text {glass /alumina }}(z)=\frac{-q_{T}}{k_{\text {glass/alumina }}} z+T\left(z_{\text {glass / alumina }}=0\right)
$$

Heater Layer: The governing equation for the heater layer was found from Equation C5 above and includes the internal heat generation produced by the resistor. The first boundary condition was found from Equation $\mathrm{C} 7$ above and involves one-dimensional heat conduction at the glass/alumina and heater interface in the positive $\mathrm{z}$ direction. The second boundary condition was also found from Equation C7 and involves onedimensional heat conduction at the heater and substrate interface in the negative $\mathrm{z}$ 
direction. Contact resistances were neglected. These equations can be used to produce the temperature distribution solution in the heater layer, Equation C13.

\section{Governing Equation}

$$
\frac{d^{2} T_{h t r}}{d z_{h t r}{ }^{2}}+\frac{g}{k_{h t r}}=0
$$

Boundary Conditions

$$
\begin{aligned}
& \frac{d T_{h t r}}{d z_{h t r}}=\frac{-q_{T}}{k_{h t r}}, \mathrm{z}_{\mathrm{htr}}=\mathrm{H}_{\mathrm{htr}} \\
& \frac{d T_{h t r}}{d z_{h t r}}=\frac{q_{L}}{k_{h t r}} \quad, \mathrm{Z}_{\mathrm{htr}}=0
\end{aligned}
$$

$\underline{\text { Solution }}$

$$
T_{h t r}(z)=\frac{-g}{2 k_{h t r}} z_{h t r}{ }^{2}+\frac{q_{L}}{k_{h t r}} z_{h t r}+T\left(z_{h t r}=0\right)
$$

Substrate Layer: The governing equation for the substrate layer was found from Equation $\mathrm{C} 6$ above and includes no internal heat generation. The boundary conditions at the heater and substrate and also the substrate and pedestal interfaces are identical and were found from Equation $\mathrm{C} 7$ above and involve one-dimensional heat conduction in the positive $\mathrm{z}$ direction. Contact resistances were neglected. These equations can be used to produce the temperature distribution solution in the substrate layer, Equation C14.

Governing Equation

$$
\frac{d^{2} T_{s u b}}{d z_{\text {sub }}}+\frac{g}{k_{\text {sub }}}=0
$$




\section{Boundary Condition}

$$
\frac{d T_{\text {sub }}}{d z_{\text {sub }}}=\frac{-q_{L}}{k_{\text {sub }}}, \mathrm{z}_{\mathrm{sub}}=0 \& \mathrm{H}_{\mathrm{sub}}
$$

$\underline{\text { Solution }}$

$$
T_{\text {sub }}(z)=\frac{-q_{L}}{k_{\text {sub }}} z_{\text {sub }}+T\left(z_{\text {sub }}=0\right)
$$

Since the temperatures at the layer interfaces are equal (i.e. the temperature at the bottom of the glass/alumina equals the temperature at the top of the heater, the temperature at the bottom of the heater equals the temperature at the top of the substrate, and the temperature at the bottom of the substrate equals the temperature at the top of the pedestal), the solutions can be combined to relate the surface temperature to the temperature measured at the top of the pedestal, $\mathrm{T}_{\mathrm{int}}$, as shown in Equation $\mathrm{C} 15$.

$$
T_{s}=\frac{-q_{T} H_{\text {glass / alumina }}}{k_{\text {glass / alumina }}}+\frac{-g H_{h t r}{ }^{2}}{2 k_{h t r}}+\frac{q_{L} H_{h t r}}{k_{h t r}}+\frac{q_{L} H_{\text {sub }}}{k_{\text {sub }}}+T_{\text {int }}
$$

\section{C2: Cap Electrode Pedestal}

\section{Energy Equation Derivation for Each Layer}

The energy equation for a one-dimensional, steady state, circular layer with insulated sides as shown below in Figure C3 can be found as follows.

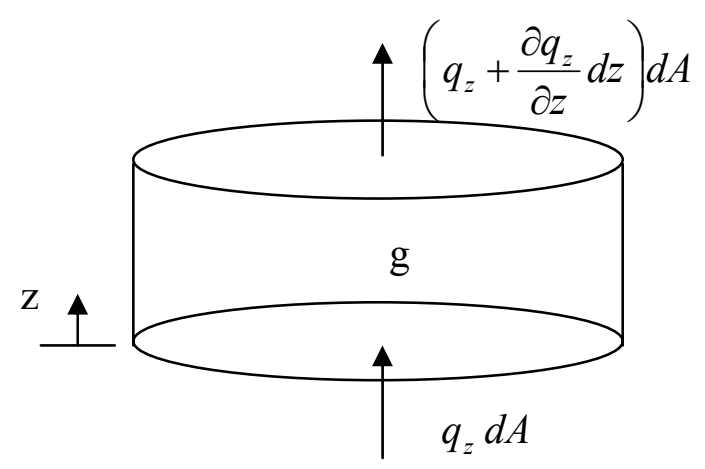

Figure C3: Energy Equation Derivation Schematic for the Cap Electrode Pedestal 
The net heat flow into the system is found by subtracting the heat flow out of the system from the heat flow into the system and including the internal heat generation rate per unit volume, g, as shown in Equation C16. Simplifying yields Equation C17.

$$
\begin{aligned}
& \frac{d q}{d t}=q_{z} d A-\left(q_{z}+\frac{\partial q_{z}}{\partial z} d z\right) d A+g d A d z \\
& \frac{d Q}{d t}=-\frac{\partial q_{z}}{\partial z}+g
\end{aligned}
$$

Using Fourier's Law in the z-direction as shown in Equation C18, assuming steady state as shown in Equation C19, and using a constant thermal conductivity reduces the equation to the energy equation needed to solve for the temperature distribution in the heater layer, Equation C20.

$$
\begin{aligned}
& q_{z}=-k \frac{\partial T}{\partial z} \\
& \frac{d Q}{d t}=0 \\
& 0=\frac{\partial^{2} T}{\partial z^{2}}+\frac{g}{k}
\end{aligned}
$$

For the substrate layer on the cap electrode pedestal, there is no internal heat generation and the equation reduces further as shown in Equation C21.

$$
0=\frac{\partial^{2} T}{\partial z^{2}}
$$




\section{Boundary Conditions Defined for Each Layer}

The boundary conditions between each layer come from Fourier's Law which is given in the z-direction as shown in Equation $\mathrm{C} 22$.

$$
q_{z}=-k \frac{d T}{d z}
$$

Solving for the heat flux and taking into account how the z-direction is defined for each of the two layers (shown in Figure C4) produces three of the four boundary conditions. The notation of $q_{T}$ and $q_{L}$ are also shown in Equations C23 and C24 to describe the amount of heat moving up and down the pedestal, respectively, from the bottom of the heater, where $\mathrm{f}$ is the heater conduction loss fraction.

$$
\begin{aligned}
& q_{T}=(1-f) q \\
& q_{L}=f q
\end{aligned}
$$

The fourth boundary condition is at the top surface of the heater layer and includes convection. The heat flux at this point can be written as shown in Equation C25.

$$
q=h\left(T_{s}-T_{\infty}\right)
$$

This equation and Fourier's Law can be related to produce the final boundary condition at the top surface of the heater and is shown in Equation C26.

$$
\frac{d T}{d z}+\frac{h}{k} T_{s}=\frac{h}{k} T_{\infty}
$$

The geometry definitions, governing equations and, boundary conditions for the cap electrode pedestal geometry are shown below in Figure C4. 

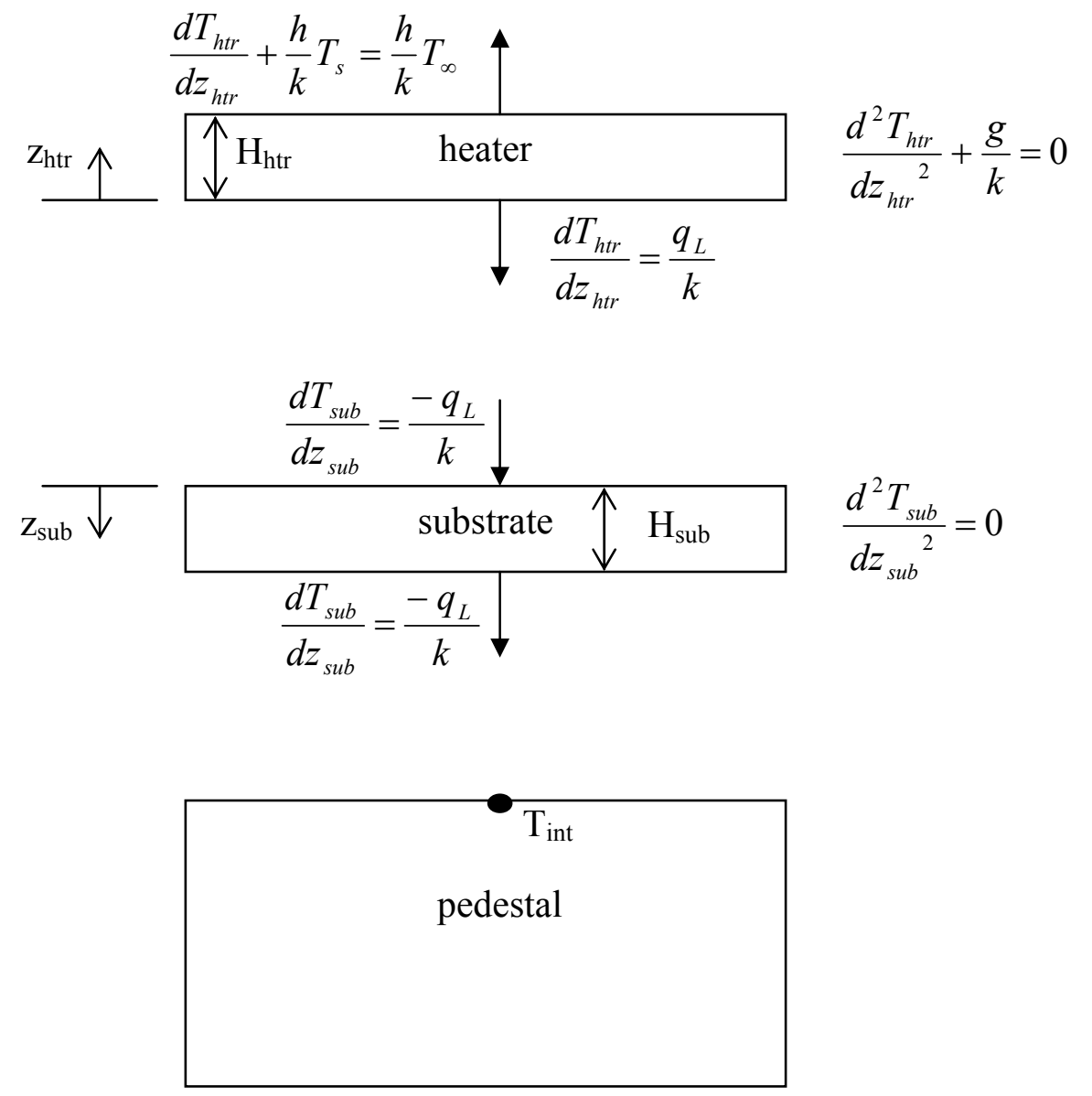

Figure C4: Respective Geometry, Equations, and Boundary Conditions for Each Layer of the Cap Electrode Study Pedestals

\section{Solutions for Each Layer}

Heater Layer: The governing equation for the heater layer was found from Equation C20 above and includes the internal heat generation produced by the resistor. The first boundary condition was found from Equation C26 and involves convection at the heater surface. The second boundary condition was found from Equation C22 above and involves one-dimensional heat conduction at the heater and substrate interface in the positive $\mathrm{z}$ direction. Contact resistances were neglected. These equations can be used to produce the temperature distribution solution in the heater layer, Equation C28. 


\section{Governing Equation}

$$
\frac{d^{2} T_{h t r}}{d z_{h t r}^{2}}+\frac{g}{k_{h t r}}=0
$$

$\underline{\text { Boundary Conditions }}$

$$
\begin{array}{ll}
\frac{d T_{h t r}}{d z_{h t r}}+\frac{h}{k_{h t r}} T_{s}=\frac{h}{k_{h t r}} T_{\infty} & , \mathrm{z}_{\mathrm{htr}}=\mathrm{H}_{\mathrm{htr}} \\
\frac{d T_{h t r}}{d z_{h t r}}=\frac{q_{L}}{k_{h t r}} & , \mathrm{z}_{\mathrm{htr}}=0
\end{array}
$$

$\underline{\text { Solution }}$

$$
T_{h t r}(z)=\frac{-g}{2 k_{h t r}} z_{h t r}^{2}+\frac{q_{L}}{k_{h t r}} z_{h t r}+T\left(z_{h t r}=0\right)
$$

Substrate Layer: The governing equation for the substrate layer was found from Equation C21 above and includes no internal heat generation. The boundary conditions at the heater and substrate and also the substrate and pedestal interfaces are identical and were found from Equation C22 above and involve one-dimensional heat conduction in the positive $\mathrm{z}$ direction. Contact resistances were neglected. These equations can be used to produce the temperature distribution solution in the substrate layer, Equation C29.

\section{Governing Equation}

$$
\frac{d^{2} T_{s u b}}{d z_{s u b}^{2}}+\frac{g}{k_{s u b}}=0
$$

\section{$\underline{\text { Boundary Condition }}$}

$$
\frac{d T_{\text {sub }}}{d z_{\text {sub }}}=\frac{-q_{L}}{k_{\text {sub }}}, \mathrm{z}_{\text {sub }}=0 \& \mathrm{H}_{\text {sub }}
$$




\section{$\underline{\text { Solution }}$}

$$
T_{\text {sub }}(z)=\frac{-q_{L}}{k_{\text {sub }}} z_{\text {sub }}+T\left(z_{\text {sub }}=0\right)
$$

Since the temperatures at the layer interfaces are equal (i.e. the temperature at the bottom of the heater equals the temperature at the top of the substrate, and the temperature at the bottom of the substrate equals the temperature at the top of the pedestal), the solutions can be combined to relate the surface temperature to the temperature measured at the top of the pedestal, $\mathrm{T}_{\mathrm{int}}$, as shown in Equation C30.

$$
T_{s}=\frac{-g H_{h t r}{ }^{2}}{2 k_{h t r}}+\frac{q_{L} H_{h t r}}{k_{h t r}}+\frac{q_{L} H_{s u b}}{k_{\text {sub }}}+T_{\text {int }}
$$




\section{Appendix D: Raw Data and Data Reduction}

This appendix explains the data reduction procedure for the three studies conducted, presents the raw data obtained, and presents the tables of reduced data. Although the data reduction steps were very similar for each, the procedure of collecting data varied. Chapter 3 explains the procedure of each test in detail. The resulting tables show the heat fluxes, subcooling, convection heat transfer coefficients, Nusselt numbers, etc., for the spacing comparison, cap electrode study, and Mehra electrode study. An error analysis is presented in Appendix E.

\section{Data Reduction Explanation}

Saturation temperatures, $\mathrm{T}_{\text {sat }}$, of the FC-72 (or HFE-7000) at each power level were found based on the recorded chamber pressures. A saturation temperature data table based on pressure was given by the manufacturer and the values were interpolated. The heat flux, q, was calculated by dividing the power levels by the estimated heater surface area. For the pedestals used for the spacing comparison and cap electrodes, this area was measured to be $1.47 \mathrm{~cm}^{2}$ (which omits the pedestal area covered by the silver conductive epoxy and the regions of the pedestal without any TFR heater present). For the Mehra electrode pedestal this area was measured to be $0.982 \mathrm{~cm}^{2}$. The surface temperature $\left(\mathrm{T}_{\mathrm{s}}\right)$ was found by deriving an equation from the energy equation to relate the surface temperature to the interface temperature $\left(\mathrm{T}_{\text {int }}\right)$ as shown in Appendix C. The relation found for the spacing comparison is shown in Equation D1, the relation for the cap electrode study is shown in Equation D2, and the relation for the Mehra electrode study is shown in Equation D3. In these equations, $\mathrm{q}$ is the heat flux in the respective direction, $\mathrm{H}$ 
is the thickness of the layer, $\mathrm{k}$ is the thermal conductivity of the layer, and $\mathrm{g}$ is the volumetric heat generation within the heater material which lies between the top glass layer and the lower alumina substrate.

$$
\begin{aligned}
& T_{s}=\frac{-q_{T} H_{\text {glass }}}{k_{\text {glass }}}+\frac{-g H_{h t r}{ }^{2}}{2 k_{h t r}}+\frac{q_{L} H_{h t r}}{k_{h t r}}+\frac{q_{L} H_{s u b}}{k_{\text {sub }}}+T_{\text {int }} \\
& T_{s}=\frac{-g H_{h t r}^{2}}{2 k_{h t r}}+\frac{q_{L} H_{h t r}}{k_{h t r}}+\frac{q_{L} H_{\text {sub }}}{k_{\text {sub }}}+T_{\text {int }} \\
& T_{s}=\frac{-q_{T} H_{\text {alumina }}}{k_{\text {alumina }}}+\frac{-g H_{h t r}{ }^{2}}{2 k_{h t r}}+\frac{q_{L} H_{h t r}}{k_{h t r}}+\frac{q_{L} H_{\text {sub }}}{k_{\text {sub }}}+T_{\text {int }}
\end{aligned}
$$

The properties of the TFR layers were assumed to be constant and are shown in Table D1. The thicknesses of each layer of the TFR were found in Appendix A. The alumina also was assumed to have constant properties with a thickness of $2 \pm 0.1 \mathrm{~mm}$ and a thermal conductivity of $33 \mathrm{~W} / \mathrm{mK}$.

Table D1: Thicknesses and Thermal Conductivity of TFR Layers

\begin{tabular}{|c|c|c|c|}
\hline Layer & $\begin{array}{c}\text { TFR Type 1 } \\
\text { H (microns) }\end{array}$ & $\begin{array}{c}\text { TFR Type 2 } \\
\text { H (microns) }\end{array}$ & k (W/mK) \\
\hline Glass & $40 \pm 5$ & --- & 1.04 \\
\hline Heater & $8 \pm 2$ & $20 \pm 5$ & 1.04 \\
\hline Substrate & $634 \pm 10$ & $634 \pm 10$ & 27 \\
\hline
\end{tabular}

Next, the average of thermocouples 10 and 11 was used to find the fluid temperature at the side of the pedestal entering the sump ( $\mathrm{T}_{\infty}$ wall) and the average of thermocouples 11 and 14 was used to find the average fluid temperature at the top of the pedestal $\left(\mathrm{T}_{\infty \text { top }}\right)$. The locations of these thermocouples can be seen in Figure 3.20.

Subcooling temperature levels at two locations were found by first finding the difference between the saturation temperature and the temperature at the side of the 
pedestal $\left(T_{\text {sat }}-T_{\infty}\right.$ wall $)$, and second finding the difference between the saturation temperature and the top of the pedestal $\left(\mathrm{T}_{\text {sat }}-\mathrm{T}_{\infty}\right.$ top $)$.

A program called f-chart was used to define the properties of the FC-72 fluid. The program provided a table of thermal conductivities at specified temperatures. The data was graphed and is shown in Figure D1 along with a linear curve fit to the data.

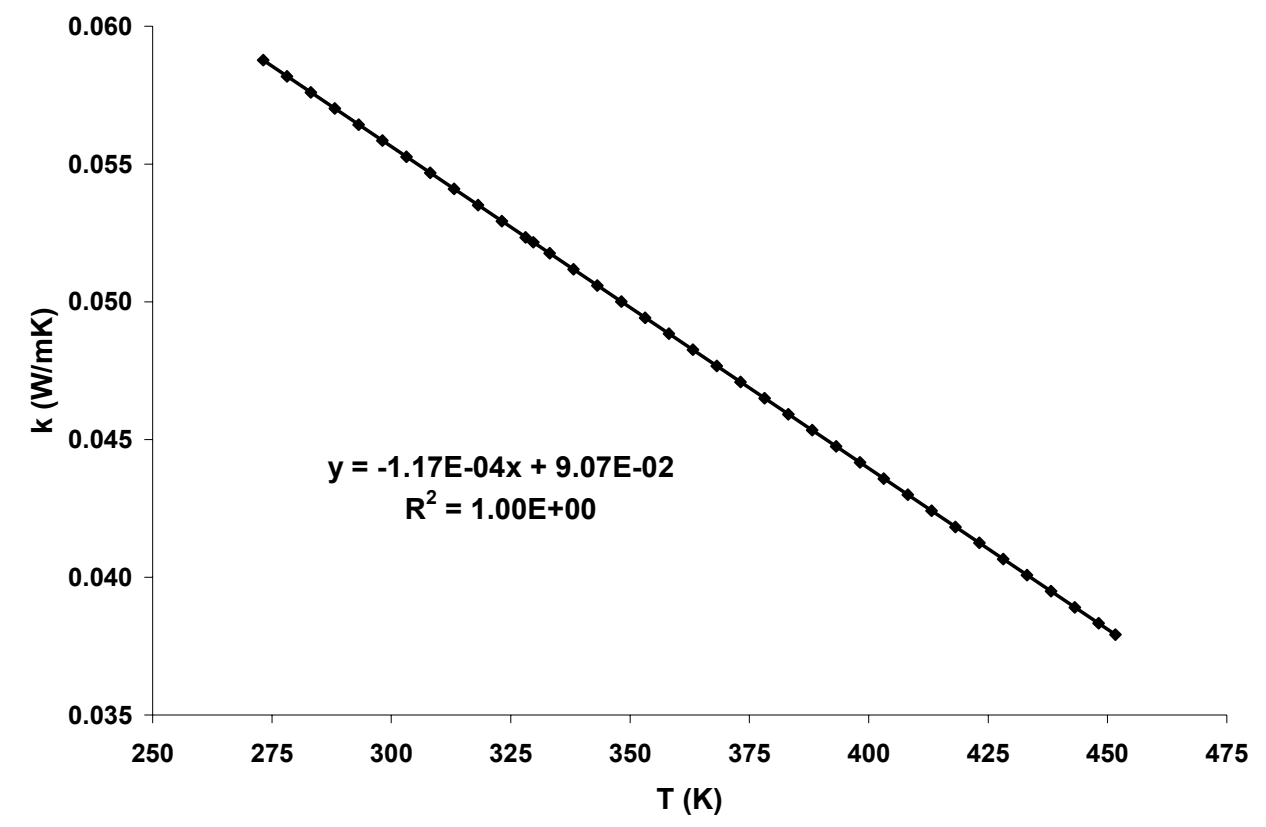

Figure D1: Plot of Thermal Conductivity vs. Temperature from the F-chart Program Data

Based on the linear curve fit of the thermal conductivity of FC-72 versus temperature obtained from the f-chart program, the thermal conductivity of the fluid at the top of the pedestal was found as in Equation D4 where $\mathrm{k}_{\text {slope }}$ is $-1.17 \mathrm{E}-04$ and $\mathrm{k}_{\text {intercept }}$ is $9.07 \mathrm{E}-02$.

$$
k_{\text {fluid }}=k_{\text {slope }} T_{\text {otop }}+k_{\text {intercept }}
$$

The thermal conductivity of the HFE-7000 fluid was found from a thermal conductivity versus temperature equation given by the manufacturer similar to Equation D4 where $\mathrm{k}_{\text {slope }}$ is $-1.96 \mathrm{E}-04$ and $\mathrm{k}_{\text {intercept }}$ is $7.98 \mathrm{E}-02$. 
Next, to find the convective heat transfer coefficient, $\mathrm{h}$, the boundary condition at the top of the pedestal, shown in Equation D5 (and also shown in Appendix C) was used.

$$
h=\frac{(1-f) q}{\left(T_{s}-T_{\text {otop }}\right)}
$$

From this, the Nusselt number was defined as shown in Equation D6.

$$
N u=\frac{h b}{k_{\text {fluid }}}
$$

Finally, the non-dimensional heat flux parameter G $\Delta$ was defined as in Equation D7.

$$
G \Delta=\frac{(1-f) Q}{\pi b\left(T_{\text {sat }}-T_{\text {sowall }}\right) k_{h t r}}
$$

For plotting purposes, the difference between the surface temperature and the temperature at the top of the pedestal $\left(\mathrm{T}_{\mathrm{s}}-\mathrm{T}_{\text {otop }}\right)$ was calculated. The non-dimensional form, $\left(\theta_{\mathrm{s}}-\theta_{\infty \mathrm{otop}}\right)$, was also found by dividing this by $\mathrm{T}_{\text {sat }}-\mathrm{T}_{\text {} \infty \text { wall }}$.

For the spacing comparison, the values obtained when each instrument was read were simply used in the subsequent data reduction. For the electrode studies, the data acquisition read values each fraction of a second. Therefore, for each test condition, values over the period of approximately one minute, while the experimental conditions (heater power, spray flow rate, electrode voltage, etc.) were all held constant, were averaged to obtain a single value for use in data reduction. A similar method was used for the Mehra electrode study, but values were averaged over a period of thirty seconds.

\section{Spacing Comparison Raw Data and Data Reduction Tables}

Raw data for the confined case of the spacing comparison is shown in Table D2 through Table D16. Similar tables for the unconfined case is shown in Table D17 
through Table D34. Table D35 through Table D45 show the reduced data for the confined and unconfined flows at each spacing.

Table D2: Power, Flow Rates, and Pressure Data for Confined $9 \mathrm{~mm}$ Spacing

\begin{tabular}{|c|c|c|c|c|c|c|c|c|c|c|}
\hline & \multicolumn{9}{|c|}{ Flow Rates $\left(\mathbf{G P H}\right.$ of $\left.\mathbf{H}_{\mathbf{2}} \mathbf{O}\right)$} & \multicolumn{5}{c|}{ Pressures } \\
\hline Sample & $\mathbf{Q}$ & $\mathbf{F C - 7 2}$ & Sump & Water & Sump & Nozzle & Chamber & Tuthill & In Res & Water \\
\hline$\#$ & $\mathbf{W}$ & $\mathbf{G P H}$ & $\mathbf{G P H}$ & $\mathbf{G P H}$ & $\mathbf{p s i a}$ & psia & psia & psia & psia & psia \\
\hline $\mathbf{1}$ & 0.00 & 9.26 & 9.07 & 12.00 & 13.28 & 21.51 & 13.28 & 22.81 & 15.01 & 15.31 \\
\hline $\mathbf{2}$ & 0.00 & 9.26 & 9.07 & 12.00 & 13.23 & 21.51 & 14.91 & 22.81 & 15.01 & 15.31 \\
\hline $\mathbf{3}$ & 10.03 & 9.27 & 9.07 & 12.00 & 13.32 & 21.56 & 14.91 & 22.81 & 15.01 & 15.31 \\
\hline $\mathbf{4}$ & 20.02 & 9.27 & 9.07 & 12.00 & 13.32 & 21.61 & 15.01 & 22.81 & 15.01 & 15.31 \\
\hline $\mathbf{5}$ & 29.89 & 9.27 & 9.08 & 12.00 & 13.23 & 21.71 & 15.01 & 23.06 & 15.01 & 15.31 \\
\hline $\mathbf{6}$ & 39.93 & 9.27 & 9.08 & 12.00 & 13.32 & 21.81 & 15.06 & 23.06 & 15.01 & 15.31 \\
\hline $\mathbf{7}$ & 49.85 & 9.28 & 9.08 & 12.00 & 13.32 & 21.91 & 15.21 & 23.31 & 15.01 & 15.31 \\
\hline $\mathbf{8}$ & 59.89 & 9.28 & 9.09 & 12.00 & 13.52 & 21.91 & 15.31 & 23.31 & 15.01 & 15.31 \\
\hline $\mathbf{9}$ & 70.07 & 9.28 & 9.28 & 12.00 & 13.57 & 22.06 & 15.51 & 23.31 & 14.91 & 15.31 \\
\hline
\end{tabular}

Table D3: Room and Pedestal Temperatures for Confined $9 \mathrm{~mm}$ Spacing

\begin{tabular}{|c|c|c|c|c|c|c|c|c|}
\hline Sample & $\mathbf{Q}$ & $\mathbf{R o o m}$ Temp & $\mathbf{T 1}$ & $\mathbf{T 2}$ & $\mathbf{T 3}$ & $\mathbf{T 4}$ & $\mathbf{T 5}$ & T6 \\
\hline$\#$ & $\mathbf{W}$ & ${ }^{\circ} \mathbf{C}$ & ${ }^{\circ} \mathbf{C}$ & ${ }^{\circ} \mathbf{C}$ & ${ }^{\circ} \mathbf{C}$ & ${ }^{\circ} \mathbf{C}$ & ${ }^{\circ} \mathbf{C}$ & ${ }^{\circ} \mathbf{C}$ \\
\hline $\mathbf{1}$ & 0.00 & 26.10 & 29.98 & 29.43 & 29.44 & 29.43 & 29.47 & 29.29 \\
\hline $\mathbf{2}$ & 0.00 & 26.10 & 29.98 & 29.43 & 29.34 & 29.33 & 29.47 & 29.19 \\
\hline $\mathbf{3}$ & 10.03 & 26.10 & 40.81 & 37.03 & 33.74 & 34.01 & 35.85 & 33.95 \\
\hline $\mathbf{4}$ & 20.02 & 26.10 & 51.29 & 44.63 & 37.93 & 38.60 & 42.23 & 38.72 \\
\hline $\mathbf{5}$ & 29.89 & 26.20 & 61.36 & 51.73 & 42.13 & 43.18 & 48.21 & 43.38 \\
\hline $\mathbf{6}$ & 39.93 & 26.30 & 70.69 & 58.23 & 46.22 & 47.36 & 53.79 & 47.85 \\
\hline $\mathbf{7}$ & 49.85 & 26.40 & 79.21 & 64.13 & 50.12 & 51.35 & 58.98 & 52.02 \\
\hline $\mathbf{8}$ & 59.89 & 26.50 & 86.63 & 69.13 & 53.41 & 54.83 & 63.37 & 55.29 \\
\hline $\mathbf{9}$ & 70.07 & 26.40 & 93.25 & 73.33 & 55.81 & 57.52 & 67.05 & 57.87 \\
\hline
\end{tabular}

Table D4: Chamber Temperatures for Confined $9 \mathrm{~mm}$ Spacing

\begin{tabular}{|c|c|c|c|c|c|c|c|c|}
\hline Sample & $\mathbf{Q}$ & $\mathbf{T 8}$ & $\mathbf{T 9}$ & $\mathbf{T 1 0}$ & $\mathbf{T 1 1}$ & $\mathbf{T 1 2}$ & $\mathbf{T 1 3}$ & $\mathbf{T 1 4}$ \\
\hline$\#$ & $\mathbf{W}$ & ${ }^{\circ} \mathbf{C}$ & ${ }^{\circ} \mathbf{C}$ & ${ }^{\circ} \mathbf{C}$ & ${ }^{\circ} \mathbf{C}$ & ${ }^{\circ} \mathbf{C}$ & ${ }^{\circ} \mathbf{C}$ & ${ }^{\circ} \mathbf{C}$ \\
\hline $\mathbf{1}$ & 0.00 & 27.77 & 28.74 & 29.38 & 29.25 & 29.26 & 28.06 & 29.38 \\
\hline $\mathbf{2}$ & 0.00 & 27.67 & 28.55 & 29.38 & 29.25 & 29.16 & 28.06 & 29.38 \\
\hline $\mathbf{3}$ & 10.03 & 27.67 & 28.55 & 30.08 & 29.75 & 30.05 & 27.96 & 29.48 \\
\hline $\mathbf{4}$ & 20.02 & 27.77 & 28.65 & 30.87 & 30.44 & 31.04 & 27.96 & 29.48 \\
\hline $\mathbf{5}$ & 29.89 & 27.77 & 28.74 & 31.87 & 31.23 & 32.14 & 29.45 & 29.48 \\
\hline $\mathbf{6}$ & 39.93 & 27.87 & 28.94 & 32.96 & 32.02 & 33.43 & 28.06 & 29.48 \\
\hline $\mathbf{7}$ & 49.85 & 28.07 & 29.14 & 34.05 & 32.91 & 34.42 & 28.26 & 29.58 \\
\hline $\mathbf{8}$ & 59.89 & 28.17 & 29.44 & 35.04 & 34.00 & 36.01 & 28.26 & 29.58 \\
\hline $\mathbf{9}$ & 70.07 & 28.26 & 29.64 & 36.14 & 35.29 & 37.30 & 28.26 & 29.58 \\
\hline
\end{tabular}


Table D5: Power, Flow Rates, and Pressure Data for Confined $11 \mathrm{~mm}$ Spacing

\begin{tabular}{|c|c|c|c|c|c|c|c|c|c|c|}
\hline & \multicolumn{3}{|c|}{ Flow Rates (GPH of $\left.\mathbf{H}_{\mathbf{2}} \mathbf{O}\right)$} & \multicolumn{5}{|c|}{ Pressures } \\
\hline Sample & $\mathbf{Q}$ & FC-72 & Sump & Water & Sump & Nozzle & Chamber & Tuthill & In Res & Water \\
\hline$\#$ & W & GPH & GPH & GPH & psia & psia & psia & psia & psia & psia \\
\hline $\mathbf{1}$ & 0.00 & 9.20 & 9.01 & 12.00 & 13.23 & 21.81 & 14.91 & 23.06 & 15.11 & 15.21 \\
\hline $\mathbf{2}$ & 0.00 & 9.20 & 9.01 & 12.00 & 13.23 & 21.71 & 14.91 & 23.06 & 15.11 & 15.21 \\
\hline $\mathbf{3}$ & 10.03 & 9.20 & 9.01 & 12.00 & 13.23 & 21.71 & 14.91 & 23.06 & 15.11 & 15.21 \\
\hline $\mathbf{4}$ & 19.89 & 9.20 & 9.01 & 12.00 & 13.32 & 21.71 & 15.01 & 23.06 & 15.11 & 15.21 \\
\hline $\mathbf{5}$ & 30.08 & 9.20 & 9.01 & 12.00 & 13.32 & 21.91 & 15.06 & 23.06 & 15.11 & 15.21 \\
\hline $\mathbf{6}$ & 40.15 & 9.20 & 9.01 & 12.00 & 13.32 & 22.01 & 15.21 & 23.31 & 15.11 & 15.21 \\
\hline $\mathbf{7}$ & 50.09 & 9.20 & 9.01 & 12.00 & 13.47 & 22.11 & 15.31 & 23.31 & 15.11 & 15.21 \\
\hline $\mathbf{8}$ & 59.89 & 9.20 & 9.01 & 12.00 & 13.62 & 22.11 & 15.41 & 23.31 & 15.11 & 15.21 \\
\hline $\mathbf{9}$ & 70.07 & 9.20 & 9.20 & 12.00 & 13.77 & 22.21 & 15.56 & 23.56 & 15.11 & 15.31 \\
\hline
\end{tabular}

Table D6: Room and Pedestal Temperatures for Confined $11 \mathrm{~mm}$ Spacing

\begin{tabular}{|c|c|c|c|c|c|c|c|c|}
\hline Sample & $\mathbf{Q}$ & $\mathbf{R o o m} \mathbf{T e m p}$ & $\mathbf{T 1}$ & ${ }^{\mathbf{T}}$ & ${ }^{\mathbf{T} 3}$ & ${ }^{\mathbf{T}}$ & ${ }^{\mathbf{T} 5}$ & T6 \\
\hline$\#$ & $\mathbf{W}$ & ${ }^{\circ} \mathbf{C}$ & ${ }^{\circ} \mathbf{C}$ & ${ }^{\circ} \mathbf{C}$ & ${ }^{\circ} \mathbf{C}$ & ${ }^{\circ} \mathbf{C}$ & ${ }^{\circ} \mathbf{C}$ & ${ }^{\circ} \mathbf{C}$ \\
\hline $\mathbf{1}$ & 0.00 & 25.90 & 29.48 & 28.83 & 28.85 & 28.83 & 28.97 & 28.69 \\
\hline $\mathbf{2}$ & 0.00 & 25.90 & 29.48 & 28.93 & 28.94 & 28.93 & 28.97 & 28.79 \\
\hline $\mathbf{3}$ & 10.03 & 25.90 & 39.61 & 35.93 & 32.94 & 33.32 & 34.85 & 33.36 \\
\hline $\mathbf{4}$ & 19.89 & 25.90 & 49.23 & 43.03 & 36.83 & 37.30 & 40.63 & 37.43 \\
\hline $\mathbf{5}$ & 30.08 & 25.90 & 58.81 & 49.83 & 40.83 & 41.48 & 46.42 & 41.79 \\
\hline $\mathbf{6}$ & 40.15 & 26.00 & 67.88 & 56.13 & 44.72 & 45.47 & 51.80 & 46.06 \\
\hline $\mathbf{7}$ & 50.09 & 26.00 & 76.05 & 61.73 & 48.32 & 49.16 & 56.59 & 49.83 \\
\hline $\mathbf{8}$ & 59.89 & 26.00 & 83.32 & 66.53 & 51.41 & 52.54 & 60.97 & 53.11 \\
\hline $\mathbf{9}$ & 70.07 & 26.00 & 90.04 & 71.03 & 54.01 & 55.33 & 64.76 & 55.99 \\
\hline
\end{tabular}

Table D7: Chamber Temperatures for Confined $11 \mathrm{~mm}$ Spacing

\begin{tabular}{|c|c|c|c|c|c|c|c|c|}
\hline Sample & $\mathbf{Q}$ & $\mathbf{T 8}$ & ${ }^{\mathbf{T} 9}$ & ${ }^{\mathbf{T 1 0}}$ & ${ }^{\mathbf{T 1 1}}$ & $\mathbf{T 1 2}$ & ${ }^{\mathbf{T 1 3}}$ & ${ }^{\mathbf{T 1 4}}$ \\
\hline $\boldsymbol{\#}$ & $\mathbf{W}$ & ${ }^{\circ} \mathbf{C}$ & ${ }^{\circ} \mathbf{C}$ & ${ }^{\circ} \mathbf{C}$ & ${ }^{\circ} \mathbf{C}$ & ${ }^{\circ} \mathbf{C}$ & ${ }^{\circ} \mathbf{C}$ & ${ }^{\circ} \mathbf{C}$ \\
\hline $\mathbf{1}$ & 0.00 & 27.97 & 28.15 & 28.79 & 28.76 & 28.76 & 27.86 & 28.98 \\
\hline $\mathbf{2}$ & 0.00 & 27.97 & 28.05 & 28.79 & 28.85 & 28.76 & 27.86 & 28.98 \\
\hline $\mathbf{3}$ & 10.03 & 28.46 & 28.15 & 29.68 & 29.55 & 29.65 & 27.86 & 29.08 \\
\hline $\mathbf{5}$ & 19.89 & 28.76 & 28.65 & 30.48 & 30.14 & 30.65 & 27.86 & 28.98 \\
\hline $\mathbf{6}$ & 40.08 & 29.16 & 29.14 & 31.47 & 30.93 & 31.74 & 27.96 & 29.08 \\
\hline $\mathbf{7}$ & 50.09 & 28.96 & 30.33 & 33.46 & 32.62 & 33.92 & 28.16 & 29.18 \\
\hline $\mathbf{8}$ & 59.89 & 28.96 & 30.92 & 33.95 & 33.41 & 35.02 & 28.16 & 29.18 \\
\hline $\mathbf{9}$ & 70.07 & 29.06 & 31.52 & 34.75 & 34.30 & 36.31 & 28.16 & 29.18 \\
\hline
\end{tabular}


Table D8: Power, Flow Rates, and Pressure Data for Confined $13 \mathrm{~mm}$ Spacing

\begin{tabular}{|c|c|c|c|c|c|c|c|c|c|c|}
\hline & & \multicolumn{2}{|c|}{ Flow Rates $\left(\mathbf{G P H}\right.$ of $\left.\mathbf{H}_{\mathbf{2}} \mathbf{O}\right)$} & \multicolumn{5}{|c|}{ Pressures } \\
\hline Sample & $\mathbf{Q}$ & $\mathbf{F C - 7 2}$ & Sump & Water & Sump & Nozzle & Chamber & Tuthill & In Res & Water \\
\hline$\#$ & W & GPH & GPH & GPH & psia & psia & psia & psia & psia & psia \\
\hline $\mathbf{1}$ & 0.00 & 9.20 & 9.01 & 12.00 & 13.13 & 21.51 & 14.81 & 22.81 & 15.11 & 15.21 \\
\hline $\mathbf{2}$ & 0.00 & 9.20 & 9.01 & 12.00 & 13.13 & 21.51 & 14.81 & 22.81 & 15.11 & 15.21 \\
\hline $\mathbf{3}$ & 10.03 & 9.20 & 9.01 & 12.00 & 13.13 & 21.56 & 14.91 & 22.81 & 15.11 & 15.21 \\
\hline $\mathbf{4}$ & 19.89 & 9.20 & 9.01 & 12.00 & 13.18 & 21.71 & 15.01 & 22.81 & 15.11 & 15.21 \\
\hline $\mathbf{5}$ & 29.89 & 9.20 & 9.01 & 12.00 & 13.32 & 21.71 & 15.06 & 23.06 & 15.11 & 15.21 \\
\hline $\mathbf{6}$ & 39.93 & 9.20 & 9.01 & 12.00 & 13.32 & 21.81 & 15.21 & 23.06 & 15.11 & 15.21 \\
\hline $\mathbf{7}$ & 50.06 & 9.20 & 9.01 & 12.00 & 13.52 & 21.91 & 15.31 & 23.31 & 15.11 & 15.21 \\
\hline $\mathbf{8}$ & 60.12 & 9.20 & 9.01 & 12.00 & 13.67 & 22.01 & 15.41 & 23.31 & 15.11 & 15.21 \\
\hline $\mathbf{9}$ & 70.07 & 9.20 & 9.20 & 12.00 & 13.77 & 22.06 & 15.51 & 23.31 & 15.11 & 15.21 \\
\hline
\end{tabular}

Table D9: Room and Pedestal Temperatures for Confined $13 \mathrm{~mm}$ Spacing

\begin{tabular}{|c|c|c|c|c|c|c|c|c|}
\hline Sample & $\mathbf{Q}$ & Room Temp & ${ }^{\mathbf{T 1}}$ & ${ }^{\mathbf{T}}$ & ${ }^{\mathbf{T 3}}$ & ${ }^{\mathbf{T} 4}$ & ${ }^{\mathbf{T} 5}$ & ${ }^{\mathbf{T} 6}$ \\
\hline$\#$ & $\mathbf{W}$ & ${ }^{\circ} \mathbf{C}$ & ${ }^{\circ} \mathbf{C}$ & ${ }^{\circ} \mathbf{C}$ & ${ }^{\circ} \mathbf{C}$ & ${ }^{\circ} \mathbf{C}$ & ${ }^{\circ} \mathbf{C}$ & ${ }^{\circ} \mathbf{C}$ \\
\hline $\mathbf{1}$ & 0.00 & 25.60 & 28.88 & 28.33 & 28.25 & 28.34 & 28.37 & 28.20 \\
\hline $\mathbf{2}$ & 0.00 & 25.60 & 28.98 & 28.33 & 28.35 & 28.34 & 28.37 & 28.20 \\
\hline $\mathbf{3}$ & 10.03 & 25.60 & 39.20 & 35.63 & 32.54 & 32.82 & 34.35 & 32.86 \\
\hline $\mathbf{4}$ & 19.89 & 25.60 & 49.03 & 42.63 & 36.43 & 36.90 & 40.23 & 36.93 \\
\hline $\mathbf{5}$ & 29.89 & 25.60 & 58.56 & 49.33 & 40.43 & 40.99 & 45.92 & 41.10 \\
\hline $\mathbf{6}$ & 39.93 & 25.60 & 67.78 & 55.83 & 44.32 & 45.07 & 51.40 & 45.37 \\
\hline $\mathbf{7}$ & 50.06 & 25.70 & 76.00 & 61.53 & 48.02 & 48.96 & 56.29 & 49.34 \\
\hline $\mathbf{8}$ & 60.12 & 25.70 & 83.62 & 66.63 & 51.51 & 52.54 & 60.77 & 52.71 \\
\hline $\mathbf{9}$ & 70.07 & 25.70 & 90.44 & 71.13 & 54.21 & 55.33 & 64.76 & 55.59 \\
\hline
\end{tabular}

Table D10: Chamber Temperatures for Confined $13 \mathrm{~mm}$ Spacing

\begin{tabular}{|c|c|c|c|c|c|c|c|c|}
\hline Sample & $\mathbf{Q}$ & $\mathbf{T 8}$ & $\mathbf{T 9}$ & ${ }^{\mathbf{T 1 0}}$ & $\mathbf{T 1 1}$ & $\mathbf{T 1 2}$ & $\mathbf{T 1 3}$ & $\mathbf{T 1 4}$ \\
\hline$\#$ & $\mathbf{W}$ & ${ }^{\circ} \mathbf{C}$ & ${ }^{\circ} \mathbf{C}$ & ${ }^{\circ} \mathbf{C}$ & ${ }^{\circ} \mathbf{C}$ & ${ }^{\circ} \mathbf{C}$ & ${ }^{\circ} \mathbf{C}$ & ${ }^{\circ} \mathbf{C}$ \\
\hline $\mathbf{1}$ & 0.00 & 27.37 & 27.55 & 28.19 & 28.16 & 28.16 & 27.56 & 28.39 \\
\hline $\mathbf{2}$ & 0.00 & 27.37 & 27.55 & 28.29 & 28.26 & 28.16 & 27.56 & 28.49 \\
\hline $\mathbf{3}$ & 10.03 & 28.07 & 27.95 & 29.09 & 29.15 & 29.16 & 27.76 & 28.59 \\
\hline $\mathbf{4}$ & 19.89 & 28.66 & 28.45 & 29.88 & 29.84 & 30.15 & 27.76 & 28.49 \\
\hline $\mathbf{5}$ & 29.89 & 29.16 & 28.84 & 30.77 & 30.74 & 31.24 & 27.76 & 28.49 \\
\hline $\mathbf{6}$ & 39.93 & 29.66 & 29.34 & 31.67 & 31.63 & 32.34 & 27.76 & 28.49 \\
\hline $\mathbf{7}$ & 50.06 & 30.06 & 29.83 & 32.56 & 32.52 & 33.43 & 27.96 & 28.59 \\
\hline $\mathbf{8}$ & 60.12 & 30.36 & 30.43 & 33.55 & 33.41 & 34.62 & 28.06 & 28.69 \\
\hline $\mathbf{9}$ & 70.07 & 30.36 & 31.02 & 34.25 & 34.50 & 35.91 & 28.06 & 28.79 \\
\hline
\end{tabular}


Table D11: Power, Flow Rates, and Pressure Data for Confined $15 \mathrm{~mm}$ Spacing

\begin{tabular}{|c|c|c|c|c|c|c|c|c|c|c|}
\hline & \multicolumn{9}{|c|}{ Flow Rates (GPH of $\left.\mathbf{H}_{\mathbf{2}} \mathbf{O}\right)$} & \multicolumn{5}{|c|}{ Pressures } \\
\hline Sample & $\mathbf{Q}$ & $\mathbf{F C - 7 2}$ & Sump & Water & Sump & Nozzle & Chamber & Tuthill & In Res & Water \\
\hline$\#$ & $\mathbf{W}$ & $\mathbf{G P H}$ & $\mathbf{G P H}$ & $\mathbf{G P H}$ & $\mathbf{p s i a}$ & psia & psia & psia & psia & psia \\
\hline $\mathbf{1}$ & 0.00 & 9.20 & 9.01 & 12.00 & 13.23 & 21.56 & 14.91 & 22.81 & 14.81 & 15.31 \\
\hline $\mathbf{2}$ & 0.00 & 9.20 & 9.01 & 12.00 & 13.13 & 21.51 & 14.91 & 22.81 & 14.81 & 15.31 \\
\hline $\mathbf{3}$ & 10.03 & 9.20 & 9.01 & 12.00 & 13.08 & 21.61 & 14.91 & 22.81 & 14.81 & 15.31 \\
\hline $\mathbf{4}$ & 19.89 & 9.20 & 9.01 & 12.00 & 13.13 & 21.61 & 15.01 & 22.81 & 14.81 & 15.31 \\
\hline $\mathbf{5}$ & 29.89 & 9.20 & 9.01 & 12.00 & 13.23 & 21.71 & 15.11 & 23.06 & 14.81 & 15.31 \\
\hline $\mathbf{6}$ & 39.93 & 9.20 & 9.01 & 12.00 & 13.32 & 21.71 & 15.11 & 23.06 & 14.81 & 15.31 \\
\hline $\mathbf{7}$ & 49.85 & 9.20 & 9.01 & 12.00 & 13.32 & 21.81 & 15.31 & 23.06 & 14.81 & 15.31 \\
\hline $\mathbf{8}$ & 59.89 & 9.20 & 9.01 & 12.00 & 13.52 & 21.91 & 15.41 & 23.06 & 14.81 & 15.31 \\
\hline $\mathbf{9}$ & 70.07 & 9.20 & 9.20 & 12.00 & 13.67 & 22.01 & 15.51 & 23.31 & 14.81 & 15.31 \\
\hline
\end{tabular}

Table D12: Room and Pedestal Temperatures for Confined $15 \mathrm{~mm}$ Spacing

\begin{tabular}{|c|c|c|c|c|c|c|c|c|}
\hline Sample & $\mathbf{Q}$ & $\mathbf{R o o m} \mathbf{T e m p}$ & $\mathbf{T 1}$ & $\mathbf{T 2}^{\mathbf{T}}$ & ${ }^{\mathbf{T}}$ & ${ }^{\mathbf{T} 4}$ & ${ }^{\mathbf{T} 5}$ & ${ }^{\mathbf{T} 6}$ \\
\hline$\#$ & $\mathbf{W}$ & ${ }^{\circ} \mathbf{C}$ & ${ }^{\circ} \mathbf{C}$ & ${ }^{\circ} \mathbf{C}$ & ${ }^{\circ} \mathbf{C}$ & ${ }^{\circ} \mathbf{C}$ & ${ }^{\circ} \mathbf{C}$ & ${ }^{\circ} \mathbf{C}$ \\
\hline $\mathbf{1}$ & 0.00 & 26.40 & 30.18 & 29.63 & 29.54 & 29.63 & 29.66 & 29.49 \\
\hline $\mathbf{2}$ & 0.00 & 26.30 & 30.28 & 29.73 & 29.64 & 29.63 & 29.66 & 29.49 \\
\hline $\mathbf{3}$ & 10.03 & 26.30 & 40.41 & 37.03 & 33.74 & 34.11 & 35.85 & 34.15 \\
\hline $\mathbf{4}$ & 19.89 & 26.30 & 50.38 & 44.23 & 37.53 & 38.50 & 41.93 & 38.72 \\
\hline $\mathbf{5}$ & 29.89 & 26.20 & 60.16 & 51.53 & 41.83 & 42.98 & 48.01 & 43.48 \\
\hline $\mathbf{6}$ & 39.93 & 26.10 & 69.29 & 57.83 & 45.62 & 47.16 & 53.49 & 47.85 \\
\hline $\mathbf{7}$ & 49.85 & 26.20 & 77.26 & 63.53 & 49.32 & 51.10 & 58.48 & 51.72 \\
\hline $\mathbf{8}$ & 59.89 & 26.20 & 84.78 & 68.43 & 52.51 & 54.44 & 62.77 & 54.80 \\
\hline $\mathbf{9}$ & 70.07 & 26.20 & 91.29 & 72.63 & 54.91 & 56.93 & 66.36 & 57.08 \\
\hline
\end{tabular}

Table D13: Chamber Temperatures for Confined $15 \mathrm{~mm}$ Spacing

\begin{tabular}{|c|c|c|c|c|c|c|c|c|}
\hline Sample & $\mathbf{Q}$ & $\mathbf{T 8}$ & ${ }^{\mathbf{T 9}}$ & ${ }^{\mathbf{T 1 0}}$ & ${ }^{\mathbf{T 1 1}}$ & $\mathbf{T 1 2}$ & $\mathbf{T 1 3}$ & $\mathbf{T 1 4}$ \\
\hline$\#$ & $\mathbf{W}$ & ${ }^{\circ} \mathbf{C}$ & ${ }^{\circ} \mathbf{C}$ & ${ }^{\circ} \mathbf{C}$ & ${ }^{\circ} \mathbf{C}$ & ${ }^{\circ} \mathbf{C}$ & ${ }^{\circ} \mathbf{C}$ & ${ }^{\circ} \mathbf{C}$ \\
\hline $\mathbf{1}$ & 0.00 & 28.76 & 28.74 & 29.38 & 29.55 & 29.36 & 28.85 & 29.98 \\
\hline $\mathbf{2}$ & 0.00 & 28.76 & 28.74 & 29.48 & 29.55 & 29.36 & 28.75 & 29.98 \\
\hline $\mathbf{3}$ & 10.03 & 28.96 & 29.04 & 30.08 & 30.04 & 30.25 & 28.95 & 29.98 \\
\hline $\mathbf{4}$ & 19.89 & 29.76 & 29.44 & 30.68 & 30.64 & 31.24 & 29.15 & 30.18 \\
\hline $\mathbf{5}$ & 29.89 & 30.46 & 30.13 & 31.37 & 31.43 & 32.53 & 29.25 & 29.98 \\
\hline $\mathbf{6}$ & 39.93 & 30.76 & 30.53 & 32.26 & 32.32 & 33.73 & 29.25 & 29.98 \\
\hline $\mathbf{7}$ & 49.85 & 30.86 & 30.92 & 33.06 & 33.11 & 34.72 & 29.25 & 29.98 \\
\hline $\mathbf{8}$ & 59.89 & 31.15 & 31.42 & 33.95 & 33.80 & 36.21 & 29.25 & 29.98 \\
\hline $\mathbf{9}$ & 70.07 & 31.85 & 32.11 & 34.55 & 34.50 & 37.70 & 29.25 & 29.98 \\
\hline
\end{tabular}


Table D14: Power, Flow Rates, and Pressure Data for Confined 17 mm Spacing

\begin{tabular}{|c|c|c|c|c|c|c|c|c|c|c|}
\hline & \multicolumn{3}{|c|}{ Flow Rates $\left(\mathbf{G P H}\right.$ of $\left.\mathbf{H}_{\mathbf{2}} \mathbf{O}\right)$} & \multicolumn{5}{c|}{ Pressures } \\
\hline Sample & $\mathbf{Q}$ & $\mathbf{F C - 7 2}$ & Sump & Water & Sump & Nozzle & Chamber & Tuthill & In Res & Water \\
\hline$\#$ & $\mathbf{W}$ & GPH & GPH & GPH & psia & psia & psia & psia & psia & psia \\
\hline $\mathbf{1}$ & 0.00 & 9.20 & 9.01 & 12.00 & 12.98 & 21.11 & 14.71 & 22.81 & 14.81 & 15.31 \\
\hline $\mathbf{2}$ & 0.00 & 9.20 & 9.01 & 12.00 & 12.98 & 21.11 & 14.71 & 22.56 & 14.81 & 15.31 \\
\hline $\mathbf{3}$ & 10.03 & 9.20 & 9.01 & 12.00 & 12.98 & 21.21 & 14.81 & 22.56 & 14.81 & 15.31 \\
\hline $\mathbf{4}$ & 20.02 & 9.20 & 9.01 & 12.00 & 12.98 & 21.21 & 14.91 & 22.56 & 14.81 & 15.31 \\
\hline $\mathbf{5}$ & 29.89 & 9.20 & 9.01 & 12.00 & 13.13 & 21.31 & 14.91 & 22.56 & 14.81 & 15.31 \\
\hline $\mathbf{6}$ & 39.93 & 9.20 & 9.01 & 12.00 & 13.28 & 21.31 & 15.01 & 22.56 & 14.81 & 15.31 \\
\hline $\mathbf{7}$ & 49.85 & 9.20 & 9.01 & 12.00 & 13.32 & 21.41 & 15.11 & 22.81 & 14.81 & 15.31 \\
\hline $\mathbf{8}$ & 59.89 & 9.20 & 9.01 & 12.00 & 13.37 & 21.51 & 15.21 & 22.81 & 14.81 & 15.31 \\
\hline $\mathbf{9}$ & 69.78 & 9.20 & 9.20 & 12.00 & 13.57 & 21.91 & 15.41 & 23.31 & 14.81 & 15.31 \\
\hline
\end{tabular}

Table D15: Room and Pedestal Temperatures for Confined $17 \mathrm{~mm}$ Spacing

\begin{tabular}{|c|c|c|c|c|c|c|c|c|}
\hline Sample & $\mathbf{Q}$ & $\mathbf{R o o m} \mathbf{T e m p}$ & ${ }^{\mathbf{T} 1}$ & ${ }^{\mathbf{T}}$ & ${ }^{\mathbf{T}}$ & ${ }^{\mathbf{T} 4}$ & ${ }^{\mathbf{T} 5}$ & $\mathbf{T 6}$ \\
\hline$\#$ & $\mathbf{W}$ & ${ }^{\circ} \mathbf{C}$ & ${ }^{\circ} \mathbf{C}$ & ${ }^{\circ} \mathbf{C}$ & ${ }^{\circ} \mathbf{C}$ & ${ }^{\circ} \mathbf{C}$ & ${ }^{\circ} \mathbf{C}$ & ${ }^{\circ} \mathbf{C}$ \\
\hline $\mathbf{1}$ & 0.00 & 26.20 & 30.08 & 29.53 & 29.54 & 29.53 & 29.57 & 29.39 \\
\hline $\mathbf{2}$ & 0.00 & 26.10 & 29.98 & 29.53 & 29.54 & 29.53 & 29.57 & 29.39 \\
\hline $\mathbf{3}$ & 10.03 & 26.00 & 39.81 & 35.53 & 32.64 & 32.42 & 34.35 & 32.76 \\
\hline $\mathbf{4}$ & 20.02 & 26.00 & 49.43 & 41.43 & 35.54 & 35.21 & 39.14 & 36.04 \\
\hline $\mathbf{5}$ & 29.89 & 26.00 & 58.86 & 47.43 & 38.58 & 38.20 & 43.92 & 39.51 \\
\hline $\mathbf{6}$ & 39.93 & 26.00 & 67.68 & 53.03 & 41.63 & 41.09 & 48.51 & 42.89 \\
\hline $\mathbf{7}$ & 49.85 & 25.90 & 76.30 & 58.63 & 44.92 & 44.07 & 53.20 & 46.26 \\
\hline $\mathbf{8}$ & 59.89 & 25.90 & 83.72 & 63.33 & 47.62 & 46.67 & 57.28 & 49.34 \\
\hline $\mathbf{9}$ & 69.78 & 25.90 & 90.44 & 67.83 & 50.22 & 49.16 & 61.07 & 52.02 \\
\hline
\end{tabular}

Table D16: Chamber Temperatures for Confined $17 \mathrm{~mm}$ Spacing

\begin{tabular}{|c|c|c|c|c|c|c|c|c|}
\hline Sample & $\mathbf{Q}$ & $\mathbf{T 8}$ & ${ }^{\mathbf{T} 9}$ & ${ }^{\mathbf{T 1 0}}$ & ${ }^{\mathbf{T 1 1}}$ & $\mathbf{T 1 2}$ & ${ }^{\mathbf{T 1 3}}$ & ${ }^{\mathbf{T 1 4}}$ \\
\hline $\boldsymbol{\#}$ & $\mathbf{W}$ & ${ }^{\circ} \mathbf{C}$ & ${ }^{\circ} \mathbf{C}$ & ${ }^{\circ} \mathbf{C}$ & ${ }^{\circ} \mathbf{C}$ & ${ }^{\circ} \mathbf{C}$ & ${ }^{\circ} \mathbf{C}$ & ${ }^{\circ} \mathbf{C}$ \\
\hline $\mathbf{1}$ & 0.00 & 28.76 & 28.84 & 29.38 & 29.35 & 29.36 & 29.05 & 29.98 \\
\hline $\mathbf{2}$ & 0.00 & 28.76 & 28.84 & 29.38 & 29.35 & 29.26 & 28.95 & 29.98 \\
\hline $\mathbf{3}$ & 10.03 & 28.96 & 29.14 & 30.08 & 30.14 & 30.05 & 29.15 & 29.88 \\
\hline $\mathbf{4}$ & 20.02 & 29.46 & 29.54 & 30.87 & 30.93 & 30.85 & 29.45 & 29.88 \\
\hline $\mathbf{5}$ & 29.89 & 29.96 & 30.03 & 31.77 & 31.82 & 31.84 & 29.55 & 29.88 \\
\hline $\mathbf{6}$ & 39.93 & 30.46 & 30.43 & 32.46 & 32.72 & 32.73 & 29.65 & 29.78 \\
\hline $\mathbf{7}$ & 49.85 & 31.45 & 31.12 & 33.46 & 33.80 & 33.73 & 29.75 & 29.68 \\
\hline $\mathbf{8}$ & 59.89 & 32.15 & 31.72 & 34.15 & 34.84 & 34.72 & 29.84 & 29.58 \\
\hline $\mathbf{9}$ & 69.78 & 32.75 & 32.41 & 35.04 & 35.93 & 35.71 & 29.84 & 29.58 \\
\hline
\end{tabular}


Table D17: Power, Flow Rates, and Pressure Data for Unconfined $9 \mathrm{~mm}$ Spacing

\begin{tabular}{|c|c|c|c|c|c|c|c|c|c|c|}
\hline & & \multicolumn{3}{|c|}{ Flow Rates (GPH of $\left.\mathbf{H}_{\mathbf{2}} \mathbf{O}\right)$} & \multicolumn{5}{c|}{ Pressures } \\
\hline Sample & $\mathbf{Q}$ & $\mathbf{F C - 7 2}$ & Sump & Water & Sump & Nozzle & Chamber & Tuthill & In Res & Water \\
\hline$\#$ & $\mathbf{W}$ & GPH & GPH & GPH & psia & psia & psia & psia & psia & psia \\
\hline $\mathbf{1}$ & 0.00 & 9.20 & 9.01 & 12.00 & 13.81 & 22.05 & 15.40 & 23.30 & 17.10 & 15.10 \\
\hline $\mathbf{2}$ & 0.00 & 9.20 & 9.01 & 12.00 & 13.81 & 21.90 & 15.40 & 23.30 & 17.10 & 15.10 \\
\hline $\mathbf{3}$ & 10.03 & 9.20 & 9.01 & 12.00 & 13.81 & 21.90 & 15.40 & 23.30 & 17.10 & 15.10 \\
\hline $\mathbf{4}$ & 20.02 & 9.20 & 9.01 & 12.00 & 13.86 & 22.10 & 15.50 & 23.30 & 17.10 & 15.10 \\
\hline $\mathbf{5}$ & 29.89 & 9.20 & 9.01 & 12.00 & 13.86 & 22.10 & 15.50 & 23.30 & 17.10 & 15.10 \\
\hline $\mathbf{6}$ & 39.93 & 9.20 & 9.01 & 12.00 & 14.06 & 22.20 & 15.70 & 23.30 & 17.10 & 15.10 \\
\hline $\mathbf{7}$ & 50.06 & 9.20 & 9.01 & 12.00 & 14.20 & 22.30 & 15.80 & 23.55 & 17.10 & 15.10 \\
\hline $\mathbf{8}$ & 59.89 & 9.20 & 9.01 & 12.00 & 14.30 & 22.40 & 15.90 & 23.80 & 17.10 & 15.10 \\
\hline $\mathbf{9}$ & 70.03 & 9.20 & 9.20 & 12.00 & 14.30 & 22.50 & 16.10 & 23.80 & 17.10 & 15.10 \\
\hline
\end{tabular}

Table D18: Room and Pedestal Temperatures for Unconfined $9 \mathrm{~mm}$ Spacing

\begin{tabular}{|c|c|c|c|c|c|c|c|c|}
\hline Sample & $\mathbf{Q}$ & $\mathbf{R o o m} \mathbf{T e m p}$ & ${ }^{\mathbf{T} 1}$ & ${ }^{\mathbf{T}}$ & ${ }^{\mathbf{T}}$ & ${ }^{\mathbf{T} 4}$ & ${ }^{\mathbf{T} 5}$ & ${ }^{\mathbf{T} 6}$ \\
\hline$\#$ & $\mathbf{W}$ & ${ }^{\circ} \mathbf{C}$ & ${ }^{\circ} \mathbf{C}$ & ${ }^{\circ} \mathbf{C}$ & ${ }^{\circ} \mathbf{C}$ & ${ }^{\circ} \mathbf{C}$ & ${ }^{\circ} \mathbf{C}$ & ${ }^{\circ} \mathbf{C}$ \\
\hline $\mathbf{1}$ & 0.00 & 25.10 & 29.08 & 28.53 & 28.55 & 28.53 & 28.62 & 28.39 \\
\hline $\mathbf{3}$ & 0.00 & 25.10 & 29.08 & 28.53 & 28.55 & 28.53 & 28.57 & 28.39 \\
\hline $\mathbf{4}$ & 10.03 & 25.10 & 39.81 & 35.63 & 32.54 & 32.82 & 34.55 & 32.86 \\
\hline $\mathbf{5}$ & 20.02 & 25.10 & 50.23 & 42.73 & 36.43 & 36.80 & 40.33 & 37.13 \\
\hline $\mathbf{6}$ & 39.93 & 25.10 & 69.79 & 56.13 & 44.62 & 45.07 & 51.60 & 45.91 \\
\hline $\mathbf{7}$ & 50.06 & 25.10 & 78.61 & 61.83 & 48.32 & 48.76 & 56.49 & 49.74 \\
\hline $\mathbf{8}$ & 59.89 & 25.10 & 86.23 & 67.03 & 51.71 & 52.14 & 61.07 & 53.16 \\
\hline $\mathbf{9}$ & 70.03 & 25.10 & 93.05 & 71.68 & 54.81 & 55.23 & 65.16 & 56.14 \\
\hline
\end{tabular}

Table D19: Chamber Temperatures for Unconfined $9 \mathrm{~mm}$ Spacing

\begin{tabular}{|c|c|c|c|c|c|c|c|c|}
\hline Sample & $\mathbf{Q}$ & $\mathbf{T 8}$ & $\mathbf{T 9}^{\mathbf{T}}$ & ${ }^{\mathbf{T 1 0}}$ & $\mathbf{T 1 1}$ & $\mathbf{T 1 2}$ & $\mathbf{T 1 3}$ & $\mathbf{T 1 4}$ \\
\hline$\#$ & $\mathbf{W}$ & ${ }^{\circ} \mathbf{C}$ & ${ }^{\circ} \mathbf{C}$ & ${ }^{\circ} \mathbf{C}$ & ${ }^{\circ} \mathbf{C}$ & ${ }^{\circ} \mathbf{C}$ & ${ }^{\circ} \mathbf{C}$ & ${ }^{\circ} \mathbf{C}$ \\
\hline $\mathbf{1}$ & 0.00 & 28.07 & 28.20 & 28.29 & 28.46 & 28.36 & 28.26 & 28.09 \\
\hline $\mathbf{2}$ & 0.00 & 27.87 & 28.05 & 28.29 & 28.46 & 28.26 & 28.16 & 28.19 \\
\hline $\mathbf{3}$ & 10.03 & 28.07 & 28.25 & 28.99 & 28.95 & 28.76 & 28.35 & 28.24 \\
\hline $\mathbf{4}$ & 20.02 & 28.36 & 28.65 & 29.78 & 29.45 & 29.36 & 28.75 & 28.19 \\
\hline $\mathbf{5}$ & 29.89 & 28.66 & 29.14 & 30.68 & 30.04 & 29.95 & 29.05 & 28.19 \\
\hline $\mathbf{6}$ & 39.93 & 29.46 & 29.64 & 31.57 & 31.03 & 30.85 & 29.55 & 28.19 \\
\hline $\mathbf{7}$ & 50.06 & 29.76 & 30.03 & 32.36 & 31.73 & 31.54 & 30.04 & 28.19 \\
\hline $\mathbf{8}$ & 59.89 & 30.56 & 30.83 & 33.36 & 32.91 & 32.34 & 30.64 & 28.19 \\
\hline $\mathbf{9}$ & 70.03 & 31.25 & 31.42 & 33.85 & 34.20 & 33.33 & 30.64 & 28.19 \\
\hline
\end{tabular}


Table D20: Power, Flow Rates, and Pressure Data for Unconfined $11 \mathrm{~mm}$ Spacing

\begin{tabular}{|c|c|c|c|c|c|c|c|c|c|c|}
\hline & & \multicolumn{2}{|c|}{ Flow Rates (GPH of $\left.\mathbf{H}_{2} \mathbf{O}\right)$} & \multicolumn{5}{|c|}{ Pressures } \\
\hline Sample & $\mathbf{Q}$ & FC-72 & Sump & Water & Sump & Nozzle & Chamber & Tuthill & In Res & Water \\
\hline$\#$ & $\mathbf{W}$ & GPH & GPH & GPH & psia & psia & psia & psia & psia & psia \\
\hline $\mathbf{1}$ & 0.00 & 9.20 & 9.01 & 12.00 & 13.91 & 22.40 & 15.50 & 23.80 & 17.10 & 15.10 \\
\hline $\mathbf{2}$ & 0.00 & 9.20 & 9.01 & 12.00 & 13.86 & 22.10 & 15.40 & 23.55 & 17.10 & 15.10 \\
\hline $\mathbf{3}$ & 10.03 & 9.20 & 9.01 & 12.00 & 13.96 & 22.20 & 15.50 & 23.55 & 17.10 & 15.10 \\
\hline $\mathbf{4}$ & 20.02 & 9.20 & 9.01 & 12.00 & 13.96 & 22.10 & 15.55 & 23.55 & 17.10 & 15.10 \\
\hline $\mathbf{5}$ & 29.89 & 9.20 & 9.01 & 12.00 & 14.06 & 22.30 & 15.70 & 23.80 & 17.10 & 15.10 \\
\hline $\mathbf{6}$ & 39.93 & 9.20 & 9.01 & 12.00 & 14.20 & 22.40 & 15.80 & 23.80 & 17.10 & 15.10 \\
\hline $\mathbf{7}$ & 50.06 & 9.20 & 9.01 & 12.00 & 14.30 & 22.50 & 15.90 & 23.80 & 17.10 & 15.10 \\
\hline $\mathbf{8}$ & 59.89 & 9.20 & 9.01 & 12.00 & 14.30 & 22.55 & 16.05 & 23.80 & 17.10 & 15.10 \\
\hline $\mathbf{9}$ & 70.03 & 9.20 & 9.20 & 12.00 & 14.50 & 22.60 & 16.20 & 23.80 & 17.10 & 15.10 \\
\hline
\end{tabular}

Table D21: Room and Pedestal Temperatures for Unconfined $11 \mathrm{~mm}$ Spacing

\begin{tabular}{|c|c|c|c|c|c|c|c|c|}
\hline Sample & $\mathbf{Q}$ & $\mathbf{R o o m} \mathbf{T e m p}$ & $\mathbf{T 1}$ & ${ }^{\mathbf{T}}$ & ${ }^{\mathbf{T}}$ & ${ }^{\mathbf{T}}$ & ${ }^{\mathbf{T} 5}$ & $\mathbf{T 6}$ \\
\hline$\#$ & $\mathbf{W}$ & ${ }^{\circ} \mathbf{C}$ & ${ }^{\circ} \mathbf{C}$ & ${ }^{\circ} \mathbf{C}$ & ${ }^{\circ} \mathbf{C}$ & ${ }^{\circ} \mathbf{C}$ & ${ }^{\circ} \mathbf{C}$ & ${ }^{\circ} \mathbf{C}$ \\
\hline $\mathbf{1}$ & 0.00 & 24.90 & 28.88 & 28.33 & 28.25 & 28.24 & 28.37 & 28.20 \\
\hline $\mathbf{2}$ & 0.00 & 24.90 & 28.83 & 28.33 & 28.25 & 28.24 & 28.32 & 28.20 \\
\hline $\mathbf{3}$ & 10.03 & 24.90 & 38.35 & 34.93 & 32.04 & 32.32 & 33.95 & 32.36 \\
\hline $\mathbf{4}$ & 20.02 & 25.00 & 47.68 & 41.73 & 35.59 & 36.16 & 39.64 & 36.53 \\
\hline $\mathbf{5}$ & 29.89 & 25.10 & 56.65 & 48.23 & 39.38 & 40.19 & 45.02 & 40.60 \\
\hline $\mathbf{6}$ & 39.93 & 25.10 & 65.53 & 54.53 & 43.33 & 44.17 & 50.40 & 44.72 \\
\hline $\mathbf{7}$ & 50.06 & 25.00 & 73.90 & 60.33 & 47.12 & 47.76 & 55.49 & 48.74 \\
\hline $\mathbf{8}$ & 59.89 & 25.00 & 81.42 & 65.43 & 50.52 & 51.25 & 59.88 & 52.22 \\
\hline $\mathbf{9}$ & 70.03 & 25.10 & 88.44 & 70.33 & 53.31 & 54.44 & 64.06 & 55.39 \\
\hline
\end{tabular}

Table D22: Chamber Temperatures for Unconfined $11 \mathrm{~mm}$ Spacing

\begin{tabular}{|c|c|c|c|c|c|c|c|c|}
\hline Sample & $\mathbf{Q}$ & $\mathbf{T 8}$ & ${ }^{\mathbf{T} 9}$ & ${ }^{\mathbf{T 1 0}}$ & $\mathbf{T 1 1}$ & $\mathbf{T 1 2}$ & ${ }^{\mathbf{T 1 3}}$ & ${ }^{\mathbf{T 1 4}}$ \\
\hline$\#$ & $\mathbf{W}$ & ${ }^{\circ} \mathbf{C}$ & ${ }^{\circ} \mathbf{C}$ & ${ }^{\circ} \mathbf{C}$ & ${ }^{\circ} \mathbf{C}$ & ${ }^{\circ} \mathbf{C}$ & ${ }^{\circ} \mathbf{C}$ & ${ }^{\circ} \mathbf{C}$ \\
\hline $\mathbf{1}$ & 0.00 & 27.67 & 27.85 & 27.99 & 28.16 & 28.06 & 27.86 & 27.99 \\
\hline $\mathbf{2}$ & 0.00 & 27.57 & 27.65 & 27.89 & 28.16 & 27.87 & 27.76 & 28.04 \\
\hline $\mathbf{3}$ & 10.03 & 27.87 & 27.95 & 28.54 & 28.76 & 28.46 & 28.06 & 27.99 \\
\hline $\mathbf{4}$ & 20.02 & 28.17 & 28.40 & 29.28 & 29.35 & 29.06 & 28.45 & 28.09 \\
\hline $\mathbf{5}$ & 29.89 & 28.76 & 29.04 & 30.08 & 29.94 & 29.65 & 28.95 & 28.09 \\
\hline $\mathbf{6}$ & 39.93 & 29.36 & 29.54 & 31.07 & 30.74 & 30.45 & 29.55 & 28.09 \\
\hline $\mathbf{7}$ & 50.06 & 29.76 & 30.13 & 31.87 & 31.43 & 31.24 & 29.94 & 28.09 \\
\hline $\mathbf{8}$ & 59.89 & 30.36 & 30.92 & 32.76 & 32.32 & 32.04 & 30.64 & 28.09 \\
\hline $\mathbf{9}$ & 70.03 & 31.40 & 31.42 & 33.46 & 33.11 & 32.93 & 30.64 & 28.19 \\
\hline
\end{tabular}


Table D23: Power, Flow Rates, and Pressure Data for Unconfined $13 \mathrm{~mm}$ Spacing

\begin{tabular}{|c|c|c|c|c|c|c|c|c|c|c|}
\hline & & \multicolumn{2}{|c|}{ Flow Rates (GPH of $\left.\mathbf{H}_{2} \mathbf{O}\right)$} & \multicolumn{5}{|c|}{ Pressures } \\
\hline Sample & $\mathbf{Q}$ & $\mathbf{F C - 7 2}$ & Sump & Water & Sump & Nozzle & Chamber & Tuthill & In Res & Water \\
\hline$\#$ & $\mathbf{W}$ & GPH & GPH & GPH & psia & psia & psia & psia & psia & psia \\
\hline $\mathbf{1}$ & 0.00 & 9.20 & 9.01 & 12.00 & 13.86 & 22.30 & 15.30 & 23.80 & 16.90 & 15.10 \\
\hline $\mathbf{2}$ & 0.00 & 9.20 & 9.01 & 12.00 & 13.81 & 22.10 & 15.30 & 23.30 & 17.00 & 15.10 \\
\hline $\mathbf{3}$ & 10.03 & 9.20 & 9.01 & 12.00 & 13.86 & 22.20 & 15.40 & 23.30 & 17.00 & 15.10 \\
\hline $\mathbf{4}$ & 20.02 & 9.20 & 9.01 & 12.00 & 13.96 & 22.30 & 15.50 & 23.55 & 17.00 & 15.10 \\
\hline $\mathbf{5}$ & 29.89 & 9.20 & 9.01 & 12.00 & 14.01 & 22.40 & 15.55 & 23.55 & 17.00 & 15.10 \\
\hline $\mathbf{6}$ & 39.93 & 9.20 & 9.01 & 12.00 & 14.15 & 22.50 & 15.70 & 23.80 & 17.00 & 15.10 \\
\hline $\mathbf{7}$ & 50.06 & 9.20 & 9.01 & 12.00 & 14.20 & 22.70 & 15.90 & 23.80 & 17.10 & 15.10 \\
\hline $\mathbf{8}$ & 59.89 & 9.20 & 9.01 & 12.00 & 14.50 & 22.80 & 16.00 & 24.05 & 17.10 & 15.10 \\
\hline $\mathbf{9}$ & 70.03 & 9.20 & 9.20 & 12.00 & 14.60 & 23.00 & 16.20 & 24.30 & 17.10 & 15.10 \\
\hline
\end{tabular}

Table D24: Room and Pedestal Temperatures for Unconfined $13 \mathrm{~mm}$ Spacing

\begin{tabular}{|c|c|c|c|c|c|c|c|c|}
\hline Sample & $\mathbf{Q}$ & $\mathbf{R o o m} \mathbf{T e m p}$ & $\mathbf{T 1}$ & ${ }^{\mathbf{T}}$ & ${ }^{\mathbf{T}}$ & ${ }^{\mathbf{T}}$ & ${ }^{\mathbf{T} 5}$ & $\mathbf{T 6}$ \\
\hline$\#$ & $\mathbf{W}$ & ${ }^{\circ} \mathbf{C}$ & ${ }^{\circ} \mathbf{C}$ & ${ }^{\circ} \mathbf{C}$ & ${ }^{\circ} \mathbf{C}$ & ${ }^{\circ} \mathbf{C}$ & ${ }^{\circ} \mathbf{C}$ & ${ }^{\circ} \mathbf{C}$ \\
\hline $\mathbf{1}$ & 0.00 & 24.70 & 27.97 & 27.33 & 27.25 & 27.34 & 27.37 & 27.20 \\
\hline $\mathbf{2}$ & 0.00 & 24.70 & 27.97 & 27.43 & 27.35 & 27.34 & 27.37 & 27.30 \\
\hline $\mathbf{3}$ & 10.03 & 24.80 & 36.50 & 33.63 & 30.94 & 31.32 & 32.86 & 31.47 \\
\hline $\mathbf{4}$ & 20.02 & 24.80 & 27.77 & 40.13 & 34.34 & 35.11 & 38.34 & 35.54 \\
\hline $\mathbf{5}$ & 29.89 & 24.80 & 53.34 & 46.13 & 37.83 & 38.89 & 43.62 & 39.41 \\
\hline $\mathbf{6}$ & 39.93 & 24.90 & 61.36 & 52.18 & 41.43 & 42.68 & 48.81 & 43.53 \\
\hline $\mathbf{7}$ & 50.06 & 24.90 & 70.99 & 57.83 & 45.02 & 46.32 & 53.69 & 47.45 \\
\hline $\mathbf{8}$ & 59.89 & 24.90 & 76.71 & 63.13 & 48.42 & 50.00 & 58.38 & 51.27 \\
\hline $\mathbf{9}$ & 70.03 & 25.00 & 83.82 & 68.13 & 51.66 & 53.19 & 62.67 & 54.40 \\
\hline
\end{tabular}

Table D25: Chamber Temperatures for Unconfined $13 \mathrm{~mm}$ Spacing

\begin{tabular}{|c|c|c|c|c|c|c|c|c|}
\hline Sample & $\mathbf{Q}$ & $\mathbf{T 8}$ & ${ }^{\mathbf{T} 9}$ & ${ }^{\mathbf{T 1 0}}$ & ${ }^{\mathbf{T 1 1}}$ & $\mathbf{T 1 2}$ & $\mathbf{T 1 3}$ & ${ }^{\mathbf{T 1 4}}$ \\
\hline$\#$ & $\mathbf{W}$ & ${ }^{\circ} \mathbf{C}$ & ${ }^{\circ} \mathbf{C}$ & ${ }^{\circ} \mathbf{C}$ & ${ }^{\circ} \mathbf{C}$ & ${ }^{\circ} \mathbf{C}$ & ${ }^{\circ} \mathbf{C}$ & ${ }^{\circ} \mathbf{C}$ \\
\hline $\mathbf{1}$ & 0.00 & 26.57 & 26.66 & 27.10 & 27.37 & 26.97 & 26.77 & 27.39 \\
\hline $\mathbf{2}$ & 0.00 & 26.57 & 26.76 & 27.10 & 27.27 & 26.97 & 26.77 & 27.39 \\
\hline $\mathbf{3}$ & 10.03 & 26.97 & 27.16 & 27.80 & 28.06 & 27.67 & 27.26 & 27.39 \\
\hline $\mathbf{4}$ & 20.02 & 27.57 & 27.75 & 28.59 & 28.85 & 28.36 & 27.86 & 27.59 \\
\hline $\mathbf{5}$ & 29.89 & 28.26 & 28.35 & 29.24 & 29.55 & 29.06 & 28.45 & 27.59 \\
\hline $\mathbf{6}$ & 39.93 & 28.86 & 28.94 & 30.08 & 30.44 & 29.85 & 28.95 & 27.69 \\
\hline $\mathbf{7}$ & 50.06 & 29.56 & 29.64 & 30.87 & 35.09 & 30.65 & 29.65 & 27.79 \\
\hline $\mathbf{8}$ & 59.89 & 30.56 & 30.33 & 31.67 & 32.02 & 31.44 & 30.24 & 27.79 \\
\hline $\mathbf{9}$ & 70.03 & 31.25 & 31.02 & 32.56 & 32.77 & 32.43 & 30.24 & 27.79 \\
\hline
\end{tabular}


Table D26: Power, Flow Rates, and Pressure Data for Unconfined $15 \mathrm{~mm}$ Spacing

\begin{tabular}{|c|c|c|c|c|c|c|c|c|c|c|}
\hline & & \multicolumn{2}{|c|}{ Flow Rates (GPH of $\left.\mathbf{H}_{\mathbf{2}} \mathbf{O}\right)$} & \multicolumn{5}{|c|}{ Pressures } \\
\hline Sample & $\mathbf{Q}$ & FC-72 & Sump & Water & Sump & Nozzle & Chamber & Tuthill & In Res & Water \\
\hline$\#$ & $\mathbf{W}$ & GPH & GPH & GPH & psia & psia & psia & psia & psia & psia \\
\hline $\mathbf{1}$ & 0.00 & 9.20 & 9.01 & 12.00 & 13.22 & 21.60 & 14.90 & 22.80 & 17.10 & 15.10 \\
\hline $\mathbf{2}$ & 0.00 & 9.20 & 9.01 & 12.00 & 13.27 & 21.60 & 14.80 & 22.80 & 16.90 & 15.10 \\
\hline $\mathbf{3}$ & 10.03 & 9.20 & 9.01 & 12.00 & 13.32 & 21.60 & 14.90 & 22.70 & 16.70 & 15.10 \\
\hline $\mathbf{4}$ & 20.02 & 9.20 & 9.01 & 12.00 & 13.37 & 21.70 & 15.00 & 22.80 & 16.70 & 15.10 \\
\hline $\mathbf{5}$ & 29.89 & 9.20 & 9.01 & 12.00 & 13.57 & 21.80 & 15.10 & 23.05 & 16.70 & 15.10 \\
\hline $\mathbf{6}$ & 39.93 & 9.20 & 9.01 & 12.00 & 13.71 & 21.90 & 15.30 & 23.30 & 16.70 & 15.10 \\
\hline $\mathbf{7}$ & 50.06 & 9.20 & 9.01 & 12.00 & 13.81 & 22.00 & 15.50 & 23.30 & 16.70 & 15.17 \\
\hline $\mathbf{8}$ & 60.12 & 9.20 & 9.01 & 12.00 & 13.91 & 22.20 & 15.60 & 23.30 & 16.70 & 15.10 \\
\hline $\mathbf{9}$ & 70.03 & 9.20 & 9.20 & 12.00 & 14.11 & 22.20 & 15.80 & 23.55 & 16.70 & 15.10 \\
\hline
\end{tabular}

Table D27: Room and Pedestal Temperatures for Unconfined $15 \mathrm{~mm}$ Spacing

\begin{tabular}{|c|c|c|c|c|c|c|c|c|}
\hline Sample & $\mathbf{Q}$ & $\mathbf{R o o m} \mathbf{T e m p}$ & $\mathbf{T 1}$ & ${ }^{\mathbf{T}}$ & ${ }^{\mathbf{T}}$ & ${ }^{\mathbf{T}}$ & ${ }^{\mathbf{T} 5}$ & $\mathbf{T 6}$ \\
\hline$\#$ & $\mathbf{W}$ & ${ }^{\circ} \mathbf{C}$ & ${ }^{\circ} \mathbf{C}$ & ${ }^{\circ} \mathbf{C}$ & ${ }^{\circ} \mathbf{C}$ & ${ }^{\circ} \mathbf{C}$ & ${ }^{\circ} \mathbf{C}$ & ${ }^{\circ} \mathbf{C}$ \\
\hline $\mathbf{1}$ & 0.00 & 25.10 & 29.18 & 28.63 & 28.65 & 28.63 & 28.67 & 28.49 \\
\hline $\mathbf{2}$ & 0.00 & 25.20 & 29.13 & 28.63 & 28.55 & 28.53 & 28.67 & 28.39 \\
\hline $\mathbf{3}$ & 10.03 & 25.20 & 37.90 & 34.73 & 31.94 & 32.12 & 33.75 & 32.26 \\
\hline $\mathbf{4}$ & 20.02 & 25.30 & 46.62 & 40.93 & 35.24 & 35.51 & 39.04 & 35.94 \\
\hline $\mathbf{5}$ & 29.89 & 25.30 & 55.10 & 46.93 & 38.68 & 39.09 & 44.02 & 39.81 \\
\hline $\mathbf{6}$ & 39.93 & 25.30 & 63.52 & 52.93 & 42.18 & 42.68 & 49.11 & 43.68 \\
\hline $\mathbf{7}$ & 50.06 & 25.40 & 71.44 & 58.63 & 45.62 & 46.27 & 53.99 & 47.45 \\
\hline $\mathbf{8}$ & 60.12 & 25.40 & 78.96 & 63.73 & 48.92 & 49.35 & 58.28 & 50.83 \\
\hline $\mathbf{9}$ & 70.03 & 25.50 & 85.83 & 68.43 & 51.76 & 52.34 & 62.37 & 53.85 \\
\hline
\end{tabular}

Table D28: Chamber Temperatures for Unconfined $15 \mathrm{~mm}$ Spacing

\begin{tabular}{|c|c|c|c|c|c|c|c|c|}
\hline Sample & $\mathbf{Q}$ & $\mathbf{T 8}$ & $\mathbf{T 9}$ & ${ }^{\mathbf{T 1 0}}$ & $\mathbf{T 1 1}$ & $\mathbf{T 1 2}$ & $\mathbf{T 1 3}$ & $\mathbf{T 1 4}$ \\
\hline$\#$ & $\mathbf{W}$ & ${ }^{\circ} \mathbf{C}$ & ${ }^{\circ} \mathbf{C}$ & ${ }^{\circ} \mathbf{C}$ & ${ }^{\circ} \mathbf{C}$ & ${ }^{\circ} \mathbf{C}$ & ${ }^{\circ} \mathbf{C}$ & ${ }^{\circ} \mathbf{C}$ \\
\hline $\mathbf{1}$ & 0.00 & 28.17 & 28.25 & 28.49 & 28.56 & 28.36 & 28.35 & 28.29 \\
\hline $\mathbf{2}$ & 0.00 & 27.97 & 28.05 & 28.39 & 28.46 & 28.26 & 28.16 & 28.39 \\
\hline $\mathbf{3}$ & 10.03 & 28.07 & 28.45 & 28.89 & 29.25 & 28.86 & 28.55 & 28.29 \\
\hline $\mathbf{4}$ & 20.02 & 28.56 & 28.94 & 29.68 & 29.94 & 29.45 & 29.05 & 28.39 \\
\hline $\mathbf{5}$ & 29.89 & 29.16 & 29.44 & 30.18 & 30.74 & 50.02 & 29.45 & 28.39 \\
\hline $\mathbf{6}$ & 39.93 & 29.76 & 30.03 & 30.87 & 31.63 & 30.94 & 29.94 & 28.39 \\
\hline $\mathbf{7}$ & 50.06 & 30.66 & 30.63 & 31.67 & 32.42 & 31.74 & 30.54 & 28.29 \\
\hline $\mathbf{8}$ & 60.12 & 31.55 & 31.42 & 32.56 & 33.41 & 32.53 & 31.23 & 28.39 \\
\hline $\mathbf{9}$ & 70.03 & 32.25 & 32.02 & 33.46 & 34.10 & 32.43 & 31.23 & 28.49 \\
\hline
\end{tabular}


Table D29: Power, Flow Rates, and Pressure Data for Unconfined $17 \mathrm{~mm}$ Spacing

\begin{tabular}{|c|c|c|c|c|c|c|c|c|c|c|}
\hline & & \multicolumn{2}{|c|}{ Flow Rates (GPH of $\left.\mathbf{H}_{2} \mathbf{O}\right)$} & \multicolumn{5}{c|}{ Pressures } \\
\hline Sample & $\mathbf{Q}$ & FC-72 & Sump & Water & Sump & Nozzle & Chamber & Tuthill & In Res & Water \\
\hline$\#$ & $\mathbf{W}$ & GPH & GPH & GPH & psia & psia & psia & psia & psia & psia \\
\hline $\mathbf{1}$ & 0.00 & 9.20 & 9.01 & 12.00 & 13.37 & 21.70 & 15.00 & 23.05 & 16.70 & 15.20 \\
\hline $\mathbf{2}$ & 0.00 & 9.20 & 9.01 & 12.00 & 13.37 & 21.70 & 15.00 & 23.05 & 16.70 & 15.20 \\
\hline $\mathbf{3}$ & 10.03 & 9.20 & 9.01 & 12.00 & 13.37 & 21.70 & 15.05 & 22.80 & 16.60 & 15.20 \\
\hline $\mathbf{4}$ & 20.02 & 9.20 & 9.01 & 12.00 & 13.47 & 21.70 & 15.10 & 22.80 & 16.60 & 15.20 \\
\hline $\mathbf{5}$ & 29.89 & 9.20 & 9.01 & 12.00 & 13.61 & 21.80 & 15.30 & 23.05 & 16.60 & 15.20 \\
\hline $\mathbf{6}$ & 39.93 & 9.20 & 9.01 & 12.00 & 13.71 & 21.90 & 15.40 & 23.30 & 16.60 & 15.20 \\
\hline $\mathbf{7}$ & 50.06 & 9.20 & 9.01 & 12.00 & 13.76 & 22.00 & 15.50 & 23.30 & 16.60 & 15.20 \\
\hline $\mathbf{8}$ & 59.89 & 9.20 & 9.01 & 12.00 & 13.91 & 22.10 & 15.70 & 23.30 & 16.60 & 15.20 \\
\hline $\mathbf{9}$ & 70.03 & 9.20 & 9.20 & 12.00 & 14.11 & 22.40 & 15.90 & 23.80 & 16.60 & 15.20 \\
\hline
\end{tabular}

Table D30: Room and Pedestal Temperatures for Unconfined $17 \mathrm{~mm}$ Spacing

\begin{tabular}{|c|c|c|c|c|c|c|c|c|}
\hline Sample & $\mathbf{Q}$ & Room Temp & $\mathbf{T 1}$ & $\mathbf{T 2}$ & $\mathbf{T 3}$ & $\mathbf{T 4}$ & $\mathbf{T 5}$ & T6 \\
\hline$\#$ & $\mathbf{W}$ & ${ }^{\circ} \mathbf{C}$ & ${ }^{\circ} \mathbf{C}$ & ${ }^{\circ} \mathbf{C}$ & ${ }^{\circ} \mathbf{C}$ & ${ }^{\circ} \mathbf{C}$ & ${ }^{\circ} \mathbf{C}$ & ${ }^{\circ} \mathbf{C}$ \\
\hline $\mathbf{1}$ & 0.00 & 25.50 & 29.48 & 28.93 & 28.94 & 28.93 & 28.97 & 28.79 \\
\hline $\mathbf{2}$ & 0.00 & 25.40 & 29.48 & 28.93 & 28.94 & 28.83 & 28.97 & 28.74 \\
\hline $\mathbf{3}$ & 10.03 & 25.40 & 38.40 & 35.23 & 32.29 & 32.42 & 34.15 & 32.66 \\
\hline $\mathbf{4}$ & 20.02 & 25.40 & 47.18 & 41.38 & 35.34 & 35.81 & 39.34 & 36.14 \\
\hline $\mathbf{5}$ & 29.89 & 25.40 & 55.80 & 47.53 & 38.73 & 39.29 & 44.52 & 39.91 \\
\hline $\mathbf{6}$ & 39.93 & 25.40 & 64.17 & 53.53 & 42.03 & 42.78 & 49.56 & 43.53 \\
\hline $\mathbf{7}$ & 50.06 & 25.40 & 72.24 & 59.23 & 45.32 & 46.07 & 54.39 & 47.25 \\
\hline $\mathbf{8}$ & 59.89 & 25.40 & 79.66 & 64.33 & 48.52 & 49.30 & 58.78 & 50.63 \\
\hline $\mathbf{9}$ & 70.03 & 25.40 & 86.58 & 69.03 & 51.26 & 52.14 & 62.87 & 53.46 \\
\hline
\end{tabular}

Table D31: Chamber Temperatures for Unconfined $17 \mathrm{~mm}$ Spacing

\begin{tabular}{|c|c|c|c|c|c|c|c|c|}
\hline Sample & $\mathbf{Q}$ & $\mathbf{T 8}$ & $\mathbf{T 9}$ & $\mathbf{T 1 0}$ & $\mathbf{T 1 1}$ & $\mathbf{T 1 2}$ & $\mathbf{T 1 3}$ & T14 \\
\hline$\#$ & $\mathbf{W}$ & ${ }^{\circ} \mathbf{C}$ & ${ }^{\circ} \mathbf{C}$ & ${ }^{\circ} \mathbf{C}$ & ${ }^{\circ} \mathbf{C}$ & ${ }^{\circ} \mathbf{C}$ & ${ }^{\circ} \mathbf{C}$ & ${ }^{\circ} \mathbf{C}$ \\
\hline $\mathbf{1}$ & 0.00 & 28.56 & 28.55 & 28.84 & 28.85 & 28.76 & 28.75 & 28.54 \\
\hline $\mathbf{2}$ & 0.00 & 28.46 & 28.45 & 28.79 & 28.76 & 28.66 & 28.55 & 28.59 \\
\hline $\mathbf{3}$ & 10.03 & 28.56 & 28.74 & 29.28 & 29.45 & 29.16 & 28.85 & 28.59 \\
\hline $\mathbf{4}$ & 20.02 & 29.16 & 29.14 & 29.88 & 30.14 & 29.75 & 29.00 & 28.59 \\
\hline $\mathbf{5}$ & 29.89 & 29.71 & 29.64 & 30.48 & 30.93 & 30.35 & 29.65 & 28.49 \\
\hline $\mathbf{6}$ & 39.93 & 30.31 & 30.23 & 31.27 & 31.87 & 30.99 & 30.24 & 28.49 \\
\hline $\mathbf{7}$ & 50.06 & 30.91 & 30.73 & 31.92 & 32.62 & 31.84 & 30.84 & 28.59 \\
\hline $\mathbf{8}$ & 59.89 & 31.65 & 31.42 & 32.66 & 33.56 & 32.53 & 31.58 & 28.59 \\
\hline $\mathbf{9}$ & 70.03 & 32.35 & 32.11 & 33.46 & 34.15 & 33.43 & 31.58 & 28.59 \\
\hline
\end{tabular}


Table D32: Power, Flow Rates, and Pressure Data for Unconfined 13 mm \#2 Spacing

\begin{tabular}{|c|c|c|c|c|c|c|c|c|c|c|}
\hline & & \multicolumn{2}{|c|}{ Flow Rates $\left(\mathbf{G P H}\right.$ of $\left.\mathbf{H}_{\mathbf{2}} \mathbf{O}\right)$} & \multicolumn{5}{|c|}{ Pressures } \\
\hline Sample & $\mathbf{Q}$ & $\mathbf{F C - 7 2}$ & Sump & Water & Sump & Nozzle & Chamber & Tuthill & In Res & Water \\
\hline$\#$ & $\mathbf{W}$ & $\mathbf{G P H}$ & $\mathbf{G P H}$ & $\mathbf{G P H}$ & psia & psia & psia & psia & psia & psia \\
\hline $\mathbf{1}$ & 0.00 & 9.20 & 9.01 & 12.00 & 13.12 & 21.60 & 14.80 & 23.05 & 16.60 & 15.20 \\
\hline $\mathbf{2}$ & 0.00 & 9.20 & 9.01 & 12.00 & 13.17 & 21.60 & 14.70 & 22.80 & 16.50 & 15.20 \\
\hline $\mathbf{3}$ & 10.03 & 9.20 & 9.01 & 12.00 & 13.22 & 21.55 & 14.90 & 22.80 & 16.50 & 15.20 \\
\hline $\mathbf{4}$ & 20.02 & 9.20 & 9.01 & 12.00 & 13.27 & 21.70 & 14.90 & 22.80 & 16.40 & 15.20 \\
\hline $\mathbf{5}$ & 29.89 & 9.20 & 9.01 & 12.00 & 13.37 & 21.70 & 15.05 & 23.05 & 16.40 & 15.20 \\
\hline $\mathbf{6}$ & 39.93 & 9.20 & 9.01 & 12.00 & 13.52 & 21.80 & 15.20 & 23.05 & 16.40 & 15.20 \\
\hline $\mathbf{7}$ & 50.06 & 9.20 & 9.01 & 12.00 & 13.61 & 21.90 & 15.30 & 23.30 & 16.40 & 15.20 \\
\hline $\mathbf{8}$ & 59.89 & 9.20 & 9.01 & 12.00 & 13.76 & 22.00 & 15.50 & 23.30 & 16.40 & 15.20 \\
\hline $\mathbf{9}$ & 70.03 & 9.20 & 9.20 & 12.00 & 13.86 & 22.10 & 15.60 & 23.30 & 16.40 & 15.20 \\
\hline $\mathbf{1 0}$ & 86.50 & 9.20 & 9.01 & 12.00 & 14.11 & 22.30 & 15.90 & 23.55 & 16.40 & 15.20 \\
\hline
\end{tabular}

Table D33: Room and Pedestal Temperatures for Unconfined $13 \mathrm{~mm}$ \#2 Spacing

\begin{tabular}{|c|c|c|c|c|c|c|c|c|}
\hline Sample & $\mathbf{Q}$ & $\mathbf{R o o m} \mathbf{T e m p}$ & $\mathbf{T 1}$ & $\mathbf{T 2}$ & $\mathbf{T 3}$ & $\mathbf{T 4}$ & $\mathbf{T 5}$ & T6 \\
\hline$\#$ & $\mathbf{W}$ & ${ }^{\circ} \mathbf{C}$ & ${ }^{\circ} \mathbf{C}$ & ${ }^{\circ} \mathbf{C}$ & ${ }^{\circ} \mathbf{C}$ & ${ }^{\circ} \mathbf{C}$ & ${ }^{\circ} \mathbf{C}$ & ${ }^{\circ} \mathbf{C}$ \\
\hline $\mathbf{1}$ & 0.00 & 25.40 & 29.58 & 29.03 & 29.04 & 29.03 & 29.07 & 28.89 \\
\hline $\mathbf{2}$ & 0.00 & 25.40 & 29.58 & 29.03 & 29.04 & 28.93 & 29.07 & 28.79 \\
\hline $\mathbf{3}$ & 10.03 & 25.40 & 39.20 & 35.63 & 32.74 & 32.82 & 34.35 & 32.86 \\
\hline $\mathbf{4}$ & 20.02 & 25.50 & 48.73 & 42.13 & 36.24 & 36.50 & 39.83 & 36.68 \\
\hline $\mathbf{5}$ & 29.89 & 25.50 & 57.90 & 48.63 & 40.03 & 40.29 & 45.22 & 40.70 \\
\hline $\mathbf{6}$ & 39.93 & 25.50 & 66.68 & 54.73 & 43.82 & 44.07 & 50.45 & 44.67 \\
\hline $\mathbf{7}$ & 50.06 & 25.50 & 75.10 & 60.53 & 47.47 & 47.76 & 55.29 & 48.59 \\
\hline $\mathbf{8}$ & 59.89 & 25.50 & 82.42 & 65.53 & 50.72 & 51.05 & 59.68 & 51.82 \\
\hline $\mathbf{9}$ & 70.03 & 25.60 & 89.44 & 70.23 & 53.56 & 54.04 & 63.76 & 54.80 \\
\hline $\mathbf{1 0}$ & 86.50 & 25.50 & 99.37 & 76.63 & 56.86 & 57.82 & 69.25 & 58.07 \\
\hline
\end{tabular}

Table D34: Chamber Temperatures for Unconfined 13 mm \#2 Spacing

\begin{tabular}{|c|c|c|c|c|c|c|c|c|}
\hline Sample & $\mathbf{Q}$ & $\mathbf{T 8}$ & $\mathbf{T 9}$ & ${ }^{\mathbf{T 1 0}}$ & $\mathbf{T 1 1}$ & $\mathbf{T 1 2}$ & $\mathbf{T 1 3}$ & $\mathbf{T 1 4}$ \\
\hline$\#$ & $\mathbf{W}$ & ${ }^{\circ} \mathbf{C}$ & ${ }^{\circ} \mathbf{C}$ & ${ }^{\circ} \mathbf{C}$ & ${ }^{\circ} \mathbf{C}$ & ${ }^{\circ} \mathbf{C}$ & ${ }^{\circ} \mathbf{C}$ & ${ }^{\circ} \mathbf{C}$ \\
\hline $\mathbf{1}$ & 0.00 & 28.51 & 28.69 & 28.79 & 28.90 & 28.86 & 28.75 & 28.69 \\
\hline $\mathbf{2}$ & 0.00 & 28.36 & 28.55 & 28.79 & 28.85 & 28.76 & 28.65 & 28.69 \\
\hline $\mathbf{3}$ & 10.03 & 28.56 & 28.84 & 29.43 & 29.55 & 29.16 & 28.95 & 28.59 \\
\hline $\mathbf{4}$ & 20.02 & 28.96 & 29.24 & 30.18 & 30.24 & 29.85 & 29.35 & 28.59 \\
\hline $\mathbf{5}$ & 29.89 & 29.46 & 29.69 & 30.68 & 31.03 & 30.45 & 29.75 & 28.59 \\
\hline $\mathbf{6}$ & 39.93 & 29.96 & 30.23 & 31.57 & 31.87 & 31.24 & 30.34 & 28.59 \\
\hline $\mathbf{7}$ & 50.06 & 30.71 & 30.83 & 32.26 & 32.81 & 32.04 & 30.84 & 28.69 \\
\hline $\mathbf{8}$ & 59.89 & 31.55 & 31.52 & 32.91 & 33.61 & 32.83 & 31.48 & 28.59 \\
\hline $\mathbf{9}$ & 70.03 & 32.25 & 32.21 & 33.85 & 34.65 & 33.63 & 32.08 & 28.69 \\
\hline $\mathbf{1 0}$ & 86.50 & 33.55 & 33.40 & 34.99 & 36.68 & 34.72 & 33.12 & 28.69 \\
\hline
\end{tabular}


Table D35: Reduced Data for Confined $9 \mathrm{~mm}$ Spacing

\begin{tabular}{|c|c|c|c|c|c|c|c|c|c|c|c|c|c|c|c|c|c|}
\hline sample & $\mathbf{T}_{\text {sat }}$ & $\mathbf{Q}$ & $\mathbf{q}$ & $\Delta \mathbf{T}_{\text {sub }}$ & $\Delta \mathbf{T}_{\mathrm{htr}}$ & $\Delta \mathbf{T}_{\text {glass }}$ & $\mathbf{T}_{\mathrm{s}}$ & $\mathbf{T}_{\infty_{\text {wall }}}$ & $\mathbf{T}_{\infty \mathrm{otop}}$ & $\begin{array}{c}\mathbf{T}_{\mathrm{s}^{-}} \\
\mathbf{T}_{\boldsymbol{c o t} \mathbf{p}}\end{array}$ & $\begin{array}{c}\mathbf{T}_{\text {sat }}{ }^{-} \\
\mathbf{T}_{\text {owall }}\end{array}$ & $\begin{array}{c}\mathbf{T}_{\text {sat }}{ }^{-} \\
\mathbf{T}_{\text {otop }}\end{array}$ & $\mathbf{k}_{\text {fluid }}$ & h & $\begin{array}{c}\theta_{\mathrm{s}^{-}} \\
\theta_{\infty} \mathrm{t}_{\mathrm{op}}\end{array}$ & $\mathrm{Nu}$ & GD \\
\hline \# & ${ }^{\circ} \mathrm{C}$ & W & $\begin{array}{c}10^{4} \\
W / \mathbf{m}^{2} \\
\end{array}$ & ${ }^{\circ} \mathrm{C}$ & ${ }^{\circ} \mathrm{C}$ & ${ }^{\circ} \mathrm{C}$ & ${ }^{\circ} \mathrm{C}$ & ${ }^{\circ} \mathrm{C}$ & ${ }^{\circ} \mathrm{C}$ & ${ }^{\circ} \mathrm{C}$ & ${ }^{\circ} \mathrm{C}$ & ${ }^{\circ} \mathrm{C}$ & $\begin{array}{c}\mathbf{W} / \\
\mathbf{m}-\mathbf{K}\end{array}$ & $\begin{array}{c}\mathbf{W} / \\
\mathbf{m}^{2} \mathbf{K}\end{array}$ & --- & --- & --- \\
\hline 1 & 57.06 & 0.00 & 0.00 & 0.00 & 0.00 & 0.00 & 29.98 & 29.32 & 29.32 & 0.66 & 24.32 & 24.32 & 0.055 & 0 & 0.03 & 0 & 0 \\
\hline 2 & 57.06 & 10.03 & 0.00 & 0.00 & 0.00 & 0.00 & 29.98 & 29.32 & 29.32 & 0.66 & 27.74 & 27.74 & 0.055 & 0 & 0.02 & 0 & 0 \\
\hline 3 & 57.27 & 20.02 & 6.82 & 0.02 & -0.18 & -2.58 & 38.06 & 29.91 & 29.61 & 8.45 & 27.14 & 27.44 & 0.055 & 7951 & 0.31 & 1150 & 14 \\
\hline 4 & 57.27 & 29.89 & 13.62 & 0.05 & -0.37 & -5.16 & 45.81 & 30.66 & 29.96 & 15.85 & 26.61 & 27.31 & 0.055 & 8465 & 0.60 & 1225 & 28 \\
\hline 5 & 57.37 & 39.93 & 20.33 & 0.07 & -0.55 & -7.70 & 53.18 & 31.55 & 30.36 & 22.83 & 25.72 & 26.91 & 0.055 & 8774 & 0.89 & 1271 & 44 \\
\hline 7 & 57.90 & 59.89 & 33.91 & 0.12 & -0.91 & -12.85 & 65.57 & 33.48 & 31.25 & 34.32 & 24.20 & 26.44 & 0.055 & 9733 & 1.42 & 1413 & 78 \\
\hline 8 & 58.32 & 70.07 & 40.74 & 0.14 & -1.10 & -15.43 & 70.24 & 34.52 & 31.79 & 38.45 & 23.37 & 26.10 & 0.055 & 10437 & 1.65 & 1517 & 97 \\
\hline 9 & 57.06 & 0.00 & 47.67 & 0.17 & -1.29 & -18.06 & 74.07 & 35.71 & 32.44 & 41.64 & 22.60 & 25.88 & 0.055 & 11276 & 1.84 & 1641 & 117 \\
\hline
\end{tabular}

Table D36: Reduced Data for Confined $11 \mathrm{~mm}$ Spacing

\begin{tabular}{|c|c|c|c|c|c|c|c|c|c|c|c|c|c|c|c|c|c|}
\hline sample & $\mathbf{T}_{\text {sat }}$ & $\mathbf{Q}$ & $\mathbf{q}$ & $\Delta \mathbf{T}_{\text {sub }}$ & $\Delta \mathbf{T}_{\mathrm{htr}}$ & $\Delta \mathbf{T}_{\text {glass }}$ & $\mathbf{T}_{\mathbf{s}}$ & $\mathbf{T}_{\infty_{\text {wall }}}$ & $\mathbf{T}_{\text {cotop }}$ & $\begin{array}{c}\mathbf{T}_{\mathbf{s}^{-}} \\
\mathbf{T}_{\text {o }} \mathbf{t}_{\text {op }}\end{array}$ & $\begin{array}{c}\mathbf{T}_{\text {sat }} \\
\mathbf{T}_{\text {owall }}\end{array}$ & $\begin{array}{c}\mathbf{T}_{\text {sat- }} \\
\mathbf{T}_{\text {otop }}\end{array}$ & $\mathbf{k}_{\text {fluid }}$ & h & $\begin{array}{c}\theta_{\mathrm{s}^{-}} \\
\theta_{\text {otop }}\end{array}$ & $\mathbf{N u}$ & GD \\
\hline \# & ${ }^{\circ} \mathrm{C}$ & W & $\begin{array}{c}10^{4} \\
\mathrm{~W} / \mathrm{m}^{2}\end{array}$ & ${ }^{\circ} \mathrm{C}$ & ${ }^{\circ} \mathrm{C}$ & ${ }^{\circ} \mathrm{C}$ & ${ }^{\circ} \mathrm{C}$ & ${ }^{\circ} \mathrm{C}$ & ${ }^{\circ} \mathrm{C}$ & ${ }^{\circ} \mathrm{C}$ & ${ }^{\circ} \mathrm{C}$ & ${ }^{\circ} \mathbf{C}$ & $\begin{array}{c}\mathbf{W} / \\
\mathbf{m}-\mathbf{K}\end{array}$ & $\begin{array}{c}\mathbf{W} / \\
\mathbf{m}^{2} \mathbf{K}\end{array}$ & --- & --- & --- \\
\hline 1 & 57.06 & 0.00 & 0.00 & 0.00 & 0.00 & 0.00 & 29.48 & 28.77 & 28.87 & 0.61 & 28.28 & 28.19 & 0.055 & 0 & 0.02 & 0 & 0 \\
\hline 2 & 57.06 & 0.00 & 0.00 & 0.00 & 0.00 & 0.00 & 29.48 & 28.82 & 28.92 & 0.56 & 28.23 & 28.14 & 0.055 & 0 & 0.02 & 0 & 0 \\
\hline 3 & 57.06 & 10.03 & 6.82 & 0.02 & -0.18 & -2.58 & 36.86 & 29.61 & 29.32 & 7.55 & 27.44 & 27.74 & 0.055 & 8905 & 0.27 & 1287 & 14 \\
\hline 4 & 57.27 & 19.89 & 13.53 & 0.05 & -0.36 & -5.13 & 43.79 & 30.31 & 29.56 & 14.23 & 26.96 & 27.70 & 0.055 & 9369 & 0.53 & 1355 & 28 \\
\hline 5 & 57.37 & 30.08 & 20.46 & 0.07 & -0.55 & -7.75 & 50.58 & 31.20 & 30.01 & 20.57 & 26.17 & 27.36 & 0.055 & 9800 & 0.79 & 1419 & 43 \\
\hline 6 & 57.69 & 40.15 & 27.31 & 0.10 & -0.74 & -10.35 & 56.90 & 32.04 & 30.45 & 26.44 & 25.64 & 27.23 & 0.055 & 10174 & 1.03 & 1474 & 59 \\
\hline 7 & 57.90 & 50.09 & 34.08 & 0.12 & -0.92 & -12.91 & 62.34 & 33.04 & 30.90 & 31.44 & 24.86 & 27.00 & 0.055 & 10675 & 1.26 & 1548 & 76 \\
\hline 8 & 58.11 & 59.89 & 40.74 & 0.14 & -1.10 & -15.43 & 66.93 & 33.68 & 31.30 & 35.64 & 24.43 & 26.81 & 0.055 & 11261 & 1.46 & 1635 & 92 \\
\hline 9 & 58.42 & 70.07 & 47.67 & 0.17 & -1.29 & -18.06 & 70.87 & 34.52 & 31.74 & 39.12 & 23.90 & 26.68 & 0.055 & 12001 & 1.64 & 1744 & 110 \\
\hline
\end{tabular}


Table D37: Reduced Data for Confined $13 \mathrm{~mm}$ Spacing

\begin{tabular}{|c|c|c|c|c|c|c|c|c|c|c|c|c|c|c|c|c|c|}
\hline sample & $\mathbf{T}_{\text {sat }}$ & $\mathbf{Q}$ & $\mathbf{q}$ & $\Delta \mathbf{T}_{\text {sub }}$ & $\Delta \mathbf{T}_{\mathrm{htr}}$ & $\Delta \mathbf{T}_{\text {glass }}$ & $\mathbf{T}_{\mathbf{s}}$ & $\mathbf{T}_{\infty_{\text {wall }}}$ & $\mathbf{T}_{\text {otop }}$ & $\begin{array}{c}\mathbf{T}_{\mathrm{s}^{-}} \\
\mathbf{T}_{\text {ot }} \text { top }^{2}\end{array}$ & $\begin{array}{c}\mathbf{T}_{\text {sat }} \\
\mathbf{T}_{\text {owall }}\end{array}$ & $\begin{array}{c}\mathbf{T}_{\text {sat }} \\
\mathbf{T}_{\text {cotop }}\end{array}$ & $\mathbf{k}_{\text {fluid }}$ & $\mathbf{h}$ & $\begin{array}{c}\theta_{\mathrm{s}^{-}} \\
\theta_{\infty} \text { top }_{\text {op }}\end{array}$ & $\mathrm{Nu}$ & GA \\
\hline \# & ${ }^{\circ} \mathrm{C}$ & W & $\begin{array}{c}10^{4} \\
\mathrm{~W} / \mathrm{m}^{2}\end{array}$ & ${ }^{\circ} \mathrm{C}$ & ${ }^{\circ} \mathrm{C}$ & ${ }^{\circ} \mathrm{C}$ & ${ }^{\circ} \mathrm{C}$ & ${ }^{\circ} \mathrm{C}$ & ${ }^{\circ} \mathrm{C}$ & ${ }^{\circ} \mathrm{C}$ & ${ }^{\circ} \mathrm{C}$ & ${ }^{\circ} \mathrm{C}$ & $\underset{/ \mathbf{m}-\mathbf{K}}{\mathbf{W}}$ & $\begin{array}{c}\mathbf{W} \\
/ \mathbf{m}^{2} \mathbf{K}\end{array}$ & colop & -- & --- \\
\hline 1 & 56.85 & 0.00 & 0.00 & 0.00 & 0.00 & 0.00 & 28.88 & 28.18 & 28.27 & 0.60 & 28.67 & 28.57 & 0.055 & 0 & 0.02 & 0 & 0 \\
\hline 2 & 56.85 & 0.00 & 0.00 & 0.00 & 0.00 & 0.00 & 28.98 & 28.28 & 28.37 & 0.60 & 28.57 & 28.47 & 0.055 & 0 & 0.02 & 0 & 0 \\
\hline 3 & 57.06 & 10.03 & 6.82 & 0.02 & -0.18 & -2.58 & 36.46 & 29.12 & 28.87 & 7.59 & 27.94 & 28.19 & 0.055 & 8851 & 0.27 & 1278 & 14 \\
\hline 4 & 57.27 & 19.89 & 13.53 & 0.05 & -0.36 & -5.13 & 43.59 & 29.86 & 29.17 & 14.42 & 27.40 & 28.10 & 0.055 & 9241 & 0.53 & 1335 & 27 \\
\hline 5 & 57.37 & 29.89 & 20.33 & 0.07 & -0.55 & -7.70 & 50.38 & 30.75 & 29.61 & 20.77 & 26.62 & 27.76 & 0.055 & 9646 & 0.78 & 1395 & 42 \\
\hline 6 & 57.69 & 39.93 & 27.16 & 0.10 & -0.73 & -10.29 & 56.85 & 31.65 & 30.06 & 26.80 & 26.04 & 27.63 & 0.055 & 9984 & 1.03 & 1445 & 58 \\
\hline 7 & 57.90 & 50.06 & 34.05 & 0.12 & -0.92 & -12.90 & 62.30 & 32.54 & 30.55 & 31.75 & 25.36 & 27.34 & 0.055 & 10564 & 1.25 & 1531 & 74 \\
\hline 8 & 58.11 & 60.12 & 40.90 & 0.14 & -1.10 & -15.49 & 67.17 & 33.48 & 31.05 & 36.13 & 24.62 & 27.06 & 0.055 & 11151 & 1.47 & 1618 & 92 \\
\hline 9 & 58.32 & 70.07 & 47.67 & 0.17 & -1.29 & -18.06 & 71.27 & 34.37 & 31.64 & 39.63 & 23.94 & 26.67 & 0.055 & 11849 & 1.66 & 1721 & 110 \\
\hline
\end{tabular}

Table D38: Reduced Data for Confined 15 mm Spacing

\begin{tabular}{|c|c|c|c|c|c|c|c|c|c|c|c|c|c|c|c|c|c|}
\hline sample & $\mathbf{T}_{\text {sat }}$ & $\mathbf{Q}$ & $\mathbf{q}$ & $\Delta \mathbf{T}_{\text {sub }}$ & $\Delta \mathbf{T}_{\mathrm{htr}}$ & $\Delta \mathbf{T}_{\text {glass }}$ & $\mathbf{T}_{\mathrm{s}}$ & $\mathbf{T}_{\infty_{\text {wall }}}$ & $\mathbf{T}_{\text {otop }}$ & $\begin{array}{c}\mathbf{T}_{\mathrm{s}^{-}} \\
\mathbf{T}_{\text {ot }} \\
\end{array}$ & $\begin{array}{c}\mathbf{T}_{\text {sat }^{-}} \\
\mathbf{T}_{\text {owall }}\end{array}$ & $\begin{array}{c}\mathbf{T}_{\text {sat- }} \\
\mathbf{T}_{\text {otop }} \\
\end{array}$ & $\mathbf{k}_{\text {fluid }}$ & h & $\begin{array}{c}\theta_{\mathrm{s}^{-}} \\
\theta_{\text {sotop }}\end{array}$ & $\mathrm{Nu}$ & GD \\
\hline \# & ${ }^{\circ} \mathrm{C}$ & $\mathbf{W}$ & $\begin{array}{c}10^{4} \\
W / m^{2}\end{array}$ & ${ }^{\circ} \mathrm{C}$ & ${ }^{\circ} \mathrm{C}$ & ${ }^{\circ} \mathrm{C}$ & ${ }^{\circ} \mathrm{C}$ & ${ }^{\circ} \mathrm{C}$ & ${ }^{\circ} \mathrm{C}$ & ${ }^{\circ} \mathrm{C}$ & ${ }^{\circ} \mathrm{C}$ & ${ }^{\circ} \mathrm{C}$ & $\underset{/ \mathbf{m}-\mathbf{K}}{\mathbf{W}}$ & $\begin{array}{c}\mathbf{W} \\
/ \mathbf{m}^{2} \mathbf{K}\end{array}$ & -- & --- & --- \\
\hline 1 & 57.06 & 0.00 & 0.00 & 0.00 & 0.00 & 0.00 & 30.18 & 29.47 & 29.76 & 0.42 & 27.59 & 27.29 & 0.055 & 0 & 0.02 & 0 & 0 \\
\hline 2 & 57.06 & 0.00 & 0.00 & 0.00 & 0.00 & 0.00 & 30.28 & 29.52 & 29.76 & 0.52 & 27.54 & 27.29 & 0.055 & 0 & 0.02 & 0 & 0 \\
\hline 3 & 57.06 & 10.03 & 6.82 & 0.02 & -0.18 & -2.58 & 37.66 & 30.06 & 30.01 & 7.65 & 26.99 & 27.04 & 0.055 & 8781 & 0.28 & 1271 & 14 \\
\hline 4 & 57.27 & 19.89 & 13.53 & 0.05 & -0.36 & -5.13 & 44.94 & 30.66 & 30.41 & 14.53 & 26.61 & 26.86 & 0.055 & 9170 & 0.55 & 1329 & 28 \\
\hline 5 & 57.48 & 29.89 & 20.33 & 0.07 & -0.55 & -7.70 & 51.98 & 31.40 & 30.70 & 21.28 & 26.08 & 26.77 & 0.055 & 9414 & 0.82 & 1365 & 43 \\
\hline 6 & 57.48 & 39.93 & 27.16 & 0.10 & -0.73 & -10.29 & 58.36 & 32.29 & 31.15 & 27.21 & 25.18 & 26.33 & 0.055 & 9833 & 1.08 & 1427 & 60 \\
\hline 7 & 57.90 & 49.85 & 33.91 & 0.12 & -0.91 & -12.85 & 63.61 & 33.08 & 31.55 & 32.07 & 24.81 & 26.35 & 0.055 & 10417 & 1.29 & 1513 & 76 \\
\hline 8 & 58.11 & 59.89 & 40.74 & 0.14 & -1.10 & -15.43 & 68.39 & 33.88 & 31.89 & 36.49 & 24.23 & 26.21 & 0.055 & 10996 & 1.51 & 1598 & 93 \\
\hline 9 & 58.32 & 70.07 & 47.67 & 0.17 & -1.29 & -18.06 & 72.12 & 34.52 & 32.24 & 39.88 & 23.79 & 26.08 & 0.055 & 11773 & 1.68 & 1712 & 111 \\
\hline
\end{tabular}


Table D39: Reduced Data for Confined $17 \mathrm{~mm}$ Spacing

\begin{tabular}{|c|c|c|c|c|c|c|c|c|c|c|c|c|c|c|c|c|c|}
\hline sample & $\mathbf{T}_{\text {sat }}$ & $\mathbf{Q}$ & $\mathbf{q}$ & $\Delta \mathbf{T}_{\text {sub }}$ & $\Delta \mathbf{T}_{\mathrm{htr}}$ & $\Delta \mathbf{T}_{\text {glass }}$ & $\mathbf{T}_{\mathrm{s}}$ & $\mathbf{T}_{\infty_{\text {wall }}}$ & $\mathbf{T}_{\text {otop }}$ & $\begin{array}{c}\mathbf{T}_{\mathbf{s}^{-}} \\
\mathbf{T}_{\text {otop }}\end{array}$ & $\begin{array}{c}\mathbf{T}_{\text {sat }} \\
\mathbf{T}_{\text {cowall }}\end{array}$ & $\begin{array}{c}\mathbf{T}_{\text {sat- }} \\
\mathbf{T}_{\text {cotop }}\end{array}$ & $\mathbf{k}_{\text {fluid }}$ & h & $\begin{array}{c}\theta_{\mathrm{s}^{-}} \\
\theta_{\infty} \text { otop }_{\text {on }}\end{array}$ & Nu & GD \\
\hline \# & ${ }^{\circ} \mathrm{C}$ & $\mathbf{W}$ & $\begin{array}{c}10^{4} \\
W / m^{2}\end{array}$ & ${ }^{\circ} \mathrm{C}$ & ${ }^{\circ} \mathrm{C}$ & ${ }^{\circ} \mathrm{C}$ & ${ }^{\circ} \mathrm{C}$ & ${ }^{\circ} \mathrm{C}$ & ${ }^{\circ} \mathrm{C}$ & ${ }^{\circ} \mathrm{C}$ & ${ }^{\circ} \mathrm{C}$ & ${ }^{\circ} \mathrm{C}$ & $\begin{array}{c}\mathbf{W} / \\
\mathbf{m}-\mathbf{K}\end{array}$ & $\begin{array}{c}\mathbf{W} / \\
\mathbf{m}^{2} \mathbf{K}\end{array}$ & -- & --- & --- \\
\hline 1 & 56.64 & 0.00 & 0.00 & 0.00 & 0.00 & 0.00 & 30.08 & 29.37 & 29.66 & 0.42 & 27.27 & 26.97 & 0.055 & 0 & 0.02 & 0 & 0 \\
\hline 2 & 56.64 & 0.00 & 0.00 & 0.00 & 0.00 & 0.00 & 29.98 & 29.37 & 29.66 & 0.31 & 27.27 & 26.97 & 0.055 & 0 & 0.01 & 0 & 0 \\
\hline 3 & 56.85 & 10.03 & 6.82 & 0.02 & -0.18 & -2.58 & 37.06 & 30.11 & 30.01 & 7.05 & 26.74 & 26.83 & 0.055 & 9530 & 0.26 & 1380 & 14 \\
\hline 4 & 57.06 & 20.02 & 13.62 & 0.05 & -0.37 & -5.16 & 43.95 & 30.90 & 30.41 & 13.55 & 26.15 & 26.65 & 0.055 & 9903 & 0.52 & 1435 & 29 \\
\hline 5 & 57.06 & 29.89 & 20.33 & 0.07 & -0.55 & -7.70 & 50.68 & 31.80 & 30.85 & 19.82 & 25.26 & 26.20 & 0.055 & 10104 & 0.78 & 1465 & 45 \\
\hline 6 & 57.27 & 39.93 & 27.16 & 0.10 & -0.73 & $\begin{array}{l}-10.29 \\
\end{array}$ & 56.75 & 32.59 & 31.25 & 25.51 & 24.68 & 26.02 & 0.055 & 10490 & 1.03 & 1522 & 61 \\
\hline 7 & 57.48 & 49.85 & 33.91 & 0.12 & -0.91 & -12.85 & 62.66 & 33.63 & 31.74 & 30.92 & 23.85 & 25.73 & 0.055 & 10804 & 1.30 & 1570 & 79 \\
\hline 8 & 57.69 & 59.89 & 40.74 & 0.14 & -1.10 & -15.43 & 67.33 & 34.50 & 32.21 & 35.12 & 23.19 & 25.47 & 0.055 & 11426 & 1.51 & 1662 & 97 \\
\hline 9 & 58.11 & 69.78 & 47.47 & 0.17 & -1.28 & -17.98 & 71.34 & 35.49 & 32.76 & 38.59 & 22.62 & 25.35 & 0.055 & 12118 & 1.71 & 1764 & 116 \\
\hline
\end{tabular}

Table D40: Reduced Data for Unconfined $9 \mathrm{~mm}$ Spacing

\begin{tabular}{|c|c|c|c|c|c|c|c|c|c|c|c|c|c|c|c|c|c|}
\hline sample & $\mathbf{T}_{\text {sat }}$ & $\mathbf{Q}$ & $\mathbf{q}$ & $\Delta \mathbf{T}_{\text {sub }}$ & $\Delta \mathbf{T}_{\mathrm{htr}}$ & $\Delta \mathbf{T}_{\text {glass }}$ & $\mathbf{T}_{\mathbf{s}}$ & $\mathbf{T}_{\infty_{\text {wall }}}$ & $\mathbf{T}_{\text {otop }}$ & $\begin{array}{c}\mathbf{T}_{\mathrm{s}^{-}} \\
\mathbf{T}_{\text {ot }} \mathbf{t}_{\mathrm{op}}\end{array}$ & $\begin{array}{c}\mathbf{T}_{\text {sat }}{ }^{-} \\
\mathbf{T}_{\text {owall }}\end{array}$ & $\begin{array}{c}\mathbf{T}_{\text {sat }}{ }^{-} \\
\mathbf{T}_{\text {otop }} \\
\end{array}$ & $\mathbf{k}_{\text {fluid }}$ & h & $\begin{array}{c}\theta_{s^{-}} \\
\theta_{\text {otop }}\end{array}$ & $\mathrm{Nu}$ & GA \\
\hline \# & ${ }^{\circ} \mathrm{C}$ & W & $\begin{array}{c}10^{4} \\
\mathrm{~W} / \mathrm{m}^{2}\end{array}$ & ${ }^{\circ} \mathrm{C}$ & ${ }^{\circ} \mathrm{C}$ & ${ }^{\circ} \mathrm{C}$ & ${ }^{\circ} \mathrm{C}$ & ${ }^{\circ} \mathrm{C}$ & ${ }^{\circ} \mathrm{C}$ & ${ }^{\circ} \mathrm{C}$ & ${ }^{\circ} \mathrm{C}$ & ${ }^{\circ} \mathrm{C}$ & $\begin{array}{c}\mathbf{W} / \\
\mathbf{m}-\mathbf{K}\end{array}$ & $\begin{array}{c}\mathbf{W} / \\
\mathbf{m}^{2} \mathbf{K}\end{array}$ & --- & --- & --- \\
\hline 1 & 58.11 & 0.00 & 0.00 & 0.00 & 0.00 & 0.00 & 29.08 & 28.37 & 28.27 & 0.80 & 29.73 & 29.83 & 0.055 & 0 & 0.03 & 0 & 0 \\
\hline 2 & 58.11 & 0.00 & 0.00 & 0.00 & 0.00 & 0.00 & 29.08 & 28.37 & 28.32 & 0.75 & 29.73 & 29.78 & 0.055 & 0 & 0.03 & 0 & 0 \\
\hline 3 & 58.11 & 10.03 & 6.82 & 0.02 & -0.18 & -2.58 & 37.06 & 28.97 & 28.60 & 8.47 & 29.14 & 29.51 & 0.055 & 7936 & 0.29 & 1145 & 13 \\
\hline 4 & 58.32 & 20.02 & 13.62 & 0.05 & -0.37 & -5.16 & 44.76 & 29.61 & 28.82 & 15.94 & 28.70 & 29.50 & 0.055 & 8417 & 0.56 & 1215 & 26 \\
\hline 5 & 58.32 & 29.89 & 20.33 & 0.07 & -0.55 & -7.70 & 52.03 & 30.36 & 29.11 & 22.92 & 27.96 & 29.20 & 0.055 & 8741 & 0.82 & 1263 & 40 \\
\hline 6 & 58.74 & 39.93 & 27.16 & 0.10 & -0.73 & -10.29 & 58.86 & 31.30 & 29.61 & 29.25 & 27.43 & 29.13 & 0.055 & 9147 & 1.07 & 1323 & 55 \\
\hline 7 & 58.95 & 50.06 & 34.05 & 0.12 & -0.92 & -12.90 & 64.91 & 32.04 & 29.96 & 34.95 & 26.90 & 28.99 & 0.055 & 9596 & 1.30 & 1389 & 70 \\
\hline 8 & 59.16 & 59.89 & 40.74 & 0.14 & -1.10 & -15.43 & 69.84 & 33.13 & 30.55 & 39.29 & 26.02 & 28.60 & 0.055 & 10214 & 1.51 & 1480 & 87 \\
\hline 9 & 59.58 & 70.03 & 47.64 & 0.17 & -1.28 & -18.05 & 73.89 & 34.03 & 31.19 & 42.69 & 25.55 & 28.38 & 0.055 & 10991 & 1.67 & 1595 & 103 \\
\hline
\end{tabular}


Table D41: Reduced Data for Unconfined $11 \mathrm{~mm}$ Spacing

\begin{tabular}{|c|c|c|c|c|c|c|c|c|c|c|c|c|c|c|c|c|c|}
\hline sample & $\mathbf{T}_{\text {sat }}$ & $\mathbf{Q}$ & $\mathbf{q}$ & $\Delta \mathbf{T}_{\text {sub }}$ & $\Delta \mathbf{T}_{\mathrm{htr}}$ & $\Delta \mathbf{T}_{\text {glass }}$ & $\mathbf{T}_{\mathrm{s}}$ & $\mathbf{T}_{\infty_{\text {wall }}}$ & $\mathbf{T}_{\text {otop }}$ & $\begin{array}{c}\mathbf{T}_{\mathbf{s}^{-}} \\
\mathbf{T}_{\text {ot }} \text { ot }_{\text {op }}\end{array}$ & $\begin{array}{c}\mathbf{T}_{\text {sat }}{ }^{-} \\
\mathbf{T}_{\text {cowall }}\end{array}$ & $\begin{array}{c}\mathbf{T}_{\text {sat }} \\
\mathbf{T}_{\text {cotop }}\end{array}$ & $\mathbf{k}_{\text {fluid }}$ & h & $\begin{array}{c}\theta_{\mathrm{s}^{-}} \\
\theta_{\text {ot top }}\end{array}$ & $\mathrm{Nu}$ & GA \\
\hline \# & ${ }^{\circ} \mathrm{C}$ & W & $\begin{array}{c}10^{4} \\
W / m^{2}\end{array}$ & ${ }^{\circ} \mathrm{C}$ & ${ }^{\circ} \mathrm{C}$ & ${ }^{\circ} \mathrm{C}$ & ${ }^{\circ} \mathrm{C}$ & ${ }^{\circ} \mathrm{C}$ & ${ }^{\circ} \mathrm{C}$ & ${ }^{\circ} \mathrm{C}$ & ${ }^{\circ} \mathrm{C}$ & ${ }^{\circ} \mathrm{C}$ & $\begin{array}{c}\mathbf{W} / \\
\mathbf{m}-\mathbf{K}\end{array}$ & $\begin{array}{c}\mathbf{W} / \\
\mathbf{m}^{2} \mathbf{K}\end{array}$ & --- & --- & --- \\
\hline 1 & 58.32 & 0.00 & 0.00 & 0.00 & 0.00 & 0.00 & 28.88 & 28.08 & 28.07 & 0.80 & 30.24 & 30.24 & 0.055 & 0 & 0.03 & 0 & 0 \\
\hline 2 & 58.11 & 0.00 & 0.00 & 0.00 & 0.00 & 0.00 & 28.83 & 28.03 & 28.10 & 0.73 & 30.08 & 30.01 & 0.055 & 0 & 0.02 & 0 & 0 \\
\hline 3 & 58.32 & 10.03 & 6.82 & 0.02 & -0.18 & -2.58 & 35.61 & 28.65 & 28.37 & 7.24 & 29.67 & 29.94 & 0.055 & 9286 & 0.24 & 1340 & 13 \\
\hline 4 & 58.42 & 20.02 & 13.62 & 0.05 & -0.37 & -5.16 & 42.20 & 29.32 & 28.72 & 13.48 & 29.10 & 29.70 & 0.055 & 9952 & 0.46 & 1437 & 26 \\
\hline 5 & 58.74 & 29.89 & 20.33 & 0.07 & -0.55 & -7.70 & 48.47 & 30.01 & 29.02 & 19.46 & 28.72 & 29.72 & 0.055 & 10295 & 0.68 & 1487 & 39 \\
\hline 6 & 58.95 & 39.93 & 27.16 & 0.10 & -0.73 & -10.29 & 54.60 & 30.90 & 29.41 & 25.19 & 28.04 & 29.53 & 0.055 & 10622 & 0.90 & 1536 & 54 \\
\hline 7 & 59.16 & 50.06 & 34.05 & 0.12 & -0.92 & -12.90 & 60.20 & 31.65 & 29.76 & 30.44 & 27.51 & 29.40 & 0.055 & 11019 & 1.11 & 1594 & 69 \\
\hline 8 & 59.47 & 59.89 & 40.74 & 0.14 & -1.10 & -15.43 & 65.03 & 32.54 & 30.20 & 34.82 & 26.93 & 29.27 & 0.055 & 11524 & 1.29 & 1669 & 84 \\
\hline 9 & 59.79 & 70.03 & 47.64 & 0.17 & -1.28 & -18.05 & 69.27 & 33.28 & 30.65 & 38.62 & 26.50 & 29.14 & 0.055 & 12149 & 1.46 & 1761 & 100 \\
\hline
\end{tabular}

Table D42: Reduced Data for Unconfined $13 \mathrm{~mm}$ Spacing

\begin{tabular}{|c|c|c|c|c|c|c|c|c|c|c|c|c|c|c|c|c|c|}
\hline sample & $\mathbf{T}_{\text {sat }}$ & $\mathbf{Q}$ & $\mathbf{q}$ & $\Delta \mathbf{T}_{\text {sub }}$ & $\Delta \mathbf{T}_{\mathrm{htr}}$ & $\Delta \mathbf{T}_{\text {glass }}$ & $\mathbf{T}_{\mathrm{s}}$ & $\mathbf{T}_{\infty_{\text {wall }}}$ & $\mathbf{T}_{\text {otop }}$ & $\begin{array}{c}\mathbf{T}_{\mathrm{s}^{-}} \\
\mathbf{T}_{\text {otop }}\end{array}$ & $\begin{array}{c}\mathbf{T}_{\text {sat }} \\
\mathbf{T}_{\text {owall }}\end{array}$ & $\begin{array}{c}\mathbf{T}_{\text {sat }} \\
\mathbf{T}_{\text {otop }}\end{array}$ & $\mathbf{k}_{\text {fluid }}$ & h & $\theta_{\mathrm{s}}-\theta_{\infty t_{\mathrm{op}}}$ & $\mathbf{N u}$ & GA \\
\hline \# & ${ }^{\circ} \mathrm{C}$ & $\mathbf{W}$ & $\begin{array}{c}10^{4} \\
W / \mathbf{m}^{2} \\
\end{array}$ & ${ }^{\circ} \mathrm{C}$ & ${ }^{\circ} \mathrm{C}$ & ${ }^{\circ} \mathrm{C}$ & ${ }^{\circ} \mathrm{C}$ & ${ }^{\circ} \mathrm{C}$ & ${ }^{\circ} \mathrm{C}$ & ${ }^{\circ} \mathrm{C}$ & ${ }^{\circ} \mathrm{C}$ & ${ }^{\circ} \mathrm{C}$ & $\begin{array}{c}\mathbf{W} / \\
\mathbf{m}-\mathbf{K}\end{array}$ & $\begin{array}{c}\mathbf{W} / \\
\mathbf{m}^{2} \mathbf{K} \\
\end{array}$ & --- & --- & --- \\
\hline 1 & 57.90 & 0.00 & 0.00 & 0.00 & 0.00 & 0.00 & 27.97 & 27.23 & 27.38 & 0.59 & 30.66 & 30.52 & 0.056 & 0 & 0.02 & 0 & 0 \\
\hline 2 & 57.90 & 0.00 & 0.00 & 0.00 & 0.00 & 0.00 & 27.97 & 27.19 & 27.33 & 0.64 & 30.71 & 30.57 & 0.056 & 0 & 0.02 & 0 & 0 \\
\hline 3 & 58.11 & 10.03 & 6.82 & 0.02 & -0.18 & -2.58 & 33.75 & 27.93 & 27.73 & 6.03 & 30.18 & 30.38 & 0.056 & 11150 & 0.20 & 1606 & 13 \\
\hline 4 & 58.32 & 20.02 & 13.62 & --- & --- & --- & --- & --- & --- & --- & --- & --- & --- & --- & --- & --- & --- \\
\hline 5 & 58.42 & 29.89 & 20.33 & 0.07 & -0.55 & -7.70 & 45.16 & 29.39 & 28.57 & 16.59 & 29.03 & 29.85 & 0.055 & 12071 & 0.57 & 1742 & 39 \\
\hline 6 & 58.74 & 39.93 & 27.16 & 0.10 & -0.73 & -10.29 & 50.44 & 30.26 & 29.06 & 21.37 & 28.48 & 29.67 & 0.055 & 12518 & 0.75 & 1808 & 53 \\
\hline 7 & 59.16 & 50.06 & 34.05 & 0.12 & -0.92 & -12.90 & 57.29 & 32.98 & 31.44 & 25.85 & 26.17 & 27.71 & 0.055 & 12975 & 0.99 & 1884 & 72 \\
\hline 8 & 59.37 & 59.89 & 40.74 & 0.14 & -1.10 & -15.43 & 60.32 & 31.85 & 29.91 & 30.41 & 27.52 & 29.46 & 0.055 & 13197 & 1.11 & 1910 & 82 \\
\hline 9 & 59.79 & 70.03 & 47.64 & 0.17 & -1.28 & -18.05 & 64.66 & 32.66 & 30.28 & 34.38 & 27.12 & 29.51 & 0.055 & 13647 & 1.27 & 1977 & 97 \\
\hline
\end{tabular}


Table D43: Reduced Data for Unconfined $15 \mathrm{~mm}$ Spacing

\begin{tabular}{|c|c|c|c|c|c|c|c|c|c|c|c|c|c|c|c|c|c|}
\hline sample & $\mathbf{T}_{\text {sat }}$ & $\mathbf{Q}$ & $\mathbf{q}$ & $\Delta \mathbf{T}_{\text {sub }}$ & $\Delta \mathbf{T}_{\mathrm{htr}}$ & $\Delta \mathbf{T}_{\text {glass }}$ & $\mathbf{T}_{\mathrm{s}}$ & $\mathbf{T}_{\infty_{\text {wall }}}$ & $\mathbf{T}_{\text {cotop }}$ & $\begin{array}{c}\mathbf{T}_{\mathbf{s}^{-}} \\
\mathbf{T}_{\text {cotop }}\end{array}$ & $\begin{array}{c}\mathbf{T}_{\text {sat }} \\
\mathbf{T}_{\text {cowall }}\end{array}$ & $\begin{array}{c}\mathbf{T}_{\text {sat }} \\
\mathbf{T}_{\text {otop }}\end{array}$ & $\mathbf{k}_{\text {fluid }}$ & h & $\begin{array}{c}\theta_{s^{-}} \\
\theta_{\infty t_{o p}}\end{array}$ & $\mathbf{N u}$ & GD \\
\hline \# & ${ }^{\circ} \mathrm{C}$ & $\mathbf{W}$ & $\begin{array}{c}10^{4} \\
W / m^{2}\end{array}$ & ${ }^{\circ} \mathrm{C}$ & ${ }^{\circ} \mathrm{C}$ & ${ }^{\circ} \mathrm{C}$ & ${ }^{\circ} \mathrm{C}$ & ${ }^{\circ} \mathrm{C}$ & ${ }^{\circ} \mathrm{C}$ & ${ }^{\circ} \mathrm{C}$ & ${ }^{\circ} \mathrm{C}$ & ${ }^{\circ} \mathrm{C}$ & $\begin{array}{c}\mathbf{W} / \\
\mathbf{m}-\mathbf{K}\end{array}$ & $\begin{array}{c}\mathbf{W} / \\
\mathbf{m}^{2} \mathbf{K}\end{array}$ & -- & --- & --- \\
\hline 1 & 57.06 & 0.00 & 0.00 & 0.00 & 0.00 & 0.00 & 29.18 & 28.52 & 28.42 & 0.76 & 28.53 & 28.63 & 0.055 & 0 & 0.03 & 0 & 0 \\
\hline 2 & 56.85 & 0.00 & 0.00 & 0.00 & 0.00 & 0.00 & 29.13 & 28.42 & 28.42 & 0.71 & 28.42 & 28.42 & 0.055 & 0 & 0.02 & 0 & 0 \\
\hline 3 & 57.06 & 10.03 & 6.82 & 0.02 & -0.18 & -2.58 & 35.16 & 29.07 & 28.77 & 6.39 & 27.99 & 28.29 & 0.055 & 10519 & 0.23 & 1519 & 14 \\
\hline 4 & 57.27 & 20.02 & 13.62 & 0.05 & -0.37 & -5.16 & 41.15 & 29.81 & 29.16 & 11.98 & 27.45 & 28.10 & 0.055 & 11197 & 0.44 & 1618 & 27 \\
\hline 5 & 57.48 & 29.89 & 20.33 & 0.07 & -0.55 & -7.70 & 46.92 & 30.46 & 29.56 & 17.36 & 27.02 & 27.91 & 0.055 & 11541 & 0.64 & 1669 & 42 \\
\hline 6 & 57.90 & 39.93 & 27.16 & 0.10 & -0.73 & -10.29 & 52.59 & 31.25 & 30.01 & 22.59 & 26.65 & 27.89 & 0.055 & 11845 & 0.85 & 1715 & 56 \\
\hline 7 & 58.32 & 50.06 & 34.05 & 0.12 & -0.92 & -12.90 & 57.74 & 32.04 & 30.35 & 27.39 & 26.27 & 27.96 & 0.055 & 12246 & 1.04 & 1774 & 72 \\
\hline 8 & 58.53 & 60.12 & 40.90 & 0.14 & -1.10 & -15.49 & 62.51 & 32.99 & 30.90 & 31.61 & 25.54 & 27.63 & 0.055 & 12742 & 1.24 & 1848 & 89 \\
\hline 9 & 58.95 & 70.03 & 47.64 & 0.17 & -1.28 & -18.05 & 66.67 & 33.78 & 31.29 & 35.37 & 25.17 & 27.65 & 0.055 & 13266 & 1.41 & 1926 & 105 \\
\hline
\end{tabular}

Table D44: Reduced Data for Unconfined $17 \mathrm{~mm}$ Spacing

\begin{tabular}{|c|c|c|c|c|c|c|c|c|c|c|c|c|c|c|c|c|c|}
\hline sample & $\mathbf{T}_{\text {sat }}$ & $\mathbf{Q}$ & $\mathbf{q}$ & $\Delta \mathbf{T}_{\text {sub }}$ & $\Delta \mathbf{T}_{\mathrm{htr}}$ & $\Delta \mathbf{T}_{\text {glass }}$ & $\mathbf{T}_{\mathrm{s}}$ & $\mathbf{T}_{\infty_{\text {wall }}}$ & $\mathbf{T}_{\text {otop }}$ & $\begin{array}{c}\mathbf{T}_{\mathbf{s}^{-}} \\
\mathbf{T}_{\text {ot top }}\end{array}$ & $\begin{array}{c}\mathbf{T}_{\text {sat }^{-}} \\
\mathbf{T}_{\text {owwall }}\end{array}$ & $\begin{array}{c}\mathbf{T}_{\text {sat }}{ }^{-} \\
\mathbf{T}_{\text {otop }}\end{array}$ & $\mathbf{k}_{\text {fluid }}$ & h & $\begin{array}{c}\theta_{s^{-}} \\
\theta_{\text {stop }}\end{array}$ & $\mathrm{Nu}$ & GD \\
\hline \# & ${ }^{\circ} \mathrm{C}$ & $\mathbf{W}$ & $\begin{array}{c}10^{4} \\
\text { W/m } \\
\end{array}$ & ${ }^{\circ} \mathrm{C}$ & ${ }^{\circ} \mathrm{C}$ & ${ }^{\circ} \mathrm{C}$ & ${ }^{\circ} \mathrm{C}$ & ${ }^{\circ} \mathrm{C}$ & ${ }^{\circ} \mathrm{C}$ & ${ }^{\circ} \mathrm{C}$ & ${ }^{\circ} \mathrm{C}$ & ${ }^{\circ} \mathrm{C}$ & $\begin{array}{c}\mathbf{W} / \\
\mathbf{m}-\mathbf{K}\end{array}$ & $\begin{array}{c}\mathbf{W} / \\
\mathbf{m}^{2} \mathbf{K}\end{array}$ & -- & --- & --- \\
\hline 1 & 57.27 & 0.00 & 0.00 & 0.00 & 0.00 & 0.00 & 29.48 & 28.85 & 28.70 & 0.78 & 28.42 & 28.57 & 0.055 & 0 & 0.03 & 0 & 0 \\
\hline 2 & 57.27 & 0.00 & 0.00 & 0.00 & 0.00 & 0.00 & 29.48 & 28.77 & 28.67 & 0.81 & 28.49 & 28.60 & 0.055 & 0 & 0.03 & 0 & 0 \\
\hline 3 & 57.37 & 10.03 & 6.82 & 0.02 & -0.18 & -2.58 & 35.66 & 29.37 & 29.02 & 6.64 & 28.00 & 28.35 & 0.055 & 10118 & 0.24 & 1462 & 13 \\
\hline 4 & 57.48 & 20.02 & 13.62 & 0.05 & -0.37 & -5.16 & 41.70 & 30.01 & 29.36 & 12.33 & 27.46 & 28.11 & 0.055 & 10877 & 0.45 & 1572 & 27 \\
\hline 5 & 57.90 & 29.89 & 20.33 & 0.07 & -0.55 & -7.70 & 47.62 & 30.70 & 29.71 & 17.91 & 27.19 & 28.19 & 0.055 & 11184 & 0.66 & 1618 & 41 \\
\hline 6 & 58.11 & 39.93 & 27.16 & 0.10 & -0.73 & -10.29 & 53.24 & 31.57 & 30.18 & 23.06 & 26.53 & 27.93 & 0.055 & 11600 & 0.87 & 1680 & 57 \\
\hline 7 & 58.32 & 50.06 & 34.05 & 0.12 & -0.92 & -12.90 & 58.54 & 32.27 & 30.60 & 27.94 & 26.05 & 27.71 & 0.055 & 12004 & 1.07 & 1740 & 72 \\
\hline 8 & 58.74 & 59.89 & 40.74 & 0.14 & -1.10 & -15.43 & 63.27 & 33.11 & 31.07 & 32.20 & 25.63 & 27.66 & 0.055 & 12462 & 1.26 & 1808 & 88 \\
\hline 9 & 59.16 & 70.03 & 47.64 & 0.17 & -1.28 & -18.05 & 67.42 & 33.80 & 31.37 & 36.05 & 25.35 & 27.79 & 0.055 & 13017 & 1.42 & 1890 & 104 \\
\hline
\end{tabular}


Table D45: Reduced Data for Unconfined 13 mm \#2 Spacing

\begin{tabular}{|c|c|c|c|c|c|c|c|c|c|c|c|c|c|c|c|c|c|}
\hline sample & $\mathbf{T}_{\text {sat }}$ & $\mathbf{Q}$ & $\mathbf{q}$ & $\Delta \mathbf{T}_{\text {sub }}$ & $\Delta \mathbf{T}_{\mathrm{htr}}$ & $\Delta \mathbf{T}_{\text {glass }}$ & $\mathbf{T}_{\mathrm{s}}$ & $\mathbf{T}_{\infty_{\text {wall }}}$ & $\mathbf{T}_{\text {otop }}$ & $\begin{array}{c}\mathbf{T}_{\mathbf{s}^{-}} \\
\mathbf{T}_{\text {otop }}\end{array}$ & $\begin{array}{c}\mathbf{T}_{\text {sat }} \\
\mathbf{T}_{\text {owall }} \\
\end{array}$ & $\begin{array}{c}\mathbf{T}_{\text {sat }^{-}} \\
\mathbf{T}_{\text {cotop }}\end{array}$ & $\mathbf{k}_{\text {fluid }}$ & h & $\begin{array}{c}\theta_{\mathrm{s}^{-}} \\
\theta_{\infty} \text { top }_{\text {op }}\end{array}$ & Nu & GD \\
\hline \# & ${ }^{\circ} \mathrm{C}$ & W & $\begin{array}{c}10^{4} \\
W / m^{2}\end{array}$ & ${ }^{\circ} \mathrm{C}$ & ${ }^{\circ} \mathrm{C}$ & ${ }^{\circ} \mathrm{C}$ & ${ }^{\circ} \mathrm{C}$ & ${ }^{\circ} \mathrm{C}$ & ${ }^{\circ} \mathrm{C}$ & ${ }^{\circ} \mathbf{C}$ & ${ }^{\circ} \mathrm{C}$ & ${ }^{\circ} \mathrm{C}$ & $\begin{array}{c}\mathbf{W} / \\
\mathbf{m}-\mathbf{K}\end{array}$ & $\begin{array}{c}\mathbf{W} / \\
\mathbf{m}^{2} \mathbf{K}\end{array}$ & --- & -- & --- \\
\hline 1 & 56.85 & 0.00 & 0.00 & 0.00 & 0.00 & 0.00 & 29.58 & 28.85 & 28.79 & 0.78 & 28.00 & 28.05 & 0.055 & 0 & 0.03 & 0 & 0 \\
\hline 2 & 56.64 & 0.00 & 0.00 & 0.00 & 0.00 & 0.00 & 29.58 & 28.82 & 28.77 & 0.81 & 27.81 & 27.87 & 0.055 & 0 & 0.03 & 0 & 0 \\
\hline 3 & 57.06 & 10.03 & 6.82 & 0.02 & -0.18 & -2.58 & 36.46 & 29.49 & 29.07 & 7.39 & 27.57 & 27.99 & 0.055 & 9088 & 0.27 & 1313 & 14 \\
\hline 4 & 57.06 & 20.02 & 13.62 & 0.05 & -0.37 & -5.16 & 43.25 & 30.21 & 29.41 & 13.84 & 26.85 & 27.64 & 0.055 & 9694 & 0.52 & 1402 & 28 \\
\hline 5 & 57.37 & 29.89 & 20.33 & 0.07 & -0.55 & -7.70 & 49.72 & 30.85 & 29.81 & 19.92 & 26.52 & 27.56 & 0.055 & 10057 & 0.75 & 1455 & 42 \\
\hline 6 & 57.69 & 39.93 & 27.16 & 0.10 & -0.73 & -10.29 & 55.75 & 31.72 & 30.23 & 25.52 & 25.96 & 27.46 & 0.055 & 10483 & 0.98 & 1518 & 58 \\
\hline 7 & 57.90 & 50.06 & 34.05 & 0.12 & -0.92 & -12.90 & 61.40 & 32.54 & 30.75 & 30.65 & 25.36 & 27.15 & 0.055 & 10943 & 1.21 & 1587 & 74 \\
\hline 8 & 58.32 & 59.89 & 40.74 & 0.14 & -1.10 & -15.43 & 66.03 & 33.26 & 31.10 & 34.93 & 25.06 & 27.22 & 0.055 & 11487 & 1.39 & 1667 & 90 \\
\hline 9 & 58.53 & 70.03 & 47.64 & 0.17 & -1.28 & -18.05 & 70.28 & 34.25 & 31.67 & 38.61 & 24.28 & 26.86 & 0.055 & 12153 & 1.59 & 1765 & 109 \\
\hline 10 & 59.16 & 86.50 & 58.84 & 0.21 & -1.59 & -22.29 & 75.70 & 35.84 & 32.68 & 43.01 & 23.32 & 26.47 & 0.055 & 13474 & 1.84 & 1962 & 140 \\
\hline
\end{tabular}




\section{Cap Electrode Averaged Raw Data and Data Reduction Tables}

The raw data for the cap electrode study consisted of values read by the data acquisition system each fraction of a second and is therefore left out of this appendix. The single numbers used in data reduction for each test condition using FC-72 are shown in Table D46 through Table D57 and were found by averaging a one minute steady state portion of the raw data. Tests of the full length electrode with HFE-7000 as the working fluid are shown in Table D58 through Table D60. Table D61 through Table D72 show the data reduction values for the electrode study using FC-72 at each high voltage level. Table D73 through Table D75 show the reduced data values for the HFE-7000 test.

Table D46: Averaged Heater and Pressure Raw Data for Bottom Half Electrode

\begin{tabular}{|c|c|c|c|c|c|c|}
\hline $\mathbf{H V}$ & $\mathbf{Q}$ & $\begin{array}{c}\text { Heater Voltage } \\
\text { after } \\
\text { precision resistor }\end{array}$ & $\begin{array}{c}\text { Heater Voltage } \\
\text { before } \\
\text { precision resistor }\end{array}$ & $\begin{array}{c}\text { Heater } \\
\text { Current }\end{array}$ & $\begin{array}{c}\text { Chamber } \\
\text { Pressure }\end{array}$ & $\begin{array}{c}\text { Nozzle } \\
\text { Pressure }\end{array}$ \\
\hline $\mathbf{k V}$ & $\mathbf{W}$ & $\mathbf{V o l t s}$ & $\mathbf{V o l t s}$ & Amps & psia & psia \\
\hline $\mathbf{0}$ & 0.00 & 0.00 & 0.00 & 0.00 & 13.61 & 21.96 \\
\hline $\mathbf{0}$ & 10.02 & 9.79 & 13.95 & 1.01 & 13.68 & 22.08 \\
\hline $\mathbf{0}$ & 20.04 & 13.80 & 17.05 & 1.74 & 13.78 & 22.22 \\
\hline $\mathbf{0}$ & 30.00 & 16.88 & 19.70 & 2.03 & 13.87 & 22.34 \\
\hline $\mathbf{0}$ & 40.04 & 19.49 & 22.02 & 2.27 & 14.07 & 22.45 \\
\hline $\mathbf{0}$ & 50.07 & 21.79 & 24.08 & 2.49 & 14.15 & 22.50 \\
\hline $\mathbf{0}$ & 59.98 & 23.83 & 26.01 & 2.70 & 14.24 & 22.54 \\
\hline $\mathbf{0}$ & 70.12 & 25.75 & 27.81 & 2.88 & 14.36 & 22.67 \\
\hline $\mathbf{0}$ & 80.02 & 27.52 & 29.52 & 3.04 & 14.47 & 22.68 \\
\hline $\mathbf{0}$ & 89.88 & 29.21 & 0.00 & 0.00 & 14.06 & 22.42 \\
\hline $\mathbf{0}$ & 0.00 & 0.00 & 22.19 & 2.26 & 14.10 & 22.35 \\
\hline $\mathbf{0}$ & 50.09 & 21.96 & 22.18 & 14.11 & 22.37 & 2.26 \\
\hline $\mathbf{3}$ & 50.18 & 21.96 & 22.19 & 14.13 & 22.38 & 2.26 \\
\hline $\mathbf{6}$ & 50.15 & 21.96 & 24.28 & 2.47 & 14.19 & 22.43 \\
\hline $\mathbf{0}$ & 60.02 & 24.03 & 24.28 & 14.22 & 22.46 & 2.47 \\
\hline $\mathbf{3}$ & 60.05 & 24.03 & 24.28 & 14.24 & 22.48 & 2.47 \\
\hline $\mathbf{6}$ & 60.05 & 24.03 & 26.23 & 2.67 & 14.34 & 22.57 \\
\hline $\mathbf{0}$ & 70.00 & 25.96 & 26.23 & 14.36 & 22.59 & 2.67 \\
\hline $\mathbf{3}$ & 69.96 & 25.96 & 26.23 & 14.38 & 22.62 & 2.67 \\
\hline $\mathbf{6}$ & 69.93 & 25.96 & 28.05 & 2.85 & 14.44 & 22.68 \\
\hline $\mathbf{0}$ & 79.88 & 27.76 & 28.05 & 14.46 & 22.69 & 2.85 \\
\hline $\mathbf{3}$ & 79.84 & 27.76 & 28.05 & 14.48 & 22.70 & 2.85 \\
\hline $\mathbf{6}$ & 79.83 & 27.76 & & & & \\
\hline & & & & & & \\
\hline
\end{tabular}


Table D47: Averaged Pedestal Temperature Raw Data for Bottom Half Electrode

\begin{tabular}{|c|c|c|c|c|c|c|c|c|}
\hline $\mathbf{H V}$ & $\mathbf{Q}$ & $\mathbf{T} 1$ & $\mathbf{T} 2$ & $\mathbf{T 3}$ & $\mathbf{T 4}$ & $\mathbf{T 5}$ & $\mathbf{T 6}$ & $\mathbf{T} 7$ \\
\hline $\mathbf{~ k V}$ & $\mathbf{W}$ & ${ }^{\circ} \mathbf{C}$ & ${ }^{\circ} \mathbf{C}$ & ${ }^{\circ} \mathbf{C}$ & ${ }^{\circ} \mathbf{C}$ & ${ }^{\circ} \mathbf{C}$ & ${ }^{\circ} \mathbf{C}$ & ${ }^{\circ} \mathbf{C}$ \\
\hline $\mathbf{0}$ & 0.00 & 23.23 & 22.82 & 22.85 & 22.85 & 23.02 & 23.07 & 24.03 \\
\hline $\mathbf{0}$ & 10.02 & 30.32 & 26.23 & 24.88 & 25.64 & 24.67 & 25.08 & 24.58 \\
\hline $\mathbf{0}$ & 20.04 & 37.38 & 30.25 & 27.24 & 29.64 & 26.89 & 27.57 & 25.69 \\
\hline $\mathbf{0}$ & 30.00 & 44.19 & 33.87 & 29.40 & 32.89 & 28.77 & 29.70 & 26.68 \\
\hline $\mathbf{0}$ & 40.04 & 50.46 & 37.02 & 31.38 & 35.39 & 30.29 & 31.41 & 27.47 \\
\hline $\mathbf{0}$ & 50.07 & 55.93 & 40.69 & 33.71 & 38.78 & 32.37 & 33.48 & 28.88 \\
\hline $\mathbf{0}$ & 59.98 & 59.70 & 43.40 & 35.65 & 41.17 & 33.98 & 35.17 & 30.21 \\
\hline $\mathbf{0}$ & 70.12 & 62.49 & 45.50 & 37.26 & 43.02 & 35.29 & 36.66 & 31.32 \\
\hline $\mathbf{0}$ & 80.02 & 65.22 & 47.44 & 38.77 & 44.84 & 36.57 & 38.16 & 32.54 \\
\hline $\mathbf{0}$ & 89.88 & 68.00 & 49.18 & 39.91 & 46.54 & 37.64 & 39.59 & 33.70 \\
\hline $\mathbf{0}$ & 0.00 & 25.40 & 27.13 & 25.54 & 30.66 & 27.20 & 26.91 & 29.09 \\
\hline $\mathbf{0}$ & 50.09 & 52.18 & 40.49 & 34.05 & 38.82 & 32.96 & 34.17 & 29.53 \\
\hline $\mathbf{3}$ & 50.18 & 52.38 & 40.82 & 34.26 & 39.38 & 33.27 & 34.43 & 30.07 \\
\hline $\mathbf{6}$ & 50.15 & 52.45 & 40.97 & 34.38 & 39.61 & 33.42 & 34.56 & 30.42 \\
\hline $\mathbf{0}$ & 60.02 & 55.58 & 42.75 & 35.72 & 40.50 & 34.25 & 35.52 & 30.94 \\
\hline $\mathbf{3}$ & 60.05 & 55.70 & 42.97 & 35.86 & 40.80 & 34.43 & 35.67 & 31.25 \\
\hline $\mathbf{6}$ & 60.05 & 55.77 & 43.08 & 35.94 & 40.92 & 34.49 & 35.72 & 31.44 \\
\hline $\mathbf{0}$ & 70.00 & 58.25 & 44.65 & 37.25 & 42.05 & 35.46 & 36.89 & 32.30 \\
\hline $\mathbf{3}$ & 69.96 & 58.28 & 44.73 & 37.29 & 42.18 & 35.55 & 36.98 & 32.49 \\
\hline $\mathbf{6}$ & 69.93 & 58.28 & 44.78 & 37.34 & 42.26 & 35.61 & 37.04 & 32.63 \\
\hline $\mathbf{0}$ & 79.88 & 60.69 & 46.06 & 38.42 & 43.12 & 36.34 & 38.03 & 33.11 \\
\hline $\mathbf{3}$ & 79.84 & 60.79 & 46.23 & 38.52 & 43.37 & 36.47 & 38.14 & 33.37 \\
\hline $\mathbf{6}$ & 79.83 & 60.86 & 46.35 & 38.62 & 43.51 & 36.58 & 38.25 & 33.52 \\
\hline
\end{tabular}


Table D48: Averaged Chamber Temperature Raw Data for Bottom Half Electrode

\begin{tabular}{|c|c|c|c|c|c|c|c|c|}
\hline $\mathbf{H V}$ & $\mathbf{Q}$ & $\mathbf{A m b}$ & $\mathbf{T 8}$ & $\mathbf{T 9}$ & $\mathbf{T 1 0}$ & $\mathbf{T 1 1}$ & $\mathbf{T 1 2}$ & ${ }^{\mathbf{T} 14}$ \\
\hline $\mathbf{k V}$ & $\mathbf{W}$ & ${ }^{\circ} \mathbf{C}$ & ${ }^{\circ} \mathbf{C}$ & ${ }^{\circ} \mathbf{C}$ & ${ }^{\circ} \mathbf{C}$ & ${ }^{\circ} \mathbf{C}$ & ${ }^{\circ} \mathbf{C}$ & ${ }^{\circ} \mathbf{C}$ \\
\hline $\mathbf{0}$ & 0.00 & 20.83 & 21.92 & 21.69 & 23.24 & 22.88 & 21.83 & 22.07 \\
\hline $\mathbf{0}$ & 10.02 & 20.96 & 22.30 & 22.23 & 24.15 & 23.54 & 22.21 & 22.11 \\
\hline $\mathbf{0}$ & 20.04 & 20.89 & 22.81 & 22.63 & 25.20 & 24.28 & 22.73 & 22.17 \\
\hline $\mathbf{0}$ & 30.00 & 20.94 & 23.32 & 23.06 & 26.23 & 24.99 & 23.24 & 22.20 \\
\hline $\mathbf{0}$ & 40.04 & 20.93 & 23.78 & 23.41 & 27.24 & 25.70 & 23.71 & 22.22 \\
\hline $\mathbf{0}$ & 50.07 & 21.00 & 24.34 & 23.80 & 28.45 & 26.58 & 24.25 & 22.27 \\
\hline $\mathbf{0}$ & 59.98 & 20.99 & 25.05 & 24.24 & 29.68 & 27.50 & 24.95 & 22.33 \\
\hline $\mathbf{0}$ & 70.12 & 21.19 & 25.69 & 24.67 & 30.85 & 28.46 & 25.61 & 22.38 \\
\hline $\mathbf{0}$ & 80.02 & 21.26 & 26.47 & 25.19 & 32.08 & 29.52 & 26.35 & 22.48 \\
\hline $\mathbf{0}$ & 89.88 & 21.38 & 27.11 & 25.55 & 32.88 & 30.81 & 27.19 & 22.57 \\
\hline $\mathbf{0}$ & 0.00 & 21.33 & 23.75 & 23.92 & 24.70 & 24.19 & 26.91 & 22.71 \\
\hline $\mathbf{0}$ & 50.09 & 21.41 & 25.17 & 24.76 & 29.17 & 27.34 & 34.17 & 22.82 \\
\hline $\mathbf{3}$ & 50.18 & 21.42 & 25.26 & 24.81 & 29.25 & 27.40 & 25.36 & 22.82 \\
\hline $\mathbf{6}$ & 50.15 & 21.57 & 25.30 & 24.83 & 29.32 & 27.48 & 25.36 & 22.85 \\
\hline $\mathbf{0}$ & 60.02 & 21.54 & 25.68 & 25.05 & 30.25 & 28.14 & 35.52 & 22.83 \\
\hline $\mathbf{3}$ & 60.05 & 21.60 & 25.80 & 25.10 & 30.28 & 28.16 & 25.90 & 22.78 \\
\hline $\mathbf{6}$ & 60.05 & 21.53 & 25.85 & 25.15 & 30.28 & 28.18 & 25.90 & 22.74 \\
\hline $\mathbf{0}$ & 70.00 & 21.50 & 26.40 & 25.44 & 31.41 & 29.09 & 36.89 & 22.83 \\
\hline $\mathbf{3}$ & 69.96 & 21.72 & 26.46 & 25.50 & 31.47 & 29.12 & 26.55 & 22.86 \\
\hline $\mathbf{6}$ & 69.93 & 21.36 & 26.49 & 25.54 & 31.50 & 29.16 & 26.55 & 22.87 \\
\hline $\mathbf{0}$ & 79.88 & 21.38 & 26.94 & 25.75 & 32.47 & 29.98 & 38.03 & 22.90 \\
\hline $\mathbf{3}$ & 79.84 & 21.58 & 27.03 & 25.80 & 32.54 & 30.03 & 27.12 & 22.91 \\
\hline $\mathbf{6}$ & 79.83 & 21.38 & 27.07 & 25.86 & 32.58 & 30.10 & 27.12 & 22.90 \\
\hline & & & & & & & & \\
\hline
\end{tabular}


Table D49: Averaged Heater and Pressure Raw Data for Thick Top Half Electrode

\begin{tabular}{|c|c|c|c|c|c|c|}
\hline $\mathbf{H V}$ & $\mathbf{Q}$ & $\begin{array}{c}\text { Heater Voltage } \\
\text { after } \\
\text { precision resistor }\end{array}$ & $\begin{array}{c}\text { Heater Voltage } \\
\text { before } \\
\text { precision resistor }\end{array}$ & $\begin{array}{c}\text { Heater } \\
\text { Current }\end{array}$ & $\begin{array}{c}\text { Chamber } \\
\text { Pressure }\end{array}$ & $\begin{array}{c}\text { Nozzle } \\
\text { Pressure }\end{array}$ \\
\hline $\mathbf{k V}$ & $\mathbf{W}$ & $\mathbf{V o l t s}$ & $\mathbf{V o l t s}$ & Amps & psia & psia \\
\hline $\mathbf{0}$ & 0.00 & 0.00 & 0.00 & 13.89 & 22.37 & 0.00 \\
\hline $\mathbf{0}$ & 10.01 & 9.57 & 9.68 & 13.93 & 22.46 & 1.03 \\
\hline $\mathbf{0}$ & 20.09 & 13.50 & 13.65 & 14.00 & 22.57 & 1.47 \\
\hline $\mathbf{0}$ & 30.03 & 16.50 & 16.68 & 14.09 & 22.68 & 1.80 \\
\hline $\mathbf{0}$ & 40.09 & 19.11 & 19.32 & 14.19 & 22.81 & 2.07 \\
\hline $\mathbf{0}$ & 50.06 & 21.33 & 21.56 & 14.32 & 22.96 & 2.32 \\
\hline $\mathbf{0}$ & 60.07 & 23.38 & 23.63 & 14.44 & 23.10 & 2.54 \\
\hline $\mathbf{0}$ & 70.19 & 25.22 & 25.49 & 14.56 & 23.24 & 2.75 \\
\hline $\mathbf{0}$ & 0.00 & 0.00 & 0.00 & 0.00 & 14.02 & 22.41 \\
\hline $\mathbf{3}$ & 0.00 & 0.00 & 0.00 & 0.00 & 13.97 & 22.37 \\
\hline $\mathbf{6}$ & 0.00 & 0.00 & 0.00 & 0.00 & 13.94 & 22.35 \\
\hline $\mathbf{0}$ & 10.02 & 9.50 & 9.61 & 1.04 & 13.97 & 22.37 \\
\hline $\mathbf{3}$ & 10.02 & 9.50 & 9.61 & 1.04 & 13.96 & 22.38 \\
\hline $\mathbf{6}$ & 10.02 & 9.50 & 9.61 & 1.04 & 13.96 & 22.37 \\
\hline $\mathbf{0}$ & 20.11 & 13.47 & 13.61 & 1.48 & 14.03 & 22.43 \\
\hline $\mathbf{3}$ & 20.12 & 13.46 & 13.61 & 1.48 & 14.04 & 22.40 \\
\hline $\mathbf{6}$ & 20.13 & 13.46 & 13.61 & 1.48 & 14.06 & 22.33 \\
\hline $\mathbf{0}$ & 30.00 & 16.45 & 16.63 & 1.80 & 14.14 & 22.39 \\
\hline $\mathbf{3}$ & 30.01 & 16.45 & 16.63 & 1.80 & 14.17 & 22.41 \\
\hline $\mathbf{6}$ & 30.01 & 16.45 & 16.63 & 1.80 & 14.18 & 22.44 \\
\hline $\mathbf{0}$ & 40.00 & 19.01 & 19.22 & 2.08 & 14.25 & 22.50 \\
\hline $\mathbf{3}$ & 40.00 & 19.01 & 19.21 & 2.08 & 14.28 & 22.53 \\
\hline $\mathbf{6}$ & 40.02 & 19.01 & 19.21 & 2.08 & 14.29 & 22.55 \\
\hline $\mathbf{0}$ & 50.00 & 21.29 & 21.52 & 2.32 & 14.37 & 22.62 \\
\hline $\mathbf{3}$ & 50.01 & 21.29 & 21.52 & 2.32 & 14.40 & 22.65 \\
\hline $\mathbf{6}$ & 50.01 & 21.29 & 21.52 & 2.32 & 14.42 & 22.66 \\
\hline $\mathbf{0}$ & 60.07 & 23.34 & 23.59 & 2.55 & 14.50 & 22.74 \\
\hline $\mathbf{3}$ & 60.09 & 23.34 & 23.59 & 2.55 & 14.52 & 22.76 \\
\hline $\mathbf{6}$ & 60.08 & 23.34 & 23.59 & 2.55 & 14.54 & 22.79 \\
\hline $\mathbf{0}$ & 70.32 & 25.18 & 25.46 & 2.76 & 14.62 & 22.86 \\
\hline $\mathbf{3}$ & 70.20 & 25.18 & 25.46 & 2.76 & 14.64 & 22.87 \\
\hline $\mathbf{6}$ & 70.14 & 25.18 & 25.46 & 2.75 & 14.65 & 22.90 \\
\hline
\end{tabular}


Table D50: Averaged Pedestal Temperature Raw Data for Thick Top Half Electrode

\begin{tabular}{|c|c|c|c|c|c|c|c|c|}
\hline HV & $\mathbf{Q}$ & T1 & T2 & T3 & T4 & T5 & T6 & T7 \\
\hline $\mathbf{k V}$ & W & ${ }^{\circ} \mathrm{C}$ & ${ }^{\circ} \mathbf{C}$ & ${ }^{\circ} \mathbf{C}$ & ${ }^{\circ} \mathbf{C}$ & ${ }^{\circ} \mathbf{C}$ & ${ }^{\circ} \mathbf{C}$ & ${ }^{\circ} \mathbf{C}$ \\
\hline 0 & 0.00 & 24.93 & 24.56 & 24.57 & 24.59 & 24.76 & 24.79 & 25.88 \\
\hline $\mathbf{0}$ & 10.01 & 31.87 & 28.04 & 26.78 & 27.51 & 26.72 & 27.08 & 26.48 \\
\hline $\mathbf{0}$ & 20.09 & 38.71 & 31.67 & 29.06 & 30.79 & 28.87 & 29.53 & 27.39 \\
\hline 0 & 30.03 & 45.40 & 35.33 & 31.38 & 34.37 & 31.12 & 32.04 & 28.44 \\
\hline $\mathbf{0}$ & 40.09 & 51.71 & 38.83 & 33.58 & 37.69 & 33.23 & 34.32 & 29.52 \\
\hline 0 & 50.06 & 57.38 & 42.23 & 35.79 & 40.85 & 35.31 & 36.47 & 30.86 \\
\hline O & 60.07 & 60.75 & 44.71 & 37.53 & 43.16 & 37.00 & 38.37 & 32.10 \\
\hline $\mathbf{0}$ & 70.19 & 63.15 & 46.60 & 38.96 & 45.01 & 38.42 & 40.13 & 33.26 \\
\hline $\mathbf{0}$ & 0.00 & 25.92 & 25.62 & 25.57 & 25.73 & 25.74 & 25.72 & 27.12 \\
\hline 3 & 0.00 & 25.92 & 25.58 & 25.54 & 25.63 & 25.69 & 25.67 & 26.85 \\
\hline 6 & 0.00 & 25.90 & 25.53 & 25.52 & 25.55 & 25.64 & 25.63 & 26.74 \\
\hline $\mathbf{0}$ & 10.02 & 32.79 & 28.81 & 27.57 & 28.24 & 27.45 & 27.79 & 27.20 \\
\hline 3 & 10.02 & 32.87 & 29.22 & 27.79 & 29.02 & 27.82 & 28.12 & 27.48 \\
\hline 6 & 10.02 & 32.89 & 29.32 & 27.84 & 29.22 & 27.92 & 28.20 & 27.64 \\
\hline $\mathbf{0}$ & 20.11 & 39.80 & 32.62 & 29.92 & 31.97 & 29.75 & 30.38 & 28.28 \\
\hline 3 & 20.12 & 40.03 & 33.19 & 30.35 & 32.80 & 30.20 & 30.77 & 28.65 \\
\hline 6 & 20.13 & 40.43 & 34.10 & 31.35 & 33.05 & 30.53 & 30.89 & 29.05 \\
\hline $\mathbf{0}$ & 30.00 & 47.12 & 37.80 & 33.94 & 35.87 & 32.53 & 33.08 & 29.88 \\
\hline 3 & 30.01 & 47.22 & 38.19 & 34.16 & 36.55 & 32.89 & 33.39 & 30.24 \\
\hline 6 & 30.01 & 47.29 & 38.31 & 34.25 & 36.72 & 32.99 & 33.46 & 30.44 \\
\hline $\mathbf{0}$ & 40.00 & 53.51 & 41.55 & 36.58 & 38.97 & 34.68 & 35.37 & 31.08 \\
\hline 3 & 40.00 & 53.62 & 42.04 & 36.89 & 39.80 & 35.13 & 35.74 & 31.51 \\
\hline 6 & 40.02 & 53.66 & 42.16 & 36.96 & 40.01 & 35.24 & 35.83 & 31.74 \\
\hline $\mathbf{0}$ & 50.00 & 58.61 & 44.96 & 39.09 & 42.02 & 36.80 & 37.57 & 32.45 \\
\hline 3 & 50.01 & 58.62 & 45.28 & 39.29 & 42.57 & 37.12 & 37.84 & 32.83 \\
\hline 6 & 50.01 & 58.66 & 45.37 & 39.35 & 42.72 & 37.20 & 37.91 & 33.04 \\
\hline $\mathbf{0}$ & 60.07 & 61.55 & 47.44 & 41.12 & 44.42 & 38.58 & 39.56 & 33.76 \\
\hline 3 & 60.09 & 61.58 & 47.64 & 41.26 & 44.78 & 38.79 & 39.73 & 34.08 \\
\hline 6 & 60.08 & 61.62 & 47.73 & 41.33 & 44.91 & 38.89 & 39.82 & 34.26 \\
\hline $\mathbf{0}$ & 70.32 & 63.92 & 49.45 & 42.89 & 46.37 & 40.12 & 41.30 & 34.99 \\
\hline 3 & 70.20 & 63.91 & 49.60 & 43.01 & 46.64 & 40.29 & 41.44 & 35.25 \\
\hline 6 & 70.14 & 63.91 & 49.64 & 43.03 & 46.75 & 40.37 & 41.54 & 35.39 \\
\hline
\end{tabular}


Table D51: Averaged Chamber Temperature Raw Data for Thick Top Half Electrode

\begin{tabular}{|c|c|c|c|c|c|c|c|c|}
\hline HV & $\mathbf{Q}$ & Amb & T8 & T9 & T10 & T11 & T12 & T14 \\
\hline kV & $\mathbf{W}$ & ${ }^{\circ} \mathbf{C}$ & ${ }^{\circ} \mathbf{C}$ & ${ }^{\circ} \mathbf{C}$ & ${ }^{\circ} \mathrm{C}$ & ${ }^{\circ} \mathbf{C}$ & ${ }^{\circ} \mathbf{C}$ & ${ }^{\circ} \mathbf{C}$ \\
\hline 0 & 0.00 & 22.46 & 23.64 & 23.42 & 25.00 & 24.60 & 23.55 & 23.93 \\
\hline 0 & 10.01 & 22.57 & 24.11 & 24.00 & 25.92 & 25.12 & 23.92 & 23.93 \\
\hline $\mathbf{0}$ & 20.09 & 22.78 & 24.57 & 24.29 & 26.96 & 25.73 & 24.30 & 23.99 \\
\hline $\mathbf{0}$ & 30.03 & 22.79 & 25.09 & 24.64 & 28.02 & 26.28 & 24.78 & 24.01 \\
\hline 0 & 40.09 & 22.94 & 25.67 & 25.15 & 29.06 & 26.85 & 25.30 & 23.99 \\
\hline 0 & 50.06 & 22.98 & 26.39 & 25.76 & 30.13 & 27.54 & 25.90 & 24.00 \\
\hline $\mathbf{0}$ & 60.07 & 22.98 & 27.08 & 26.35 & 31.30 & 28.32 & 26.51 & 24.13 \\
\hline $\mathbf{0}$ & 70.19 & 22.93 & 27.75 & 26.84 & 32.43 & 29.10 & 27.12 & 24.21 \\
\hline $\mathbf{0}$ & 0.00 & 23.19 & 24.74 & 24.64 & 25.67 & 25.32 & 24.75 & 24.37 \\
\hline 3 & 0.00 & 23.11 & 24.70 & 24.60 & 25.63 & 25.29 & 24.69 & 24.38 \\
\hline 6 & 0.00 & 23.19 & 24.68 & 24.56 & 25.62 & 25.28 & 24.65 & 24.39 \\
\hline $\mathbf{0}$ & 10.02 & 23.37 & 24.97 & 24.96 & 26.54 & 25.76 & 24.85 & 24.42 \\
\hline 3 & 10.02 & 23.32 & 25.05 & 25.02 & 26.56 & 25.78 & 24.88 & 24.42 \\
\hline 6 & 10.02 & 23.17 & 25.07 & 25.03 & 26.58 & 25.79 & 24.90 & 24.44 \\
\hline $\mathbf{0}$ & 20.11 & 23.30 & 25.40 & 25.20 & 27.55 & 26.32 & 25.17 & 24.43 \\
\hline 3 & 20.12 & 23.06 & 25.45 & 25.22 & 27.61 & 26.49 & 25.22 & 24.45 \\
\hline 6 & 20.13 & 23.13 & 25.46 & 25.30 & 27.61 & 26.82 & 25.31 & 24.46 \\
\hline $\mathbf{0}$ & 30.00 & 23.35 & 25.84 & 25.62 & 28.63 & 27.56 & 25.63 & 24.47 \\
\hline 3 & 30.01 & 23.20 & 25.94 & 25.71 & 28.70 & 27.63 & 25.70 & 24.48 \\
\hline 6 & 30.01 & 23.34 & 25.99 & 25.74 & 28.74 & 27.67 & 25.75 & 24.49 \\
\hline $\mathbf{0}$ & 40.00 & 23.30 & 26.35 & 25.94 & 29.77 & 28.44 & 26.09 & 24.53 \\
\hline 3 & 40.00 & 23.33 & 26.49 & 26.05 & 29.87 & 28.52 & 26.18 & 24.55 \\
\hline 6 & 40.02 & 23.25 & 26.55 & 26.09 & 29.91 & 28.57 & 26.25 & 24.56 \\
\hline $\mathbf{0}$ & 50.00 & 23.28 & 27.03 & 26.42 & 30.92 & 29.37 & 26.69 & 24.59 \\
\hline 3 & 50.01 & 23.38 & 27.16 & 26.50 & 31.02 & 29.46 & 26.80 & 24.61 \\
\hline 6 & 50.01 & 23.37 & 27.21 & 26.56 & 31.06 & 29.51 & 26.83 & 24.62 \\
\hline $\mathbf{0}$ & 60.07 & 23.39 & 27.71 & 26.90 & 32.09 & 30.40 & 27.26 & 24.65 \\
\hline 3 & 60.09 & 23.60 & 27.83 & 27.00 & 32.18 & 30.48 & 27.37 & 24.67 \\
\hline 6 & 60.08 & 23.60 & 27.90 & 27.07 & 32.25 & 30.56 & 27.44 & 24.69 \\
\hline 0 & 70.32 & 23.64 & 28.48 & 27.42 & 33.30 & 31.55 & 27.92 & 24.72 \\
\hline 3 & 70.20 & 23.60 & 28.61 & 27.53 & 33.36 & 31.61 & 28.02 & 24.74 \\
\hline 6 & 70.14 & 23.37 & 28.67 & 27.60 & 33.42 & 31.67 & 28.08 & 24.76 \\
\hline
\end{tabular}


Table D52: Averaged Heater and Pressure Raw Data for Full Length Electrode

\begin{tabular}{|c|c|c|c|c|c|c|}
\hline HV & $\mathbf{Q}$ & $\begin{array}{c}\text { Heater Voltage } \\
\text { after } \\
\text { precision resistor }\end{array}$ & $\begin{array}{l}\text { Heater Voltage } \\
\text { before } \\
\text { precision resistor }\end{array}$ & $\begin{array}{l}\text { Heater } \\
\text { Current }\end{array}$ & $\begin{array}{c}\text { Chamber } \\
\text { Pressure }\end{array}$ & $\begin{array}{c}\text { Nozzle } \\
\text { Pressure }\end{array}$ \\
\hline $\mathbf{k V}$ & $\mathbf{W}$ & Volts & Volts & Amps & psia & psia \\
\hline $\mathbf{0}$ & 0.00 & 0.00 & 0.00 & 0.00 & 14.34 & 22.64 \\
\hline $\mathbf{0}$ & 10.09 & 9.59 & 9.69 & 1.04 & 14.36 & 22.74 \\
\hline $\mathbf{0}$ & 20.10 & 13.53 & 13.68 & 1.47 & 14.42 & 22.78 \\
\hline 0 & 30.05 & 16.54 & 16.72 & 1.80 & 14.48 & 22.78 \\
\hline 0 & 39.99 & 19.08 & 19.29 & 2.07 & 14.56 & 22.85 \\
\hline 0 & 50.02 & 21.34 & 21.57 & 2.32 & 14.64 & 22.94 \\
\hline $\mathbf{0}$ & 60.00 & 23.34 & 23.60 & 2.54 & 14.74 & 23.04 \\
\hline $\mathbf{0}$ & 70.04 & 25.20 & 25.47 & 2.75 & 14.84 & 23.13 \\
\hline 0 & 0.00 & 0.00 & 0.00 & 0.00 & 14.31 & 22.56 \\
\hline 3 & 0.00 & 0.00 & 0.00 & 0.00 & 14.28 & 22.56 \\
\hline 6 & 0.00 & 0.00 & 0.00 & 0.00 & 14.26 & 22.55 \\
\hline O & 10.03 & 9.56 & 9.67 & 1.04 & 14.30 & 22.58 \\
\hline 3 & 10.03 & 9.56 & 9.67 & 1.04 & 14.31 & 22.59 \\
\hline 6 & 10.03 & 9.56 & 9.67 & 1.04 & 14.31 & 22.61 \\
\hline $\mathbf{0}$ & 19.98 & 13.50 & 13.65 & 1.46 & 14.37 & 22.66 \\
\hline 3 & 19.98 & 13.50 & 13.65 & 1.46 & 14.38 & 22.67 \\
\hline 6 & 19.99 & 13.50 & 13.65 & 1.46 & 14.40 & 22.69 \\
\hline $\mathbf{0}$ & 30.04 & 16.55 & 16.73 & 1.80 & 14.46 & 22.75 \\
\hline 3 & 30.05 & 16.55 & 16.73 & 1.80 & 14.48 & 22.77 \\
\hline 6 & 30.05 & 16.55 & 16.73 & 1.80 & 14.49 & 22.78 \\
\hline 0 & 39.98 & 19.08 & 19.29 & 2.07 & 14.56 & 22.84 \\
\hline 3 & 39.98 & 19.08 & 19.29 & 2.07 & 14.58 & 22.83 \\
\hline 6 & 39.98 & 19.08 & 19.29 & 2.07 & 14.60 & 22.87 \\
\hline $\mathbf{0}$ & 49.98 & 21.27 & 21.51 & 2.32 & 14.66 & 22.95 \\
\hline 3 & 49.80 & 21.28 & 21.51 & 2.32 & 14.69 & 22.96 \\
\hline 6 & 49.78 & 21.28 & 21.51 & 2.31 & 14.70 & 22.97 \\
\hline $\mathbf{0}$ & 60.05 & 23.35 & 23.60 & 2.54 & 14.77 & 23.03 \\
\hline 3 & 60.07 & 23.35 & 23.60 & 2.55 & 14.80 & 23.03 \\
\hline 6 & 60.05 & 23.35 & 23.60 & 2.54 & 14.81 & 23.03 \\
\hline 0 & 69.99 & 25.19 & 25.47 & 2.75 & 14.89 & 23.10 \\
\hline 3 & 70.06 & 25.19 & 25.47 & 2.75 & 14.91 & 23.13 \\
\hline 6 & 70.10 & 25.19 & 25.47 & 2.75 & 14.93 & 23.15 \\
\hline $\mathbf{0}$ & 75.07 & 26.07 & 26.35 & 2.85 & 14.97 & 23.17 \\
\hline 3 & 75.20 & 26.06 & 26.35 & 2.85 & 14.98 & 23.19 \\
\hline 6 & 75.16 & 26.07 & 26.35 & 2.85 & 14.99 & 23.20 \\
\hline
\end{tabular}


Table D53: Averaged Pedestal Temperature Raw Data for Full Length Electrode

\begin{tabular}{|c|c|c|c|c|c|c|c|c|}
\hline HV & $\mathbf{Q}$ & $\mathrm{T1}$ & T2 & T3 & T4 & T5 & T6 & T7 \\
\hline $\mathbf{k V}$ & W & ${ }^{\circ} \mathbf{C}$ & ${ }^{\circ} \mathbf{C}$ & ${ }^{\circ} \mathbf{C}$ & ${ }^{\circ} \mathbf{C}$ & ${ }^{\circ} \mathbf{C}$ & ${ }^{\circ} \mathbf{C}$ & ${ }^{\circ} \mathbf{C}$ \\
\hline 0 & 0.00 & 26.44 & 26.07 & 26.06 & 26.10 & 26.19 & 26.19 & 27.24 \\
\hline 0 & 0.09 & 4.10 & 30.10 & 28.94 & 28.73 & 28.08 & 28.25 & 28.04 \\
\hline $\mathbf{0}$ & .10 & 41.76 & 34.49 & 32.02 & 32.06 & 30.30 & 30.58 & \\
\hline 0 & .05 & 49.10 & 38.89 & 35.08 & 35.59 & 32.66 & 33.00 & 30.40 \\
\hline $\mathbf{0}$ & 39.99 & 55.56 & 42.89 & 37.94 & 38.92 & 34.88 & 35.29 & 31.65 \\
\hline 0 & 50.02 & 60.13 & 46.18 & 40.44 & 41.84 & 36.90 & 37.37 & 32.93 \\
\hline 0 & 0.00 & 62.86 & 48.56 & 42.47 & 44.06 & 38.56 & 39.17 & 34.17 \\
\hline 0 & 70.04 & 65.07 & 50.47 & 44.26 & 45.94 & 40.06 & 40.92 & 35.35 \\
\hline 0 & 0.00 & 26.75 & 26.41 & 26.34 & 26.46 & 26.49 & 26.48 & 27.76 \\
\hline 3 & 0.00 & 26.75 & 26.39 & 26.34 & 26.42 & 26.47 & 26.47 & 27.65 \\
\hline 6 & 0.00 & 26.72 & 26.35 & 26.30 & 26.36 & 26.43 & 26.42 & 27.53 \\
\hline 0 & 93 & 34.30 & 30.33 & 29.17 & 28.93 & 28.30 & 28.47 & 28.24 \\
\hline 3 & 10.03 & 34.39 & 30.77 & 29.39 & 29.68 & 28.65 & 28.76 & 28.54 \\
\hline 6 & 10.03 & 34.38 & 30.85 & 29.43 & 29.88 & 28.74 & 28.83 & 28.69 \\
\hline 0 & 19.98 & 41.92 & 34.92 & 32.33 & 32.71 & 30.72 & 30.96 & 29.57 \\
\hline 3 & 19.98 & 42.01 & 35.28 & 32.56 & 33.35 & 31.07 & 31.27 & 29.95 \\
\hline 6 & .99 & 42.04 & 35.38 & 32.61 & 33.53 & 31.16 & 31.34 & 30.12 \\
\hline 0 & 30.04 & 49.29 & 39.41 & 35.48 & 36.40 & 33.19 & 33.51 & 31.02 \\
\hline 3 & 30.05 & 49.38 & 39.72 & 35.66 & 36.95 & 33.48 & 33.75 & 31.35 \\
\hline 6 & 30.05 & 49.43 & 39.85 & 35.76 & 37.15 & 33.61 & 33.86 & 31.59 \\
\hline 0 & 39.98 & 55.78 & 43.39 & 38.35 & 39.68 & 35.44 & 35.84 & 32.35 \\
\hline 3 & 39.98 & 55.89 & 43.73 & 38.58 & 40.27 & 35.76 & 36.12 & 32.76 \\
\hline 6 & 39.98 & 55.86 & 43.81 & 38.63 & 40.42 & 35.85 & 36.20 & 32.94 \\
\hline 0 & 49.98 & 60.40 & 46.60 & 40.88 & 42.38 & 37.40 & 37.85 & 33.68 \\
\hline 3 & 49.80 & 60.30 & 46.85 & 41.02 & 42.92 & 37.69 & 38.13 & 34.04 \\
\hline 6 & 49.78 & 60.35 & 46.98 & 41.12 & 43.11 & 37.82 & 38.26 & 34.27 \\
\hline $\mathbf{0}$ & 60.05 & 63.04 & 48.96 & 42.93 & 44.66 & 39.11 & 39.75 & 34.91 \\
\hline 3 & 60.07 & 63.13 & 49.28 & 43.16 & 45.16 & 39.43 & 40.05 & 35.32 \\
\hline 6 & 60.05 & 63.00 & 49.31 & 43.16 & 45.24 & 39.43 & 40.02 & 35.49 \\
\hline 0 & 69.99 & 65.23 & 51.07 & 44.88 & 46.81 & 40.81 & 41.62 & 36.32 \\
\hline 3 & 70.06 & 65.25 & 51.19 & 44.95 & 47.04 & 40.95 & 41.76 & 36.57 \\
\hline 6 & 70.10 & 65.32 & 51.29 & 45.04 & 47.15 & 41.03 & 41.82 & 36.71 \\
\hline 0 & 75.07 & 66.42 & 52.10 & 45.82 & 47.85 & 41.66 & 42.57 & 37.12 \\
\hline 3 & 75.20 & 66.48 & 52.21 & 45.91 & 48.00 & 41.74 & 42.64 & 37.26 \\
\hline 6 & 75.16 & 66.52 & 52.28 & 45.98 & 48.07 & 41.81 & 42.70 & 37.37 \\
\hline
\end{tabular}


Table D54: Averaged Chamber Temperature Raw Data for Full Length Electrode

\begin{tabular}{|c|c|c|c|c|c|c|c|c|}
\hline HV & $\mathbf{Q}$ & Amb & T8 & T9 & T10 & T11 & T12 & T14 \\
\hline $\mathbf{k V}$ & W & ${ }^{\circ} \mathbf{C}$ & ${ }^{\circ} \mathbf{C}$ & ${ }^{\circ} \mathbf{C}$ & ${ }^{\circ} \mathbf{C}$ & ${ }^{\circ} \mathbf{C}$ & ${ }^{\circ} \mathbf{C}$ & ${ }^{\circ} \mathbf{C}$ \\
\hline 0 & 0.00 & 23.40 & 24.89 & 24.77 & 26.41 & 25.95 & 24.85 & 25.40 \\
\hline $\mathbf{0}$ & 10.09 & 23.67 & 25.28 & 25.23 & 27.33 & 26.67 & 25.13 & 25.34 \\
\hline 0 & 20.10 & 23.46 & 25.71 & 25.42 & 28.35 & 27.44 & 25.46 & 25.32 \\
\hline $\mathbf{0}$ & 30.05 & 23.59 & 26.15 & 25.65 & 29.38 & 28.23 & 25.79 & 25.27 \\
\hline $\mathbf{0}$ & 39.99 & 23.60 & 26.71 & 25.98 & 30.45 & 29.06 & 26.21 & 25.23 \\
\hline $\mathbf{0}$ & 50.02 & 23.68 & 27.32 & 26.38 & 31.55 & 29.96 & 26.68 & 25.19 \\
\hline 0 & 60.00 & 23.55 & 27.94 & 26.72 & 32.68 & 30.91 & 27.12 & 25.18 \\
\hline 0 & 70.04 & 23.70 & 28.62 & 27.06 & 33.83 & 31.96 & 27.62 & 25.21 \\
\hline 0 & 0.00 & 23.57 & 25.32 & 25.24 & 26.54 & 26.14 & 25.31 & 25.24 \\
\hline 3 & 0.00 & 23.47 & 25.35 & 25.29 & 26.54 & 26.15 & 25.31 & 25.29 \\
\hline 6 & 0.00 & 23.62 & 25.26 & 25.19 & 26.49 & 26.11 & 25.23 & 25.26 \\
\hline 0 & 10.03 & 23.81 & 25.61 & 25.60 & 27.45 & 26.85 & 25.46 & 25.31 \\
\hline 3 & 10.03 & 23.49 & 25.66 & 25.59 & 27.44 & 26.83 & 25.47 & 25.26 \\
\hline 6 & 10.03 & 23.60 & 25.74 & 25.70 & 27.45 & 26.85 & 25.52 & 25.27 \\
\hline $\mathbf{0}$ & 19.98 & 23.70 & 26.04 & 25.74 & 28.42 & 27.55 & 25.76 & 25.25 \\
\hline 3 & 19.98 & 23.68 & 26.13 & 25.80 & 28.53 & 27.65 & 25.82 & 25.32 \\
\hline 6 & 19.99 & 23.81 & 26.15 & 25.82 & 28.52 & 27.67 & 25.84 & 25.29 \\
\hline $\mathbf{0}$ & 30.04 & 23.98 & 26.60 & 26.09 & 29.59 & 28.46 & 26.20 & 25.33 \\
\hline 3 & 30.05 & 23.83 & 26.67 & 26.15 & 29.62 & 28.49 & 26.24 & 25.30 \\
\hline 6 & 30.05 & 23.78 & 26.75 & 26.23 & 29.70 & 28.59 & 26.33 & 25.36 \\
\hline $\mathbf{0}$ & 39.98 & 23.84 & 27.15 & 26.46 & 30.70 & 29.33 & 26.64 & 25.33 \\
\hline 3 & 39.98 & 23.77 & 27.32 & 26.61 & 30.83 & 29.48 & 26.77 & 25.39 \\
\hline 6 & 39.98 & 23.84 & 27.35 & 26.65 & 30.83 & 29.50 & 26.80 & 25.38 \\
\hline 0 & 49.98 & 23.81 & 27.82 & 26.96 & 31.92 & 30.34 & 27.19 & 25.44 \\
\hline 3 & 49.80 & 23.79 & 27.95 & 27.03 & 31.99 & 30.37 & 27.25 & 25.43 \\
\hline 6 & 49.78 & 23.82 & 28.75 & 27.51 & 33.28 & 31.64 & 27.90 & 25.52 \\
\hline $\mathbf{0}$ & 60.05 & 23.86 & 28.49 & 27.33 & 33.10 & 31.37 & 27.69 & 25.46 \\
\hline 3 & 60.07 & 23.95 & 28.71 & 27.50 & 33.25 & 31.50 & 27.83 & 25.51 \\
\hline 6 & 60.05 & 23.98 & 28.04 & 27.14 & 32.05 & 30.49 & 27.36 & 25.48 \\
\hline $\mathbf{0}$ & 69.99 & 24.09 & 29.43 & 27.85 & 34.38 & 32.57 & 28.37 & 25.58 \\
\hline 3 & 70.06 & 23.98 & 29.53 & 27.93 & 34.46 & 32.63 & 28.46 & 25.59 \\
\hline 6 & 70.10 & 24.15 & 29.61 & 28.02 & 34.50 & 32.74 & 28.55 & 25.63 \\
\hline 0 & 75.07 & 24.14 & 29.98 & 28.20 & 35.05 & 33.25 & 28.83 & 25.69 \\
\hline 3 & 75.20 & 24.09 & 30.01 & 28.24 & 35.11 & 33.30 & 28.88 & 25.69 \\
\hline 6 & 75.16 & 24.10 & 30.12 & 28.35 & 35.15 & 33.41 & 28.97 & 25.72 \\
\hline
\end{tabular}


Table D55: Averaged Heater and Pressure Raw Data for Top Half Electrode

\begin{tabular}{|c|c|c|c|c|c|c|}
\hline HV & $\mathbf{Q}$ & $\begin{array}{c}\text { Heater Voltage } \\
\text { after } \\
\text { precision resistor }\end{array}$ & $\begin{array}{c}\text { Heater Voltage } \\
\text { before } \\
\text { precision resistor }\end{array}$ & $\begin{array}{c}\text { Heater } \\
\text { Current }\end{array}$ & $\begin{array}{l}\text { Chamber } \\
\text { Pressure }\end{array}$ & $\begin{array}{c}\text { Nozzle } \\
\text { Pressure }\end{array}$ \\
\hline $\mathrm{kV}$ & $\mathbf{W}$ & Volts & Volts & Amps & psia & psia \\
\hline $\mathbf{0}$ & 0.00 & 0.00 & 0.00 & 0.00 & 13.81 & 22.10 \\
\hline $\mathbf{0}$ & 10.07 & 9.62 & 9.73 & 1.04 & 13.85 & 21.97 \\
\hline 0 & 20.09 & 13.59 & 13.74 & 1.46 & 13.90 & 22.04 \\
\hline 0 & 30.11 & 16.60 & 16.78 & 1.79 & 13.98 & 22.14 \\
\hline 0 & 40.10 & 19.10 & 19.31 & 2.08 & 14.08 & 22.26 \\
\hline $\mathbf{0}$ & 50.01 & 21.29 & 21.53 & 2.32 & 14.18 & 22.39 \\
\hline $\mathbf{0}$ & 60.09 & 23.29 & 23.55 & 2.55 & 14.29 & 22.52 \\
\hline $\mathbf{0}$ & 70.01 & 25.12 & 25.40 & 2.76 & 14.39 & 22.65 \\
\hline $\mathbf{0}$ & 0.00 & 0.00 & 0.00 & 0.00 & 14.08 & 22.49 \\
\hline 3 & 0.00 & 0.00 & 0.00 & 0.00 & 14.07 & 22.52 \\
\hline 6 & 0.00 & 0.00 & 0.00 & 0.00 & 14.06 & 22.55 \\
\hline 0 & 10.03 & 9.48 & 9.59 & 1.05 & 14.11 & 22.61 \\
\hline 3 & 10.04 & 9.48 & 9.59 & 1.05 & 14.13 & 22.64 \\
\hline 6 & 10.04 & 9.48 & 9.59 & 1.05 & 14.14 & 22.69 \\
\hline $\mathbf{0}$ & 19.99 & 13.39 & 13.54 & 1.48 & 14.22 & 22.78 \\
\hline 3 & 20.00 & 13.39 & 13.54 & 1.48 & 14.24 & 22.83 \\
\hline 6 & 20.01 & 13.39 & 13.54 & 1.48 & 14.27 & 22.88 \\
\hline $\mathbf{0}$ & 30.06 & 16.43 & 16.61 & 1.81 & 14.35 & 22.98 \\
\hline 3 & 30.07 & 16.43 & 16.62 & 1.81 & 14.37 & 23.02 \\
\hline 6 & 30.08 & 16.43 & 16.62 & 1.81 & 14.40 & 23.06 \\
\hline $\mathbf{0}$ & 40.01 & 18.97 & 19.18 & 2.09 & 14.48 & 23.16 \\
\hline 3 & 40.01 & 18.97 & 19.18 & 2.09 & 14.51 & 23.18 \\
\hline 6 & 40.02 & 18.97 & 19.18 & 2.09 & 14.54 & 23.21 \\
\hline $\mathbf{0}$ & 50.00 & 21.21 & 21.45 & 2.33 & 14.60 & 23.25 \\
\hline 3 & 50.01 & 21.21 & 21.45 & 2.33 & 14.64 & 23.36 \\
\hline 6 & 50.06 & 21.21 & 21.45 & 2.33 & 14.67 & 23.39 \\
\hline 0 & 60.07 & 23.25 & 23.50 & 2.56 & 14.74 & 23.46 \\
\hline 3 & 60.09 & 23.25 & 23.50 & 2.56 & 14.76 & 23.50 \\
\hline 6 & 60.12 & 23.25 & 23.50 & 2.56 & 14.79 & 23.55 \\
\hline $\mathbf{0}$ & 70.03 & 25.10 & 25.37 & 2.76 & 14.86 & 23.60 \\
\hline 3 & 70.04 & 25.10 & 25.37 & 2.76 & 14.89 & 23.65 \\
\hline 6 & 70.06 & 25.10 & 25.37 & 2.76 & 14.90 & 23.66 \\
\hline $\mathbf{0}$ & 75.00 & 25.97 & 26.25 & 2.86 & 14.94 & 23.70 \\
\hline 3 & 75.02 & 25.97 & 26.25 & 2.86 & 14.95 & 23.66 \\
\hline 6 & 74.99 & 25.97 & 26.25 & 2.86 & 14.94 & 23.63 \\
\hline
\end{tabular}


Table D56: Averaged Pedestal Temperature Raw Data for Top Half Electrode

\begin{tabular}{|c|c|c|c|c|c|c|c|c|}
\hline & $\mathbf{Q}$ & 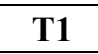 & 2 & T3 & 4 & T5 & T6 & 1 \\
\hline $\mathbf{k V}$ & $\mathbf{W}$ & ${ }^{\circ} \mathbf{C}$ & ${ }^{\circ} \mathrm{C}$ & ${ }^{\circ} \mathbf{C}$ & ${ }^{\circ} \mathbf{C}$ & ${ }^{\circ} \mathbf{C}$ & ${ }^{\circ} \mathbf{C}$ & ${ }^{\circ} \mathbf{C}$ \\
\hline 0 & 00 & 15 & .92 & 97 & 21.96 & 2.14 & 2.18 & 23.12 \\
\hline 0 & & 2 & & 10 & & 3 & 44 & 4. \\
\hline 0 & & & 89 & 7.90 & 0 & U. & 6.45 & 5. \\
\hline 0 & .11 & 5.89 & 5.27 & 31.51 & 1.81 & 8.96 & 29.34 & 26.75 \\
\hline 0 & & .61 & & 57 & & 1.4 & & 8.32 \\
\hline 0 & 0.01 & 57.87 & 43.16 & 37.28 & 8.55 & 33.58 & 4.11 & 29.72 \\
\hline 0 & .09 & 1.00 & 45.85 & 39.55 & 10 & 5.45 & 6.08 & 31.05 \\
\hline 0 & .0 & .36 & 77 & 1.47 & & & 83 & 32.29 \\
\hline 0 & 00 & 3.57 & 23.42 & 23.29 & 23.77 & 23.59 & 23.57 & 25.34 \\
\hline 3 & & 60 & & .26 & 16 & 47 & 3.47 & 24.8 \\
\hline 0 & 00 & 3.67 & 30 & 23.29 & 0 & 3.46 & 3.48 & 4.66 \\
\hline 0 & 0.03 & 1.44 & 27.23 & 26.15 & 25.76 & 25.25 & 25.43 & 25.28 \\
\hline 3 & (1) & & 1. & 6.49 & 6. & 5. & 0.80 & 5.68 \\
\hline 6 & .04 & 31.60 & 27.94 & 26.56 & 26.95 & 25.87 & 25.96 & 25.86 \\
\hline 0 & .9 & 0 & & 29.33 & & 27. & 7.88 & 6.65 \\
\hline 3 & .00 & $T$ & 9 & 29.69 & 33 & 8.16 & 8.35 & 27.14 \\
\hline 6 & .01 & 39.27 & 32. & 29.80 & 30.62 & 28.33 & 28.48 & 27.38 \\
\hline 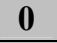 & 1.0 & 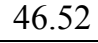 & 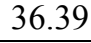 & 2.58 & 5.17 & 0. & 30.51 & 28.22 \\
\hline 3 & .07 & 6.65 & 36.89 & 32.87 & 34.01 & 30.64 & 30.89 & 28.67 \\
\hline 6 & .0 & 5.66 & 37.00 & 32.95 & 34.25 & 0. & 31.01 & 28.91 \\
\hline 0 & 0.0 & 3.04 & 0 & 5.51 & 36.66 & 32.5 & 32.97 & 29.70 \\
\hline 3 & 0.01 & 3.20 & 40.96 & 35.81 & 37.40 & 2.98 & 3.31 & 30.14 \\
\hline 6 & .02 & 0 & & 35.94 & 37.63 & 3. & 33.44 & 30.38 \\
\hline 0 & 50 & 22 & 44.22 & 38.26 & 39.89 & 34.75 & 5.21 & 31.18 \\
\hline 3 & 0.01 & 3 & & 8.50 & 40.44 & 35. & 35.54 & 31.58 \\
\hline 6 & .06 & 58.70 & 44.67 & 38.63 & 40.65 & 35.30 & 35.68 & 31.80 \\
\hline $\mathbf{0}$ & 007 & & 46.84 & 40.54 & 42.28 & 36.63 & 37.21 & 32.46 \\
\hline 5 & .09 & 3 & 71.21 & 40.77 & 42.81 & 36.97 & 37.50 & 32.89 \\
\hline 6 & 60.1 & 1.98 & 47. & 40.87 & $T$ & 37 & 37.59 & 33.11 \\
\hline 0 & 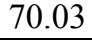 & 4.29 & 49.08 & 42.53 & 44.43 & 38.29 & 39.08 & 33.78 \\
\hline 3 & 70.04 & 5 & 49.33 & 42.70 & 88 & 38.56 & 39.31 & 34.16 \\
\hline 6 & 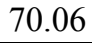 & 4.38 & 49.42 & 2.77 & 44.99 & 86 & 9.34 & 34.33 \\
\hline $\mathbf{0}$ & 00 & 14 & 50.19 & 43.50 & 45.63 & 39.18 & 40.05 & 34.66 \\
\hline 3 & 0 & & & 43.54 & 45.81 & 39.23 & 0.08 & 34.84 \\
\hline 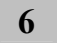 & 74.99 & 65.65 & 50.37 & 43.54 & 45.70 & 39.03 & 39.87 & 35.16 \\
\hline
\end{tabular}


Table D57: Averaged Chamber Temperature Raw Data for Top Half Electrode

\begin{tabular}{|c|c|c|c|c|c|c|c|c|}
\hline HV & $\mathbf{Q}$ & Amb & T8 & T9 & T10 & T11 & T12 & T14 \\
\hline $\mathbf{k V}$ & W & ${ }^{\circ} \mathrm{C}$ & ${ }^{\circ} \mathbf{C}$ & ${ }^{\circ} \mathbf{C}$ & ${ }^{\circ} \mathbf{C}$ & ${ }^{\circ} \mathbf{C}$ & ${ }^{\circ} \mathbf{C}$ & ${ }^{\circ} \mathbf{C}$ \\
\hline 0 & 0.00 & 19.90 & 21.12 & 20.90 & 22.36 & 21.90 & 21.00 & 21.34 \\
\hline 0 & 10.07 & 20.03 & 21.54 & 21.42 & 23.44 & 22.75 & 21.37 & 21.43 \\
\hline 0 & 20.09 & 20.11 & 21.93 & 21.61 & 24.49 & 23.55 & 21.70 & 21.48 \\
\hline $\mathbf{0}$ & 30.11 & 20.24 & 22.58 & 22.08 & 25.74 & 24.52 & 22.21 & 21.55 \\
\hline $\mathbf{0}$ & 40.10 & 20.38 & 23.21 & 22.52 & 26.97 & 25.51 & 22.71 & 21.61 \\
\hline $\mathbf{0}$ & 50.01 & 20.45 & 23.93 & 23.02 & 28.22 & 26.54 & 23.26 & 21.67 \\
\hline 0 & 60.09 & 20.58 & 24.64 & 23.49 & 29.46 & 27.66 & 23.79 & 21.72 \\
\hline 0 & 70.01 & 20.69 & 25.39 & 23.98 & 30.66 & 28.80 & 24.34 & 21.77 \\
\hline 0 & 0.00 & 20.96 & 22.37 & 22.24 & 23.40 & 23.00 & 22.38 & 21.98 \\
\hline 3 & 0.00 & 21.03 & 22.37 & 22.23 & 23.41 & 23.02 & 22.39 & 22.05 \\
\hline 6 & 0.00 & 21.09 & 22.42 & 22.27 & 23.45 & 23.08 & 22.42 & 22.11 \\
\hline $\mathbf{0}$ & 10.03 & 21.14 & 22.67 & 22.60 & 24.41 & 23.85 & 22.60 & 22.21 \\
\hline 3 & 10.04 & 21.20 & 22.78 & 22.65 & 24.52 & 23.94 & 22.66 & 22.27 \\
\hline 6 & 10.04 & 21.25 & 22.88 & 22.79 & 24.54 & 23.96 & 22.74 & 22.31 \\
\hline $\mathbf{0}$ & 19.99 & 21.32 & 23.22 & 22.99 & 25.54 & 24.74 & 23.03 & 22.37 \\
\hline 3 & 20.00 & 21.37 & 23.38 & 23.14 & 25.65 & 24.82 & 23.13 & 22.43 \\
\hline 6 & 20.01 & 21.44 & 23.45 & 23.23 & 25.71 & 24.89 & 23.19 & 22.48 \\
\hline $\mathbf{0}$ & 30.06 & 21.51 & 23.84 & 23.50 & 26.75 & 25.67 & 23.52 & 22.50 \\
\hline 3 & 30.07 & 21.55 & 23.99 & 23.60 & 26.85 & 25.76 & 23.61 & 22.53 \\
\hline 6 & 30.08 & 21.59 & 24.05 & 23.69 & 26.93 & 25.83 & 23.67 & 22.56 \\
\hline $\mathbf{0}$ & 40.01 & 21.67 & 24.46 & 23.98 & 28.02 & 26.69 & 24.04 & 22.64 \\
\hline 3 & 40.01 & 21.71 & 24.63 & 24.09 & 28.13 & 26.78 & 24.16 & 22.68 \\
\hline 6 & 40.02 & 21.74 & 24.72 & 24.17 & 28.23 & 26.90 & 24.24 & 22.72 \\
\hline $\mathbf{0}$ & 50.00 & 21.81 & 25.20 & 24.48 & 29.34 & 27.85 & 24.63 & 22.76 \\
\hline 3 & 50.01 & 21.85 & 25.36 & 24.66 & 29.41 & 27.84 & 24.75 & 22.79 \\
\hline 6 & 50.06 & 21.91 & 25.44 & 24.75 & 29.51 & 27.96 & 24.82 & 22.84 \\
\hline $\mathbf{0}$ & 60.07 & 21.96 & 25.92 & 25.12 & 30.57 & 28.86 & 25.22 & 22.91 \\
\hline 3 & 60.09 & 21.99 & 26.12 & 25.28 & 30.70 & 28.97 & 25.35 & 22.94 \\
\hline 6 & 60.12 & 22.03 & 26.20 & 25.35 & 30.78 & 29.09 & 25.41 & 22.98 \\
\hline $\mathbf{0}$ & 70.03 & 22.08 & 26.71 & 25.73 & 31.83 & 30.03 & 25.79 & 23.04 \\
\hline 3 & 70.04 & 22.10 & 26.88 & 25.82 & 31.94 & 30.14 & 25.91 & 23.10 \\
\hline 6 & 70.06 & 22.13 & 26.96 & 25.89 & 31.97 & 30.24 & 25.98 & 23.10 \\
\hline $\mathbf{0}$ & 75.00 & 22.18 & 27.23 & 26.14 & 32.45 & 30.66 & 26.21 & 23.10 \\
\hline 3 & 75.02 & 22.21 & 27.34 & 26.21 & 32.48 & 30.82 & 26.30 & 23.11 \\
\hline 6 & 74.99 & 22.24 & 27.43 & 25.80 & 32.64 & 31.09 & 26.36 & 23.19 \\
\hline
\end{tabular}


Table D58: Averaged Heater and Pressure Raw Data for Full Length Electrode with HFE-7000

\begin{tabular}{|c|c|c|c|c|c|}
\hline HV & $\mathbf{Q}$ & $\begin{array}{c}\text { Heater Voltage } \\
\text { after } \\
\text { precision resistor }\end{array}$ & $\begin{array}{c}\text { Heater Voltage } \\
\text { before } \\
\text { precision resistor }\end{array}$ & $\begin{array}{c}\text { Heater } \\
\text { Current }\end{array}$ & $\begin{array}{c}\text { Chamber } \\
\text { Pressure }\end{array}$ \\
\hline $\mathbf{k V}$ & $\mathbf{W}$ & $\mathbf{V o l t s}$ & $\mathbf{V o l t s}$ & Amps & psia \\
\hline $\mathbf{0}$ & 0 & 0.00 & 0.00 & 0.00 & 17.23 \\
\hline $\mathbf{0}$ & 10.01 & 9.82 & 9.82 & 1.02 & 17.48 \\
\hline $\mathbf{0}$ & 19.96 & 13.96 & 13.96 & 1.43 & 17.73 \\
\hline $\mathbf{0}$ & 29.93 & 17.10 & 17.10 & 1.75 & 17.90 \\
\hline $\mathbf{0}$ & 39.87 & 19.74 & 19.74 & 2.02 & 18.06 \\
\hline $\mathbf{0}$ & 44.58 & 20.83 & 20.83 & 2.14 & 18.14 \\
\hline $\mathbf{0}$ & 0 & 0.00 & 0.00 & 0.00 & 17.44 \\
\hline $\mathbf{3}$ & 0 & 0.00 & 0.00 & 0.00 & 17.35 \\
\hline $\mathbf{6}$ & 0 & 0.00 & 0.00 & 0.00 & 17.28 \\
\hline $\mathbf{0}$ & 9.908 & 9.81 & 9.81 & 1.01 & 17.32 \\
\hline $\mathbf{3}$ & 9.908 & 9.81 & 9.81 & 1.01 & 17.30 \\
\hline $\mathbf{6}$ & 9.908 & 9.81 & 9.81 & 1.01 & 17.27 \\
\hline $\mathbf{0}$ & 19.88 & 13.90 & 13.90 & 1.43 & 17.35 \\
\hline $\mathbf{3}$ & 19.88 & 13.90 & 13.90 & 1.43 & 17.34 \\
\hline $\mathbf{6}$ & 19.88 & 13.90 & 13.90 & 1.43 & 17.34 \\
\hline $\mathbf{0}$ & 29.84 & 17.05 & 17.05 & 1.75 & 17.43 \\
\hline $\mathbf{3}$ & 29.84 & 17.05 & 17.05 & 1.75 & 17.44 \\
\hline $\mathbf{6}$ & 29.84 & 17.05 & 17.05 & 1.75 & 17.45 \\
\hline $\mathbf{0}$ & 39.71 & 19.66 & 19.66 & 2.02 & 17.54 \\
\hline $\mathbf{3}$ & 39.71 & 19.66 & 19.66 & 2.02 & 17.55 \\
\hline $\mathbf{6}$ & 39.71 & 19.66 & 19.66 & 2.02 & 17.57 \\
\hline $\mathbf{0}$ & 44.78 & 20.83 & 20.83 & 2.15 & 17.61 \\
\hline $\mathbf{3}$ & 44.78 & 20.83 & 20.83 & 2.15 & 17.62 \\
\hline $\mathbf{6}$ & 44.78 & 20.83 & 20.83 & 2.15 & 17.64 \\
\hline & & & & & \\
\hline
\end{tabular}


Table D59: Averaged Pedestal Temperature Raw Data for Full Length Electrode with HFE-7000

\begin{tabular}{|c|c|c|c|c|c|c|c|c|}
\hline $\mathbf{H V}$ & $\mathbf{Q}$ & $\mathbf{T} 1$ & $\mathbf{T} 2$ & $\mathbf{T 3}$ & $\mathbf{T 4}$ & $\mathbf{T 5}$ & $\mathbf{T 6}$ & $\mathbf{T} 7$ \\
\hline $\mathbf{k V}$ & $\mathbf{W}$ & ${ }^{\circ} \mathbf{C}$ & ${ }^{\circ} \mathbf{C}$ & ${ }^{\circ} \mathbf{C}$ & ${ }^{\circ} \mathbf{C}$ & ${ }^{\circ} \mathbf{C}$ & ${ }^{\circ} \mathbf{C}$ & ${ }^{\circ} \mathbf{C}$ \\
\hline $\mathbf{0}$ & 0 & 23.49 & 22.99 & 23.03 & 23.01 & 23.18 & 23.21 & 24.27 \\
\hline $\mathbf{0}$ & 10.01 & 30.20 & 26.53 & 25.36 & 25.96 & 25.28 & 25.51 & 25.03 \\
\hline $\mathbf{0}$ & 19.96 & 37.27 & 30.47 & 27.90 & 29.51 & 27.69 & 28.00 & 26.24 \\
\hline $\mathbf{0}$ & 29.93 & 43.78 & 33.97 & 30.18 & 32.44 & 29.75 & 30.16 & 27.19 \\
\hline $\mathbf{0}$ & 39.87 & 47.56 & 36.57 & 32.02 & 34.76 & 31.49 & 32.01 & 28.15 \\
\hline $\mathbf{0}$ & 44.58 & 48.74 & 37.63 & 32.84 & 35.88 & 32.36 & 32.92 & 28.93 \\
\hline $\mathbf{0}$ & 0 & 24.26 & 24.31 & 24.05 & 24.88 & 24.43 & 24.35 & 26.05 \\
\hline $\mathbf{3}$ & 0 & 24.17 & 23.90 & 23.82 & 24.13 & 24.04 & 24.02 & 25.51 \\
\hline $\mathbf{6}$ & 0 & 24.09 & 23.76 & 23.75 & 23.89 & 23.93 & 23.94 & 25.28 \\
\hline $\mathbf{0}$ & 9.908 & 31.02 & 27.11 & 25.86 & 26.44 & 25.72 & 25.90 & 25.64 \\
\hline $\mathbf{3}$ & 9.908 & 31.08 & 27.30 & 25.96 & 26.78 & 25.89 & 26.03 & 25.79 \\
\hline $\mathbf{6}$ & 9.908 & 30.94 & 27.24 & 25.89 & 26.73 & 25.83 & 25.80 & 25.82 \\
\hline $\mathbf{0}$ & 19.88 & 37.97 & 30.67 & 28.09 & 29.42 & 27.74 & 28.06 & 26.39 \\
\hline $\mathbf{3}$ & 19.88 & 38.02 & 30.94 & 28.22 & 29.90 & 27.96 & 28.22 & 26.60 \\
\hline $\mathbf{6}$ & 19.88 & 37.81 & 30.85 & 28.12 & 29.79 & 27.86 & 27.77 & 26.69 \\
\hline $\mathbf{0}$ & 29.84 & 44.18 & 34.25 & 30.39 & 32.65 & 29.94 & 30.32 & 27.42 \\
\hline $\mathbf{3}$ & 29.84 & 44.19 & 34.42 & 30.48 & 32.98 & 30.10 & 30.45 & 27.64 \\
\hline $\mathbf{6}$ & 29.84 & 44.04 & 34.27 & 30.32 & 32.76 & 29.92 & 29.75 & 27.71 \\
\hline $\mathbf{0}$ & 39.71 & 47.52 & 36.72 & 32.19 & 34.97 & 31.70 & 32.19 & 28.47 \\
\hline $\mathbf{3}$ & 39.71 & 47.43 & 36.77 & 32.20 & 35.11 & 31.76 & 32.21 & 28.66 \\
\hline $\mathbf{6}$ & 39.71 & 47.27 & 36.53 & 31.97 & 34.75 & 31.49 & 31.29 & 28.70 \\
\hline $\mathbf{0}$ & 44.78 & 48.50 & 37.69 & 32.99 & 35.95 & 32.53 & 33.05 & 29.17 \\
\hline $\mathbf{3}$ & 44.78 & 48.50 & 37.72 & 33.00 & 36.02 & 32.55 & 33.03 & 29.31 \\
\hline $\mathbf{6}$ & 44.78 & 48.32 & 37.45 & 32.73 & 35.60 & 32.23 & 32.03 & 29.32 \\
\hline
\end{tabular}


Table D60: Averaged Chamber Temperature Raw Data for Full Length Electrode with HFE-7000

\begin{tabular}{|c|c|c|c|c|c|c|c|c|}
\hline $\mathbf{H V}$ & $\mathbf{Q}$ & $\mathbf{A m b}$ & $\mathbf{T} 8$ & $\mathbf{T 9}$ & $\mathbf{T 1 0}$ & $\mathbf{T 1 1}$ & $\mathbf{T 1 2}$ & $\mathbf{T 1 4}$ \\
\hline $\mathbf{k V}$ & $\mathbf{W}$ & ${ }^{\circ} \mathbf{C}$ & ${ }^{\circ} \mathbf{C}$ & ${ }^{\circ} \mathbf{C}$ & ${ }^{\circ} \mathbf{C}$ & ${ }^{\circ} \mathbf{C}$ & ${ }^{\circ} \mathbf{C}$ & ${ }^{\circ} \mathbf{C}$ \\
\hline $\mathbf{0}$ & 0 & 23.22 & 23.30 & 22.96 & 23.52 & 23.11 & 23.49 & 23.63 \\
\hline $\mathbf{0}$ & 10.01 & 23.35 & 23.73 & 23.54 & 24.35 & 23.95 & 23.88 & 23.72 \\
\hline $\mathbf{0}$ & 19.96 & 23.38 & 24.35 & 24.00 & 25.22 & 24.83 & 24.36 & 23.85 \\
\hline $\mathbf{0}$ & 29.93 & 23.69 & 24.95 & 24.50 & 26.05 & 25.68 & 24.81 & 23.95 \\
\hline $\mathbf{0}$ & 39.87 & 23.51 & 25.61 & 25.05 & 26.88 & 26.58 & 25.32 & 23.94 \\
\hline $\mathbf{0}$ & 44.58 & 23.29 & 25.99 & 25.38 & 27.42 & 27.14 & 25.64 & 23.95 \\
\hline $\mathbf{0}$ & 0 & 23.28 & 24.14 & 23.94 & 24.26 & 23.85 & 24.34 & 23.96 \\
\hline $\mathbf{0}$ & 9.908 & 23.30 & 24.16 & 24.05 & 24.75 & 24.37 & 24.32 & 23.89 \\
\hline $\mathbf{0}$ & 19.88 & 23.21 & 24.56 & 24.29 & 25.43 & 25.05 & 24.58 & 23.88 \\
\hline $\mathbf{0}$ & 29.84 & 23.35 & 25.16 & 24.80 & 26.26 & 25.91 & 25.00 & 23.93 \\
\hline $\mathbf{0}$ & 39.71 & 23.40 & 25.80 & 25.30 & 27.18 & 26.90 & 25.49 & 24.01 \\
\hline $\mathbf{0}$ & 44.78 & 23.55 & 26.27 & 25.73 & 27.78 & 27.52 & 25.88 & 24.14 \\
\hline $\mathbf{3}$ & 0 & 23.25 & 23.94 & 23.77 & 24.17 & 23.76 & 24.21 & 23.93 \\
\hline $\mathbf{3}$ & 9.908 & 23.38 & 24.23 & 24.09 & 24.83 & 24.43 & 24.33 & 23.92 \\
\hline $\mathbf{3}$ & 19.88 & 23.27 & 24.69 & 24.39 & 25.51 & 25.12 & 24.63 & 23.87 \\
\hline $\mathbf{3}$ & 29.84 & 23.33 & 25.32 & 24.90 & 26.41 & 26.05 & 25.05 & 23.96 \\
\hline $\mathbf{3}$ & 39.71 & 23.43 & 25.99 & 25.45 & 27.32 & 27.02 & 25.56 & 24.04 \\
\hline $\mathbf{3}$ & 44.78 & 23.80 & 26.47 & 25.86 & 27.93 & 27.65 & 25.95 & 24.16 \\
\hline $\mathbf{6}$ & 0 & 23.24 & 23.89 & 23.72 & 24.19 & 23.82 & 24.15 & 23.95 \\
\hline $\mathbf{6}$ & 9.908 & 23.21 & 24.28 & 24.12 & 24.79 & 24.58 & 24.32 & 23.88 \\
\hline $\mathbf{6}$ & 19.88 & 23.27 & 24.79 & 24.43 & 25.50 & 25.44 & 24.66 & 23.89 \\
\hline $\mathbf{6}$ & 29.84 & 23.38 & 25.44 & 24.94 & 26.34 & 26.46 & 25.09 & 23.98 \\
\hline $\mathbf{6}$ & 39.71 & 23.45 & 26.14 & 25.57 & 27.19 & 27.50 & 25.63 & 24.07 \\
\hline $\mathbf{6}$ & 44.78 & 23.63 & 26.61 & 26.09 & 27.76 & 28.14 & 26.03 & 24.20 \\
\hline
\end{tabular}


Table D61: Reduced Data for Bottom Half Electrode 0 kV Runs

\begin{tabular}{|c|c|c|c|c|c|c|c|c|c|c|c|c|c|c|c|c|}
\hline HV & $\mathbf{T}_{\text {sat }}$ & $\mathbf{Q}$ & $\mathbf{q}$ & $\Delta \mathbf{T}_{\text {sub }}$ & $\Delta \mathbf{T}_{\mathrm{htr}}$ & $\mathbf{T}_{\mathrm{s}}$ & $\mathbf{T}_{\infty \text { wall }}$ & $\mathbf{T}_{\text {otop }}$ & $\begin{array}{c}\mathbf{T}_{\mathrm{s}^{-}} \\
\mathbf{T}_{\text {otop }}\end{array}$ & $\begin{array}{c}\mathbf{T}_{\text {sat }^{-}} \\
\mathbf{T}_{\text {cowall }}\end{array}$ & $\begin{array}{c}\mathbf{T}_{\text {sat }}{ }^{-} \\
\mathbf{T}_{\text {cotop }}\end{array}$ & $\mathbf{k}_{\text {fluid }}$ & $\mathbf{h}$ & $\theta_{\mathrm{s}}-\theta_{\infty \mathrm{top}}$ & $\mathbf{N u}$ & G $\Delta$ \\
\hline & ${ }^{\circ} \mathrm{C}$ & $\mathbf{W}$ & $\begin{array}{c}10^{4} \\
W / m^{2}\end{array}$ & ${ }^{\circ} \mathrm{C}$ & ${ }^{\circ} \mathrm{C}$ & ${ }^{\circ} \mathrm{C}$ & ${ }^{\circ} \mathrm{C}$ & ${ }^{\circ} \mathrm{C}$ & ${ }^{\circ} \mathrm{C}$ & ${ }^{\circ} \mathrm{C}$ & ${ }^{\circ} \mathrm{C}$ & $\begin{array}{c}\mathbf{W} / \\
\mathbf{m}-\mathbf{K}\end{array}$ & $\begin{array}{c}\mathbf{W} / \\
\mathbf{m}^{2}-\mathbf{K}\end{array}$ & -- & --- & --- \\
\hline $\mathbf{0}$ & 54.35 & 0.00 & 0.00 & 0.00 & 0.00 & 23.23 & 23.06 & 22.47 & 0.75 & 31.29 & 31.87 & 0.056 & 0 & 0.02 & 0.00 & 0.00 \\
\hline $\mathbf{0}$ & 54.47 & 10.02 & 6.82 & 0.02 & -0.46 & 29.88 & 23.85 & 22.82 & 7.06 & 30.63 & 31.65 & 0.056 & 9516 & 0.23 & 1356.86 & 12.33 \\
\hline $\mathbf{0}$ & 54.68 & 20.04 & 13.63 & 0.05 & -0.92 & 36.51 & 24.74 & 23.22 & 13.29 & 29.94 & 31.46 & 0.056 & 10104 & 0.44 & 1441.93 & 25.22 \\
\hline $\mathbf{0}$ & 54.87 & 30.00 & 20.41 & 0.07 & -1.38 & 42.89 & 25.61 & 23.60 & 19.29 & 29.26 & 31.27 & 0.056 & 10423 & 0.66 & 1488.67 & 38.64 \\
\hline $\mathbf{0}$ & 55.04 & 40.04 & 27.24 & 0.10 & -1.84 & 48.72 & 26.47 & 23.96 & 24.76 & 28.57 & 31.08 & 0.056 & 10836 & 0.87 & 1548.85 & 52.82 \\
\hline $\mathbf{0}$ & 55.29 & 50.07 & 34.06 & 0.12 & -2.30 & 53.76 & 27.51 & 24.43 & 29.33 & 27.78 & 30.87 & 0.056 & 11439 & 1.06 & 1636.64 & 67.93 \\
\hline $\mathbf{0}$ & 55.48 & 59.98 & 40.80 & 0.14 & -2.75 & 57.09 & 28.59 & 24.92 & 32.18 & 26.89 & 30.57 & 0.056 & 12490 & 1.20 & 1788.77 & 84.05 \\
\hline $\mathbf{0}$ & 55.67 & 70.12 & 47.70 & 0.17 & -3.22 & 59.45 & 29.65 & 25.42 & 34.03 & 26.02 & 30.25 & 0.056 & 13809 & 1.31 & 1979.73 & 101.57 \\
\hline $\mathbf{0}$ & 55.92 & 80.02 & 54.43 & 0.19 & -3.67 & 61.74 & 30.80 & 26.00 & 35.74 & 25.13 & 29.92 & 0.056 & 15002 & 1.42 & 2153.48 & 120.02 \\
\hline $\mathbf{0}$ & 56.13 & 89.88 & 61.14 & 0.22 & -4.12 & 64.09 & 31.84 & 26.69 & 37.39 & 24.29 & 29.44 & 0.056 & 16105 & 1.54 & 2315.17 & 139.46 \\
\hline $\mathbf{0}$ & 55.27 & 0.00 & 0.00 & 0.00 & 0.00 & 25.40 & 24.45 & 23.45 & 1.95 & 30.82 & 31.82 & 0.056 & 0 & 0.06 & 0.00 & 0.00 \\
\hline $\mathbf{0}$ & 55.38 & 50.09 & 34.08 & 0.12 & -2.30 & 50.01 & 28.26 & 25.08 & 24.93 & 27.12 & 30.30 & 0.056 & 13465 & 0.92 & 1929.06 & 69.60 \\
\hline $\mathbf{0}$ & 55.54 & 60.02 & 40.83 & 0.14 & -2.75 & 52.97 & 29.20 & 25.48 & 27.48 & 26.35 & 30.06 & 0.056 & 14634 & 1.04 & 2098.34 & 85.85 \\
\hline $\mathbf{0}$ & 55.86 & 70.00 & 47.62 & 0.17 & -3.21 & 55.21 & 30.25 & 25.96 & 29.25 & 25.61 & 29.90 & 0.056 & 16038 & 1.14 & 2302.01 & 103.01 \\
\hline 0 & 56.09 & 79.88 & 54.34 & 0.19 & -3.66 & 57.21 & 31.23 & 26.44 & 30.77 & 24.86 & 29.65 & 0.056 & 17396 & 1.24 & 2499.42 & 121.09 \\
\hline
\end{tabular}

Table D62: Reduced Data for Bottom Half Electrode $3 \mathrm{kV}$ Run

\begin{tabular}{|c|c|c|c|c|c|c|c|c|c|c|c|c|c|c|c|c|}
\hline HV & $\mathbf{T}_{\text {sat }}$ & $\mathbf{Q}$ & $\mathbf{q}$ & $\Delta \mathbf{T}_{\text {sub }}$ & $\Delta \mathbf{T}_{\mathrm{htr}}$ & $\mathbf{T}_{\mathrm{s}}$ & $\mathbf{T}_{\infty \text { wall }}$ & $\mathbf{T}_{\text {otop }}$ & $\begin{array}{c}\mathbf{T}_{\mathbf{s}^{-}} \\
\mathbf{T}_{\text {otop }}\end{array}$ & $\begin{array}{c}\mathbf{T}_{\text {sat }}{ }^{-} \\
\mathbf{T}_{\text {owall }}\end{array}$ & $\begin{array}{c}\mathbf{T}_{\text {sat }}{ }^{-} \\
\mathbf{T}_{\text {ctop }}\end{array}$ & $\mathbf{k}_{\text {fluid }}$ & h & $\theta_{\mathrm{s}}-\theta_{\infty \mathrm{top}}$ & $\mathbf{N u}$ & GD \\
\hline & ${ }^{\circ} \mathrm{C}$ & $\mathbf{W}$ & $\begin{array}{c}10^{4} \\
W / m^{2}\end{array}$ & ${ }^{\circ} \mathrm{C}$ & ${ }^{\circ} \mathrm{C}$ & ${ }^{\circ} \mathrm{C}$ & ${ }^{\circ} \mathrm{C}$ & ${ }^{\circ} \mathrm{C}$ & ${ }^{\circ} \mathrm{C}$ & ${ }^{\circ} \mathrm{C}$ & ${ }^{\circ} \mathrm{C}$ & $\begin{array}{c}\mathbf{W} / \\
\mathbf{m}-\mathbf{K}\end{array}$ & $\begin{array}{c}\mathbf{W} / \\
\mathbf{m}^{2}-\mathbf{K}\end{array}$ & --- & -- & --- \\
\hline 3 & 55.40 & 50.18 & 34.13 & 0.12 & -2.30 & 50.20 & 28.33 & 25.11 & 25.09 & 27.07 & 30.29 & 0.056 & 13399 & 0.93 & 1919.82 & 69.85 \\
\hline 3 & 55.61 & 60.05 & 40.85 & 0.14 & -2.75 & 53.09 & 29.22 & 25.47 & 27.62 & 26.39 & 30.14 & 0.056 & 14569 & 1.05 & 2088.97 & 85.75 \\
\hline 3 & 55.90 & 69.96 & 47.59 & 0.17 & -3.21 & 55.24 & 30.29 & 25.99 & 29.25 & 25.61 & 29.91 & 0.056 & 16029 & 1.14 & 2300.76 & 102.96 \\
\hline 3 & 56.13 & 79.84 & 54.31 & 0.19 & -3.66 & 57.32 & 31.28 & 26.47 & 30.85 & 24.85 & 29.66 & 0.056 & 17338 & 1.24 & 2491.25 & 121.08 \\
\hline
\end{tabular}


Table D63: Reduced Data for Bottom Half Electrode 6 kV Run

\begin{tabular}{|c|c|c|c|c|c|c|c|c|c|c|c|c|c|c|c|c|}
\hline HV & $\mathbf{T}_{\text {sat }}$ & $\mathbf{Q}$ & $\mathbf{q}$ & $\Delta \mathbf{T}_{\text {sub }}$ & $\Delta \mathbf{T}_{\mathrm{htr}}$ & $\mathbf{T}_{\mathrm{s}}$ & $\mathbf{T}_{\infty \text { wall }}$ & $\mathbf{T}_{\text {otop }}$ & $\begin{array}{c}\mathbf{T}_{\mathbf{s}^{-}} \\
\mathbf{T}_{\text {otop }}\end{array}$ & $\begin{array}{c}\mathbf{T}_{\text {sat- }} \\
\mathbf{T}_{\text {cowall }}\end{array}$ & $\begin{array}{c}\mathbf{T}_{\text {sat }} \\
\mathbf{T}_{\text {otop }}\end{array}$ & $\mathbf{k}_{\text {fluid }}$ & $\mathbf{h}$ & $\theta_{\mathrm{s}}-\theta_{\infty \mathrm{otop}}$ & $\mathbf{N u}$ & G $\Delta$ \\
\hline & ${ }^{\circ} \mathrm{C}$ & $\mathbf{W}$ & $\begin{array}{c}10^{4} \\
W / m^{2}\end{array}$ & ${ }^{\circ} \mathrm{C}$ & ${ }^{\circ} \mathrm{C}$ & ${ }^{\circ} \mathrm{C}$ & ${ }^{\circ} \mathrm{C}$ & ${ }^{\circ} \mathrm{C}$ & ${ }^{\circ} \mathrm{C}$ & ${ }^{\circ} \mathrm{C}$ & ${ }^{\circ} \mathrm{C}$ & $\begin{array}{c}\mathbf{W} / \\
\mathbf{m}-\mathbf{K}\end{array}$ & $\begin{array}{c}\mathbf{W} / \\
\mathbf{m}^{2}-K\end{array}$ & --- & --- & --- \\
\hline 6 & 55.42 & 50.15 & 34.12 & 0.12 & -2.30 & 50.27 & 28.40 & 25.17 & 25.10 & 27.02 & 30.25 & 0.056 & 13389 & 0.93 & 1918.48 & 69.95 \\
\hline 6 & 55.67 & 60.05 & 40.85 & 0.14 & -2.75 & 53.16 & 29.23 & 25.46 & 27.70 & 26.44 & 30.21 & 0.056 & 14525 & 1.05 & 2082.64 & 85.60 \\
\hline 6 & 55.94 & 69.93 & 47.57 & 0.17 & -3.21 & 55.24 & 30.33 & 26.02 & 29.23 & 25.61 & 29.93 & 0.056 & 16032 & 1.14 & 2301.42 & 102.91 \\
\hline 6 & 56.15 & 79.83 & 54.31 & 0.19 & -3.66 & 57.39 & 31.34 & 26.50 & 30.89 & 24.81 & 29.65 & 0.056 & 17320 & 1.24 & 2488.85 & 121.26 \\
\hline
\end{tabular}

Table D64: Reduced Data for Thick Top Half Electrode $0 \mathrm{kV}$ Runs

\begin{tabular}{|c|c|c|c|c|c|c|c|c|c|c|c|c|c|c|c|c|}
\hline HV & $\mathbf{T}_{\text {sat }}$ & $\mathbf{Q}$ & $\mathbf{q}$ & $\Delta \mathbf{T}_{\text {sub }}$ & $\Delta \mathbf{T}_{\mathrm{htr}}$ & $\mathbf{T}_{\mathrm{s}}$ & $\mathbf{T}_{\text {cowall }}$ & $\mathbf{T}_{\text {cotop }}$ & $\begin{array}{c}\mathbf{T}_{\mathrm{s}^{-}} \\
\mathbf{T}_{\text {ootop }}\end{array}$ & $\begin{array}{c}\mathbf{T}_{\text {sat }^{-}} \\
\mathbf{T}_{\text {cowall }}\end{array}$ & $\begin{array}{c}\mathbf{T}_{\text {sat }}{ }^{-} \\
\mathbf{T}_{\text {otop }} \\
\end{array}$ & $\mathbf{k}_{\text {fluid }}$ & h & $\theta_{\mathrm{s}}-\theta_{\infty \mathrm{otop}}$ & $\mathbf{N u}$ & G $\Delta$ \\
\hline & ${ }^{\circ} \mathrm{C}$ & $\mathbf{W}$ & $\begin{array}{c}10^{4} \\
W / m^{2} \\
\end{array}$ & ${ }^{\circ} \mathrm{C}$ & ${ }^{\circ} \mathrm{C}$ & ${ }^{\circ} \mathrm{C}$ & ${ }^{\circ} \mathrm{C}$ & ${ }^{\circ} \mathrm{C}$ & ${ }^{\circ} \mathrm{C}$ & ${ }^{\circ} \mathrm{C}$ & ${ }^{\circ} \mathrm{C}$ & $\begin{array}{c}\text { W/ } \\
\text { m-K }\end{array}$ & $\begin{array}{c}\mathbf{W} / \\
\mathbf{m}^{2}-K \\
\end{array}$ & --- & - & --- \\
\hline $\mathbf{0}$ & 54.91 & 0.00 & 0.00 & 0.00 & 0.00 & 24.93 & 24.80 & 24.27 & 0.66 & 30.11 & 30.65 & 0.056 & 0 & 0.02 & 0.00 & 0.00 \\
\hline $\mathbf{0}$ & 55.02 & 10.01 & 6.81 & 0.02 & -0.46 & 31.43 & 25.52 & 24.53 & 6.91 & 29.50 & 30.49 & 0.056 & 9717 & 0.23 & 1390.58 & 12.79 \\
\hline O & 55.17 & 20.09 & 13.67 & 0.05 & -0.92 & 37.84 & 26.34 & 24.86 & 12.98 & 28.82 & 30.31 & 0.056 & 10374 & 0.45 & 1485.57 & 26.27 \\
\hline $\mathbf{0}$ & 55.33 & 30.03 & 20.43 & 0.07 & -1.38 & 44.10 & 27.15 & 25.15 & 18.95 & 28.18 & 30.19 & 0.056 & 10617 & 0.67 & 1521.30 & 40.15 \\
\hline $\mathbf{0}$ & 55.54 & 40.09 & 27.27 & 0.10 & -1.84 & 49.97 & 27.95 & 25.42 & 24.54 & 27.59 & 30.12 & 0.056 & 10945 & 0.89 & 1569.20 & 54.76 \\
\hline $\mathbf{0}$ & 55.82 & 50.06 & 34.05 & 0.12 & -2.30 & 55.20 & 28.83 & 25.77 & 29.43 & 26.98 & 30.05 & 0.056 & 11396 & 1.09 & 1635.01 & 69.91 \\
\hline $\mathbf{0}$ & 56.07 & 60.07 & 40.87 & 0.14 & -2.76 & 58.13 & 29.81 & 26.23 & 31.91 & 26.26 & 29.84 & 0.056 & 12615 & 1.22 & 1811.73 & 86.21 \\
\hline $\mathbf{0}$ & 56.32 & 70.19 & 47.75 & 0.17 & -3.22 & 60.10 & 30.76 & 26.65 & 33.45 & 25.56 & 29.67 & 0.056 & 14061 & 1.31 & 2021.17 & 103.49 \\
\hline $\mathbf{0}$ & 55.21 & 0.00 & 0.00 & 0.00 & 0.00 & 25.92 & 25.50 & 24.84 & 1.08 & 29.71 & 30.36 & 0.056 & 0 & 0.04 & 0.00 & 0.00 \\
\hline $\mathbf{0}$ & 55.08 & 10.02 & 6.82 & 0.02 & -0.46 & 32.36 & 26.15 & 25.09 & 7.27 & 28.93 & 29.99 & 0.056 & 9237 & 0.25 & 1323.44 & 13.05 \\
\hline $\mathbf{0}$ & 55.21 & 20.11 & 13.68 & 0.05 & -0.92 & 38.93 & 26.94 & 25.38 & 13.55 & 28.27 & 29.83 & 0.056 & 9946 & 0.48 & 1425.83 & 26.81 \\
\hline $\mathbf{0}$ & 55.46 & 30.00 & 20.41 & 0.07 & -1.38 & 45.81 & 28.09 & 26.01 & 19.80 & 27.37 & 29.45 & 0.056 & 10156 & 0.72 & 1457.82 & 41.32 \\
\hline $\mathbf{0}$ & 55.67 & 40.00 & 27.21 & 0.10 & -1.83 & 51.77 & 29.10 & 26.49 & 25.28 & 26.57 & 29.18 & 0.056 & 10601 & 0.95 & 1523.27 & 56.74 \\
\hline $\mathbf{0}$ & 55.94 & 50.00 & 34.02 & 0.12 & -2.29 & 56.43 & 30.15 & 26.98 & 29.45 & 25.80 & 28.96 & 0.056 & 11377 & 1.14 & 1636.45 & 73.05 \\
\hline $\mathbf{0}$ & 56.19 & 60.07 & 40.86 & 0.14 & -2.75 & 58.94 & 31.25 & 27.52 & 31.42 & 24.95 & 28.67 & 0.056 & 12812 & 1.26 & 1845.01 & 90.74 \\
\hline $\mathbf{0}$ & 56.45 & 70.32 & 47.83 & 0.17 & -3.22 & 60.86 & 32.43 & 28.14 & 32.72 & 24.02 & 28.31 & 0.055 & 14400 & 1.36 & 2076.28 & 110.32 \\
\hline
\end{tabular}


Table D65: Reduced Data for Thick Top Half Electrode 3 kV Run

\begin{tabular}{|c|c|c|c|c|c|c|c|c|c|c|c|c|c|c|c|c|}
\hline HV & $\mathbf{T}_{\text {sat }}$ & $\mathbf{Q}$ & $\mathbf{q}$ & $\Delta \mathbf{T}_{\text {sub }}$ & $\Delta \mathbf{T}_{\mathrm{htr}}$ & $\mathbf{T}_{\mathrm{s}}$ & $\mathbf{T}_{\text {owall }}$ & $\mathbf{T}_{\text {otop }}$ & $\begin{array}{c}\mathbf{T}_{\mathrm{s}^{-}} \\
\mathbf{T}_{\text {otop }} \\
\end{array}$ & $\begin{array}{c}\mathbf{T}_{\text {sat- }} \\
\mathbf{T}_{\text {owall }}\end{array}$ & $\begin{array}{c}\mathbf{T}_{\text {sat }^{-}} \\
\mathbf{T}_{\text {otop }}\end{array}$ & $\mathbf{k}_{\text {fluid }}$ & h & $\theta_{\mathrm{s}}-\theta_{\infty \mathrm{otop}}$ & $\mathbf{N u}$ & G $\Delta$ \\
\hline & ${ }^{\circ} \mathrm{C}$ & $\mathbf{W}$ & $\begin{array}{c}10^{4} \\
W / m^{2} \\
\end{array}$ & ${ }^{\circ} \mathrm{C}$ & ${ }^{\circ} \mathrm{C}$ & ${ }^{\circ} \mathrm{C}$ & ${ }^{\circ} \mathrm{C}$ & ${ }^{\circ} \mathrm{C}$ & ${ }^{\circ} \mathrm{C}$ & ${ }^{\circ} \mathrm{C}$ & ${ }^{\circ} \mathrm{C}$ & $\begin{array}{c}\mathbf{W} / \\
\mathbf{m}-\mathbf{K}\end{array}$ & $\begin{array}{c}\mathbf{W} / \\
\mathbf{m}^{2}-\mathbf{K}\end{array}$ & --- & --- & --- \\
\hline 3 & 55.08 & 0.00 & 0.00 & 0.00 & 0.00 & 25.92 & 25.46 & 24.83 & 1.08 & 29.62 & 30.25 & 0.056 & 0 & 0.04 & 0.00 & 0.00 \\
\hline 3 & 55.08 & 10.02 & 6.82 & 0.02 & -0.46 & 32.44 & 26.17 & 25.10 & 7.34 & 28.91 & 29.98 & 0.056 & 9153 & 0.25 & 1311.40 & 13.06 \\
\hline 3 & 55.25 & 20.12 & 13.68 & 0.05 & -0.92 & 39.16 & 27.05 & 25.47 & 13.69 & 28.20 & 29.78 & 0.056 & 9848 & 0.49 & 1412.01 & 26.88 \\
\hline 3 & 55.50 & 30.01 & 20.42 & 0.07 & -1.38 & 45.92 & 28.16 & 26.05 & 19.86 & 27.34 & 29.45 & 0.056 & 10123 & 0.73 & 1453.29 & 41.37 \\
\hline 3 & 55.73 & 40.00 & 27.21 & 0.10 & -1.83 & 51.88 & 29.19 & 26.54 & 25.35 & 26.54 & 29.20 & 0.056 & 10576 & 0.96 & 1519.78 & 56.80 \\
\hline 3 & 55.98 & 50.01 & 34.02 & 0.12 & -2.29 & 56.45 & 30.24 & 27.03 & 29.41 & 25.75 & 28.95 & 0.056 & 11392 & 1.14 & 1638.76 & 73.20 \\
\hline 3 & 56.24 & 60.09 & 40.88 & 0.14 & -2.76 & 58.97 & 31.33 & 27.57 & 31.39 & 24.91 & 28.66 & 0.056 & 12827 & 1.26 & 1847.32 & 90.92 \\
\hline 3 & 56.49 & 70.20 & 47.75 & 0.17 & -3.22 & 60.86 & 32.49 & 28.18 & 32.68 & 24.00 & 28.31 & 0.055 & 14393 & 1.36 & 2075.44 & 110.21 \\
\hline
\end{tabular}

Table D66: Reduced Data for Thick Top Half Electrode 6 kV Run

\begin{tabular}{|c|c|c|c|c|c|c|c|c|c|c|c|c|c|c|c|c|}
\hline HV & $\mathbf{T}_{\text {sat }}$ & $\mathbf{Q}$ & $\mathbf{q}$ & $\Delta \mathbf{T}_{\mathrm{sub}}$ & $\Delta \mathrm{T}_{\mathrm{htr}}$ & $\mathbf{T}_{\mathbf{s}}$ & $\mathbf{T}_{\infty \text { wall }}$ & $\mathbf{T}_{\text {otop }}$ & $\begin{array}{c}\mathbf{T}_{\mathrm{s}^{-}} \\
\mathbf{T}_{\text {otop }}\end{array}$ & $\begin{array}{c}\mathbf{T}_{\text {sat }}{ }^{-} \\
\mathbf{T}_{\text {owall }}\end{array}$ & $\begin{array}{c}\mathbf{T}_{\text {sat }^{-}} \\
\mathbf{T}_{\text {ootop }}\end{array}$ & $\mathbf{k}_{\text {fluid }}$ & h & $\theta_{\mathrm{s}}-\theta_{\infty \mathrm{otop}}$ & Nu & G $\Delta$ \\
\hline & ${ }^{\circ} \mathrm{C}$ & $\mathbf{W}$ & $\begin{array}{c}10^{4} \\
W / m^{2} \\
\end{array}$ & ${ }^{\circ} \mathrm{C}$ & ${ }^{\circ} \mathrm{C}$ & ${ }^{\circ} \mathrm{C}$ & ${ }^{\circ} \mathrm{C}$ & ${ }^{\circ} \mathrm{C}$ & ${ }^{\circ} \mathrm{C}$ & ${ }^{\circ} \mathrm{C}$ & ${ }^{\circ} \mathrm{C}$ & $\begin{array}{c}\mathbf{W} / \\
\mathbf{m}-\mathbf{K}\end{array}$ & $\begin{array}{c}\text { W/ } \\
\mathbf{m}^{2}-K\end{array}$ & --- & --- & --- \\
\hline 6 & 55.02 & 0.00 & 0.00 & 0.00 & 0.00 & 25.90 & 25.45 & 24.83 & 1.06 & 29.57 & 30.19 & 0.056 & 0 & 0.04 & 0.00 & 0.00 \\
\hline 6 & 55.08 & 10.02 & 6.82 & 0.02 & -0.46 & 32.45 & 26.19 & 25.11 & 7.33 & 28.89 & 29.97 & 0.056 & 9157 & 0.25 & 1311.93 & 13.07 \\
\hline 6 & 55.29 & 20.13 & 13.69 & 0.05 & -0.92 & 39.55 & 27.22 & 25.64 & 13.91 & 28.08 & 29.65 & 0.056 & 9698 & 0.50 & 1390.99 & 27.02 \\
\hline 6 & 55.54 & 30.01 & 20.41 & 0.07 & -1.38 & 45.99 & 28.21 & 26.08 & 19.90 & 27.34 & 29.46 & 0.056 & 10102 & 0.73 & 1450.36 & 41.37 \\
\hline 6 & 55.77 & 40.02 & 27.23 & 0.10 & -1.84 & 51.92 & 29.24 & 26.57 & 25.36 & 26.53 & 29.21 & 0.056 & 10575 & 0.96 & 1519.82 & 56.84 \\
\hline 6 & 56.03 & 50.01 & 34.02 & 0.12 & -2.29 & 56.48 & 30.28 & 27.06 & 29.42 & 25.74 & 28.96 & 0.056 & 11392 & 1.14 & 1638.86 & 73.21 \\
\hline 6 & 56.28 & 60.08 & 40.87 & 0.14 & -2.76 & 59.01 & 31.40 & 27.63 & 31.38 & 24.87 & 28.65 & 0.056 & 12828 & 1.26 & 1847.65 & 91.02 \\
\hline 6 & 56.53 & 70.14 & 47.72 & 0.17 & -3.22 & 60.86 & 32.54 & 28.21 & 32.65 & 23.99 & 28.32 & 0.055 & 14397 & 1.36 & 2076.16 & 110.19 \\
\hline
\end{tabular}


Table D67: Reduced Data for Full Length Electrode 0 kV Runs

\begin{tabular}{|c|c|c|c|c|c|c|c|c|c|c|c|c|c|c|c|c|}
\hline HV & $\mathbf{T}_{\text {sat }}$ & $\mathbf{Q}$ & $\mathbf{q}$ & $\Delta \mathbf{T}_{\text {sub }}$ & $\Delta \mathbf{T}_{\text {htr }}$ & $\mathbf{T}_{\mathrm{s}}$ & $\mathbf{T}_{\text {cwall }}$ & $\mathbf{T}_{\infty \mathrm{top}}$ & $\begin{array}{c}\mathbf{T}_{\mathbf{s}^{-}} \\
\mathbf{T}_{\text {otop }}\end{array}$ & $\begin{array}{c}\mathbf{T}_{\text {sat }^{-}} \\
\mathbf{T}_{\text {owall }} \\
\end{array}$ & $\begin{array}{c}\mathbf{T}_{\text {sat }^{-}} \\
\mathbf{T}_{\text {otop }}\end{array}$ & $\mathbf{k}_{\text {fluid }}$ & h & $\theta_{\mathrm{s}}-\theta_{\text {otop }}$ & $\mathbf{N u}$ & GD \\
\hline & ${ }^{\circ} \mathrm{C}$ & $\mathbf{W}$ & $10^{4} \mathrm{~W} / \mathrm{m}^{2}$ & ${ }^{\circ} \mathrm{C}$ & ${ }^{\circ} \mathrm{C}$ & ${ }^{\circ} \mathrm{C}$ & ${ }^{\circ} \mathrm{C}$ & ${ }^{\circ} \mathrm{C}$ & ${ }^{\circ} \mathrm{C}$ & ${ }^{\circ} \mathrm{C}$ & ${ }^{\circ} \mathrm{C}$ & $\begin{array}{c}\mathbf{W} / \\
\mathbf{m}-\mathbf{K}\end{array}$ & $\begin{array}{c}\mathbf{W} / \\
\mathbf{m}^{2}-K\end{array}$ & --- & --- & --- \\
\hline 0 & 55.88 & 0.00 & 0.00 & 0.00 & 0.00 & 26.44 & 26.18 & 25.68 & 0.76 & 29.70 & 30.20 & 0.056 & 0 & 0.03 & 0.00 & 0.00 \\
\hline 0 & 55.92 & 10.09 & 6.86 & 0.02 & -0.46 & 33.66 & 27.00 & 26.00 & 7.66 & 28.92 & 29.92 & 0.056 & 8828 & 0.26 & 1267.17 & 13.14 \\
\hline $\mathbf{0}$ & 56.05 & 20.10 & 13.67 & 0.05 & -0.92 & 40.88 & 27.89 & 26.38 & 14.50 & 28.15 & 29.67 & 0.056 & 9287 & 0.52 & 1334.11 & 26.90 \\
\hline 0 & 56.17 & 30.05 & 20.45 & 0.07 & -1.38 & 47.80 & 28.81 & 26.75 & 21.05 & 27.37 & 29.42 & 0.056 & 9569 & 0.77 & 1375.70 & 41.38 \\
\hline 0 & 56.32 & 39.99 & 27.21 & 0.10 & -1.83 & 53.82 & 29.75 & 27.14 & 26.68 & 26.57 & 29.18 & 0.056 & 10045 & 1.00 & 1445.38 & 56.72 \\
\hline 0 & 56.51 & 50.02 & 34.03 & 0.12 & -2.29 & 57.95 & 30.75 & 27.58 & 30.38 & 25.76 & 28.93 & 0.056 & 11034 & 1.18 & 1589.15 & 73.19 \\
\hline 0 & 56.72 & 60.00 & 40.82 & 0.14 & -2.75 & 60.25 & 31.80 & 28.05 & 32.21 & 24.92 & 28.67 & 0.055 & 12482 & 1.29 & 1799.49 & 90.72 \\
\hline 0 & 56.93 & 70.04 & 47.64 & 0.17 & -3.21 & 62.02 & 32.90 & 28.59 & 33.44 & 24.03 & 28.34 & 0.055 & 14034 & 1.39 & 2025.47 & 109.81 \\
\hline $\mathbf{0}$ & 55.80 & 0.00 & 0.00 & 0.00 & 0.00 & 26.75 & 26.34 & 25.69 & 1.05 & 29.45 & 30.10 & 0.056 & 0 & 0.04 & 0.00 & 0.00 \\
\hline 0 & 55.77 & 10.03 & 6.82 & 0.02 & -0.46 & 33.86 & 27.15 & 26.08 & 7.79 & 28.63 & 29.70 & 0.056 & 8630 & 0.27 & 1238.99 & 13.20 \\
\hline $\mathbf{0}$ & 55.92 & 19.98 & 13.59 & 0.05 & -0.92 & 41.05 & 27.99 & 26.40 & 14.64 & 27.93 & 29.52 & 0.056 & 9142 & 0.52 & 1313.38 & 26.95 \\
\hline 0 & 56.13 & 30.04 & 20.44 & 0.07 & -1.38 & 47.99 & 29.03 & 26.90 & 21.09 & 27.10 & 29.24 & 0.056 & 9543 & 0.78 & 1372.45 & 41.77 \\
\hline $\mathbf{0}$ & 56.34 & 39.98 & 27.20 & 0.10 & -1.83 & 54.04 & 30.02 & 27.33 & 26.71 & 26.33 & 29.01 & 0.056 & 10031 & 1.01 & 1443.97 & 57.23 \\
\hline 0 & 56.55 & 49.98 & 34.00 & 0.12 & -2.29 & 58.23 & 31.13 & 27.89 & 30.34 & 25.42 & 28.66 & 0.056 & 11038 & 1.19 & 1590.65 & 74.08 \\
\hline 0 & 56.78 & 60.05 & 40.85 & 0.14 & -2.75 & 60.43 & 32.24 & 28.42 & 32.01 & 24.55 & 28.37 & 0.055 & 12571 & 1.30 & 1813.67 & 92.19 \\
\hline $\mathbf{0}$ & 57.03 & 69.99 & 47.61 & 0.17 & -3.21 & 62.19 & 33.47 & 29.07 & 33.12 & 23.56 & 27.96 & 0.055 & 14161 & 1.41 & 2045.91 & 111.94 \\
\hline 0 & 57.20 & 75.07 & 51.07 & 0.18 & -3.44 & 63.15 & 34.15 & 29.47 & 33.69 & 23.05 & 27.73 & 0.055 & 14932 & 1.46 & 2159.13 & 122.71 \\
\hline
\end{tabular}


Table D68: Reduced Data for Full Length Electrode 3 kV Run

\begin{tabular}{|c|c|c|c|c|c|c|c|c|c|c|c|c|c|c|c|c|}
\hline HV & $\mathbf{T}_{\text {sat }}$ & $\mathbf{Q}$ & $\mathbf{q}$ & $\Delta \mathbf{T}_{\text {sub }}$ & $\Delta \mathbf{T}_{\text {htr }}$ & $\mathbf{T}_{\mathrm{s}}$ & $\mathbf{T}_{\infty \text { wall }}$ & $\mathbf{T}_{\text {otop }}$ & $\begin{array}{c}\mathbf{T}_{\mathbf{s}^{-}} \\
\mathbf{T}_{\text {otop }}\end{array}$ & $\begin{array}{c}\mathbf{T}_{\text {sat }^{-}} \\
\mathbf{T}_{\text {cowall }}\end{array}$ & $\begin{array}{c}\mathbf{T}_{\text {sat }}{ }^{-} \\
\mathbf{T}_{\text {otop }}\end{array}$ & $\mathbf{k}_{\text {fluid }}$ & h & $\theta_{\mathrm{s}}-\theta_{\text {ootop }}$ & Nu & GD \\
\hline & ${ }^{\circ} \mathrm{C}$ & $\mathbf{W}$ & $10^{4} \mathrm{~W} / \mathrm{m}^{2}$ & ${ }^{\circ} \mathrm{C}$ & ${ }^{\circ} \mathrm{C}$ & ${ }^{\circ} \mathrm{C}$ & ${ }^{\circ} \mathrm{C}$ & ${ }^{\circ} \mathrm{C}$ & ${ }^{\circ} \mathrm{C}$ & ${ }^{\circ} \mathrm{C}$ & ${ }^{\circ} \mathrm{C}$ & $\begin{array}{c}\mathbf{W} / \\
\mathbf{m}-\mathbf{K}\end{array}$ & $\begin{array}{c}\mathbf{W} / \\
\mathbf{m}^{2}-K\end{array}$ & --- & --- & --- \\
\hline 3 & 55.75 & 0.00 & 0.00 & 0.00 & 0.00 & 26.75 & 26.35 & 25.72 & 1.03 & 29.41 & 30.04 & 0.056 & 0 & 0.03 & 0.00 & 0.00 \\
\hline 3 & 55.80 & 10.03 & 6.82 & 0.02 & -0.46 & 33.95 & 27.14 & 26.05 & 7.91 & 28.66 & 29.75 & 0.056 & 8502 & 0.28 & 1220.49 & 13.19 \\
\hline 3 & 55.96 & 19.98 & 13.60 & 0.05 & -0.92 & 41.14 & 28.09 & 26.49 & 14.65 & 27.87 & 29.48 & 0.056 & 9139 & 0.53 & 1313.24 & 27.02 \\
\hline 3 & 56.17 & 30.05 & 20.44 & 0.07 & -1.38 & 48.07 & 29.05 & 26.89 & 21.18 & 27.12 & 29.28 & 0.056 & 9507 & 0.78 & 1367.22 & 41.75 \\
\hline 3 & 56.38 & 39.98 & 27.20 & 0.10 & -1.83 & 54.15 & 30.15 & 27.44 & 26.72 & 26.23 & 28.95 & 0.056 & 10027 & 1.02 & 1443.71 & 57.44 \\
\hline 3 & 56.59 & 49.80 & 33.88 & 0.12 & -2.28 & 58.13 & 31.18 & 27.90 & 30.23 & 25.41 & 28.69 & 0.056 & 11038 & 1.19 & 1590.76 & 73.84 \\
\hline 3 & 56.82 & 60.07 & 40.86 & 0.14 & -2.75 & 60.52 & 32.37 & 28.51 & 32.01 & 24.45 & 28.32 & 0.055 & 12573 & 1.31 & 1814.34 & 92.58 \\
\hline 3 & 57.08 & 70.06 & 47.66 & 0.17 & -3.21 & 62.21 & 33.55 & 29.11 & 33.09 & 23.53 & 27.96 & 0.055 & 14186 & 1.41 & 2049.73 & 112.21 \\
\hline 3 & 57.22 & 75.20 & 51.15 & 0.18 & -3.45 & 63.21 & 34.20 & 29.49 & 33.72 & 23.02 & 27.73 & 0.055 & 14942 & 1.46 & 2160.62 & 123.09 \\
\hline
\end{tabular}

Table D69: Reduced Data for Full Length Electrode 6 kV Run

\begin{tabular}{|c|c|c|c|c|c|c|c|c|c|c|c|c|c|c|c|c|}
\hline HV & $\mathbf{T}_{\text {sat }}$ & $\mathbf{Q}$ & $\mathbf{q}$ & $\Delta \mathbf{T}_{\text {sub }}$ & $\Delta \mathbf{T}_{\mathrm{htr}}$ & $\mathbf{T}_{\mathrm{s}}$ & $\mathbf{T}_{\text {owall }}$ & $\mathbf{T}_{\text {cotop }}$ & $\begin{array}{c}\mathbf{T}_{\mathrm{s}^{-}} \\
\mathbf{T}_{\text {otop }}\end{array}$ & $\begin{array}{c}\mathbf{T}_{\text {sat }^{-}} \\
\mathbf{T}_{\text {cwall }}\end{array}$ & $\begin{array}{c}\mathbf{T}_{\text {sat }^{-}} \\
\mathbf{T}_{\text {otop }}\end{array}$ & $\mathbf{k}_{\text {fluid }}$ & h & $\theta_{\mathrm{s}}-\theta_{\text {otop }}$ & $\mathbf{N u}$ & G $\Delta$ \\
\hline & ${ }^{\circ} \mathrm{C}$ & $\mathbf{W}$ & $10^{4} \mathrm{~W} / \mathrm{m}^{2}$ & ${ }^{\circ} \mathrm{C}$ & ${ }^{\circ} \mathrm{C}$ & ${ }^{\circ} \mathrm{C}$ & ${ }^{\circ} \mathrm{C}$ & ${ }^{\circ} \mathrm{C}$ & ${ }^{\circ} \mathrm{C}$ & ${ }^{\circ} \mathrm{C}$ & ${ }^{\circ} \mathrm{C}$ & $\begin{array}{c}\mathbf{W} / \\
\mathbf{m}-\mathbf{K}\end{array}$ & $\begin{array}{c}\mathbf{W} / \\
\mathbf{m}^{2}-K\end{array}$ & --- & --- & --- \\
\hline 6 & 55.69 & 0.00 & 0.00 & 0.00 & 0.00 & 26.72 & 26.30 & 25.68 & 1.04 & 29.39 & 30.01 & 0.056 & 0 & 0.04 & 0.00 & 0.00 \\
\hline 6 & 55.80 & 10.03 & 6.82 & 0.02 & -0.46 & 33.95 & 27.15 & 26.06 & 7.89 & 28.65 & 29.74 & 0.056 & 8524 & 0.28 & 1223.71 & 13.20 \\
\hline 6 & 55.98 & 19.99 & 13.60 & 0.05 & -0.92 & 41.17 & 28.10 & 26.48 & 14.69 & 27.89 & 29.51 & 0.056 & 9116 & 0.53 & 1309.81 & 27.01 \\
\hline 6 & 56.19 & 30.05 & 20.44 & 0.07 & -1.38 & 48.12 & 29.14 & 26.97 & 21.15 & 27.05 & 29.22 & 0.056 & 9522 & 0.78 & 1369.64 & 41.87 \\
\hline 6 & 56.40 & 39.98 & 27.20 & 0.10 & -1.83 & 54.13 & 30.17 & 27.44 & 26.69 & 26.24 & 28.96 & 0.056 & 10040 & 1.02 & 1445.49 & 57.43 \\
\hline 6 & 56.64 & 49.78 & 33.87 & 0.12 & -2.28 & 58.19 & 31.27 & 27.98 & 30.21 & 25.37 & 28.65 & 0.056 & 11043 & 1.19 & 1591.71 & 73.96 \\
\hline 6 & 56.87 & 60.05 & 40.85 & 0.14 & -2.75 & 60.39 & 32.46 & 28.58 & 31.81 & 24.41 & 28.29 & 0.055 & 12648 & 1.30 & 1825.47 & 92.71 \\
\hline 6 & 57.12 & 70.10 & 47.69 & 0.17 & -3.21 & 62.27 & 33.62 & 29.19 & 33.08 & 23.50 & 27.93 & 0.055 & 14198 & 1.41 & 2051.71 & 112.42 \\
\hline 6 & 57.24 & 75.16 & 51.13 & 0.18 & -3.45 & 63.25 & 34.28 & 29.57 & 33.69 & 22.96 & 27.68 & 0.055 & 14950 & 1.47 & 2162.12 & 123.34 \\
\hline
\end{tabular}


Table D70: Reduced Data for Top Half Electrode 0 kV Runs

\begin{tabular}{|c|c|c|c|c|c|c|c|c|c|c|c|c|c|c|c|c|}
\hline HV & $\mathbf{T}_{\text {sat }}$ & $\mathbf{Q}$ & $\mathbf{q}$ & $\Delta T_{\text {sub }}$ & $\Delta \mathbf{T}_{\mathrm{htr}}$ & $\mathbf{T}_{\mathrm{s}}$ & $\mathbf{T}_{\text {owall }}$ & $\mathbf{T}_{\infty \mathrm{top}}$ & $\begin{array}{c}\mathbf{T}_{\mathbf{s}^{-}} \\
\mathbf{T}_{\text {otop }}\end{array}$ & $\begin{array}{c}\mathbf{T}_{\text {sat }^{-}} \\
\mathbf{T}_{\text {cowall }}\end{array}$ & $\begin{array}{c}\mathbf{T}_{\text {sat }} \\
\mathbf{T}_{\text {cotop }}\end{array}$ & $\mathbf{k}_{\text {fluid }}$ & h & $\theta_{\mathrm{s}}-\theta_{\infty \mathrm{top}}$ & $\mathrm{Nu}$ & GD \\
\hline & ${ }^{\circ} \mathrm{C}$ & $\mathbf{W}$ & $10^{4} \mathrm{~W} / \mathrm{m}^{2}$ & ${ }^{\circ} \mathrm{C}$ & ${ }^{\circ} \mathrm{C}$ & ${ }^{\circ} \mathrm{C}$ & ${ }^{\circ} \mathrm{C}$ & ${ }^{\circ} \mathrm{C}$ & ${ }^{\circ} \mathrm{C}$ & ${ }^{\circ} \mathrm{C}$ & ${ }^{\circ} \mathrm{C}$ & $\begin{array}{c}\mathbf{W} / \\
\mathbf{m}-\mathbf{K}\end{array}$ & $\begin{array}{c}\mathbf{W} / \\
\mathbf{m}^{2}-K\end{array}$ & --- & --- & --- \\
\hline $\mathbf{0}$ & 54.75 & 0.00 & 0.00 & 0.00 & 0.00 & 22.45 & 22.13 & 21.62 & 0.83 & 32.61 & 33.12 & 0.056 & 0 & 0.03 & 0.00 & 0.00 \\
\hline $\mathbf{0}$ & 54.83 & 10.07 & 6.85 & 0.02 & -0.46 & 29.98 & 23.09 & 22.09 & 7.89 & 31.74 & 32.74 & 0.056 & 8557 & 0.25 & 1218.34 & 11.96 \\
\hline 0 & 54.96 & 20.09 & 13.67 & 0.05 & -0.92 & 37.29 & 24.02 & 22.52 & 14.77 & 30.93 & 32.44 & 0.056 & 9112 & 0.48 & 1298.53 & 24.47 \\
\hline 0 & 55.12 & 30.11 & 20.48 & 0.07 & -1.38 & 44.58 & 25.13 & 23.03 & 21.55 & 29.99 & 32.09 & 0.056 & 9363 & 0.72 & 1335.70 & 37.83 \\
\hline $\mathbf{0}$ & 55.31 & 40.10 & 27.28 & 0.10 & -1.84 & 50.87 & 26.24 & 23.56 & 27.31 & 29.07 & 31.75 & 0.056 & 9837 & 0.94 & 1404.88 & 51.97 \\
\hline 0 & 55.52 & 50.01 & 34.02 & 0.12 & -2.29 & 55.70 & 27.38 & 24.10 & 31.60 & 28.14 & 31.42 & 0.056 & 10606 & 1.12 & 1516.44 & 66.97 \\
\hline $\mathbf{0}$ & 55.75 & 60.09 & 40.88 & 0.14 & -2.76 & 58.39 & 28.56 & 24.69 & 33.70 & 27.19 & 31.06 & 0.056 & 11948 & 1.24 & 1710.43 & 83.28 \\
\hline $\mathbf{0}$ & 55.98 & 70.01 & 47.62 & 0.17 & -3.21 & 60.31 & 29.73 & 25.28 & 35.03 & 26.26 & 30.70 & 0.056 & 13392 & 1.33 & 1919.40 & 100.48 \\
\hline $\mathbf{0}$ & 55.31 & 0.00 & 0.00 & 0.00 & 0.00 & 23.57 & 23.20 & 22.49 & 1.08 & 32.11 & 32.82 & 0.056 & 0 & 0.03 & 0.00 & 0.00 \\
\hline 0 & 55.40 & 10.03 & 6.82 & 0.02 & -0.46 & 31.00 & 24.13 & 23.03 & 7.98 & 31.27 & 32.37 & 0.056 & 8427 & 0.26 & 1202.10 & 12.09 \\
\hline $\mathbf{0}$ & 55.61 & 19.99 & 13.60 & 0.05 & -0.92 & 38.24 & 25.14 & 23.56 & 14.68 & 30.46 & 32.05 & 0.056 & 9126 & 0.48 & 1303.31 & 24.73 \\
\hline $\mathbf{0}$ & 55.88 & 30.06 & 20.45 & 0.07 & -1.38 & 45.22 & 26.21 & 24.09 & 21.13 & 29.67 & 31.79 & 0.056 & 9534 & 0.71 & 1363.06 & 38.18 \\
\hline $\mathbf{0}$ & 56.15 & 40.01 & 27.21 & 0.10 & -1.83 & 51.30 & 27.35 & 24.66 & 26.64 & 28.80 & 31.49 & 0.056 & 10064 & 0.92 & 1440.58 & 52.35 \\
\hline $\mathbf{0}$ & 56.43 & 50.00 & 34.01 & 0.12 & -2.29 & 56.45 & 28.59 & 25.30 & 31.14 & 27.83 & 31.12 & 0.056 & 10757 & 1.12 & 1541.87 & 67.69 \\
\hline $\mathbf{0}$ & 56.70 & 60.07 & 40.87 & 0.14 & -2.76 & 59.25 & 29.72 & 25.88 & 33.37 & 26.98 & 30.81 & 0.056 & 12064 & 1.24 & 1731.32 & 83.91 \\
\hline $\mathbf{0}$ & 56.97 & 70.03 & 47.64 & 0.17 & -3.21 & 61.24 & 30.93 & 26.53 & 34.71 & 26.04 & 30.44 & 0.056 & 13520 & 1.33 & 1942.92 & 101.33 \\
\hline $\mathbf{0}$ & 57.14 & 75.00 & 51.02 & 0.18 & -3.44 & 62.19 & 31.55 & 26.88 & 35.31 & 25.59 & 30.26 & 0.056 & 14233 & 1.38 & 2046.80 & 110.45 \\
\hline
\end{tabular}


Table D71: Reduced Data for Top Half Electrode $3 \mathrm{kV}$ Run

\begin{tabular}{|c|c|c|c|c|c|c|c|c|c|c|c|c|c|c|c|c|}
\hline HV & $\mathbf{T}_{\text {sat }}$ & $\mathbf{Q}$ & $\mathbf{q}$ & $\Delta \mathbf{T}_{\text {sub }}$ & $\Delta \mathbf{T}_{\mathrm{htr}}$ & $\mathbf{T}_{\mathrm{s}}$ & $\mathbf{T}_{\text {cwall }}$ & $\mathbf{T}_{\text {otop }}$ & $\begin{array}{c}\mathbf{T}_{\mathrm{s}^{-}} \\
\mathbf{T}_{\text {otop }}\end{array}$ & $\begin{array}{c}\mathbf{T}_{\text {sat- }} \\
\mathbf{T}_{\text {owall }}\end{array}$ & $\begin{array}{c}\mathbf{T}_{\text {sat- }} \\
\mathbf{T}_{\text {otop }}\end{array}$ & $\mathbf{k}_{\text {fluid }}$ & h & $\theta_{\mathrm{s}}-\theta_{\infty \mathrm{top}}$ & $\mathbf{N u}$ & GD \\
\hline & ${ }^{\circ} \mathrm{C}$ & $\mathbf{W}$ & $10^{4} \mathrm{~W} / \mathrm{m}^{2}$ & ${ }^{\circ} \mathrm{C}$ & ${ }^{\circ} \mathrm{C}$ & ${ }^{\circ} \mathrm{C}$ & ${ }^{\circ} \mathrm{C}$ & ${ }^{\circ} \mathrm{C}$ & ${ }^{\circ} \mathrm{C}$ & ${ }^{\circ} \mathrm{C}$ & ${ }^{\circ} \mathrm{C}$ & $\begin{array}{c}\text { W/ } \\
\text { m-K }\end{array}$ & $\begin{array}{c}\mathbf{W} / \\
\mathbf{m}^{2}-\mathbf{K}\end{array}$ & -- & -- & --- \\
\hline 3 & 55.29 & 0.00 & 0.00 & 0.00 & 0.00 & 23.60 & 23.22 & 22.53 & 1.07 & 32.08 & 32.76 & 0.056 & 0 & 0.03 & 0.00 & 0.00 \\
\hline 3 & 55.42 & 10.04 & 6.83 & 0.02 & -0.46 & 31.14 & 24.23 & 23.10 & 8.03 & 31.19 & 32.31 & 0.056 & 8372 & 0.26 & 1194.50 & 12.13 \\
\hline 3 & 55.67 & 20.00 & 13.61 & 0.05 & -0.92 & 38.37 & 25.23 & 23.62 & 14.75 & 30.44 & 32.05 & 0.056 & 9088 & 0.48 & 1298.01 & 24.76 \\
\hline 3 & 55.94 & 30.07 & 20.46 & 0.07 & -1.38 & 45.34 & 26.31 & 24.15 & 21.19 & 29.63 & 31.80 & 0.056 & 9508 & 0.72 & 1359.58 & 38.24 \\
\hline 3 & 56.24 & 40.01 & 27.22 & 0.10 & -1.83 & 51.46 & 27.46 & 24.73 & 26.73 & 28.78 & 31.50 & 0.056 & 10030 & 0.93 & 1435.87 & 52.39 \\
\hline 3 & 56.49 & 50.01 & 34.02 & 0.12 & -2.29 & 56.46 & 28.62 & 25.31 & 31.14 & 27.87 & 31.17 & 0.056 & 10761 & 1.12 & 1542.51 & 67.64 \\
\hline 3 & 56.76 & 60.09 & 40.88 & 0.14 & -2.76 & 59.32 & 29.83 & 25.96 & 33.36 & 26.93 & 30.81 & 0.056 & 12069 & 1.24 & 1732.34 & 84.10 \\
\hline 3 & 57.01 & 70.04 & 47.65 & 0.17 & -3.21 & 61.30 & 31.04 & 26.62 & 34.68 & 25.97 & 30.39 & 0.056 & 13532 & 1.34 & 1945.04 & 101.62 \\
\hline 3 & 57.16 & 75.02 & 51.04 & 0.18 & -3.44 & 62.29 & 31.65 & 26.96 & 35.33 & 25.51 & 30.20 & 0.056 & 14231 & 1.38 & 2046.87 & 110.83 \\
\hline
\end{tabular}

Table D72: Reduced Data for Top Half Electrode 6 kV Run

\begin{tabular}{|c|c|c|c|c|c|c|c|c|c|c|c|c|c|c|c|c|}
\hline HV & $\mathbf{T}_{\text {sat }}$ & $\mathbf{Q}$ & $\mathbf{q}$ & $\Delta \mathbf{T}_{\text {sub }}$ & $\Delta \mathbf{T}_{\mathrm{htr}}$ & $\mathbf{T}_{\mathrm{s}}$ & $\mathbf{T}_{\text {owall }}$ & $\mathbf{T}_{\text {otop }}$ & $\begin{array}{c}\mathbf{T}_{\mathbf{s}^{-}} \\
\mathbf{T}_{\text {otop }}\end{array}$ & $\begin{array}{c}\mathbf{T}_{\text {sat- }} \\
\mathbf{T}_{\text {owall }}\end{array}$ & $\begin{array}{c}\mathbf{T}_{\text {sat }^{-}} \\
\mathbf{T}_{\text {otop }}\end{array}$ & $\mathbf{k}_{\text {fluid }}$ & h & $\theta_{\mathrm{s}}-\theta_{\infty \mathrm{top}}$ & $\mathbf{N u}$ & GD \\
\hline & ${ }^{\circ} \mathrm{C}$ & $\mathbf{W}$ & $10^{4} \mathrm{~W} / \mathrm{m}^{2}$ & ${ }^{\circ} \mathrm{C}$ & ${ }^{\circ} \mathrm{C}$ & ${ }^{\circ} \mathrm{C}$ & ${ }^{\circ} \mathrm{C}$ & ${ }^{\circ} \mathrm{C}$ & ${ }^{\circ} \mathrm{C}$ & ${ }^{\circ} \mathrm{C}$ & ${ }^{\circ} \mathrm{C}$ & $\begin{array}{c}\mathbf{W} / \\
\mathbf{m}-\mathbf{K}\end{array}$ & $\begin{array}{c}\mathbf{W} / \\
\mathbf{m}^{2}-\mathbf{K}\end{array}$ & --- & --- & --- \\
\hline 6 & 55.29 & 0.00 & 0.00 & 0.00 & 0.00 & 23.67 & 23.26 & 22.59 & 1.07 & 32.03 & 32.70 & 0.056 & 0 & 0.03 & 0.00 & 0.00 \\
\hline 6 & 55.44 & 10.04 & 6.83 & 0.02 & -0.46 & 31.17 & 24.25 & 23.14 & 8.03 & 31.19 & 32.30 & 0.056 & 8379 & 0.26 & 1195.59 & 12.13 \\
\hline 6 & 55.71 & 20.01 & 13.61 & 0.05 & -0.92 & 38.40 & 25.30 & 23.68 & 14.72 & 30.41 & 32.03 & 0.056 & 9107 & 0.48 & 1300.87 & 24.79 \\
\hline 6 & 55.98 & 30.08 & 20.46 & 0.07 & -1.38 & 45.35 & 26.38 & 24.20 & 21.15 & 29.61 & 31.79 & 0.056 & 9527 & 0.71 & 1362.39 & 38.28 \\
\hline 6 & 56.28 & 40.02 & 27.22 & 0.10 & -1.84 & 51.56 & 27.56 & 24.81 & 26.75 & 28.72 & 31.47 & 0.056 & 10023 & 0.93 & 1435.22 & 52.52 \\
\hline 6 & 56.55 & 50.06 & 34.05 & 0.12 & -2.30 & 56.52 & 28.73 & 25.40 & 31.13 & 27.82 & 31.15 & 0.056 & 10776 & 1.12 & 1544.91 & 67.81 \\
\hline 6 & 56.82 & 60.12 & 40.90 & 0.14 & -2.76 & 59.37 & 29.93 & 26.03 & 33.34 & 26.89 & 30.79 & 0.056 & 12084 & 1.24 & 1734.64 & 84.24 \\
\hline 6 & 57.06 & 70.06 & 47.66 & 0.17 & -3.21 & 61.34 & 31.10 & 26.67 & 34.67 & 25.95 & 30.38 & 0.056 & 13541 & 1.34 & 1946.52 & 101.73 \\
\hline 6 & 57.14 & 74.99 & 51.01 & 0.18 & -3.44 & 62.40 & 31.87 & 27.14 & 35.25 & 25.27 & 30.00 & 0.056 & 14254 & 1.40 & 2050.95 & 111.83 \\
\hline
\end{tabular}


Table D73: Reduced Data for Full Length Electrode 0 kV Runs with HFE-7000

\begin{tabular}{|c|c|c|c|c|c|c|c|c|c|c|c|c|c|c|c|c|}
\hline HV & $\mathbf{T}_{\text {sat }}$ & $\mathbf{Q}$ & $\mathbf{q}$ & $\Delta T_{\text {sub }}$ & $\Delta \mathbf{T}_{\mathrm{htr}}$ & $\mathbf{T}_{\mathrm{s}}$ & $\mathbf{T}_{\text {owall }}$ & $\mathbf{T}_{\text {ootop }}$ & $\begin{array}{c}\mathbf{T}_{\mathrm{s}^{-}} \\
\mathbf{T}_{\text {otop }} \\
\end{array}$ & $\begin{array}{c}\mathbf{T}_{\text {sat }}{ }^{-} \\
\mathbf{T}_{\text {owall }}\end{array}$ & $\begin{array}{c}\mathbf{T}_{\text {sat }} \\
\mathbf{T}_{\text {otop }} \\
\end{array}$ & $\mathbf{k}_{\text {fluid }}$ & h & $\theta_{\mathrm{s}}-\theta_{\infty \mathrm{top}}$ & $\mathbf{N u}$ & GD \\
\hline & ${ }^{\circ} \mathrm{C}$ & $\mathbf{W}$ & $10^{4} \mathrm{~W} / \mathrm{m}^{2}$ & ${ }^{\circ} \mathrm{C}$ & ${ }^{\circ} \mathrm{C}$ & ${ }^{\circ} \mathrm{C}$ & ${ }^{\circ} \mathrm{C}$ & ${ }^{\circ} \mathrm{C}$ & ${ }^{\circ} \mathrm{C}$ & ${ }^{\circ} \mathrm{C}$ & ${ }^{\circ} \mathrm{C}$ & $\begin{array}{c}\mathbf{W} / \\
\mathbf{m}-\mathbf{K}\end{array}$ & $\begin{array}{c}\mathbf{W} / \\
\mathbf{m}^{2}-\mathrm{K}\end{array}$ & --- & --- & --- \\
\hline $\mathbf{0}$ & 39.5 & 0.00 & 0.00 & 0.00 & 0.00 & 23.49 & 23.32 & 23.37 & 0.11 & 16.23 & 16.17 & 0.075 & 0 & 0.01 & 0.00 & 0.00 \\
\hline $\mathbf{0}$ & 39.5 & 10.01 & 6.81 & 0.02 & -0.46 & 29.77 & 24.15 & 23.84 & 5.93 & 15.40 & 15.71 & 0.075 & 11311 & 0.39 & 1206.56 & 24.51 \\
\hline $\mathbf{0}$ & 39.5 & 19.96 & 13.58 & 0.05 & -0.92 & 36.40 & 25.02 & 24.34 & 12.06 & 14.52 & 15.21 & 0.075 & 11093 & 0.83 & 1183.24 & 51.79 \\
\hline 0 & 39.5 & 29.93 & 20.36 & 0.07 & -1.37 & 42.48 & 25.87 & 24.81 & 17.67 & 13.68 & 14.73 & 0.075 & 11348 & 1.29 & 1210.50 & 82.42 \\
\hline $\mathbf{0}$ & 39.5 & 39.87 & 27.12 & 0.10 & -1.83 & 45.83 & 26.73 & 25.26 & 20.57 & 12.82 & 14.29 & 0.075 & 12990 & 1.60 & 1385.58 & 117.19 \\
\hline $\mathbf{0}$ & 39.5 & 44.58 & 30.32 & 0.11 & -2.04 & 46.80 & 27.28 & 25.55 & 21.25 & 12.27 & 14.00 & 0.075 & 14054 & 1.73 & 1499.09 & 136.95 \\
\hline $\mathbf{0}$ & 39.5 & 0.00 & 0.00 & 0.00 & 0.00 & 24.26 & 24.05 & 23.90 & 0.36 & 15.49 & 15.64 & 0.075 & 0 & 0.02 & 0.00 & 0.00 \\
\hline $\mathbf{0}$ & 39.5 & 9.91 & 6.74 & 0.02 & -0.45 & 30.59 & 24.56 & 24.13 & 6.46 & 14.99 & 15.42 & 0.075 & 10276 & 0.43 & 1096.08 & 24.91 \\
\hline $\mathbf{0}$ & 39.5 & 19.88 & 13.53 & 0.05 & -0.91 & 37.10 & 25.24 & 24.47 & 12.64 & 14.30 & 15.08 & 0.075 & 10543 & 0.88 & 1124.60 & 52.38 \\
\hline 0 & 39.5 & 29.84 & 20.30 & 0.07 & -1.37 & 42.88 & 26.08 & 24.92 & 17.96 & 13.46 & 14.63 & 0.075 & 11130 & 1.33 & 1187.24 & 83.51 \\
\hline $\mathbf{0}$ & 39.5 & 39.71 & 27.01 & 0.10 & -1.82 & 45.79 & 27.04 & 25.46 & 20.34 & 12.51 & 14.09 & 0.075 & 13084 & 1.63 & 1395.66 & 119.63 \\
\hline $\mathbf{0}$ & 39.5 & 44.78 & 30.46 & 0.11 & -2.05 & 46.55 & 27.65 & 25.83 & 20.72 & 11.90 & 13.72 & 0.075 & 14480 & 1.74 & 1544.56 & 141.82 \\
\hline
\end{tabular}

Table D74: Reduced Data for Full Length Electrode 3 kV Run with HFE-7000

\begin{tabular}{|c|c|c|c|c|c|c|c|c|c|c|c|c|c|c|c|c|}
\hline HV & $\mathbf{T}_{\text {sat }}$ & $\mathbf{Q}$ & $\mathbf{q}$ & $\Delta \mathbf{T}_{\text {sub }}$ & $\Delta \mathbf{T}_{\mathrm{htr}}$ & $\mathbf{T}_{\mathrm{s}}$ & $\mathbf{T}_{\text {cwall }}$ & $\mathbf{T}_{\text {cotop }}$ & $\begin{array}{c}\mathbf{T}_{\mathbf{s}^{-}} \\
\mathbf{T}_{\text {cotop }} \\
\end{array}$ & $\begin{array}{c}\mathbf{T}_{\text {sat }^{-}} \\
\mathbf{T}_{\text {owall }} \\
\end{array}$ & $\begin{array}{c}\mathbf{T}_{\text {sat }}{ }^{-} \\
\mathbf{T}_{\text {otop }}\end{array}$ & $\mathbf{k}_{\text {fluid }}$ & h & $\theta_{\mathrm{s}}-\theta_{\infty \mathrm{otop}}$ & $\mathrm{Nu}$ & GD \\
\hline & ${ }^{\circ} \mathrm{C}$ & $\mathbf{W}$ & $10^{4} \mathrm{~W} / \mathrm{m}^{2}$ & ${ }^{\circ} \mathrm{C}$ & ${ }^{\circ} \mathrm{C}$ & ${ }^{\circ} \mathrm{C}$ & ${ }^{\circ} \mathrm{C}$ & ${ }^{\circ} \mathrm{C}$ & ${ }^{\circ} \mathrm{C}$ & ${ }^{\circ} \mathrm{C}$ & ${ }^{\circ} \mathrm{C}$ & $\begin{array}{c}\mathbf{W} / \\
\mathbf{m}-\mathbf{K}\end{array}$ & $\begin{array}{c}\mathbf{W} / \\
\mathbf{m}^{2}-K\end{array}$ & --- & --- & --- \\
\hline 3 & 39.5 & 0.00 & 0.00 & 0.00 & 0.00 & 24.17 & 23.97 & 23.84 & 0.32 & 15.58 & 15.70 & 0.075 & 0 & 0.02 & 0.00 & 0.00 \\
\hline 3 & 39.5 & 9.91 & 6.74 & 0.02 & -0.45 & 30.65 & 24.63 & 24.17 & 6.48 & 14.92 & 15.38 & 0.075 & 10252 & 0.43 & 1093.56 & 25.03 \\
\hline 3 & 39.5 & 19.88 & 13.53 & 0.05 & -0.91 & 37.16 & 25.32 & 24.49 & 12.66 & 14.23 & 15.06 & 0.075 & 10522 & 0.89 & 1122.30 & 52.65 \\
\hline 3 & 39.5 & 29.84 & 20.30 & 0.07 & -1.37 & 42.90 & 26.23 & 25.01 & 17.89 & 13.32 & 14.54 & 0.075 & 11176 & 1.34 & 1192.09 & 84.42 \\
\hline 3 & 39.5 & 39.71 & 27.01 & 0.10 & -1.82 & 45.70 & 27.17 & 25.53 & 20.17 & 12.38 & 14.02 & 0.075 & 13190 & 1.63 & 1406.93 & 120.88 \\
\hline 3 & 39.5 & 44.78 & 30.46 & 0.11 & -2.05 & 46.56 & 27.79 & 25.90 & 20.65 & 11.76 & 13.64 & 0.075 & 14529 & 1.76 & 1549.73 & 143.49 \\
\hline
\end{tabular}


Table D75: Reduced Data for Full Length Electrode 6 kV Run with HFE-7000

\begin{tabular}{|c|c|c|c|c|c|c|c|c|c|c|c|c|c|c|c|c|}
\hline HV & $\mathbf{T}_{\text {sat }}$ & $\mathbf{Q}$ & $\mathbf{q}$ & $\Delta \mathbf{T}_{\text {sub }}$ & $\Delta \mathbf{T}_{\mathrm{htr}}$ & $\mathbf{T}_{\mathrm{s}}$ & $\mathbf{T}_{\text {owall }}$ & $\mathbf{T}_{\text {otop }}$ & $\begin{array}{c}\mathbf{T}_{\mathrm{s}^{-}} \\
\mathbf{T}_{\text {otop }} \\
\end{array}$ & $\begin{array}{c}\mathbf{T}_{\text {sat }^{-}} \\
\mathbf{T}_{\text {cowall }}\end{array}$ & $\begin{array}{c}\mathbf{T}_{\text {sat }^{-}} \\
\mathbf{T}_{\text {otop }}\end{array}$ & $\mathbf{k}_{\text {fluid }}$ & $\mathbf{h}$ & $\theta_{\mathrm{s}}-\theta_{\infty \mathrm{top}}$ & $\mathbf{N u}$ & G $\Delta$ \\
\hline & ${ }^{\circ} \mathrm{C}$ & $\mathbf{W}$ & $10^{4} \mathrm{~W} / \mathrm{m}^{2}$ & ${ }^{\circ} \mathrm{C}$ & ${ }^{\circ} \mathrm{C}$ & ${ }^{\circ} \mathrm{C}$ & ${ }^{\circ} \mathrm{C}$ & ${ }^{\circ} \mathrm{C}$ & ${ }^{\circ} \mathrm{C}$ & ${ }^{\circ} \mathrm{C}$ & ${ }^{\circ} \mathrm{C}$ & $\begin{array}{c}\mathbf{W} / \\
\mathbf{m}-\mathbf{K}\end{array}$ & $\begin{array}{c}\mathbf{W} / \\
\mathbf{m}^{2}-K\end{array}$ & --- & -- & --- \\
\hline 6 & 39.5 & 0.00 & 0.00 & 0.00 & 0.00 & 24.09 & 24.00 & 23.89 & 0.21 & 15.55 & 15.66 & 0.075 & 0 & 0.01 & 0.00 & 0.00 \\
\hline 6 & 39.5 & 9.91 & 6.74 & 0.02 & -0.45 & 30.51 & 24.68 & 24.23 & 6.28 & 14.86 & 15.32 & 0.075 & 10572 & 0.42 & 1127.67 & 25.12 \\
\hline 6 & 39.5 & 19.88 & 13.53 & 0.05 & -0.91 & 36.95 & 25.47 & 24.67 & 12.28 & 14.07 & 14.88 & 0.075 & 10852 & 0.87 & 1157.56 & 53.24 \\
\hline 6 & 39.5 & 29.84 & 20.30 & 0.07 & -1.37 & 42.74 & 26.40 & 25.22 & 17.53 & 13.15 & 14.33 & 0.075 & 11409 & 1.33 & 1216.96 & 85.51 \\
\hline 6 & 39.5 & 39.71 & 27.01 & 0.10 & -1.82 & 45.55 & 27.35 & 25.78 & 19.76 & 12.20 & 13.76 & 0.075 & 13464 & 1.62 & 1436.21 & 122.65 \\
\hline 6 & 39.5 & 44.78 & 30.46 & 0.11 & -2.05 & 46.38 & 27.95 & 26.17 & 20.21 & 11.60 & 13.38 & 0.075 & 14849 & 1.74 & 1583.87 & 145.49 \\
\hline
\end{tabular}




\section{Mehra Electrode Averaged Raw Data and Data Reduction Tables}

The raw data for the Mehra electrode study consisted of values read by the data acquisition system each fraction of a second and is therefore left out of this appendix. The single numbers that were used in data reduction for each test condition are shown in Table D76 through Table D89 and were found by averaging a thirty second steady state portion of the raw data. Table D90 through Table D102 show the data reduction values for the electrode study at each high voltage level. 
Table D76: Averaged Raw Data for Mehra Electrode at $0 \mathrm{~W}$

\begin{tabular}{|c|c|c|c|c|c|c|c|c|c|c|c|c|c|c|c|c|c|}
\hline HV & Q & $\begin{array}{c}\text { Heater } \\
\text { Voltage } \\
\text { after } \\
\text { precision } \\
\text { resistor }\end{array}$ & $\begin{array}{c}\text { Heater } \\
\text { Voltage } \\
\text { before } \\
\text { precision } \\
\text { resistor }\end{array}$ & $\begin{array}{c}\text { Heater } \\
\text { Current }\end{array}$ & $\begin{array}{l}\text { Chamber } \\
\text { Pressure }\end{array}$ & Amb & T1 & T2 & T3 & T4 & T5 & T8 & T9 & T10 & T11 & T12 & T14 \\
\hline $\mathbf{k V}$ & W & Volts & Volts & Amps & psia & ${ }^{\circ} \mathrm{C}$ & ${ }^{\circ} \mathrm{C}$ & ${ }^{\circ} \mathrm{C}$ & ${ }^{\circ} \mathrm{C}$ & ${ }^{\circ} \mathrm{C}$ & ${ }^{\circ} \mathrm{C}$ & ${ }^{\circ} \mathbf{C}$ & ${ }^{\circ} \mathrm{C}$ & ${ }^{\circ} \mathrm{C}$ & ${ }^{\circ} \mathrm{C}$ & ${ }^{\circ} \mathbf{C}$ & ${ }^{\circ} \mathrm{C}$ \\
\hline 0 & 0.00 & 0.00 & 0.00 & 0.00 & 14.50 & 20.86 & 21.87 & 21.41 & 21.48 & 21.49 & 21.62 & 21.23 & 20.83 & 22.00 & 21.56 & 21.38 & 22.03 \\
\hline 4 & 0.00 & 0.00 & 0.00 & 0.00 & 14.50 & 21.12 & 21.88 & 21.44 & 21.50 & 21.51 & 21.64 & 21.26 & 20.86 & 22.02 & 21.58 & 21.42 & 22.06 \\
\hline 8 & 0.00 & 0.00 & 0.00 & 0.00 & 14.51 & 20.97 & 21.94 & 21.48 & 21.53 & 21.54 & 21.67 & 21.31 & 20.89 & 22.07 & 21.63 & 21.48 & 22.08 \\
\hline 12 & 0.00 & 0.00 & 0.00 & 0.00 & 14.52 & 20.94 & 22.01 & 21.56 & 21.60 & 21.62 & 21.74 & 21.34 & 20.86 & 22.15 & 21.71 & 21.52 & 22.11 \\
\hline 16 & 0.00 & 0.00 & 0.00 & 0.00 & 14.53 & 21.05 & 22.05 & 21.58 & 21.63 & 21.63 & 21.76 & 21.39 & 20.89 & 22.15 & 21.72 & 21.56 & 22.10 \\
\hline 18 & 0.00 & 0.00 & 0.00 & 0.00 & 14.55 & 21.24 & 22.09 & 21.65 & 21.69 & 21.70 & 21.83 & 21.46 & 20.98 & 22.24 & 21.81 & 21.61 & 22.15 \\
\hline 20 & 0.00 & 0.00 & 0.00 & 0.00 & 14.56 & 21.12 & 22.15 & 21.67 & 21.69 & 21.71 & 21.83 & 21.54 & 21.06 & 22.23 & 21.80 & 21.69 & 22.15 \\
\hline 21 & 0.00 & 0.00 & 0.00 & 0.00 & 14.57 & 21.49 & 22.21 & 21.76 & 21.78 & 21.80 & 21.92 & 21.61 & 21.14 & 22.31 & 21.89 & 21.75 & 22.20 \\
\hline 22 & 0.00 & 0.00 & 0.00 & 0.00 & 14.59 & 21.39 & 22.20 & 21.75 & 21.78 & 21.80 & 21.92 & 21.62 & 21.16 & 22.33 & 21.90 & 21.77 & 22.22 \\
\hline 23 & 0.00 & 0.00 & 0.00 & 0.00 & 14.59 & 21.44 & 22.25 & 21.80 & 21.83 & 21.84 & 21.97 & 21.68 & 21.24 & 22.38 & 21.95 & 21.83 & 22.23 \\
\hline 22 & 0.00 & 0.00 & 0.00 & 0.00 & 14.60 & 21.52 & 22.24 & 21.79 & 21.82 & 21.84 & 21.97 & 21.71 & 21.25 & 22.39 & 21.96 & 21.86 & 22.25 \\
\hline 21 & 0.00 & 0.00 & 0.00 & 0.00 & 14.60 & 21.69 & 22.28 & 21.85 & 21.88 & 21.90 & 22.03 & 21.76 & 21.29 & 22.46 & 22.03 & 21.91 & 22.32 \\
\hline 20 & 0.00 & 0.00 & 0.00 & 0.00 & 14.59 & 21.37 & 22.24 & 21.78 & 21.79 & 21.81 & 21.94 & 21.82 & 21.33 & 22.34 & 21.92 & 21.96 & 22.24 \\
\hline 18 & 0.00 & 0.00 & 0.00 & 0.00 & 14.58 & 21.32 & 22.23 & 21.78 & 21.80 & 21.81 & 21.94 & 21.81 & 21.36 & 22.32 & 21.91 & 21.93 & 22.26 \\
\hline 16 & 0.00 & 0.00 & 0.00 & 0.00 & 14.58 & 21.60 & 22.30 & 21.88 & 21.89 & 21.91 & 22.03 & 21.77 & 21.33 & 22.42 & 22.00 & 21.91 & 22.31 \\
\hline 12 & 0.00 & 0.00 & 0.00 & 0.00 & 14.58 & 21.61 & 22.34 & 21.90 & 21.91 & 21.93 & 22.05 & 21.85 & 21.40 & 22.41 & 22.00 & 22.00 & 22.30 \\
\hline 8 & 0.00 & 0.00 & 0.00 & 0.00 & 14.58 & 21.54 & 22.33 & 21.89 & 21.90 & 21.91 & 22.03 & 21.88 & 21.41 & 22.41 & 21.99 & 22.05 & 22.32 \\
\hline 4 & 0.00 & 0.00 & 0.00 & 0.00 & 14.57 & 21.31 & 22.31 & 21.86 & 21.87 & 21.89 & 22.00 & 21.92 & 21.43 & 22.36 & 21.95 & 22.06 & 22.30 \\
\hline 0 & 0.00 & 0.00 & 0.00 & 0.00 & 14.56 & 21.59 & 22.33 & 21.89 & 21.89 & 21.91 & 22.02 & 21.91 & 21.48 & 22.37 & 21.96 & 22.04 & 22.31 \\
\hline
\end{tabular}


Table D77: Averaged Raw Data for Mehra Electrode at $1 \mathrm{~W}$

\begin{tabular}{|c|c|c|c|c|c|c|c|c|c|c|c|c|c|c|c|c|c|}
\hline HV & $\mathbf{Q}$ & $\begin{array}{c}\text { Heater Voltage } \\
\text { after } \\
\text { precision } \\
\text { resistor } \\
\end{array}$ & $\begin{array}{c}\text { Heater Voltage } \\
\text { before } \\
\text { precision } \\
\text { resistor } \\
\end{array}$ & $\begin{array}{l}\text { Heater } \\
\text { Current }\end{array}$ & $\begin{array}{l}\text { Chamber } \\
\text { Pressure }\end{array}$ & Amb & T1 & T2 & T3 & T4 & T5 & T8 & T9 & T10 & T11 & T12 & T14 \\
\hline $\mathrm{kV}$ & $\mathbf{W}$ & Volts & Volts & Amps & psia & ${ }^{\circ} \mathbf{C}$ & ${ }^{\circ} \mathbf{C}$ & ${ }^{\circ} \mathrm{C}$ & ${ }^{\circ} \mathrm{C}$ & ${ }^{\circ} \mathrm{C}$ & ${ }^{\circ} \mathbf{C}$ & ${ }^{\circ} \mathrm{C}$ & ${ }^{\circ} \mathbf{C}$ & ${ }^{\circ} \mathrm{C}$ & ${ }^{\circ} \mathbf{C}$ & ${ }^{\circ} \mathbf{C}$ & ${ }^{\circ} \mathbf{C}$ \\
\hline 0 & 0.98 & 2.98 & 2.98 & 0.33 & 14.55 & 21.40 & 33.61 & 27.54 & 24.76 & 25.71 & 24.45 & 21.81 & 21.49 & 22.56 & 22.15 & 21.99 & 22.35 \\
\hline 4 & 0.98 & 2.98 & 2.98 & 0.33 & 14.56 & 21.56 & 33.62 & 27.52 & 24.72 & 25.67 & 24.39 & 21.86 & 21.50 & 22.49 & 22.08 & 22.03 & 22.28 \\
\hline 8 & 0.98 & 2.98 & 2.98 & 0.33 & 14.55 & 21.27 & 33.55 & 27.46 & 24.66 & 25.61 & 24.33 & 21.88 & 21.51 & 22.43 & 22.01 & 22.03 & 22.24 \\
\hline 12 & 0.98 & 2.98 & 2.98 & 0.33 & 14.56 & 21.25 & 33.55 & 27.48 & 24.68 & 25.63 & 24.35 & 21.84 & 21.51 & 22.44 & 22.03 & 21.98 & 22.23 \\
\hline 16 & 0.98 & 2.98 & 2.98 & 0.33 & 14.56 & 21.28 & 33.55 & 27.48 & 24.68 & 25.62 & 24.33 & 21.83 & 21.54 & 22.40 & 22.00 & 21.96 & 22.17 \\
\hline 18 & 0.98 & 2.98 & 2.98 & 0.33 & 14.56 & 21.23 & 33.54 & 27.47 & 24.68 & 25.62 & 24.33 & 21.80 & 21.53 & 22.43 & 22.02 & 21.95 & 22.18 \\
\hline 20 & 0.98 & 2.98 & 2.98 & 0.33 & 14.55 & 21.08 & 33.51 & 27.43 & 24.64 & 25.58 & 24.29 & 21.77 & 21.53 & 22.39 & 21.99 & 21.92 & 22.16 \\
\hline 21 & 0.98 & 2.98 & 2.98 & 0.33 & 14.54 & 21.33 & 33.51 & 27.45 & 24.67 & 25.60 & 24.31 & 21.70 & 21.49 & 22.40 & 22.01 & 21.85 & 22.17 \\
\hline 22 & 0.98 & 2.98 & 2.98 & 0.33 & 14.54 & 21.17 & 33.55 & 27.48 & 24.70 & 25.63 & 24.33 & 21.67 & 21.44 & 22.42 & 22.04 & 21.83 & 22.15 \\
\hline 23 & 0.98 & 2.98 & 2.98 & 0.33 & 14.55 & 21.48 & 33.55 & 27.48 & 24.70 & 25.64 & 24.34 & 21.73 & 21.48 & 22.46 & 22.08 & 21.93 & 22.17 \\
\hline 22 & 0.98 & 2.98 & 2.98 & 0.33 & 14.55 & 20.99 & 33.51 & 27.44 & 24.65 & 25.58 & 24.29 & 21.80 & 21.53 & 22.40 & 22.01 & 21.93 & 22.14 \\
\hline 21 & 0.98 & 2.98 & 2.98 & 0.33 & 14.54 & 21.31 & 33.54 & 27.45 & 24.66 & 25.59 & 24.30 & 21.76 & 21.49 & 22.40 & 22.01 & 21.92 & 22.15 \\
\hline 20 & 0.98 & 2.98 & 2.98 & 0.33 & 14.54 & 21.51 & 33.60 & 27.56 & 24.77 & 25.69 & 24.41 & 21.74 & 21.47 & 22.49 & 22.12 & 21.91 & 22.22 \\
\hline 18 & 0.98 & 2.98 & 2.98 & 0.33 & 14.54 & 21.05 & 33.53 & 27.47 & 24.67 & 25.60 & 24.31 & 21.76 & 21.48 & 22.40 & 22.00 & 21.93 & 22.16 \\
\hline 16 & 0.98 & 2.98 & 2.98 & 0.33 & 14.53 & 21.45 & 33.54 & 27.51 & 24.70 & 25.63 & 24.36 & 21.78 & 21.50 & 22.43 & 22.05 & 21.94 & 22.21 \\
\hline 12 & 0.98 & 2.98 & 2.98 & 0.33 & 14.53 & 21.36 & 33.56 & 27.51 & 24.70 & 25.63 & 24.35 & 21.83 & 21.53 & 22.41 & 22.03 & 22.00 & 22.22 \\
\hline 8 & 0.98 & 2.98 & 2.98 & 0.33 & 14.52 & 21.63 & 33.59 & 27.53 & 24.72 & 25.65 & 24.37 & 21.79 & 21.47 & 22.42 & 22.05 & 21.97 & 22.27 \\
\hline 4 & 0.98 & 2.98 & 2.98 & 0.33 & 14.52 & 21.32 & 33.60 & 27.52 & 24.70 & 25.62 & 24.34 & 21.83 & 21.51 & 22.39 & 22.01 & 22.04 & 22.23 \\
\hline O & 0.98 & 2.98 & 2.98 & 0.33 & 14.52 & 21.56 & 33.63 & 27.56 & 24.73 & 25.65 & 24.37 & 21.88 & 21.53 & 22.38 & 22.02 & 22.07 & 22.23 \\
\hline
\end{tabular}


Table D78: Averaged Raw Data for Mehra Electrode at $2 \mathrm{~W}$

\begin{tabular}{|c|c|c|c|c|c|c|c|c|c|c|c|c|c|c|c|c|c|}
\hline HV & $\mathbf{Q}$ & $\begin{array}{c}\text { Heater Voltage } \\
\text { after } \\
\text { precision } \\
\text { resistor } \\
\end{array}$ & $\begin{array}{c}\text { Heater Voltage } \\
\text { before } \\
\text { precision } \\
\text { resistor } \\
\end{array}$ & $\begin{array}{c}\text { Heater } \\
\text { Current }\end{array}$ & $\begin{array}{l}\text { Chamber } \\
\text { Pressure }\end{array}$ & Amb & T1 & T2 & T3 & T4 & T5 & T8 & T9 & T10 & T11 & T12 & T14 \\
\hline $\mathrm{kV}$ & $\mathbf{W}$ & Volts & Volts & Amps & psia & ${ }^{\circ} \mathbf{C}$ & ${ }^{\circ} \mathbf{C}$ & ${ }^{\circ} \mathbf{C}$ & ${ }^{\circ} \mathbf{C}$ & ${ }^{\circ} \mathrm{C}$ & ${ }^{\circ} \mathrm{C}$ & ${ }^{\circ} \mathrm{C}$ & ${ }^{\circ} \mathrm{C}$ & ${ }^{\circ} \mathrm{C}$ & ${ }^{\circ} \mathbf{C}$ & ${ }^{\circ} \mathrm{C}$ & ${ }^{\circ} \mathbf{C}$ \\
\hline 0 & 2.05 & 4.27 & 4.27 & 0.48 & 14.54 & 21.38 & 45.59 & 33.70 & 27.95 & 29.82 & 26.95 & 21.88 & 21.54 & 22.55 & 22.22 & 22.05 & 22.28 \\
\hline 4 & 2.05 & 4.27 & 4.27 & 0.48 & 14.54 & 21.11 & 45.63 & 33.71 & 27.95 & 29.84 & 26.95 & 21.95 & 21.62 & 22.60 & 22.24 & 22.13 & 22.31 \\
\hline 8 & 2.05 & 4.27 & 4.27 & 0.48 & 14.39 & 21.55 & 45.69 & 33.81 & 28.04 & 29.90 & 27.05 & 21.93 & 21.53 & 22.69 & 22.30 & 22.12 & 22.34 \\
\hline 12 & 2.05 & 4.27 & 4.27 & 0.48 & 14.43 & 21.43 & 45.67 & 33.81 & 28.05 & 29.91 & 27.05 & 22.02 & 21.61 & 22.71 & 22.31 & 22.17 & 22.33 \\
\hline 16 & 2.05 & 4.27 & 4.27 & 0.48 & 14.46 & 21.36 & 45.62 & 33.78 & 28.03 & 29.88 & 27.03 & 22.07 & 21.69 & 22.70 & 22.31 & 22.17 & 22.34 \\
\hline 18 & 2.05 & 4.27 & 4.27 & 0.48 & 14.47 & 21.46 & 45.64 & 33.82 & 28.09 & 29.93 & 27.09 & 21.97 & 21.63 & 22.79 & 22.41 & 22.10 & 22.40 \\
\hline 20 & 2.05 & 4.27 & 4.27 & 0.48 & 14.48 & 21.51 & 45.69 & 33.83 & 28.10 & 29.95 & 27.08 & 22.04 & 21.71 & 22.79 & 22.41 & 22.17 & 22.37 \\
\hline 21 & 2.05 & 4.27 & 4.27 & 0.48 & 14.49 & 21.49 & 45.66 & 33.81 & 28.09 & 29.93 & 27.08 & 22.02 & 21.67 & 22.83 & 22.45 & 22.17 & 22.43 \\
\hline 22 & 2.05 & 4.27 & 4.27 & 0.48 & 14.49 & 21.53 & 45.70 & 33.85 & 28.13 & 29.98 & 27.12 & 22.09 & 21.76 & 22.88 & 22.50 & 22.22 & 22.43 \\
\hline 23 & 2.05 & 4.27 & 4.27 & 0.48 & 14.50 & 21.53 & 45.75 & 33.89 & 28.16 & 30.01 & 27.15 & 22.09 & 21.75 & 22.91 & 22.53 & 22.22 & 22.44 \\
\hline 22 & 2.05 & 4.27 & 4.27 & 0.48 & 14.51 & 21.32 & 45.73 & 33.85 & 28.12 & 29.96 & 27.10 & 22.12 & 21.79 & 22.87 & 22.48 & 22.21 & 22.42 \\
\hline 21 & 2.05 & 4.27 & 4.27 & 0.48 & 14.50 & 21.24 & 45.68 & 33.80 & 28.06 & 29.89 & 27.05 & 22.08 & 21.79 & 22.79 & 22.41 & 22.16 & 22.37 \\
\hline 20 & 2.05 & 4.27 & 4.27 & 0.48 & 14.49 & 21.42 & 45.74 & 33.88 & 28.12 & 29.95 & 27.11 & 22.05 & 21.80 & 22.82 & 22.44 & 22.15 & 22.39 \\
\hline 18 & 2.05 & 4.27 & 4.27 & 0.48 & 14.48 & 21.52 & 45.75 & 33.88 & 28.11 & 29.94 & 27.11 & 22.02 & 21.77 & 22.82 & 22.44 & 22.16 & 22.43 \\
\hline 16 & 2.05 & 4.27 & 4.27 & 0.48 & 14.48 & 21.10 & 45.68 & 33.79 & 28.00 & 29.84 & 27.01 & 22.04 & 21.76 & 22.71 & 22.31 & 22.17 & 22.35 \\
\hline 12 & 2.05 & 4.27 & 4.27 & 0.48 & 14.47 & 21.33 & 45.74 & 33.84 & 28.03 & 29.87 & 27.05 & 21.98 & 21.69 & 22.73 & 22.33 & 22.12 & 22.40 \\
\hline 8 & 2.05 & 4.27 & 4.27 & 0.48 & 14.47 & 21.62 & 45.80 & 33.86 & 28.04 & 29.88 & 27.06 & 22.06 & 21.78 & 22.72 & 22.33 & 22.18 & 22.39 \\
\hline 4 & 2.05 & 4.27 & 4.27 & 0.48 & 14.46 & 21.45 & 45.82 & 33.86 & 28.04 & 29.87 & 27.05 & 22.02 & 21.71 & 22.71 & 22.31 & 22.17 & 22.38 \\
\hline 0 & 2.05 & 4.27 & 4.27 & 0.48 & 14.46 & 21.43 & 45.84 & 33.88 & 28.07 & 29.89 & 27.06 & 22.11 & 21.81 & 22.72 & 22.32 & 22.22 & 22.36 \\
\hline
\end{tabular}


Table D79: Averaged Raw Data for Mehra Electrode at $3 \mathrm{~W}$

\begin{tabular}{|c|c|c|c|c|c|c|c|c|c|c|c|c|c|c|c|c|c|}
\hline HV & $\mathbf{Q}$ & $\begin{array}{c}\text { Heater Voltage } \\
\text { after } \\
\text { precision } \\
\text { resistor }\end{array}$ & $\begin{array}{c}\text { Heater Voltage } \\
\text { before } \\
\text { precision } \\
\text { resistor }\end{array}$ & $\begin{array}{c}\text { Heater } \\
\text { Current }\end{array}$ & $\begin{array}{l}\text { Chamber } \\
\text { Pressure }\end{array}$ & Amb & T1 & T2 & T3 & T4 & T5 & T8 & T9 & T10 & T11 & T12 & T14 \\
\hline $\mathbf{k V}$ & W & Volts & Volts & Amps & psia & ${ }^{\circ} \mathrm{C}$ & ${ }^{\circ} \mathrm{C}$ & ${ }^{\circ} \mathrm{C}$ & ${ }^{\circ} \mathbf{C}$ & ${ }^{\circ} \mathbf{C}$ & ${ }^{\circ} \mathbf{C}$ & ${ }^{\circ} \mathbf{C}$ & ${ }^{\circ} \mathrm{C}$ & ${ }^{\circ} \mathbf{C}$ & ${ }^{\circ} \mathbf{C}$ & ${ }^{\circ} \mathbf{C}$ & ${ }^{\circ} \mathbf{C}$ \\
\hline 0 & 2.99 & 5.16 & 5.16 & 0.58 & 0.72 & 21.22 & 56.66 & 39.44 & 31.01 & 33.61 & 29.26 & 22.07 & 21.81 & 22.75 & 22.35 & 22.19 & 22.26 \\
\hline 4 & 2.99 & 5.16 & 5.16 & 0.58 & 0.72 & 21.24 & 56.68 & 39.46 & 31.03 & 33.65 & 29.32 & 22.03 & 21.79 & 22.82 & 22.41 & 22.15 & 22.34 \\
\hline 8 & 2.99 & 5.16 & 5.16 & 0.58 & 0.72 & 21.55 & 56.67 & 39.45 & 31.02 & 33.64 & 29.33 & 22.02 & 21.77 & 22.81 & 22.41 & 22.16 & 22.34 \\
\hline 12 & 2.99 & 5.16 & 5.16 & 0.58 & 0.72 & 21.47 & 56.57 & 39.39 & 30.98 & 33.59 & 29.29 & 22.00 & 21.73 & 22.79 & 22.38 & 22.15 & 22.32 \\
\hline 16 & 2.99 & 5.16 & 5.16 & 0.58 & 0.72 & 21.22 & 56.57 & 39.41 & 31.02 & 33.62 & 29.32 & 22.11 & 21.85 & 22.84 & 22.44 & 22.22 & 22.32 \\
\hline 18 & 2.99 & 5.16 & 5.16 & 0.58 & 0.72 & 21.33 & 56.57 & 39.40 & 31.01 & 33.61 & 29.31 & 22.07 & 21.81 & 22.84 & 22.45 & 22.19 & 22.32 \\
\hline 20 & 2.99 & 5.16 & 5.16 & 0.58 & 0.72 & 21.48 & 56.55 & 39.39 & 31.00 & 33.59 & 29.30 & 22.06 & 21.79 & 22.87 & 22.48 & 22.18 & 22.34 \\
\hline 21 & 2.99 & 5.16 & 5.16 & 0.58 & 0.72 & 21.43 & 56.56 & 39.41 & 31.05 & 33.64 & 29.36 & 22.07 & 21.81 & 22.97 & 22.58 & 22.17 & 22.39 \\
\hline 22 & 2.99 & 5.16 & 5.16 & 0.58 & 0.72 & 20.83 & 56.56 & 39.38 & 31.01 & 33.59 & 29.30 & 22.07 & 21.82 & 22.90 & 22.52 & 22.13 & 22.30 \\
\hline 23 & 2.99 & 5.16 & 5.16 & 0.58 & 0.72 & 21.22 & 56.56 & 39.40 & 31.01 & 33.60 & 29.30 & 22.09 & 21.87 & 22.92 & 22.54 & 22.18 & 22.32 \\
\hline 22 & 2.99 & 5.16 & 5.16 & 0.58 & 0.72 & 21.29 & 56.58 & 39.41 & 31.02 & 33.61 & 29.32 & 22.04 & 21.83 & 22.91 & 22.54 & 22.11 & 22.32 \\
\hline 21 & 2.99 & 5.16 & 5.16 & 0.58 & 0.72 & 21.37 & 56.61 & 39.41 & 31.00 & 33.58 & 29.31 & 21.99 & 21.77 & 22.86 & 22.48 & 22.10 & 22.30 \\
\hline 20 & 2.99 & 5.16 & 5.16 & 0.58 & 0.72 & 21.24 & 56.58 & 39.37 & 30.95 & 33.53 & 29.26 & 21.96 & 21.75 & 22.83 & 22.45 & 22.07 & 22.28 \\
\hline 18 & 2.99 & 5.16 & 5.16 & 0.58 & 0.72 & 21.30 & 56.62 & 39.42 & 31.00 & 33.57 & 29.32 & 21.99 & 21.77 & 22.86 & 22.48 & 22.12 & 22.33 \\
\hline 16 & 2.99 & 5.16 & 5.16 & 0.58 & 0.72 & 21.31 & 56.61 & 39.38 & 30.95 & 33.54 & 29.29 & 22.06 & 21.85 & 22.81 & 22.41 & 22.18 & 22.31 \\
\hline 12 & 2.99 & 5.16 & 5.16 & 0.58 & 0.72 & 21.19 & 56.69 & 39.41 & 30.94 & 33.53 & 29.29 & 22.01 & 21.76 & 22.74 & 22.34 & 22.15 & 22.25 \\
\hline 8 & 2.99 & 5.16 & 5.16 & 0.58 & 0.72 & 21.41 & 56.75 & 39.44 & 30.95 & 33.56 & 29.32 & 21.98 & 21.74 & 22.79 & 22.39 & 22.11 & 22.34 \\
\hline 4 & 2.99 & 5.16 & 5.16 & 0.58 & 0.72 & 21.39 & 56.79 & 39.45 & 30.96 & 33.56 & 29.32 & 21.97 & 21.72 & 22.80 & 22.39 & 22.10 & 22.32 \\
\hline 0 & 2.99 & 5.16 & 5.16 & 0.58 & 0.72 & 21.36 & 56.81 & 39.44 & 30.93 & 33.52 & 29.28 & 22.08 & 21.83 & 22.75 & 22.34 & 22.19 & 22.26 \\
\hline
\end{tabular}


Table D80: Averaged Raw Data for Mehra Electrode at $4 \mathrm{~W}$

\begin{tabular}{|c|c|c|c|c|c|c|c|c|c|c|c|c|c|c|c|c|c|}
\hline HV & $\mathbf{Q}$ & $\begin{array}{c}\text { Heater Voltage } \\
\text { after } \\
\text { precision } \\
\text { resistor } \\
\end{array}$ & $\begin{array}{c}\text { Heater Voltage } \\
\text { before } \\
\text { precision } \\
\text { resistor } \\
\end{array}$ & $\begin{array}{l}\text { Heater } \\
\text { Current }\end{array}$ & $\begin{array}{l}\text { Chamber } \\
\text { Pressure }\end{array}$ & Amb & T1 & T2 & T3 & T4 & T5 & T8 & T9 & T10 & T11 & T12 & T14 \\
\hline $\mathrm{kV}$ & $\mathbf{W}$ & Volts & Volts & Amps & psia & ${ }^{\circ} \mathrm{C}$ & ${ }^{\circ} \mathrm{C}$ & ${ }^{\circ} \mathrm{C}$ & ${ }^{\circ} \mathrm{C}$ & ${ }^{\circ} \mathrm{C}$ & ${ }^{\circ} \mathbf{C}$ & ${ }^{\circ} \mathrm{C}$ & ${ }^{\circ} \mathrm{C}$ & ${ }^{\circ} \mathrm{C}$ & ${ }^{\circ} \mathbf{C}$ & ${ }^{\circ} \mathrm{C}$ & ${ }^{\circ} \mathbf{C}$ \\
\hline 0 & 3.98 & 5.95 & 5.95 & 0.67 & 0.72 & 21.28 & 68.13 & 45.32 & 34.10 & 37.42 & 31.73 & 22.03 & 21.78 & 22.90 & 22.51 & 22.17 & 22.27 \\
\hline 4 & 3.98 & 5.95 & 5.95 & 0.67 & 0.72 & 21.29 & 68.14 & 45.31 & 34.08 & 37.42 & 31.74 & 22.02 & 21.75 & 22.93 & 22.53 & 22.16 & 22.31 \\
\hline 8 & 3.98 & 5.95 & 5.95 & 0.67 & 0.72 & 21.37 & 68.06 & 45.24 & 34.01 & 37.35 & 31.69 & 22.02 & 21.77 & 22.87 & 22.48 & 22.16 & 22.31 \\
\hline 12 & 3.98 & 5.95 & 5.95 & 0.67 & 0.72 & 21.24 & 67.97 & 45.21 & 34.02 & 37.34 & 31.70 & 22.02 & 21.78 & 22.89 & 22.49 & 22.15 & 22.29 \\
\hline 16 & 3.98 & 5.95 & 5.95 & 0.67 & 0.72 & 21.16 & 67.90 & 45.18 & 33.99 & 37.31 & 31.66 & 22.08 & 21.89 & 22.89 & 22.50 & 22.19 & 22.27 \\
\hline 18 & 3.98 & 5.95 & 5.95 & 0.67 & 0.72 & 21.25 & 67.96 & 45.23 & 34.05 & 37.37 & 31.71 & 22.02 & 21.83 & 22.98 & 22.60 & 22.14 & 22.32 \\
\hline 20 & 3.98 & 5.95 & 5.95 & 0.67 & 0.72 & 21.21 & 67.95 & 45.21 & 34.04 & 37.36 & 31.71 & 22.03 & 21.82 & 23.02 & 22.64 & 22.15 & 22.31 \\
\hline 21 & 3.98 & 5.95 & 5.95 & 0.67 & 0.72 & 21.07 & 67.91 & 45.15 & 33.98 & 37.31 & 31.65 & 22.03 & 21.82 & 22.98 & 22.61 & 22.12 & 22.27 \\
\hline 22 & 3.98 & 5.95 & 5.95 & 0.67 & 0.72 & 20.89 & 67.89 & 45.15 & 33.98 & 37.31 & 31.64 & 22.07 & 21.88 & 23.00 & 22.63 & 22.14 & 22.29 \\
\hline 23 & 3.98 & 5.95 & 5.95 & 0.67 & 0.72 & 21.44 & 68.00 & 45.26 & 34.08 & 37.42 & 31.74 & 22.02 & 21.81 & 23.09 & 22.72 & 22.12 & 22.35 \\
\hline 22 & 3.98 & 5.95 & 5.95 & 0.67 & 0.72 & 21.32 & 67.96 & 45.20 & 34.03 & 37.35 & 31.69 & 22.03 & 21.82 & 23.03 & 22.66 & 22.13 & 22.32 \\
\hline 21 & 3.98 & 5.95 & 5.95 & 0.67 & 0.72 & 21.11 & 67.96 & 45.20 & 34.01 & 37.33 & 31.69 & 22.10 & 21.90 & 23.01 & 22.63 & 22.18 & 22.29 \\
\hline 20 & 3.98 & 5.95 & 5.95 & 0.67 & 0.72 & 21.43 & 68.03 & 45.26 & 34.05 & 37.37 & 31.74 & 22.06 & 21.85 & 23.04 & 22.66 & 22.18 & 22.34 \\
\hline 18 & 3.98 & 5.95 & 5.95 & 0.67 & 0.72 & 21.17 & 68.07 & 45.29 & 34.04 & 37.36 & 31.74 & 22.08 & 21.84 & 22.98 & 22.60 & 22.22 & 22.32 \\
\hline 16 & 3.98 & 5.95 & 5.95 & 0.67 & 0.72 & 20.98 & 68.09 & 45.28 & 34.01 & 37.34 & 31.72 & 22.06 & 21.82 & 22.93 & 22.54 & 22.19 & 22.29 \\
\hline 12 & 3.98 & 5.94 & 5.94 & 0.67 & 0.72 & 21.27 & 68.17 & 45.33 & 34.03 & 37.37 & 31.77 & 22.03 & 21.81 & 22.94 & 22.55 & 22.17 & 22.35 \\
\hline 8 & 3.98 & 5.94 & 5.94 & 0.67 & 0.72 & 21.30 & 68.27 & 45.35 & 34.01 & 37.35 & 31.76 & 22.09 & 21.82 & 22.92 & 22.52 & 22.22 & 22.33 \\
\hline 4 & 3.98 & 5.94 & 5.94 & 0.67 & 0.72 & 21.45 & 68.35 & 45.42 & 34.04 & 37.39 & 31.79 & 22.08 & 21.79 & 22.95 & 22.56 & 22.20 & 22.34 \\
\hline 0 & 3.98 & 5.94 & 5.94 & 0.67 & 0.72 & 21.14 & 68.35 & 45.40 & 34.02 & 37.36 & 31.77 & 22.15 & 21.87 & 22.97 & 22.55 & 22.27 & 22.37 \\
\hline
\end{tabular}


Table D81: Averaged Raw Data for Mehra Electrode at $5 \mathrm{~W}$

\begin{tabular}{|c|c|c|c|c|c|c|c|c|c|c|c|c|c|c|c|c|c|}
\hline HV & $\mathbf{Q}$ & $\begin{array}{c}\text { Heater Voltage } \\
\text { after } \\
\text { precision } \\
\text { resistor }\end{array}$ & $\begin{array}{c}\text { Heater Voltage } \\
\text { before } \\
\text { precision } \\
\text { resistor } \\
\end{array}$ & $\begin{array}{c}\text { Heater } \\
\text { Current }\end{array}$ & $\begin{array}{l}\text { Chamber } \\
\text { Pressure }\end{array}$ & Amb & T1 & T2 & T3 & T4 & T5 & T8 & T9 & T10 & T11 & T12 & T14 \\
\hline $\mathrm{kV}$ & $\mathbf{W}$ & Volts & Volts & Amps & psia & ${ }^{\circ} \mathbf{C}$ & ${ }^{\circ} \mathbf{C}$ & ${ }^{\circ} \mathbf{C}$ & ${ }^{\circ} \mathrm{C}$ & ${ }^{\circ} \mathrm{C}$ & ${ }^{\circ} \mathrm{C}$ & ${ }^{\circ} \mathrm{C}$ & ${ }^{\circ} \mathrm{C}$ & ${ }^{\circ} \mathrm{C}$ & ${ }^{\circ} \mathrm{C}$ & ${ }^{\circ} \mathrm{C}$ & ${ }^{\circ} \mathrm{C}$ \\
\hline 0 & 4.91 & 6.64 & 6.64 & 0.74 & 0.72 & 20.70 & 79.33 & 51.22 & 37.14 & 41.13 & 34.12 & 22.00 & 21.77 & 23.08 & 22.67 & 22.15 & 22.34 \\
\hline 4 & 4.91 & 6.64 & 6.64 & 0.74 & 0.72 & 21.10 & 79.28 & 51.22 & 37.09 & 41.12 & 34.10 & 22.09 & 21.86 & 23.03 & 22.63 & 22.22 & 22.30 \\
\hline 8 & 4.91 & 6.64 & 6.64 & 0.74 & 0.72 & 21.08 & 79.15 & 51.14 & 36.97 & 40.99 & 34.00 & 22.03 & 21.82 & 22.90 & 22.52 & 22.14 & 22.25 \\
\hline 12 & 4.91 & 6.64 & 6.64 & 0.74 & 0.72 & 21.26 & 79.10 & 51.18 & 37.04 & 41.05 & 34.08 & 21.98 & 21.82 & 22.99 & 22.60 & 22.12 & 22.29 \\
\hline 16 & 4.91 & 6.64 & 6.64 & 0.74 & 0.72 & 21.30 & 79.03 & 51.15 & 37.05 & 41.07 & 34.09 & 21.99 & 21.78 & 23.09 & 22.70 & 22.16 & 22.32 \\
\hline 18 & 4.91 & 6.64 & 6.64 & 0.74 & 0.72 & 21.37 & 79.03 & 51.17 & 37.05 & 41.07 & 34.11 & 22.02 & 21.79 & 23.14 & 22.76 & 22.16 & 22.36 \\
\hline 20 & 4.91 & 6.64 & 6.64 & 0.74 & 0.72 & 21.18 & 79.00 & 51.11 & 37.02 & 41.05 & 34.07 & 22.04 & 21.78 & 23.17 & 22.79 & 22.19 & 22.34 \\
\hline 21 & 4.91 & 6.64 & 6.64 & 0.74 & 0.72 & 20.98 & 78.73 & 51.05 & 36.96 & 41.02 & 34.02 & 22.06 & 21.77 & 23.16 & 22.78 & 22.19 & 22.33 \\
\hline 22 & 4.91 & 6.64 & 6.64 & 0.74 & 0.72 & 21.16 & 78.64 & 51.05 & 36.94 & 41.01 & 34.01 & 22.05 & 21.78 & 23.16 & 22.79 & 22.18 & 22.35 \\
\hline 23 & 4.91 & 6.64 & 6.64 & 0.74 & 0.72 & 21.17 & 78.68 & 51.10 & 36.97 & 41.06 & 34.05 & 22.06 & 21.81 & 23.21 & 22.84 & 22.18 & 22.36 \\
\hline 22 & 4.91 & 6.64 & 6.64 & 0.74 & 0.72 & 21.00 & 78.65 & 51.07 & 36.93 & 41.01 & 34.01 & 22.07 & 21.83 & 23.17 & 22.80 & 22.20 & 22.34 \\
\hline 21 & 4.91 & 6.64 & 6.64 & 0.74 & 0.72 & 21.03 & 78.72 & 51.12 & 36.95 & 41.03 & 34.04 & 22.07 & 21.83 & 23.18 & 22.80 & 22.19 & 22.34 \\
\hline 20 & 4.91 & 6.64 & 6.64 & 0.74 & 0.72 & 21.26 & 78.76 & 51.17 & 36.97 & 41.04 & 34.08 & 22.07 & 21.82 & 23.18 & 22.80 & 22.20 & 22.39 \\
\hline 18 & 4.91 & 6.64 & 6.64 & 0.74 & 0.72 & 20.99 & 78.83 & 51.26 & 37.02 & 41.08 & 34.14 & 22.07 & 21.80 & 23.21 & 22.82 & 22.20 & 22.43 \\
\hline 16 & 4.91 & 6.64 & 6.64 & 0.74 & 0.72 & 21.34 & 78.86 & 51.32 & 37.03 & 41.12 & 34.17 & 22.06 & 21.78 & 23.20 & 22.81 & 22.20 & 22.45 \\
\hline 12 & 4.91 & 6.64 & 6.64 & 0.74 & 0.72 & 21.51 & 79.00 & 51.40 & 37.06 & 41.14 & 34.21 & 22.12 & 21.84 & 23.17 & 22.77 & 22.26 & 22.45 \\
\hline 8 & 4.91 & 6.64 & 6.64 & 0.74 & 0.72 & 21.53 & 79.13 & 51.48 & 37.08 & 41.18 & 34.24 & 22.18 & 21.89 & 23.16 & 22.77 & 22.30 & 22.44 \\
\hline 4 & 4.91 & 6.64 & 6.64 & 0.74 & 0.72 & 21.04 & 79.17 & 51.48 & 37.06 & 41.16 & 34.21 & 22.20 & 21.89 & 23.14 & 22.74 & 22.32 & 22.41 \\
\hline 0 & 4.91 & 6.64 & 6.64 & 0.74 & 0.72 & 21.37 & 79.20 & 51.55 & 37.12 & 41.20 & 34.27 & 22.15 & 21.83 & 23.23 & 22.83 & 22.27 & 22.51 \\
\hline
\end{tabular}


Table D82: Averaged Raw Data for Mehra Electrode at $6 \mathrm{~W}$

\begin{tabular}{|c|c|c|c|c|c|c|c|c|c|c|c|c|c|c|c|c|c|}
\hline HV & Q & $\begin{array}{c}\text { Heater Voltage } \\
\text { after } \\
\text { precision } \\
\text { resistor } \\
\end{array}$ & $\begin{array}{c}\text { Heater Voltage } \\
\text { before } \\
\text { precision } \\
\text { resistor } \\
\end{array}$ & $\begin{array}{c}\text { Heater } \\
\text { Current }\end{array}$ & $\begin{array}{l}\text { Chamber } \\
\text { Pressure }\end{array}$ & Amb & T1 & T2 & T3 & T4 & T5 & T8 & T9 & T10 & T11 & T12 & T14 \\
\hline $\mathbf{k V}$ & $\mathbf{W}$ & Volts & Volts & Amps & psia & ${ }^{\circ} \mathrm{C}$ & ${ }^{\circ} \mathbf{C}$ & ${ }^{\circ} \mathbf{C}$ & ${ }^{\circ} \mathrm{C}$ & ${ }^{\circ} \mathrm{C}$ & ${ }^{\circ} \mathrm{C}$ & ${ }^{\circ} \mathrm{C}$ & ${ }^{\circ} \mathrm{C}$ & ${ }^{\circ} \mathrm{C}$ & ${ }^{\circ} \mathbf{C}$ & ${ }^{\circ} \mathrm{C}$ & ${ }^{\circ} \mathrm{C}$ \\
\hline 0 & 6.04 & 7.37 & 7.37 & 0.82 & 0.72 & 21.35 & 90.86 & 58.90 & 40.94 & 45.60 & 37.11 & 22.17 & 21.96 & 23.37 & 22.96 & 22.31 & 22.50 \\
\hline 4 & 6.04 & 7.37 & 7.37 & 0.82 & 0.73 & 21.50 & 90.79 & 58.95 & 40.91 & 45.60 & 37.11 & 22.15 & 21.88 & 23.37 & 22.96 & 22.32 & 22.47 \\
\hline 8 & 6.04 & 7.37 & 7.37 & 0.82 & 0.73 & 21.41 & 90.62 & 58.93 & 40.90 & 45.57 & 37.11 & 22.29 & 22.00 & 23.34 & 22.95 & 22.45 & 22.47 \\
\hline 12 & 6.04 & 7.37 & 7.37 & 0.82 & 0.73 & 21.29 & 90.45 & 58.91 & 40.88 & 45.53 & 37.10 & 22.30 & 22.00 & 23.38 & 22.98 & 22.44 & 22.50 \\
\hline 16 & 6.04 & 7.37 & 7.37 & 0.82 & 0.73 & 21.46 & 90.25 & 58.87 & 40.96 & 45.53 & 37.10 & 22.29 & 22.02 & 23.40 & 23.02 & 22.39 & 22.49 \\
\hline 18 & 6.04 & 7.37 & 7.37 & 0.82 & 0.73 & 21.48 & 90.16 & 58.81 & 40.86 & 45.48 & 37.08 & 22.30 & 22.06 & 23.48 & 23.10 & 22.40 & 22.53 \\
\hline 20 & 6.04 & 7.37 & 7.37 & 0.82 & 0.73 & 21.47 & 90.09 & 58.73 & 40.84 & 45.47 & 37.05 & 22.30 & 22.07 & 23.50 & 23.13 & 22.37 & 22.51 \\
\hline 21 & 6.04 & 7.37 & 7.37 & 0.82 & 0.73 & 21.51 & 89.99 & 58.68 & 40.83 & 45.43 & 37.00 & 22.29 & 22.06 & 23.48 & 23.12 & 22.36 & 22.50 \\
\hline 22 & 6.04 & 7.37 & 7.37 & 0.82 & 0.73 & 21.35 & 89.90 & 58.65 & 40.81 & 45.44 & 37.01 & 22.27 & 22.03 & 23.54 & 23.17 & 22.31 & 22.53 \\
\hline 23 & 6.04 & 7.37 & 7.37 & 0.82 & 0.73 & 21.41 & 89.87 & 58.66 & 40.78 & 45.44 & 37.00 & 22.30 & 22.06 & 23.55 & 23.18 & 22.34 & 22.54 \\
\hline 22 & 6.04 & 7.37 & 7.37 & 0.82 & 0.73 & 21.43 & 89.83 & 58.66 & 40.78 & 45.42 & 37.01 & 22.28 & 22.05 & 23.55 & 23.18 & 22.32 & 22.54 \\
\hline 21 & 6.04 & 7.37 & 7.37 & 0.82 & 0.73 & 21.42 & 89.82 & 58.68 & 40.76 & 45.42 & 37.01 & 22.29 & 22.06 & 23.53 & 23.16 & 22.33 & 22.52 \\
\hline 20 & 6.04 & 7.37 & 7.37 & 0.82 & 0.73 & 21.38 & 89.85 & 58.75 & 40.78 & 45.43 & 37.04 & 22.28 & 22.05 & 23.52 & 23.15 & 22.37 & 22.52 \\
\hline 18 & 6.04 & 7.37 & 7.37 & 0.82 & 0.73 & 21.26 & 89.91 & 58.81 & 40.78 & 45.42 & 37.05 & 22.35 & 22.12 & 23.43 & 23.06 & 22.45 & 22.49 \\
\hline 16 & 6.04 & 7.37 & 7.37 & 0.82 & 0.73 & 21.30 & 89.93 & 58.90 & 40.80 & 45.47 & 37.09 & 22.37 & 22.15 & 23.42 & 23.03 & 22.49 & 22.49 \\
\hline 12 & 6.04 & 7.37 & 7.37 & 0.82 & 0.73 & 21.40 & 90.00 & 58.99 & 40.78 & 45.44 & 37.09 & 22.35 & 22.13 & 23.36 & 22.96 & 22.48 & 22.47 \\
\hline 8 & 6.04 & 7.37 & 7.37 & 0.82 & 0.73 & 21.42 & 90.13 & 59.07 & 40.77 & 45.46 & 37.10 & 22.31 & 22.08 & 23.34 & 22.95 & 22.42 & 22.47 \\
\hline 4 & 6.04 & 7.37 & 7.37 & 0.82 & 0.73 & 21.60 & 90.17 & 59.14 & 40.79 & 45.48 & 37.13 & 22.34 & 22.10 & 23.40 & 23.00 & 22.45 & 22.49 \\
\hline 0 & 6.04 & 7.37 & 7.37 & 0.82 & 0.72 & 21.55 & 90.16 & 59.16 & 40.80 & 45.45 & 37.11 & 22.27 & 22.01 & 23.40 & 22.99 & 22.40 & 22.50 \\
\hline
\end{tabular}


Table D83: Averaged Raw Data for Mehra Electrode at $7 \mathrm{~W}$

\begin{tabular}{|c|c|c|c|c|c|c|c|c|c|c|c|c|c|c|c|c|c|}
\hline HV & $\mathbf{Q}$ & $\begin{array}{c}\text { Heater Voltage } \\
\text { after } \\
\text { precision } \\
\text { resistor } \\
\end{array}$ & $\begin{array}{c}\text { Heater Voltage } \\
\text { before } \\
\text { precision } \\
\text { resistor } \\
\end{array}$ & $\begin{array}{c}\text { Heater } \\
\text { Current }\end{array}$ & $\begin{array}{l}\text { Chamber } \\
\text { Pressure }\end{array}$ & Amb & T1 & T2 & T3 & T4 & T5 & T8 & T9 & T10 & T11 & T12 & T14 \\
\hline $\mathrm{kV}$ & W & Volts & Volts & Amps & psia & ${ }^{\circ} \mathrm{C}$ & ${ }^{\circ} \mathbf{C}$ & ${ }^{\circ} \mathbf{C}$ & ${ }^{\circ} \mathrm{C}$ & ${ }^{\circ} \mathbf{C}$ & ${ }^{\circ} \mathbf{C}$ & ${ }^{\circ} \mathbf{C}$ & ${ }^{\circ} \mathbf{C}$ & ${ }^{\circ} \mathrm{C}$ & ${ }^{\circ} \mathbf{C}$ & ${ }^{\circ} \mathbf{C}$ & ${ }^{\circ} \mathbf{C}$ \\
\hline O & 7.01 & 8.01 & 8.01 & 0.88 & 0.73 & 21.39 & 99.56 & 66.27 & 44.56 & 49.68 & 39.91 & 22.33 & 22.11 & 23.59 & 23.19 & 22.45 & 22.53 \\
\hline 4 & 7.01 & 8.01 & 8.01 & 0.88 & 0.73 & 21.51 & 99.37 & 66.19 & 44.41 & 49.58 & 39.81 & 22.29 & 22.08 & 23.48 & 23.08 & 22.42 & 22.50 \\
\hline 8 & 7.01 & 8.01 & 8.01 & 0.88 & 0.73 & 21.62 & 99.21 & 66.23 & 44.46 & 49.61 & 39.88 & 22.30 & 22.11 & 23.52 & 23.14 & 22.43 & 22.54 \\
\hline 12 & 7.01 & 8.01 & 8.01 & 0.88 & 0.73 & 21.76 & 99.03 & 66.18 & 44.47 & 49.63 & 39.92 & 22.31 & 22.09 & 23.62 & 23.22 & 22.49 & 22.60 \\
\hline 16 & 7.01 & 8.01 & 8.01 & 0.88 & 0.73 & 21.66 & 98.82 & 66.08 & 44.43 & 49.59 & 39.85 & 22.36 & 22.14 & 23.59 & 23.22 & 22.50 & 22.55 \\
\hline 18 & 7.01 & 8.01 & 8.01 & 0.88 & 0.73 & 21.76 & 98.66 & 65.93 & 44.44 & 49.61 & 39.87 & 22.33 & 22.11 & 23.68 & 23.31 & 22.48 & 22.60 \\
\hline 20 & 7.01 & 8.01 & 8.01 & 0.88 & 0.73 & 21.63 & 98.49 & 65.79 & 44.36 & 49.55 & 39.81 & 22.37 & 22.14 & 23.72 & 23.36 & 22.51 & 22.62 \\
\hline 21 & 7.01 & 8.01 & 8.01 & 0.88 & 0.73 & 21.75 & 98.46 & 65.77 & 44.32 & 49.54 & 39.80 & 22.38 & 22.14 & 23.77 & 23.40 & 22.49 & 22.63 \\
\hline 22 & 7.01 & 8.01 & 8.01 & 0.88 & 0.73 & 21.66 & 98.34 & 65.70 & 44.30 & 49.53 & 39.75 & 22.42 & 22.18 & 23.72 & 23.35 & 22.52 & 22.61 \\
\hline 23 & 7.01 & 8.01 & 8.01 & 0.88 & 0.73 & 21.73 & 98.40 & 65.79 & 44.28 & 49.61 & 39.80 & 22.42 & 22.20 & 23.76 & 23.40 & 22.54 & 22.63 \\
\hline 22 & 7.01 & 8.01 & 8.01 & 0.88 & 0.73 & 21.74 & 98.32 & 65.76 & 44.15 & 49.59 & 39.82 & 22.43 & 22.20 & 23.81 & 23.44 & 22.50 & 22.69 \\
\hline 21 & 7.01 & 8.01 & 8.01 & 0.88 & 0.73 & 21.74 & 98.31 & 65.77 & 44.06 & 49.55 & 39.80 & 22.50 & 22.29 & 23.77 & 23.40 & 22.55 & 22.66 \\
\hline 20 & 7.01 & 8.01 & 8.01 & 0.88 & 0.73 & 21.46 & 98.38 & 65.84 & 44.10 & 49.61 & 39.87 & 22.50 & 22.29 & 23.78 & 23.42 & 22.60 & 22.65 \\
\hline 18 & 7.01 & 8.01 & 8.01 & 0.88 & 0.73 & 21.64 & 98.45 & 65.95 & 44.06 & 49.60 & 39.88 & 22.45 & 22.22 & 23.69 & 23.33 & 22.57 & 22.64 \\
\hline 16 & 7.01 & 8.01 & 8.01 & 0.88 & 0.73 & 21.89 & 98.54 & 66.10 & 44.16 & 49.73 & 40.02 & 22.47 & 22.23 & 23.79 & 23.41 & 22.61 & 22.75 \\
\hline 12 & 7.01 & 8.01 & 8.01 & 0.88 & 0.73 & 21.73 & 98.72 & 66.28 & 44.16 & 49.75 & 40.06 & 22.53 & 22.28 & 23.73 & 23.34 & 22.70 & 22.70 \\
\hline 8 & 7.01 & 8.01 & 8.01 & 0.88 & 0.73 & 21.63 & 98.81 & 66.35 & 44.12 & 49.75 & 40.06 & 22.56 & 22.28 & 23.71 & 23.32 & 22.70 & 22.71 \\
\hline 4 & 7.01 & 8.01 & 8.01 & 0.88 & 0.73 & 21.57 & 98.89 & 66.40 & 44.10 & 49.77 & 40.06 & 22.57 & 22.28 & 23.73 & 23.33 & 22.69 & 22.71 \\
\hline 0 & 7.01 & 8.01 & 8.01 & 0.88 & 0.73 & 21.71 & 98.93 & 66.45 & 44.14 & 49.78 & 40.08 & 22.51 & 22.21 & 23.75 & 23.35 & 22.66 & 22.73 \\
\hline
\end{tabular}


Table D84: Averaged Raw Data for Mehra Electrode at $8 \mathrm{~W}$

\begin{tabular}{|c|c|c|c|c|c|c|c|c|c|c|c|c|c|c|c|c|c|}
\hline HV & $\mathbf{Q}$ & $\begin{array}{c}\text { Heater Voltage } \\
\text { after } \\
\text { precision } \\
\text { resistor } \\
\end{array}$ & $\begin{array}{c}\text { Heater Voltage } \\
\text { before } \\
\text { precision } \\
\text { resistor } \\
\end{array}$ & $\begin{array}{c}\text { Heater } \\
\text { Current }\end{array}$ & $\begin{array}{l}\text { Chamber } \\
\text { Pressure }\end{array}$ & Amb & T1 & $\mathrm{T} 2$ & T3 & $T 4$ & T5 & T8 & T9 & T10 & T11 & T12 & T14 \\
\hline $\mathrm{kV}$ & W & Volts & Volts & Amps & psia & ${ }^{\circ} \mathrm{C}$ & ${ }^{\circ} \mathrm{C}$ & ${ }^{\circ} \mathbf{C}$ & ${ }^{\circ} \mathrm{C}$ & ${ }^{\circ} \mathbf{C}$ & ${ }^{\circ} \mathbf{C}$ & ${ }^{\circ} \mathbf{C}$ & ${ }^{\circ} \mathbf{C}$ & ${ }^{\circ} \mathbf{C}$ & ${ }^{\circ} \mathbf{C}$ & ${ }^{\circ} \mathbf{C}$ & ${ }^{\circ} \mathbf{C}$ \\
\hline 0 & 8.00 & 8.60 & 8.60 & 0.93 & 0.73 & 21.56 & 109.18 & 73.57 & 48.05 & 54.01 & 43.10 & 22.55 & 22.27 & 23.98 & 23.58 & 22.70 & 22.80 \\
\hline 4 & 8.00 & 8.60 & 8.60 & 0.93 & 0.73 & 21.85 & 109.08 & 73.52 & 47.92 & 53.99 & 43.08 & 22.60 & 22.31 & 23.96 & 23.56 & 22.74 & 22.80 \\
\hline 8 & 8.00 & 8.60 & 8.60 & 0.93 & 0.73 & 21.87 & 108.94 & 75.05 & 47.88 & 53.91 & 43.06 & 22.68 & 22.38 & 23.92 & 23.54 & 22.81 & 22.78 \\
\hline 12 & 8.00 & 8.60 & 8.60 & 0.93 & 0.73 & 21.97 & 108.88 & 75.21 & 47.96 & 53.97 & 43.13 & 22.65 & 22.37 & 23.96 & 23.57 & 22.81 & 22.81 \\
\hline 16 & 7.92 & 8.60 & 8.60 & 0.92 & 0.73 & 21.38 & 108.75 & 69.60 & 47.93 & 53.95 & 43.08 & 22.69 & 22.42 & 23.96 & 23.59 & 22.81 & 22.77 \\
\hline 18 & 7.91 & 8.60 & 8.60 & 0.92 & 0.73 & 21.81 & 108.55 & 69.24 & 47.81 & 53.88 & 43.02 & 22.62 & 22.40 & 24.03 & 23.67 & 22.75 & 22.81 \\
\hline 20 & 7.91 & 8.60 & 8.60 & 0.92 & 0.73 & 21.69 & 108.44 & 69.08 & 47.81 & 53.89 & 43.02 & 22.63 & 22.40 & 24.13 & 23.76 & 22.77 & 22.86 \\
\hline 21 & 7.91 & 8.60 & 8.60 & 0.92 & 0.73 & 21.70 & 108.32 & 68.97 & 47.80 & 53.88 & 43.00 & 22.62 & 22.39 & 24.16 & 23.79 & 22.79 & 22.87 \\
\hline 22 & 7.91 & 8.60 & 8.60 & 0.92 & 0.73 & 21.45 & 108.23 & 68.92 & 47.74 & 53.85 & 42.95 & 22.64 & 22.40 & 24.13 & 23.75 & 22.80 & 22.85 \\
\hline 23 & 7.91 & 8.60 & 8.60 & 0.92 & 0.73 & 21.71 & 108.17 & 68.89 & 47.68 & 53.88 & 42.95 & 22.67 & 22.43 & 24.15 & 23.77 & 22.80 & 22.85 \\
\hline 22 & 7.96 & 8.60 & 8.60 & 0.93 & 0.73 & 21.71 & 108.09 & 68.84 & 47.81 & 53.89 & 42.98 & 22.72 & 22.47 & 24.12 & 23.75 & 22.75 & 22.82 \\
\hline 21 & 7.91 & 8.60 & 8.60 & 0.92 & 0.73 & 21.65 & 108.01 & 68.82 & 47.86 & 53.78 & 42.93 & 22.73 & 22.51 & 24.09 & 23.73 & 22.75 & 22.82 \\
\hline 20 & 7.91 & 8.60 & 8.60 & 0.92 & 0.73 & 21.63 & 108.02 & 68.85 & 47.97 & 53.81 & 42.98 & 22.72 & 22.49 & 24.07 & 23.72 & 22.77 & 22.81 \\
\hline 18 & 7.91 & 8.60 & 8.60 & 0.92 & 0.73 & 21.83 & 108.12 & 69.02 & 47.96 & 53.83 & 43.05 & 22.66 & 22.42 & 24.09 & 23.73 & 22.79 & 22.88 \\
\hline 16 & 7.91 & 8.60 & 8.60 & 0.92 & 0.73 & 21.70 & 108.24 & 69.21 & 47.99 & 53.87 & 43.08 & 22.64 & 22.42 & 24.04 & 23.66 & 22.79 & 22.89 \\
\hline 12 & 7.91 & 8.60 & 8.60 & 0.92 & 0.73 & 21.90 & 108.37 & 69.35 & 48.02 & 53.88 & 43.13 & 22.63 & 22.39 & 24.02 & 23.63 & 22.80 & 22.89 \\
\hline 8 & 7.91 & 8.60 & 8.60 & 0.92 & 0.73 & 21.72 & 108.46 & 69.51 & 48.00 & 53.87 & 43.11 & 22.65 & 22.41 & 23.97 & 23.59 & 22.81 & 22.88 \\
\hline 4 & 7.92 & 8.61 & 8.61 & 0.92 & 0.73 & 21.60 & 108.58 & 69.60 & 48.03 & 53.93 & 43.13 & 22.64 & 22.40 & 23.98 & 23.58 & 22.77 & 22.87 \\
\hline 0 & 7.92 & 8.61 & 8.61 & 0.92 & 0.73 & 21.79 & 108.68 & 69.70 & 48.12 & 53.94 & 43.17 & 22.60 & 22.37 & 24.04 & 23.63 & 22.73 & 22.90 \\
\hline
\end{tabular}


Table D85: Averaged Raw Data for Mehra Electrode at $9 \mathrm{~W}$

\begin{tabular}{|c|c|c|c|c|c|c|c|c|c|c|c|c|c|c|c|c|c|}
\hline HV & $\mathbf{Q}$ & $\begin{array}{c}\text { Heater Voltage } \\
\text { after } \\
\text { precision } \\
\text { resistor }\end{array}$ & $\begin{array}{c}\text { Heater Voltage } \\
\text { before } \\
\text { precision } \\
\text { resistor }\end{array}$ & $\begin{array}{c}\text { Heater } \\
\text { Current }\end{array}$ & $\begin{array}{l}\text { Chamber } \\
\text { Pressure }\end{array}$ & Amb & T1 & T2 & T3 & T4 & T5 & T8 & T9 & T10 & T11 & T12 & T14 \\
\hline $\mathrm{kV}$ & $\mathbf{W}$ & Volts & Volts & Amps & psia & ${ }^{\circ} \mathbf{C}$ & ${ }^{\circ} \mathrm{C}$ & ${ }^{\circ} \mathbf{C}$ & ${ }^{\circ} \mathbf{C}$ & ${ }^{\circ} \mathbf{C}$ & ${ }^{\circ} \mathbf{C}$ & ${ }^{\circ} \mathbf{C}$ & ${ }^{\circ} \mathbf{C}$ & ${ }^{\circ} \mathbf{C}$ & ${ }^{\circ} \mathrm{C}$ & ${ }^{\circ} \mathbf{C}$ & ${ }^{\circ} \mathbf{C}$ \\
\hline 0 & 8.93 & 9.30 & 9.30 & 0.96 & 0.73 & 21.52 & 118.68 & 74.93 & 51.96 & 59.20 & 46.12 & 22.53 & 22.33 & 24.12 & 23.71 & 22.68 & 22.83 \\
\hline 4 & 8.92 & 9.30 & 9.30 & 0.96 & 0.73 & 21.65 & 118.31 & 74.72 & 51.76 & 59.12 & 46.05 & 22.55 & 22.36 & 24.09 & 23.68 & 22.70 & 22.83 \\
\hline 8 & 8.92 & 9.30 & 9.30 & 0.96 & 0.73 & 21.54 & 118.19 & 74.62 & 51.63 & 59.12 & 46.03 & 22.54 & 22.35 & 24.04 & 23.65 & 22.70 & 22.79 \\
\hline 12 & 8.92 & 9.30 & 9.30 & 0.96 & 0.73 & 21.53 & 117.91 & 74.50 & 51.59 & 59.11 & 46.01 & 22.53 & 22.35 & 24.06 & 23.66 & 22.72 & 22.80 \\
\hline 16 & 8.92 & 9.30 & 9.30 & 0.96 & 0.73 & 21.58 & 117.51 & 74.32 & 51.44 & 59.02 & 45.93 & 22.55 & 22.39 & 24.09 & 23.71 & 22.72 & 22.76 \\
\hline 18 & 8.92 & 9.30 & 9.30 & 0.96 & 0.73 & 21.59 & 117.23 & 74.08 & 51.34 & 58.96 & 45.88 & 22.60 & 22.43 & 24.17 & 23.81 & 22.74 & 22.80 \\
\hline 20 & 8.92 & 9.30 & 9.30 & 0.96 & 0.73 & 21.65 & 116.84 & 73.63 & 51.10 & 58.81 & 45.71 & 22.55 & 22.38 & 24.15 & 23.80 & 22.67 & 22.75 \\
\hline 21 & 8.92 & 9.30 & 9.30 & 0.96 & 0.73 & 21.63 & 116.61 & 73.49 & 50.98 & 58.71 & 45.64 & 22.56 & 22.38 & 24.14 & 23.78 & 22.68 & 22.73 \\
\hline 22 & 8.92 & 9.30 & 9.30 & 0.96 & 0.73 & 21.59 & 116.55 & 73.41 & 50.97 & 58.75 & 45.65 & 22.57 & 22.38 & 24.19 & 23.82 & 22.68 & 22.77 \\
\hline 23 & 8.92 & 9.30 & 9.30 & 0.96 & 0.73 & 21.62 & 116.45 & 73.35 & 50.90 & 58.75 & 45.62 & 22.56 & 22.38 & 24.17 & 23.79 & 22.67 & 22.74 \\
\hline 22 & 8.92 & 9.30 & 9.30 & 0.96 & 0.73 & 21.58 & 116.42 & 73.29 & 50.84 & 58.70 & 45.56 & 22.57 & 22.40 & 24.08 & 23.71 & 22.63 & 22.69 \\
\hline 21 & 8.92 & 9.30 & 9.30 & 0.96 & 0.72 & 21.61 & 116.36 & 73.20 & 50.84 & 58.70 & 45.59 & 22.49 & 22.33 & 24.11 & 23.74 & 22.54 & 22.73 \\
\hline 20 & 8.92 & 9.30 & 9.30 & 0.96 & 0.72 & 21.52 & 116.45 & 73.09 & 50.83 & 58.76 & 45.61 & 22.51 & 22.37 & 24.09 & 23.73 & 22.62 & 22.72 \\
\hline 18 & 8.92 & 9.30 & 9.30 & 0.96 & 0.72 & 21.43 & 116.55 & 73.17 & 50.88 & 58.80 & 45.68 & 22.53 & 22.38 & 24.04 & 23.67 & 22.63 & 22.69 \\
\hline 16 & 8.92 & 9.30 & 9.30 & 0.96 & 0.72 & 21.40 & 116.53 & 73.46 & 50.78 & 58.71 & 45.65 & 22.48 & 22.32 & 23.98 & 23.60 & 22.62 & 22.67 \\
\hline 12 & 8.83 & 9.30 & 9.30 & 0.95 & 0.72 & 21.56 & 116.68 & 74.14 & 50.78 & 58.67 & 45.70 & 22.46 & 22.35 & 23.92 & 23.53 & 22.61 & 22.66 \\
\hline 8 & 8.83 & 9.30 & 9.30 & 0.95 & 0.72 & 21.61 & 116.72 & 74.20 & 50.78 & 58.69 & 45.70 & 22.40 & 22.26 & 23.91 & 23.52 & 22.54 & 22.68 \\
\hline 4 & 8.86 & 9.32 & 9.32 & 0.95 & 0.72 & 21.69 & 117.14 & 74.48 & 50.87 & 58.83 & 45.74 & 22.43 & 22.29 & 23.89 & 23.49 & 22.58 & 22.63 \\
\hline 0 & 8.90 & 9.37 & 9.37 & 0.95 & 0.72 & 21.31 & 118.05 & 75.01 & 51.29 & 59.22 & 45.97 & 22.46 & 22.28 & 23.89 & 23.49 & 22.59 & 22.62 \\
\hline
\end{tabular}


Table D86: Averaged Raw Data for Mehra Electrode at $10 \mathrm{~W}$

\begin{tabular}{|c|c|c|c|c|c|c|c|c|c|c|c|c|c|c|c|c|c|}
\hline HV & $\mathbf{Q}$ & $\begin{array}{c}\text { Heater Voltage } \\
\text { after } \\
\text { precision } \\
\text { resistor }\end{array}$ & $\begin{array}{c}\text { Heater Voltage } \\
\text { before } \\
\text { precision } \\
\text { resistor }\end{array}$ & $\begin{array}{c}\text { Heater } \\
\text { Current }\end{array}$ & $\begin{array}{l}\text { Chamber } \\
\text { Pressure }\end{array}$ & Amb & T1 & T2 & T3 & T4 & T5 & T8 & T9 & T10 & T11 & T12 & T14 \\
\hline $\mathbf{k V}$ & $\mathbf{W}$ & Volts & Volts & Amps & psia & ${ }^{\circ} \mathrm{C}$ & ${ }^{\circ} \mathrm{C}$ & ${ }^{\circ} \mathrm{C}$ & ${ }^{\circ} \mathrm{C}$ & ${ }^{\circ} \mathrm{C}$ & ${ }^{\circ} \mathrm{C}$ & ${ }^{\circ} \mathrm{C}$ & ${ }^{\circ} \mathbf{C}$ & ${ }^{\circ} \mathrm{C}$ & ${ }^{\circ} \mathrm{C}$ & ${ }^{\circ} \mathrm{C}$ & ${ }^{\circ} \mathrm{C}$ \\
\hline 0 & 10.07 & 9.97 & 9.97 & 1.01 & 0.72 & 21.10 & 128.41 & 81.34 & 56.56 & 64.20 & 49.61 & 22.36 & 22.22 & 24.09 & 23.70 & 22.50 & 22.64 \\
\hline 4 & 9.97 & 9.97 & 9.97 & 1.00 & 0.72 & 21.48 & 127.37 & 79.70 & 56.31 & 64.20 & 49.54 & 22.43 & 22.31 & 24.07 & 23.68 & 22.57 & 22.64 \\
\hline 8 & 10.07 & 9.97 & 9.97 & 1.01 & 0.72 & 21.59 & 127.27 & 78.11 & 55.95 & 64.06 & 49.53 & 22.39 & 22.25 & 24.05 & 23.68 & 22.56 & 22.65 \\
\hline 12 & 10.06 & 9.96 & 9.96 & 1.01 & 0.72 & 21.64 & 126.96 & 77.88 & 55.87 & 63.89 & 49.53 & 22.40 & 22.26 & 24.08 & 23.69 & 22.62 & 22.65 \\
\hline 16 & 10.09 & 9.99 & 9.99 & 1.01 & 0.72 & 21.69 & 126.70 & 77.33 & 55.67 & 64.01 & 49.51 & 22.44 & 22.30 & 24.13 & 23.78 & 22.61 & 22.67 \\
\hline 18 & 10.10 & 10.00 & 10.00 & 1.01 & 0.72 & 21.40 & 126.14 & 75.93 & 55.61 & 64.09 & 49.44 & 22.45 & 22.29 & 24.17 & 23.83 & 22.60 & 22.65 \\
\hline 20 & 10.00 & 10.00 & 10.00 & 1.00 & 0.72 & 21.60 & 125.34 & 74.93 & 55.36 & 63.94 & 49.27 & 22.45 & 22.25 & 24.21 & 23.87 & 22.55 & 22.67 \\
\hline 21 & 9.99 & 9.99 & 9.99 & 1.00 & 0.72 & 21.23 & 125.01 & 74.85 & 55.14 & 63.80 & 49.14 & 22.44 & 22.24 & 24.20 & 23.84 & 22.58 & 22.63 \\
\hline 22 & 9.99 & 9.99 & 9.99 & 1.00 & 0.72 & 20.90 & 124.62 & 75.07 & 55.13 & 63.85 & 49.11 & 22.45 & 22.28 & 24.16 & 23.80 & 22.55 & 22.58 \\
\hline 23 & 9.99 & 9.99 & 9.99 & 1.00 & 0.72 & 21.24 & 124.37 & 74.97 & 54.97 & 63.78 & 49.04 & 22.36 & 22.19 & 24.18 & 23.81 & 22.50 & 22.59 \\
\hline 22 & 9.99 & 9.99 & 9.99 & 1.00 & 0.72 & 21.08 & 124.66 & 75.55 & 55.26 & 63.53 & 49.07 & 22.39 & 22.24 & 24.11 & 23.76 & 22.52 & 22.55 \\
\hline 21 & 9.99 & 9.99 & 9.99 & 1.00 & 0.72 & 21.00 & 124.62 & 75.58 & 55.19 & 63.51 & 49.05 & 22.35 & 22.22 & 24.11 & 23.76 & 22.44 & 22.52 \\
\hline 20 & 10.01 & 10.01 & 10.01 & 1.00 & 0.72 & 20.88 & 124.74 & 76.03 & 55.20 & 63.57 & 49.10 & 22.38 & 22.25 & 24.06 & 23.72 & 22.51 & 22.49 \\
\hline 18 & 10.01 & 10.01 & 10.01 & 1.00 & 0.72 & 21.04 & 124.98 & 77.64 & 55.22 & 63.61 & 49.19 & 22.35 & 22.25 & 24.01 & 23.68 & 22.49 & 22.50 \\
\hline 16 & 10.04 & 10.04 & 10.04 & 1.00 & 0.72 & 21.00 & 125.47 & 79.33 & 55.33 & 63.86 & 49.36 & 22.33 & 22.22 & 23.97 & 23.62 & 22.50 & 22.49 \\
\hline 12 & 10.06 & 10.06 & 10.06 & 1.00 & 0.72 & 21.32 & 126.30 & 80.35 & 55.77 & 64.10 & 49.60 & 22.31 & 22.20 & 23.92 & 23.54 & 22.50 & 22.49 \\
\hline 8 & 10.06 & 10.06 & 10.06 & 1.00 & 0.72 & 21.13 & 126.40 & 80.50 & 55.66 & 64.13 & 49.61 & 22.28 & 22.15 & 23.91 & 23.56 & 22.46 & 22.50 \\
\hline 4 & 10.09 & 10.09 & 10.09 & 1.00 & 0.72 & 20.90 & 126.74 & 80.40 & 55.61 & 64.27 & 49.62 & 22.31 & 22.19 & 23.84 & 23.47 & 22.47 & 22.42 \\
\hline 0 & 10.11 & 10.11 & 10.11 & 1.00 & 0.72 & 20.98 & 127.38 & 81.13 & 56.07 & 64.40 & 49.82 & 22.22 & 22.10 & 23.88 & 23.50 & 22.38 & 22.44 \\
\hline
\end{tabular}


Table D87: Averaged Raw Data for Mehra Electrode at $11 \mathrm{~W}$ (first)

\begin{tabular}{|c|c|c|c|c|c|c|c|c|c|c|c|c|c|c|c|c|c|}
\hline HV & $\mathbf{Q}$ & $\begin{array}{c}\text { Heater Voltage } \\
\text { after } \\
\text { precision } \\
\text { resistor }\end{array}$ & $\begin{array}{c}\text { Heater Voltage } \\
\text { before } \\
\text { precision } \\
\text { resistor }\end{array}$ & $\begin{array}{c}\text { Heater } \\
\text { Current }\end{array}$ & $\begin{array}{l}\text { Chamber } \\
\text { Pressure }\end{array}$ & Amb & T1 & T2 & T3 & T4 & T5 & T8 & T9 & T10 & T11 & T12 & T14 \\
\hline $\mathbf{k V}$ & $\mathbf{W}$ & Volts & Volts & Amps & psia & ${ }^{\circ} \mathrm{C}$ & ${ }^{\circ} \mathrm{C}$ & ${ }^{\circ} \mathbf{C}$ & ${ }^{\circ} \mathrm{C}$ & ${ }^{\circ} \mathrm{C}$ & ${ }^{\circ} \mathrm{C}$ & ${ }^{\circ} \mathrm{C}$ & ${ }^{\circ} \mathrm{C}$ & ${ }^{\circ} \mathrm{C}$ & ${ }^{\circ} \mathrm{C}$ & ${ }^{\circ} \mathbf{C}$ & ${ }^{\circ} \mathrm{C}$ \\
\hline 0 & 10.96 & 10.54 & 10.54 & 1.04 & 0.72 & 20.92 & 135.01 & 85.54 & 58.22 & 67.56 & 52.42 & 22.09 & 22.00 & 23.78 & 23.39 & 22.25 & 22.23 \\
\hline 4 & 10.95 & 10.53 & 10.53 & 1.04 & 0.72 & 20.79 & 134.93 & 85.55 & 58.70 & 67.50 & 52.54 & 22.08 & 22.01 & 23.77 & 23.41 & 22.24 & 22.21 \\
\hline 8 & 10.95 & 10.53 & 10.53 & 1.04 & 0.71 & 20.94 & 133.75 & 84.61 & 58.33 & 67.52 & 52.32 & 22.08 & 22.01 & 23.68 & 23.34 & 22.25 & 22.18 \\
\hline 12 & 11.00 & 10.57 & 10.57 & 1.04 & 0.71 & 20.82 & 133.03 & 83.52 & 58.34 & 67.49 & 52.14 & 22.01 & 21.97 & 23.62 & 23.26 & 22.20 & 22.11 \\
\hline 16 & 11.03 & 10.61 & 10.61 & 1.04 & 0.71 & 20.64 & 132.24 & 82.46 & 58.91 & 67.60 & 52.00 & 21.94 & 21.88 & 23.58 & 23.24 & 22.12 & 22.04 \\
\hline 18 & 10.90 & 10.58 & 10.58 & 1.03 & 0.71 & 20.90 & 131.17 & 80.92 & 58.81 & 67.45 & 51.81 & 21.81 & 21.76 & 23.63 & 23.32 & 21.99 & 22.03 \\
\hline 20 & 10.91 & 10.59 & 10.59 & 1.03 & 0.71 & 20.78 & 130.45 & 80.35 & 58.58 & 67.36 & 51.60 & 21.82 & 21.76 & 23.68 & 23.36 & 22.03 & 22.01 \\
\hline 21 & 10.92 & 10.60 & 10.60 & 1.03 & 0.71 & 20.74 & 130.04 & 79.69 & 58.35 & 67.05 & 51.35 & 21.85 & 21.78 & 23.64 & 23.30 & 22.06 & 21.97 \\
\hline 22 & 10.93 & 10.61 & 10.61 & 1.03 & 0.71 & 20.52 & 129.74 & 79.26 & 58.01 & 66.88 & 51.11 & 21.70 & 21.67 & 23.53 & 23.17 & 21.90 & 21.91 \\
\hline 23 & 10.93 & 10.61 & 10.61 & 1.03 & 0.71 & 20.85 & 130.00 & 79.24 & 57.94 & 66.89 & 51.10 & 21.73 & 21.65 & 23.66 & 23.29 & 21.97 & 21.97 \\
\hline 22 & 11.02 & 10.70 & 10.70 & 1.03 & 0.71 & 20.24 & 130.48 & 79.90 & 58.31 & 67.00 & 51.23 & 21.73 & 21.62 & 23.47 & 23.13 & 21.92 & 21.85 \\
\hline 21 & 10.93 & 10.71 & 10.71 & 1.02 & 0.71 & 20.33 & 130.51 & 79.76 & 58.19 & 67.23 & 51.21 & 21.61 & 21.54 & 23.45 & 23.13 & 21.80 & 21.83 \\
\hline 20 & 10.93 & 10.71 & 10.71 & 1.02 & 0.71 & 20.72 & 131.04 & 80.10 & 58.21 & 67.23 & 51.33 & 21.53 & 21.48 & 23.46 & 23.15 & 21.72 & 21.81 \\
\hline 18 & 10.93 & 10.72 & 10.72 & 1.02 & 0.71 & 20.08 & 130.85 & 80.25 & 58.17 & 67.37 & 51.27 & 21.54 & 21.45 & 23.30 & 22.98 & 21.74 & 21.74 \\
\hline 16 & 10.99 & 10.77 & 10.77 & 1.02 & 0.71 & 20.12 & 131.25 & 81.31 & 58.53 & 67.75 & 51.43 & 21.40 & 21.36 & 23.20 & 22.86 & 21.60 & 21.72 \\
\hline 12 & 11.06 & 10.84 & 10.84 & 1.02 & 0.71 & 20.71 & 133.26 & 82.72 & 59.46 & 68.38 & 52.07 & 21.40 & 21.41 & 23.30 & 22.95 & 21.62 & 21.81 \\
\hline 8 & 11.08 & 10.86 & 10.86 & 1.02 & 0.71 & 20.75 & 133.47 & 82.68 & 59.71 & 68.59 & 52.18 & 21.48 & 21.45 & 23.27 & 22.94 & 21.69 & 21.77 \\
\hline 4 & 11.10 & 10.88 & 10.89 & 1.02 & 0.71 & 20.26 & 133.14 & 82.60 & 59.68 & 68.69 & 52.05 & 21.52 & 21.47 & 23.17 & 22.82 & 21.70 & 21.68 \\
\hline 0 & 10.98 & 10.76 & 10.76 & 1.02 & 0.71 & 20.04 & 133.34 & 82.59 & 60.35 & 68.32 & 52.24 & 21.46 & 21.42 & 23.16 & 22.79 & 21.62 & 21.65 \\
\hline
\end{tabular}


Table D88: Averaged Raw Data for Mehra Electrode at $11 \mathrm{~W}$ (second)

\begin{tabular}{|c|c|c|c|c|c|c|c|c|c|c|c|c|c|c|c|c|c|}
\hline HV & $\mathbf{Q}$ & $\begin{array}{c}\text { Heater Voltage } \\
\text { after } \\
\text { precision } \\
\text { resistor }\end{array}$ & $\begin{array}{c}\text { Heater Voltage } \\
\text { before } \\
\text { precision } \\
\text { resistor }\end{array}$ & $\begin{array}{c}\text { Heater } \\
\text { Current }\end{array}$ & $\begin{array}{l}\text { Chamber } \\
\text { Pressure }\end{array}$ & Amb & T1 & $\mathrm{T} 2$ & T3 & T4 & T5 & T8 & T9 & T10 & T11 & T12 & T14 \\
\hline $\mathrm{kV}$ & $\mathbf{W}$ & Volts & Volts & Amps & psia & ${ }^{\circ} \mathbf{C}$ & ${ }^{\circ} \mathrm{C}$ & ${ }^{\circ} \mathbf{C}$ & ${ }^{\circ} \mathrm{C}$ & ${ }^{\circ} \mathbf{C}$ & ${ }^{\circ} \mathrm{C}$ & ${ }^{\circ} \mathbf{C}$ & ${ }^{\circ} \mathbf{C}$ & ${ }^{\circ} \mathbf{C}$ & ${ }^{\circ} \mathbf{C}$ & ${ }^{\circ} \mathbf{C}$ & ${ }^{\circ} \mathbf{C}$ \\
\hline 0 & 11.00 & 10.68 & 10.68 & 1.03 & 0.72 & 19.71 & 127.25 & 76.17 & 59.65 & 68.69 & 50.41 & 20.13 & 19.78 & 22.04 & 21.70 & 20.31 & 19.78 \\
\hline 4 & 11.01 & 10.69 & 10.69 & 1.03 & 0.72 & 20.08 & 127.00 & 76.66 & 59.86 & 67.61 & 50.53 & 20.29 & 19.93 & 22.14 & 21.77 & 20.45 & 19.93 \\
\hline 8 & 10.99 & 10.67 & 10.67 & 1.03 & 0.72 & 19.84 & 127.04 & 76.36 & 58.81 & 66.71 & 50.35 & 20.43 & 20.00 & 22.14 & 21.75 & 20.63 & 20.00 \\
\hline 12 & 10.88 & 10.67 & 10.67 & 1.02 & 0.73 & 20.20 & 126.43 & 75.65 & 58.29 & 66.32 & 50.20 & 20.44 & 20.08 & 22.22 & 21.88 & 20.53 & 20.08 \\
\hline 16 & 10.88 & 10.67 & 10.67 & 1.02 & 0.73 & 20.35 & 126.07 & 74.98 & 57.92 & 66.15 & 50.05 & 20.48 & 20.16 & 22.31 & 21.98 & 20.59 & 20.16 \\
\hline 18 & 10.79 & 10.68 & 10.68 & 1.01 & 0.73 & 20.32 & 124.79 & 74.03 & 57.49 & 65.67 & 49.74 & 20.60 & 20.23 & 22.39 & 22.05 & 20.79 & 20.23 \\
\hline 20 & 10.85 & 10.63 & 10.63 & 1.02 & 0.73 & 20.07 & 125.94 & 74.36 & 56.95 & 64.77 & 49.50 & 20.72 & 20.37 & 22.46 & 22.28 & 20.86 & 20.37 \\
\hline 21 & 10.84 & 10.63 & 10.63 & 1.02 & 0.73 & 20.33 & 125.57 & 74.14 & 56.75 & 64.68 & 49.43 & 20.75 & 20.43 & 22.62 & 22.47 & 21.03 & 20.43 \\
\hline 22 & 10.87 & 10.65 & 10.65 & 1.02 & 0.73 & 20.10 & 125.65 & 74.46 & 56.74 & 64.80 & 49.44 & 20.83 & 20.48 & 22.61 & 22.50 & 21.12 & 20.48 \\
\hline 23 & 10.88 & 10.67 & 10.67 & 1.02 & 0.73 & 20.37 & 125.99 & 74.50 & 56.83 & 64.86 & 49.58 & 20.85 & 20.47 & 22.74 & 22.77 & 21.17 & 20.47 \\
\hline 22 & 10.89 & 10.68 & 10.68 & 1.02 & 0.73 & 20.34 & 125.99 & 74.24 & 56.76 & 64.85 & 49.56 & 20.80 & 20.37 & 22.72 & 22.53 & 21.06 & 20.37 \\
\hline 21 & 10.92 & 10.70 & 10.70 & 1.02 & 0.73 & 20.78 & 126.40 & 74.53 & 56.92 & 64.97 & 49.74 & 20.80 & 20.37 & 22.84 & 22.59 & 21.08 & 20.37 \\
\hline 20 & 10.94 & 10.72 & 10.72 & 1.02 & 0.73 & 20.62 & 126.82 & 75.07 & 56.94 & 65.08 & 49.87 & 20.99 & 20.51 & 22.85 & 22.60 & 21.22 & 20.51 \\
\hline 18 & 10.98 & 10.76 & 10.76 & 1.02 & 0.73 & 20.32 & 127.51 & 75.59 & 57.29 & 65.31 & 50.07 & 21.04 & 20.57 & 22.74 & 22.44 & 21.24 & 20.57 \\
\hline 16 & 10.91 & 10.80 & 10.80 & 1.01 & 0.73 & 20.39 & 127.47 & 76.34 & 57.74 & 65.66 & 50.33 & 21.04 & 20.62 & 22.76 & 22.45 & 21.22 & 20.62 \\
\hline 12 & 10.91 & 10.80 & 10.80 & 1.01 & 0.73 & 20.56 & 127.93 & 77.77 & 58.13 & 65.47 & 50.60 & 21.00 & 20.61 & 22.74 & 22.38 & 21.21 & 20.61 \\
\hline 8 & 10.92 & 10.82 & 10.82 & 1.01 & 0.73 & 20.93 & 127.89 & 77.88 & 58.14 & 65.69 & 50.72 & 20.99 & 20.60 & 22.94 & 22.55 & 21.24 & 20.60 \\
\hline 4 & 11.00 & 10.89 & 10.89 & 1.01 & 0.73 & 21.07 & 128.82 & 78.57 & 58.69 & 66.16 & 51.02 & 21.09 & 20.61 & 23.06 & 22.63 & 21.37 & 20.61 \\
\hline 0 & 10.98 & 10.87 & 10.87 & 1.01 & 0.73 & 20.87 & 128.36 & 78.33 & 58.44 & 65.93 & 50.91 & 21.20 & 20.65 & 23.01 & 22.68 & 21.46 & 20.65 \\
\hline
\end{tabular}


Table D89: Averaged Raw Data for Mehra Electrode at $12 \mathrm{~W}$

\begin{tabular}{|c|c|c|c|c|c|c|c|c|c|c|c|c|c|c|c|c|c|}
\hline HV & $\mathbf{Q}$ & $\begin{array}{c}\text { Heater Voltage } \\
\text { after } \\
\text { precision } \\
\text { resistor }\end{array}$ & $\begin{array}{c}\text { Heater Voltage } \\
\text { before } \\
\text { precision } \\
\text { resistor }\end{array}$ & $\begin{array}{l}\text { Heater } \\
\text { Current }\end{array}$ & $\begin{array}{l}\text { Chamber } \\
\text { Pressure }\end{array}$ & Amb & T1 & T2 & T3 & T4 & T5 & T8 & T9 & T10 & T11 & T12 & T14 \\
\hline $\mathbf{k V}$ & $\mathbf{W}$ & Volts & Volts & Amps & psia & ${ }^{\circ} \mathrm{C}$ & ${ }^{\circ} \mathrm{C}$ & ${ }^{\circ} \mathrm{C}$ & ${ }^{\circ} \mathrm{C}$ & ${ }^{\circ} \mathrm{C}$ & ${ }^{\circ} \mathrm{C}$ & ${ }^{\circ} \mathbf{C}$ & ${ }^{\circ} \mathrm{C}$ & ${ }^{\circ} \mathbf{C}$ & ${ }^{\circ} \mathrm{C}$ & ${ }^{\circ} \mathrm{C}$ & ${ }^{\circ} \mathbf{C}$ \\
\hline 0 & 12.07 & 11.39 & 11.39 & 1.06 & 0.74 & 21.03 & 139.42 & 85.71 & 63.52 & 70.32 & 54.59 & 21.57 & 20.91 & 23.48 & 23.12 & 22.00 & 21.81 \\
\hline 4 & 11.96 & 11.39 & 11.39 & 1.05 & 0.74 & 21.22 & 139.15 & 85.40 & 62.90 & 70.32 & 54.49 & 21.62 & 20.96 & 23.50 & 23.15 & 22.01 & 21.89 \\
\hline 8 & 11.96 & 11.39 & 11.39 & 1.05 & 0.74 & 21.32 & 139.00 & 85.15 & 62.67 & 70.44 & 54.50 & 21.67 & 21.02 & 23.46 & 23.12 & 22.00 & 21.93 \\
\hline 12 & 11.96 & 11.39 & 11.39 & 1.05 & 0.74 & 21.45 & 139.16 & 84.85 & 62.66 & 70.29 & 54.55 & 21.71 & 21.05 & 23.47 & 23.15 & 22.02 & 21.99 \\
\hline 16 & 11.96 & 11.39 & 11.39 & 1.05 & 0.74 & 21.26 & 137.92 & 83.23 & 62.29 & 70.23 & 54.25 & 21.84 & 21.15 & 23.57 & 23.27 & 22.14 & 22.01 \\
\hline 18 & 11.96 & 11.39 & 11.39 & 1.05 & 0.74 & 21.52 & 137.25 & 82.79 & 62.04 & 69.92 & 54.23 & 21.82 & 21.14 & 23.69 & 23.41 & 22.21 & 22.08 \\
\hline 20 & 11.85 & 11.39 & 11.39 & 1.04 & 0.74 & 21.33 & 136.49 & 82.04 & 61.62 & 69.79 & 53.90 & 21.84 & 21.18 & 23.78 & 23.56 & 22.47 & 22.12 \\
\hline 21 & 11.85 & 11.39 & 11.39 & 1.04 & 0.74 & 21.49 & 136.34 & 81.90 & 61.51 & 69.81 & 53.90 & 21.89 & 21.23 & 23.89 & 23.63 & 22.40 & 22.18 \\
\hline 22 & 11.96 & 11.39 & 11.39 & 1.05 & 0.74 & 21.23 & 137.15 & 82.88 & 61.70 & 69.44 & 54.03 & 21.92 & 21.30 & 24.00 & 23.62 & 22.32 & 22.16 \\
\hline 23 & 11.96 & 11.39 & 11.39 & 1.05 & 0.74 & 21.34 & 137.78 & 82.79 & 61.56 & 69.80 & 53.60 & 21.96 & 21.37 & 24.05 & 23.85 & 22.12 & 22.25 \\
\hline 22 & 11.96 & 11.39 & 11.39 & 1.05 & 0.74 & 21.56 & 137.69 & 82.87 & 61.65 & 69.83 & 53.63 & 21.99 & 21.41 & 24.11 & 23.77 & 22.16 & 22.31 \\
\hline 21 & 11.85 & 11.39 & 11.39 & 1.04 & 0.74 & 21.46 & 137.20 & 82.58 & 61.58 & 69.84 & 53.49 & 22.03 & 21.44 & 24.02 & 23.73 & 22.34 & 22.26 \\
\hline 20 & 11.85 & 11.39 & 11.39 & 1.04 & 0.74 & 21.42 & 136.90 & 82.36 & 61.52 & 69.97 & 53.48 & 22.06 & 21.47 & 23.97 & 23.71 & 22.35 & 22.28 \\
\hline 18 & 11.85 & 11.39 & 11.39 & 1.04 & 0.74 & 21.57 & 138.15 & 82.86 & 61.38 & 70.48 & 53.63 & 22.09 & 21.50 & 24.00 & 23.70 & 22.29 & 22.34 \\
\hline 16 & 11.85 & 11.39 & 11.39 & 1.04 & 0.74 & 21.47 & 138.39 & 83.52 & 61.65 & 70.70 & 53.81 & 22.13 & 21.54 & 23.91 & 23.62 & 22.38 & 22.30 \\
\hline 12 & 11.96 & 11.39 & 11.39 & 1.05 & 0.74 & 21.44 & 139.61 & 85.90 & 62.20 & 70.59 & 54.13 & 22.12 & 21.56 & 23.90 & 23.57 & 22.36 & 22.34 \\
\hline 8 & 11.85 & 11.39 & 11.39 & 1.04 & 0.74 & 21.50 & 139.52 & 85.92 & 62.07 & 70.65 & 54.10 & 22.13 & 21.60 & 24.00 & 23.62 & 22.39 & 22.35 \\
\hline 4 & 11.85 & 11.39 & 11.39 & 1.04 & 0.74 & 21.35 & 139.42 & 85.63 & 62.05 & 70.67 & 54.00 & 22.14 & 21.62 & 24.00 & 23.59 & 22.40 & 22.37 \\
\hline 0 & 11.85 & 11.39 & 11.39 & 1.04 & 0.74 & 21.80 & 139.24 & 85.50 & 62.10 & 70.75 & 54.04 & 22.07 & 21.55 & 24.04 & 23.73 & 22.33 & 22.49 \\
\hline
\end{tabular}


Table D90: Reduced Data for Mehra Electrode at $0 \mathrm{~W}$

\begin{tabular}{|c|c|c|c|c|c|c|c|c|c|c|c|c|c|c|c|c|c|}
\hline HV & $\mathbf{T}_{\text {sat }}$ & $\mathbf{Q}$ & $\mathbf{q}$ & $\begin{array}{l}\text { T2 thru T5 } \\
\text { Average }\end{array}$ & $\Delta \mathbf{T}_{\text {alumina }}$ & $\mathbf{T}_{\mathrm{s}}$ & $\mathbf{T}_{\infty_{\text {wall }}}$ & $\mathbf{T}_{\text {otop }}$ & $T \infty_{\text {top }}$ & $\begin{array}{c}\mathbf{T}_{\mathbf{s}^{-}} \\
\mathbf{T}_{\text {otop }}\end{array}$ & $\begin{array}{l}\mathbf{T}_{\text {sat }} \\
\mathbf{T}_{\text {owall }}\end{array}$ & $\begin{array}{l}\mathbf{T}_{\text {sat }} \\
\mathbf{T}_{\text {otop }}\end{array}$ & $\mathbf{k}_{\text {fluid }}$ & h & $\begin{array}{c}\theta_{\mathrm{s}^{-}} \\
\theta_{\text {otop }}\end{array}$ & $\mathrm{Nu}$ & GD \\
\hline $\mathrm{kV}$ & ${ }^{\circ} \mathrm{C}$ & W & $10^{4} \mathrm{~W} / \mathrm{m}^{2}$ & ${ }^{\circ} \mathrm{C}$ & ${ }^{\circ} \mathrm{C}$ & ${ }^{\circ} \mathrm{C}$ & ${ }^{\circ} \mathrm{C}$ & ${ }^{\circ} \mathrm{C}$ & $\mathbf{K}$ & ${ }^{\circ} \mathrm{C}$ & ${ }^{\circ} \mathrm{C}$ & ${ }^{\circ} \mathrm{C}$ & $\mathbf{W} / \mathbf{m}-\mathbf{K}$ & $\begin{array}{c}\mathbf{W} / \mathbf{m}^{2}- \\
\mathbf{K}\end{array}$ & -- & --- & --- \\
\hline 0 & 36.36 & 0.00 & 0.00 & 21.50 & 0.00 & 21.50 & 21.78 & 21.79 & 294.94 & -0.29 & 14.58 & 14.56 & 0.076 & 0 & -0.02 & 0 & 0.00 \\
\hline 4 & 36.36 & 0.00 & 0.00 & 21.52 & 0.00 & 21.52 & 21.80 & 21.82 & 294.97 & -0.30 & 14.56 & 14.54 & 0.076 & 0 & -0.02 & 0 & 0.00 \\
\hline 8 & 36.38 & 0.00 & 0.00 & 21.56 & 0.00 & 21.56 & 21.85 & 21.86 & 295.01 & -0.30 & 14.52 & 14.52 & 0.076 & 0 & -0.02 & 0 & 0.00 \\
\hline 12 & 36.39 & 0.00 & 0.00 & 21.63 & 0.00 & 21.63 & 21.93 & 21.91 & 295.06 & -0.28 & 14.47 & 14.49 & 0.076 & 0 & -0.02 & 0 & 0.00 \\
\hline 16 & 36.39 & 0.00 & 0.00 & 21.65 & 0.00 & 21.65 & 21.93 & 21.91 & 295.06 & -0.26 & 14.46 & 14.48 & 0.076 & 0 & -0.02 & 0 & 0.00 \\
\hline 18 & 36.43 & 0.00 & 0.00 & 21.72 & 0.00 & 21.72 & 22.03 & 21.98 & 295.13 & -0.27 & 14.41 & 14.45 & 0.075 & 0 & -0.02 & 0 & 0.00 \\
\hline 20 & 36.45 & 0.00 & 0.00 & 21.72 & 0.00 & 21.72 & 22.02 & 21.97 & 295.12 & -0.25 & 14.43 & 14.48 & 0.075 & 0 & -0.02 & 0 & 0.00 \\
\hline 21 & 36.49 & 0.00 & 0.00 & 21.82 & 0.00 & 21.82 & 22.10 & 22.05 & 295.20 & -0.23 & 14.39 & 14.44 & 0.075 & 0 & -0.02 & 0 & 0.00 \\
\hline 22 & 36.51 & 0.00 & 0.00 & 21.81 & 0.00 & 21.81 & 22.11 & 22.06 & 295.21 & -0.24 & 14.39 & 14.45 & 0.075 & 0 & -0.02 & 0 & 0.00 \\
\hline 23 & 36.52 & 0.00 & 0.00 & 21.86 & 0.00 & 21.86 & 22.17 & 22.09 & 295.24 & -0.23 & 14.36 & 14.43 & 0.075 & 0 & -0.02 & 0 & 0.00 \\
\hline 22 & 36.52 & 0.00 & 0.00 & 21.85 & 0.00 & 21.85 & 22.17 & 22.11 & 295.26 & -0.25 & 14.35 & 14.42 & 0.075 & 0 & -0.02 & 0 & 0.00 \\
\hline 21 & 36.52 & 0.00 & 0.00 & 21.92 & 0.00 & 21.92 & 22.25 & 22.18 & 295.33 & -0.26 & 14.28 & 14.35 & 0.075 & 0 & -0.02 & 0 & 0.00 \\
\hline 20 & 36.52 & 0.00 & 0.00 & 21.83 & 0.00 & 21.83 & 22.13 & 22.08 & 295.23 & -0.25 & 14.40 & 14.45 & 0.075 & 0 & -0.02 & 0 & 0.00 \\
\hline 18 & 36.51 & 0.00 & 0.00 & 21.83 & 0.00 & 21.83 & 22.12 & 22.08 & 295.23 & -0.25 & 14.39 & 14.42 & 0.075 & 0 & -0.02 & 0 & 0.00 \\
\hline 16 & 36.49 & 0.00 & 0.00 & 21.93 & 0.00 & 21.93 & 22.21 & 22.16 & 295.31 & -0.23 & 14.27 & 14.33 & 0.075 & 0 & -0.02 & 0 & 0.00 \\
\hline 12 & 36.49 & 0.00 & 0.00 & 21.95 & 0.00 & 21.95 & 22.20 & 22.15 & 295.30 & -0.20 & 14.28 & 14.34 & 0.075 & 0 & -0.01 & 0 & 0.00 \\
\hline 8 & 36.49 & 0.00 & 0.00 & 21.93 & 0.00 & 21.93 & 22.20 & 22.16 & 295.31 & -0.22 & 14.29 & 14.33 & 0.075 & 0 & -0.02 & 0 & 0.00 \\
\hline 4 & 36.49 & 0.00 & 0.00 & 21.90 & 0.00 & 21.90 & 22.16 & 22.12 & 295.27 & -0.22 & 14.33 & 14.37 & 0.075 & 0 & -0.02 & 0 & 0.00 \\
\hline 0 & 36.47 & 0.00 & 0.00 & 21.93 & 0.00 & 21.93 & 22.17 & 22.14 & 295.29 & -0.21 & 14.30 & 14.33 & 0.075 & 0 & -0.01 & 0 & 0.00 \\
\hline
\end{tabular}


Table D91: Reduced Data for Mehra Electrode at $1 \mathrm{~W}$

\begin{tabular}{|c|c|c|c|c|c|c|c|c|c|c|c|c|c|c|c|c|c|}
\hline HV & $\mathbf{T}_{\text {sat }}$ & $\mathbf{Q}$ & $\mathbf{q}$ & $\begin{array}{l}\text { T2 thru } \\
\text { T5 } \\
\text { Average }\end{array}$ & $\Delta \mathbf{T}_{\text {alumina }}$ & $\mathbf{T}_{\mathrm{s}}$ & $\mathbf{T}_{\text {owall }}$ & $\mathbf{T}_{\text {otop }}$ & $\mathbf{T} \infty_{\text {top }}$ & $\begin{array}{c}T_{s^{-}} \\
T_{\text {otop }}\end{array}$ & $\begin{array}{c}\mathbf{T}_{\text {sat }} \\
\mathbf{T}_{\text {owall }}\end{array}$ & $\begin{array}{l}\mathbf{T}_{\text {sat }} \\
\mathbf{T}_{\text {otop }}\end{array}$ & $\mathbf{k}_{\text {fluid }}$ & $\mathbf{h}$ & $\begin{array}{c}\theta_{\mathrm{s}}= \\
\theta_{\text {otop }}\end{array}$ & $\mathrm{Nu}$ & GD \\
\hline $\mathbf{k V}$ & ${ }^{\circ} \mathrm{C}$ & $\mathbf{W}$ & $10^{4} \mathrm{~W} / \mathrm{m}^{2}$ & ${ }^{\circ} \mathrm{C}$ & ${ }^{\circ} \mathrm{C}$ & ${ }^{\circ} \mathrm{C}$ & ${ }^{\circ} \mathrm{C}$ & ${ }^{\circ} \mathrm{C}$ & $\mathbf{K}$ & ${ }^{\circ} \mathrm{C}$ & ${ }^{\circ} \mathbf{C}$ & ${ }^{\circ} \mathrm{C}$ & W/m-K & $\begin{array}{c}\mathbf{W} / \mathbf{m}^{2}- \\
\mathbf{K}\end{array}$ & --- & --- & --- \\
\hline 0 & 36.45 & 0.98 & 0.67 & 25.62 & 0.00 & 25.62 & 22.36 & 22.25 & 295.40 & 3.36 & 14.09 & 14.20 & 0.075 & 1961 & 0.24 & 208 & 2.63 \\
\hline 4 & 36.45 & 0.98 & 0.67 & 25.57 & 0.00 & 25.57 & 22.29 & 22.18 & 295.33 & 3.40 & 14.16 & 14.27 & 0.075 & 1943 & 0.24 & 206 & 2.62 \\
\hline 8 & 36.45 & 0.98 & 0.67 & 25.52 & 0.00 & 25.52 & 22.22 & 22.12 & 295.27 & 3.39 & 14.23 & 14.33 & 0.075 & 1945 & 0.24 & 206 & 2.61 \\
\hline 12 & 36.45 & 0.98 & 0.67 & 25.54 & 0.00 & 25.54 & 22.24 & 22.13 & 295.28 & 3.41 & 14.21 & 14.32 & 0.075 & 1937 & 0.24 & 205 & 2.61 \\
\hline 16 & 36.45 & 0.98 & 0.67 & 25.53 & 0.00 & 25.53 & 22.20 & 22.08 & 295.23 & 3.45 & 14.25 & 14.37 & 0.075 & 1914 & 0.24 & 203 & 2.60 \\
\hline 18 & 36.45 & 0.98 & 0.67 & 25.52 & 0.00 & 25.52 & 22.23 & 22.10 & 295.25 & 3.42 & 14.22 & 14.35 & 0.075 & 1929 & 0.24 & 205 & 2.61 \\
\hline 20 & 36.43 & 0.98 & 0.67 & 25.48 & 0.00 & 25.48 & 22.19 & 22.07 & 295.22 & 3.41 & 14.24 & 14.36 & 0.075 & 1933 & 0.24 & 205 & 2.60 \\
\hline 21 & 36.41 & 0.98 & 0.67 & 25.51 & 0.00 & 25.51 & 22.21 & 22.09 & 295.24 & 3.42 & 14.21 & 14.32 & 0.075 & 1931 & 0.24 & 205 & 2.61 \\
\hline 22 & 36.43 & 0.98 & 0.67 & 25.53 & 0.00 & 25.53 & 22.23 & 22.09 & 295.24 & 3.44 & 14.20 & 14.34 & 0.075 & 1918 & 0.24 & 203 & 2.61 \\
\hline 23 & 36.43 & 0.98 & 0.67 & 25.54 & 0.00 & 25.54 & 22.27 & 22.13 & 295.28 & 3.41 & 14.16 & 14.31 & 0.075 & 1933 & 0.24 & 205 & 2.62 \\
\hline 22 & 36.43 & 0.98 & 0.67 & 25.49 & 0.00 & 25.49 & 22.20 & 22.08 & 295.23 & 3.41 & 14.23 & 14.35 & 0.075 & 1934 & 0.24 & 205 & 2.61 \\
\hline 21 & 36.43 & 0.98 & 0.67 & 25.50 & 0.00 & 25.50 & 22.21 & 22.08 & 295.23 & 3.42 & 14.23 & 14.35 & 0.075 & 1929 & 0.24 & 204 & 2.61 \\
\hline 20 & 36.43 & 0.98 & 0.67 & 25.61 & 0.00 & 25.61 & 22.30 & 22.17 & 295.32 & 3.44 & 14.13 & 14.26 & 0.075 & 1919 & 0.24 & 203 & 2.63 \\
\hline 18 & 36.41 & 0.98 & 0.67 & 25.51 & 0.00 & 25.51 & 22.20 & 22.08 & 295.23 & 3.43 & 14.21 & 14.33 & 0.075 & 1924 & 0.24 & 204 & 2.61 \\
\hline 16 & 36.39 & 0.98 & 0.67 & 25.55 & 0.00 & 25.55 & 22.24 & 22.13 & 295.28 & 3.42 & 14.15 & 14.26 & 0.075 & 1931 & 0.24 & 205 & 2.62 \\
\hline 12 & 36.39 & 0.98 & 0.67 & 25.55 & 0.00 & 25.55 & 22.22 & 22.12 & 295.27 & 3.42 & 14.18 & 14.27 & 0.075 & 1927 & 0.24 & 204 & 2.62 \\
\hline 8 & 36.38 & 0.98 & 0.67 & 25.57 & 0.00 & 25.57 & 22.24 & 22.16 & 295.31 & 3.41 & 14.14 & 14.22 & 0.075 & 1935 & 0.24 & 205 & 2.62 \\
\hline 4 & 36.38 & 0.98 & 0.67 & 25.54 & 0.00 & 25.54 & 22.20 & 22.12 & 295.27 & 3.43 & 14.18 & 14.26 & 0.075 & 1925 & 0.24 & 204 & 2.62 \\
\hline 0 & 36.38 & 0.98 & 0.67 & 25.58 & 0.00 & 25.58 & 22.20 & 22.13 & 295.28 & 3.45 & 14.18 & 14.25 & 0.075 & 1912 & 0.24 & 203 & 2.62 \\
\hline
\end{tabular}


Table D92: Reduced Data for Mehra Electrode at $2 \mathrm{~W}$

\begin{tabular}{|c|c|c|c|c|c|c|c|c|c|c|c|c|c|c|c|c|c|}
\hline HV & $\mathbf{T}_{\text {sat }}$ & $\mathbf{Q}$ & $\mathbf{q}$ & $\begin{array}{c}\text { T2 thru } \\
\text { T5 } \\
\text { Average }\end{array}$ & $\Delta \mathbf{T}_{\text {alumina }}$ & $\mathbf{T}_{\mathrm{s}}$ & $\mathbf{T}_{\infty_{\text {wall }}}$ & $\mathbf{T}_{\text {otop }}$ & $T \infty_{\text {top }}$ & $\mathbf{T}_{\mathrm{s}}-\mathbf{T}_{\text {otop }}$ & $\mathbf{T}_{\text {sat }}-\mathbf{T}_{\text {cowall }}$ & $\mathbf{T}_{\text {sat }}-\mathbf{T}_{\text {cotop }}$ & $\mathbf{k}_{\text {fluid }}$ & $\mathbf{h}$ & $\theta_{\mathrm{s}}-\theta_{\text {ootop }}$ & $\mathrm{Nu}$ & G $\Delta$ \\
\hline $\mathbf{k V}$ & ${ }^{\circ} \mathrm{C}$ & W & $\begin{array}{c}10^{4} \\
W / m^{2}\end{array}$ & ${ }^{\circ} \mathrm{C}$ & ${ }^{\circ} \mathrm{C}$ & ${ }^{\circ} \mathrm{C}$ & ${ }^{\circ} \mathbf{C}$ & ${ }^{\circ} \mathrm{C}$ & $\mathbf{K}$ & ${ }^{\circ} \mathrm{C}$ & ${ }^{\circ} \mathrm{C}$ & ${ }^{\circ} \mathrm{C}$ & W/m-K & $\begin{array}{c}\mathbf{W} / \mathbf{m}^{2}- \\
\mathbf{K}\end{array}$ & --- & -- & --- \\
\hline 0 & 36.41 & 2.05 & 1.39 & 29.61 & 0.00 & 29.61 & 22.39 & 22.25 & 295.40 & 7.35 & 14.02 & 14.16 & 0.075 & 1867 & 0.52 & 198 & 5.51 \\
\hline 4 & 36.43 & 2.05 & 1.39 & 29.61 & 0.00 & 29.61 & 22.42 & 22.27 & 295.42 & 7.34 & 14.01 & 14.16 & 0.075 & 1871 & 0.52 & 198 & 5.51 \\
\hline 8 & 36.13 & 2.05 & 1.39 & 29.70 & 0.00 & 29.70 & 22.49 & 22.32 & 295.47 & 7.38 & 13.64 & 13.81 & 0.075 & 1861 & 0.54 & 197 & 5.66 \\
\hline 12 & 36.21 & 2.05 & 1.39 & 29.71 & 0.00 & 29.71 & 22.51 & 22.32 & 295.47 & 7.38 & 13.70 & 13.88 & 0.075 & 1860 & 0.54 & 197 & 5.64 \\
\hline 16 & 36.26 & 2.05 & 1.39 & 29.68 & 0.00 & 29.68 & 22.51 & 22.33 & 295.48 & 7.36 & 13.76 & 13.94 & 0.075 & 1866 & 0.53 & 198 & 5.61 \\
\hline 18 & 36.30 & 2.05 & 1.39 & 29.73 & 0.00 & 29.73 & 22.60 & 22.40 & 295.55 & 7.33 & 13.70 & 13.90 & 0.075 & 1873 & 0.53 & 199 & 5.63 \\
\hline 20 & 36.32 & 2.05 & 1.39 & 29.74 & 0.00 & 29.74 & 22.60 & 22.39 & 295.54 & 7.35 & 13.72 & 13.93 & 0.075 & 1867 & 0.54 & 198 & 5.63 \\
\hline 21 & 36.32 & 2.05 & 1.39 & 29.72 & 0.00 & 29.72 & 22.64 & 22.44 & 295.59 & 7.29 & 13.68 & 13.88 & 0.075 & 1884 & 0.53 & 200 & 5.64 \\
\hline 22 & 36.34 & 2.05 & 1.39 & 29.77 & 0.00 & 29.77 & 22.69 & 22.47 & 295.62 & 7.30 & 13.65 & 13.87 & 0.075 & 1879 & 0.54 & 199 & 5.66 \\
\hline 23 & 36.36 & 2.05 & 1.39 & 29.80 & 0.00 & 29.80 & 22.72 & 22.48 & 295.63 & 7.32 & 13.64 & 13.87 & 0.075 & 1875 & 0.54 & 199 & 5.66 \\
\hline 22 & 36.36 & 2.05 & 1.39 & 29.76 & 0.00 & 29.76 & 22.68 & 22.45 & 295.60 & 7.31 & 13.68 & 13.91 & 0.075 & 1878 & 0.53 & 199 & 5.64 \\
\hline 21 & 36.34 & 2.05 & 1.39 & 29.70 & 0.00 & 29.70 & 22.60 & 22.39 & 295.54 & 7.31 & 13.74 & 13.95 & 0.075 & 1878 & 0.53 & 199 & 5.62 \\
\hline 20 & 36.32 & 2.05 & 1.39 & 29.76 & 0.00 & 29.76 & 22.63 & 22.41 & 295.56 & 7.35 & 13.69 & 13.91 & 0.075 & 1868 & 0.54 & 198 & 5.64 \\
\hline 18 & 36.32 & 2.05 & 1.39 & 29.76 & 0.00 & 29.76 & 22.63 & 22.43 & 295.58 & 7.33 & 13.69 & 13.89 & 0.075 & 1874 & 0.54 & 199 & 5.64 \\
\hline 16 & 36.30 & 2.05 & 1.39 & 29.66 & 0.00 & 29.66 & 22.51 & 22.33 & 295.48 & 7.33 & 13.79 & 13.97 & 0.075 & 1874 & 0.53 & 199 & 5.60 \\
\hline 12 & 36.30 & 2.05 & 1.39 & 29.70 & 0.00 & 29.70 & 22.53 & 22.36 & 295.51 & 7.34 & 13.77 & 13.94 & 0.075 & 1871 & 0.53 & 198 & 5.60 \\
\hline 8 & 36.28 & 2.05 & 1.39 & 29.71 & 0.00 & 29.71 & 22.52 & 22.36 & 295.51 & 7.35 & 13.76 & 13.93 & 0.075 & 1867 & 0.53 & 198 & 5.61 \\
\hline 4 & 36.28 & 2.05 & 1.39 & 29.71 & 0.00 & 29.71 & 22.51 & 22.35 & 295.50 & 7.36 & 13.77 & 13.93 & 0.075 & 1865 & 0.53 & 198 & 5.61 \\
\hline 0 & 36.26 & 2.05 & 1.39 & 29.73 & 0.00 & 29.73 & 22.52 & 22.34 & 295.49 & 7.39 & 13.75 & 13.92 & 0.075 & 1859 & 0.54 & 197 & 5.62 \\
\hline
\end{tabular}


Table D93: Reduced Data for Mehra Electrode at $3 \mathrm{~W}$

\begin{tabular}{|c|c|c|c|c|c|c|c|c|c|c|c|c|c|c|c|c|c|}
\hline HV & $\mathbf{T}_{\text {sat }}$ & $\mathbf{Q}$ & $\mathbf{q}$ & $\begin{array}{c}\text { T2 thru } \\
\text { T5 } \\
\text { Average }\end{array}$ & $\Delta \mathbf{T}_{\text {alumina }}$ & $\mathbf{T}_{\mathrm{s}}$ & $\mathbf{T}_{\text {owall }_{\text {I }}}$ & $\mathbf{T}_{\text {otop }}$ & $\mathbf{T} \infty_{\text {top }}$ & $T_{s}-T_{\text {otop }}$ & $\mathbf{T}_{\text {sat }}-\mathbf{T}_{\text {owall }}$ & $\mathbf{T}_{\text {sat }}-\mathbf{T}_{\text {otop }}$ & $\mathbf{k}_{\text {fluid }}$ & h & $\theta_{\mathrm{s}}-\theta_{\text {otop }}$ & $\mathrm{Nu}$ & G $\Delta$ \\
\hline $\mathbf{k V}$ & ${ }^{\circ} \mathrm{C}$ & W & $\begin{array}{c}10^{4} \\
W / \mathbf{m}^{2}\end{array}$ & ${ }^{\circ} \mathrm{C}$ & ${ }^{\circ} \mathrm{C}$ & ${ }^{\circ} \mathrm{C}$ & ${ }^{\circ} \mathbf{C}$ & ${ }^{\circ} \mathrm{C}$ & $\mathbf{K}$ & ${ }^{\circ} \mathrm{C}$ & ${ }^{\circ} \mathrm{C}$ & ${ }^{\circ} \mathrm{C}$ & $\mathbf{W} / \mathbf{m}-\mathbf{K}$ & $\begin{array}{c}\mathbf{W} / \mathbf{m}^{2}- \\
\mathbf{K}\end{array}$ & --- & -- & --- \\
\hline $\mathbf{0}$ & 36.28 & 2.99 & 2.04 & 33.33 & 0.00 & 33.33 & 22.55 & 22.30 & 295.45 & 11.03 & 13.74 & 13.98 & 0.075 & 1820 & 0.80 & 193 & 8.22 \\
\hline 4 & 36.28 & 2.99 & 2.04 & 33.36 & 0.00 & 33.36 & 22.61 & 22.37 & 295.52 & 10.99 & 13.67 & 13.91 & 0.075 & 1826 & 0.80 & 194 & 8.26 \\
\hline 8 & 36.28 & 2.99 & 2.04 & 33.36 & 0.00 & 33.36 & 22.61 & 22.37 & 295.52 & 10.99 & 13.68 & 13.91 & 0.075 & 1826 & 0.80 & 194 & 8.25 \\
\hline 12 & 36.30 & 2.99 & 2.04 & 33.31 & 0.00 & 33.31 & 22.58 & 22.35 & 295.50 & 10.96 & 13.72 & 13.95 & 0.075 & 1830 & 0.80 & 194 & 8.23 \\
\hline 16 & 36.32 & 2.99 & 2.04 & 33.34 & 0.00 & 33.34 & 22.64 & 22.38 & 295.53 & 10.96 & 13.68 & 13.94 & 0.075 & 1830 & 0.80 & 194 & 8.25 \\
\hline 18 & 36.32 & 2.99 & 2.04 & 33.33 & 0.00 & 33.33 & 22.64 & 22.38 & 295.53 & 10.95 & 13.68 & 13.94 & 0.075 & 1833 & 0.80 & 194 & 8.25 \\
\hline 20 & 36.32 & 2.99 & 2.04 & 33.32 & 0.00 & 33.32 & 22.68 & 22.41 & 295.56 & 10.91 & 13.64 & 13.91 & 0.075 & 1839 & 0.80 & 195 & 8.27 \\
\hline 21 & 36.34 & 2.99 & 2.04 & 33.36 & 0.00 & 33.36 & 22.78 & 22.49 & 295.64 & 10.87 & 13.56 & 13.85 & 0.075 & 1845 & 0.80 & 196 & 8.32 \\
\hline 22 & 36.34 & 2.99 & 2.04 & 33.32 & 0.00 & 33.32 & 22.71 & 22.41 & 295.56 & 10.91 & 13.63 & 13.93 & 0.075 & 1839 & 0.80 & 195 & 8.28 \\
\hline 23 & 36.34 & 2.99 & 2.04 & 33.33 & 0.00 & 33.33 & 22.73 & 22.43 & 295.58 & 10.90 & 13.61 & 13.91 & 0.075 & 1840 & 0.80 & 195 & 8.29 \\
\hline 22 & 36.34 & 2.99 & 2.04 & 33.34 & 0.00 & 33.34 & 22.73 & 22.43 & 295.58 & 10.91 & 13.61 & 13.91 & 0.075 & 1839 & 0.80 & 195 & 8.29 \\
\hline 21 & 36.32 & 2.99 & 2.04 & 33.32 & 0.00 & 33.32 & 22.67 & 22.39 & 295.54 & 10.93 & 13.65 & 13.93 & 0.075 & 1835 & 0.80 & 195 & 8.27 \\
\hline 20 & 36.32 & 2.99 & 2.04 & 33.28 & 0.00 & 33.28 & 22.64 & 22.36 & 295.51 & 10.92 & 13.68 & 13.96 & 0.075 & 1838 & 0.80 & 195 & 8.25 \\
\hline 18 & 36.32 & 2.99 & 2.04 & 33.33 & 0.00 & 33.33 & 22.67 & 22.40 & 295.55 & 10.92 & 13.65 & 13.92 & 0.075 & 1836 & 0.80 & 195 & 8.27 \\
\hline 16 & 36.30 & 2.99 & 2.04 & 33.29 & 0.00 & 33.29 & 22.61 & 22.36 & 295.51 & 10.93 & 13.69 & 13.94 & 0.075 & 1835 & 0.80 & 195 & 8.24 \\
\hline 12 & 36.28 & 2.99 & 2.04 & 33.29 & 0.00 & 33.29 & 22.54 & 22.30 & 295.45 & 10.99 & 13.74 & 13.98 & 0.075 & 1825 & 0.80 & 194 & 8.21 \\
\hline 8 & 36.28 & 2.99 & 2.04 & 33.32 & 0.00 & 33.32 & 22.59 & 22.37 & 295.52 & 10.95 & 13.69 & 13.92 & 0.075 & 1832 & 0.80 & 194 & 8.24 \\
\hline 4 & 36.26 & 2.99 & 2.04 & 33.32 & 0.00 & 33.32 & 22.59 & 22.36 & 295.51 & 10.96 & 13.67 & 13.91 & 0.075 & 1830 & 0.80 & 194 & 8.25 \\
\hline $\mathbf{0}$ & 36.26 & 2.99 & 2.04 & 33.29 & 0.00 & 33.29 & 22.54 & 22.30 & 295.45 & 10.99 & 13.72 & 13.96 & 0.075 & 1825 & 0.80 & 194 & 8.22 \\
\hline
\end{tabular}


Table D94: Reduced Data for Mehra Electrode at $4 \mathrm{~W}$

\begin{tabular}{|c|c|c|c|c|c|c|c|c|c|c|c|c|c|c|c|c|c|}
\hline HV & $T_{\text {sat }}$ & $\mathbf{Q}$ & $\mathbf{q}$ & $\begin{array}{c}\text { T2 thru } \\
\text { T5 } \\
\text { Average }\end{array}$ & $\Delta \mathbf{T}_{\text {alumina }}$ & $\mathbf{T}_{\mathrm{s}}$ & $\mathbf{T}_{\infty_{\text {wall }}}$ & $\mathbf{T}_{\text {otop }}$ & $\mathbf{T} \infty_{\text {top }}$ & $\mathbf{T}_{\mathrm{s}}-\mathbf{T}_{\text {otop }}$ & $\mathbf{T}_{\text {sat }}-\mathbf{T}_{\text {owall }}$ & $\mathbf{T}_{\text {sat }}-\mathbf{T}_{\text {otop }}$ & $\mathbf{k}_{\text {fluid }}$ & h & $\theta_{\mathrm{s}}-\theta_{\text {otop }}$ & $\mathrm{Nu}$ & G $\Delta$ \\
\hline $\mathrm{kV}$ & ${ }^{\circ} \mathbf{C}$ & W & $\begin{array}{c}10^{4} \\
W / m^{2}\end{array}$ & ${ }^{\circ} \mathrm{C}$ & ${ }^{\circ} \mathrm{C}$ & ${ }^{\circ} \mathbf{C}$ & ${ }^{\circ} \mathbf{C}$ & ${ }^{\circ} \mathrm{C}$ & K & ${ }^{\circ} \mathrm{C}$ & ${ }^{\circ} \mathrm{C}$ & ${ }^{\circ} \mathrm{C}$ & $\mathbf{W} / \mathbf{m}-\mathbf{K}$ & $\begin{array}{c}\mathbf{W} / \mathbf{m}^{2}- \\
\mathbf{K}\end{array}$ & -- & --- & -- \\
\hline 0 & 36.26 & 3.98 & 2.71 & 37.14 & 0.00 & 37.14 & 22.71 & 22.39 & 295.54 & 14.75 & 13.56 & 13.87 & 0.075 & 1810 & 1.09 & 192 & 11.07 \\
\hline 4 & 36.28 & 3.98 & 2.71 & 37.14 & 0.00 & 37.14 & 22.73 & 22.42 & 295.57 & 14.72 & 13.55 & 13.87 & 0.075 & 1813 & 1.09 & 192 & 11.08 \\
\hline 8 & 36.28 & 3.98 & 2.71 & 37.07 & 0.00 & 37.07 & 22.67 & 22.39 & 295.54 & 14.68 & 13.61 & 13.89 & 0.075 & 1818 & 1.08 & 193 & 11.03 \\
\hline 12 & 36.28 & 3.98 & 2.71 & 37.07 & 0.00 & 37.07 & 22.69 & 22.39 & 295.54 & 14.67 & 13.59 & 13.89 & 0.075 & 1819 & 1.08 & 193 & 11.05 \\
\hline 16 & 36.30 & 3.98 & 2.71 & 37.04 & 0.00 & 37.04 & 22.70 & 22.38 & 295.53 & 14.65 & 13.60 & 13.92 & 0.075 & 1822 & 1.08 & 193 & 11.04 \\
\hline 18 & 36.30 & 3.98 & 2.71 & 37.09 & 0.00 & 37.09 & 22.79 & 22.46 & 295.61 & 14.63 & 13.52 & 13.84 & 0.075 & 1824 & 1.08 & 194 & 11.11 \\
\hline 20 & 36.32 & 3.98 & 2.71 & 37.08 & 0.00 & 37.08 & 22.83 & 22.48 & 295.63 & 14.60 & 13.49 & 13.84 & 0.075 & 1828 & 1.08 & 194 & 11.13 \\
\hline 21 & 36.32 & 3.98 & 2.71 & 37.02 & 0.00 & 37.02 & 22.80 & 22.44 & 295.59 & 14.58 & 13.52 & 13.88 & 0.075 & 1831 & 1.08 & 194 & 11.10 \\
\hline 22 & 36.32 & 3.98 & 2.71 & 37.02 & 0.00 & 37.02 & 22.82 & 22.46 & 295.61 & 14.56 & 13.50 & 13.86 & 0.075 & 1834 & 1.08 & 195 & 11.12 \\
\hline 23 & 36.32 & 3.98 & 2.71 & 37.13 & 0.00 & 37.13 & 22.90 & 22.53 & 295.68 & 14.59 & 13.42 & 13.79 & 0.075 & 1829 & 1.09 & 194 & 11.19 \\
\hline 22 & 36.32 & 3.98 & 2.71 & 37.07 & 0.00 & 37.07 & 22.85 & 22.49 & 295.64 & 14.58 & 13.47 & 13.83 & 0.075 & 1831 & 1.08 & 194 & 11.14 \\
\hline 21 & 36.32 & 3.98 & 2.71 & 37.06 & 0.00 & 37.06 & 22.82 & 22.46 & 295.61 & 14.60 & 13.50 & 13.86 & 0.075 & 1828 & 1.08 & 194 & 11.12 \\
\hline 20 & 36.32 & 3.98 & 2.71 & 37.10 & 0.00 & 37.10 & 22.85 & 22.50 & 295.65 & 14.60 & 13.47 & 13.82 & 0.075 & 1828 & 1.08 & 194 & 11.14 \\
\hline 18 & 36.32 & 3.98 & 2.71 & 37.11 & 0.00 & 37.11 & 22.79 & 22.46 & 295.61 & 14.65 & 13.53 & 13.86 & 0.075 & 1822 & 1.08 & 193 & 11.10 \\
\hline 16 & 36.30 & 3.98 & 2.71 & 37.09 & 0.00 & 37.09 & 22.74 & 22.42 & 295.57 & 14.67 & 13.56 & 13.88 & 0.075 & 1819 & 1.08 & 193 & 11.07 \\
\hline 12 & 36.28 & 3.98 & 2.71 & 37.13 & 0.00 & 37.13 & 22.74 & 22.45 & 295.60 & 14.68 & 13.54 & 13.84 & 0.075 & 1818 & 1.08 & 193 & 11.09 \\
\hline 8 & 36.28 & 3.98 & 2.71 & 37.12 & 0.00 & 37.12 & 22.72 & 22.43 & 295.58 & 14.69 & 13.56 & 13.86 & 0.075 & 1817 & 1.08 & 193 & 11.07 \\
\hline 4 & 36.28 & 3.98 & 2.71 & 37.16 & 0.00 & 37.16 & 22.75 & 22.45 & 295.60 & 14.72 & 13.53 & 13.84 & 0.075 & 1814 & 1.09 & 192 & 11.10 \\
\hline $\mathbf{0}$ & 36.28 & 3.98 & 2.71 & 37.14 & 0.00 & 37.14 & 22.76 & 22.46 & 295.61 & 14.68 & 13.52 & 13.82 & 0.075 & 1818 & 1.09 & 193 & 11.10 \\
\hline
\end{tabular}


Table D95: Reduced Data for Mehra Electrode at $5 \mathrm{~W}$

\begin{tabular}{|c|c|c|c|c|c|c|c|c|c|c|c|c|c|c|c|c|c|}
\hline HV & $\mathbf{T}_{\text {sat }}$ & $\mathbf{Q}$ & $\mathbf{q}$ & $\begin{array}{c}\text { T2 thru } \\
\text { T5 } \\
\text { Average }\end{array}$ & $\Delta T_{\text {alumina }}$ & $\mathbf{T}_{\mathrm{s}}$ & $\mathbf{T}_{\infty_{\text {wall }}}$ & $\mathbf{T}_{\text {otop }}$ & $T \infty_{\text {top }}$ & $T_{s}-T_{\text {otop }}$ & $\mathbf{T}_{\text {sat }}-\mathbf{T}_{\text {owall }}$ & $\mathbf{T}_{\text {sat }}-\mathbf{T}_{\text {otop }}$ & $\mathbf{k}_{\text {fluid }}$ & h & $\theta_{\mathrm{s}}-\theta_{\text {otop }}$ & $\mathrm{Nu}$ & G $\Delta$ \\
\hline kV & ${ }^{\circ} \mathbf{C}$ & $\mathbf{W}$ & $\begin{array}{c}10^{4} \\
\mathrm{~W} / \mathrm{m}^{2}\end{array}$ & ${ }^{\circ} \mathrm{C}$ & ${ }^{\circ} \mathbf{C}$ & ${ }^{\circ} \mathrm{C}$ & ${ }^{\circ} \mathrm{C}$ & ${ }^{\circ} \mathbf{C}$ & $\mathbf{K}$ & ${ }^{\circ} \mathrm{C}$ & ${ }^{\circ} \mathrm{C}$ & ${ }^{\circ} \mathrm{C}$ & $\mathbf{W} / \mathbf{m}-\mathbf{K}$ & $\begin{array}{c}\mathbf{W} / \mathbf{m}^{2}- \\
\mathbf{K}\end{array}$ & -- & --- & -- \\
\hline 0 & 36.28 & 4.91 & 3.34 & 40.90 & 0.00 & 40.90 & 22.87 & 22.50 & 295.65 & 18.40 & 13.41 & 13.78 & 0.075 & 1789 & 1.37 & 190 & 13.80 \\
\hline 4 & 36.28 & 4.91 & 3.34 & 40.88 & 0.00 & 40.88 & 22.83 & 22.47 & 295.62 & 18.42 & 13.45 & 13.82 & 0.075 & 1787 & 1.37 & 190 & 13.76 \\
\hline 8 & 36.28 & 4.91 & 3.34 & 40.78 & 0.00 & 40.78 & 22.71 & 22.39 & 295.54 & 18.39 & 13.57 & 13.90 & 0.075 & 1789 & 1.35 & 190 & 13.64 \\
\hline 12 & 36.28 & 4.91 & 3.34 & 40.84 & 0.00 & 40.84 & 22.80 & 22.45 & 295.60 & 18.39 & 13.48 & 13.84 & 0.075 & 1789 & 1.36 & 190 & 13.73 \\
\hline 16 & 36.28 & 4.91 & 3.34 & 40.84 & 0.00 & 40.84 & 22.89 & 22.51 & 295.66 & 18.33 & 13.39 & 13.77 & 0.075 & 1795 & 1.37 & 190 & 13.82 \\
\hline 18 & 36.30 & 4.91 & 3.34 & 40.85 & 0.00 & 40.85 & 22.95 & 22.56 & 295.71 & 18.29 & 13.35 & 13.74 & 0.075 & 1799 & 1.37 & 191 & 13.86 \\
\hline 20 & 36.30 & 4.91 & 3.34 & 40.81 & 0.00 & 40.81 & 22.98 & 22.57 & 295.72 & 18.25 & 13.32 & 13.74 & 0.075 & 1803 & 1.37 & 191 & 13.89 \\
\hline 21 & 36.30 & 4.91 & 3.34 & 40.76 & 0.00 & 40.76 & 22.97 & 22.56 & 295.71 & 18.21 & 13.33 & 13.75 & 0.075 & 1807 & 1.37 & 192 & 13.88 \\
\hline 22 & 36.30 & 4.91 & 3.34 & 40.75 & 0.00 & 40.75 & 22.98 & 22.57 & 295.72 & 18.18 & 13.32 & 13.73 & 0.075 & 1810 & 1.36 & 192 & 13.89 \\
\hline 23 & 36.30 & 4.91 & 3.34 & 40.80 & 0.00 & 40.80 & 23.03 & 22.60 & 295.75 & 18.20 & 13.27 & 13.70 & 0.075 & 1808 & 1.37 & 192 & 13.94 \\
\hline 22 & 36.30 & 4.91 & 3.34 & 40.76 & 0.00 & 40.75 & 22.99 & 22.57 & 295.72 & 18.18 & 13.32 & 13.73 & 0.075 & 1810 & 1.37 & 192 & 13.90 \\
\hline 21 & 36.32 & 4.91 & 3.34 & 40.79 & 0.00 & 40.79 & 22.99 & 22.57 & 295.72 & 18.21 & 13.33 & 13.75 & 0.075 & 1807 & 1.37 & 192 & 13.88 \\
\hline 20 & 36.32 & 4.91 & 3.34 & 40.82 & 0.00 & 40.82 & 22.99 & 22.59 & 295.74 & 18.22 & 13.33 & 13.72 & 0.075 & 1806 & 1.37 & 192 & 13.89 \\
\hline 18 & 36.32 & 4.91 & 3.34 & 40.88 & 0.00 & 40.88 & 23.01 & 22.63 & 295.78 & 18.25 & 13.31 & 13.69 & 0.075 & 1803 & 1.37 & 191 & 13.91 \\
\hline 16 & 36.32 & 4.91 & 3.34 & 40.91 & 0.00 & 40.91 & 23.01 & 22.63 & 295.78 & 18.28 & 13.31 & 13.69 & 0.075 & 1800 & 1.37 & 191 & 13.90 \\
\hline 12 & 36.32 & 4.91 & 3.34 & 40.95 & 0.00 & 40.95 & 22.97 & 22.61 & 295.76 & 18.34 & 13.35 & 13.71 & 0.075 & 1794 & 1.37 & 190 & 13.86 \\
\hline 8 & 36.32 & 4.91 & 3.34 & 40.99 & 0.00 & 40.99 & 22.96 & 22.61 & 295.76 & 18.39 & 13.36 & 13.71 & 0.075 & 1789 & 1.38 & 190 & 13.85 \\
\hline 4 & 36.32 & 4.91 & 3.34 & 40.98 & 0.00 & 40.98 & 22.94 & 22.58 & 295.73 & 18.40 & 13.38 & 13.74 & 0.075 & 1788 & 1.38 & 190 & 13.83 \\
\hline 0 & 36.32 & 4.91 & 3.34 & 41.04 & 0.00 & 41.04 & 23.03 & 22.67 & 295.82 & 18.37 & 13.29 & 13.65 & 0.075 & 1791 & 1.38 & 190 & 13.92 \\
\hline
\end{tabular}


Table D96: Reduced Data for Mehra Electrode at $6 \mathrm{~W}$

\begin{tabular}{|c|c|c|c|c|c|c|c|c|c|c|c|c|c|c|c|c|c|}
\hline HV & $\mathbf{T}_{\text {sat }}$ & $\mathbf{Q}$ & $\mathbf{q}$ & $\begin{array}{c}\text { T2 thru } \\
\text { T5 } \\
\text { Average }\end{array}$ & $\Delta \mathbf{T}_{\text {alumina }}$ & $\mathbf{T}_{\mathrm{s}}$ & $\mathbf{T}_{\text {}_{\text {wall }}}$ & $\mathbf{T}_{\text {otop }}$ & $\mathbf{T} \infty_{\text {top }}$ & $\mathbf{T}_{\mathrm{s}}-\mathbf{T}_{\text {otop }}$ & $\mathbf{T}_{\text {sat }}-\mathbf{T}_{\text {owall }}$ & $\mathbf{T}_{\text {sat }}-\mathbf{T}_{\text {otop }}$ & $\mathbf{k}_{\text {fluid }}$ & h & $\theta_{\mathrm{s}}-\theta_{\text {otop }}$ & $\mathrm{Nu}$ & G $\Delta$ \\
\hline $\mathrm{kV}$ & ${ }^{\circ} \mathbf{C}$ & W & $\begin{array}{c}10^{4} \\
W / \mathbf{m}^{2}\end{array}$ & ${ }^{\circ} \mathrm{C}$ & ${ }^{\circ} \mathrm{C}$ & ${ }^{\circ} \mathrm{C}$ & ${ }^{\circ} \mathrm{C}$ & ${ }^{\circ} \mathrm{C}$ & K & ${ }^{\circ} \mathbf{C}$ & ${ }^{\circ} \mathrm{C}$ & ${ }^{\circ} \mathrm{C}$ & W/m-K & $\begin{array}{c}\mathbf{W} / \mathbf{m}^{2}- \\
\mathbf{K}\end{array}$ & -- & -- & -- \\
\hline $\mathbf{0}$ & 36.34 & 6.04 & 4.11 & 45.64 & 0.00 & 45.64 & 23.16 & 22.73 & 295.88 & 22.91 & 13.17 & 13.61 & 0.075 & 1767 & 1.74 & 188 & 17.28 \\
\hline 4 & 36.36 & 6.04 & 4.11 & 45.65 & 0.00 & 45.64 & 23.17 & 22.72 & 295.87 & 22.93 & 13.19 & 13.64 & 0.075 & 1766 & 1.74 & 187 & 17.26 \\
\hline 8 & 36.36 & 6.04 & 4.11 & 45.63 & 0.00 & 45.63 & 23.15 & 22.71 & 295.86 & 22.91 & 13.21 & 13.64 & 0.075 & 1767 & 1.73 & 188 & 17.24 \\
\hline 12 & 36.38 & 6.04 & 4.11 & 45.60 & 0.00 & 45.60 & 23.18 & 22.74 & 295.89 & 22.87 & 13.20 & 13.64 & 0.075 & 1770 & 1.73 & 188 & 17.25 \\
\hline 16 & 36.41 & 6.04 & 4.11 & 45.61 & 0.00 & 45.61 & 23.21 & 22.75 & 295.90 & 22.86 & 13.21 & 13.66 & 0.075 & 1771 & 1.73 & 188 & 17.24 \\
\hline 18 & 36.41 & 6.04 & 4.11 & 45.56 & 0.00 & 45.56 & 23.29 & 22.82 & 295.97 & 22.74 & 13.12 & 13.59 & 0.075 & 1780 & 1.73 & 189 & 17.35 \\
\hline 20 & 36.43 & 6.04 & 4.11 & 45.52 & 0.00 & 45.52 & 23.32 & 22.82 & 295.97 & 22.70 & 13.11 & 13.61 & 0.075 & 1783 & 1.73 & 189 & 17.36 \\
\hline 21 & 36.43 & 6.04 & 4.11 & 45.49 & 0.00 & 45.49 & 23.30 & 22.81 & 295.96 & 22.68 & 13.13 & 13.62 & 0.075 & 1785 & 1.73 & 190 & 17.34 \\
\hline 22 & 36.43 & 6.04 & 4.11 & 45.48 & 0.00 & 45.48 & 23.36 & 22.85 & 296.00 & 22.63 & 13.07 & 13.58 & 0.075 & 1789 & 1.73 & 190 & 17.41 \\
\hline 23 & 36.43 & 6.04 & 4.11 & 45.47 & 0.00 & 45.47 & 23.36 & 22.86 & 296.01 & 22.61 & 13.07 & 13.57 & 0.075 & 1790 & 1.73 & 190 & 17.42 \\
\hline 22 & 36.41 & 6.04 & 4.11 & 45.47 & 0.00 & 45.47 & 23.36 & 22.86 & 296.01 & 22.61 & 13.05 & 13.55 & 0.075 & 1790 & 1.73 & 190 & 17.45 \\
\hline 21 & 36.41 & 6.04 & 4.11 & 45.47 & 0.00 & 45.47 & 23.35 & 22.84 & 295.99 & 22.62 & 13.07 & 13.57 & 0.075 & 1789 & 1.73 & 190 & 17.42 \\
\hline 20 & 36.39 & 6.04 & 4.11 & 45.50 & 0.00 & 45.50 & 23.33 & 22.83 & 295.98 & 22.67 & 13.06 & 13.56 & 0.075 & 1786 & 1.74 & 190 & 17.43 \\
\hline 18 & 36.39 & 6.04 & 4.11 & 45.51 & 0.00 & 45.51 & 23.25 & 22.77 & 295.92 & 22.74 & 13.15 & 13.62 & 0.075 & 1780 & 1.73 & 189 & 17.31 \\
\hline 16 & 36.39 & 6.04 & 4.11 & 45.56 & 0.00 & 45.56 & 23.23 & 22.76 & 295.91 & 22.80 & 13.17 & 13.64 & 0.075 & 1775 & 1.73 & 188 & 17.29 \\
\hline 12 & 36.38 & 6.04 & 4.11 & 45.57 & 0.00 & 45.57 & 23.16 & 22.71 & 295.86 & 22.86 & 13.22 & 13.66 & 0.075 & 1770 & 1.73 & 188 & 17.22 \\
\hline 8 & 36.36 & 6.04 & 4.11 & 45.60 & 0.00 & 45.60 & 23.14 & 22.71 & 295.86 & 22.89 & 13.21 & 13.65 & 0.075 & 1768 & 1.73 & 188 & 17.23 \\
\hline 4 & 36.36 & 6.04 & 4.11 & 45.64 & 0.00 & 45.64 & 23.20 & 22.74 & 295.89 & 22.89 & 13.16 & 13.61 & 0.075 & 1768 & 1.74 & 188 & 17.30 \\
\hline 0 & 36.34 & 6.04 & 4.11 & 45.63 & 0.00 & 45.63 & 23.19 & 22.75 & 295.90 & 22.88 & 13.14 & 13.59 & 0.075 & 1769 & 1.74 & 188 & 17.32 \\
\hline
\end{tabular}


Table D97: Reduced Data for Mehra Electrode at $7 \mathrm{~W}$

\begin{tabular}{|c|c|c|c|c|c|c|c|c|c|c|c|c|c|c|c|c|c|}
\hline HV & $\mathbf{T}_{\text {sat }}$ & $\mathbf{Q}$ & $q$ & $\begin{array}{c}\text { T2 thru } \\
\text { T5 } \\
\text { Average }\end{array}$ & $\Delta \mathbf{T}_{\text {alumina }}$ & $\mathbf{T}_{\mathrm{s}}$ & $\mathbf{T}_{\text {}_{\text {wall }}}$ & $\mathbf{T}_{\text {otop }}$ & $\mathbf{T} \infty_{\text {top }}$ & $\mathbf{T}_{\mathrm{s}}-\mathbf{T}_{\text {otop }}$ & $\mathbf{T}_{\text {sat }}-\mathbf{T}_{\text {cowall }}$ & $\mathbf{T}_{\text {sat }}-\mathbf{T}_{\text {cotop }}$ & $\mathbf{k}_{\text {fluid }}$ & h & $\theta_{\mathrm{s}}-\theta_{\text {ostop }}$ & $\mathrm{Nu}$ & G $\Delta$ \\
\hline $\mathbf{k V}$ & ${ }^{\circ} \mathrm{C}$ & W & $\begin{array}{c}10^{4} \\
\mathrm{~W} / \mathrm{m}^{2}\end{array}$ & ${ }^{\circ} \mathrm{C}$ & ${ }^{\circ} \mathrm{C}$ & ${ }^{\circ} \mathrm{C}$ & ${ }^{\circ} \mathbf{C}$ & ${ }^{\circ} \mathrm{C}$ & $\mathbf{K}$ & ${ }^{\circ} \mathbf{C}$ & ${ }^{\circ} \mathrm{C}$ & ${ }^{\circ} \mathrm{C}$ & $\mathbf{W} / \mathbf{m}-\mathbf{K}$ & $\begin{array}{c}\mathbf{W} / \mathbf{m}^{2}- \\
\mathbf{K}\end{array}$ & -- & -- & --- \\
\hline 0 & 36.36 & 7.01 & 4.77 & 50.10 & 0.00 & 50.10 & 23.39 & 22.86 & 296.01 & 27.24 & 12.97 & 13.50 & 0.075 & 1723 & 2.10 & 183 & 20.36 \\
\hline 4 & 36.36 & 7.01 & 4.77 & 50.00 & 0.00 & 50.00 & 23.28 & 22.79 & 295.94 & 27.21 & 13.08 & 13.57 & 0.075 & 1726 & 2.08 & 183 & 20.19 \\
\hline 8 & 36.36 & 7.01 & 4.77 & 50.04 & 0.00 & 50.04 & 23.33 & 22.84 & 295.99 & 27.20 & 13.03 & 13.52 & 0.075 & 1726 & 2.09 & 183 & 20.27 \\
\hline 12 & 36.38 & 7.01 & 4.77 & 50.05 & 0.00 & 50.05 & 23.42 & 22.91 & 296.06 & 27.14 & 12.96 & 13.47 & 0.075 & 1730 & 2.09 & 184 & 20.37 \\
\hline 16 & 36.39 & 7.01 & 4.77 & 49.99 & 0.00 & 49.99 & 23.41 & 22.89 & 296.04 & 27.10 & 12.99 & 13.51 & 0.075 & 1732 & 2.09 & 184 & 20.33 \\
\hline 18 & 36.41 & 7.01 & 4.77 & 49.96 & 0.00 & 49.96 & 23.50 & 22.96 & 296.11 & 27.00 & 12.92 & 13.45 & 0.075 & 1739 & 2.09 & 185 & 20.44 \\
\hline 20 & 36.41 & 7.01 & 4.77 & 49.88 & 0.00 & 49.88 & 23.54 & 22.99 & 296.14 & 26.89 & 12.87 & 13.43 & 0.075 & 1746 & 2.09 & 186 & 20.51 \\
\hline 21 & 36.41 & 7.01 & 4.77 & 49.86 & 0.00 & 49.86 & 23.58 & 23.02 & 296.17 & 26.84 & 12.83 & 13.40 & 0.075 & 1749 & 2.09 & 186 & 20.58 \\
\hline 22 & 36.43 & 7.01 & 4.77 & 49.82 & 0.00 & 49.82 & 23.53 & 22.98 & 296.13 & 26.84 & 12.90 & 13.45 & 0.075 & 1749 & 2.08 & 186 & 20.47 \\
\hline 23 & 36.41 & 7.01 & 4.77 & 49.87 & 0.00 & 49.87 & 23.58 & 23.01 & 296.16 & 26.85 & 12.83 & 13.40 & 0.075 & 1748 & 2.09 & 186 & 20.57 \\
\hline 22 & 36.41 & 7.01 & 4.77 & 49.83 & 0.00 & 49.83 & 23.62 & 23.06 & 296.21 & 26.77 & 12.79 & 13.35 & 0.075 & 1754 & 2.09 & 186 & 20.64 \\
\hline 21 & 36.41 & 7.01 & 4.77 & 49.80 & 0.00 & 49.79 & 23.59 & 23.03 & 296.18 & 26.76 & 12.83 & 13.38 & 0.075 & 1754 & 2.09 & 186 & 20.58 \\
\hline 20 & 36.41 & 7.01 & 4.77 & 49.85 & 0.00 & 49.85 & 23.60 & 23.04 & 296.19 & 26.82 & 12.81 & 13.38 & 0.075 & 1751 & 2.09 & 186 & 20.61 \\
\hline 18 & 36.41 & 7.01 & 4.77 & 49.87 & 0.00 & 49.87 & 23.51 & 22.99 & 296.14 & 26.88 & 12.90 & 13.42 & 0.075 & 1746 & 2.08 & 186 & 20.47 \\
\hline 16 & 36.41 & 7.01 & 4.77 & 50.00 & 0.00 & 50.00 & 23.60 & 23.08 & 296.23 & 26.92 & 12.82 & 13.33 & 0.075 & 1744 & 2.10 & 185 & 20.60 \\
\hline 12 & 36.41 & 7.01 & 4.77 & 50.06 & 0.00 & 50.06 & 23.53 & 23.02 & 296.17 & 27.04 & 12.88 & 13.39 & 0.075 & 1736 & 2.10 & 184 & 20.50 \\
\hline 8 & 36.41 & 7.01 & 4.77 & 50.07 & 0.00 & 50.07 & 23.51 & 23.02 & 296.17 & 27.05 & 12.90 & 13.40 & 0.075 & 1735 & 2.10 & 184 & 20.47 \\
\hline 4 & 36.41 & 7.01 & 4.77 & 50.08 & 0.00 & 50.08 & 23.53 & 23.02 & 296.17 & 27.06 & 12.88 & 13.39 & 0.075 & 1735 & 2.10 & 184 & 20.50 \\
\hline 0 & 36.41 & 7.01 & 4.77 & 50.11 & 0.00 & 50.11 & 23.55 & 23.04 & 296.19 & 27.07 & 12.87 & 13.37 & 0.075 & 1734 & 2.10 & 184 & 20.52 \\
\hline
\end{tabular}


Table D98: Reduced Data for Mehra Electrode at $8 \mathrm{~W}$

\begin{tabular}{|c|c|c|c|c|c|c|c|c|c|c|c|c|c|c|c|c|c|}
\hline HV & $\mathbf{T}_{\text {sat }}$ & Q & $\mathbf{q}$ & $\begin{array}{c}\text { T2 thru } \\
\text { T5 } \\
\text { Average }\end{array}$ & $\Delta \mathbf{T}_{\text {alumina }}$ & $\mathbf{T}_{\mathrm{s}}$ & $\mathbf{T}_{\infty_{\text {wall }}}$ & $\mathbf{T}_{\text {otop }}$ & $T \infty_{\text {top }}$ & $\mathbf{T}_{\mathbf{s}}-\mathbf{T}_{\text {otop }}$ & $\mathbf{T}_{\text {sat }}-\mathbf{T}_{\text {cowall }}$ & $\mathbf{T}_{\text {sat }}-\mathbf{T}_{\text {otop }}$ & $\mathbf{k}_{\text {fluid }}$ & $\mathbf{h}$ & $\theta_{\mathrm{s}}-\theta_{\text {sotop }}$ & $\mathrm{Nu}$ & G $\Delta$ \\
\hline $\mathbf{k V}$ & ${ }^{\circ} \mathrm{C}$ & $\mathbf{W}$ & $\begin{array}{c}10^{4} \\
W / \mathbf{m}^{2}\end{array}$ & ${ }^{\circ} \mathrm{C}$ & ${ }^{\circ} \mathrm{C}$ & ${ }^{\circ} \mathbf{C}$ & ${ }^{\circ} \mathrm{C}$ & ${ }^{\circ} \mathrm{C}$ & $\mathbf{K}$ & ${ }^{\circ} \mathbf{C}$ & ${ }^{\circ} \mathrm{C}$ & ${ }^{\circ} \mathrm{C}$ & $\mathbf{W} / \mathbf{m}-\mathbf{K}$ & $\begin{array}{c}\mathbf{W} / \mathbf{m}^{2}- \\
\mathbf{K}\end{array}$ & -- & -- & -- \\
\hline 0 & 36.41 & 8.00 & 5.44 & 54.68 & 0.00 & 54.68 & 23.78 & 23.19 & 296.34 & 31.49 & 12.63 & 13.22 & 0.075 & 1701 & 2.49 & 181 & 23.85 \\
\hline 4 & 36.41 & 8.00 & 5.44 & 54.63 & 0.00 & 54.63 & 23.76 & 23.18 & 296.33 & 31.45 & 12.65 & 13.24 & 0.075 & 1704 & 2.49 & 181 & 23.81 \\
\hline 8 & 36.43 & 8.00 & 5.44 & 54.98 & 0.00 & 54.98 & 23.73 & 23.16 & 296.31 & 31.82 & 12.70 & 13.27 & 0.075 & 1684 & 2.51 & 179 & 23.73 \\
\hline 12 & 36.43 & 8.00 & 5.44 & 55.06 & 0.00 & 55.06 & 23.77 & 23.19 & 296.34 & 31.87 & 12.67 & 13.24 & 0.075 & 1681 & 2.52 & 179 & 23.80 \\
\hline 16 & 36.47 & 7.92 & 5.38 & 53.64 & 0.00 & 53.64 & 23.78 & 23.18 & 296.33 & 30.46 & 12.69 & 13.29 & 0.075 & 1741 & 2.40 & 185 & 23.51 \\
\hline 18 & 36.47 & 7.91 & 5.38 & 53.49 & 0.00 & 53.49 & 23.85 & 23.24 & 296.39 & 30.25 & 12.62 & 13.23 & 0.075 & 1753 & 2.40 & 186 & 23.64 \\
\hline 20 & 36.47 & 7.91 & 5.38 & 53.45 & 0.00 & 53.45 & 23.95 & 23.31 & 296.46 & 30.14 & 12.52 & 13.16 & 0.075 & 1760 & 2.41 & 187 & 23.82 \\
\hline 21 & 36.49 & 7.91 & 5.38 & 53.41 & 0.00 & 53.41 & 23.97 & 23.33 & 296.48 & 30.08 & 12.51 & 13.16 & 0.075 & 1763 & 2.40 & 187 & 23.83 \\
\hline 22 & 36.47 & 7.91 & 5.38 & 53.36 & 0.00 & 53.36 & 23.94 & 23.30 & 296.45 & 30.06 & 12.53 & 13.16 & 0.075 & 1764 & 2.40 & 188 & 23.81 \\
\hline 23 & 36.47 & 7.91 & 5.38 & 53.35 & 0.00 & 53.35 & 23.96 & 23.31 & 296.46 & 30.04 & 12.51 & 13.16 & 0.075 & 1765 & 2.40 & 188 & 23.84 \\
\hline 22 & 36.47 & 7.96 & 5.41 & 53.38 & 0.00 & 53.38 & 23.94 & 23.28 & 296.43 & 30.10 & 12.53 & 13.18 & 0.075 & 1772 & 2.40 & 188 & 23.93 \\
\hline 21 & 36.47 & 7.91 & 5.38 & 53.35 & 0.00 & 53.35 & 23.91 & 23.28 & 296.43 & 30.07 & 12.56 & 13.19 & 0.075 & 1763 & 2.39 & 187 & 23.75 \\
\hline 20 & 36.45 & 7.91 & 5.38 & 53.40 & 0.00 & 53.40 & 23.89 & 23.26 & 296.41 & 30.14 & 12.56 & 13.19 & 0.075 & 1759 & 2.40 & 187 & 23.75 \\
\hline 18 & 36.45 & 7.91 & 5.38 & 53.46 & 0.00 & 53.46 & 23.91 & 23.30 & 296.45 & 30.16 & 12.54 & 13.15 & 0.075 & 1758 & 2.40 & 187 & 23.78 \\
\hline 16 & 36.43 & 7.91 & 5.38 & 53.54 & 0.00 & 53.54 & 23.85 & 23.28 & 296.43 & 30.26 & 12.58 & 13.16 & 0.075 & 1752 & 2.41 & 186 & 23.70 \\
\hline 12 & 36.41 & 7.91 & 5.38 & 53.59 & 0.00 & 53.59 & 23.83 & 23.26 & 296.41 & 30.34 & 12.59 & 13.15 & 0.075 & 1748 & 2.41 & 186 & 23.69 \\
\hline 8 & 36.41 & 7.91 & 5.38 & 53.62 & 0.00 & 53.62 & 23.78 & 23.23 & 296.38 & 30.39 & 12.63 & 13.18 & 0.075 & 1745 & 2.41 & 186 & 23.61 \\
\hline 4 & 36.39 & 7.92 & 5.39 & 53.67 & 0.00 & 53.67 & 23.78 & 23.22 & 296.37 & 30.45 & 12.62 & 13.17 & 0.075 & 1744 & 2.41 & 185 & 23.67 \\
\hline 0 & 36.39 & 7.92 & 5.39 & 53.73 & 0.00 & 53.73 & 23.83 & 23.27 & 296.42 & 30.47 & 12.56 & 13.13 & 0.075 & 1743 & 2.43 & 185 & 23.77 \\
\hline
\end{tabular}


Table D99: Reduced Data for Mehra Electrode at $9 \mathrm{~W}$

\begin{tabular}{|c|c|c|c|c|c|c|c|c|c|c|c|c|c|c|c|c|c|}
\hline HV & $\mathbf{T}_{\text {sat }}$ & Q & $\mathbf{q}$ & $\begin{array}{c}\text { T2 thru } \\
\text { T5 } \\
\text { Average }\end{array}$ & $\Delta \mathbf{T}_{\text {alumina }}$ & $\mathbf{T}_{\mathrm{s}}$ & $\mathbf{T}_{\infty_{\text {wall }}}$ & $\mathbf{T}_{\text {otop }}$ & $T \infty_{\text {top }}$ & $\mathbf{T}_{\mathbf{s}}-\mathbf{T}_{\text {otop }}$ & $\mathbf{T}_{\text {sat }}-\mathbf{T}_{\text {cowall }}$ & $\mathbf{T}_{\text {sat }}-\mathbf{T}_{\text {otop }}$ & $\mathbf{k}_{\text {fluid }}$ & $\mathbf{h}$ & $\theta_{\mathrm{s}}-\theta_{\text {otop }}$ & $\mathrm{Nu}$ & G $\Delta$ \\
\hline $\mathbf{k V}$ & ${ }^{\circ} \mathrm{C}$ & $\mathbf{W}$ & $\begin{array}{c}10^{4} \\
W / \mathbf{m}^{2}\end{array}$ & ${ }^{\circ} \mathrm{C}$ & ${ }^{\circ} \mathrm{C}$ & ${ }^{\circ} \mathbf{C}$ & ${ }^{\circ} \mathrm{C}$ & ${ }^{\circ} \mathrm{C}$ & $\mathbf{K}$ & ${ }^{\circ} \mathbf{C}$ & ${ }^{\circ} \mathrm{C}$ & ${ }^{\circ} \mathrm{C}$ & $\mathbf{W} / \mathbf{m}-\mathbf{K}$ & $\begin{array}{c}\mathbf{W} / \mathbf{m}^{2}- \\
\mathbf{K}\end{array}$ & -- & -- & -- \\
\hline 0 & 36.38 & 8.93 & 6.08 & 58.05 & 0.00 & 58.05 & 23.92 & 23.27 & 296.42 & 34.78 & 12.46 & 13.10 & 0.075 & 1721 & 2.79 & 183 & 27.02 \\
\hline 4 & 36.38 & 8.92 & 6.07 & 57.91 & 0.00 & 57.91 & 23.89 & 23.26 & 296.41 & 34.65 & 12.49 & 13.12 & 0.075 & 1726 & 2.77 & 183 & 26.93 \\
\hline 8 & 36.38 & 8.92 & 6.07 & 57.85 & 0.00 & 57.85 & 23.85 & 23.22 & 296.37 & 34.62 & 12.53 & 13.15 & 0.075 & 1727 & 2.76 & 184 & 26.84 \\
\hline 12 & 36.38 & 8.92 & 6.07 & 57.80 & 0.00 & 57.80 & 23.86 & 23.23 & 296.38 & 34.57 & 12.52 & 13.15 & 0.075 & 1729 & 2.76 & 184 & 26.86 \\
\hline 16 & 36.39 & 8.92 & 6.07 & 57.68 & 0.00 & 57.68 & 23.90 & 23.24 & 296.39 & 34.44 & 12.49 & 13.16 & 0.075 & 1736 & 2.76 & 185 & 26.92 \\
\hline 18 & 36.39 & 8.92 & 6.07 & 57.57 & 0.00 & 57.56 & 23.99 & 23.30 & 296.45 & 34.26 & 12.40 & 13.09 & 0.075 & 1745 & 2.76 & 186 & 27.11 \\
\hline 20 & 36.39 & 8.92 & 6.07 & 57.31 & 0.00 & 57.31 & 23.97 & 23.27 & 296.42 & 34.04 & 12.42 & 13.12 & 0.075 & 1757 & 2.74 & 187 & 27.08 \\
\hline 21 & 36.39 & 8.92 & 6.07 & 57.21 & 0.00 & 57.21 & 23.96 & 23.26 & 296.41 & 33.95 & 12.43 & 13.14 & 0.075 & 1761 & 2.73 & 187 & 27.05 \\
\hline 22 & 36.38 & 8.92 & 6.07 & 57.20 & 0.00 & 57.20 & 24.00 & 23.29 & 296.44 & 33.90 & 12.37 & 13.08 & 0.075 & 1764 & 2.74 & 188 & 27.18 \\
\hline 23 & 36.38 & 8.92 & 6.07 & 57.15 & 0.00 & 57.15 & 23.98 & 23.26 & 296.41 & 33.89 & 12.40 & 13.11 & 0.075 & 1764 & 2.73 & 188 & 27.13 \\
\hline 22 & 36.36 & 8.92 & 6.07 & 57.10 & 0.00 & 57.10 & 23.89 & 23.20 & 296.35 & 33.90 & 12.46 & 13.16 & 0.075 & 1764 & 2.72 & 188 & 26.98 \\
\hline 21 & 36.34 & 8.92 & 6.07 & 57.08 & 0.00 & 57.08 & 23.92 & 23.24 & 296.39 & 33.85 & 12.42 & 13.10 & 0.075 & 1767 & 2.73 & 188 & 27.08 \\
\hline 20 & 36.32 & 8.92 & 6.07 & 57.07 & 0.00 & 57.07 & 23.91 & 23.22 & 296.37 & 33.84 & 12.41 & 13.09 & 0.075 & 1767 & 2.73 & 188 & 27.10 \\
\hline 18 & 36.30 & 8.92 & 6.07 & 57.13 & 0.00 & 57.13 & 23.86 & 23.18 & 296.33 & 33.95 & 12.45 & 13.12 & 0.075 & 1761 & 2.73 & 187 & 27.02 \\
\hline 16 & 36.28 & 8.92 & 6.07 & 57.15 & 0.00 & 57.15 & 23.79 & 23.14 & 296.29 & 34.01 & 12.49 & 13.15 & 0.075 & 1758 & 2.72 & 187 & 26.91 \\
\hline 12 & 36.26 & 8.83 & 6.01 & 57.32 & 0.00 & 57.32 & 23.73 & 23.10 & 296.25 & 34.23 & 12.54 & 13.17 & 0.075 & 1729 & 2.73 & 184 & 26.55 \\
\hline 8 & 36.25 & 8.83 & 6.01 & 57.34 & 0.00 & 57.34 & 23.71 & 23.10 & 296.25 & 34.24 & 12.53 & 13.15 & 0.075 & 1729 & 2.73 & 184 & 26.56 \\
\hline 4 & 36.23 & 8.86 & 6.03 & 57.48 & 0.00 & 57.48 & 23.69 & 23.06 & 296.21 & 34.42 & 12.54 & 13.16 & 0.075 & 1725 & 2.75 & 183 & 26.63 \\
\hline 0 & 36.23 & 8.90 & 6.06 & 57.87 & 0.00 & 57.87 & 23.69 & 23.05 & 296.20 & 34.82 & 12.54 & 13.17 & 0.075 & 1713 & 2.78 & 182 & 26.76 \\
\hline
\end{tabular}


Table D100: Reduced Data for Mehra Electrode at $10 \mathrm{~W}$

\begin{tabular}{|c|c|c|c|c|c|c|c|c|c|c|c|c|c|c|c|c|c|}
\hline HV & $\mathbf{T}_{\text {sat }}$ & Q & $\mathbf{q}$ & $\begin{array}{c}\text { T2 thru } \\
\text { T5 } \\
\text { Average }\end{array}$ & $\Delta \mathbf{T}_{\text {alumina }}$ & $\mathbf{T}_{\mathrm{s}}$ & $\mathbf{T}_{\infty_{\text {wall }}}$ & $\mathbf{T}_{\text {otop }}$ & $T \infty_{\text {top }}$ & $\mathbf{T}_{\mathbf{s}}-\mathbf{T}_{\text {otop }}$ & $\mathbf{T}_{\text {sat }}-\mathbf{T}_{\text {cowall }}$ & $\mathbf{T}_{\text {sat }}-\mathbf{T}_{\text {otop }}$ & $\mathbf{k}_{\text {fluid }}$ & $\mathbf{h}$ & $\theta_{\mathrm{s}}-\theta_{\text {cotop }}$ & $\mathrm{Nu}$ & G $\Delta$ \\
\hline $\mathbf{k V}$ & ${ }^{\circ} \mathrm{C}$ & $\mathbf{W}$ & $\begin{array}{c}10^{4} \\
W / \mathbf{m}^{2}\end{array}$ & ${ }^{\circ} \mathrm{C}$ & ${ }^{\circ} \mathrm{C}$ & ${ }^{\circ} \mathrm{C}$ & ${ }^{\circ} \mathrm{C}$ & ${ }^{\circ} \mathrm{C}$ & $\mathbf{K}$ & ${ }^{\circ} \mathbf{C}$ & ${ }^{\circ} \mathrm{C}$ & ${ }^{\circ} \mathrm{C}$ & $\mathbf{W} / \mathbf{m}-\mathbf{K}$ & $\begin{array}{c}\mathbf{W} / \mathbf{m}^{2}- \\
\mathbf{K}\end{array}$ & -- & -- & -- \\
\hline 0 & 36.23 & 10.07 & 6.85 & 62.92 & 0.00 & 62.92 & 23.90 & 23.17 & 296.32 & 39.75 & 12.33 & 13.06 & 0.075 & 1698 & 3.22 & 180 & 30.78 \\
\hline 4 & 36.21 & 9.97 & 6.78 & 62.44 & 0.00 & 62.44 & 23.88 & 23.16 & 296.31 & 39.28 & 12.33 & 13.05 & 0.075 & 1701 & 3.18 & 181 & 30.47 \\
\hline 8 & 36.23 & 10.07 & 6.85 & 61.91 & 0.00 & 61.91 & 23.86 & 23.17 & 296.32 & 38.75 & 12.36 & 13.06 & 0.075 & 1742 & 3.13 & 185 & 30.71 \\
\hline 12 & 36.25 & 10.06 & 6.84 & 61.79 & 0.00 & 61.79 & 23.88 & 23.17 & 296.32 & 38.62 & 12.36 & 13.08 & 0.075 & 1745 & 3.12 & 185 & 30.65 \\
\hline 16 & 36.25 & 10.09 & 6.87 & 61.63 & 0.00 & 61.63 & 23.96 & 23.22 & 296.37 & 38.40 & 12.29 & 13.02 & 0.075 & 1761 & 3.12 & 187 & 30.95 \\
\hline 18 & 36.26 & 10.10 & 6.87 & 61.27 & 0.00 & 61.27 & 24.00 & 23.24 & 296.39 & 38.03 & 12.26 & 13.02 & 0.075 & 1780 & 3.10 & 189 & 31.05 \\
\hline 20 & 36.26 & 10.00 & 6.80 & 60.88 & 0.00 & 60.88 & 24.04 & 23.27 & 296.42 & 37.61 & 12.22 & 12.99 & 0.075 & 1781 & 3.08 & 189 & 30.82 \\
\hline 21 & 36.26 & 9.99 & 6.80 & 60.73 & 0.00 & 60.73 & 24.02 & 23.23 & 296.38 & 37.50 & 12.24 & 13.03 & 0.075 & 1786 & 3.06 & 190 & 30.76 \\
\hline 22 & 36.25 & 9.99 & 6.80 & 60.79 & 0.00 & 60.79 & 23.98 & 23.19 & 296.34 & 37.60 & 12.26 & 13.05 & 0.075 & 1781 & 3.07 & 189 & 30.70 \\
\hline 23 & 36.23 & 9.99 & 6.80 & 60.69 & 0.00 & 60.69 & 23.99 & 23.20 & 296.35 & 37.49 & 12.23 & 13.03 & 0.075 & 1786 & 3.06 & 190 & 30.79 \\
\hline 22 & 36.21 & 9.99 & 6.80 & 60.85 & 0.00 & 60.85 & 23.94 & 23.16 & 296.31 & 37.70 & 12.27 & 13.05 & 0.075 & 1776 & 3.07 & 189 & 30.68 \\
\hline 21 & 36.21 & 9.99 & 6.80 & 60.83 & 0.00 & 60.83 & 23.93 & 23.14 & 296.29 & 37.69 & 12.28 & 13.07 & 0.075 & 1777 & 3.07 & 189 & 30.68 \\
\hline 20 & 36.19 & 10.01 & 6.81 & 60.98 & 0.00 & 60.98 & 23.89 & 23.11 & 296.26 & 37.87 & 12.30 & 13.08 & 0.075 & 1772 & 3.08 & 188 & 30.69 \\
\hline 18 & 36.15 & 10.01 & 6.81 & 61.41 & 0.00 & 61.41 & 23.84 & 23.09 & 296.24 & 38.33 & 12.31 & 13.07 & 0.075 & 1751 & 3.11 & 186 & 30.66 \\
\hline 16 & 36.15 & 10.04 & 6.83 & 61.97 & 0.00 & 61.97 & 23.79 & 23.05 & 296.20 & 38.92 & 12.36 & 13.10 & 0.075 & 1729 & 3.15 & 184 & 30.62 \\
\hline 12 & 36.13 & 10.06 & 6.84 & 62.45 & 0.00 & 62.45 & 23.73 & 23.02 & 296.17 & 39.44 & 12.41 & 13.12 & 0.075 & 1709 & 3.18 & 182 & 30.55 \\
\hline 8 & 36.13 & 10.06 & 6.84 & 62.48 & 0.00 & 62.48 & 23.73 & 23.03 & 296.18 & 39.45 & 12.40 & 13.10 & 0.075 & 1708 & 3.18 & 182 & 30.56 \\
\hline 4 & 36.13 & 10.09 & 6.86 & 62.47 & 0.00 & 62.47 & 23.66 & 22.94 & 296.09 & 39.53 & 12.48 & 13.19 & 0.075 & 1710 & 3.17 & 182 & 30.47 \\
\hline 0 & 36.10 & 10.11 & 6.88 & 62.86 & 0.00 & 62.86 & 23.69 & 22.97 & 296.12 & 39.89 & 12.41 & 13.13 & 0.075 & 1698 & 3.21 & 180 & 30.71 \\
\hline
\end{tabular}


Table D101: Reduced Data for Mehra Electrode at $11 \mathrm{~W}$ (averaged)

\begin{tabular}{|c|c|c|c|c|c|c|c|c|c|c|c|c|c|c|c|c|c|}
\hline HV & $\mathbf{T}_{\text {sat }}$ & Q & $\mathbf{q}$ & $\begin{array}{c}\text { T2 thru } \\
\text { T5 } \\
\text { Average }\end{array}$ & $\Delta \mathbf{T}_{\text {alumina }}$ & $\mathbf{T}_{\mathrm{s}}$ & $\mathbf{T}_{\text {owwall }}$ & $\mathbf{T}_{\text {ootop }}$ & $T \infty_{\text {top }}$ & $\mathbf{T}_{\mathbf{s}}-\mathbf{T}_{\text {otop }}$ & $\mathbf{T}_{\text {sat }}-\mathbf{T}_{\text {owwall }}$ & $\mathbf{T}_{\text {sat }}-\mathbf{T}_{\text {ootop }}$ & $\mathbf{k}_{\text {fluid }}$ & h & $\theta_{\mathrm{s}}-\theta_{\text {otop }}$ & Nu & GA \\
\hline $\mathbf{k V}$ & ${ }^{\circ} \mathrm{C}$ & W & $\begin{array}{c}10^{4} \\
\mathrm{~W} / \mathrm{m}^{2}\end{array}$ & ${ }^{\circ} \mathbf{C}$ & ${ }^{\circ} \mathrm{C}$ & ${ }^{\circ} \mathbf{C}$ & ${ }^{\circ} \mathrm{C}$ & ${ }^{\circ} \mathbf{C}$ & $\mathbf{K}$ & ${ }^{\circ} \mathrm{C}$ & ${ }^{\circ} \mathrm{C}$ & ${ }^{\circ} \mathbf{C}$ & $\mathbf{W} / \mathbf{m}-\mathbf{K}$ & $\begin{array}{c}\mathbf{W} / \mathbf{m}^{2}- \\
\mathbf{K}\end{array}$ & --- & --- & --- \\
\hline 0 & 36.10 & 10.98 & 7.47 & 64.83 & 0.00 & 64.83 & 22.73 & 21.98 & 295.13 & 42.85 & 13.37 & 14.11 & 0.075 & 1716.57 & 3.22 & 182 & 31.09 \\
\hline 4 & 36.10 & 10.98 & 7.47 & 64.87 & 0.00 & 64.87 & 22.77 & 22.03 & 295.18 & 42.84 & 13.33 & 14.07 & 0.075 & 1717.99 & 3.23 & 182 & 31.20 \\
\hline 8 & 36.13 & 10.97 & 7.47 & 64.38 & 0.00 & 64.38 & 22.73 & 22.00 & 295.15 & 42.37 & 13.40 & 14.13 & 0.075 & 1735.73 & 3.18 & 184 & 31.01 \\
\hline 12 & 36.17 & 10.94 & 7.44 & 63.99 & 0.00 & 63.99 & 22.74 & 22.03 & 295.18 & 41.97 & 13.43 & 14.14 & 0.075 & 1747.00 & 3.14 & 185 & 30.86 \\
\hline 16 & 36.17 & 10.96 & 7.45 & 63.76 & 0.00 & 63.76 & 22.78 & 22.04 & 295.19 & 41.72 & 13.39 & 14.13 & 0.075 & 1760.46 & 3.13 & 187 & 30.99 \\
\hline 18 & 36.14 & 10.84 & 7.38 & 63.24 & 0.00 & 63.24 & 22.85 & 22.09 & 295.24 & 41.15 & 13.29 & 14.05 & 0.075 & 1765.92 & 3.12 & 187 & 30.90 \\
\hline 20 & 36.21 & 10.88 & 7.40 & 62.94 & 0.00 & 62.93 & 22.95 & 22.16 & 295.31 & 40.78 & 13.26 & 14.05 & 0.075 & 1788.44 & 3.10 & 190 & 31.08 \\
\hline 21 & 36.21 & 10.88 & 7.40 & 62.68 & 0.00 & 62.68 & 23.01 & 22.21 & 295.36 & 40.47 & 13.20 & 14.00 & 0.075 & 1802.31 & 3.08 & 191 & 31.19 \\
\hline 22 & 36.18 & 10.90 & 7.41 & 62.59 & 0.00 & 62.59 & 22.95 & 22.16 & 295.31 & 40.42 & 13.22 & 14.02 & 0.075 & 1806.92 & 3.07 & 192 & 31.17 \\
\hline 23 & 36.17 & 10.91 & 7.42 & 62.62 & 0.00 & 62.62 & 23.12 & 22.30 & 295.45 & 40.32 & 13.05 & 13.87 & 0.075 & 1812.96 & 3.10 & 192 & 31.59 \\
\hline 22 & 36.17 & 10.96 & 7.45 & 62.73 & 0.00 & 62.73 & 22.96 & 22.17 & 295.32 & 40.56 & 13.20 & 14.00 & 0.075 & 1811.27 & 3.09 & 192 & 31.38 \\
\hline 21 & 36.16 & 10.92 & 7.43 & 62.82 & 0.00 & 62.82 & 23.00 & 22.21 & 295.36 & 40.61 & 13.16 & 13.95 & 0.075 & 1803.07 & 3.10 & 191 & 31.37 \\
\hline 20 & 36.16 & 10.93 & 7.44 & 62.98 & 0.00 & 62.98 & 23.02 & 22.22 & 295.37 & 40.76 & 13.14 & 13.94 & 0.075 & 1798.11 & 3.12 & 191 & 31.44 \\
\hline 18 & 36.16 & 10.96 & 7.45 & 63.17 & 0.00 & 63.17 & 22.87 & 22.11 & 295.26 & 41.06 & 13.29 & 14.05 & 0.075 & 1788.92 & 3.10 & 190 & 31.15 \\
\hline 16 & 36.12 & 10.95 & 7.45 & 63.64 & 0.00 & 63.64 & 22.82 & 22.08 & 295.23 & 41.55 & 13.30 & 14.04 & 0.075 & 1766.18 & 3.14 & 187 & 31.10 \\
\hline 12 & 36.10 & 10.98 & 7.47 & 64.32 & 0.00 & 64.32 & 22.84 & 22.12 & 295.27 & 42.21 & 13.26 & 13.98 & 0.075 & 1744.45 & 3.20 & 185 & 31.33 \\
\hline 8 & 36.13 & 11.00 & 7.48 & 64.45 & 0.00 & 64.45 & 22.92 & 22.18 & 295.33 & 42.27 & 13.21 & 13.95 & 0.075 & 1745.26 & 3.21 & 185 & 31.48 \\
\hline 4 & 36.15 & 11.05 & 7.52 & 64.68 & 0.00 & 64.68 & 22.92 & 22.15 & 295.30 & 42.53 & 13.23 & 14.00 & 0.075 & 1742.23 & 3.22 & 185 & 31.55 \\
\hline 0 & 36.15 & 10.98 & 7.47 & 64.64 & 0.00 & 64.64 & 22.91 & 22.16 & 295.31 & 42.48 & 13.24 & 13.99 & 0.075 & 1733.36 & 3.22 & 184 & 31.31 \\
\hline
\end{tabular}


Table D102: Reduced Data for Mehra Electrode at $12 \mathrm{~W}$

\begin{tabular}{|c|c|c|c|c|c|c|c|c|c|c|c|c|c|c|c|c|c|}
\hline HV & $\mathbf{T}_{\text {sat }}$ & $\mathbf{Q}$ & $\mathbf{q}$ & $\begin{array}{c}\text { T2 thru } \\
\text { T5 } \\
\text { Average }\end{array}$ & $\Delta \mathbf{T}_{\text {alumina }}$ & $\mathbf{T}_{\mathrm{s}}$ & $\mathbf{T}_{\infty \text { wall }}$ & $\mathbf{T}_{\text {otop }}$ & $T \infty_{\text {top }}$ & $\mathbf{T}_{\mathrm{s}}-\mathbf{T}_{\text {otop }}$ & $\mathbf{T}_{\text {sat }}-\mathbf{T}_{\text {cowall }}$ & $\mathbf{T}_{\text {sat }}-\mathbf{T}_{\text {otop }}$ & $\mathbf{k}_{\text {fluid }}$ & h & $\theta_{\mathrm{s}}-\theta_{\text {otop }}$ & $\mathrm{Nu}$ & G $\Delta$ \\
\hline kV & ${ }^{\circ} \mathbf{C}$ & W & $\begin{array}{c}10^{4} \\
W / \mathbf{m}^{2}\end{array}$ & ${ }^{\circ} \mathrm{C}$ & ${ }^{\circ} \mathrm{C}$ & ${ }^{\circ} \mathrm{C}$ & ${ }^{\circ} \mathrm{C}$ & ${ }^{\circ} \mathrm{C}$ & K & ${ }^{\circ} \mathrm{C}$ & ${ }^{\circ} \mathrm{C}$ & ${ }^{\circ} \mathrm{C}$ & $\mathbf{W} / \mathbf{m}-\mathbf{K}$ & $\begin{array}{c}\mathbf{W} / \mathbf{m}^{2}- \\
\mathbf{K}\end{array}$ & -- & -- & -- \\
\hline 0 & 36.75 & 12.07 & 8.21 & 68.54 & 0.00 & 68.54 & 23.30 & 22.46 & 295.61 & 46.07 & 13.45 & 14.28 & 0.075 & 1755 & 3.43 & 186 & 33.82 \\
\hline 4 & 36.78 & 11.96 & 8.14 & 68.28 & 0.00 & 68.28 & 23.33 & 22.52 & 295.67 & 45.76 & 13.46 & 14.26 & 0.075 & 1752 & 3.40 & 186 & 33.51 \\
\hline 8 & 36.78 & 11.96 & 8.14 & 68.19 & 0.00 & 68.19 & 23.29 & 22.52 & 295.67 & 45.66 & 13.50 & 14.26 & 0.075 & 1755 & 3.38 & 186 & 33.40 \\
\hline 12 & 36.82 & 11.96 & 8.14 & 68.09 & 0.00 & 68.09 & 23.31 & 22.57 & 295.72 & 45.52 & 13.51 & 14.25 & 0.075 & 1761 & 3.37 & 187 & 33.37 \\
\hline 16 & 36.84 & 11.96 & 8.14 & 67.50 & 0.00 & 67.50 & 23.42 & 22.64 & 295.79 & 44.86 & 13.42 & 14.20 & 0.075 & 1787 & 3.34 & 190 & 33.60 \\
\hline 18 & 36.82 & 11.96 & 8.14 & 67.25 & 0.00 & 67.24 & 23.55 & 22.74 & 295.89 & 44.50 & 13.27 & 14.08 & 0.075 & 1801 & 3.35 & 191 & 33.97 \\
\hline 20 & 36.80 & 11.85 & 8.06 & 66.84 & 0.00 & 66.84 & 23.67 & 22.84 & 295.99 & 44.00 & 13.13 & 13.96 & 0.075 & 1805 & 3.35 & 192 & 34.01 \\
\hline 21 & 36.80 & 11.85 & 8.06 & 66.78 & 0.00 & 66.78 & 23.76 & 22.90 & 296.05 & 43.88 & 13.04 & 13.90 & 0.075 & 1810 & 3.37 & 192 & 34.25 \\
\hline 22 & 36.80 & 11.96 & 8.14 & 67.01 & 0.00 & 67.01 & 23.81 & 22.89 & 296.04 & 44.12 & 12.99 & 13.91 & 0.075 & 1817 & 3.40 & 193 & 34.71 \\
\hline 23 & 36.84 & 11.96 & 8.14 & 66.94 & 0.00 & 66.94 & 23.95 & 23.05 & 296.20 & 43.89 & 12.89 & 13.79 & 0.075 & 1826 & 3.41 & 194 & 34.98 \\
\hline 22 & 36.86 & 11.96 & 8.14 & 66.99 & 0.00 & 66.99 & 23.94 & 23.04 & 296.19 & 43.95 & 12.92 & 13.81 & 0.075 & 1824 & 3.40 & 194 & 34.90 \\
\hline 21 & 36.86 & 11.85 & 8.06 & 66.87 & 0.00 & 66.87 & 23.87 & 22.99 & 296.14 & 43.88 & 12.98 & 13.86 & 0.075 & 1809 & 3.38 & 192 & 34.39 \\
\hline 20 & 36.87 & 11.85 & 8.06 & 66.83 & 0.00 & 66.83 & 23.84 & 23.00 & 296.15 & 43.83 & 13.03 & 13.88 & 0.075 & 1811 & 3.36 & 192 & 34.26 \\
\hline 18 & 36.89 & 11.85 & 8.06 & 67.09 & 0.00 & 67.08 & 23.85 & 23.02 & 296.17 & 44.06 & 13.04 & 13.87 & 0.075 & 1802 & 3.38 & 191 & 34.23 \\
\hline 16 & 36.89 & 11.85 & 8.06 & 67.42 & 0.00 & 67.42 & 23.77 & 22.96 & 296.11 & 44.46 & 13.13 & 13.93 & 0.075 & 1786 & 3.39 & 190 & 34.01 \\
\hline 12 & 36.89 & 11.96 & 8.14 & 68.20 & 0.00 & 68.20 & 23.74 & 22.96 & 296.11 & 45.25 & 13.16 & 13.94 & 0.075 & 1772 & 3.44 & 188 & 34.27 \\
\hline 8 & 36.89 & 11.85 & 8.06 & 68.19 & 0.00 & 68.18 & 23.81 & 22.99 & 296.14 & 45.20 & 13.08 & 13.91 & 0.075 & 1757 & 3.45 & 187 & 34.13 \\
\hline 4 & 36.89 & 11.85 & 8.06 & 68.09 & 0.00 & 68.09 & 23.80 & 22.98 & 296.13 & 45.11 & 13.09 & 13.91 & 0.075 & 1760 & 3.44 & 187 & 34.10 \\
\hline $\mathbf{0}$ & 36.89 & 11.85 & 8.06 & 68.10 & 0.00 & 68.10 & 23.89 & 23.11 & 296.26 & 44.99 & 13.01 & 13.78 & 0.075 & 1765 & 3.46 & 188 & 34.33 \\
\hline
\end{tabular}




\section{Appendix E: Error Analysis}

An error analysis was also performed for the three studies. It was based on the initial specified or estimated accuracy of each measurement device and calculated based on the propagation of those errors through the data reduction equations. Since the spacing study was performed before the modifications were made to the apparatus as discussed in Chapter 3, the error analysis for the spacing comparison study is slightly different than for the two electrode studies. These differences are explained below. Actual computed error values for the three studies are also given.

\section{Error Analysis Explanation}

Table E1 shows a list of the equipment used in calculating values and their respective errors found from manufacturer's information or from estimating the accuracy. The errors shown for the electrode studies include the measurement error as well as the conversion error from the data acquisition system where applicable.

Table E1: Equipment and Errors Used in Spacing Comparison and Electrode Studies

\begin{tabular}{|c|c|c|c|}
\hline \multirow{2}{*}{ Measurement } & \multicolumn{3}{|c|}{ Error } \\
\cline { 2 - 4 } & Spacing & Cap & Mehra \\
\hline Chamber Pressure Gauge & $\pm 1 \% \mathrm{FS}$ & --- & --- \\
\hline Chamber Pressure Transducer & --- & $\pm 0.25 \% \mathrm{FS}$ & $\pm 0.25 \% \mathrm{FS}$ \\
\hline TFR Voltage Input (LED) & $\pm 0.2 \%+2$ digits & --- & $\pm 0.2 \%+2$ digits \\
\hline TFR Voltage Input (data acq.) & --- & $\pm 0.0026 \mathrm{~V}$ & --- \\
\hline TFR Current Input (LED) & $\pm 1 \%+3$ digits & --- & $\pm 1 \%+3$ digits \\
\hline Precision Resistor & --- & $2 \mathrm{E} 10-5 \Omega$ & --- \\
\hline TFR Width & $\pm 0.0051 \mathrm{~cm}$ & $\pm 0.0051 \mathrm{~cm}$ & $\pm 0.0051 \mathrm{~cm}$ \\
\hline TFR Length & $\pm 0.015 \mathrm{~cm}$ & $\pm 0.015 \mathrm{~cm}$ & $\pm 0.015 \mathrm{~cm}$ \\
\hline Thermocouples (calibrated) & $\pm 0.1{ }^{\circ} \mathrm{C}$ & --- & --- \\
\hline Thermocouples (uncalibrated) & --- & $\pm 0.61{ }^{\circ} \mathrm{C}$ & $\pm 0.61{ }^{\circ} \mathrm{C}$ \\
\hline 2 mm Alumina Layer Thickness & --- & --- & $\pm 0.1 \mathrm{~mm}$ \\
\hline TFR Glass Layer Thickness & \pm 5 microns & --- & --- \\
\hline TFR Heater Layer Thickness & \pm 20 microns & \pm 2 microns & $\pm 2 \mathrm{microns}$ \\
\hline TFR Substrate Layer Thickness & \pm 10 microns & \pm 10 microns & $\pm 10 \mathrm{microns}$ \\
\hline Heat Fraction Lost Down Pedestal & \pm 0.01 & \pm 0.01 & \pm 0.01 \\
\hline Rotameters & $3 \%$ & $3 \%$ & $5 \%$ \\
\hline
\end{tabular}


The error saturation temperature, $\mathrm{T}_{\text {sat }}$, was found from the error of the chamber pressure. For the spacing comparison this was read directly from the chamber pressure gauge. The maximum and minimum chamber pressures were calculated from the specified percent error for the gauge. The corresponding saturation temperatures were then retrieved from a table of $\mathrm{T}_{\text {sat }}$ versus $\mathrm{P}_{\text {sat }}$ and the values were averaged. For the cap electrode and Mehra electrode studies, the chamber pressure was found from the pressure transducer. The data acquisition system accuracy was found to be very small $( \pm 0.00026$ psia) compared to the accuracy of the transducer itself and was therefore ignored. The maximum and minimum chamber pressures were then calculated from the specified percent error for the transducer.

The error of the power input, Q, to the TFR heater was found based on the specified error in the input voltage and current data. For the spacing comparison and Mehra electrode study, these values were read directly from the LED display. For the cap electrode studies, the voltage was read from the heater power supply by the data acquisition system and the reading error was given by the manufacturer. The current was calculated by dividing the voltage difference across a precision resistor by the resistance. The current error was found from the voltage error given and the accuracy of the precision resistor. The corresponding error in the heat flux, $\mathrm{q}$, was found from the heater power and the estimated TFR surface area (not including the silver conductive epoxy). A manufacturer's specification of $1.2573 \pm 0.0051 \mathrm{~cm}$ was given for the width of both types of TFR heaters, and the lengths of the TFR used for the spacing and cap electrode studies (not including the silver conductive epoxy) were estimated to be $1.175 \pm .015 \mathrm{~cm}$ based 
on measurements using a scale. This gives a TFR surface area of $1.47 \pm 0.025 \mathrm{~cm}^{2}$. The area of the TFR for the Mehra electrode study was found to be $0.982 \pm 0.025 \mathrm{~cm}^{2}$.

For the spacing comparison, the interface temperature, $\mathrm{T}_{\text {int }}$, the pedestal wall temperature, $\mathrm{T}_{\text {cowall, }}$ and the pedestal top temperature, $\mathrm{T}_{\infty \text { otop }}$, were simply based on thermocouple readings. The manufacturer specified that the thermocouples used had an accuracy of $1.0^{\circ} \mathrm{C}$, but after calibrating them for the spacing comparison using precision mercury thermometers with a reading error of $\pm 0.05{ }^{\circ} \mathrm{C}$ in a water bath, the accuracies were assumed to be $0.1{ }^{\circ} \mathrm{C}$. Different pedestals were used for the cap and Mehra electrode studies, and the thermocouples embedded within them were not calibrated. However, calibration data from the thermocouples used in the spacing comparison and others used by AFRL showed that these thermocouples were all accurate to at least \pm 0.5 ${ }^{\circ} \mathrm{C}$ and therefore the uncalibrated thermocouples in the pedestals used for the electrode studies were assumed to be accurate to $\pm 0.5^{\circ} \mathrm{C}$ as well. The data acquisition system error of $\pm 0.11{ }^{\circ} \mathrm{C}$ for the thermocouples was added to this to give the overall thermocouple error of $\pm 0.61^{\circ} \mathrm{C}$.

The temperature differences across the TFR glass (for the spacing comparison only), conductive heater, and substrate layers were found based on the heat fraction lost down the pedestal, heat flux, thickness of each of these layers, and thermal conductivity of each layer. The heat lost down the pedestal was assumed to be $0.015 \pm 0.01$ from values obtained by Baysinger (2005). The error for the thickness of each layer was estimated from scanning electron microscope images. (See Appendix A for more discussion.) The thermal conductivities of each layer were assumed constant with no 
error. The surface temperature, $\mathrm{T}_{\mathrm{s}}$, error was found by adding the temperature errors of each layer and the error of the interface temperature, $\mathrm{T}_{\mathrm{int}}$.

The thermal conductivity of the FC-72 fluid, $\mathrm{k}_{\text {fluid, }}$ is based on information from a program called f-chart that gave a plot of fluid temperature versus thermal conductivity. The thermal conductivity of the HFE-7000 fluid is based on manufacturer's information. Therefore, the errors of the thermal conductivities of the two fluids were found from the thermocouple error.

The convection heat transfer coefficient was found from the heat fraction lost down the pedestal, the heat flux, and the difference in surface temperature and pedestal top temperature. The fraction of heat lost down the pedestal was again assumed to be $0.015 \pm 0.01$

For the Nusselt number and G $\Delta$ error calculations, the pedestal radius, $\mathrm{b}$, was assumed constant with no error.

\section{Spacing Comparison Error Analysis}

Table E2 and Table E3 show the errors calculated for the confined case at a nozzle-to-heater distance of $9 \mathrm{~mm}$ in units and in percentages, respectively. Table E4 and Table E5 show the errors calculated for the unconfined case also at a nozzle-to-heater distance of $9 \mathrm{~mm}$ in units and in percentages, respectively. Errors for the other spacings for both cases were assumed to be very similar and were therefore not calculated individually. 
Table E2: Error Values for Confined $9 \mathrm{~mm}$ Spacing with Respective Units

\begin{tabular}{|c|c|c|c|c|c|c|c|c|c|c|c|c|c|c|}
\hline Sample & $\mathbf{T}_{\text {sat }}$ & $\mathbf{Q}$ & $q$ & $\mathbf{T}_{\mathrm{s}}$ & $\mathbf{T}_{\infty \text { wall }}$ & $T_{\text {otop }}$ & $\mathbf{T}_{\mathbf{s}}-\mathbf{T}_{\text {cotop }}$ & $\mathbf{T}_{\text {sat }}-\mathbf{T}_{\text {owall }}$ & $T_{\text {sat }}-T_{\text {otop }}$ & $\mathbf{k}_{\text {fluid }}$ & $\mathbf{h}$ & $\theta_{\mathrm{s}}-\theta_{\text {otop }}$ & $\mathbf{N u}$ & GD \\
\hline \# & ${ }^{\circ} \mathrm{C}$ & $\mathbf{W}$ & $\begin{array}{c}10^{4} \\
W / m^{2}\end{array}$ & ${ }^{\circ} \mathrm{C}$ & ${ }^{\circ} \mathrm{C}$ & ${ }^{\circ} \mathrm{C}$ & ${ }^{\circ} \mathrm{C}$ & ${ }^{\circ} \mathrm{C}$ & ${ }^{\circ} \mathrm{C}$ & $\mathbf{W} / \mathbf{m}-\mathbf{K}$ & $W / m^{2}-K$ & -- & --- & --- \\
\hline 1 & 0.31 & 0.00 & 0.00 & 0.10 & 0.10 & 0.10 & 0.20 & 0.41 & 0.41 & $1.2 \mathrm{E}-05$ & 0.00 & 0.01 & 0.00 & 0.00 \\
\hline 2 & 0.31 & 0.00 & 0.00 & 0.10 & 0.10 & 0.10 & 0.20 & 0.41 & 0.41 & $1.2 \mathrm{E}-05$ & 0.00 & 0.01 & 0.00 & 0.00 \\
\hline 3 & 0.31 & 0.12 & 0.20 & 0.15 & 0.10 & 0.10 & 0.25 & 0.41 & 0.41 & $1.2 \mathrm{E}-05$ & 320.75 & 0.01 & 46.64 & 0.52 \\
\hline 4 & 0.31 & 0.24 & 0.40 & 0.21 & 0.10 & 0.10 & 0.31 & 0.41 & 0.41 & $1.2 \mathrm{E}-05$ & 251.28 & 0.02 & 36.63 & 1.08 \\
\hline 5 & 0.31 & 0.36 & 0.59 & 0.26 & 0.10 & 0.10 & 0.36 & 0.41 & 0.41 & $1.2 \mathrm{E}-05$ & 228.79 & 0.03 & 33.41 & 1.68 \\
\hline 6 & 0.31 & 0.48 & 0.79 & 0.32 & 0.10 & 0.10 & 0.42 & 0.41 & 0.41 & $1.2 \mathrm{E}-05$ & 226.61 & 0.04 & 33.14 & 2.36 \\
\hline 7 & 0.31 & 0.61 & 0.99 & 0.37 & 0.10 & 0.10 & 0.47 & 0.41 & 0.41 & $1.2 \mathrm{E}-05$ & 232.81 & 0.04 & 34.09 & 3.06 \\
\hline 8 & 0.31 & 0.72 & 1.19 & 0.43 & 0.10 & 0.10 & 0.53 & 0.41 & 0.41 & $1.2 \mathrm{E}-05$ & 249.12 & 0.05 & 36.52 & 3.86 \\
\hline 9 & 0.31 & 0.85 & 1.39 & 0.48 & 0.10 & 0.10 & 0.58 & 0.41 & 0.41 & $1.2 \mathrm{E}-05$ & 272.37 & 0.06 & 39.98 & 4.75 \\
\hline
\end{tabular}

Table E3: Error Values for Confined $9 \mathrm{~mm}$ Spacing in Percentages

\begin{tabular}{|c|c|c|c|c|c|c|c|c|c|c|c|c|c|c|}
\hline Sample & $\mathbf{T}_{\text {sat }}$ & $\mathbf{Q}$ & $\mathbf{q}$ & $\mathbf{T}_{\mathrm{s}}$ & $\mathbf{T}_{\text {owall }}$ & $\mathbf{T}_{\text {ootop }}$ & $\mathbf{T}_{\mathbf{s}}-\mathbf{T}_{\text {cotop }}$ & $\mathbf{T}_{\text {sat }}-\mathbf{T}_{\text {owall }}$ & $\mathbf{T}_{\text {sat }}-\mathbf{T}_{\text {cotop }}$ & $\mathbf{k}_{\text {fluid }}$ & $\mathbf{h}$ & $\theta_{\mathrm{s}}-\theta_{\text {ootop }}$ & $\mathbf{N u}$ & GD \\
\hline \# & $\%$ & $\%$ & $\%$ & $\%$ & $\%$ & $\%$ & $\%$ & $\%$ & $\%$ & $\%$ & $\%$ & $\%$ & $\%$ & $\%$ \\
\hline 1 & 0.59 & --- & --- & 0.33 & 0.34 & 0.34 & --- & 1.71 & 1.71 & 0.02 & --- & --- & --- & --- \\
\hline 2 & 0.55 & --- & --- & 0.33 & 0.34 & 0.34 & --- & 1.50 & 1.50 & 0.02 & --- & --- & --- & --- \\
\hline 3 & 0.55 & 1.22 & 2.93 & 0.41 & 0.33 & 0.34 & 3.01 & 1.53 & 1.51 & 0.02 & 4.03 & 4.54 & 4.06 & 3.77 \\
\hline 4 & 0.55 & 1.22 & 2.92 & 0.46 & 0.33 & 0.33 & 1.95 & 1.56 & 1.52 & 0.02 & 2.97 & 3.51 & 2.99 & 3.79 \\
\hline 5 & 0.55 & 1.21 & 2.92 & 0.50 & 0.32 & 0.33 & 1.59 & 1.61 & 1.54 & 0.02 & 2.61 & 3.21 & 2.63 & 3.84 \\
\hline 6 & 0.55 & 1.21 & 2.91 & 0.53 & 0.31 & 0.33 & 1.44 & 1.67 & 1.56 & 0.02 & 2.46 & 3.11 & 2.48 & 3.90 \\
\hline 7 & 0.55 & 1.22 & 2.92 & 0.57 & 0.30 & 0.32 & 1.38 & 1.71 & 1.57 & 0.02 & 2.39 & 3.09 & 2.41 & 3.95 \\
\hline 8 & 0.54 & 1.21 & 2.91 & 0.61 & 0.29 & 0.31 & 1.37 & 1.78 & 1.59 & 0.02 & 2.39 & 3.15 & 2.41 & 4.00 \\
\hline 9 & 0.54 & 1.21 & 2.91 & 0.65 & 0.28 & 0.31 & 1.40 & 1.84 & 1.60 & 0.02 & 2.42 & 3.24 & 2.44 & 4.06 \\
\hline
\end{tabular}


Table E4: Error Values for Unconfined $9 \mathrm{~mm}$ Spacing with Respective Units

\begin{tabular}{|c|c|c|c|c|c|c|c|c|c|c|c|c|c|c|}
\hline Sample & $\mathbf{T}_{\text {sat }}$ & $\mathbf{Q}$ & $q$ & $\mathbf{T}_{\mathrm{s}}$ & $\mathbf{T}_{\infty \text { wall }}$ & $T_{\text {otop }}$ & $T_{s}-T_{\text {otop }}$ & $\mathbf{T}_{\text {sat }}-\mathbf{T}_{\text {owall }}$ & $T_{\text {sat }}-T_{\text {otop }}$ & $\mathbf{k}_{\text {fluid }}$ & $\mathbf{h}$ & $\theta_{\mathrm{s}}-\theta_{\text {otop }}$ & $\mathbf{N u}$ & GA \\
\hline \# & ${ }^{\circ} \mathrm{C}$ & $\mathbf{W}$ & $\begin{array}{c}10^{4} \\
W / m^{2}\end{array}$ & ${ }^{\circ} \mathrm{C}$ & ${ }^{\circ} \mathrm{C}$ & ${ }^{\circ} \mathrm{C}$ & ${ }^{\circ} \mathrm{C}$ & ${ }^{\circ} \mathrm{C}$ & ${ }^{\circ} \mathrm{C}$ & $\mathbf{W} / \mathbf{m}-\mathbf{K}$ & $\mathbf{W} / \mathbf{m}^{2}-K$ & -- & --- & --- \\
\hline 1 & 0.31 & 0.00 & 0.00 & 0.10 & 0.10 & 0.10 & 0.20 & 0.41 & 0.41 & $1.2 \mathrm{E}-05$ & 0.00 & 0.01 & 0.00 & 0.00 \\
\hline 2 & 0.31 & 0.00 & 0.00 & 0.10 & 0.10 & 0.10 & 0.20 & 0.41 & 0.41 & $1.2 \mathrm{E}-05$ & 0.00 & 0.01 & 0.00 & 0.00 \\
\hline 3 & 0.31 & 0.12 & 0.20 & 0.15 & 0.10 & 0.10 & 0.25 & 0.41 & 0.41 & $1.2 \mathrm{E}-05$ & 319.70 & 0.01 & 46.38 & 0.48 \\
\hline 4 & 0.31 & 0.24 & 0.40 & 0.21 & 0.10 & 0.10 & 0.31 & 0.41 & 0.41 & $1.2 \mathrm{E}-05$ & 248.94 & 0.02 & 36.20 & 0.97 \\
\hline 5 & 0.31 & 0.36 & 0.59 & 0.26 & 0.10 & 0.10 & 0.36 & 0.41 & 0.41 & $1.2 \mathrm{E}-05$ & 227.37 & 0.03 & 33.12 & 1.50 \\
\hline 6 & 0.31 & 0.48 & 0.79 & 0.32 & 0.10 & 0.10 & 0.42 & 0.41 & 0.41 & $1.2 \mathrm{E}-05$ & 223.67 & 0.03 & 32.63 & 2.05 \\
\hline 7 & 0.31 & 0.61 & 0.99 & 0.37 & 0.10 & 0.10 & 0.47 & 0.41 & 0.41 & $1.2 \mathrm{E}-05$ & 227.45 & 0.04 & 33.22 & 2.64 \\
\hline 8 & 0.31 & 0.72 & 1.19 & 0.43 & 0.10 & 0.10 & 0.53 & 0.41 & 0.41 & $1.2 \mathrm{E}-05$ & 240.79 & 0.04 & 35.21 & 3.31 \\
\hline 9 & 0.31 & 0.85 & 1.39 & 0.48 & 0.10 & 0.10 & 0.58 & 0.41 & 0.41 & $1.2 \mathrm{E}-05$ & 261.63 & 0.05 & 38.31 & 3.98 \\
\hline
\end{tabular}

Table E5: Error Values for Unconfined $9 \mathrm{~mm}$ Spacing in Percentages

\begin{tabular}{|c|c|c|c|c|c|c|c|c|c|c|c|c|c|c|}
\hline Sample & $\mathbf{T}_{\text {sat }}$ & $\mathbf{Q}$ & $\mathbf{q}$ & $\mathbf{T}_{\mathrm{s}}$ & $\mathbf{T}_{\text {owall }}$ & $\mathbf{T}_{\text {otop }}$ & $T_{s}-T_{\text {otop }}$ & $\mathbf{T}_{\text {sat }}-\mathbf{T}_{\text {owall }}$ & $\mathbf{T}_{\text {sat }}-\mathbf{T}_{\text {cotop }}$ & $\mathbf{k}_{\text {fluid }}$ & h & $\theta_{\mathrm{s}}-\theta_{\infty \mathrm{top}}$ & $\mathbf{N u}$ & G $\Delta$ \\
\hline$\#$ & $\%$ & $\%$ & $\%$ & $\%$ & $\%$ & $\%$ & $\%$ & $\%$ & $\%$ & $\%$ & $\%$ & $\%$ & $\%$ & $\%$ \\
\hline 1 & 0.54 & --- & --- & 0.34 & 0.35 & 0.35 & 24.87 & 1.40 & 1.39 & 0.02 & --- & 26.27 & --- & --- \\
\hline 2 & 0.54 & --- & --- & 0.34 & 0.35 & 0.35 & 26.51 & 1.40 & 1.39 & 0.02 & --- & 27.91 & --- & --- \\
\hline 3 & 0.54 & 1.22 & 2.93 & 0.42 & 0.35 & 0.35 & 3.01 & 1.42 & 1.41 & 0.02 & 4.03 & 4.43 & 4.05 & 3.67 \\
\hline 4 & 0.54 & 1.22 & 2.92 & 0.47 & 0.34 & 0.35 & 1.94 & 1.45 & 1.41 & 0.02 & 2.96 & 3.39 & 2.98 & 3.68 \\
\hline 5 & 0.54 & 1.21 & 2.92 & 0.51 & 0.33 & 0.34 & 1.59 & 1.48 & 1.42 & 0.02 & 2.60 & 3.07 & 2.62 & 3.71 \\
\hline 6 & 0.54 & 1.21 & 2.91 & 0.54 & 0.32 & 0.34 & 1.43 & 1.51 & 1.42 & 0.02 & 2.45 & 2.94 & 2.47 & 3.74 \\
\hline 7 & 0.53 & 1.21 & 2.91 & 0.58 & 0.31 & 0.33 & 1.35 & 1.54 & 1.43 & 0.02 & 2.37 & 2.90 & 2.39 & 3.77 \\
\hline 8 & 0.53 & 1.21 & 2.91 & 0.61 & 0.30 & 0.33 & 1.34 & 1.59 & 1.45 & 0.02 & 2.36 & 2.94 & 2.38 & 3.82 \\
\hline 9 & 0.53 & 1.21 & 2.91 & 0.65 & 0.29 & 0.32 & 1.36 & 1.62 & 1.46 & 0.02 & 2.38 & 2.99 & 2.40 & 3.85 \\
\hline
\end{tabular}




\section{Cap Electrode Error Analysis}

Table E6 and Table E7 show the errors calculated for the top half electrode in units and in percentages, respectively. Errors for the other cap electrode geometries were assumed to be very similar and were therefore not calculated individually. The error analysis for this study is slightly different than for the spacing comparison. First, the thermocouples on the PTFE pedestal used were not calibrated. Second, the data acquisition system was installed and the heater power setting was read by the computer. Also the chamber pressure was read by the data acquisition system from the output of the chamber pressure transducer. 
Table E6: Error Values for Top Half Cap Electrode with Respective Units at $0 \mathrm{kV}$

\begin{tabular}{|c|c|c|c|c|c|c|c|c|c|c|c|c|c|c|c|}
\hline HV & $\mathbf{T}_{\text {sat }}$ & I & $\mathbf{Q}$ & $\mathbf{q}$ & $\mathbf{T}_{\mathrm{s}}$ & $\mathbf{T}_{\text {cowall }}$ & $\mathbf{T}_{\text {otop }}$ & $\mathbf{T}_{\mathbf{s}}-\mathbf{T}_{\infty \mathrm{top}}$ & $\mathbf{T}_{\text {sat }}-\mathbf{T}_{\text {owall }}$ & $\mathbf{T}_{\text {sat }}-\mathbf{T}_{\text {cotop }}$ & $\mathbf{k}_{\text {fluid }}$ & h & $\theta_{\mathrm{s}}-\theta_{\text {otop }}$ & $\mathrm{Nu}$ & Gu \\
\hline $\mathbf{k V}$ & ${ }^{\circ} \mathrm{C}$ & $\mathbf{A}$ & $\mathbf{W}$ & $\begin{array}{c}10^{4} \\
W / m^{2} \\
\end{array}$ & ${ }^{\circ} \mathrm{C}$ & ${ }^{\circ} \mathrm{C}$ & ${ }^{\circ} \mathrm{C}$ & ${ }^{\circ} \mathrm{C}$ & ${ }^{\circ} \mathrm{C}$ & ${ }^{\circ} \mathrm{C}$ & $\mathbf{W} / \mathbf{m}-\mathbf{K}$ & $W / \mathbf{m}^{2}-K$ & -- & --- & --- \\
\hline 0 & 1.05 & 0.026 & 0.00 & 0.00 & 0.61 & 0.61 & 0.61 & 1.22 & 1.66 & 1.66 & 7.1E-05 & 0.00 & 0.04 & 0.00 & 0.00 \\
\hline 0 & 1.05 & 0.026 & 0.25 & 0.29 & 0.64 & 0.61 & 0.61 & 1.25 & 1.66 & 1.66 & 7.1E-05 & 1485.51 & 0.05 & 213.05 & 1.05 \\
\hline 0 & 1.05 & 0.026 & 0.36 & 0.48 & 0.68 & 0.61 & 0.61 & 1.29 & 1.66 & 1.66 & 7.1E-05 & 894.43 & 0.07 & 129.11 & 2.01 \\
\hline 0 & 1.05 & 0.026 & 0.44 & 0.65 & 0.71 & 0.61 & 0.61 & 1.32 & 1.66 & 1.66 & 7.1E-05 & 672.71 & 0.08 & 97.66 & 3.04 \\
\hline 0 & 1.05 & 0.026 & 0.51 & 0.81 & 0.75 & 0.61 & 0.61 & 1.36 & 1.66 & 1.66 & 7.1E-05 & 590.38 & 0.10 & 86.10 & 4.17 \\
\hline $\mathbf{0}$ & 1.05 & 0.026 & 0.57 & 0.97 & 0.78 & 0.61 & 0.61 & 1.39 & 1.66 & 1.66 & 7.1E-05 & 576.01 & 0.12 & 84.29 & 5.41 \\
\hline 0 & 1.05 & 0.027 & 0.62 & 1.12 & 0.82 & 0.61 & 0.61 & 1.43 & 1.66 & 1.66 & 7.1E-05 & 628.21 & 0.13 & 92.11 & 6.82 \\
\hline 0 & 1.05 & 0.027 & 0.67 & 1.27 & 0.85 & 0.61 & 0.61 & 1.46 & 1.66 & 1.66 & 7.1E-05 & 695.51 & 0.14 & 102.14 & 8.37 \\
\hline
\end{tabular}

Table E7: Error Values for Top Half Cap Electrode in Percentages at $0 \mathrm{kV}$

\begin{tabular}{|c|c|c|c|c|c|c|c|c|c|c|c|c|c|c|c|}
\hline HV & $\mathbf{T}_{\text {sat }}$ & I & $\mathbf{Q}$ & $\mathbf{q}$ & $\mathbf{T}_{\mathrm{s}}$ & $\mathbf{T}_{\infty \text { wall }}$ & $\mathbf{T}_{\infty \mathrm{top}}$ & $\mathbf{T}_{\mathrm{s}}-\mathbf{T}_{\infty \mathrm{top}}$ & $\mathbf{T}_{\text {sat }}-\mathbf{T}_{\text {cwall }}$ & $\mathbf{T}_{\text {sat }}-\mathbf{T}_{\text {otop }}$ & $\mathbf{k}_{\text {fluid }}$ & h & $\theta_{\mathrm{s}}-\theta_{\infty \mathrm{top}}$ & $\mathrm{Nu}$ & GA \\
\hline $\mathbf{k V}$ & $\%$ & $\%$ & $\%$ & $\%$ & $\%$ & $\%$ & $\%$ & $\%$ & $\%$ & $\%$ & $\%$ & $\%$ & $\%$ & $\%$ & $\%$ \\
\hline 0 & 1.92 & --- & --- & --- & 2.72 & 2.76 & 2.82 & --- & 5.09 & 5.01 & 0.13 & --- & --- & --- & --- \\
\hline 0 & 1.91 & 2.53 & 2.53 & 4.23 & 2.15 & 2.64 & 2.76 & 15.91 & 5.23 & 5.07 & 0.13 & 17.36 & 21.19 & 17.49 & 8.80 \\
\hline 0 & 1.91 & 1.80 & 1.80 & 3.50 & 1.82 & 2.54 & 2.71 & 8.73 & 5.37 & 5.12 & 0.13 & 9.82 & 14.13 & 9.94 & 8.20 \\
\hline 0 & 1.90 & 1.47 & 1.47 & 3.17 & 1.60 & 2.43 & 2.65 & 6.14 & 5.53 & 5.17 & 0.13 & 7.18 & 11.71 & 7.31 & 8.04 \\
\hline $\mathbf{0}$ & 1.90 & 1.27 & 1.27 & 2.97 & 1.47 & 2.32 & 2.59 & 4.97 & 5.71 & 5.23 & 0.13 & 6.00 & 10.71 & 6.13 & 8.02 \\
\hline $\mathbf{0}$ & 1.89 & 1.14 & 1.14 & 2.84 & 1.40 & 2.23 & 2.53 & 4.40 & 5.90 & 5.28 & 0.13 & 5.43 & 10.34 & 5.56 & 8.08 \\
\hline $\mathbf{0}$ & 1.88 & 1.04 & 1.04 & 2.74 & 1.40 & 2.14 & 2.47 & 4.23 & 6.10 & 5.34 & 0.13 & 5.26 & 10.38 & 5.39 & 8.19 \\
\hline 0 & 1.88 & 0.96 & 0.96 & 2.66 & 1.41 & 2.05 & 2.41 & 4.17 & 6.32 & 5.41 & 0.13 & 5.19 & 10.53 & 5.32 & 8.33 \\
\hline
\end{tabular}




\section{Mehra Electrode Error Analysis}

Table E8 and Table E9 show the errors calculated for the Mehra electrodes in units and in percentages, respectively. The error analysis for this study is very similar to the cap electrode comparison. However, the heater power error was calculated differently. Although the data acquisition system was installed, the output reading of the heater power was not working correctly as the data was being taken and was therefore read from the LED display as in the spacing comparison tests. 
Table E8: Error Values for Mehra Electrode with Respective Units at $23 \mathrm{kV}$

\begin{tabular}{|c|c|c|c|c|c|c|c|c|c|c|c|c|c|c|c|}
\hline HV & $\mathbf{T}_{\text {sat }}$ & I & $\mathbf{Q}$ & $\mathbf{q}$ & $\mathbf{T}_{\mathrm{s}}$ & $\mathbf{T}_{\text {owall }}$ & $\mathbf{T}_{\text {otop }}$ & $T_{s}-T_{\text {otop }}$ & $\mathbf{T}_{\text {sat }}-\mathbf{T}_{\text {owall }}$ & $\mathbf{T}_{\text {sat }}-\mathbf{T}_{\text {ootop }}$ & $\mathbf{k}_{\text {fluid }}$ & h & $\theta_{\mathrm{s}}-\theta_{\infty \mathrm{otop}}$ & $\mathrm{Nu}$ & G $\Delta$ \\
\hline $\mathbf{k V}$ & ${ }^{\circ} \mathrm{C}$ & $\mathbf{A}$ & $\mathbf{W}$ & $\begin{array}{c}10^{4} \\
W / m^{2} \\
\end{array}$ & ${ }^{\circ} \mathrm{C}$ & ${ }^{\circ} \mathrm{C}$ & ${ }^{\circ} \mathrm{C}$ & ${ }^{\circ} \mathrm{C}$ & ${ }^{\circ} \mathrm{C}$ & ${ }^{\circ} \mathrm{C}$ & $\mathbf{W} / \mathbf{m}-\mathbf{K}$ & $W / m^{2}-K$ & --- & -- & -- \\
\hline 23 & 0.93 & 0.030 & 0.00 & 0.00 & 0.61 & 0.61 & 0.61 & 1.22 & 1.54 & 1.54 & $1.20 \mathrm{E}-04$ & 0.00 & 0.08 & 0.00 & 0.00 \\
\hline 23 & 0.93 & 0.030 & 0.09 & 0.12 & 0.62 & 0.61 & 0.61 & 1.23 & 1.54 & 1.54 & $1.20 \mathrm{E}-04$ & 1931.11 & 0.11 & 205.31 & 0.56 \\
\hline 23 & 0.93 & 0.030 & 0.13 & 0.18 & 0.62 & 0.61 & 0.61 & 1.23 & 1.54 & 1.54 & $1.20 \mathrm{E}-04$ & 751.93 & 0.14 & 80.36 & 1.07 \\
\hline 23 & 0.93 & 0.030 & 0.16 & 0.24 & 0.63 & 0.61 & 0.61 & 1.24 & 1.54 & 1.54 & $1.20 \mathrm{E}-04$ & 494.23 & 0.17 & 52.99 & 1.48 \\
\hline 23 & 0.93 & 0.030 & 0.18 & 0.29 & 0.64 & 0.61 & 0.61 & 1.25 & 1.54 & 1.54 & $1.20 \mathrm{E}-04$ & 373.53 & 0.20 & 40.19 & 1.93 \\
\hline 23 & 0.93 & 0.030 & 0.20 & 0.33 & 0.64 & 0.61 & 0.61 & 1.25 & 1.54 & 1.54 & $1.20 \mathrm{E}-04$ & 301.52 & 0.23 & 32.55 & 2.37 \\
\hline 23 & 0.93 & 0.030 & 0.22 & 0.38 & 0.65 & 0.61 & 0.61 & 1.26 & 1.54 & 1.54 & $1.20 \mathrm{E}-04$ & 246.43 & 0.27 & 26.71 & 2.91 \\
\hline 23 & 0.93 & 0.030 & 0.24 & 0.43 & 0.66 & 0.61 & 0.61 & 1.27 & 1.54 & 1.54 & $1.20 \mathrm{E}-04$ & 206.57 & 0.31 & 22.47 & 3.44 \\
\hline 23 & 0.93 & 0.030 & 0.26 & 0.47 & 0.66 & 0.61 & 0.61 & 1.27 & 1.54 & 1.54 & $1.20 \mathrm{E}-04$ & 191.14 & 0.36 & 20.86 & 4.02 \\
\hline 23 & 0.93 & 0.030 & 0.28 & 0.52 & 0.67 & 0.61 & 0.61 & 1.28 & 1.54 & 1.54 & $1.20 \mathrm{E}-04$ & 173.61 & 0.39 & 18.99 & 4.57 \\
\hline 23 & 0.93 & 0.030 & 0.30 & 0.57 & 0.68 & 0.61 & 0.61 & 1.29 & 1.54 & 1.54 & $1.20 \mathrm{E}-04$ & 163.37 & 0.44 & 17.91 & 5.22 \\
\hline 23 & 0.94 & 0.03 & 0.32 & 0.61 & 0.68 & 0.61 & 0.61 & 1.29 & 1.55 & 1.55 & $1.20 \mathrm{E}-04$ & 157.97 & 0.42 & 17.30 & 5.10 \\
\hline 23 & 0.92 & 0.030 & 0.34 & 0.66 & 0.69 & 0.61 & 0.61 & 1.30 & 1.53 & 1.53 & $1.20 \mathrm{E}-04$ & 149.76 & 0.44 & 16.47 & 5.59 \\
\hline
\end{tabular}


Table E9: Error Values for Mehra Electrode in Percentages at $23 \mathrm{kV}$

\begin{tabular}{|c|c|c|c|c|c|c|c|c|c|c|c|c|c|c|c|}
\hline HV & $\mathbf{T}_{\text {sat }}$ & $I$ & $\mathbf{Q}$ & $q$ & $\mathbf{T}_{\mathrm{s}}$ & $\mathbf{T}_{\text {owall }}$ & $\mathbf{T}_{\text {otop }}$ & $\mathbf{T}_{\mathrm{s}}-\mathbf{T}_{\text {otop }}$ & $\mathbf{T}_{\text {sat }}-T_{\infty \text { wall }}$ & $\mathbf{T}_{\text {sat }}-\mathbf{T}_{\text {otop }}$ & $\mathbf{k}_{\text {fluid }}$ & $\mathbf{h}$ & $\theta_{\mathrm{s}}-\theta_{\text {ctop }}$ & $\mathbf{N u}$ & GD \\
\hline $\mathbf{k V}$ & $\%$ & $\%$ & $\%$ & $\%$ & $\%$ & $\%$ & $\%$ & $\%$ & $\%$ & $\%$ & $\%$ & $\%$ & $\%$ & $\%$ & $\%$ \\
\hline 23 & 2.54 & --- & --- & --- & 2.79 & 2.75 & 2.76 & $\begin{array}{l}-- \\
--\end{array}$ & 10.70 & 10.65 & 0.16 & --- & --- & --- & $\begin{array}{l}-- \\
--\end{array}$ \\
\hline 23 & 2.55 & 9.09 & 9.18 & 11.73 & 2.47 & 2.74 & 2.76 & 43.57 & 10.87 & 10.76 & 0.16 & 55.04 & 55.09 & 55.20 & 21.32 \\
\hline 23 & 2.56 & 6.25 & 6.31 & 8.86 & 2.18 & 2.69 & 2.71 & 20.30 & 11.30 & 11.11 & 0.16 & 22.23 & 32.01 & 22.39 & 18.87 \\
\hline 23 & 2.56 & 5.17 & 5.22 & 7.77 & 2.00 & 2.68 & 2.72 & 13.65 & 11.33 & 11.08 & 0.16 & 14.95 & 25.30 & 15.11 & 17.80 \\
\hline 23 & 2.57 & 4.48 & 4.52 & 7.07 & 1.83 & 2.66 & 2.71 & 10.24 & 11.49 & 11.19 & 0.16 & 11.38 & 22.03 & 11.54 & 17.26 \\
\hline 23 & 2.57 & 4.05 & 4.09 & 6.64 & 1.70 & 2.65 & 2.70 & 8.23 & 11.62 & 11.26 & 0.16 & 9.31 & 20.13 & 9.47 & 16.96 \\
\hline 23 & 2.55 & 3.66 & 3.69 & 6.24 & 1.56 & 2.61 & 2.67 & 6.65 & 11.78 & 11.34 & 0.16 & 7.70 & 18.69 & 7.86 & 16.72 \\
\hline 23 & 2.55 & 3.43 & 3.46 & 6.01 & 1.44 & 2.59 & 2.65 & 5.61 & 12.00 & 11.49 & 0.16 & 6.64 & 17.86 & 6.80 & 16.72 \\
\hline 23 & 2.54 & 3.26 & 3.29 & 5.84 & 1.36 & 2.55 & 2.62 & 5.04 & 12.29 & 11.69 & 0.16 & 6.07 & 17.60 & 6.23 & 16.86 \\
\hline 23 & 2.56 & 3.13 & 3.15 & 5.70 & 1.29 & 2.54 & 2.62 & 4.49 & 12.43 & 11.75 & 0.16 & 5.52 & 17.19 & 5.68 & 16.86 \\
\hline 23 & 2.58 & 3.00 & 3.03 & 5.58 & 1.24 & 2.54 & 2.63 & 4.09 & 12.63 & 11.86 & 0.16 & 5.12 & 16.99 & 5.28 & 16.94 \\
\hline 23 & 2.59 & 2.93 & 2.95 & 5.50 & 1.22 & 2.64 & 2.74 & 3.84 & 11.85 & 11.15 & 0.16 & 4.86 & 16.01 & 5.02 & 16.14 \\
\hline 23 & 2.49 & 2.86 & 2.88 & 5.43 & 1.16 & 2.55 & 2.65 & 3.55 & 11.85 & 11.08 & 0.16 & 4.57 & 15.62 & 4.73 & 15.98 \\
\hline
\end{tabular}

